\title{
Kohlenstoffspeicherung als Teilziel der strategischen Waldbauplanung
}

erläutert an Reinbeständen verschiedener Baumarten in

Niedersachsen

\author{
Dissertation \\ zur Erlangung des Doktorgrades \\ der Fakultät für Forstwissenschaften und Waldökologie \\ der Georg-August-Universität Göttingen
}

\author{
vorgelegt von \\ René Wördehoff \\ geboren in Mühlhausen/Thüringen
}

Göttingen, 2016 
1. Gutachter: Herr Prof. Dr. Hermann Spellmann

2. Gutachter: Herr Prof. Dr. Christian Ammer

Tag der mündlichen Prüfung: 08.04.2016 
Für meine Familie, Freunde und Kollegen.

„Der Forstmann ... darf nie vergessen, dass es keine Regel gibt, die überall richtig ist, und dass Ausnahmen eintreten können, wo gerade das, was man im allgemeinen als Fehler ansieht, sich vollständig rechtfertigt. ..."

Wilhelm Leopold Pfeil (1783 - 1859) 



\section{Inhaltsverzeichnis}

1 Einleitung 1

1.1 Anlass der Arbeit . . . . . . . . . . . . . . . . . . . . 1

1.2 Ausgangssituation . . . . . . . . . . . . . . . . . 3

1.2.1 Betrachtete Kohlenstoffspeicher . . . . . . . . . . . . 5

1.2.2 Literaturstudie zur lebenden Baumbiomasse . . . . . . . . . . 6

1.2.3 Literaturstudie zur toten Baumbiomasse . . . . . . . . . . . . . . 10

1.2.4 Literaturstudie zur Bodenvegetation . . . . . . . . . . . . 11

1.2.5 Literaturstudie zu Mineralboden und Auflagehumus . . . . . . . . 11

1.2.6 Literaturstudie zu Holzprodukten . . . . . . . . . . . . . . . . . . 14

1.3 Strategische Waldbauplanung . . . . . . . . . . . . . . . . . . . 16

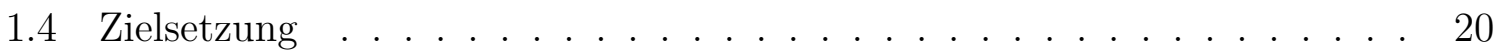

2 Material 22

2.1 Untersuchte Baumarten . . . . . . . . . . . . . . . . . . . . . 22

2.2 Ausgesuchte Standorte im niedersächsischen Landeswald . . . . . . . . . 24

2.2.1 Allgemeine Charakterisierung . . . . . . . . . . . . . . . 24

2.2 .2 Identifikation der wichtigsten Standorte . . . . . . . . . . . . 28

2.3 Weiserbestände zur Überprüfung von Waldbaustrategien . . . . . . . . . 31

2.3.1 Methodisches Vorgehen und Modellentwicklung . . . . . . . . . . 32

2.3.2 Ergebnisse der entwickelten Modelle zur Bestandesgenerierung . . 36

3 Methoden 42

3.1 Waldbaustrategien . . . . . . . . . . . . . . . . . . . . . . 42

3.2 Simulation der Waldentwicklung . . . . . . . . . . . . . . . . . 43

3.2.1 Prognosewerkzeug WaldPlaner . . . . . . . . . . . . . . . . . 43

3.2 .2 Waldbauliche Steuerung . . . . . . . . . . . . . . . 45

3.2.3 Herleitung der maximalen Bestandesgrundfläche . . . . . . . . . . 45

3.2.3.1 Methodisches Vorgehen und Modellentwicklung . . . . . 45

3.2.3.2 Ergebnisse der entwickelten Modelle zur Bestimmung der maximalen Bestandesgrundfläche . . . . . . . . . 56

3.2 .4 Sortierungsvorgaben . . . . . . . . . . . . . . . 59

3.3 Ermittlung der Kohlenstoffvorräte der betrachteten Speicher . . . . . . . 59

3.3.1 Lebende Baumbiomasse . . . . . . . . . . . . . . 59

3.3.2 Tote Baumbiomasse . . . . . . . . . . . . . . . 61 
3.3.3 Holzprodukte . . . . . . . . . . . . . . . . . . . 62

3.3.4 Ableitung der Speicherraten . . . . . . . . . . . . . 66

4 Kohlenstoffvorräte und Speicherraten der betrachteten Speicher $\quad 68$

4.1 Lebende Baumbiomasse . . . . . . . . . . . . . . . . . . 68

4.2 Tote Baumbiomasse . . . . . . . . . . . . . . . . . . 71

4.3 Holzprodukte . . . . . . . . . . . . . . . . . . . . . . . 73

4.4 Speicherraten der betrachteten Speicher . . . . . . . . . . . . . . . 77

5 Diskussion $\quad \mathbf{8 7}$

5.1 Material und Methoden . . . . . . . . . . . . . . . 87

5.2 Entwicklung der Kohlenstoffspeicher . . . . . . . . . . . . . . . . . . . . 89

6 Schlussfolgerungen $\quad 96$

6.1 Kohlenstoffspeicherung . . . . . . . . . . . . . . . . . . . 96

6.2 Forstbetriebliche Ebene . . . . . . . . . . . . . . . . . . . . . . . . . . . . . . 98

6.3 Forstpolitische Ebene . . . . . . . . . . . . . . . . . . . . . 100

$\begin{array}{ll}\text { Literaturverzeichnis } & 105\end{array}$

$\begin{array}{ll}\text { A Anhang } & 126\end{array}$

A.1 Leistungsklassen der Hauptwirtschaftsbaumarten auf ausgesuchten Standorten im niedersächsischen Landeswald . . . . . . . . . . . . . . . . . . . 126

A.2 Generierung von Weiserbeständen . . . . . . . . . . . . . . . . . 133

A.2.1 Angaben zu den Durchmesserverteilungen . . . . . . . . . . . 133

A.2.2 Angaben zu den Grundflächenmodellen . . . . . . . . . . . . . . . 141

A.3 Herleitung der maximalen Bestandesgrundfläche . . . . . . . . . . . . . . 146

A.4 Kennwerte der Weiserbestände . . . . . . . . . . . . . . . . . . . . 154

A.5 Entwicklung des Kohlenstoffvorrates der lebenden Biomasse . . . . . . . 177

A.6 Entwicklung des Kohlenstoffvorrates der toten Baumbiomasse . . . . . . 179

A.7 Kohlenstoffspeicherraten . . . . . . . . . . . . . . 180 


\section{Abbildungsverzeichnis}

1.1 Kohlenstoffspeicher im Wald . . . . . . . . . . . . . . . . . 6

1.2 Kohlenstoffvorrat der lebenden Baumbiomasse auf Grundlage von Ertragstafelangaben $($ Schober, 1995) . . . . . . . . . . . . . 9

2.1 Durchmesserverteilungen von Douglasienreinbeständen mit guter Bonität für verschiedene Alter . . . . . . . . . . . . . . . . . . 34

2.2 Effekt des Alters und der absoluten Mittelhöhenbonität auf die Schätzung der Bestandesgrundfläche von Douglasienreinbeständen . . . . . . . . . . . .

2.3 Geschätzte Bestandesgrundflächen von Douglasienreinbeständen über dem Bestandesalter bei unterschiedlichen Ertragsklassen und die geschätzte Durchmesserverteilung eines jungen Douglasienreinbestandes mit guter Bonität . .

2.4 Absolute Häufigkeitsverteilung der Durchmesser und Baumhöhen der Hauptwirtschaftsbaumarten in den Wuchsbezirken Ems-Hase-Hunte-Geest auf Geschiebelehm und Göttinger Wald auf Kalkgesteinen . . . . . . . . . . . . . .

3.1 Vergleich der Versuchsflächendaten mit dem Verlauf der Funktion zur Schätzung der maximalen Bestandesgrundfläche über der Spitzenhöhe nach dem Ansatz von Sterba $(1975,1981,1987)$ mit den Koeffizienten von Döbbeler (2004) und hier neu geschätzten für Reinbestände verschiedener Baumarten . . . . . . . . . . . . . . . . . . . .

3.2 Quantile-Quantile-Plots zur Validierung der Box-Cox-Cole-Green-Verteilung für die Schätzung der Bestandesgrundfläche der Hauptwirtschaftsbaumarten . . . . . . . . . . . . . . . . . . . 53

3.3 Vergleich der Versuchsflächendaten mit dem Funktionsverlauf des 0,95-Quantils der Box-Cox-Cole-Green-Verteilung zur Schätzung der maximalen Bestandesgrundfläche sowie der ausgleichenden Funktion über der Spitzenhöhe für Reinbestände der Hauptwirtschaftsbaumarten in Nordwestdeutschland . . .

3.4 Zeitliche Entwicklung des Holzdichteanteils des im Bestand verbleibenden Totholzes getrennt nach Baumarten . . . . . . . . . . . . . .

3.5 Aufteilung der Sortimente auf Produktklassen und das entwickelte Modell zur Kaskadennutzung von Holzprodukten . . . . . . . . . . . . . . . .

4.1 Entwicklung des Kohlenstoffvorrates der lebenden Baumbiomasse von Buchenreinbeständen bei naturnaher und kohlenstofforientierter Behandlung getrennt nach Wuchsbezirken . . . . . . . . . . . . . . . . 
4.2 Entwicklung des Kohlenstoffvorrates der toten Baumbiomasse in Reinbeständen aus Buche und Fichte in verschiedenen Wuchsbezirken bei unterschied-

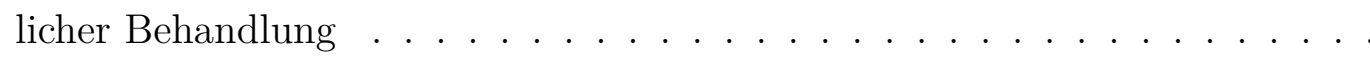

4.3 Im Verlauf der Simulationen anfallende Nutzungsmengen aus den Weiserbeständen, getrennt nach Laub- und Nadelholz sowie waldbaulicher Behandlung . . . . . . . . . . . . . . . . . . . .

4.4 Entwicklung des gesamten Kohlenstoffvorrates im Produktspeicher, basierend auf den anfallenden Nutzungsmengen der jeweils ein Hektar großen Weiserbestände, getrennt nach Produktklassen sowie Laub- und Nadelholz bei naturnaher und kohlenstofforientierter waldbaulicher Behandlung . . . .

4.5 Summarische Entwicklung der Substitutionswirkung des Produktspeichers, basierend auf den angefallenen, den Produktklassen zugeordneten Nutzungsmengen der jeweils ein Hektar großen Weiserbestände, getrennt nach Laubund Nadelholz bei naturnaher und kohlenstofforientierter waldbaulicher Behandlung . . . . . . . . . . . . . . . . . . .

4.6 Kohlenstoffspeicherrate der lebenden Baumbiomasse in Abhängigkeit von Wasser- und Nährstoffversorgung, getrennt nach Baumart, Behandlung und Standortstypenobergruppe in den ausgewählten Wuchsbezirken des niedersächsischen Berglandes . . . . . . . . . . . . . . . . . . . . .

4.7 Kohlenstoffspeicherrate der lebenden Baumbiomasse in Abhängigkeit von Wasser- und Nährstoffversorgung, getrennt nach Baumart, Behandlung und Standortstypenobergruppe in ausgewählten Wuchsbezirken des niedersächsischen Tieflandes . . . . . . . . . . . . . . . . . . . . .

4.8 Kohlenstoffspeicherraten der analysierten Speicher von zwei Buchenbeständen auf ausgewählten Standorten bei unterschiedlicher waldbaulicher Be-

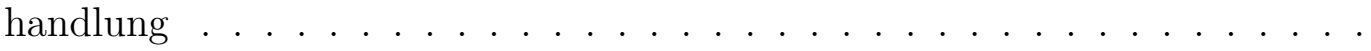

5.1 Relativer Anteil der baumartenspezifischen mittleren Kohlenstoffbindungsraten der lebenden Baumbiomasse in Bezug zur Fichte bei unterschiedlicher Wasserversorgung sowie mäßiger, ziemlich guter und sehr guter Nährstoffversorgung im niedersächsischen Bergland . . . . . . . . . . . . . . .

5.2 Relativer Anteil der baumartenspezifischen mittleren Kohlenstoffbindungsraten der lebenden Baumbiomasse in Bezug zur Kiefer bei unterschiedlicher Wasserversorgung sowie schwacher, mäßiger und ziemlich guter Nährstoffversorgung im niedersächsischen Tiefland . . . . . . . . . . . . .

A.1 Effekt des Alters und der absoluten Mittelhöhenbonität auf die Parameter der Box-Cox-Cole-Green-Verteilung zur Schätzung der Durchmesserverteilung von Eichenreinbeständen . . . . . . . . . . . . . . . . . .

A.2 Effekt des Alters und der absoluten Mittelhöhenbonität auf die Parameter der Box-Cox-Cole-Green-Verteilung zur Schätzung der Durchmesserverteilung von Buchenreinbeständen . . . . . . . . . . . . . . 137 
A.3 Effekt des Alters und der absoluten Mittelhöhenbonität auf die Parameter der Box-Cox-Cole-Green-Verteilung zur Schätzung der Durchmesserverteilung von Fichtenreinbeständen . . . . . . . . . . . . . . . . . . . . . 138

A.4 Effekt des Alters und der absoluten Mittelhöhenbonität auf die Parameter der Box-Cox-Cole-Green-Verteilung zur Schätzung der Durchmesserverteilung von Douglasienreinbeständen . . . . . . . . . . . . . . . . . 139

A.5 Effekt des Alters und der absoluten Mittelhöhenbonität auf die Parameter der Box-Cox-Cole-Green-Verteilung zur Schätzung der Durchmesserverteilung von Kiefernreinbeständen . . . . . . . . . . . . . . . . . . . . . . . . . 140

A.6 Effekt des Alters und der absoluten Mittelhöhenbonität auf die Schätzung der Bestandesgrundfläche von Eichenreinbeständen . . . . . . . . . . . . . . 141

A.7 Effekt des Alters und der absoluten Mittelhöhenbonität auf die Schätzung der Bestandesgrundfläche von Buchenreinbeständen . . . . . . . . . . . . . . 142

A.8 Effekt des Alters und der absoluten Mittelhöhenbonität auf die Schätzung der Bestandesgrundfläche von Fichtenreinbeständen . . . . . . . . . . . . . . 143

A.9 Effekt des Alters und der absoluten Mittelhöhenbonität auf die Schätzung der Bestandesgrundfläche von Kiefernreinbeständen . . . . . . . . . . . . . . 144

A.10 Geschätzte Bestandesgrundflächen von Reinbeständen über dem Bestandesalter bei unterschiedlichen Ertragsklassen . . . . . . . . . . . . . . . . . . . 145

A.11 Effekt der Spitzenhöhe auf Parameter der Box-Cox-Cole-Green-Verteilung zur Schätzung der maximalen Bestandesgrundfläche von Eichenreinbeständen . . . . . . . . . . . . . . . . . . 1 151

A.12 Effekt der Spitzenhöhe auf Parameter der Box-Cox-Cole-Green-Verteilung zur Schätzung der maximalen Bestandesgrundfläche von Buchenreinbeständen . . . . . . . . . . . . . . . . . . .

A.13 Effekt der Spitzenhöhe auf Parameter der Box-Cox-Cole-Green-Verteilung zur Schätzung der maximalen Bestandesgrundfläche von Fichtenreinbeständen . . . . . . . . . . . . . . . . . . . . . 152

A.14 Effekt der Spitzenhöhe auf Parameter der Box-Cox-Cole-Green-Verteilung zur Schätzung der maximalen Bestandesgrundfläche von Douglasienreinbeständen . . . . . . . . . . . . . . . . . . .

A.15 Effekt der Spitzenhöhe auf Parameter der Box-Cox-Cole-Green-Verteilung zur Schätzung der maximalen Bestandesgrundfläche von Kiefernreinbeständen . . . . . . . . . . . . . . . . . . . . 153

A.16 Entwicklung des Kohlenstoffvorrates der lebenden Baumbiomasse von Eichenund Fichtenreinbeständen bei naturnaher und kohlenstofforientierter Behandlung getrennt nach Wuchsbezirken . . . . . . . . . . . . . . . . 177

A.17 Entwicklung des Kohlenstoffvorrates der lebenden Baummasse von Douglasienund Kiefernreinbeständen bei naturnaher und kohlenstofforientierter Behandlung getrennt nach Wuchsbezirken . . . . . . . . . . . . . . . . 178 
A.18 Entwicklung des Kohlenstoffvorrates der toten Baumbiomasse in Reinbeständen aus Eiche, Douglasie und Kiefer, getrennt nach Wuchsbezirken und waldbaulicher Behandlung . . . . . . . . . . . . . . . . . . . . 179 


\section{Tabellenverzeichnis}

1.1 Vergleich der Kohlenstoffvorräte der lebenden Baumbiomasse der untersuchten Baumarten in Niedersachsen, Sachsen-Anhalt und Schleswig-Holstein . . 8

1.2 Baumartenspezifische Raumdichten und Biomasse-Expansionsfaktoren . . . 9

2.1 Flächengrößen der wichtigen Standortstypenobergruppen in ausgewählten niedersächsischen Wuchsbezirken . . . . . . . . . . . . . . 25

2.2 Mittlere Niederschlagssumme und Jahrestemperatur sowie klimatische, geographische und geologische Merkmale der ausgewählten Wuchsbezirke im niedersächsischen Tiefland . . . . . . . . . . . . . . . . . . 26

2.3 Mittlere Niederschlagssumme und Jahrestemperatur sowie klimatische, geographische und geologische Merkmale der ausgewählten Wuchsbezirke im niedersächsischen Bergland . . . . . . . . . . . . . . . .

2.4 Einteilung der Nährstoffziffern in Gruppen getrennt nach Berg- und Tiefland in Niedersachsen . . . . . . . . . . . . . . . . . . . . . . . . . . . . 29

2.5 Einteilung der grundwasserunbeeinflussten Standorte in Gruppen mit Hilfe der nutzbaren Feldkapazität im niedersächsischen Berg- und Tiefland in Anlehnung an Overbeck et al. (2011) . . . . . . . . . . . . . . . .

2.6 Mittlere Leistungsklassen der Hauptwirtschaftsbaumarten auf wichtigen Standorten getrennt nach Wasserhaushalt und Nährstoffversorgung im Wuchsbezirk Göttinger Wald . . . . . . . . . . . . . . . . . . . . . . . 29

2.7 Mittlere Leistungsklassen der Hauptwirtschaftsbaumarten auf wichtigen Standorten getrennt nach Wasserhaushalt und Nährstoffversorgung im Wuchsbezirk Ems-Hase-Hunte-Geest . . . . . . . . . . . . . . . . . . . . . 30

2.8 Baumartenspezifische Koeffizienten der Einheitshöhenkurve . . . . . . . . . 33

2.9 Koeffizienten der verwendeten Funktionen zur Schätzung der Bonität und der Höhe des Grundflächenmittelstammes entsprechend der Bonität in beliebigen

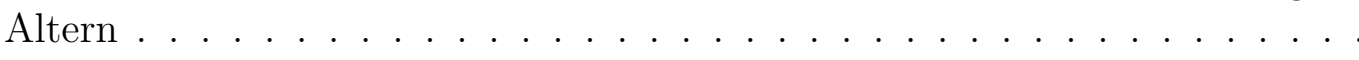

2.10 Parameter und statistische Eigenschaften des Grundflächenmodells für Douglasienreinbestände . . . . . . . . . . . . . . . . . . . 36

2.11 Kennwerte einiger Weiserbestände in den Wuchsbezirken Ems-Hase-HunteGeest und Göttinger Wald, getrennt nach Standortstypenobergruppe, Wasserhaushalts- und Nährstoffgruppe . . . . . . . . . . . . . . . .

2.12 Mittlere Bestandeskennwerte der jungen Weiserbestände getrennt nach Region und Art . . . . . . . . . . . . . . . . . . . 
2.13 Bestandeskennwerte generierter Kiefernweiserbestände im Wuchsbezirk OstHeide auf trockenen Sanden mit schwacher Nährstoffversorgung . . . . . . .

3.1 Baumartenabhängige Verjüngungsgänge . . . . . . . . . . . . . . . . . 44

3.2 Baumartenspezifische Übersicht einiger Rahmenwerte der Simulation . . . . 46

3.3 Produktions- und Simulationszeiträume der untersuchten Baumarten . . . . 46

3.4 Anzahl der Weiserbestände getrennt nach Bonitätsgruppe und Baumart . 46

3.5 Baumartenspezifische konstante Zieldurchmesser bei naturnaher und gestaffelte, bonitätsabhängige Zieldurchmesser bei kohlenstofforientierter Behandlung bei mittleren Risiken und Qualitäten . . . . . . . . . . . . . .

3.6 Ermittelte Koeffizienten zur Schätzung der maximalen Bestandesgrundfläche von Reinbeständen der Hauptwirtschaftsbaumarten in Nordwestdeutschland

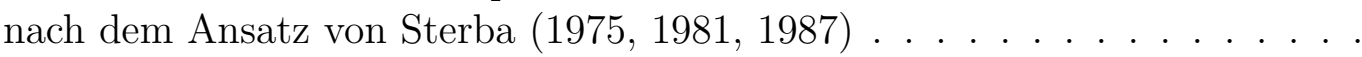

3.7 Koeffizienten der kumulativen Weibullverteilungsfunktion zur Schätzung der maximalen Bestandesgrundfläche für die Hauptwirtschaftsbaumarten in Nordwestdeutschland . . . . . . . . . . . . . . . . .

3.8 Steuerung der Durchforstung von Reinbeständen der Hauptwirtschaftsbaumarten mit Hilfe des natürlichen Bestockungsgrades in bestimmten Pflegephasen, definiert über Spitzenhöhenbereiche . . . . . . . . . . . . . 55

3.9 Verwendete Sortierungsvorgaben . . . . . . . . . . . . . . . . . . . 59

3.10 Koeffizienten der verwendeten Funktion zur Schätzung der unterirdisch lebenden Baumbiomasse . . . . . . . . . . . . . . . . . 61

3.11 Totholzzersetzungskonstanten und Lebensdauern des Totholzes der Hauptwirtschaftsbaumarten, bezogen auf die Holzdichte . . . . . . . . . . . . . 62

3.12 Holzproduktklassen mit entsprechenden Zersetzungskonstanten, Halbwertszeiten, Lebensdauern und Hauptprodukten . . . . . . . . . . . . .

3.13 Holzverwendungsschlüssel zur Verteilung von Laub- und Nadelholz auf die Produktklassen in Niedersachsen . . . . . . . . . . . . . . . . . . .

4.1 Mittlere ober- und unterirdisch lebende Baumbiomassen sowie daraus resultierende Kohlenstoffvorräte der erstellten Weiserbestände getrennt nach Region, Art und Alter . . . . . . . . . . . . . . . .

$4.210 \%$ - und 90\%-Quantil der Kohlenstoffbindungsrate der lebenden und toten Baumbiomasse, getrennt nach Art und waldbaulicher Behandlung für die Weiserbestände . . . . . . . . . . . . . . . . .

$4.310 \%$ - und 90\%-Quantil der Kohlenstoffbindungsrate des Holzproduktspeichers und dessen Substitutionswirkung, getrennt nach Art und waldbaulicher Behandlung für die Weiserbestände . . . . . . . . . . . . . . .

A.1 Mittlere Leistungsklassen der Hauptwirtschaftsbaumarten auf ausgesuchten Standorten getrennt nach Wasserhaushalt und Nährstoffversorgung im Wuchsbezirk Montaner Mittel- und Oberharz . . . . . . . . . . . . . . . . 
A.2 Mittlere Leistungsklassen der Hauptwirtschaftsbaumarten auf ausgesuchten Standorten getrennt nach Wasserhaushalt und Nährstoffversorgung im Wuchsbezirk Unterer Solling . . . . . . . . . . . . . . . . . . . . . 127

A.3 Mittlere Leistungsklassen der Hauptwirtschaftsbaumarten auf ausgesuchten Standorten getrennt nach Wasserhaushalt und Nährstoffversorgung im Wuchsbezirk Weser-Leine-Bergland . . . . . . . . . . . . . . . . . . . . . 128

A.4 Mittlere Leistungsklassen der Hauptwirtschaftsbaumarten auf ausgesuchten Standorten getrennt nach Wasserhaushalt und Nährstoffversorgung im Wuchsbezirk Ost-Heide . . . . . . . . . . . . . . . . . . . . . . . .

A.5 Mittlere Leistungsklassen der Hauptwirtschaftsbaumarten auf ausgesuchten Standorten getrennt nach Wasserhaushalt und Nährstoffversorgung im Wuchsbezirk Geest-Mitte

A.6 Mittlere Leistungsklassen der Hauptwirtschaftsbaumarten auf ausgesuchten Standorten getrennt nach Wasserhaushalt und Nährstoffversorgung im Wuchsbezirk Hohe Heide

A.7 Koeffizienten und statistische Eigenschaften der Parameter der Box-CoxCole-Green-Verteilung zur Schätzung der Durchmesserverteilung von Eichenreinbeständen . . . . . . . . . . . . . . . . . . .

A.8 Koeffizienten und statistische Eigenschaften der Parameter der Box-CoxCole-Green-Verteilung zur Schätzung der Durchmesserverteilung von Buchenreinbeständen

A.9 Koeffizienten und statistische Eigenschaften der Parameter der Box-CoxCole-Green-Verteilung zur Schätzung der Durchmesserverteilung von Fichtenreinbeständen

A.10 Koeffizienten und statistische Eigenschaften der Parameter der Box-CoxCole-Green-Verteilung zur Schätzung der Durchmesserverteilung von Douglasienreinbeständen . . . . . . . . . . . . . . . . .

A.11 Koeffizienten und statistische Eigenschaften der Parameter der Box-CoxCole-Green-Verteilung zur Schätzung der Durchmesserverteilung von Kiefernreinbeständen . . . . . . . . . . . . . . . . 135

A.12 Parameter und statistische Eigenschaften des Grundflächenmodells für Eichenreinbestände . . . . . . . . . . . . . . . . . . 14

A.13 Parameter und statistische Eigenschaften des Grundflächenmodells für $\mathrm{Bu}-$ chenreinbestände . . . . . . . . . . . . . . . . . . . . . . . 142

A.14 Parameter und statistische Eigenschaften des Grundflächenmodells für Fichtenreinbestände . . . . . . . . . . . . . . . . . . . . . . . . . . . 143

A.15 Parameter und statistische Eigenschaften des Grundflächenmodells für Kiefernreinbestände . . . . . . . . . . . . . . . . .

A.16 Verwendete Versuchsflächen der Nordwestdeutschen Forstlichen Versuchsanstalt zur Ermittlung der maximalen Bestandesgrundfläche von Reinbeständen der Hauptwirtschaftsbaumarten in Nordwestdeutschland . . . . . . . . . 146 
A.17 Koeffizienten und statistische Eigenschaften der Parameter der Box-CoxCole-Green-Verteilung zur Schätzung der maximalen Bestandesgrundfläche von Eichenreinbeständen . . . . . . . . . . . . . . . . . . . . . . . . . 148

A.18 Koeffizienten und statistische Eigenschaften der Parameter der Box-CoxCole-Green-Verteilung zur Schätzung der maximalen Bestandesgrundfläche von Buchenreinbeständen . . . . . . . . . . . . . . . . . . . . . . . . 149

A.19 Koeffizienten und statistische Eigenschaften der Parameter der Box-CoxCole-Green-Verteilung zur Schätzung der maximalen Bestandesgrundfläche von Fichtenreinbeständen . . . . . . . . . . . . . . . . . . . . . . . . . . . 149

A.20 Koeffizienten und statistische Eigenschaften der Parameter der Box-CoxCole-Green-Verteilung zur Schätzung der maximalen Bestandesgrundfläche von Douglasienreinbeständen . . . . . . . . . . . . . . . . . . . . . 150

A.21 Koeffizienten und statistische Eigenschaften der Parameter der Box-CoxCole-Green-Verteilung zur Schätzung der maximalen Bestandesgrundfläche von Kiefernreinbeständen . . . . . . . . . . . . . . . . . . . . . . . . 150

A.22 Kennwerte der erstellten Weiserbestände getrennt nach Wuchsbezirk, Standortstypenobergruppe, Wasserhaushalts- und Nährstoffgruppe sowie Art, Alter und Bonitätsgruppe . . . . . . . . . . . . . . . . . . . . . 154

A.23 Kohlenstoffbindungsraten der einzelnen Speicher getrennt nach Wuchsbezirk, Standortstypenobergruppe, Wasserhaushalts- und Nährstoffgruppe sowie Art und waldbauliche Behandlung . . . . . . . . . . . . . . . 180 


\section{Zusammenfassung}

Die Speicherung von Kohlenstoff im Wald ist ein hochaktuelles, klimapolitisches Thema. Dabei werden als Speicher die lebende und die tote Baumbiomasse sowie die aus dem geernteten Holz hergestellten Produkte und deren Substitutionseffekte betrachtet. Die Kohlenstoffbindung in der lebenden Baumbiomasse der Wälder ist vornehmlich von der Baumart, dem Standort und der Waldbehandlung abhängig. Außerhalb des Waldes, bei den Holzprodukten und deren Substitutionspotenzial, ist die Art und Dauer der Verwendung maßgeblich für die Kohlenstoffbindung. Forstbetriebe können durch ihre strategische Ausrichtung das Teilziel der Kohlenstoffspeicherung stärker gewichten und somit zum gesellschaftlich geforderten Klimaschutz einen Beitrag leisten. Dazu sind jedoch Kenntnisse über baumarten-, standort- und behandlungsspezifische Effekte notwendig, welche die Kohlenstoffspeicherung beeinflussen. Zu diesem Zweck wurden Simulationen einer naturnahen und einer kohlenstofforientierten waldbaulichen Behandlung der fünf wichtigsten Baumarten im niedersächsischen Landeswald auf bedeutenden Standortseinheiten durchgeführt.

Die Hauptwirtschaftsbaumarten im Landeswald von Niedersachsen sind Eiche, Buche, Fichte, Douglasie und Kiefer. Die analysierten Standorte verteilen sich auf jeweils vier Wuchsbezirke im Tief- und Bergland. Für die Identifikation wichtiger Kombinationen aus Wasser- und Nährstoffversorgung sowie der entsprechenden Leistungsfähigkeit der Baumarten wurden Informationen der Standortskartierung sowie der Forsteinrichtung der Niedersächsischen Landesforsten genutzt.

Auf der Grundlage der Betriebsinventur der Niedersächsischen Landesforsten konnten Modelle zur Generierung von Einzelbaumdaten erstellt werden, die insbesondere zur Schätzung der aktuellen Bestandesgrundflächen und der zu Grunde liegenden Durchmesserverteilungen genutzt wurden. Mit ihrer Hilfe konnten realitätsnahe Weiserbestände als Grundlage der Simulationen, entsprechend den Informationen aus Forsteinrichtung und Standortskartierung, generiert werden.

Damit die waldbauliche Behandlung abgebildet werden kann, ist es notwendig die Durchforstungsstärke und -art nachzubilden. Unter Verwendung von ertragskundlichen Versuchsflächendaten der Nordwestdeutschen Forstlichen Versuchsanstalt wurden erstmalig mittels Quantilsregression baumartenspezifische Funktionen zur Bestimmung der maximalen Bestandesgrundfläche für Nordwestdeutschland hergeleitet. Diese stellen aufgrund der größeren Datengrundlage und der verwendeten Methoden eine Verbesserung im Vergleich zum vorher benutzten Ansatz der Nordwestdeutschen Forstlichen Versuchsanstalt dar. Mit ihnen lässt sich die maximale Bestandesgrundfläche sicherer schätzen. Zur Definition eines praxisnahen Nutzungskonzeptes mit unterschiedlichen Pflegephasen, 
abgegrenzt durch bestimmte Höhenbereiche, wird die aktuelle Bestandesgrundfläche ins Verhältnis zur maximal möglichen Grundfläche des Bestandes gesetzt. Mit dem neu erstellten Konzept kann nun eine grundflächengesteuerte, gestaffelte Durchforstung abgebildet werden. Wobei mit den entwickelten Methoden auch die Nachbildung anderer Nutzungskonzepte möglich ist.

Mit den generierten Weiserbeständen und dem Waldwachstumssimulator WaldPlaner wurden die Auswirkungen der verschiedenen waldbaulichen Behandlungen auf die Bestandesentwicklung und die Kohlenstoffspeicherung untersucht. Dazu wurden u. a. die Einzelbaumdaten mittels bekannter Funktionen aus der Literatur in Biomasse umgerechnet, ein neuer Holzverwendungsschlüssel zur Verteilung des eingeschlagenen Rohholzes zu Produktklassen aufgestellt und ein Modell zur Kaskadennutzung in die Auswertung integriert.

Durch die Bildung einer Kohlenstoffspeicherrate sind Rückschlüsse über den Einfluss der Wasser- und Nährstoffversorgung auf die Kohlenstoffspeicherung möglich. Sie erlaubt die Vergleichbarkeit der Baumarten, der Standorte sowie der einzelnen Speicher. Es zeigt sich bei einer Gesamtbetrachtung der untersuchten Pools (lebende und tote Baumbiomasse, Holzprodukte sowie deren Substitutionspotenzial), dass sich die Kohlenstoffspeicherraten deutlich zwischen den Baumarten unterscheiden und sich folgende Reihung ergibt: Douglasie ${ }^{1}$, Fichte $>$ Kiefer $>$ Buche, Eiche.

Der Forstbetrieb kann durch eine standortgemäße Baumartenwahl und -mischung, die Waldbehandlung und der Berücksichtigung der erwartbaren Holzqualitäten und Risiken das Teilziel der Kohlenstoffspeicherung im Rahmen des strategischen Managements stärker gewichten. Dabei haben standortgerechte Nadelbaumarten eine große Klimaschutzwirkung. Sie sollten allerdings nur insoweit angebaut werden, als das die multifunktionale Nachhaltigkeit der Wälder nicht verletzt wird. Im Bereich der Holzverwendung ist die Kaskadennutzung und stoffliche Nutzung weiter auszubauen, um möglichst viele Holzprodukte im Zivilisationskreislauf zu halten und abschließend energetisch zu nutzen. Auf forstpolitischer Ebene ergeben sich verschiedene Handlungsfelder. Einerseits sind konkrete Wege zur Lösung von Zielkonflikten zwischen nationaler Klimapolitik und anderen Strategien (z. B. Nationale Biodiversitätsstrategie, Waldstrategie 2020) zu entwickeln. Andererseits ist die große Bedeutung der Wälder als Kohlenstoffspeicher und nachhaltiger Rohstofflieferant, intensiver als bisher, der Gesellschaft näher zu bringen. Dessen ungeachtet besteht noch enormer Forschungsbedarf über den Einfluss des Klimawandels und verschiedener Risiken sowie der Kaskadennutzung auf die Kohlenstoffspeicherung im Forst-Holz-Sektor.

\footnotetext{
${ }^{1}$ Das allgemein sehr hohe Leistungspotenzial der Douglasie wird aufgrund der Vorauswahl von Standorten nicht zutreffend dargestellt.
} 


\section{Abstract}

Carbon sequestration in forests is a highly topical issue in the current climate change debate. The most important carbon pools are living and dead tree biomass, timber products and their substitution effects. The carbon sequestration of the living tree biomass mainly depends on tree species, site and silvicultural management. Type and duration of use are the main determinants of carbon sequestration in timber products and their potential for substitution. Forest management strategies can focus on the aim of carbon storage and thereby contribute to the socially demanded climate protection. However, for this to be achieved, knowledge about the effects of tree species, site and silvicultural management on carbon sequestration is required. For that purpose, the development of the five main tree species in Lower Saxony, Germany, was simulated under a near-natural and a carbon-oriented management at selected sites.

The main tree species in the Lower Saxony State Forest are Oak, European beech, Norway spruce, Douglas fir and Scots pine. The selected sites are distributed in four growth districts in the lowlands and the mountainous regions of Lower Saxony, respectively. In order to identify important combinations of water and nutrient supply as well as the corresponding productivity of the tree species, information was gathered from site mapping and forest planing of the forest administration.

Based on the operating inventory of the Lower Saxony State Forest, models for the generation of single tree data were created. These models were particularly used for the estimation of the current basal area and the underlying diameter distribution of the simulation stands.

To represent silvicultural management, it is necessary to model intensity and the type of thinning. Using data from long-term thinning experiments of the Northwest German Forest Research Institute and quantile regression, it was possible to create species specific functions for maximum basal area, which are valid for Northwest Germany. Due to the larger database and the applied methods, these functions allow for more accurate estimates and can thus be regarded as improvements over the previously utilized methods. The ratio of current to maximum basal stand area was used for defining a realistic concept of utilization with height dependent thinnings. With help of this new concept, it is possible to simulate a graduated thinning, controlled by basal area. Furthermore, other utilization concepts can be modelled with the developed methods.

The effects of silvicultural management on stand development were simulated with the forest growth simulator WaldPlaner. In order to estimate the consequences for carbon storage, single-tree data were converted to biomass with published functions. Additionally, harvested timber was allocated to product groups with a new allocation formula 
and a model for cascade use was integrated in the analysis.

By forming a carbon sequestration rate, conclusions about the impact of water and nutrients on carbon storage are possible. Further, this rate allows for the comparison of tree species, sites and individual carbon pools. With respect to the considered pools, the sum of the carbon rates differs clearly among tree species and results in the following order: Douglas fir $^{2}$, Norway spruce $>$ Scots pine $>$ Beech, Oak.

More weight can be given to carbon sequestration, as a subgoal of forest management, by selecting and mixing site adapted tree species, silvicultural management and consideration of the expectable wood qualities and risks. Site adapted coniferous species have a strong potential for climate protection. However, their cultivation should not harm the multifunctional sustainability of forests. Cascade and material usage of wood, followed by energetic utilization, are to be expanded, in order to keep as many wood products as possible in use. Forest policy has to manage several fields of action. On the one hand, concrete measures for resolving target conflicts between national climate policy and other strategies have to be developed (e.g., Nationale Biodiversitätsstrategie, Waldstrategie 2020). On the other hand, it is an important task to give the society a better understanding of the great importance forests have for carbon sequestration and sustainable provision of raw material. Nevertheless, there is still an enormous need for research on the impact of climate change and various risks and multiple use of wood on carbon sequestration in the forestry-wood-sector.

\footnotetext{
${ }^{2}$ The generally very high productivity of Douglas fir is not presented accurately due to the pre-selection of sites.
} 


\section{Einleitung}

\subsection{Anlass der Arbeit}

Der aktuelle fünfte Sachstandsbericht des Intergovernmental Panel on Climate Change führt den Klimawandel und seine Auswirkungen auf den Menschen zurück (IPCC, 2013). Angetrieben wird er durch die anthropogene Freisetzung von Treibhausgasen, wobei dem Treibhausgas Kohlenstoffdioxid $\left(\mathrm{CO}_{2}\right)$ eine besondere Rolle zukommt (IPCC, 2007). Seine Konzentration in der Atmosphäre ist seit 1750 von vorher 280 ppm (parts per million) auf 391 ppm im Jahr 2011 angestiegen (IPCC, 2013). Ein weiterer Anstieg ist anzunehmen, wie andere Messungen bestätigen (NOAA, 2016). Kohlenstoffdioxid ist das am häufigsten auftretende Treibhausgas und es hat darum einen entscheidenden Einfluss auf die vorausgesagten Änderungen des Klimas. Die Vermeidung von Kohlenstoffdioxidemissionen bzw. deren Speicherung ist daher ein hochaktuelles, vor allem politisches Thema. Wälder spielen dabei eine wichtige Rolle als Speicher für Kohlenstoff (C) und als Bestandteil des globalen Kohlenstoffkreislaufes (Schulze, 2000; Pistorius, 2007a; Lorenz und Lal, 2010).

Das Kyoto-Protokoll, welches auch von Deutschland ratifiziert wurde, ist darauf ausgelegt die Treibhausgasemissionen zu verringern. In Artikel 3.4 wird den Vertragsstaaten die Möglichkeit eingeräumt, die Senkeneffekte der Waldbewirtschaftung in der Treibhausgasbilanz anrechnen zu lassen. Deutschland macht von dieser Option seit 2006 Gebrauch, wobei bisher der Waldökosystemansatz zur Bilanzierung genutzt wurde. Hierbei wird das Ökosystem Wald innerhalb seiner Grenzen betrachtet und jede Holznutzung kommt einer Freisetzung von Kohlenstoff gleich. Das allerdings der Kohlenstoff weiterhin in den verschiedensten Holzprodukten mit teilweise sehr langen Lebensdauern gespeichert ist, wird dabei außer Acht gelassen. Um den Holzsektor und seine Möglichkeiten, Kohlenstoff zu speichern, in den Treibhausgasbilanzen zu berücksichtigen, wurde bei der 17. Vertragsstaatenkonferenz der Klimarahmenkonvention in Durban beschlossen, die stofflich genutzten Holzprodukte mit in die Bilanzierung einzubeziehen (BMELV, 2012a). Damit ergibt sich die Notwendigkeit, den Beitrag der Holzprodukte zur Kohlenstoffsequestrierung besser zu quantifizieren.

Das vor dreihundert Jahren in der Forstwirtschaft eingeführte Prinzip der Nachhaltigkeit unterlag im Laufe der Zeit einigen Änderungen. Anfangs bezog es sich auf die Holzerzeugung und die Holzerträge, später auf die Gelderträge der Forstbetriebe und seit den siebziger Jahren des letzten Jahrhunderts auf die Vielfachnutzungen der Wälder. Dies spiegelt sich bis heute im Konzept einer nachhaltigen, multifunktionalen Forstwirtschaft 
wider, welches alle Waldbesitzarten eint (Spellmann et al., 2004). Es fand auch Eingang in die Helsinki-Resolution von 1993 zum Schutz der Wälder in Europa. Dort lautet die Definition: Nachhaltige Waldbewirtschaftung ist die Pflege und Nutzung von Wäldern und Waldflächen auf eine Weise und in einem Ausmaß, das deren biologische Vielfalt, Produktivität, Verjüngungsfähigkeit und Vitalität erhalten bleibt sowie deren Potenzial gesichert ist, jetzt und in Zukunft die entsprechenden ökologischen, wirtschaftlichen und sozialen Funktionen auf lokaler, nationaler und globaler Ebene zu erfüllen, ohne anderen Ökosystemen Schaden zuzufügen (MCPFE, 1993).

Bei der vierten Ministerkonferenz zum Schutz der Wälder in Europa wurden 2003 in Wien 35 quantitative und 17 qualitative Kriterien für eine nachhaltige Forstwirtschaft in Europa verabschiedet (MCPFE, 2003), um die Definition von Helsinki zu operationalisieren, ohne allerdings bestimmte waldbauliche Empfehlungen zu geben (Röhrig et al., 2006). Das Ziel dieser Kriterien, mit den ihnen zugeordneten Indikatoren, ist es, die Umsetzung des Nachhaltigkeitsprinzips von der Ebene des einzelnen Forstbetriebes bis zur Landesebene zu fördern. Sie können zur Entwicklung von Zielsystemen, zur Definition angestrebter Zielzustände und zur Feststellung von Zielerreichungsgraden genutzt werden (Spellmann, 2003). Zum Kriterium I, Forstliche Ressourcen, zählt unter anderem der quantitative Indikator Kohlenstoffvorrat, der sich auf die Kohlenstoffspeicherung in der Holzbiomasse und im Waldboden bezieht. Durch den Zuwachs der Bäume wird Kohlenstoffdioxid aus der Atmosphäre gebunden. Wälder können allerdings auch zu einer Quelle für Kohlenstoffdioxid werden, wenn die Respiration der Pflanzen deren Fotosyntheseleistung übersteigt, sie verbrennen oder sich zersetzen (Nabuurs et al., 2008). In der internationalen Klimapolitik wird diese Senken- bzw. Quellenfunktion heftig diskutiert.

Die Speicherung von Kohlenstoff in der Biomasse wird allein durch die Fotosynthesereaktionen der Pflanzen ermöglicht. Dabei werden organische Substanzen aus Kohlenstoffdioxid und Wasser unter Nutzung von Strahlungsenergie aufgebaut. Es wird dementsprechend Strahlung absorbiert und in einer chemischen Verbindung gespeichert. Diese wird gebildet, indem der Wasserstoff vom Wasser abgespalten und gleichzeitig Sauerstoff freigesetzt wird. Der Wasserstoff reagiert mit dem Kohlenstoffdioxid und wird in einer metastabilen Kohlenstoffverbindung festgelegt (Nultsch, 2001). In dieser aufgebauten organischen Substanz ist die Energie der Sonne gespeichert. So sind in 2,5 kg lufttrockenem Holz, die dem Brennwert von einem Liter Heizöl entsprechen, etwa $10 \mathrm{kWh}$ bzw. 36 MJ Strahlungsenergie gespeichert, wobei der Heizwert von Nadelholz im Vergleich zu Laubholz ca. $2 \%$ höher ist (Hartmann, 2009).

Der Forstwirtschaft und den aus geerntetem Holz hergestellten Produkten kommt somit für die Senkung der Kohlenstoffdioxidkonzentration in der Atmosphäre eine wichtige Rolle zu. Gleichzeitig erwächst daraus die Verpflichtung, Wälder so zu bewirtschaften, dass sie diese Funktion möglichst erfüllen und nicht zur Treibhausgasquelle werden. 


\subsection{Ausgangssituation}

Die grundsätzlichen Prozesse des Kohlenstoffkreislaufes sind gut bekannt, dennoch bestehen wesentliche Unsicherheiten über das Verhalten vieler seiner Quellen und Senken. Seine Erforschung in Waldökosystemen ist aufgrund biogeochemischer Prozesse, des Klimas und seiner zukünftigen Entwicklung, von Störungen und der Bewirtschaftung, genauso wie der räumlichen sowie der zeitlichen Heterogenität der Kohlenstoffspeicherung verschiedener Regionen und Waldtypen, eine besondere Herausforderung (Ashton et al., 2012).

In Wäldern sind $77 \%$ der weltweiten oberirdischen terrestrischen Kohlenstoffvorräte gespeichert (IPCC, 2000). Die globale Waldfläche betrug 2005 ca. 3,69 Mrd. ha (FAO, 2012), was einer Landbedeckung von etwa $30 \%$ entspricht. Allerdings nimmt diese Fläche schon seit geraumer Zeit ab. Zwischen 1990 und 2005 betrug die jährliche Rate der Waldverluste etwa 4,9 Mio. ha (FAO, 2012). Im Vergleich zu ihrer historischen Ausdehnung ist somit die globale Waldfläche mittlerweile um rund $50 \%$ geschrumpft (WRI, 2011).

Aktuelle Untersuchungen deuten darauf hin, dass die Wälder der Erde von 1990 bis 2007 für eine jährliche Speicherrate von 2,7 Mrd. t C verantwortlich sind. Im europäischen Raum betrug 2007 der Kohlenstoffvorrat der lebenden Baumbiomasse, des Totholzes, der Humusauflage und des Mineralbodens bis $1 \mathrm{~m}$ Tiefe insgesamt etwa 24,0 $\pm 3,0 \mathrm{Mrd}$.t C bzw. 166,4 t C ha ${ }^{-1}$ (Pan et al., 2011). Luyssaert et al. (2010) geben eine jährliche Kohlenstoffspeicherrate von rund 0,75 $\pm 0,20 \mathrm{t} \mathrm{Cha}^{-1}$ an, bezogen auf die Wälder in der Europäischen Union mit einer Fläche von 132 bis 155 Mio. ha und den Zeitraum von 1990 bis 2005 .

Die dritte Bundeswaldinventur ergab für die lebende und tote Baumbiomasse der Wälder in Deutschland einen Kohlenstoffvorrat von ca. $150 \mathrm{t} \mathrm{C} \mathrm{ha}^{-1}$ (1.169 Mio. t C). In der Streuauflage und im Mineralboden bis $30 \mathrm{~cm}$ sind nochmals 850 Mio. t $\mathrm{C}$ gespeichert. Momentan werden im Wald jährlich rund 52 Mio. t C gespeichert (UBA, 2014). Im Vergleich dazu verursacht Deutschland als hochtechnisiertes Industrieland enorme Treibhausgasemissionen mit einem Kohlenstoffdioxidanteil von rund $88 \%$, wobei in 2014 etwa 912 Mio. t Kohlenstoffdioxid-Äquivalente emittiert wurden (UBA, 2015). Daraus ergibt sich für die Wälder in Deutschland eine Minderungsrate der atmosphärischen Kohlenstoffdioxidemissionen von etwa $6 \%$.

In Deutschland wurden schon einige Studien zur Kohlenstoffspeicherung in Wäldern durchgeführt, sei es auf nationaler Ebene (Burschel et al., 1993; Dieter und Elsasser, 2002; Oehmichen et al., 2011), regionaler Ebene (Böswald, 1996; Böswald und Wierling, 1997; Klein und Schulz, 2012; Knauf et al., 2013; Mund et al., 2015; Pistorius und Zell, 2005; Profft et al., 2009; Wirth et al., 2004a; Wördehoff et al., 2011, 2012b,a) oder Bestandesebene (Borys et al., 2013; Fürstenau et al., 2007; Höllerl und Neuner, 2011; Höllerl und Bork, 2013; Mund et al., 2002; Mund und Schulze, 2006). Dabei wurden vielfach unterschiedliche Methoden und Datenquellen zur Berechnung des Kohlenstoffvorrates der verschiedenen Speicher angewandt, wodurch direkte Vergleiche oft nicht 
möglich sind. Auch wurde der Produktspeicher und dessen Substitutionswirkung nicht immer mit in die Betrachtungen einbezogen.

Im Kyoto-Protokoll wurde die Speicherung und Freisetzung von Kohlenstoff nur unvollständig betrachtet. Durch den benutzten Waldökosystemansatz, mit der Systemgrenze Waldrand, kommt eine Holznutzung einer Freisetzung von Kohlenstoff gleich. Dies führte in der Vergangenheit dazu, dass die Erhöhung des Kohlenstoffvorrates in Wäldern durch die Verlängerung der Umtriebszeit und ein Verzicht der Holznutzung gefordert wurden (Köhl et al., 2009). Das Konzept der Nullnutzung hat Relevanz in besonders geschützten Waldbiotopen ${ }^{1}$ oder Urwäldern, jedoch nicht für die überwiegend bewirtschafteten Wälder in Deutschland ${ }^{2}$. Köhl et al. (2009) haben sich intensiv mit der Kohlenstoffspeicherung in Wäldern in Abhängigkeit von unterschiedlichen Nutzungskonzepten auseinandergesetzt, wobei die angewandten Szenarien von einem generellen Nutzungsverzicht bis zu einer Maximierung der Gewinne aus der Waldbewirtschaftung mit einer starken Ausrichtung auf die Fichte reichen. Sie kommen zu dem Ergebnis, dass die gewinnmaximierende Variante im Zeitraum von 30 Jahren zu einer Speicherleistung im Bestand von ca. $110 \mathrm{t} \mathrm{C}^{-1}$ führt. Im Gegensatz dazu führt der Nutzungsverzicht zu einer Erhöhung des Vorrates an Kohlenstoff im Bestand auf $245 \mathrm{t} \mathrm{Cha}^{-1}$. Die Differenz der Kohlenstoffvorräte zwischen der gewinnmaximierenden Behandlung und dem Nutzungsverzicht der Wälder verringert sich allerdings, wenn der energetische Substitutionseffekt der Holzprodukte mit in die Betrachtung einbezogen wird. Dieser beträgt bei der gewinnmaximierenden Variante ca. $84 \mathrm{t} \mathrm{Cha}^{-1}$. Der Effekt der Materialsubstitution ist ungleich höher, da Holzprodukte, abhängig von ihrer Lebensdauer und Verwendung, mehr Kohlenstoff als vergleichbare Produkte aus anderen Materialien enthalten und gleichzeitig um ein Vielfaches weniger Energie zu ihrer Produktion benötigen (Albrecht et al., 2008). Es ist somit notwendig die Substitutionseffekte der Holzprodukte mit in die Betrachtung einzubeziehen, um ein vollständiges Bild der Kohlenstoffspeicherung im Forst-Holz-Sektor zu erhalten. In den Untersuchungen von Wördehoff et al. (2011, 2012b,a) kommen die Autoren bei 30-jährigen Simulationsläufen zu dem Ergebnis, dass durch eine naturschutzorientierte Behandlung der Kohlenstoffvorrat im Bestand am höchsten ist. Dabei werden allerdings die Nutzungen größtenteils jenseits des Betrachtungszeitraumes verschoben und gleichzeitig auch die Risiken erhöht. Darüber hinaus leistet diese Variante den geringsten Beitrag zur Rohholzversorgung und damit zur materiellen sowie energetischen Substitution. Auch in der Kohlenstoffbilanz der bayerischen Forst- und Holzwirtschaft wird geschlussfolgert, dass eine Nichtnutzung bei günstiger Entwicklung einen Beitrag zum Klimaschutz leistet. Jedoch ist aufgrund des hohen Fichtenanteils der bayerischen Wälder davon auszugehen, dass mit zunehmenden Alter auch extreme Schadereignisse öfter auftreten. Durch die typischerweise steigende Mortalitätsrate bei einer natürlichen Entwicklung, ist die Nichtnutzung einer nachhaltigen Nutzung unterlegen (Klein und

\footnotetext{
${ }^{1}$ rund $5 \%$ des begehbaren Waldes in Deutschland (BMEL, 2014)

${ }^{2}$ lediglich auf $4 \%$ der Fläche des bestockten Holzbodens in Deutschland ist Holznutzung unzulässig bzw. nicht zu erwarten (BMEL, 2014)
} 
Schulz, 2012). Hasenauer (2011) kommt in einem Vergleich von Simulationsrechnungen für von Fichten und Buchen dominierte Urwälder sowie einem bewirtschafteten Fichtenbestand zu dem Ergebnis, dass bewirtschafteter Wald, welcher vor dem Erreichen der physiologischen Altersgrenze und dem Eintritt in die Zerfallsphase genutzt wird, eine Kohlenstoffsenke darstellt, während der Urwald mit dem Eintritt in die Zerfallsphase zur Quelle wird. Des Weiteren haben Nadelwälder größere biotische und abiotische Risiken als Laubwälder, wobei sie ohne Nutzung in der Zerfallsphase großflächig zusammenbrechen und somit zu einer Quelle werden. Auch wenn Wälder bis in ein hohes Alter Kohlenstoff aufnehmen können (Knohl et al., 2003; Luyssaert et al., 2008), müssen der jetzige Waldzustand sowie etwaige Umweltveränderungen mit beachtet werden. Diese haben in den letzten 50 Jahren zu einer deutlichen Zunahme des Wachstums geführt, ungeachtet ob es sich um unbewirtschafteten oder bewirtschafteten Wald handelt. Ursächlich dafür sind vor allem die hohen Stickstoffeinträge, der Anstieg des Kohlenstoffdioxidgehaltes in der Atmosphäre, die steigenden Temperaturen, eine verlängerte Vegetationszeit sowie eine veränderte Verteilung der Niederschläge (Krug et al., 2012).

\subsubsection{Betrachtete Kohlenstoffspeicher}

In dieser Arbeit werden die folgenden Kohlenstoffspeicher, auch Pools genannt, im ForstHolz-Sektor berücksichtigt bzw. deren Entwicklung unter verschiedenen Behandlungsszenarien betrachtet (s. Abb. 1.1):

- lebende Baumbiomasse:

- die oberirdische, lebende Biomasse der Bäume wie Stämme, Äste, Rinde, und Stümpfe sowie die Nadeln beim Nadelholz,

- die unterirdisch lebenden Grobwurzeln, jedoch nicht die Feinwurzeln (Durchmesser $<2 \mathrm{~mm})$,

- tote Baumbiomasse:

- das oberirdische, tote Derbholz ab 7 cm Durchmesser,

- Holzprodukte:

- Produkte aus Holz wie Bauholz, Holzwerkstoffe, Papier und Pappe bis hin zu Brennholz.

$\mathrm{Zu}$ den weiteren Kohlenstoffspeichern im Wald, der Bodenvegetation und dem Boden, wurden keine eigenen Analysen durchgeführt. 


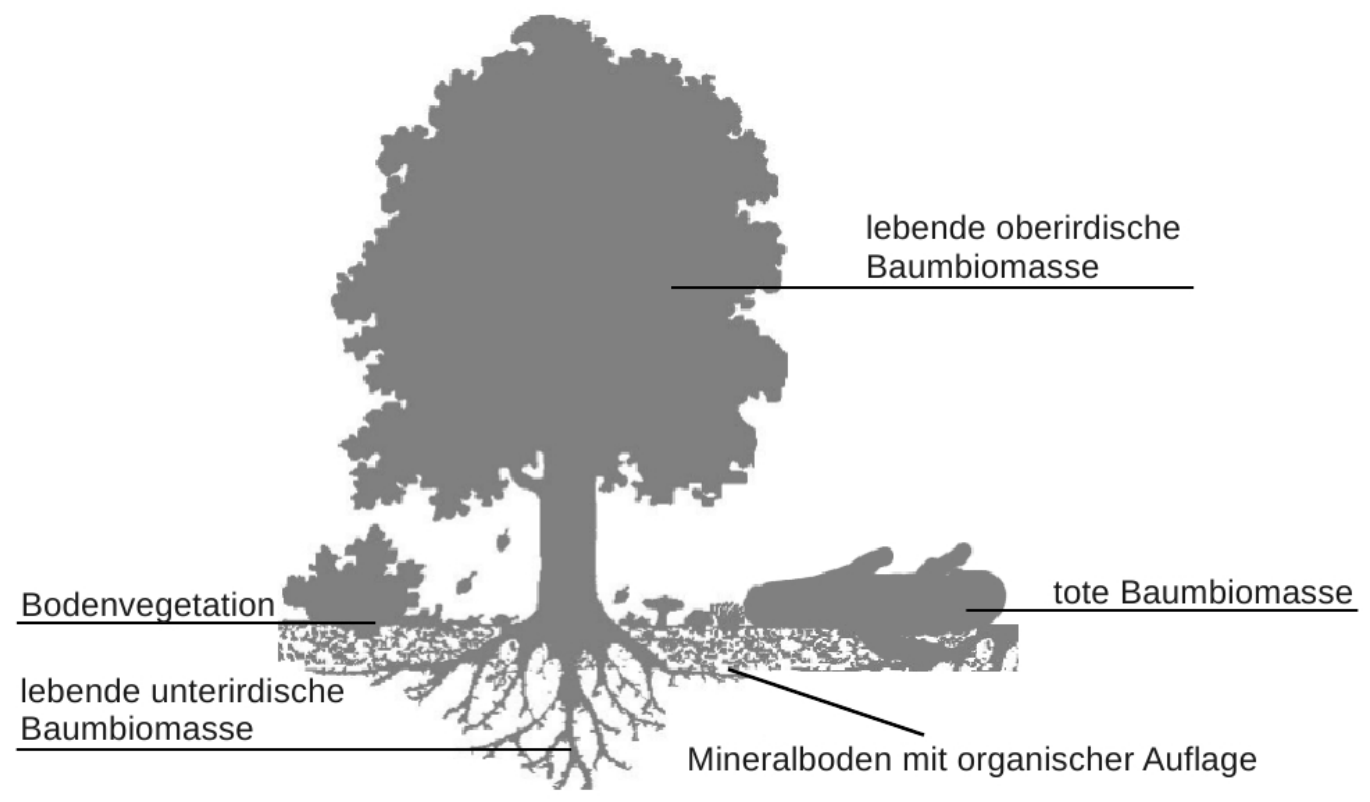

Abbildung 1.1: Kohlenstoffspeicher im Wald (verändert nach WBGU (1998)) (Quelle: Wördehoff et al. (2011)).

\subsubsection{Literaturstudie zur lebenden Baumbiomasse}

Die lebende Baumbiomasse wird eingeteilt in ober- und unterirdische Kompartimente. Weltweit sind ca. $44 \%$ des Kohlenstoffvorrates der Wälder in der Biomasse gespeichert und in Europa, ohne das Gebiet der Russischen Föderation, sind es rund 36\%. Absolut hat sich der weltweite Kohlenstoffvorrat in der Waldbiomasse zwischen 1990 (71,9 t C ha $\left.{ }^{-1}\right)$ und 2010 (71,6 t C ha $\left.{ }^{-1}\right)$ kaum verändert, während er in Europa ohne Russland von 58,5 $\mathrm{t} \mathrm{ha}^{-1}$ im Jahr 1990 auf 63,9 $\mathrm{t} \mathrm{Cha}^{-1}$ in 2010 gestiegen ist (FAO, 2010). Dabei sind in Europa 79,8\% des Kohlenstoffvorrates in der oberirdischen und 20,2\% in der unterirdischen Baumbiomasse gespeichert (MCPFE, 2011). Betrachtet man die Wälder der gesamten gemäßigten Klimazone, so beläuft sich der Kohlenstoffvorrat in der lebenden oberirdischen Dendromasse der Nadelbäume auf schätzungsweise 149 t C ha ${ }^{-1}$ und in der unterirdischen Dendromasse auf $46 \mathrm{t} \mathrm{Cha}^{-1}$, gesamt rund $195 \mathrm{t} \mathrm{C} \mathrm{ha}^{-1}$. Die analogen Werte für Laubwälder betragen oberirdisch rund $109 \mathrm{t} \mathrm{C} \mathrm{ha}^{-1} \mathrm{bzw}$. unterirdisch ca. 26 t C ha-1, gesamt etwa $135 \mathrm{t} \mathrm{Cha}^{-1}$ (Luyssaert et al., 2007). Derzeit stellen die Wälder der gemäßigten Zone eine Kohlenstoffsenke mit einer jährlichen Aufnahme in Höhe von 0,37 Mrd. t C dar (Robinson, 2007). Dies deckt sich mit den Ergebnissen der Ernährungsund Landwirtschaftsorganisation der Vereinten Nationen (FAO, 2010).

In Deutschland wurden Anfang der 1990er Jahre erste Studien zur Speicherung von Kohlenstoff in Wäldern durchgeführt. Burschel et al. (1993) kalkulierten auf Grundlage der ersten Bundeswaldinventur (BWI) und des Datenspeichers Waldfonds einen mittle- 
ren Kohlenstoffvorrat in der lebenden Baumbiomasse von rund $89 \mathrm{t} \mathrm{Cha}^{-1}$. Dieter und Elsasser (2002) kamen, basierend auf der ersten Bundeswaldinventur und verschiedenen Datenquellen für die neuen Bundesländer, je nach Altersklasse und Baumart, zu Werten zwischen 120 bis $190 \mathrm{t} \mathrm{Cha}^{-1}$. In der Inventurstudie 2008, als deutschlandweite Analyse der Kohlenstoffvorräte in den Wäldern zur Erfüllung der Kyoto-ProtokollBerichtspflichten, konnte ein durchschnittlicher Vorrat von 114 t C ha ${ }^{-1}$ (1,28 Mrd.t C) ermittelt werden. Dabei entfielen $81 \%$ (1,04 Mrd.t C) auf den oberirdischen und $19 \%$ (0,24 Mrd.t C) auf den unterirdischen Teil der Biomasse. Wobei der größte Teil des Kohlenstoffs (92\%) in der Oberschicht des Baumbestandes bzw. im Hauptbestand gebunden ist (Oehmichen et al., 2011). Aus den Ergebnissen der Inventurstudie 2008 können deutschlandweit mittlere Kohlenstoffvorräte von $148 \mathrm{t} \mathrm{Cha}^{-1}$ für Eichenbestände, $153 \mathrm{t} \mathrm{C} \mathrm{ha}^{-1}$ für Buchenbestände, $138 \mathrm{t} \mathrm{C} \mathrm{ha}^{-1}$ für Fichtenbestände, $119 \mathrm{t} \mathrm{Cha}^{-1}$ für Douglasienbestände und $111 \mathrm{t} \mathrm{C} \mathrm{ha}^{-1}$ für Kiefernbestände abgeleitet werden. Die Unterschiede erklären sich durch die standörtliche Zuordnung der Baumarten, ihre Bonitäten und Rohdichten sowie durch den jeweiligen Altersklassenaufbau. Weiterhin wurden auch regionale Untersuchungen durchgeführt (vgl. Böswald (1996) sowie Klein und Schulz (2012) für Bayern, Böswald und Wierling (1997) sowie Wördehoff et al. (2011) für Niedersachsen, Knauf et al. (2013) für Nordrhein-Westfalen, Pistorius (2007a) für Baden-Württemberg, Wirth et al. (2004a) für Thüringen, Wördehoff et al. (2012a) für Schleswig-Holstein, Wördehoff et al. (2012b) für Sachsen-Anhalt), aus denen trotz aller Unterschiede in den Berechnungsweisen, ein Gradient mit steigenden Kohlenstoffvorräten in der lebenden Baumbiomasse von Norden nach Süden zu erkennen ist. Im Gegensatz zu den bisher aufgeführten Kohlenstoffvorräten in bewirtschafteten Wäldern, beträgt er in unbewirtschafteten Wäldern nach einer Literaturanalyse von Klein und Schulz (2012) im Mittel $182 \pm 49,2 \mathrm{t} \mathrm{Cha}^{-1}$, wobei er in alten Wäldern (120 bis 200 Jahre) zwischen 140 und $230 \mathrm{t} \mathrm{C} \mathrm{ha}^{-1}$ liegt.

Auch zwischen den einzelnen Baumarten bestehen deutliche Differenzen. So können die Kohlenstoffvorräte in Buchenbeständen zwischen 120 t C ha $^{-1}$ in Nordrhein-Westfalen (Joosten et al., 2004) und 248 t C ha $^{-1}$ im Göttinger Wald in Niedersachsen (Rademacher et al., 2009) schwanken. Aus der Literaturstudie von Jacobsen et al. (2003) lässt sich für Eichenbestände ein durchschnittlicher Kohlenstoffvorrat von rund $92 \mathrm{t} \mathrm{Cha}^{-1}$ ableiten, ähnlich hoch ist er mit 96,4 $\mathrm{t} \mathrm{C}^{-1}$ Ende 2006 in Niedersachsen (Wördehoff et al., 2011). Aus Untersuchungen einzelner Fichtenbestände in Bayern (Ilg, 2002; Schwarzmeier, 2000; Weis und Göttlein, 2002) können Kohlenstoffvorräte zwischen 188 und 295 t C ha $^{-1}$ geschätzt werden. In niedersächsischen Fichtenbeständen ist er mit 86,7 t C ha ${ }^{-1}$ sehr viel geringer (Wördehoff et al., 2011). In umfangreichen Untersuchungen zur Kiefer ermittelten Heinsdorf und Krauß (1990) Kohlenstoffvorräte der oberirdischen Dendromasse vollbestockter Bestände in Abhängigkeit vom Bestandesalter und der Mittelhöhe. Danach hat beispielsweise ein 60-jähriger Kiefernbestand bei einer Mittelhöhe von 24 Metern einen Vorrat von 73,62 t $\mathrm{C} \mathrm{ha}^{-1}$. In Niedersachsen wurde ein geringerer Wert für die gesamte Baumbiomasse der Kiefernbestände von $67,7 \mathrm{t} \mathrm{C} \mathrm{ha}^{-1}$ ermittelt (Wördehoff et al., 2011). Für Douglasienbestände liegen hinsichtlich der Kohlenstoffvorräte nur wenige Analysen 
vor, jedoch kann aus der Studie von Jacobsen et al. (2003) ein Wert von rund 95 t C ha ${ }^{-1}$ in der lebenden oberirdischen Baumbiomasse hergeleitet werden. Die Kohlenstoffvorräte der lebenden ober- und unterirdischen Baumbiomasse von vier untersuchten Hauptwirtschaftsbaumarten in Niedersachsen, Sachsen-Anhalt und Schleswig-Holstein sind in Tabelle $1.1 \mathrm{zu}$ finden. Dabei wird sehr deutlich, welchen Einfluss der Altersklassenaufbau sowie standörtliche und baumartenspezifische Gegebenheiten auf den Kohlenstoffvorrat im Wald haben.

Ein einfacher Vergleich der zeitlichen Entwicklung der Kohlenstoffspeicherung der Hauptwirtschaftsbaumarten ist auf Grundlage der Angaben der Ertragstafelsammlung von Schober (1995) für Bestände erster Ertragsklasse und mäßiger bzw. starker (Douglasie) Durchforstung möglich, wobei grundlegende Unterschiede bei der Speicherung von Kohlenstoff im Wald aufgedeckt werden. Es wurden die gesamten Derbholzvorräte unter Berücksichtigung der baumartenspezifischen Raumdichten von Knigge und Schulz (1966), der Biomasse-Expansionsfaktoren von Pretzsch (2009) (s. Tab. 1.2) und einem mittleren Kohlenstoffgehalt des Holzes von $50 \%$ in den Kohlenstoffvorrat der lebenden Baumbiomasse umgerechnet. Die ertragstafelgemäße Entwicklung der Kohlenstoffvorräte über dem Alter wird bestimmt durch den Wachstumsgang, das Zuwachsniveau und die Raumdichte der Baumarten. Die zuwachsstarke Fichte ist trotz ihrer geringen Raumdichte zunächst der Kiefer und den Laubbaumarten überlegen. Erst im Alter 100 wird sie von der später im Zuwachs kulminierenden Buche eingeholt. Diese ist zunächst auch den früh startenden Baumarten Kiefer und Eiche unterlegen. Die Eiche kann ab dem Alter 60 aufgrund ihrer höheren Raumdichte die etwas zuwachsstärkere Kiefer übertreffen und bis zum Alter 120 immer mehr zu den Baumarten Fichte und Buche aufschließen (Wördehoff et al., 2011). Allen anderen Baumarten ist die Douglasie aufgrund ihres großen Zuwachses bei gleicher Ertragsklasse weit überlegen (s. Abb. 1.2). Generell ist bei einem Baumartenvergleich darauf zu achten, dass der gleiche Zeitraum betrachtet wird, um Fehlinterpretationen zu vermeiden.

Tabelle 1.1: Vergleich der Kohlenstoffvorräte $\left[\mathrm{t} \mathrm{C} \mathrm{ha}^{-1}\right]$ der lebenden Baumbiomasse der untersuchten Baumarten in Niedersachsen, Sachsen-Anhalt und Schleswig-Holstein im Jahr 2007 (Quellen: Wördehoff et al. (2011, 2012a,b)).

\begin{tabular}{lccc}
\hline Baumart & Niedersachsen & Sachsen-Anhalt & Schleswig-Holstein \\
\hline Eiche & 96 & 105 & 98 \\
Buche & 133 & 110 & 126 \\
Fichte & 87 & 93 & 89 \\
Kiefer & 68 & 67 & 85 \\
\hline
\end{tabular}


Tabelle 1.2: Baumartenspezifische Raumdichten $\left[\mathrm{t} \mathrm{m}^{-3}\right]$ und Biomasse-Expansionsfaktoren (Quellen: Knigge und Schulz (1966); Pretzsch (2009)).

\begin{tabular}{lcccc}
\hline Baumart & Raumdichte & Reisigfaktor & Blattfaktor & Wurzelfaktor \\
\hline Eiche & 0,5611 & & 1,05 & \\
Buche & 0,5543 & & 1,03 & \\
Fichte & 0,3771 & 1,45 & 1,00 & 1,25 \\
Douglasie & 0,4124 & & 1,00 & \\
Kiefer & 0,4307 & & 1,00 & \\
\hline
\end{tabular}

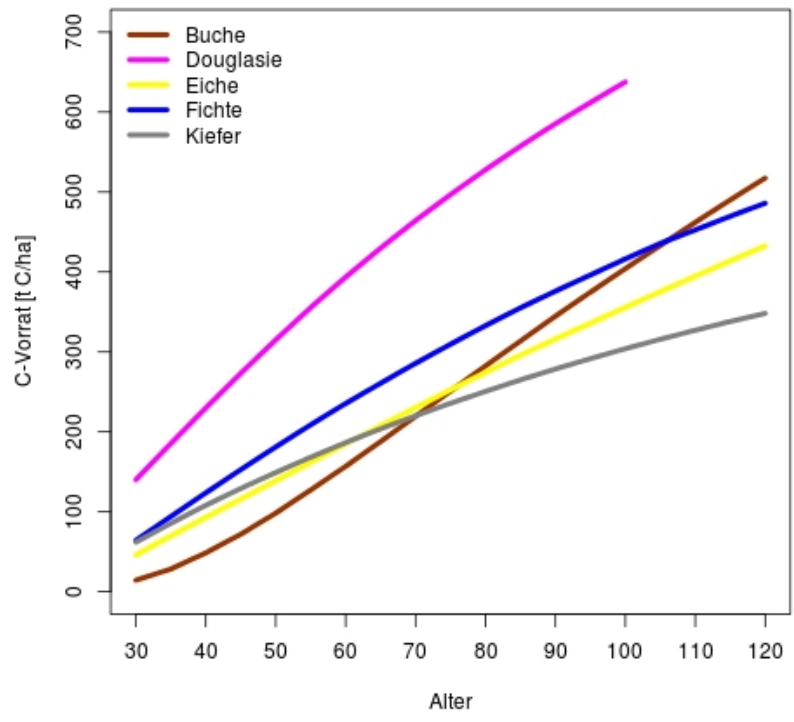

Abbildung 1.2: Kohlenstoffvorrat $\left[\mathrm{t} \mathrm{Cha}^{-1}\right]$ der lebenden Baumbiomasse der untersuchten Baumarten vom Alter 30 bis maximal 120 Jahren auf Grundlage von Ertragstafelangaben (Schober, 1995). 


\subsubsection{Literaturstudie zur toten Baumbiomasse}

Die tote Baumbiomasse ist ein wichtiger Bestandteil des Ökosystems Wald, worin sich große Mengen Kohlenstoff akkumulieren können. Pregitzer und Euskirchen (2004) geben für die Wälder der gemäßigten Zone einen Kohlenstoffvorrat im Totholzpool von $42 \mathrm{t} \mathrm{Cha}^{-1}$ an. Dieser vergleichsweise hohe Wert ist unter anderem auf das Aufnahmegebiet, die gemäßigte Zone in Nord- und Südamerika, zurückzuführen. Wirth et al. (2004a) ermittelten in ihrer Studie für extensiv genutzte Wälder in der borealen und gemäßigten Zone außerhalb Deutschlands einen Vorrat von 17,8 $\pm 16,9$ t C ha $^{-1}$. Für Nicht-Wirtschaftswälder in Mitteleuropa geben sie einen Kohlenstoffvorrat von 5,9 \pm $3,4 \mathrm{t} \mathrm{Cha}^{-1}$ und für Wirtschaftswälder von $2,1 \pm 1,4 \mathrm{t} \mathrm{C} \mathrm{ha}^{-1}$ an.

Bei der zweiten Bundeswaldinventur (Stichjahr 2002) wurde im bundesweiten Durchschnitt ein Totholzvorrat ohne Stubben von $11,5 \mathrm{~m}^{3} \mathrm{ha}^{-1}$ (Durchmesser $\geq 20 \mathrm{~cm}$ am stärkeren Ende, Wurzelstockhöhe ab $50 \mathrm{~cm}$ ) festgestellt. Für Niedersachsen, Hamburg und Bremen lag dieser Wert bei 8,2 $\mathrm{m}^{3} \mathrm{ha}^{-1}$ (BMELV, 2005). Nach den Ergebnissen der Inventurstudie 2008 hat sich der bundesweite Totholzvorrat auf insgesamt $15 \mathrm{~m}^{3} \mathrm{ha}^{-1}$ erhöht. Bei Zugrundelegung einer geringeren Aufnahmegrenze (Durchmesser $\geq 10 \mathrm{~cm}$ ) erhöht sich der Totholzvorrat auf bis zu $24 \mathrm{~m}^{3} \mathrm{ha}^{-1}$ ohne Stubben (Polley et al., 2009). Zu noch höheren Werten bei gleicher abgesenkter Aufnahmegrenze kommen Meyer et al. (2009) auf der Basis der Daten der zweiten Bodenzustandserhebung der Bundesländer Niedersachsen, Hessen und Sachsen-Anhalt. Der von ihnen bestimmte Vorrat an Totholz beläuft sich auf $28 \mathrm{~m}^{3} \mathrm{ha}^{-1}$. Die dritte Bundeswaldinventur (Stichjahr 2012, Durchmesser $\geq 10 \mathrm{~cm}$ am stärkeren Ende, Wurzelstöcke ab einem Schnittflächendurchmesser von $20 \mathrm{~cm}$ ) ermittelte bundesweit einen Totholzvorrat von 21,6 $\mathrm{m}^{3} \mathrm{ha}^{-1}$ (BMEL, 2014), in Niedersachsen beläuft er sich auf fast $17 \mathrm{~m}^{3} \mathrm{ha}^{-1}$ (ML, 2014). Geht man von den zuvor genannten Volumina aus und unterstellt eine mittlere Dichte von $0,25 \mathrm{t} \mathrm{m}^{-3}$ für Totholz sowie einen Kohlenstoffanteil von $50 \%$ an der Masse, so ergeben sich Kohlenstoffvorräte von rund 1,4 bis 3,5 t $\mathrm{Cha}^{-1}$. Dabei wird einmal der starke Einfluss der Kluppschwelle auf die Totholzvorräte und die in ihnen gespeicherten Kohlenstoffmengen deutlich, zum anderen dokumentieren sie einen Totholzanstieg zwischen 2002 und 2012 aufgrund der höheren Waldrestholzanteile, die im Zuge der stärkeren Nutzungen im Wald verblieben sind.

Die zeitliche Dynamik des Totholzpools wird bestimmt durch die Bildungs- und Zersetzungsraten des Totholzes. Eine Zunahme erfährt der Totholzvorrat durch die natürliche Mortalität und den Astabfall, abiotisch bzw. biotisch bedingte Ausfälle sowie durch menschliche Eingriffe. Die Zersetzung des Holzes ist der Hauptaustrag, welcher vor allem von Destruenten vorangetrieben wird und deren Atmung zu einer direkten Freisetzung von Kohlenstoff führt (Mackensen et al., 2003). Die Zersetzungsdauer von Totholz ist bei der Quantifizierung der Kohlenstoffspeicherung in Waldökosystemen ein wichtiger Aspekt. Beispielsweise veröffentlichte Rock (2008) kontinuierliche Zersetzungskonstanten für die Baumarten Eiche, Buche, Fichte und Kiefer in Europa. Zell et al. (2009) leiteten ein gemischtes nichtlineares Abbaumodell für Totholz auf der Basis einer Meta- 
analyse her, in das unter anderem der Ausgangsdurchmesser des Totholzes, die mittlere Temperatur im Juli und der Jahresniederschlag als erklärende Variablen eingehen. Meyer et al. (2009) haben in ihrer Arbeit ebenfalls Totholzzersetzungsfunktionen für Eiche, Buche, Fichte und Kiefer ableiten können.

\subsubsection{Literaturstudie zur Bodenvegetation}

Auch wenn zur Bodenvegetation keine eigene Untersuchung angestellt wurde, ist für diesen Pool aus Gründen der Vollständigkeit ebenfalls eine Literaturstudie durchgeführt worden. Der bestimmende Faktor für die Kohlenstoffspeicherung in der Bodenvegetation ist die auf den Waldboden treffende Strahlungsmenge, die sehr unterschiedlich sein kann (Böswald und Wierling, 1997). So erhält z. B. der Waldboden eines hallenartigen Buchenaltbestandes völlig andere Strahlungsmengen als der eines geschlossenen jungen Buchenbestandes mit tief ineinandergreifenden Kronen. Zur Ermittlung der Kohlenstoffvorräte in der Bodenvegetation liegen keine flächendeckenden Daten für Niedersachsen vor bzw. eine Regionalisierung dieser Daten aus Stichprobeninventuren ist schwierig. In einer Studie für Thüringen konnten Wirth et al. (2004a) Kohlenstoffvorräte für die Bodenvegetation berechnen. In Buchenbeständen hat dieser Speicher demnach einen Kohlenstoffvorrat von rund $0,30 \mathrm{tC} \mathrm{ha}^{-1}$, in Eichenbeständen ca. 1,69 t Cha ${ }^{-1}$, in Fichtenbeständen ca. 0,24 t C ha ${ }^{-1}$ und in Kiefernbeständen rund 1,46 t C ha-1 . Hier zeigen sich sehr deutliche Unterschiede zwischen den Lichtbaumarten und den anderen untersuchten Baumarten. Liegen flächendeckende Daten zur Bodenbedeckung mit entsprechender Datenqualität vor, wie im Falle von Wirth et al. (2004a), so ist es mit Hilfe der Funktionen von Bolte (1999) möglich, den Kohlenstoffvorrat für diesen Pool zu berechnen.

Insgesamt betrachtet ist der Kohlenstoffvorrat der Bodenvegetation im Vergleich zur lebenden Baumbiomasse sehr gering (Burschel et al., 1993; Wirth et al., 2004a). Aufgrund dessen, den fehlenden Daten sowie der geringen Lebensspanne des Großteils der Pflanzen der Bodenvegetation wird dieser Speicher oft vereinfachend als konstant angesehen und nicht weiter betrachtet (vgl. Pistorius, 2007a). Schulze et al. (2002) weisen allerdings darauf hin, dass die oberirdische Bodenvegetation in Mitteleuropa, vor allem im Frühjahr, eine Netto-Primärproduktion von 10 bis $20 \%$ derjenigen der Baumschicht erreicht, welche in Mitteleuropa bei etwa $10 \mathrm{t} \mathrm{C} \mathrm{ha}^{-1} \mathrm{a}^{-1}$ liegt (Wirth et al., 2004a). Daraus kann eine mittlere Speicherrate von $0,15 \mathrm{t} \mathrm{C} \mathrm{ha}^{-1} \mathrm{a}^{-1}$ abgeleitet werden.

\subsubsection{Literaturstudie zu Mineralboden und Auflagehumus}

Der Vollständigkeit halber wurde auch für die Speicher Mineralboden und Auflagehumus eine Literaturstudie durchgeführt. Diese sind weitere große Kohlenstoffspeicher im Wald. Der Vorrat an Kohlenstoff beträgt im Boden auf dem ersten Meter in den Wäldern der borealen Zone schätzungsweise 338 Mrd.t C, in der gemäßigten Zone etwa 153 bis $195 \mathrm{Mrd}$.t C und in der tropischen Zone rund 214 bis $435 \mathrm{Mrd}$.t C (Saugier et al., 2001). Das durchschnittliche Verhältnis des Kohlenstoffvorrates im Boden zur darauf 
befindlichen Vegetation beträgt in der borealen Zone etwa 5:1, in der gemäßigten 2:1 und in der tropischen 1:1 (Jarvis et al., 2005).

Bundesweit wurde in den ersten $30 \mathrm{~cm}$ des Mineralbodens zum Zeitpunkt der Inventurstudie 2008 ein Kohlenstoffvorrat von $68 \mathrm{t} \mathrm{C} \mathrm{ha}^{-1}$ ermittelt. Wobei er in Böden mit hohem Tongehalt größer war als in sandigen Böden. In der Streu von Laubwäldern lag der Kohlenstoffvorrat bei 12,1 t $\mathrm{Cha}^{-1}$, von Mischwäldern bei 18,3 $\mathrm{t} \mathrm{Cha}^{-1}$ und von Nadelwäldern bei 27,1 t Cha ${ }^{-1}$, im bundesweiten Mittel betrug er in der Humusauflage 19,8 t C ha-1 (Oehmichen et al., 2011). Aus den Daten der zweiten bundesweiten Bodenzustandserhebung und des BioSoil-Projektes konnten in der Streu und den ersten $30 \mathrm{~cm}$ des Mineralbodens ein Kohlenstoffvorrat von insgesamt 822 Mio. t C ermittelt werden (Wellbrock et al., 2010). Grüneberg et al. (2015) kamen auf der gleichen Datenbasis im Mineralboden bis $30 \mathrm{~cm}$ Tiefe zu einem Vorrat von $61,8 \pm 3,7 \mathrm{t} \mathrm{Cha}^{-1}$ und im Mineralboden von 18,8 \pm 0,3 $\mathrm{t} \mathrm{Cha}^{-1}$, in der Summe rund $81 \mathrm{t} \mathrm{Cha}^{-1}$ bzw. rund 826 Mio. $\mathrm{C}$.

In Thüringen wurden die Kohlenstoffvorräte in der organischen Auflage der Wälder auf rund 27,7 t C ha ${ }^{-1}$ geschätzt. Dabei war er in den Nadelwäldern im Mittel dreimal höher als in den Laubwäldern. Im Mineralboden (0 bis $60 \mathrm{~cm}$ ) waren rund 69,9 t $\mathrm{C} \mathrm{ha}^{-1}$ gespeichert, wobei die Werte unter Laubwald leicht höher waren als unter Nadelwald (Wirth et al., 2004a). In der Arbeit von Pistorius (2007a) wurden sehr viel höhere Kohlenstoffvorräte im Mineralboden von Baden-Württemberg durch Simulationsrechnungen ermittelt $\left(135,4\right.$ t $\left.\mathrm{C} \mathrm{ha}^{-1}\right)$, die sich allerdings auf keine bestimmte Tiefenstufe beziehen und daher schwer einzuordnen sind. In der Humusauflage in den niedersächsischen Wäldern waren die mittleren Kohlenstoffvorräte mit 27,6 t C ha ${ }^{-1}$ (Wördehoff et al., 2011) ähnlich hoch wie in Thüringen. Für die Mineralböden (0 bis $90 \mathrm{~cm}$ ) sowie die organische Auflage in den Wäldern von Niedersachsen konnte Evers (2016) auf Grundlage der zweiten Bodenzustandserhebung einen mittleren Kohlenstoffvorrat über alle Bestandestypen und ohne die Moorstandorte von $156 \mathrm{t} \mathrm{C} \mathrm{ha}^{-1}$ ermitteln. Auf Grundlage aller Punkte der zweiten Bodenzustandserhebung (inklusive der Moore) ergibt sich für die Auflage sowie den Mineralboden in Laubwäldern ein Kohlenstoffvorrat von insgesamt rund 164 t C, ha ${ }^{-1}$. In Nadelwäldern ist dieser Vorrat, aufgrund des größeren Kohlenstoffvorrates im Auflagehumus, rund $19 \mathrm{t} \mathrm{C}$, ha ${ }^{-1}$ höher (etwa $183 \mathrm{t} \mathrm{C} \mathrm{ha}^{-1}$ ). In Sachsen-Anhalt und Schleswig-Holstein betrugen die entsprechenden Werte in Laubwäldern rund 112 t $\mathrm{C} \mathrm{ha}^{-1}$ bzw. $155 \mathrm{t} \mathrm{Cha}^{-1}$ sowie in Nadelwäldern etwa $113 \mathrm{t} \mathrm{C} \mathrm{ha}^{-1}$ bzw. $175 \mathrm{t} \mathrm{Cha}^{-1}$ (Wördehoff et al., 2012a,b). In Mecklenburg-Vorpommern liegt der Median des Kohlenstoffvorrates im Gesamtboden bei rund $103 \mathrm{tCha}^{-1}$ (Russ et al., 2011). In der Humusauflage bzw. im Mineralboden bis $90 \mathrm{~cm}$ Tiefe der Wälder in Hessen waren zum Zeitpunkt der zweiten Bodenzustandserhebung 13,9 bzw. 68,9 t $\mathrm{C} \mathrm{ha}^{-1}$, insgesamt 82,8 t $\mathrm{C} \mathrm{ha}^{-1}$ gespeichert (Evers et al., 2011). Dabei wurde im Auflagehumus der Waldböden eine Abnahme und im Mineralboden eine Zunahme des Kohlenstoffvorrates festgestellt. Der Grund dafür ist eine höhere Abbaurate der Auflage, die durch viele unterschiedliche Faktoren (Zunahme trocken-warmer Jahre seit den 1980er Jahren, schwankende Niederschlagsmengen, hohe Stickstoffeinträge, geringere Säureeinträge, Waldkalkung) beeinflusst wurde. Im Mineralboden nehmen die Kohlenstoffvorräte aufgrund von erhöhter Bioturbation sowie 
intensiverer Durchwurzelung des Bodens durch die Naturverjüngung, und damit einem höheren Anteil toter Feinwurzeln, zu (Evers et al., 2011).

Die Kohlenstoffaufnahmerate des Bodens wird von Jandl et al. (2007) als gering eingeschätzt. Dies kann durch Auswertungen auf Grundlage der beiden bisher durchgeführten Bodenzustandserhebungen und anderer Datenquellen von verschiedenen Autoren in Deutschland nur bedingt bestätigt werden, auch wenn Inventuren des Kohlenstoffs im Boden mit großen Unsicherheiten verbunden sind (Monni et al., 2007; Peltoniemi et al., 2006; Luyssaert et al., 2010). So geben beispielsweise Russ et al. (2011) für die Böden in Mecklenburg-Vorpommern eine Fixierungsrate von 1,73 $\mathrm{t} \mathrm{Cha}^{-1} \mathrm{a}^{-1}$ an, wobei die Speicherung hauptsächlich im Mineralboden stattfand. In hessischen Wäldern konnte eine Speicherrate von etwa $0,33 \mathrm{t} \mathrm{Cha}^{-1} \mathrm{a}^{-1}$ für die Auflage und den Mineralboden bis $90 \mathrm{~cm}$ Tiefe ermittelt werden (Evers et al., 2011), während sie in Niedersachsen bei 1,35 t C ha ${ }^{-1} \mathrm{a}^{-1}$ liegt (Evers, 2015). In der Inventurstudie 2008 wurde deutschlandweit eine Speicherrate von 0,50 $\mathrm{t} \mathrm{Cha}^{-1} \mathrm{a}^{-1}$ in den ersten $30 \mathrm{~cm}$ des Bodens errechnet (Oehmichen et al., 2011). Zu dem gleichen Ergebnis kamen auch Wellbrock et al. (2010), mit einem abnehmenden Nord-Süd-Gradienten (Bolte et al., 2011; Grüneberg et al., 2015). Die organischen Waldböden (Moore u.a.) mit ihren sehr hohen Kohlenstoffvorräten emittierten allerdings gleichzeitig auch etwa $0,68 \mathrm{t} \mathrm{C} \mathrm{ha}^{-1} \mathrm{a}^{-1}$ (Oehmichen et al., 2011). Momentan geht man von einer durchschnittlichen Speicherrate des Mineralbodens bis $30 \mathrm{~cm}$ Tiefe in Höhe von 0,41 \pm 0,03 t C ha ${ }^{-1} \mathrm{a}^{-1}$ für ganz Deutschland aus. Für die Streu ergab sich eine, nicht von Null signifikant abweichende Rate, in Höhe von -0,02 $\pm 0,02$ t C ha ${ }^{-1} \mathrm{a}^{-1}$ (Grüneberg et al., 2015). Da die genannten Auswertungen nur auf zwei Zeitpunkten beruhen, bleibt abzuwarten, inwiefern diese teilweise sehr hohen Speicherraten, insbesondere der Sandböden, einmalig sind oder einen Trend darstellen.

Von verschiedenen Autoren (vgl. Liski et al., 2005; Lorenz und Lal, 2010; Pistorius et al., 2006) wird der Bodenspeicher als konstant angesehen. Sie gehen davon aus, dass er sich auch bei extremen waldbaulichen Behandlungen auf sehr lange Sicht kaum verändert. Jedoch kann innerhalb eines Bestandeslebens die Mächtigkeit der Humusauflage und damit ihr Kohlenstoffvorrat verhältnismäßig stark schwanken. Abgesehen davon gibt es eine große räumliche Heterogenität der Speicherung und anderer Faktoren, welche die Kohlenstoffspeicherung im Boden beeinflussen, die momentan nicht abgebildet werden können. Auch Wäldchen et al. (2013) kommen zu dem Schluss, dass frühere und heutige Eingriffe keinen nachweisbaren Einfluss auf die Menge des organisch gebundenen Kohlenstoffs haben. Allerdings zeigen Christophel et al. (2013) in ihrer Untersuchung der Stickstoff- und der organischen Kohlenstoffvorräte in der Auflage und dem Mineralboden in den Bayerischen Kalk-Alpen, dass durch die dortige Waldbewirtschaftung, große Mengen der untersuchten Elemente aus den flachen, kalkhaltigen Böden freigesetzt werden.

Kompensationskalkungen dienen der Verbesserung des Bodenzustandes und der Verhinderung einer weiter fortschreitenden Tiefenversauerung der Waldböden. Diese Effekte konnten Evers und Paar (2012) anhand von Daten aus Niedersachsen nachweisen. Allerdings kann eine Kalkung auch die Mineralisation anregen, d.h. den Abbau des Auf- 
lagehumus beschleunigen, was zu einer Abnahme des Kohlenstoffvorrates in diesem Teil des Bodenspeichers führt. Gleichzeitig gelangen die Abbau- sowie Umbauprodukte in den oberen Mineralboden, wo die Kohlenstoffvorräte langsam zunehmen (Evers et al., 2011). Auch Ammer und Huber (2007) konnten den Einfluss der Waldkalkung auf den Kohlenstoffvorrat der Humusauflage eines Fichtenbestandes nachweisen. Dabei sank er innerhalb von 21 Jahren nach der Kalkung von 38,1 auf 19,5 t C ha ${ }^{-1}$, wobei der Großteil (ca. $73 \%$ ) veratmet und nur ein geringer Teil in den Mineralboden (etwa 27\%) verlagert wurde. Dabei ist der überwiegende Abbau der Humusauflage kritisch zu betrachten, denn dadurch entstehen Kohlenstoffverluste und das Sickerwasser wird mit erheblich mehr Nitrat belastet (Ammer und Huber, 2007). In Buchenwäldern in Niedersachsen konnten Evers et al. (2008) den Einfluss der Kalkung auf den Kohlenstoffvorrat in der Auflage und im Mineralboden bis $10 \mathrm{~cm}$ Tiefe nachweisen. Dabei blieb der mittlere Kohlenstoffvorrat auf den gekalkten Parzellen im Auflagehumus auf ähnlichem Niveau und im Mineralboden fand eine Anreicherung statt. Somit konnten die Kohlenstoffvorräte durch die Kompensationskalkungen stabilisiert werden. Was sich dort auf Versuchsflächen zeigt, hat Evers (2015) auch in einer Auswertung der Bodenzustandserhebungen von Niedersachsen und Hessen für die Substratgruppen Buntsandstein sowie unverlehmte Sande gefunden. Es zeigt sich, dass die Waldkalkung auf den Buntsandsteinstandorten den Kohlenstoffvorrat im Auflagehumus signifikant verringert und im Mineralboden bis $30 \mathrm{~cm}$ erhöht. Auf den gekalkten Standorten aus unverlehmten Sanden fand ein Anreicherung von Kohlenstoff im Auflagehumus und im Mineralboden bis $30 \mathrm{~cm}$ statt.

\subsubsection{Literaturstudie zu Holzprodukten}

Der Produktpool stellt neben dem Wald einen weiteren wichtigen Speicher für Kohlenstoff dar, der in dieser Studie betrachtet wird. Holzprodukte leisten einen nennenswerten Beitrag zur Reduzierung der Kohlenstoffdioxidkonzentration in der Atmosphäre, indem durch die stoffliche Holznutzung die natürliche Freisetzung dieses Treibhausgases durch Zersetzung verzögert wird. Außerdem substituieren Holzprodukte sowohl andere Bauund Werkstoffe, die in der Herstellung energieintensiver sind und größere Kohlenstoffdioxidemissionen verursachen, als auch als Energieholz fossile Brennstoffe (Köhl et al., 2008; Rüter, 2010). Durch den steigenden stofflichen Holzverbrauch konnten in den vergangenen Jahren bundesweit durchschnittlich 10 Mio. t Kohlenstoffdioxidemissionen pro Jahr und durch die wachsende energetische Holznutzung jährlich weitere 30 Mio. t Kohlenstoffdioxid zusätzlich vermieden werden (Rüter, 2010). Bisher wurde der Produktpool im Zuge der Berichtspflichten zum Kyoto-Protokoll nicht in die Bilanzierung der Kohlenstoffvorräte im Forstsektor einbezogen. Dies wird jedoch im modifizierten KyotoNachfolgeprozess nicht mehr der Fall sein, da die stoffliche Nutzung von Holzprodukten bei der Anrechnung der Treibhausgasemissionen Berücksichtigung finden wird (BMELV, 2012a).

In einer Schweizer Studie wurde für die Holzprodukte im Zivilisationskreislauf ein Kohlenstoffvorrat von rund 27,5 Mio.tC (101 Mio.t $\mathrm{CO}_{2}$ ) im Jahr 2000 geschätzt (Taverna 
et al., 2007). Böswald und Wierling (1997) berechneten für den niedersächsischen Holzproduktpool eine Speichermenge von 23,8 Mio.t C im Jahr 1993. Pistorius (2007a) kam für Baden-Württemberg auf der Basis statistischer Angaben zur Gebäudezahl und den Nutzungsmengen zwischen der ersten und zweiten Bundeswaldinventur zu einer Speicherhöhe von 49,85 Mio. t C für den Holzproduktpool. Weiterhin konnte er eine jährliche zusätzliche Speicherung von etwa 0,85 Mio.t C ermitteln. Die mittlere Kohlenstoffbindung im verkauften Holz des Thüringer Landeswaldes betrug in den Jahren 2001 und 2002 rund 0,21 Mio. t C (Mund et al., 2006). In Bayern konnten im Zeitraum von 2002 bis 2008 ca. 1,3 Mio. t $\mathrm{C} \mathrm{a}^{-1}$ in Holzprodukten gespeichert werden (Klein und Schulz, 2012).

Die Absatzwege des sägefähigen und nicht sägefähigen Rohholzes sind wichtige Informationen zur Einschätzung der Kohlenstoffbindung in Holzprodukten. Holzrohstoffbilanzen für Deutschland sind von Mantau (2012) und Weimar (2014) aufgestellt worden. Erstmalig sind auch regionale Holzrohstoffbilanzen für einige Bundesländer Norddeutschlands von Mantau (2015a) verfügbar. Auch für Thüringen gibt es aus einer Untersuchung Näherungswerte zur Holzverwendung (Profft et al., 2009). Allerdings kann aus diesen Bilanzen das fertige Holzprodukt nicht direkt abgeleitet werden, sondern nur die erste Verarbeitung- bzw. Verwendungsstufe für einen bestimmten Zeitpunkt. Auch ist daraus nicht der Binnenhandel innerhalb Deutschlands erkennbar. Besser ist die Informationslage hinsichtlich der mittleren Lebensdauer einzelner Produktklassen (Wirth et al., 2004b; Profft et al., 2009; Mund et al., 2006).

Bei einer vollständigen Nutzung des Holzes als langlebiges Produkt (Materialsubstitution), mit darauf folgender energetischer Verwendung (Energiesubstitution), ergibt sich ein maximales Substitutionspotenzial von 1,238 t C pro t Holz (Rock, 2008). Dieser Wert stellt eine Obergrenze dar, da nicht alles Holz zu langlebigen Produkten verarbeitet wird, nicht immer fossile Brennstoffe ersetzt werden und eine Verbrennung nicht zwangsläufig von Nutzen sein muss (Rock, 2008). In jedem Fall ist der Trend zu beobachten, dass Holz als Energieträger immer wichtiger wird. Nach Mantau (2012) hat der Anteil der energetischen Nutzung mit 50,6\% an der gesamten Holzverwendung den Anteil der stofflichen Nutzung mit 49,4\% überholt und die Tendenz ist weiter steigend. Im Jahr 2002 betrug demgegenüber dieser Anteil lediglich 26\% (Mantau, 2004). Auch in Niedersachsen ist Holz als Energieträger immer wichtiger geworden. Allein durch den Einsatz des Energieträgers Holz konnten 2013 in etwa 1,3 Mio. Feuerungsanlagen rund 12,3 Mrd. kWh substituieren und damit den Kohlenstoffdioxidausstoß in Niedersachsen um mehr als 3,8 Mio.t verringern (3N, 2014).

Der Konflikt zwischen stofflicher und energetischer Holznutzung, bei steigender Nachfrage, kann durch die Kaskadennutzung des Holzes entschärft werden. Frühwald et al. (2010) verstehen unter einer Kaskadennutzung im klassischen Sinn die stoffliche Nutzung eines Stoffes über mehrere Stufen. Wobei im ersten Produkt die stofflichen Eigenschaften auf dem höchsten Niveau genutzt werden. Am Ende der Lebensdauer eines Produktes erfolgt eine Aufarbeitung, um es anschließend zu einem anderen Produkt zu verarbeiten. Dabei stellt das neue Produkt in der Regel geringere Anforderungen an die stofflichen Eigenschaften des Rohstoffes als das vorherige. Allerdings ist die Häufigkeit der Wieder- 
verwertung abhängig vom eingesetzten Rohstoff sowie von technischen, sozialen, ökologischen und ökonomischen Rahmenbedingungen. Durch eine Kaskadennutzung wird die stoffliche Ressource durch wiederholten Einsatz optimal genutzt und zeitgleich werden die geringer werdenden Qualitäten durch die verminderten Anforderungen an die Folgeprodukte berücksichtigt (Frühwald et al., 2010). In der Realität gibt es oftmals keine echte Kaskadennutzung, denn auf den verschiedenen Nutzungsstufen finden stoffliche und energetische Verwendung gleichzeitig statt.

Frühwald et al. (2010) haben in ihrer Arbeit eine Kaskadennutzung beispielhaft für Holz dargestellt. Für das erste Produkt, etwa konstruktives Bauholz, wird Schnittholz benötigt, welches beim kontrollierten Rückbau von Häusern als Altholz aussortiert werden kann. Dieses Altholz kann anschließend für die Spanplattenproduktion genutzt werden. In Deutschland fallen jährlich 8 Mio. t Altholz an, von denen rund $80 \%$ energetisch und $20 \%$ stofflich weiterverwendet werden. Die energetische Verwertung von Holz stellt die letzte Nutzungsstufe dar. Durch die Kaskadennutzung wird die Gesamtnutzungsdauer des Holzes und damit die Kohlenstoffspeicherung in Holzprodukten verlängert, ohne dass die Holzentnahme aus den Wäldern erhöht werden muss (Frühwald et al., 2010).

Die Herleitung des Kohlenstoffvorrates im Holzproduktpool stellt sich jedoch im Vergleich zu den anderen Speichern ungleich schwieriger dar. Die Probleme beginnen mit der Einteilung der Holzprodukte in Klassen entsprechend ihrer mittleren Lebensdauer und der Zuordnung des eingeschlagenen Holzes zu den zuvor gebildeten Kategorien. Anschließend müssen die Absatzwege und Zersetzungspfade des Holzes bzw. der Holzprodukte sowie Substitutionsfaktoren für andere Materialien und energetische Rohstoffe ermittelt werden. Dabei ist die Datengrundlage meist mangelhaft oder wenig geeignet, sowie die Zuordnung der eingeschlagenen Sortimente zu Holzprodukten nur mit starken Vereinfachungen möglich. Hinzu kommt, dass der aktuelle Kohlenstoffvorrat des Holzproduktspeichers unbekannt ist.

\subsection{Strategische Waldbauplanung}

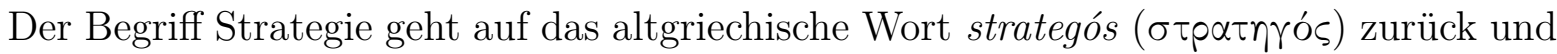
bedeutet Feldherr oder Kommandant. Diesen hochgestellten Entscheidungsträgern oblag es, militärische Aktionen vorausschauend zu planen, um zukünftige Erfolge zu sichern. Übertragen auf den Forstbetrieb bedeutet dies, dass es die Aufgabe des strategischen Managements ist, frühzeitig und systematisch Potenziale zur Sicherung künftiger Erfolge und der Entwicklungsfähigkeit aufzubauen. Es beschäftigt sich damit, „die richtigen Dinge zu tun" und ist Teil der Führung von Forstbetrieben, wobei strategische Entscheidungen vorrangig als Aufgabe des obersten Managements anzusehen sind (Oesten und Roeder, 2012).

Ein Planungsprozess beinhaltet zu Anfang eine umfassende Analyse des Ausgangszustandes (Strategische Analyse). Dazu dienen im Forstbereich im Allgemeinen verschiedene Informationsquellen: Forsteinrichtungs-, Fernerkundungs-, Boden- und Standortsda- 
ten sowie Informationen von Dauerbeobachtungsflächen. Da hier der Einfluss verschiedener waldbaulicher Konzepte auf die Kohlenstoffspeicherung im Wald und in Holzprodukten in Niedersachsen untersucht werden soll, sind die Ergebnisse der Kohlenstoffstudie Forst und Holz Niedersachsen (Wördehoff et al., 2011) die Basis dieser Arbeit. Die Bewertung von Auswirkungen waldbaulicher Handlungsalternativen kann durch die Nutzung EDV-gestützter Instrumente erfolgen, wobei es im forstlichen Bereich Wuchsmodelle sind, mit denen die Folgen strategischer Entscheidungen beurteilt werden (Schmithüsen et al., 2009). Mit ihnen und geeigneten quantitativen Indikatoren lassen sich die Auswirkungen verschiedener Behandlungsalternativen einschätzen. Im Allgemeinen sind Weiserbestände die Grundlage, um die naturalen, ökonomischen und ökologischen Konsequenzen der erarbeitenden Behandlungsszenarien zu analysieren und gegebenenfalls zu überdenken oder zu adjustieren (Pretzsch und Pott, 2004). Die Ergebnisse können als Diskussionsgrundlage zur Zielfindung und Zielvereinbarung dienen (Pretzsch, 2003), d. h. es kann die für den Betrieb beste Option gewählt werden (Strategieformulierung). Die gewählte Strategie muss danach in konkreten Handlungen des Forstbetriebes münden, wobei die Umsetzung von der Akzeptanz der gewählten Option abhängt (Strategieimplementierung). Die grundsätzlichen Auswirkungen verschiedener waldbaulicher Behandlungen auf die Kohlenstoffspeicherung in Wäldern kann anhand der Kohlenstoffstudie Forst und Holz Niedersachsen gezeigt werden (Wördehoff et al., 2011). Dabei wurden die Auswirkungen von drei unterschiedlichen Szenarien, ertragsorientiert, naturnah und naturschutzorientiert, in einem 30-jährigen Zeitraum analysiert. Das ertragsorientierte Szenario verringert durch stärkere Eingriffe die Kohlenstoffvorräte in der lebenden Baumbiomasse deutlich im Vergleich zu den anderen Varianten. Bei der naturschutzorientierten Behandlung werden die höchsten Vorräte von Kohlenstoff im Bestand akkumuliert, jedoch wird die Senkenleistung immer geringer, da der Volumenzuwachs nachlässt und die aufgeschobenen Eingriffe verzögert durchgeführt werden. Dadurch kommt es zu einem zeitlich verzögerten Abbau der Kohlenstoffvorräte. Die naturnahe Variante bleibt dem Prinzip einer nachhaltigen, multifunktionalen Forstwirtschaft treu. Durch sie werden die Kohlenstoffvorräte im Bestand auf einem hohen Niveau gehalten und gleichzeitig wird ein großer Beitrag zur Kohlenstoffsequestrierung im Produktpool geleistet (Wördehoff et al., 2011). Da sich die naturnahe Variante im Kontext dieser Arbeit als vorteilhaft erwiesen hat, soll sie als Grundlage für weitere Betrachtungen dienen.

Strategische Entscheidungen sind durch ihren Zukunftsbezug mit hohen Risiken behaftet. Für den Forstbetrieb mit seinen extrem langen Produktionszyklen ist dieser Aspekt sehr wichtig. Denn Veränderungen im Wuchsverhalten der Baumarten, bedingt durch klimatische Standortverschiebungen, in waldbaulichen Verfahren, in Marktbedingungen sowie wechselnden Waldeigentümern mit veränderlichen Zielen und sich verschiebenden Ansprüchen der Gesellschaft an den Wald, sind die mit vielen Unsicherheiten behafteten Rahmenbedingungen (Bitter, 2004). Daher bedürfen strategische Entscheidungen einer ständigen Überprüfung hinsichtlich der Realisierbarkeit sowie Eignung, um das formulierte Ziel zu erreichen (Strategische Kontrolle). Wobei diese als laufende Validierung zu verstehen ist (Oesten und Roeder, 2012). Es gilt der Grundsatz: Kontrolle 
ohne Planung ist unmöglich und Planung ohne Kontrolle ist sinnlos. Darüber hinaus sind auch längerfristige Konsequenzen der Produktionsplanung auf die Nachhaltigkeit des Forstbetriebes von Interesse (Riechsteiner und Lemm, 2009). Dass die Rahmenbedingungen nicht konstant sind, kann vortrefflich anhand der temporär unterschiedlichen gesellschaftlichen Ansprüche an den Wald gezeigt werden. Anfang des 19. Jahrhunderts stand das Gleichmaß der Holzerträge, d. h. fortwährend gleichmäßig hohe bzw. steigende Massenerträge, im Vordergrund (Hartig, 1804), gefolgt von der Anforderung stetiger Holzerzeugung. Dies bedeutet eine Dauerbestockung der Flächen der Forstbetriebe und damit die Erhaltung einer kontinuierlichen Produktion (Heyer, 1841). Die Generierung dauernder, gleichmäßig hoher Gewinne bzw. Geldbeträge wurde von Ostwald (1931) zu Beginn des 20. Jahrhunderts gefordert. Kurze Zeit später, nach dem Zweiten Weltkrieg, entwickelte sich die deutsche Forstwirtschaft, mit Fokus auf die Versorgungssicherheit, zu einer multifunktionalen Forstwirtschaft. Heute stehen die Möglichkeiten der Vielfachnutzung des Waldes im Mittelpunkt (Spellmann et al., 2004). Dazu zählt auch die Kohlenstoffspeicherung in Wäldern und in Holzprodukten bzw. deren Nutzen für jetzige und zukünftige Generationen. Daher fand dieser quantitative Indikator Eingang in den Kriterien- und Indikatorenkatalog einer nachhaltiger Forstwirtschaft in Europa (MCPFE, 2011). Denn die Kohlenstoffspeicherung im Forst- und Holzsektor ist ein Beitrag zum andauernden, gesellschaftlich geforderten Klimaschutz und daher ein wichtiges Teilziel der strategischen Waldbauplanung. Dabei dürfen allerdings andere Regelungs-, Lebensraum-, Nutz-, Kultur- und Sozialfunktionen der Wälder sowie Risiken nicht vernachlässigt werden. Auch die Niedersächsischen Landesforsten haben die Kohlenstoffspeicherung als besondere Waldfunktion erkannt. Diese soll durch eine nachhaltige sowie naturnahe Bewirtschaftung der niedersächsischen Landeswälder gesichert werden (NLF, 2011).

Um Waldentwicklung nachhaltig mittel- und langfristig zu steuern, muss der Forstbetrieb Leitbilder formulieren, periodische Nutzungsmengen bestimmen und Prinzipien der Waldbehandlung festlegen (Gadow, 2005). In Niedersachsen stellte nach dem Zweiten Weltkrieg Borchers (1952) erste konkrete Überlegungen zur waldbaulichen Planung an. Er erkannte in Betriebszieltypen ${ }^{3}$, heute auch Waldentwicklungstypen ${ }^{4}$, die Grundlage der zukünftigen Wirtschafts- sowie Betriebsführung und weist auf die Bedeutung von Forsteinrichtung und Standortskartierung als Planungsgrundlage hin. Diese Vorstellungen wurden immer weiterentwickelt bis im Jahr 1991 die niedersächsische Landesregierung das Programm zur Langfristigen, ökologischen Waldentwicklung, kurz LÖWE, beschloss und es zuletzt 2013 aktualisierte (ML, 2013). Es ist das Leitbild der Niedersächsischen Landesforsten (Merker, 2015) und wirkt in alle Bereiche der Bewirtschaftung des Landeswaldes hinein. Auch wenn das LÖWE-Programm anfangs nicht geeignet erschien

\footnotetext{
${ }^{3}$ Ein Betriebszieltyp ist eine waldbautechnische langfristige Zielsetzung für Einzelbestände, wobei sich die Wahl des Typs an standörtlichen, betriebswirtschaftlichen und forstpolitischen Kriterien orientiert (Gadow, 2005).

${ }^{4}$ Ein Waldentwicklungstyp ist durch bestimmte Ziele hinsichtlich der Mischungsanteile verschiedener Baumarten, deren Produktionszeiträume und Zielsortimente definiert (Gadow, 2005).
} 
den Reinertrag des Forstbetriebes zu maximieren (Spellmann, 1997), so konnten seit 2006 im Produktbereich 1 - Produktion von Holz und anderen Erzeugnissen - durch veränderte Rahmenbedingungen (Organisationsreform, Personalabbau, altersbedingte Sortimentsverschiebungen, höhere Rohholzpreise) Gewinne erzielt werden. Wobei gleichzeitig die Zuwendungen des Landes Niedersachsen in den anderen Produktbereichen sanken. Darüber hinaus ist dieses Leitbild eine gute Orientierungshilfe und fördert den Ausgleich zwischen Ökologie, Ökonomie und Sozioökonomie (Spellmann, 2015). Als Teilstrategie beinhaltet das LÖWE-Programm die Gewährleistung besonderer Waldfunktionen, worin die Funktion des Waldes als Kohlenstoffspeicher eingebunden ist.

Die Produktions- und Nutzungsplanung des Forstbetriebes bieten im Hinblick auf das Teilziel der Kohlenstoffspeicherung im Wald und in Holzprodukten entscheidende Steuerungsmöglichkeiten. Hierbei sind für ihre Umsetzung fundierte waldbauliche Kenntnisse nötig (Knoke et al., 2012), welche, als Kennzeichen der ordnungsgemäßen Forstwirtschaft, auf den gesicherten Erkenntnissen der Wissenschaft und den bewährten Regeln der Praxis beruhen ( $\$ 11$ NWaldLG).

In der Produktionsplanung oder -regelung sind alle Maßnahmen der Holzerzeugung und die Bereitstellung von Infrastruktur- sowie Ökosystemdienstleistungen vereint (Speidel, 1972). Die Baumartenwahl ist im Rahmen der Produktionsplanung die wichtigste langfristige ökologische sowie ökonomische Entscheidung im Forstbetrieb, welche heutige und zukünftige Generationen betrifft. Sie bestimmt wesentlich den Ertrag, den Aufwand und die Risiken des Forstbetriebes (Speidel, 1972) sowie viele andere Waldfunktionen (z. B. Bestandesaufbau und -stabilität, Biodiversität, Landschaftscharakter, etc.) (Röhrig et al., 2006) für mindestens eine Umtriebszeit. Die Entscheidung für oder gegen eine Baumart ist eine ausgeprägte lokale oder regionale Herausforderung (Mayer, 1992). Dabei sind die standörtlichen Verhältnisse, charakterisiert durch das Klima, physikalische und chemische Bodeneigenschaften sowie die Bodenvegetation, und die waldbaulichen Ausgangsbedingungen bestimmend, denn diese legen die realen Möglichkeiten fest. In der Forstlichen Standortskartierung wird der Standort ${ }^{5}$ erfasst und fließt in die Waldbauplanung mit standortgemäßen Baumarten ein. Standortgemäß sind Baumarten, deren Ansprüche an Klima und Boden den natürlichen Eigenschaften des Standorts angemessen sind, sie ein entsprechendes Wachstum zeigen und dabei ihr natürliches Lebensalter ohne erhebliche Risiken erreichen können, sie sich natürlich verjüngen lassen sowie den Bodenzustand nicht verschlechtern (Burschel und Huss, 2003; Röhrig et al., 2006). Aber auch die Ziele des Waldbesitzers, welche sich an ökonomischen, ökologischen und gesellschaftlichen Werten orientieren, sind von sehr großer Bedeutung in Bezug auf die Baumartenwahl und der waldbaulichen Behandlung der Bestände. Weitere wichtige Instrumente der Produktionsplanung sind die Jungbestandspflege, die Wertästung, die Kompensationskalkung, der Unter- und der Voranbau sowie die Umwandlung und die Überführung von Beständen (Burschel und Huss, 2003; Knoke et al., 2012; NLF, 2005;

\footnotetext{
${ }^{5}$ Der forstliche Standort ist die Gesamtheit der für das Baumwachstum bedeutsamen Umweltbedingungen (Boden, Klima, Lage).
} 
Röhrig et al., 2006; Speidel, 1972).

Alle Entscheidungen über die Art und Menge des geernteten Holzes werden der Nutzungsplanung zugerechnet (Speidel, 1972) und es wird zwischen Vor- und Endnutzungen unterschieden. Während Vornutzungen bzw. Durchforstungen das Wertholzpotenzial des vorhandenen Bestandes optimieren sollen, ist die Integration von Holzernte und Verjüngung das entscheidende Merkmal von Endnutzungen (Röhrig et al., 2006). Welche Art der Vornutzung (Jungbestandspflege, Jung- oder Altdurchforstung) Anwendung findet, ergibt sich aus dem aktuellen Zustand des Bestandes und den Abweichungen vom Produktionsziel, wobei auch Dringlichkeit und Häufigkeit der Pflegemaßnahmen eine Rolle spielen. Mit der Endnutzung beginnt die Erntephase und die Dimensionierung der Einzelbäume endet, gleichzeitig wird eine neue Verjüngung mit eigener Dynamik eingeleitet. Dabei kann die Endnutzungsentscheidung anhand folgender Kriterien geprüft werden: Produktivität: Wird der vorhandene Bestand im weiteren Verlauf einen angemessenen Zustand erreichen oder ist ein Voranbau notwendig?; Stabilität: Ist die Bestandesstabilität ausreichend oder der Bestand nicht standortgemäß?; Durchmesserentwicklung: Ist der Zieldurchmesser unter Beachtung der Bonitäten und Qualitäten auf dem Großteil der Bestandesfläche erreicht?; Verjüngungsdynamik: Bedarf es besonderer Maßnahmen, um die geplante Bestockung zu realisieren und so hohe Folgekosten zu vermeiden? (Knoke et al., 2012).

Der Waldbauplanung kommt mit den Aspekten der Produktions- und Nutzungsplanung in der strategischen Ausrichtung der Forstbetriebe eine hohe Bedeutung zu. Sie beeinflusst maßgeblich die zukünftige Entwicklung des Produktionsmittels Baum, welcher gleichzeitig auch das Produkt ist. Somit sind auch die Einnahmen der Forstbetriebe aus dem Holzverkauf (ca. $90 \%$ der Einnahmen) und die Ausgaben, welche in unmittelbarem Zusammenhang mit der Holzproduktion und -nutzung stehen (etwa 50 bis $60 \%$ der Ausgaben), betroffen (Oesten und Roeder, 2012). Das hat gleichzeitig Auswirkungen auf die Liquidität der Betriebe und andere Faktoren wie beispielsweise die Baumartenzusammensetzung oder die Kohlenstoffspeicherung der Wälder.

\subsection{Zielsetzung}

Das Thema der Kohlenstoffspeicherung ist klimapolitisch hochaktuell, wobei Wälder oft eine Senke für dieses Element darstellen. In Deutschland hat bislang ein Vorratsaufbau mit entsprechend steigender Senkenleistung stattgefunden. Ziel dieser Arbeit ist es, zur Versachlichung der Diskussionen über die Senken- bzw. Quellfunktion von Wäldern für Kohlenstoff beizutragen. Hierzu sollen die Sequestrierungsbeiträge der Hauptbaumarten in Niedersachsen in Abhängigkeit von den Standortverhältnissen, der Bonität und der waldbaulichen Behandlung exemplarisch hergeleitet werden, um so die direkten waldbaulich möglichen Einflüsse auf die Kohlenstoffspeicherung in der lebenden und toten Biomasse sowie im Produktspeicher besser quantifizieren zu können (vgl. Krug et al., 2012). Infolgedessen kann auf den Ergebnissen der Clusterstudie und der Kohlenstoffstu- 
die Forst und Holz Niedersachsen (Rüther et al., 2007; Wördehoff et al., 2011) aufgebaut werden. Da die Niedersächsischen Landesforsten eine umfangreiche Datengrundlage zur Verfügung stellen, beziehen sich die Ergebnisse vornehmlich auf den Landeswald.

Im Einzelnen sollen folgende Arbeitshypothesen überprüft werden:

- Die Höhe der Kohlenstoffbindungsraten ist baumarten-, alters- und bonitätsabhängig.

- Die waldbauliche Behandlung hat Einfluss auf die Kohlenstoffbindungsraten der Speicher im Wald.

- Die waldbauliche Behandlung beeinflusst die Kohlenstoffspeicherraten des Holzproduktpools.

Als Grundlagen für die Hypothesenüberprüfung soll folgenden Fragen nachgegangen werden:

- Was leisten unsere Hauptbaumarten auf den wichtigsten Standorten in ausgewählten niedersächsischen Wuchsbezirken?

- Wie können realitätsnahe Weiserbestände der betrachteten Baumarten mit unterschiedlicher Leistung generiert werden?

- Wie lassen sich für die Hauptbaumarten maximale Grundflächenhaltungen herleiten, die zur grundflächenbezogenen Steuerung der Durchforstung genutzt werden sollen?

- Wie lassen sich der Holzverwendungsschlüssel für Niedersachsen verbessern und die Auswirkungen einer Kaskadennutzung von Holzprodukten einschließlich ihrer Substitutionseffekte abbilden?

- Wie hoch ist die standortabhängige Senkenleistung unserer Hauptbaumarten bei unterschiedlicher Waldbehandlung?

- Welche Konsequenzen ergeben sich aus dem Baumartenvergleich für die strategische Waldbauplanung? 


\section{Material}

\subsection{Untersuchte Baumarten}

Die Untersuchungen in dieser Arbeit beziehen sich auf die fünf wirtschaftlich wichtigsten Baumarten in Niedersachsen. Hierbei handelt es sich um Eiche (Quercus robur L. und Quercus petraea (Mattuschka) Liebl.), Buche (Fagus sylvatica L.), Fichte (Picea abies [L.] Karst.), Douglasie (Pseudotsuga menziesii [Mirb.] Franco) und Kiefer (Pinus sylvestris L.). Die Flächenanteile der entsprechenden Baumartengruppen an der gesamten Holzbodenfläche (1.161.444 ha) betragen landesweit $13 \%$ für die Eiche, $14 \%$ für die Buche, $17 \%$ für die Fichte, $2 \%$ für die Douglasie und $29 \%$ für die Kiefer, wobei die geographische Verteilung sehr unterschiedlich ist. Gründe dafür sind die sehr verschiedenen natürlichen Bedingungen sowie forstgeschichtliche Entwicklungen und Eigentumsverhältnisse. Das niedersächsische Bergland wird durch Buche und Fichte geprägt. Im ost- und westniedersächsischen Tiefland ist die Charakterbaumart die Kiefer, gefolgt von anderen Laubbäumen mit niedriger Lebensdauer (vornehmlich Birke, Erle, Pappel, Weide und Eberesche) sowie Eiche. Auf ärmeren Standorten wird bei der Bestandesverjüngung verstärkt auf Douglasie zurückgegriffen und somit erreicht sie im westniedersächsischen Tiefland einen Anteil am Holzboden von rund $4 \%$ (ML, 2014).

Das Verbreitungsgebiet der Stieleiche (Quercus robur L.) liegt im mitteleuropäischen, gemäßigt kontinentalen Klima, mit einem Optimum in Auengebieten und bodenfeuchten Ebenen. Die Traubeneiche (Quercus petraea (Mattuschka) Liebl.) kommt in Deutschland typischerweise in einigen Mittelgebirgen (Pfälzer Wald, Spessart, Ostharz, Nordthüringen) und in Teilen des niedersächsischen Flachlands (z. B. Göhrde) vor, wo sie ursprünglich weiter verbreitet war. Beide Arten stellen keine großen Anforderungen an die Nähstoffversorgung, unterscheiden sich jedoch deutlich hinsichtlich der Anforderungen an die Wasserversorgung voneinander. Dies kann auch aus den Verbreitungsgebieten abgeleitet werden (Röhrig und Bartsch, 1992). Die Stieleiche gilt gegenüber der Traubeneiche generell als feuchtigkeits- und nährstoffbedürftiger (Otto et al., 2007). Beide Baumarten haben allerdings gleich hohe Ansprüche an Wärme und Licht. Im Landeswald von Niedersachsen stocken Eichen auf etwa 13\% der Holzbodenfläche (ca. 39.000 ha). Allerdings sind Reinbestände aus Trauben- oder Stieleiche nur auf rund 11.600 ha vertreten. Es überwiegen junge (Alter $\leq 40$ Jahre) und alte Eichenbestände (Alter $\geq 100$ Jahre). Von den alten Eichenbeständen weist die Mehrzahl Vorausverjüngungen vor allem aus Buche auf. Der große Anteil junger Eichenbestände erklärt sich durch Wiederaufforstungen nach dem Orkantief Quimburga vom 13. November 1972 und im Allgemeinen 
aus den Bemühungen zur Erhaltung des Flächenanteils dieser Baumart (NLF, 2014b).

Die Buche (Fagus sylvatica L.) ist eine Schattbaumart des atlantischen und subkontinentalen Klimas. Im Norden Deutschlands kommt sie bis an die Küste mit stetig steigender Vitalität vor. Im Nordharz ist sie bis auf ca. $600 \mathrm{~m}$ und im Südharz bis auf $800 \mathrm{~m}$ über Normalnull zu finden. Für ein gutes Wachstum benötigt sie tiefgründige, nachhaltig frische, gut durchlüftete, möglichst basen- und nährstoffreiche Böden ohne Stauwasser. Nährstoffarme, trockene Böden und solche mit hohem Grundwasserstand werden von ihr gemieden (Otto, 1994). Im Landeswald von Niedersachsen nimmt die Buche mit rund $21 \%$ einen bedeutenden Flächenanteil ein (NLF, 2014a), der aufgrund zahlreicher Voranbauten und vollflächiger Naturverjüngungen weiter zunehmen wird. Bestände mit führender Buche bzw. Buchenmischbestände sind mit rund 75.000 bzw. 65.000 ha in den Niedersächsischen Landesforsten vertreten. Diese Bestände sind relativ ausgeglichen über das Alter verteilt (NLF, 2014c).

Die Fichte (Picea abies [L.] Karst.) kommt natürlicherweise in drei großen Teilgebieten vor: in Mittel- und Südosteuropa, in Nordosteuropa und in Sibirien östlich des Urals. Ihr Anbauoptimum liegt im kühl-kontinentalen Klima mit guter Wasserversorgung (Otto et al., 2007; Röhrig und Bartsch, 1992). Diese große Standortsamplitude macht ihren Anbau auf geeigneten Standorten auch außerhalb ihres Verbreitungsgebietes möglich, wobei allerdings die Vor- und Nachteile insbesondere unter Beachtung von Risiken (z. B. Sturmwurf, Rotfäule, Schneebruch, etc.) abzuwägen sind (Röhrig und Bartsch, 1992). Die Fichte ist derzeit die vorherrschende Baumart in den Niedersächsischen Landesforsten mit einem Anteil von etwa $27 \%$ an der Gesamtwaldfläche. Davon sind ca. 49.000 ha gleichaltrige, einschichtige, homogene Reinbestände. Ein Großteil dieser Fichtenbestände befindet sich in der zweiten und dritten Altersklasse, d.h. sie sind zwischen 21 und 60 Jahre alt. Grundsätzlich wird angestrebt, die Fichte zukünftig in Mischbeständen anzubauen (NLF, 2014d).

Die Douglasie (Pseudotsuga menziesii [Mirb.] Franco) ist die wichtigste eingeführte Baumart in Niedersachsen, mit weiter steigender Bedeutung vor dem Hintergrund der zu erwartenden Klimaveränderungen. Sie ist ökologisch zuträglich und erbringt, bei geringen Anforderungen an den Standort (geringe Nährstoff- und mittlere Wasserversorgung), hohe Volumen- und Werterträge (Kownatzki et al., 2011; NLF, 2011; Vor et al., 2015). Ihr natürliches Verbreitungsgebiet in der pazifischen Nadelwaldregion Nordamerikas ist sehr ausgedehnt und ökologisch differenziert. Es reicht von Britisch Kolumbien in Kanada bis in die Hochgebirge von Mexiko und von der Pazifikküste bis in die Rocky Mountains (Röhrig und Bartsch, 1992). Ihr Wachstum wird in Deutschland durch milde Winter, mäßig warme Sommer und eine lange Vegetationszeit gefördert (Otto et al., 2007). In den Niedersächsischen Landesforsten ist die Douglasie auf rund 11.000 ha (4\%) im Hauptbestand vertreten und kommt zu $72 \%$ in Mischbeständen vor. Diese Bestände sind überwiegend in der zweiten und dritten Altersklasse zu finden (NLF, 2015).

Die Kiefer (Pinus sylvestris L.) ist eine anspruchslose Baumart des subkontinentalen bis kontinentalen Klimas. Ihr natürliches Verbreitungsgebiet reicht von der Türkei bis ins nördliche Norwegen und vom Osten Russlands bis zur Sierra Nevada in Spanien (Röhrig 
und Bartsch, 1992). Die Kiefer ist die wichtigste Wirtschaftsbaumart im norddeutschen Tiefland, im Bergland ist sie weniger bedeutend. Der hohe Anteil im Tiefland ist auf die Erstaufforstungen im 19. und 20. Jahrhundert, die Wiederaufforstungen nach den Reparationshieben im Anschluss an den zweiten Weltkrieg, die Sturmschäden der 1960er und 1970er Jahre sowie die Waldbrände von 1975/76 zurückzuführen. Heute sind die Kiefernwälder in den Niedersächsischen Landesforsten meist zwischen 30 bis 70 Jahre alt und ihr Flächenanteil wird sich in Zukunft zugunsten von Eiche, Buche, Douglasie und Küstentanne (Abies grandis [Douglas ex D. Don] Lindl.) verringern (NLF, 2014e).

\subsection{Ausgesuchte Standorte im niedersächsischen Landeswald}

\subsubsection{Allgemeine Charakterisierung}

Die Standorteigenschaften haben einen großen Einfluss auf das Baumwachstum (Assmann, 1961). Um diesen Effekt abbilden zu können, wurden wichtige Standorte in ausgewählten Wuchsbezirken identifiziert. In die Analyse gingen die Wuchsbezirke EmsHase-Hunte-Geest (EH), Geest-Mitte (GM), Hohe Heide (HH) und Ost-Heide (OH) im niedersächsischen Tiefland, sowie das Untere Weser-Leine-Bergland (UW), der Untere Solling (US), der Göttinger Wald (GW) und der Montane Mittel- und Oberharz (MH) im niedersächsischen Bergland ein.

Das niedersächsische Tiefland teilt sich in das Höhen- und das Talpleistozän sowie das Holozän auf. Das Höhenpleistozän besteht aus den Grundmoränen, Endmoränen und Sanderflächen. Hier finden sich vor allem Geschiebelehme, ärmere und mittlere, teilweise auch reichere Sande, Sandlöss und Lauenburger Ton als Hauptsubstrate. Im Allgemeinen haben diese Standorte keinen Grundwasseranschluss. Wasserüberschuss ist allenfalls durch Stauwasser bedingt. Im Talpleistozän und Holozän sind Talsande und Auenstandorte, Moore und Brücher die wesentlichen standörtlichen Bodeneinheiten. Diese Standorte sind mehr oder weniger stark durch Grundwasser beeinflusst (Otto, 1972).

Im Übergang zum Bergland liegt die stark lössbeeinflusste, flach wellige Berglandschwelle, die überwiegend landwirtschaftlich genutzt wird. Das geologisch vielgestaltige Bergland mit Gesteinen aus Trias, Jura und Kreide sowie dem Niedersächsischen Harz mit alten Gesteinen aus dem Paläozoikum ist bodenkundlich außerordentlich vielgestaltig und die Standorte wechseln - auch in Folge des Reliefs - auf kleinstem Raum. Häufig finden sich Lössfließerden über Buntsandstein, jedoch auch Muschelkalk und mächtige Lössdecken sind weit verbreitet. Es überwiegen schluffig-lehmige bis sandige, basenarme Silikatverwitterungsböden (Wördehoff et al., 2011).

Die ausgewählten Wuchsbezirke wurden von Otto $(1989,1991)$ ausführlich beschrieben sowie standörtlich und waldbaulich analysiert. Die wichtigsten Standortstypenobergruppen in den selektierten Wuchsbezirken des Tieflandes sind Flugsande, Sande, Sande im Grundwasserbereich, Sandlösse und Geschiebelehme. In den gewählten Wuchsbezirken 
des Berglandes sind es basenarme und -reiche Silikatgesteine, Kalkgesteine sowie Lösse. Eine detailliertere Aufstellung der Standortstypenobergruppen nach Wuchsbezirk ist in Tabelle $2.1 \mathrm{zu}$ finden und eine kurze Beschreibung der klimatischen, geographischen sowie geologischen Merkmale der einzelnen Wuchsbezirke in den Tabellen 2.2 und 2.3. Die ausgewählten Standorte repräsentieren rund $47 \%$ der niedersächsischen Landeswaldfläche von 335.813 ha.

Tabelle 2.1: Flächengrößen der wichtigen Standortstypenobergruppen in ausgewählten niedersächsischen Wuchsbezirken (Quellen: Otto $(1989,1991))$.

\begin{tabular}{llr}
\hline Wuchsbezirk & Standortstypenobergruppe & Fläche [ha] \\
\hline \multirow{3}{*}{ Ems-Hase-Hunte-Geest } & Flugsande & 3.548 \\
& Sande & 4.244 \\
& Geschiebelehme & 4.685 \\
\hline \multirow{2}{*}{ Geest-Mitte } & Sande & 3.839 \\
& Sande im Grundwasserbereich & 3.927 \\
& Sandlösse & 3.417 \\
& Geschiebelehme & 5.499 \\
\hline \multirow{3}{*}{ Hohe Heide } & Sande & 18.669 \\
& Sande im Grundwasserbereich & 1.396 \\
& Geschiebelehme & 3.967 \\
\hline \multirow{2}{*}{ Ost-Heide } & Sande & 12.300 \\
& Sande im Grundwasserbereich & 4.705 \\
& Geschiebelehme & 4.603 \\
\hline \multirow{2}{*}{ Unteres Weser-Leine-Bergland } & basenarme Silikatgesteine & 12.302 \\
& Kalkgesteine & 8.885 \\
& Lösse & 5.488 \\
\hline \multirow{2}{*}{ Unterer Solling } & basenarme Silikatgesteine & 16.545 \\
& Lösse & 2.383 \\
\hline \multirow{2}{*}{ Göttinger Wald } & basenarme Silikatgesteine & 2.180 \\
\hline \multirow{2}{*}{ Montaner Mittel- und Oberharz } & 1.944 \\
\hline & Kalkgesteine & 28.160 \\
& basenarme Silikatgesteine & 4.335 \\
\hline & & \\
& & \\
& & \\
& &
\end{tabular}


Tabelle 2.2: Mittlere Niederschlagssumme (N) und Jahrestemperatur (T) sowie klimatische, geographische und geologische Merkmale der ausgewählten Wuchsbezirke im niedersächsischen Tiefland (Quelle: Otto (1989)).

\begin{tabular}{|c|c|c|c|}
\hline Wuchsbezirk & Klima & Geographie & Geologie \\
\hline $\begin{array}{l}\text { Ems-Hase-Hunte- } \\
\text { Geest } \\
\text { N: } 760 \mathrm{~L} \mathrm{~m}^{-2} \mathrm{a}^{-1} \\
\mathrm{~T}: 8,6^{\circ} \mathrm{C}\end{array}$ & $\begin{array}{l}\text { Atlantisch, ziem- } \\
\text { lich humid und } \\
\text { regenreich, mild, } \\
\text { ohne größere Ex- } \\
\text { treme, geringe } \\
\text { Spätfrostgefahr }\end{array}$ & $\begin{array}{l}\text { Flachwellige } \\
\text { Grundmoränen, } \\
\text { ebene Talsand- } \\
\text { gebiete, einige } \\
\text { Endmoränen mit } \\
\text { stärkerer Relief- } \\
\text { energie }\end{array}$ & $\begin{array}{l}\text { Drenthestadiale } \\
\text { Grund- und End- } \\
\text { moränen, } \\
\text { gestaucht, } \\
\begin{array}{l}\text { sande, } \\
\text { gealterte }\end{array} \\
\text { und Lärker } \\
\text { und }\end{array}$ \\
\hline $\begin{array}{l}\text { Geest-Mitte } \\
\mathrm{N}: 670 \mathrm{~L} \mathrm{~m}^{-2} \mathrm{a}^{-1} \\
\mathrm{~T}: 8,6{ }^{\circ} \mathrm{C}\end{array}$ & $\begin{array}{l}\text { Übergangsklima } \\
\text { mit deutlich ozea- } \\
\text { nischer Tönung, } \\
\text { mäßig regenreich } \\
\text { und humid, mild } \\
\text { ohne größere Ex- } \\
\text { treme, geringe } \\
\text { Spätfrostgefahr }\end{array}$ & $\begin{array}{l}\text { Flachwelliges } \\
\text { Grundmoränen- } \\
\text { gebiet, im Süden } \\
\text { Endmoränen mit } \\
\text { stärkerer Relief- } \\
\text { energie }\end{array}$ & $\begin{array}{l}\text { Drenthestadiale } \\
\text { Grund- und End- } \\
\text { moränen, stärker } \\
\text { gealterte Sande } \\
\text { und Lehme, ab- } \\
\text { wechselnd mit } \\
\text { Grundwasserböden } \\
\text { und Auen }\end{array}$ \\
\hline $\begin{array}{l}\text { Hohe Heide } \\
\mathrm{N}: 730 \mathrm{~L} \mathrm{~m}^{-2} \mathrm{a}^{-1} \\
\mathrm{~T}: 8,0{ }^{\circ} \mathrm{C}\end{array}$ & $\begin{array}{l}\text { Atlantisch kühl, } \\
\text { ziemlich regenreich, } \\
\text { gemilderte Tem- } \\
\text { peraturextreme, } \\
\text { starke Spätfrostge- } \\
\text { fährdung }\end{array}$ & $\begin{array}{l}\text { Flachwelliges bis } \\
\text { hügeliges Morä- } \\
\text { nengebiet, mäßige } \\
\text { Reliefausformung }\end{array}$ & $\begin{array}{l}\text { Warthestadiale } \\
\text { Grund- und End- } \\
\text { moränen, lebhaft } \\
\text { gestaffelt, wenig } \\
\text { gealterte } \\
\text { und Lehme }\end{array}$ \\
\hline $\begin{array}{l}\mathrm{N}: 630 \mathrm{~L} \mathrm{~m}^{-2} \mathrm{a}^{-1} \\
\mathrm{~T}: 8,0{ }^{\circ} \mathrm{C}\end{array}$ & $\begin{array}{l}\text { Subkontinental, } \\
\text { begrenzte Nieder- } \\
\text { schläge, sommer- } \\
\text { warm, ausgeprägte } \\
\text { Extreme, Spät- } \\
\text { frostgefährdung }\end{array}$ & $\begin{array}{l}\text { Flachwelliges bis } \\
\text { hügeliges Morä- } \\
\text { nengebiet, Relief- } \\
\text { energie an der Elbe } \\
\text { z. T. beachtlich }\end{array}$ & $\begin{array}{l}\text { Warthestadiale } \\
\text { Grund- und End- } \\
\text { moränen sowie } \\
\text { Elbe-Urstromtal, } \\
\text { wenig gealterte } \\
\text { Sande und Lehme } \\
\text { z. T. Geschiebemer- } \\
\text { gel }\end{array}$ \\
\hline
\end{tabular}


Tabelle 2.3: Mittlere Niederschlagssumme (N) und Jahrestemperatur (T) sowie klimatische, geographische und geologische Merkmale der ausgewählten Wuchsbezirke im niedersächsischen Bergland (Quelle: Otto (1989)).

\begin{tabular}{|c|c|c|c|}
\hline Wuchsbezirk & Klima & Geographie & Geologie \\
\hline $\begin{array}{l}\text { Unteres Weser- } \\
\text { Leine-Bergland }\end{array}$ & $\begin{array}{l}\text { Typisch atlantisch, } \\
\text { regenreich, hu- } \\
\text { mid, mild, geringe } \\
\text { Temperaturextre- } \\
\text { me, Schneelagen } \\
\text { seltener }\end{array}$ & $\begin{array}{l}\text { Typische Schicht- } \\
\text { rippenlandschaft, } \\
\text { steil gestellte, } \\
\text { rippenförmig her- } \\
\text { ausmodellierte } \\
\text { Schichten, hohe } \\
\text { Reliefenergie }\end{array}$ & $\begin{array}{l}\text { Trias, Jura, Kreide, } \\
\text { vielfältige Aus- } \\
\text { gangsgesteine auf } \\
\text { kleinstem Raum, } \\
\text { dazu Lössflie- } \\
\text { Berden, extreme } \\
\text { bodenkundliche } \\
\text { Vielfalt }\end{array}$ \\
\hline $\begin{array}{l}\mathrm{N}: 900 \mathrm{~L} \mathrm{~m}^{-2} \mathrm{a}^{-1} \\
\mathrm{~T}: 7,5^{\circ} \mathrm{C}\end{array}$ & $\begin{array}{l}\text { Bergland-Klima, } \\
\text { atlantisch, re- } \\
\text { genreich, humid, } \\
\text { kühl }\end{array}$ & $\begin{array}{l}250 \text { - } 400 \mathrm{~m} \text { ü. NN, } \\
\text { Schichtstufenland- } \\
\text { schaft, } \quad \text { ruhiges } \\
\text { Relief }\end{array}$ & $\begin{array}{l}\text { Trias, mit wenigen } \\
\text { Ausnahmen Bunt- } \\
\text { sandstein, Löss }\end{array}$ \\
\hline $\begin{array}{l}\mathrm{N}: 680 \mathrm{~L} \mathrm{~m}^{-2} \mathrm{a}^{-1} \\
\mathrm{~T}: 7,8{ }^{\circ} \mathrm{C}\end{array}$ & 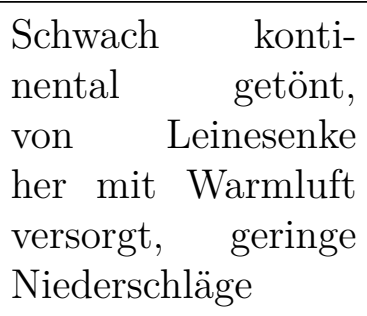 & $\begin{array}{l}\text { Sanfter } \quad \text { Schicht- } \\
\text { einfall zum Leine- } \\
\text { graben, typische } \\
\text { Schichtstufenland- } \\
\text { schaft, ruhiges } \\
\text { Relief }\end{array}$ & $\begin{array}{l}\text { Trias, Buntsand- } \\
\text { stein und Muschel- } \\
\text { kalk, verbreitet } \\
\text { Löss }\end{array}$ \\
\hline $\begin{array}{l}\text { Montaner Mittel- } \\
\text { und Oberharz }\end{array}$ & $\begin{array}{l}\text { Sehr regenreiches, } \\
\text { kühles Berglandkli- } \\
\text { ma, Hauptschnee- } \\
\text { bruchzone, höhere, } \\
\text { exponierte Lagen } \\
\text { auch duft- und } \\
\text { eisbruchgefährdet }\end{array}$ & $\begin{array}{l}500 \text { - } 700 \mathrm{mü.} \mathrm{NN}, \\
\text { in den Plateau- } \\
\text { lagen geringe, in } \\
\text { den stark zertalten } \\
\text { Plateaurändern } \\
\text { hohe Reliefenergie }\end{array}$ & $\begin{array}{l}\text { Paläozoikum, } \\
\text { Kulmgrauwacken } \\
\text { und Tonschiefer, } \\
\text { Diabas, Gabbro, } \\
\text { Granit, Kieselschie- } \\
\text { fer, Kahlebergsand- } \\
\text { stein }\end{array}$ \\
\hline
\end{tabular}




\subsubsection{Identifikation der wichtigsten Standorte}

Für die ausgewählten Wuchsbezirke und die flächenmäßig bedeutsamsten Standortstypenobergruppen wurden die Informationen der digitalen Standortskartierung und Forsteinrichtung der Niedersächsischen Landesforsten ausgewertet. Dabei wurden die mittleren Bonitäten für die auf den wichtigsten Standorte stockenden Bestände mit den mindestens führenden Hauptwirtschaftsbaumarten im Hauptbestand identifiziert. Auf Grundlage der Nährstoffziffer wird die Nährstoffversorgung der Standorte stufenweise charakterisiert (s. Tab. 2.4). Für die Bildung von Wasserhaushaltsklassen auf grundwasserunbeeinflussten Standorten wird die nutzbare Feldkapazität ${ }^{1}$ (nFK) herangezogen (s. Tab. 2.5). Grundwasserbeeinflusste Standorte im Tiefland werden mit Hilfe der Wasserhaushaltszahl in die Kategorien schwach (Wasserhaushaltszahl 35), mäßig (Wasserhaushaltszahl 34) und stark (Wasserhaushaltszahl 32 und 33) eingeteilt. Im Bergland wurden nur Sonnen- und Schatthänge sowie Plateaulagen in die Analyse einbezogen. Weiterhin wurden Moore, sowie die Baumarten Kiefer und Douglasie im Bergland nicht betrachtet. Für die Douglasie ergab sich aus der Vorauswahl von Wuchsbezirken und Standortstypenobergruppen eine geringe Anzahl von Vergleichsflächen, so dass auch nur wenige Weiserbestände generiert wurden (s. Tab. 3.4). Dies führte dazu, dass beispielsweise Douglasienbestände mit sehr guter Leistungsfähigkeit im Bergland nicht in die weiteren Betrachtungen einbezogen werden und somit das hohe Klimaschutzpotenzial dieser Baumart nur unzureichend abgebildet wird.

So haben beispielsweise Buchen- bzw. Fichtenbestände im Göttinger Wald auf mäßig frischen Kalkstandorten mit sehr guter Nährstoffversorgung eine mittlere Leistungsklasse (LKL) von 8,2 bzw. 13,0 $\mathrm{m}^{3} \mathrm{ha}^{-1} \mathrm{a}^{-1}$ (s. Tab. 2.6). Im Wuchsgebiet Ems-Hase-Hunte-Geest beträgt die mittlere Leistungsklasse von Kiefernbeständen auf trockenen und schwach versorgten Flugsandstandorten $5,9 \mathrm{~m}^{3} \mathrm{ha}^{-1} \mathrm{a}^{-1}$. Werden Wasser- und Nährstoffversorgung besser (schwach grundwasserbeeinflusst, mäßig versorgt mit Nährstoffen) erhöht sich die Leistungsklasse bei gleichem Ausgangssubstrat auf $6,2 \mathrm{~m}^{3} \mathrm{ha}^{-1} \mathrm{a}^{-1}$ (s. Tab. 2.7). Die ermittelten durchschnittlichen Leistungsklassen getrennt nach Baumart, Wasserhaushalt und Nährstoffversorgung sowie Standortstypenobergruppe in den einzelnen Wuchsbezirken sind im Anhang A.1 zu finden.

Darüber hinaus wurde die Mittelhöhe im Alter 100 ermittelt, die als Eingangsinformation für die Generierung der Weiserbestände dient (s. Kap. 2.3). Zur Wahrung der Flächenrepräsentanz der Baumarten auf den wichtigen Standorte wurden nur Bestände in die Analysen einbezogen, deren ermittelte Fläche je Baumart größer als 25 ha ist (Ausnahme Göttinger Wald: Mindestfläche 8 ha).

\footnotetext{
${ }^{1}$ Die nutzbare Feldkapazität beschreibt die Menge des für das Pflanzenwachstums frei verfügbare Wasser im Boden.
} 
Tabelle 2.4: Einteilung der Nährstoffziffern in Gruppen getrennt nach Berg- und Tiefland in Niedersachsen.

\begin{tabular}{ccc}
\hline \multirow{2}{*}{ Nährstoffgruppe } & \multicolumn{2}{c}{ Nährstoffziffer } \\
& Bergland & Tiefland \\
\hline sehr gut & $4+$ bis 6 & $4+$ bis 6 \\
ziemlich gut & $3+$ bis 4 & 4 - bis 4 \\
mäßig & 3 & 3 bis $3+$ \\
schwach & 1 bis $3-$ & 1 bis $3-$ \\
\hline
\end{tabular}

Tabelle 2.5: Einteilung der grundwasserunbeeinflussten Standorte in Gruppen mit Hilfe der nutzbaren Feldkapazität (nFK $\left[\mathrm{mm} \mathrm{l} \mathrm{m}^{-1}\right]$ ) im niedersächsischen Berg- und Tiefland in Anlehnung an Overbeck et al. (2011).

\begin{tabular}{|c|c|c|}
\hline Region & Wasserhaushaltsgruppe & $\mathrm{nFK}\left[\mathrm{mm} 1 \mathrm{~m}^{-1}\right]$ \\
\hline \multirow{5}{*}{ Bergland } & nachhaltig frisch & $>187$ \\
\hline & frisch & $129-187$ \\
\hline & mäßig frisch & $95-128$ \\
\hline & sommertrocken & $53-94$ \\
\hline & trocken & $<53$ \\
\hline \multirow{4}{*}{ Tiefland } & frisch & $>150$ \\
\hline & mäßig frisch bis frisch & $119-150$ \\
\hline & mäßig frisch & $95-118$ \\
\hline & trocken & $<95$ \\
\hline
\end{tabular}

Tabelle 2.6: Mittlere Leistungsklassen (LKL $\left[\mathrm{m}^{3} \mathrm{ha}^{-1} \mathrm{a}^{-1}\right]$ ) der Hauptwirtschaftsbaumarten (Bu $=$ Buche, $\mathrm{Fi}=$ Fichte) auf wichtigen Standorten getrennt nach Wasserhaushalt und Nährstoffversorgung im Wuchsbezirk Göttinger Wald.

\begin{tabular}{lllcc}
\hline $\begin{array}{l}\text { Standortstypen- } \\
\text { obergruppe }\end{array}$ & Wasserhaushalt & $\begin{array}{l}\text { Nährstoffver- } \\
\text { sorgung }\end{array}$ & Baumart & $\begin{array}{c}\text { LKL } \\
{\left[\mathrm{m}^{3} \mathrm{ha}^{-1} \mathrm{a}^{-1}\right]}\end{array}$ \\
\hline basenarme & \multirow{2}{*}{ frisch } & ziemlich gut & $\mathrm{Bu}$ & 8,7 \\
Silikatgesteine & \multirow{2}{*}{ mäßig frisch } & sehr gut & $\mathrm{Bu}$ & 12,4 \\
\hline Kalkgesteine & & & $\mathrm{Fi}$ & 8,2 \\
& & & & \\
\hline
\end{tabular}


Tabelle 2.7: Mittlere Leistungsklassen (LKL $\left[\mathrm{m}^{3} \mathrm{ha}^{-1} \mathrm{a}^{-1}\right]$ ) der Hauptwirtschaftsbaumarten (Ei $=$ Eiche, $\mathrm{Bu}=$ Buche, $\mathrm{Fi}=$ Fichte, $\mathrm{Dgl}=$ Douglasie, $\mathrm{Ki}=$ Kiefer $)$ auf wichtigen Standorten getrennt nach Wasserhaushalt und Nährstoffversorgung im Wuchsbezirk Ems-Hase-Hunte-Geest.

\begin{tabular}{|c|c|c|c|c|}
\hline $\begin{array}{l}\text { Standortstypen- } \\
\text { obergruppe }\end{array}$ & Wasserhaushalt & $\begin{array}{l}\text { Nährstoffver- } \\
\text { sorgung }\end{array}$ & Baumart & $\begin{array}{c}\text { LKL } \\
{\left[\mathrm{m}^{3} \mathrm{ha}^{-1} \mathrm{a}^{-1}\right]}\end{array}$ \\
\hline \multirow{2}{*}{ Flugsande } & \multirow{2}{*}{$\begin{array}{l}\text { schwach grundwasser- } \\
\text { beeinflusst }\end{array}$} & mäßig & $\begin{array}{l}\mathrm{Ei} \\
\mathrm{Ki}\end{array}$ & $\begin{array}{l}6,0 \\
6,2\end{array}$ \\
\hline & & schwach & $\begin{array}{l}\text { Dgl } \\
\text { Ki }\end{array}$ & $\begin{array}{c}14,1 \\
5,9\end{array}$ \\
\hline Flugsande & trocken & schwach & $\begin{array}{c}\text { Dgl } \\
\mathrm{Fi} \\
\mathrm{Ki}\end{array}$ & $\begin{array}{c}13,8 \\
10,4 \\
5,9\end{array}$ \\
\hline \multirow{3}{*}{ Geschiebelehme } & \multirow[b]{2}{*}{ frisch } & mäßig & $\begin{array}{c}\text { Dgl } \\
\mathrm{Ei} \\
\mathrm{Fi} \\
\mathrm{Ki}\end{array}$ & $\begin{array}{c}13,7 \\
6,0 \\
11,6 \\
6,8\end{array}$ \\
\hline & & ziemlich gut & $\begin{array}{c}\mathrm{Bu} \\
\mathrm{Dgl} \\
\mathrm{Ei} \\
\mathrm{Fi} \\
\mathrm{Ki}\end{array}$ & $\begin{array}{c}8,7 \\
14,3 \\
6,0 \\
11,8 \\
6,7\end{array}$ \\
\hline & mäßig frisch bis frisch & mäßig & $\begin{array}{c}\text { Dgl } \\
\mathrm{Ei} \\
\mathrm{Ki}\end{array}$ & $\begin{array}{c}13,7 \\
5,7 \\
6,5\end{array}$ \\
\hline \multirow{3}{*}{ Sande } & mäßig frisch bis frisch & mäßig & $\begin{array}{c}\mathrm{Bu} \\
\mathrm{Dgl} \\
\mathrm{Ei} \\
\mathrm{Fi} \\
\mathrm{Ki}\end{array}$ & $\begin{array}{c}8,0 \\
13,7 \\
6,1 \\
12,5 \\
6,9 \\
\end{array}$ \\
\hline & \multirow[t]{2}{*}{ mäßig frisch } & mäßig & $\begin{array}{c}\text { Dgl } \\
\mathrm{Ei} \\
\mathrm{Fi} \\
\mathrm{Ki}\end{array}$ & $\begin{array}{c}14,5 \\
5,7 \\
11,2 \\
6,4\end{array}$ \\
\hline & & schwach & $\begin{array}{l}\mathrm{Dgl} \\
\mathrm{Ki}\end{array}$ & $\begin{array}{c}13,5 \\
6,3\end{array}$ \\
\hline
\end{tabular}

Fortsetzung auf nächster Seite. 
Tabelle 2.7

\begin{tabular}{lllcc}
\hline $\begin{array}{l}\text { Standortstypen- } \\
\text { obergruppe }\end{array}$ & Wasserhaushalt & $\begin{array}{l}\text { Nährstoffver- } \\
\text { sorgung }\end{array}$ & Baumart & $\begin{array}{c}\text { LKL } \\
{\left[\mathrm{m}^{3} \mathrm{ha}^{-1} \mathrm{a}^{-1}\right]}\end{array}$ \\
\hline \multirow{3}{*}{ Sande } & mäßig & Dgl & 14,5 \\
& trocken & & $\mathrm{Ki}$ & 6,7 \\
\cline { 3 - 5 } & schwach & Dgl & 13,8 \\
& & Fi & 10,5 \\
& & Ki & 6,2 \\
\hline
\end{tabular}

\subsection{Weiserbestände zur Überprüfung von Waldbaustrategien}

Für die Simulation verschiedener Waldbaustrategien werden realitätsnahe Bestände unterschiedlicher Bonität benötigt (s. Kap. 1.3), die den gesamten Produktionszeitraum der jeweiligen Baumart abdecken (s. Tab. 3.3). Die Datengrundlage zur Generierung dieser Weiserbestände bilden die Informationen der Betriebsinventur und der Forsteinrichtung der Niedersächsischen Landesforsten. Die Betriebsinventur ist ein zweiphasiges Inventurverfahren mit permanenten Stichprobenpunkten. Ursprünglich wurde die Inventur mit dauerhaften Stichproben in den USA entwickelt und Anfang der 1960er Jahre in der Schweiz eingeführt. Etwas modifiziert, wird dieses Verfahren seit den 1980er Jahren im niedersächsischen Landeswald durchgeführt. Die Betriebsinventur findet in den Forstämtern in Kombination mit einer herkömmlichen Forsteinrichtung zum gleichen Stichtag mit einem zeitlichen Vorlauf statt. Dabei werden Informationen über den Waldzustand, insbesondere über die Entwicklung verschiedener Parameter (z. B. Vorrat, Baumartenanteile, Altersaufbau, Zuwachs, etc.), auf Forstamtsebene gewonnen. Mit Hilfe von dauerhaften Stichproben können wesentlich genauere Aussagen über Zustandsveränderungen getroffen werden. Es sind jedoch nur in Ausnahmefällen ausreichend genaue Ergebnisse auf Bestandesebene ableitbar, da die Stichprobenanzahl für diese Auswertungsebene zu gering ist. Ohne die räumliche Information der Bestandesinventur sind mit der Betriebsinventur eher nur Aussagen für große Gebiete möglich (NFP, 2013). Die wesentliche Aufgabe der Forsteinrichtung ist die periodische Prüfung und Sicherung der Nachhaltigkeit des Forstwirtschaftsbetriebes. Dabei wird für jedes Forstamt periodisch der Waldzustand erhoben und kritisch beurteilt (Zustandserfassung), der Betriebsvollzug und -erfolg mit Schwerpunkt im naturalen Bereich kontrolliert sowie ausgewertet (Analyse) und es werden mittelfristige Betriebspläne aufgestellt (Planung) (NFP, 2012). Für weitere Informationen sei hier für Niedersachsen auf Böckmann et al. (1998,a), im Allgemeinen auf Gadow (2005) sowie Kramer und Akca (2008), verwiesen. 


\subsubsection{Methodisches Vorgehen und Modellentwicklung}

Um einen Bestand modellhaft zu charakterisieren, werden zunächst verschiedene Einzelbaumwerte, wie die Baumhöhe, der Durchmesser oder das Alter, benötigt. Daraus können anschließend Bestandeskennwerte wie beispielsweise der Grundflächenmittelstamm, die Grundfläche oder die Mittelhöhe abgeleitet werden.

Die Einzelbaumdurchmesser werden bei der Betriebsinventur in konzentrischen Probekreisen erhoben. Die Höhe der Einzelbäume wird allerdings nur an einem Laub- und bzw. oder Nadelbaum pro Bestandesschicht, d. h. Haupt- und Nebenbestand sowie Überhalt, aufgenommen (NFP, 2009b). Die fehlenden Baumhöhen $(h[\mathrm{~m}])$ der Betriebsinventurpunkte müssen daher ergänzt werden. Dies wurde hier in Anlehnung an die Auswertungsmethoden der zweiten Bundeswaldinventur umgesetzt (Schmitz et al., 2008). Dazu wurde die Höhe des Grundflächenmittelstammes ( $h g[\mathrm{~m}])$ mit einem iterativen Verfahren schichtweise ermittelt. Anschließend wurde die Einheitshöhenkurve von Sloboda et al. (1993) (s. Gl. 2.1) mit den Koeffizienten von Nagel (1999) (s. Tab. 2.8), in Abhängigkeit von der Höhe und dem Durchmesser des Grundflächenmittelstammes (hg $[\mathrm{m}], d g[\mathrm{~m}])$ sowie dem Einzelbaumdurchmesser $(d[\mathrm{~m}])$ genutzt, um die fehlenden Einzelbaumhöhen der betreffenden Schicht zu berechnen. Wird die Einheitshöhenkurve, mit Eingangsgrößen auf Grundlage des gesamten Bestandes angewendet, wird die Bestandesstruktur nicht berücksichtigt.

Anschließend wurde über die Höhe des Grundflächenmittelstammes ( $h g[\mathrm{~m}])$ des Hauptbestandes und dem Alter ( $a$ [Jahre]) mit den Höhenbonitätsfunktionen von Nagel (1999) (s. Gl. 2.2) bonitiert. Hierfür wurden diese anhand der Mittelhöhe aus der Ertragstafelsammlung von Schober (1995) baumartenspezifisch mittels nicht linearer Regression neu parametrisiert (s. Tab. 2.9). Damit ist es auch möglich, die Höhe des Grundflächenmittelstammes entsprechend der Bonität $(s i[\mathrm{~m}])$ in beliebigen Altern ( $a$ [Jahre]) zu schätzen (s. Gl. 2.3).

$$
\begin{aligned}
& h=1.3+(h g-1.3) \times \exp \left\{p_{0} \times\left(1-\frac{d g}{d}\right)\right\} \times \exp \left\{p_{1} \times\left(\frac{1}{d g}-\frac{1}{d}\right)\right\} \\
& s i=\frac{\left(h g+m-n \times \ln (a)-o \times\left((\ln (a))^{2}\right)\right)}{(-p+q \times \ln (a))} \\
& h g=-m+n \times \ln (a)+o \times\left(\ln (a)^{2}\right)-p \times s i+q \times s i \times \ln (a)
\end{aligned}
$$

Mittels der Einzelbaumdurchmesser der Betriebsinventur ist es möglich die Bestandesgrundfläche, als häufig verwendetes, bestandesbezogenes Maß für die Dichte (Gadow, 2005; Kramer und Akca, 2008), zu ermitteln. Durch die Anwendung eines generalisierten additiven Modells (Wood, 2011), konnte die Grundfläche von Reinbeständen der 
Tabelle 2.8: Baumartenspezifische Koeffizienten der Einheitshöhenkurve (Quelle: Nagel (1999))

\begin{tabular}{lcc}
\hline Baumart & $p_{0}$ & $p_{1}$ \\
\hline Eiche & 0.14657227 & 3.78686023 \\
Buche & 0.20213328 & 5.64023296 \\
Fichte & 0.18290951 & 5.68789430 \\
Douglasie & 0.19965100 & 4.63277655 \\
Kiefer & 0.25963741 & 1.30645374 \\
\hline
\end{tabular}

Tabelle 2.9: Koeffizienten der verwendeten Funktionen zur Schätzung der Bonität und der Höhe des Grundflächenmittelstammes entsprechend der Bonität in beliebigen Altern (s. Gl. 2.2 und 2.3).

\begin{tabular}{lccccc}
\hline Baumart & $m$ & $n$ & $o$ & $p$ & $q$ \\
\hline Eiche & 28.35126268 & 5.35873918 & 0.09185592 & 0.0 & 0.23419962 \\
Buche & 56.2704814 & 12.9826098 & -0.1454899 & 0.0 & 0.2111513 \\
Fichte & 6.85431047 & -9.54322296 & 2.36998502 & 0.09728744 & 0.24329396 \\
Douglasie & 44.9611781 & 8.0999799 & 0.5099378 & 0.0 & 0.2040147 \\
Kiefer & 14.5280867 & 3.9959830 & -0.1895817 & 0.7270016 & 0.3749912 \\
\hline
\end{tabular}

untersuchten Baumarten alters- und bonitätsabhängig geschätzt werden. Da die Bestandesgrundfläche von den vorhandenen Baumarten und deren Anteilen abhängig ist, werden nur Informationen von Betriebsinventurstichprobenpunkten genutzt, die in Beständen mit einem Mischungsprozent, entnommen aus den Forsteinrichtungsdaten, von mindestens $90 \%$ für die entsprechende Baumart liegen. Damit soll sichergestellt werden, dass sich die Stichprobenpunkte der Betriebsinventur in Reinbeständen befinden. Dabei sind Reinbestände so definiert, dass sie nur aus einer Baumart bestehen oder in denen die anderen vorkommenden Arten zusammen nicht mehr als $10 \%$ der Bestandesgrundfläche einnehmen (NFP, 2009a). So ist es mit dem erstellten Modell möglich für jedes Alter und jede absolute Mittelhöhenbonität im Alter 100 (hg 100 [m]) eine Grundfläche in Reinbeständen zu schätzen.

Als Nächstes wird die zur geschätzten Grundfläche zugehörige Durchmesserverteilung benötigt. Deren Generierung erfolgt auf Grundlage von Betriebsinventurpunkten in Beständen mit einem Mischungsanteil von mindestens $50 \%$ (Douglasie) bzw. $60 \%$ (alle anderen Baumarten) der untersuchten Hauptbaumarten (führende Baumart). Dieses Vorgehen wurde gewählt, um eine ausreichend große Datengrundlage zu erhalten. Dafür wurde die Annahme getroffen, dass die Verteilung der Durchmesser, als Indikator für die Bestandesstruktur, nicht so stark von der Artenzusammensetzung und den Mischungsanteilen abhängt, wie die Bestandesgrundfläche. Die Schätzung der Durchmesserverteilung erfolgte durch ein Quantilsregressionsverfahren (Rigby und Stasinopoulos, 


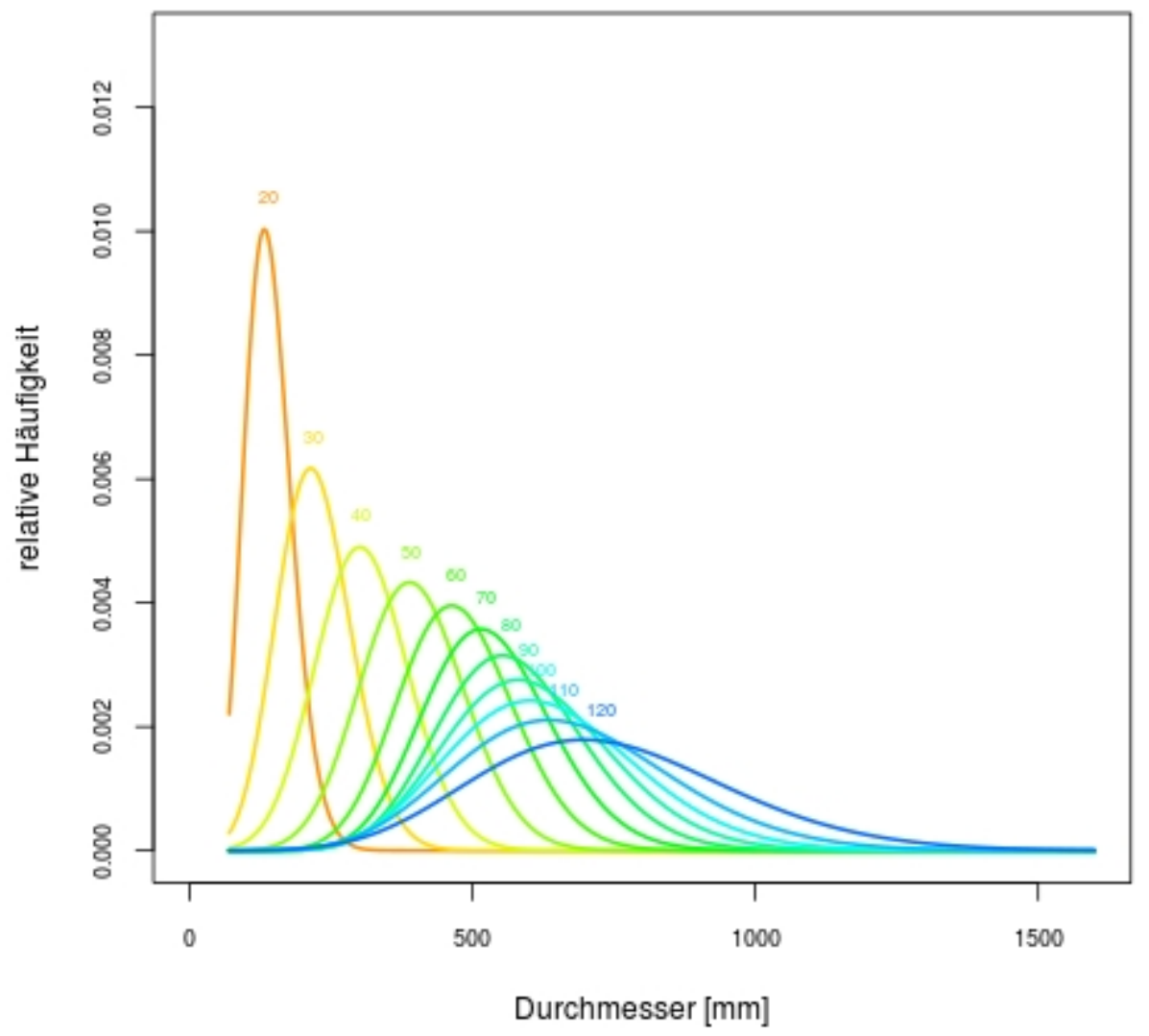

Abbildung 2.1: Durchmesserverteilungen von Douglasienreinbeständen mit guter Bonität (I. Ertragsklasse) für verschiedene Alter (von 20 bis 120 Jahren in 10Jahresabständen).

2005), wobei sich die Verteilung der abhängigen Variable durch die Box-Cox-Cole-GreenWahrscheinlichkeitsverteilung beschreiben lässt (vgl. Kap. 3.2.3). Dabei wurde hier eine linksseitig trunkierte Form der Box-Cox-Cole-Green-Verteilung verwendet, da nur Messdaten für Bäume ab sieben cm Brusthöhendurchmesser vorliegen. Des Weiteren wurden penalisierende glättende Splines zur Schätzung der Parameter der Verteilung benutzt. Die so erstellten Durchmesserverteilungen wurden auf mögliche Vereinfachungen der Modelleffekte auf die Parameter mittels sachlogischer Approximation überprüft. Die Modellparameter und -effekte sind im Anhang A.2.1 zu finden. Die Abbildung 2.1 zeigt beispielhaft die ermittelten Durchmesserverteilungen von Douglasienreinbeständen mit guter Bonität (I. Ertragsklasse) für verschiedene Alter. Es ist zu erkennen, dass sich die Durchmesserverteilung mit zunehmenden Alter verbreitert und abflacht.

Mit den eben beschriebenen Modellen ist die Generierung einzelner Weiserbestände, 
abhängig von der Baumart, dem Alter und der absoluten Mittelhöhenbonität im Alter $100\left(\mathrm{hg}_{100}[\mathrm{~m}]\right)$, möglich. Dabei wird zuerst die Bestandesgrundfläche mit dem erstellten Modell geschätzt. In Abbildung 2.2 sind beispielhaft die Effekte des Alters und der Mittelhöhe im Alter 100 auf die Schätzung der Bestandesgrundfläche von Douglasienbeständen dargestellt. Wie zu erkennen ist, wird die Grundfläche mit steigendem Alter und steigender absoluter Mittelhöhenbonität größer. In Tabelle 2.10 sind die Parameter des Grundflächenmodells für Douglasie, für die anderen Baumarten im Anhang A.2.2, zu finden. Ein iteratives Verfahren überprüft, bei welchen Werten der Parameter $\mu, \nu$ und $\sigma$ der Durchmesserverteilung, die Abweichungen zwischen der geschätzten Grundfläche und der aus der Durchmesserverteilung berechneten Grundfläche, am geringsten sind. Zur Berechnung der Grundfläche aus der Box-Cox-Cole-Green-Verteilung wird die entsprechende Quantilsfunktion, die Umkehrfunktion der Wahrscheinlichkeitsverteilung, genutzt. Somit kann die zur berechneten Grundfläche dazugehörige Durchmesserverteilung erstellt und es können Einzelbaumdaten, auf Grundlage der oben genannten Zusammenhänge, generiert werden. Die so generierten Daten wurden anschließend mittels Plugin in den WaldPlaner (s. Kap. 3.2.1, Hansen und Nagel (2014)) importiert und bilden ein Hektar große Weiserbestände.

Die Modellierung erfolgte mit dem Statistikpaket R (R Development Core Team, 2012) sowie den Bibliotheken gamlss (Rigby und Stasinopoulos, 2005), mgcv (Wood, 2011) und nls (Grothendieck, 2010).

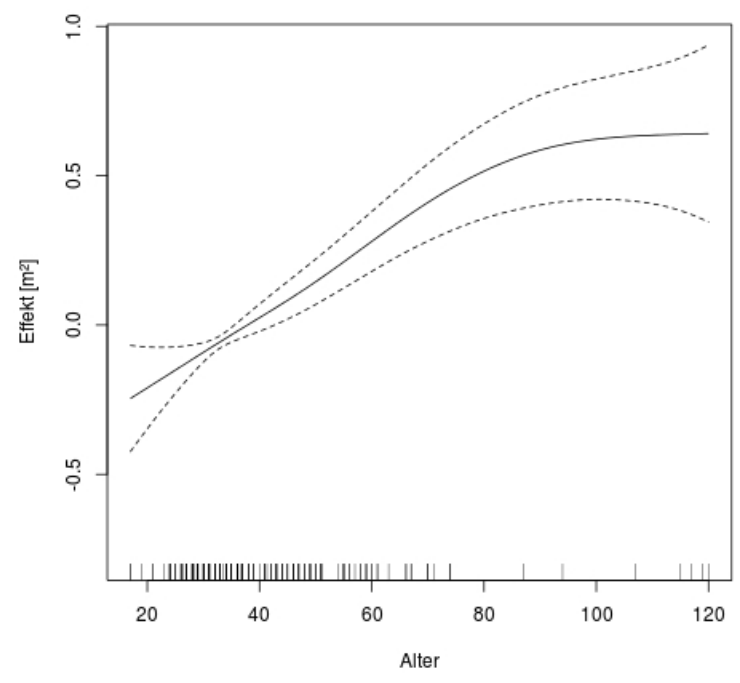

(a)

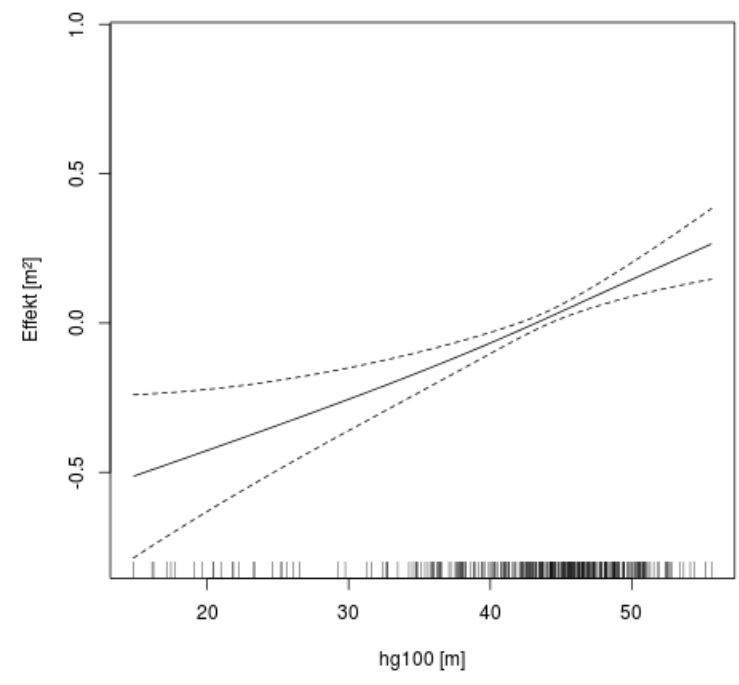

(b)

Abbildung 2.2: Effekt des Alters (a) und der absoluten Mittelhöhenbonität (hg100 [m]) (b) auf die Schätzung der Bestandesgrundfläche von Douglasienreinbeständen. 
Tabelle 2.10: Parameter und statistische Eigenschaften des Grundflächenmodells für Douglasienreinbestände.

\begin{tabular}{lllll}
\hline \multicolumn{2}{l}{ Parameterkoeffizienten } \\
& Schätzwert & Std. Fehler & t-Wert & $\operatorname{Pr}(>|\mathrm{t}|)$ \\
\hline Interzept & 3.10547 & 0.02507 & 123.9 & $<2 \mathrm{e}-16$ \\
\hline \multicolumn{5}{c}{ Approximierte Signifikanz der glättenden Terme } \\
& edf & Ref.df & F & p-Wert \\
\hline Alter & 2.613 & 3.243 & 18.72 & $1.04 \mathrm{e}-11$ \\
hg100 & 1.339 & 1.603 & 20.16 & $2.89 \mathrm{e}-07$ \\
\hline
\end{tabular}

\subsubsection{Ergebnisse der entwickelten Modelle zur Bestandesgenerierung}

Für die Simulationen wurden mit Hilfe der eben beschriebenen Methoden Weiserbestände generiert. Ausgehend vom baumartenspezifischen Startalter wurden in einem Abstand von 30 Jahren weitere Weiserbestände erstellt, die den gesamten Produktionszeitraum der jeweiligen Baumart abbilden. Das Initialalter der Bestände aus Eiche beträgt 35 Jahre, der aus Buche 40 Jahre, der aus Fichte und Kiefer jeweils 30 Jahre sowie der aus Douglasie 25 Jahre (s. Tab. 3.3). Das anfängliche Bestandesalter wurde so gewählt, dass die Bestände kurz vor der Erstdurchforstung stehen. Die Durchforstungen beginnen sobald eine Höhe von 14 bis $18 \mathrm{~m}$ erreicht ist (s. Kap. 3.2.2), dies tritt je nach Bonität früher oder später ein. Des Weiteren befindet sich ab dem festgelegten Startalter der Großteil der baumartenspezifischen Durchmesserverteilung über der Derbholzgrenze (Durchmesser $\geq 7 \mathrm{~cm}$ ) und damit wird eine inkorrekte Schätzung der Durchmesser junger Bäume unterhalb dieser Grenze vermieden.

Am Beispiel eines bewirtschafteten Douglasienreinbestandes soll das Zusammenspiel der Methoden zur Bestandesgenerierung näher beschrieben werden. In Abbildung 2.3 sind die Schätzungen der Grundflächen über dem Alter für Douglasienbestände bei unterschiedlichen Ertragsklassen zu sehen. Im Alter 25 ergeben sich voneinander abweichende Anfangsgrundflächen, für die erste Ertragsklasse sind es rund $23 \mathrm{~m}^{2} \mathrm{ha}^{-1}$. Diese wird mit steigendem Alter größer und nähert sich einer Asymptote an. Im Vergleich zur Douglasienertragstafel von Bergel (1985), starke Durchforstung und mittleres Ertragsniveau, ist die geschätzte Grundfläche rund $4 \mathrm{~m}^{2} \mathrm{ha}^{-1}$ geringer. Nun werden zufällig aus dem erstellten, alters- und bonitätsabhängigen Durchmesserverteilungsmodell solange Bäume gezogen, bis die Summe ihrer Grundflächen die zuvor berechnete Grundfläche, hier $23 \mathrm{~m}^{2}$ ha $^{-1}$, erreicht. Daraus resultiert ein Douglasienbestand mit 814 Einzelbäumen und einem Durchmesser bzw. einer Höhe des Grundflächenmittelstamms von 19,2 cm bzw. 14,8 m. Dieser Bestand, mit einer eingipfligen Durchmesserverteilung (s. Abb. 2.3), ist stammzahlärmer und etwa gleich hoch wie der vergleichbare Ertragstafelbestand. Je- 


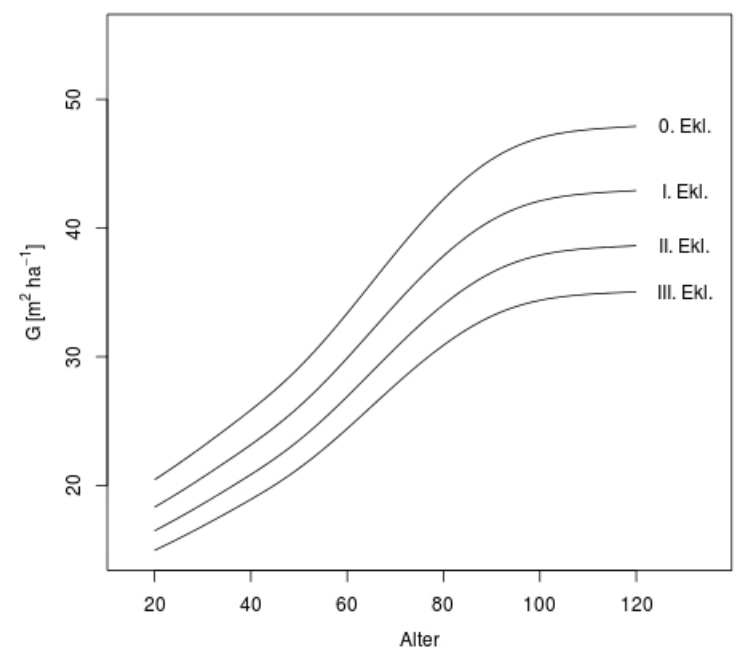

(a)

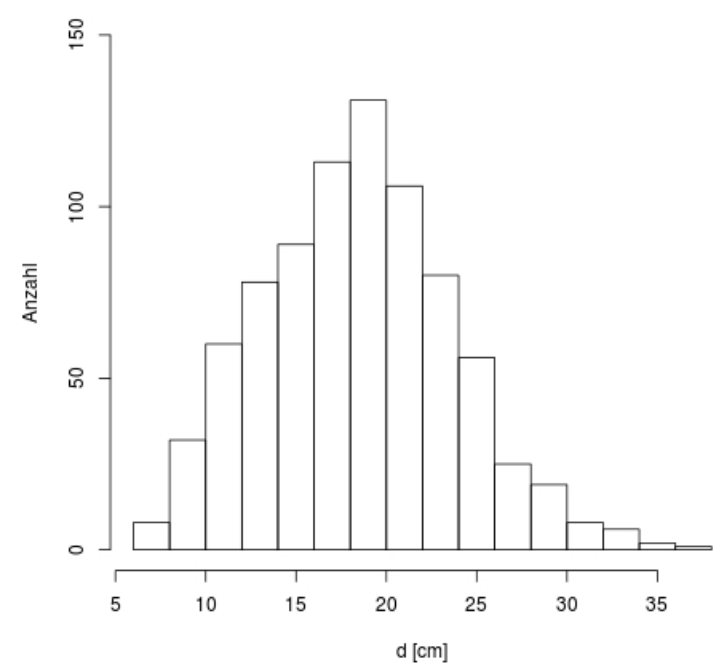

(b)

Abbildung 2.3: Geschätzte Bestandesgrundflächen von Douglasienreinbeständen über dem Bestandesalter bei unterschiedlichen Ertragsklassen (a) und die geschätzte Durchmesserverteilung eines jungen Douglasienreinbestandes mit guter Bonität (I. Ertragsklasse) (b). Erläuterungen siehe Text.

doch ist der Durchmesser des Grundflächenmittelstamms beim generierten Bestand ca. $2 \mathrm{~cm}$ größer als in der Ertragstafel angegeben.

In Tabelle 2.11 sind wichtige Kennwerte für einige Weiserbestände in den Wuchsbezirken Ems-Hase-Hunte-Geest und Göttinger Wald zu finden, eine Übersicht aller anderen Wuchsbezirke befindet sich im Anhang (s. Tab. A.22). Wobei hier nur die jungen Bestände im Startalter (s. Tab. 3.3) dargestellt sind. Auf dem beispielhaften Standort im Wuchsbezirk Ems-Hase-Hunte-Geest sind alle Hauptwirtschaftsbaumarten, im Wuchsbezirk Göttinger Wald nur Buche und Fichte vorhanden. Die hier dargestellten Leistungsklassen der Baumarten in den zwei Wuchsbezirken sind nahezu identisch mit den aus den Forsteinrichtungs- und Standortsdaten ermittelten mittleren Leistungsklassen (s. Tab. 2.6 und 2.7). Die angeführten Stammzahlen sind geringer im Vergleich zu den Angaben der Ertragstafelsammlung von Schober (1995) im gleichen Bestandesalter und ähnlicher Bonität. Der Durchmesser des Grundflächenmittelstamms wiederum ist größer als in einem vergleichbaren Ertragstafelbestand. Die dem Durchmesser und der Höhe des Grundflächenmittelstamms zugrunde liegenden Verteilungen, für die vorkommenden Hauptwirtschaftsbaumarten auf bestimmten Standorten in den zwei Wuchsbezirken, weisen eine eingipflige Verteilung auf (s. Abb. 2.4). Dabei sind hier die Durchmesserverteilungen der Fichten- und Douglasienbestände weiter nach rechts verschoben als die der anderen Arten. Bei den Höhenverteilungen fällt auf, dass im Göttinger Wald die jün- 
Tabelle 2.11: Kennwerte (LKL $=$ Leistungsklasse $\left[\mathrm{m}^{3} \mathrm{ha}^{-1} \mathrm{a}^{-1}\right], \mathrm{N}=$ Stammzahl $\left[\mathrm{nha}^{-1}\right]$, dg $=$ Durchmesser des Grundflächenmittelstamms [cm], hg = Höhe des Grundflächenmittelstamms $[\mathrm{m}], \mathrm{G}=$ Bestandesgrundfläche $\left[\mathrm{m}^{2} \mathrm{ha}^{-1}\right], \mathrm{V}=$ Derbholzvolumen $\left[\mathrm{m}^{3} \mathrm{ha}^{-1}\right], \mathrm{CV}=$ Kohlenstoffvorrat der lebenden Baumbiomasse $\left.\left[\mathrm{t} \mathrm{C} \mathrm{ha}^{-1}\right]\right)$ einiger Weiserbestände getrennt nach Wuchsbezirk (WBZ, EH = Ems-Hase-HunteGeest, GW = Göttinger Wald), Standortstypenobergruppe (SOGR, $4=$ Geschiebelehme, $5=$ Kalkgesteine), Wasserhaushalts- (WHZ, $5=$ frisch, $7=$ mäßig frisch) und Nährstoffgruppe (NZ, $1=$ sehr gut, $2=$ ziemlich gut) sowie Art (110 $=$ Eiche, $211=$ Buche, $511=$ Fichte, $611=$ Douglasie, $711=$ Kiefer $)$.

\begin{tabular}{ccccccccccccc}
\hline WBZ & SOGR & WHZ & NZ & Art & Alt & LKL & N & dg & hg & V & G & CV \\
\hline EH & 4 & 5 & 2 & 110 & 35 & 6,0 & 1179 & 12,8 & 12,0 & 86,1 & 15,1 & 36,9 \\
EH & 4 & 5 & 2 & 211 & 40 & 8,8 & 1262 & 13,6 & 14,6 & 126,0 & 18,2 & 57,9 \\
EH & 4 & 5 & 2 & 511 & 30 & 11,6 & 1140 & 18,2 & 12,1 & 167,5 & 29,8 & 82,3 \\
EH & 4 & 5 & 2 & 611 & 25 & 14,4 & 876 & 17,8 & 12,4 & 122,1 & 21,8 & 61,7 \\
EH & 4 & 5 & 2 & 711 & 30 & 6,6 & 1960 & 12,8 & 10,6 & 124,1 & 25,2 & 40,9 \\
GW & 5 & 7 & 1 & 211 & 40 & 8,2 & 1351 & 13,1 & 13,4 & 114,6 & 18,1 & 53,0 \\
GW & 5 & 7 & 1 & 511 & 30 & 12,9 & 1059 & 19,3 & 13,8 & 199,4 & 30,9 & 89,8 \\
\hline
\end{tabular}

geren Fichten im Mittel erwartungsgemäß höher sind als die 10 Jahre älteren Buchen. Auf dem Tieflandstandort sind eindeutig die Buchen im Mittel höher als die anderen Baumarten. Die größten Grundflächen werden bei dieser Standortauswahl in den Fichtenbeständen mit rund $30 \mathrm{~m}^{2} \mathrm{ha}^{-1}$ erreicht. Dann folgen Kiefer $\left(25,2 \mathrm{~m}^{2} \mathrm{ha}^{-1}\right)$, Douglasie $\left(21,8 \mathrm{~m}^{2} \mathrm{ha}^{-1}\right)$, Buche (rund $\left.18 \mathrm{~m}^{2} \mathrm{ha}^{-1}\right)$ und Eiche $\left(15,1 \mathrm{~m}^{2} \mathrm{ha}^{-1}\right)$. Das Bestandesvolumen ist hier am geringsten im Eichenbestand im Tiefland $\left(86,1 \mathrm{~m}^{3} \mathrm{ha}^{-1}\right)$ und am größten im Fichtenbestand im Bergland $\left(199,4 \mathrm{~m}^{3} \mathrm{ha}^{-1}\right)$, ebenso verhält sich der Kohlenstoffvorrat der lebenden Baumbiomasse (s. Tab. 2.11).

Ein Vergleich der mittleren Kennwerte aller jungen Weiserbestände getrennt nach Region und Baumart (s. Tab. 2.12) mit verschiedenen Ertragstafeln (Dittmar et al., 1983; Erteld, 1962; Lembcke et al., 2000; Schober, 1995; Wenk et al., 1984) führt, wie schon oben beschrieben, zu folgendem Resultat: Der Durchmesser des Grundflächenmittelstamms der generierten Bestände ist im Vergleich zu den Ertragstafeln größer und die Stammzahl geringer. Jedoch liegt die Höhe des Grundflächenmittelstamms im Bereich der Ertragstafelangaben.

Mit den entwickelten Modellen sind insgesamt 855 Weiserbestände in unterschiedlichen Altern für alle identifizierten Standorte (s. Kap. 2.2.1) bei insgesamt 198 BaumartWasserhaushalt-Nährstoffkombinationen erstellt worden. In Tabelle 2.13 sind die Bestandeskennwerte beispielhaft für einen Kiefernbestand im Wuchsbezirk Ost-Heide auf trockenen, schwach versorgten Sanden dargestellt. Die Angaben zu den anderen generierten Weiserbeständen sind in Anhang A.22 zu finden. Wie zu sehen ist, sinkt die 


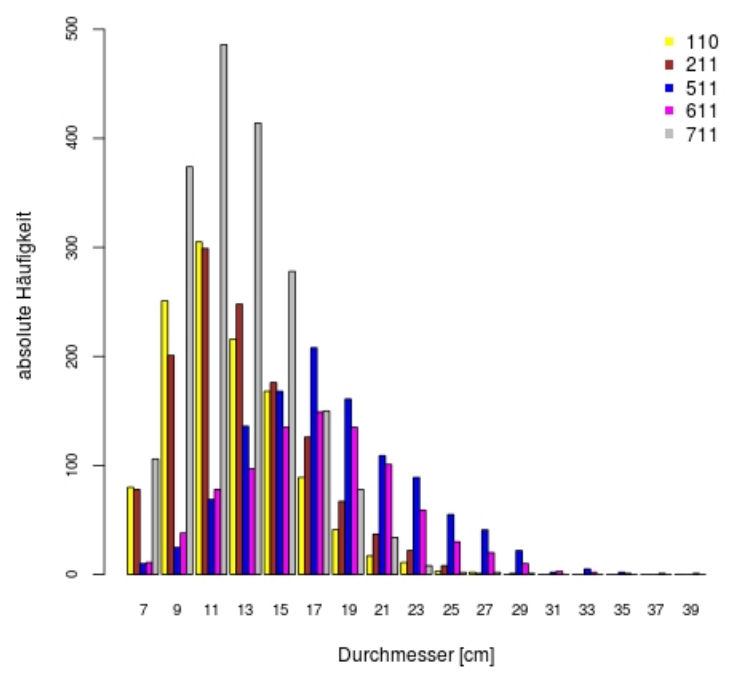

(a)

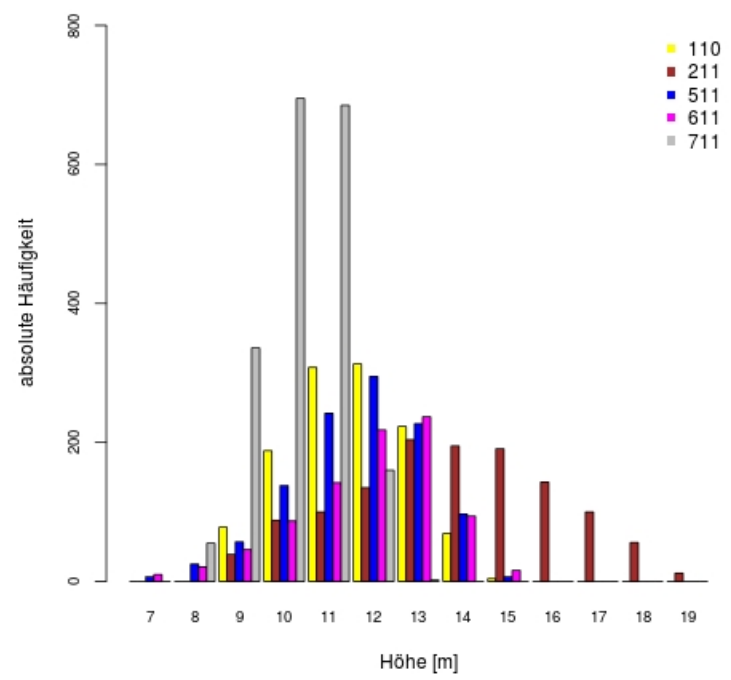

(c)

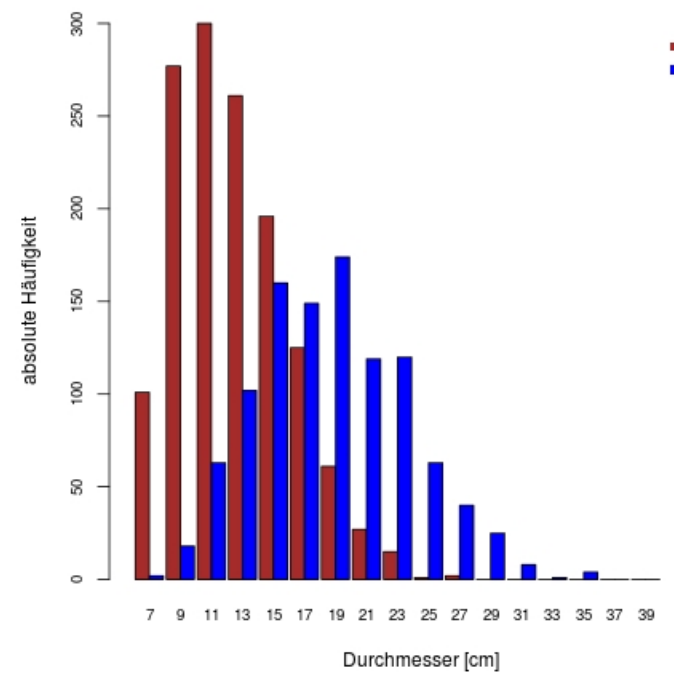

(b)

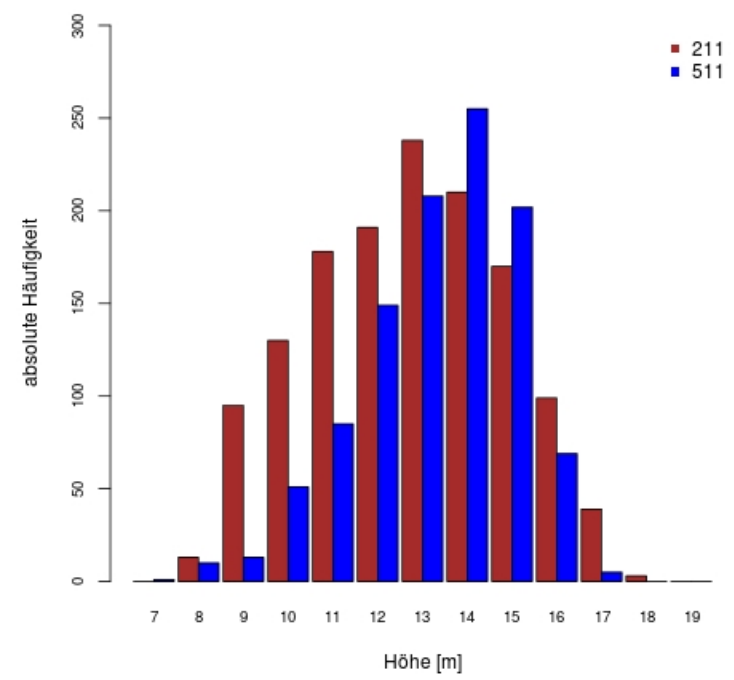

(d)

Abbildung 2.4: Absolute Häufigkeitsverteilung der Durchmesser [cm] (a, b) in $2 \mathrm{~cm}$-Stufen und Baumhöhen $[\mathrm{m}](\mathrm{c}, \mathrm{d})$ der Hauptwirtschaftsbaumarten $(110=$ Eiche, $211=$ Buche, $511=$ Fichte, $611=$ Douglasie, $711=$ Kiefer) in den Wuchsbezirken Ems-Hase-Hunte-Geest auf frischen, ziemlich gut versorgten Geschiebelehmen $(a, c)$ und Göttinger Wald auf mäßig frischen, sehr gut versorgten Kalkgesteinen $(b, d)$. 
Tabelle 2.12: Mittlere Bestandeskennwerte $\left(\mathrm{LKL}=\right.$ Leistungsklasse $\left[\mathrm{m}^{3} \mathrm{ha}^{-1} \mathrm{a}^{-1}\right], \mathrm{N}=$ Stammzahl $\left[\mathrm{n} \mathrm{ha}^{-1}\right], \mathrm{dg}=$ Durchmesser des Grundflächenmittelstamms $[\mathrm{cm}], \mathrm{hg}=$ Höhe des Grundflächenmittelstamms $[\mathrm{m}], \mathrm{G}=$ Bestandesgrundfläche $\left[\mathrm{m}^{2} \mathrm{ha}^{-1}\right], \mathrm{V}=$ Derbholzvolumen $\left.\left[\mathrm{m}^{3} \mathrm{ha}^{-1}\right]\right)$ der jungen Weiserbestände getrennt nach Region (B $=$ Bergland, $\mathrm{T}=$ Tiefland $)$ und Art $(110=$ Eiche, $211=$ Buche, $511=$ Fichte, $611=$ Douglasie, $711=$ Kiefer $)$.

\begin{tabular}{ccccccccc}
\hline Reg & Art & Alter & LKL & N & dg & hg & G & V \\
\hline \multirow{4}{*}{ B } & 110 & 35 & 6,2 & 1162 & 12,9 & 12,4 & 15,1 & 90,1 \\
& 211 & 40 & 7,5 & 1498 & 12,4 & 11,9 & 18,0 & 100,4 \\
& 511 & 30 & 10,9 & 1195 & 17,5 & 10,8 & 28,8 & 144,3 \\
\hline \multirow{6}{*}{$\mathrm{T}$} & 110 & 35 & 5,7 & 1200 & 12,5 & 11,4 & 14,7 & 79,0 \\
& 211 & 40 & 7,2 & 1577 & 12,1 & 11,0 & 17,9 & 92,7 \\
& 511 & 30 & 10,8 & 1193 & 17,5 & 10,7 & 28,7 & 142,8 \\
& 611 & 25 & 13,9 & 879 & 17,7 & 12,0 & 21,7 & 117,6 \\
& 711 & 30 & 6,9 & 1924 & 13,0 & 10,9 & 25,4 & 129,1 \\
\hline
\end{tabular}

Tabelle 2.13: Bestandeskennwerte (LKL $=$ Leistungsklasse $\left[\mathrm{m}^{3} \mathrm{ha}^{-1} \mathrm{a}^{-1}\right], \mathrm{N}=$ Stammzahl $\left[\mathrm{n} \mathrm{ha}^{-1}\right], \mathrm{dg}=$ Durchmesser des Grundflächenmittelstamms $[\mathrm{cm}], \mathrm{hg}=$ Höhe des Grundflächenmittelstamms $[\mathrm{m}], \mathrm{V}=$ Derbholzvolumen $\left[\mathrm{m}^{3} \mathrm{ha}^{-1}\right], \mathrm{G}=$ Bestandesgrundfläche $\left.\left[\mathrm{m}^{2} \mathrm{ha}^{-1}\right]\right)$ generierter Kiefernweiserbestände im Wuchsbezirk OstHeide auf trockenen Sanden mit schwacher Nährstoffversorgung.

\begin{tabular}{ccccccc}
\hline Alter & LKL & N & dg & hg & V & G \\
\hline 30 & 6,6 & 1955 & 12,9 & 10,6 & 125,5 & 25,4 \\
60 & 6,6 & 382 & 27,1 & 18,9 & 188,0 & 22,0 \\
90 & 6,6 & 226 & 35,6 & 23,7 & 235,0 & 22,6 \\
120 & 6,6 & 161 & 42,2 & 27,0 & 263,7 & 22,5 \\
150 & 6,6 & 109 & 48,6 & 29,6 & 256,4 & 20,2 \\
\hline
\end{tabular}

Stammzahl mit steigendem Alter und liegt unter den Angaben der Ertragstafel von Lembcke et al. (2000) für Kiefer im nordostdeutschen Tiefland (mittleres Ertragsniveau, Mittelhöhenbonität $24 \mathrm{~m}$, Bestockungsgrad 0,8). Dies ist auf den im Vergleich zur Ertragstafel größeren Durchmesser des Grundflächenmittelstammes zurückzuführen. Die ermittelte Höhe des Grundflächenmittelstammes ist nur geringfügig höher als in der Ertragstafel. Im Alter 30 ist die Grundfläche des Weiserbestandes höher als die Angabe von Lembcke et al. (2000). In den folgenden Altern ist es umgekehrt und die Grundfläche der Weiserbestände ist geringer als in der Ertragstafel. Dieser Effekt beruht auf dem 
Grundflächenmodell, welches anhand von regulär bewirtschafteten Flächen im nordwestdeutschen Tiefland parametrisiert wurde (s. Kap. 2.3.1). Auch das Derbholzvolumen der generierten Bestände ist anfangs höher als in der Kiefernertragstafel, ab Alter 60 jedoch geringer.

Das erarbeitete System aus Grundflächenmodell und Durchmesserverteilung auf Grundlage verallgemeinerter, additiver Regressionsmodelle sowie der Einzelbaumhöhenergänzung ist geeignet, um Reinbestände der Hauptwirtschaftsbaumarten in Nordwestdeutschland zu erstellen. Auch ist es für Anwender mit Grundkenntnissen in der Programmiersprache $\mathrm{R}$ einfach zu nutzen. 


\section{Methoden}

\subsection{Waldbaustrategien}

Bei der Speicherung von Kohlenstoff in Wäldern muss zwischen der maximalen Aufnahme und der Maximierung des Vorrates an Kohlenstoff im Bestand unterschieden werden. In jungen Beständen mit hoher Volumenproduktion ist die Kohlenstoffbindungsrate im Allgemeinen größer als in älteren Beständen, dafür ist deren Kohlenstoffvorrat höher. Die größte jährliche Volumenproduktion und damit die höchste Bindung von Kohlenstoff in Baumbiomasse wird erreicht, wenn der laufende Zuwachs (1Z) kulminiert (Kramer, 1988). Dieser Zeitpunkt ist erreicht, wenn die Differenz zwischen der Gesamtwuchsleistung eines Bestandes (GWL) in den aufeinanderfolgenden Jahren am größten ist. Nach diesem Punkt sinkt der laufende Zuwachs und die Höhe der Kohlenstoffaufnahme nimmt ab. Bis zum Erreichen der höchstmöglichen durchschnittlichen Volumenleistung (dGZmax) steigt der durchschnittliche Gesamtzuwachs und damit auch die Kohlenstoffmenge in der lebenden Baumbiomasse eines Bestandes an. Der Kulminationszeitpunkt des durchschnittlichen Gesamtzuwachses und dessen nachfolgendes, langsames Absinken ist besonders für betriebswirtschaftliche Überlegungen sehr bedeutend (Assmann, 1961). Jedoch ist zu diesem Zeitpunkt der durchschnittliche Wertzuwachs eines Bestandes nicht immer am größten. Dieser Moment wird meistens später erreicht. Der laufende Wertzuwachs eines Bestandes ist abhängig vom laufenden Volumenzuwachs, von den Veränderungen der Dimensionen der herrschenden Bäume, der Holzqualität sowie möglichen Entwertungen und Risiken die auf den Bestand einwirken können. Die Wertleistung bestimmt schlussendlich den finanziellen Ertrag der gesamten Produktion eines Bestandes. Auch hier gilt, wenn der laufende Wertzuwachs unter den durchschnittlichen Wertzuwachs eines realistischen Produktionszeitraumes sinkt, ist die Hiebsreife eines Bestandes gegeben. Allerdings kann auch anderen Aspekten, wie beispielsweise dem Verjüngungsgang, größere Bedeutung beigemessen werden (Röhrig et al., 2006).

Basierend auf diesen Überlegungen und den zuvor angesprochenen Gesichtspunkten der strategischen Waldbauplanung werden in dieser Arbeit zwei waldbauliche Szenarien analysiert. Diese beziehen sich auf gleichaltrige, einschichtige Reinbestände der Baumarten Eiche, Buche, Fichte, Douglasie und Kiefer mit variablen Produktionszeiträumen.

Die naturnahe Behandlung erfolgt in Anlehnung an die Clusterstudie Forst und Holz Niedersachsen (Rüther et al., 2007) und repräsentiert die derzeitige Waldbehandlung. Die kohlenstofforientierte Behandlung ist eine differenzierte Variante des naturnahen Szenarios, welches sich wie oben erwähnt, als vorteilhaft bei der Kohlenstoffspeicherung 
im Bestand und in den Holzprodukten erwiesen hat (s. Kap. 1.3). Das Ziel der kohlenstofforientierten Behandlung ist ein möglichst hoher Stammholzanteil in den Nutzungsmassen, um einen größtmöglichen Beitrag zur Kohlenstoffspeicherung in Holzprodukten mit langer und mittlerer Lebensdauer zu ermöglichen. Gleichzeitig soll auch der Kohlenstoffvorrat im Bestand erhöht werden.

\subsection{Simulation der Waldentwicklung}

\subsubsection{Prognosewerkzeug WaldPlaner}

Die Simulation der waldbaulichen Szenarien wird mit Hilfe des Waldwachstumsmodells WaldPlaner (Hansen, 2012; Hansen und Nagel, 2014) durchgeführt. Mit diesem Programm ist es möglich Bestände unter verschiedenen waldbaulichen Strategien fortzuschreiben. Dem Nutzer stehen als Simulationsergebnisse Informationen zur Produktion (Zuwachs, Nutzung, Sortenstruktur), zur biologischen Diversität (Laubholzanteil, Habitatbäume, Artprofil) und zu sozioökonomischen Funktionen (Erntekosten, Abtriebswert) sowie zu forstlichen Ressourcen (Vorrat, Bestandestypen) zur Verfügung. Somit ist der Anwender in der Lage, die Auswirkungen unterschiedlicher Waldbehandlungen abzuschätzen (NW-FVA, 2013a). In Verbindung mit der Programmiersprache R (R Core Team, 2015) sind umfangreiche Auswertungen möglich.

Der generelle konzeptionelle Ablauf einer Bestandesbehandlung mit dem Waldwachstumssimulator WaldPlaner ist folgendermaßen. Zu Beginn werden, falls gewünscht, Gassen angelegt und Habitatbäume ausgewählt. Anschließend wird geprüft, ob Holzerntemaßnahmen im Bestand notwendig sind, wobei bei einer Unterschreitung eines vorgegebenen Kronenschlussgrades, der gesamte Oberstand genutzt wird. Sofern ein Bestand $\mathrm{zu}$ dicht und die vorgegebene minimale Nutzungsmenge erreicht ist, findet eine Nutzung statt. Dabei wird geprüft, wie hoch die aktuelle Grundfläche des Bestandes im Vergleich zur Bestandesgrundfläche bei einem höhen- und baumartenspezifischen natürlichen Bestockungsgrad ist (s. Kap. 3.2.3). Anschließend werden zunächst die Zukunftsbäume $^{1}$ freigestellt bis sie keinen Kronenkontakt mehr zu benachbarten Bäumen aufweisen oder die Zielgrundfläche in der Entwicklungsphase erreicht ist. Sind die Zukunftsbäume freigestellt und die momentane Bestandesgrundfläche immer noch höher als die vorgegebene Zielgrundfläche, so werden schrittweise die Bäume entnommen, die den höchsten Konkurrenzdruck auf die benachbarten Bäume ausüben. Die Endnutzung setzt ein, sobald der baumartenspezifische Zieldurchmesser überschritten und eine bestimmte Erntemenge erreicht ist (Hansen und Nagel, 2014). Dabei beschränkt sich die simulierte Zielstärkennutzung nicht nur auf die Entnahme zielstarker Bäume, da der Unter- und Zwischenstand das Produktionsziel nicht oder nicht in angemessener Zeit erreichen kann. Sobald in einem Bestand $30 \%$ der Grundfläche auf zielstarke Bäume entfallen, werden

\footnotetext{
${ }^{1}$ Zukunftsbäume, auch Z-Bäume genannt, sind Bäume, von denen erwartet wird, dass sie den angestrebten Zieldurchmesser oder das beabsichtigte Alter erreichen.
} 
Tabelle 3.1: Baumartenabhängige Verjüngungsgänge, wobei der Zielbestand dem Ausgangsbestand entspricht.

\begin{tabular}{lc}
\hline Baumart & Verjüngungsgang \\
\hline Eiche & $0,6-0,3-0,0$ \\
Buche & Zielstärkennutzung \\
Fichte & $0,6-0,5-0,0$ \\
Douglasie & $0,6-0,5-0,4-0,0$ \\
Kiefer & $0,6-0,4-0,3-0,2-0,0$ \\
\hline
\end{tabular}

von diesen $50 \%$ zufallsgesteuert genutzt. Die restlichen $50 \%$ werden vom schwachen Ende kommend genutzt (Spellmann et al., 2015b). Durch eine alleinige Nutzung zielstarker Bäume verblieben im Bestand Individuen mit einer geringeren Leistungsfähigkeit, was sich auch negativ auf den Höhenzuwachs auswirkt. Zusätzlich zum Zieldurchmesser ist, mit Ausnahme der Buche, ein baumartenspezifischer Verjüngungsgang implementiert, welcher den Fortschritt der Bestandesauflichtungen in Abständen von fünf Jahren beschreibt (s. Tab. 3.1). Daraus ergibt sich ein Überlappungszeitraum von Altbestand und Nachwuchs. Dabei wird die Annahme getroffen, dass der Zielbestand aus der gleichen Baumart besteht wie der Ausgangsbestand. Daraus ergibt sich für die Baumart Eiche als Verjüngungsform ein kurzfristiger Schirmschlag. Unabhängig vom Verjüngungsgang wird ein Bestand final geräumt, wenn die aktuelle Bestandesgrundfläche $30 \%$ oder weniger der angestrebten Zielgrundfläche der momentanen Entwicklungsphase beträgt. Die Habitatbäume werden aber auf der Fläche belassen. Für weitere Details zum WaldPlaner sei hier auf Hansen und Nagel (2014) verwiesen.

Für die Simulationen wurden ausgehend vom baumartenspezifischen Startalter über den gesamten Produktionszeitraum (s. Kap. 3.2.2) verteilt ein Hektar große Weiserbestände (s. Kap. 2.3) in 30-jährigen Abständen generiert und anschließend maximal 30 Jahre für beide Behandlungsvarianten einmal fortgeschrieben. Dieses Vorgehen ist vergleichbar mit einer unechten Zeitreihe. Auch in Bezug auf die Güte der Simulationsergebnisse ist dieses Vorgehen vorteilhafter als eine durchgehende Simulation für sehr lange Zeiträume, weil mit der Zeit die Unsicherheiten einer Simulation stark ansteigen. Aufgrund des beschriebenen Vorgehens gibt es mehrere Initialzustände. Hinzu kommt, dass die Art der Holzverwendung über einen längeren Zeitraum stark von gesellschaftlichen Ansprüchen abhängt und die fortschreitenden Klimaveränderungen mit ihren Einflüssen auf die Leistungsfähigkeit der Baumarten und den Kohlenstoffkreislauf in dieser Arbeit nicht abgebildet werden können. 


\subsubsection{Waldbauliche Steuerung}

Bei der naturnahen (nn) und kohlenstofforientierten (co) Variante werden naturschutzund eingriffsspezifische Parameter zur Einsteuerung der waldbaulichen Behandlung genutzt (s. Tab. 3.2). Es werden drei Habitatbäume aus der Nutzung genommen und der natürlichen Entwicklung bis hin zur Zersetzung überlassen. Zudem wird eine Totholzmenge von $20 \mathrm{~m}^{3} \mathrm{ha}^{-1}$ angestrebt. Diese zersetzt sich und bildet so eine wichtige Lebensgrundlage für viele im Wald lebende Organismen. Zu Beginn der Behandlung werden Rückegassen im Abstand von $20 \mathrm{~m}$ und einer Breite von $4 \mathrm{~m}$ angelegt. Die sich anschließenden Durchforstungen beginnen bei einer Oberhöhe von 14 bis $18 \mathrm{~m}$, wobei die Zukunftsbäume stark freigestellt und gestaffelt in der Eingriffstärke, mit einem Übergang von starken Eingriffen zu Beginn der Durchforstung und schwachen Eingriffen an deren Ende, behandelt werden.

Der Endnutzungszeitpunkt und somit die Produktionszeit (s. Tab. 3.3) wird baumartenspezifisch über den Brusthöhendurchmesser (BHD) gesteuert. Um bei der kohlenstofforientierten Behandlung einen größtmöglichen Beitrag zur Kohlenstoffspeicherung in Holzprodukten und im Bestand zu realisieren, werden die Zieldurchmesser bei dieser Variante, bei Unterstellung mittlerer Bestandesrisiken und Qualitäten in Abhängigkeit von der Bonität, gestaffelt festgelegt. Dabei ist eine schlechte Bonität einer Ertragsklasse geringer als I,9, eine mittlere Bonität zwischen einer I,9 und I,4 Ertragsklasse und eine gute Bonität einer Ertragsklasse über I,4, nach der Ertragstafelsammlung von Schober (1995) bei mäßiger bzw. für Douglasie bei starker Durchforstung, gleichzusetzen. Demnach sind etwa jeweils $20 \%$ der Weiserbestände einer guten bzw. schlechten Bonität, die restlichen 60\% einer mittleren Bonität zuzuordnen (s. Tab. 3.4). Die Zieldurchmesser sind damit teilweise gleich, geringer oder größer als bei der naturnahen Behandlung ( $\mathrm{s}$. Tab. 3.5) und somit wird die Wuchsleistung der Bestände bei der Endnutzung beachtet.

Das Durchforstungs- und Erntevolumen wird gemäß dem Holzverwendungsschlüssel (s. Tab. 3.13) den Produktklassen zugeordnet. Bestände die bis zum Ende der baumartenspezifischen Produktionszeit nicht vom WaldPlaner geräumt waren, wurden nachträglich sortiert und in den Holzproduktspeicher überführt. Dem gesamten geernteten Holz wird eine bestimmte Verwendung unterstellt und es wird anschließend in einer Kaskade genutzt (s. Kap. 3.3.3).

\subsubsection{Herleitung der maximalen Bestandesgrundfläche}

\subsubsection{Methodisches Vorgehen und Modellentwicklung}

Zur Erfassung der Durchforstungsstärke und -art wurde 1902 im Versuchsplan des Vereins der Forstlichen Versuchsanstalten eine kategorische Abstufung in Form der Durchforstungsgrade eingeführt (Anonymus, 1902). Wiedemann (1935) hat später in Preußen damit begonnen die Durchforstungsstärke anhand der Grundflächenhaltung zu beschreiben. Die abgestufte qualitative Beschreibung der Eingriffstärke von 1902 wurde später von Assmann (1961) heftig kritisiert, da es unklar war, wie stark in die herrschenden 
Tabelle 3.2: Baumartenspezifische Übersicht einiger Rahmenwerte der Simulation.

\begin{tabular}{lccccc}
\hline Baumart & $\begin{array}{c}\text { Endnutzungsvolumen } \\
{\left[\mathrm{Vfm} \mathrm{ha}^{-1}\right]}\end{array}$ & $\begin{array}{c}\text { Durchforstungsvolumen } \\
{\left[\mathrm{Vfm} \mathrm{ha}^{-1}\right]}\end{array}$ & $\begin{array}{c}\text { Anzahl der } \\
\text { Zukunftsbäume }\end{array}$ \\
\cline { 2 - 5 } & min. & $\max$ & $\min$. & $\max$. & \\
\hline Eiche & 20 & 80 & 10 & 60 & 120 \\
Buche & 20 & 100 & 20 & 80 & 100 \\
Fichte & 20 & 80 & 20 & 80 & 150 \\
Douglasie & 20 & 120 & 20 & 100 & 120 \\
Kiefer & 20 & 80 & 20 & 80 & 180 \\
\hline
\end{tabular}

Tabelle 3.3: Produktions- und maximale Simulationszeiträume der untersuchten Baumarten sowie Alter der Weiserbestände zu Beginn der Simulationen.

\begin{tabular}{lccc}
\hline Baumart & $\begin{array}{c}\text { Produktionszeitraum } \\
\text { [Jahre] }\end{array}$ & $\begin{array}{c}\text { maximaler } \\
\text { Simulationszeitraum } \\
\text { [Jahre] }\end{array}$ & $\begin{array}{c}\text { Startalter } \\
\text { [Jahre] }\end{array}$ \\
\hline Eiche & $160-200$ & 165 & 35 \\
Buche & $100-160$ & 120 & 40 \\
Fichte & $60-120$ & 90 & 30 \\
Douglasie & $80-120$ & 105 & 25 \\
Kiefer & $120-160$ & 130 & 30 \\
\hline
\end{tabular}

Tabelle 3.4: Anzahl der Weiserbestände getrennt nach Bonitätsgruppe und Baumart.

\begin{tabular}{ccccccc}
\hline \multirow{2}{*}{ Bonitätsgruppe } & \multicolumn{5}{c}{ Baumart } & \multirow{2}{*}{ Gesamt } \\
\cline { 2 - 6 } & Eiche & Buche & Fichte & Douglasie & Kiefer \\
\hline gut & 42 & 28 & 54 & 4 & 35 & 163 \\
mittel & 150 & 84 & 75 & 88 & 115 & 512 \\
schlecht & 42 & 72 & 24 & 12 & 30 & 180 \\
\hline
\end{tabular}


Tabelle 3.5: Baumartenspezifische konstante Zieldurchmesser bei naturnaher und gestaffelte, bonitätsabhängige Zieldurchmesser bei kohlenstofforientierter Behandlung bei mittleren Risiken und Qualitäten.

\begin{tabular}{lcccc}
\hline & \multicolumn{4}{c}{ Zieldurchmesser $[\mathrm{cm}]$} \\
\cline { 2 - 5 } Baumart & naturnah & \multicolumn{3}{c}{ kohlenstofforientiert } \\
\cline { 3 - 5 } & & gute Bonität & mittlere Bonität & schlechte Bonität \\
\hline Eiche & 70 & 80 & 70 & 50 \\
Buche & 60 & 70 & 60 & 50 \\
Fichte & 45 & 55 & 45 & 40 \\
Douglasie & 70 & 80 & 70 & 60 \\
Kiefer & 45 & 55 & 45 & 40 \\
\hline
\end{tabular}

Baumklassen eingegriffen werden durfte. Daher schlug er die maximale Grundflächenhaltung als Referenz bzw. Abweichungen von dieser Referenz als quantitatives Maß zur Beurteilung der Durchforstungsstärke, mithin der Regulierung der Bestandesdichte, vor. Um dies umzusetzen ist es zunächst einmal nötig, die maximal mögliche Bestandesgrundfläche eines Standortes zu ermitteln.

Die Dichte eines Bestandes, beschrieben in Form der Grundfläche pro Hektar, ist eine Funktion der Anzahl der Bäume und des mittleren quadratischen Durchmessers. Durch das Wachstum der Bäume nimmt die Bestandesdichte über dem Alter immer weiter zu, bis erste Bäume beginnen abzusterben. Dieser Prozess wird dichteabhängige Mortalität oder Selbstdurchforstung genannt. Dabei verringert sich die Stammzahl der Bäume, der Vorrat bzw. die Biomasse pro Hektar nimmt im Allgemeinen zu. Die maximale Dichte, somit der Grenzwert der Bestandesdichte, spiegelt das Ertragsniveau eines Standortes wider (Assmann, 1961).

Zusätzlich zur maximalen Bestandesgrundfläche wird zur Festlegung der angestrebten Grundfläche am Ende des Planungszeitraumes ein Nutzungskonzept benötigt. In dieser Arbeit sind mehrere Pflegephasen vorgesehen. Diese Phasen beziehen sich wie bei Spellmann et al. (1999) auf bestimmte Spitzenhöhenbereiche. In Verbindung mit dem natürlichen Bestockungsgrad (nat. $\mathrm{B}^{\circ}$ ) ist eine objektive Grundlage für die Durchforstungsstärke vorhanden. Der natürliche Bestockungsgrad ist das Verhältnis der beobachteten Grundfläche eines Bestandes zur maximalen Grundfläche eines unbehandelten Bestandes auf gleichem Standort und bei gleicher Entwicklungsstufe. Demgegenüber gibt es auch den Bestockungsgrad auf Grundlage von Ertragstafeln, wobei die aktuell ermittelte Bestandesgrundfläche mit der entsprechenden Angabe der Ertragstafel verglichen wird. Seine unkritische Nutzung ist für die Steuerung der Eingriffstärke in Wachstumssimulatoren nicht zweckmäßig, da aus ihm zu starke Grundflächenabsenkungen resultieren können (Röhrig et al., 2006). Der natürliche Bestockungsgrad, in Zusammenhang mit der zuverlässig hergeleiteten maximalen Bestandesgrundfläche, ist als Steuerungsgröße 
besser geeignet. Er ist flexibler als der Bestockungsgrad auf Grundlage der Ertragstafeln und berücksichtigt das Ertragsniveau.

Die maximale Bestandesdichte lässt sich mit Hilfe langfristig beobachteter Versuchsflächen ohne aktive Durchforstung herleiten oder sie wird rechnerisch eingeschätzt. Nagel (1997) hat die rechnerische Herleitung nach dem Schätzverfahren von Sterba (1975, 1981, 1987) durchgeführt. Dieses Verfahren stützt sich auf die maximale Stammzahl bei gegebenem Mitteldurchmesser im Sinne des von Reineke aufgestellten maximalen Stand Density Index (Reineke, 1933). Dieser Weiser hat den Vorteil, dass er alters- und bonitätsunabhängig ist. Mit ihm und der Competition-Density-Rule der Japaner Kira et al. (1953), Tadaki (1963) und Ando (1968) lässt sich die maximale Grundflächenhaltung nach Assmann (1961) bestimmen (Spellmann et al., 1999). Dabei wird, unter der Annahme, dass die maximale Grundfläche des Standortes erreicht ist, zuerst der Zusammenhang zwischen dem Durchmesser des Grundflächenmittelstammes ( $\left.\operatorname{dg}_{\text {Gmax }}\right)$, der Spitzenhöhe (h100) und der Stammzahl (N) hergeleitet. Dabei werden mittels nichtlinearer Regression die Koeffizienten der Gleichung 3.1 für die unterschiedlichen Baumarten geschätzt. Somit kann die Stammzahl bei maximaler Grundfläche $\left(\mathrm{N}_{\mathrm{Gmax}}\right)$ in Abhängigkeit vom dazugehörigen Durchmesser des Grundflächenmittelstammes ( $\operatorname{dg}_{\text {Gmax }}$ ) ermittelt werden (s. Gl. 3.2). Die maximale Grundfläche $\left(\mathrm{G}_{\max }\right)$ wird abschließend abhängig von der Spitzenhöhe (h100) mit der Formel 3.3 berechnet.

Nagel (1997) konnte, mit Hilfe des genannten Schätzverfahrens und ertragskundlicher Daten, die maximale Bestandesgrundfläche in Nordwestdeutschland berechnen. Döbbeler (2004) erarbeitete dies für verschiedene Regionen in Deutschland. Auf Grundlage von neueren und erweiterten ertragskundlichen Versuchsflächendaten der Nordwestdeutschen Forstlichen Versuchsanstalt (s. Anh. A.3) und dem Statistikprogramm R (R Development Core Team, 2011) sowie der Bibliothek nlme (Pinheiro et al., 2011) wurde die Gleichung 3.1 baumartenspezifisch mittels nichtlinearer Regression für Nordwestdeutschland neu parametrisiert. Die benutzten Versuchsflächendaten enthalten im Gegensatz zum Datensatz von Döbbeler (2004) vor allem mehr junge Bestände. Auch sind in begrenztem Umfang Flächen aus Rheinland-Pfalz sowie dem Saarland enthalten. Darüber hinaus wurden nur Bestände der Hauptwirtschaftsbaumarten aus den vorliegenden Versuchsflächendaten genutzt, welche einen Anteil von mindestens 0,8 an der verbleibenden Bestandesgrundfläche aufwiesen, um möglichst Reinbestände in die Analyse einzubeziehen. Somit konnten die in Tabelle 3.6 aufgeführten Koeffizienten geschätzt werden.

$$
\begin{aligned}
& d g_{\text {Gmax }}=\frac{1}{a_{0} \times h 100^{a_{1}} \times N+b_{0} \times h 100^{b_{1}}} \\
& N_{\text {Gmax }}=\frac{b_{0}}{a_{0}} \times\left(2 \times b_{0} \times d g_{\text {Gmax }}\right)^{\frac{a_{1}}{b_{1}}-1} \\
& G_{\max }=\frac{\pi}{16 \times a_{0} \times b_{0} \times h 100^{\left(a_{1}+b_{1}\right)}}
\end{aligned}
$$




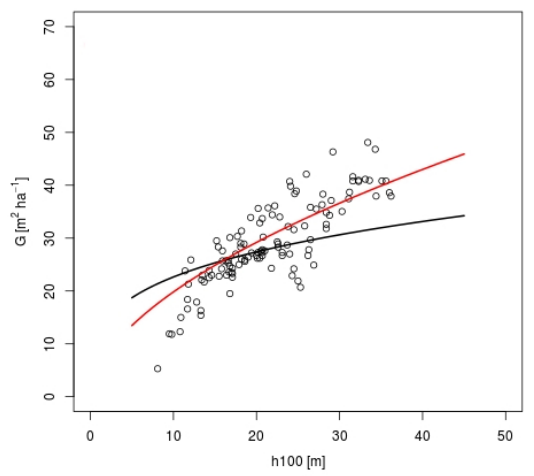

(a)

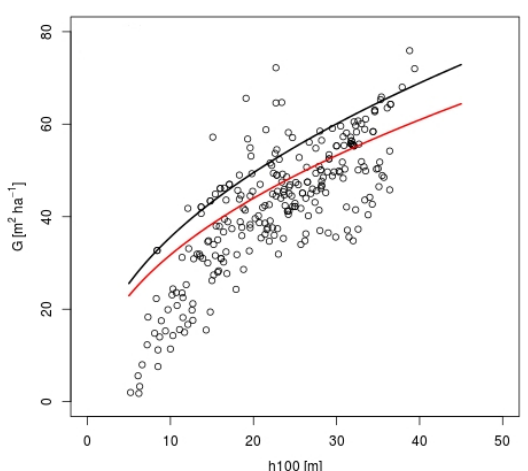

(c)

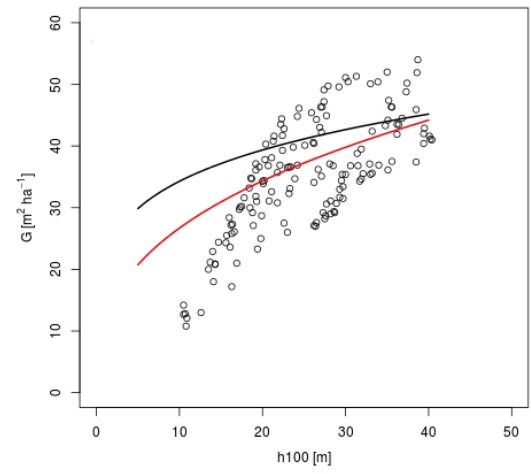

(b)

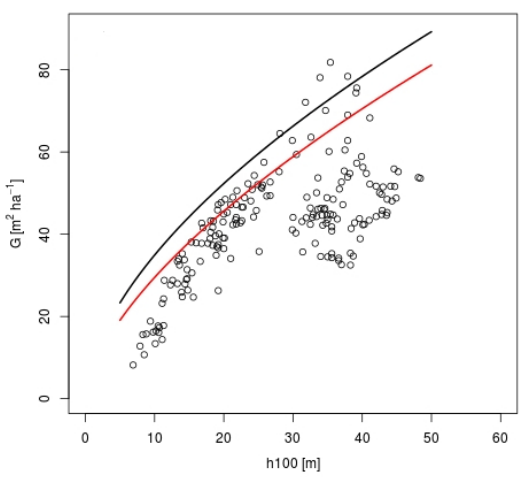

(d)

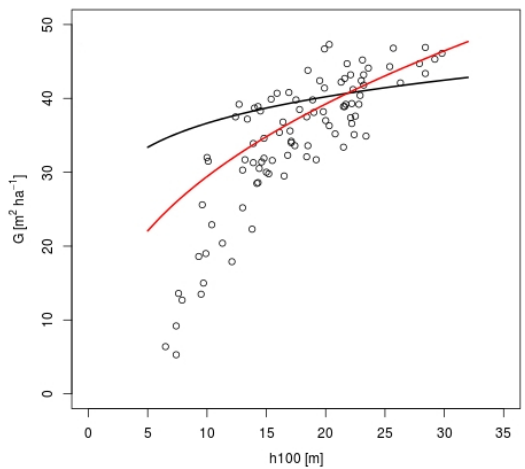

(e)

Abbildung 3.1: Vergleich der Versuchsflächendaten mit dem Verlauf der Funktion zur Schätzung der maximalen Bestandesgrundfläche $\left(\mathrm{G}\left[\mathrm{m}^{2} \mathrm{ha}^{-1}\right]\right)$ über der Spitzenhöhe (h100 [m]) nach dem Ansatz von Sterba $(1975,1981,1987)$ mit den Koeffizienten von Döbbeler (2004) (schwarze Kurve) und hier neu geschätzten (s. Tab. 3.6 , rote Kurve) für Reinbestände verschiedener Baumarten $(\mathrm{a}=$ Eiche, $\mathrm{b}=$ Buche, $\mathrm{c}=$ Fichte, $\mathrm{d}=$ Douglasie, $\mathrm{e}=$ Kiefer $)$. 
Tabelle 3.6: Ermittelte Koeffizienten zur Schätzung der maximalen Bestandesgrundfläche von Reinbeständen der Hauptwirtschaftsbaumarten in Nordwestdeutschland nach dem Ansatz von Sterba $(1975,1981,1987)$ (s. Gl. 3.3).

\begin{tabular}{lcccc}
\hline Baumart & $\mathrm{a}_{0}$ & $\mathrm{a}_{1}$ & $\mathrm{~b}_{0}$ & $\mathrm{~b}_{1}$ \\
\hline Buche & $2.616551 \mathrm{e}-07$ & 1.368151 & 6.496417 & -1.731867 \\
Douglasie & $3.478716 \mathrm{e}-06$ & 0.4525542 & 0.814011 & -1.081344 \\
Eiche & $2.407913 \mathrm{e}-07$ & 1.513792 & 14.8852 & -2.072313 \\
Fichte & $4.913256 \mathrm{e}-06$ & 0.4394706 & 0.3716977 & -0.9097641 \\
Kiefer & $7.480717 \mathrm{e}-07$ & 1.064168 & 2.321089 & -1.479456 \\
\hline
\end{tabular}

Die Abbildung 3.1 zeigt den Verlauf der Funktion 3.3 mit den Koeffizienten von Döbbeler (2004) und den hier neu ermittelten für Reinbestände der Hauptwirtschaftsbaumarten (s. Tab. 3.6) sowie als Referenz Daten weitestgehend unbehandelter Versuchsflächen in Nordwestdeutschland. Wie zu sehen ist, resultiert aus den bedingten Schätzungen der maximalen Bestandesgrundfläche basierend auf dem Ansatz von Sterba (1975, 1981, 1987) keine gute Beschreibung der beobachteten Grundflächen. Weder mit den alten, noch mit den neu ermittelten Koeffizienten. In jungen Beständen (geringe Spitzenhöhen) wird die maximal beobachtete Grundfläche überschätzt, in mittelalten sowie alten Beständen (mittlere und große Spitzenhöhen) wird sie unterschätzt. Annäherungsweise wäre eine Umhüllende zu erwarten. Bei der Betrachtung der Referenzflächen fällt auf, dass einige ältere Flächen deutlich geringere Grundflächen haben als andere in der gleichen Entwicklungsphase. Gründe dafür können Unterschiede im Ertragsniveau oder eine späte Versuchsanlage ehemals behandelter Bestände sein.

Als Alternative zur Beschreibung einer umhüllenden Funktion bzw. zur Schätzung von oberen Extremwerten stehen seit neuerem verschiedene Methoden der Quantilsregression zur Verfügung (Rigby und Stasinopoulos, 2005; Koenker, 2012). Dabei wird ein beliebiges Quantil der Zielgröße in Abhängigkeit von den erklärenden Variablen geschätzt (Fahrmeir et al., 2009). In der vorliegenden Untersuchung wird ein Ansatz verwendet, der auf einer parametrischen Verteilungsfunktion basiert. Die hier benutzte Box-CoxCole-Green-Verteilung (BCCG-Verteilung) lässt sich mit den Parametern Mittelwert $\mu$, Schiefe $\nu$ und Variationskoeffizient $\sigma$ beschreiben (Albert und Schmidt, 2009; Stasinopoulos et al., 2012). Die BCCG-Verteilung hat sich unter anderem bei der Beschreibung von unimodalen Verteilungen der Bonität verschiedener Baumarten bewährt (Albert und Schmidt, 2009). Mit Hilfe von verallgemeinerten additiven Modellen als Funktion von Kovariablen und glättender Splines wird eine sehr hohe Modellflexibilität ermöglicht (Rigby und Stasinopoulos, 2005). Ist die BCCG-Verteilung eine geeignete theoretische Approximation der Zielvariable, führt die Methode zu einer Transformation der Response-Variable in eine standard-normalverteilte Größe $Z$ (s. Gl. 3.4). 


$$
Z= \begin{cases}\frac{1}{\sigma \nu}\left[\left(\frac{Y}{\mu}\right)^{\nu}-1\right], & \text { wenn } \nu \neq 0 \\ \frac{1}{\sigma} \ln \left(\frac{Y}{\mu}\right), & \text { wenn } \nu=0\end{cases}
$$

für $0<Y<\infty$ wenn $\mu>0, \sigma>0, \nu=(-\infty,+\infty)$ und wenn die Variable $Z$ standard-normalverteilt ist. Somit ist die Wahrscheinlichkeitsdichtefunktion von $Y$ wie folgt definiert:

$$
f_{Y}(y)=\frac{y^{\nu-1} \exp \left(-\frac{1}{2} z^{2}\right)}{\mu^{\nu} \sigma \sqrt{2 \pi} \phi\left(\frac{1}{\sigma|\nu|}\right)}
$$

wobei $Z$ durch Gleichung 3.4 beschrieben und $\phi$ die Standardnormalverteilung ist. Die bedingte Quantilsfunktion wird folgendermaßen definiert:

$$
Q\left(\tau \mid X_{i}\right)=\mu\left(X_{i}\right)\left(1+\nu\left(X_{i}\right) \sigma\left(X_{i}\right) \phi^{-1}(\tau)\right)^{\frac{1}{\nu\left(X_{i}\right)}}
$$

mit:

$\tau=$ kumulierte Wahrscheinlichkeitsdichte

$X_{i}=$ Kovariablenvektor $i$ von Beobachtung $i$

$\phi^{-1}=$ inverse Standardnormalverteilung

Für die Modellierung des Grundflächenquantils werden folgende Funktionen für die Hauptwirtschaftsbaumarten in Nordwestdeutschland aufgestellt und im Statistikpaket R (R Development Core Team, 2011) unter Verwendung der Bibliothek gamlss (Rigby und Stasinopoulos, 2005) parametrisiert:

$$
\begin{aligned}
& \mu=\alpha_{1}+f_{1}(h 100) \\
& \ln (\sigma)=\alpha_{2}+f_{2}(h 100) \underset{\sigma=\text { konstant }}{\longrightarrow} \alpha_{2} \\
& \nu=\alpha_{3}+f_{3}(h 100) \\
& \text { mit: } \\
& h 100=\text { Spitzenhöhe [m] } \\
& \alpha_{1} \ldots \alpha_{3}=\text { Regressionskoeffizienten } \\
& f_{1} \ldots f_{3}=\text { glättende Splines zur Beschreibung nicht-linearer Modelleffekte }
\end{aligned}
$$


Im Verlauf der Analyse wurde der Variationskoeffizient $\sigma$ (s. Gl. 3.8) und die Schiefe $\nu$ (s. Gl. 3.9) aufgrund von nicht signifikanten Effekten oder unplausiblen Ergebnissen teilweise konstant gesetzt. Möglicherweise sind diese Effekte auf den partiell geringen Datenumfang zurückzuführen. Im Anhang A.3 sind die Parameterschätzungen der Koeffizienten und die Modelleffekte auf die Koeffizienten der dazugehörigen BCCG-Verteilung für die Hauptwirtschaftsbaumarten in Nordwestdeutschland zu finden. Die Validierung der BCCG-Verteilung erfolgt mittels sogenannter Quantile-Quantile-Plots (QQ-Plots), wobei die Modellresiduen denen einer Normalverteilung gegenübergestellt werden. Stimmen die empirischen und die theoretischen Quantile annähernd überein, liegen sie auf einer Diagonalen und stammen aus Grundgesamtheiten mit gleicher Verteilung (Sachs und Hedderich, 2006). In diesem Fall ist es die geforderte Normalverteilung, die durch die Transformation der Antwortvariable in die Variable $Z$ erreicht werden soll (s. Gl. 3.4). Wie in Abbildung 3.2 zu sehen ist, liegen die baumartenspezifischen empirischen Quantile näherungsweise auf der jeweiligen Winkelhalbierenden. Somit ist die BCCG-Verteilung eine geeignete theoretische Approximation, um die Grundfläche über der Spitzenhöhe zu schätzen.

Aufbauend auf der Modellierung der bedingten BCCG-Verteilung können beliebige Quantile mit Hilfe der dazugehörigen Quantilsverteilung in Abhängigkeit von der Spitzenhöhe geschätzt werden. Durch die Quantilsregression können die Daten besser beschrieben und plausiblere Ergebnisse erreicht werden als durch den bisherigen Ansatz, vor allem im Bereich geringerer Spitzenhöhen. Hier wird das 0,95-Quantil zur Schätzung der maximalen Bestandesgrundfläche genutzt und als Referenz für den natürlichen Bestockungsgrad herangezogen. Es entspricht demnach einem natürlichen Bestockungsgrad von 1,0. Zur einfachen Implementierung des gewählten Quantils in den Waldwachstumssimulator wurde dieses mittels nichtlinearer Regression mit Hilfe des Statistikprogramms R (R Development Core Team, 2011) sowie der Bibliothek nlme (Pinheiro et al., 2011) funktional über der Spitzenhöhe ausgeglichen (s. Abb. 3.3). Als Funktionsform dient die kumulative Weibullverteilungsfunktion (s. Gl. 3.10), deren Koeffizienten mit Hilfe der Selbststartfunktion SSweibull aus der Bibliothek nlme (Pinheiro et al., 2011) geschätzt wurden (s. Tab. 3.7).

Die so ermittelte Funktion beschreibt die maximale Bestandesgrundfläche bei gegebener Spitzenhöhe. Sie dient im Folgenden zur Ermittlung der maximalen Grundfläche und in Verbindung mit dem natürlichen Bestockungsgrad zur Quantifizierung der Eingriffstärke. Dabei wird die maximale Grundfläche anteilig auf die gewünschte Zielgrundfläche abgesenkt. Mit Hilfe von Expertenwissen der Abteilung Waldwachstum der Nordwestdeutschen Forstlichen Versuchsanstalt wurde die Steuerung der Durchforstung von Reinbeständen der Hauptwirtschaftsbaumarten in Nordwestdeutschland anhand des natürlichen Bestockungsgrades in verschiedenen Spitzenhöhenbereichen neu definiert (s. Tab. 3.8). Dabei ist zu beachten, dass die ermittelten Koeffizienten aufgrund des Datenumfangs für Kiefernbestände für Spitzenhöhenbereiche von rund 8 bis 30 m, für Eichen-, Buchen- und Fichtenbestände bis $40 \mathrm{~m}$ und für Douglasienbestände bis $50 \mathrm{~m}$ gültig sind. 


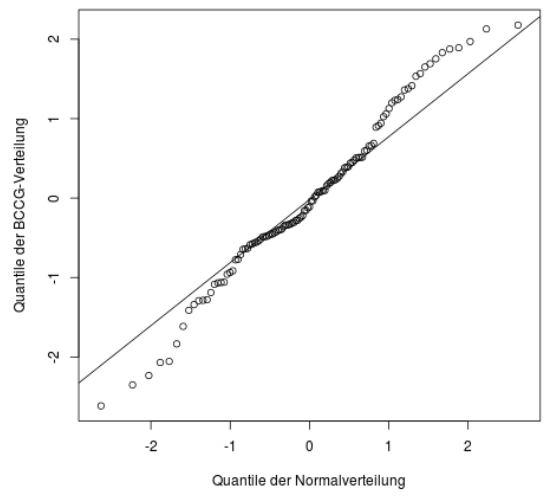

(a)

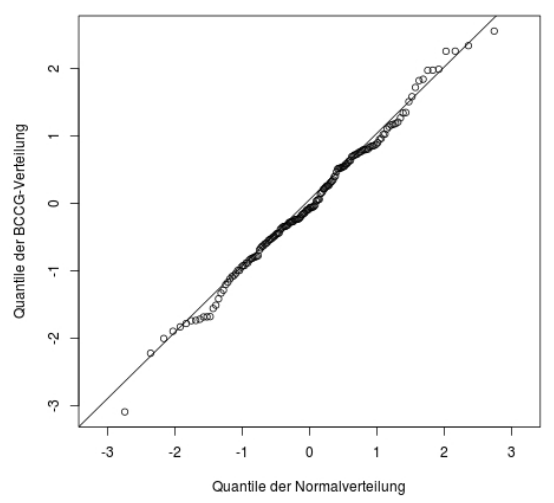

(c)

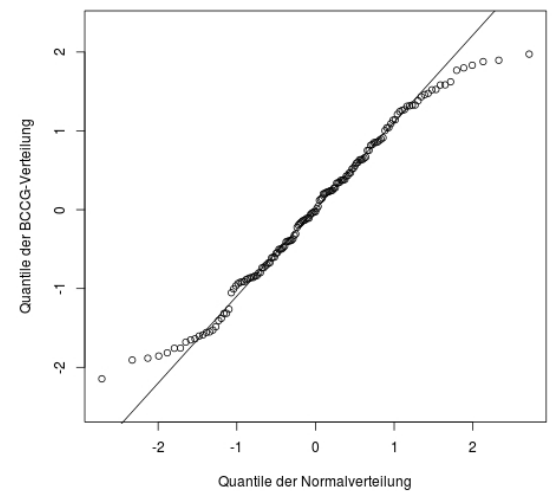

(b)

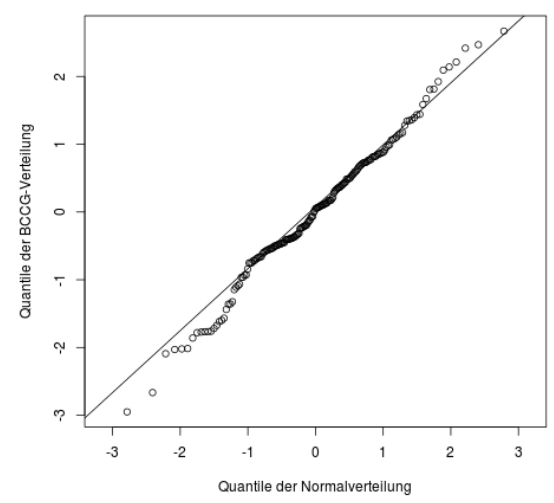

(d)

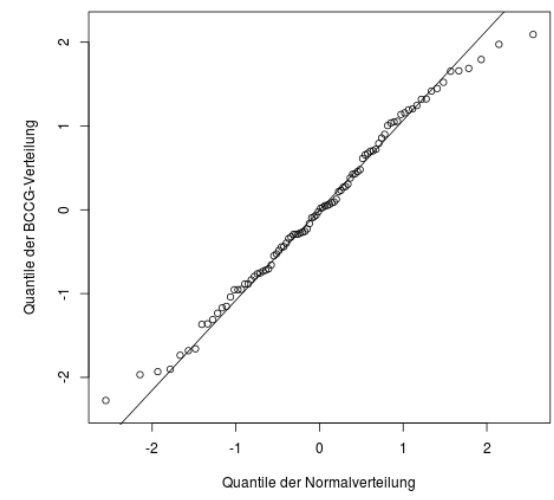

(e)

Abbildung 3.2: Quantile-Quantile-Plots zur Validierung der Box-Cox-Cole-Green-Verteilung (BCCG-Verteilung) für die Schätzung der Bestandesgrundfläche der Hauptwirtschaftsbaumarten $(\mathrm{a}=$ Eiche, $\mathrm{b}=$ Buche, $\mathrm{c}=$ Fichte, $\mathrm{d}=$ Douglasie, $\mathrm{e}=$ Kiefer). 
$G_{\text {max approx } 95}=\operatorname{asym}-a \times \exp \left(-\exp (b) \times h 100^{(c)}\right)$

mit:

$h 100=$ Spitzenhöhe $[\mathrm{m}]$

asym $=$ Asymptote

$a=$ Lageparameter

$b=$ Skalierungsparameter

$c=$ Formparameter

Tabelle 3.7: Koeffizienten der kumulativen Weibullverteilungsfunktion (s. Gl. 3.10) zur Schätzung der maximalen Bestandesgrundfäche für die Hauptwirtschaftsbaumarten in Nordwestdeutschland.

\begin{tabular}{lcccc}
\hline Baumart & asym & $\mathrm{a}$ & $\mathrm{b}$ & $\mathrm{c}$ \\
\hline Eiche & 52.5880 & 44.9760 & -4.4630 & 1.4750 \\
Buche & 55.3420 & 127.0100 & -2.3140 & 1.0250 \\
Fichte & 71.5180 & 70.4940 & -4.7660 & 1.6950 \\
Douglasie & 88.7417 & 157.5353 & -1.8518 & 0.7506 \\
Kiefer & 47.0250 & 184.8930 & -1.4110 & 1.0000 \\
\hline
\end{tabular}


Tabelle 3.8: Steuerung der Durchforstung von Reinbeständen der Hauptwirtschaftsbaumarten in Nordwestdeutschland mit Hilfe des natürlichen Bestockungsgrades (nat. $\mathrm{B}^{\circ}$ ) in bestimmten Pflegephasen (DF-Phase), definiert über Spitzenhöhenbereiche $(\mathrm{h} 100[\mathrm{~m}])$.

\begin{tabular}{lcccccccccc}
\hline $\begin{array}{l}\text { Pflegephase } \\
\text { Baumart }\end{array}$ & \multicolumn{2}{c}{ DF-Phase 1} & \multicolumn{2}{c}{ DF-Phase 2} & \multicolumn{2}{c}{ DF-Phase 3} & \multicolumn{2}{c}{ DF-Phase 4} & \multicolumn{2}{c}{ DF-Phase 5 } \\
h100 & nat. B & h100 & nat. B & h100 & nat. B & h100 & nat. B & h100 & nat. B \\
\hline Eiche & $14-18$ & 0,55 & $18-20$ & 0,60 & $20-24$ & 0,65 & $>24$ & 0,70 & & \\
Buche & $16-22$ & 0,55 & $22-26$ & 0,60 & $26-32$ & 0,65 & $>32$ & 0,70 & & \\
Fichte & $14-20$ & 0,50 & $20-26$ & 0,55 & $26-32$ & 0,60 & $>32$ & 0,65 & & \\
Douglasie & $14-20$ & 0,50 & $20-26$ & 0,55 & $26-32$ & 0,60 & $32-38$ & 0,65 & $>38$ & 0,70 \\
Kiefer & $12-18$ & 0,60 & $18-22$ & 0,65 & $>22$ & 0,70 & & & & \\
\hline
\end{tabular}




\subsubsection{Ergebnisse der entwickelten Modelle zur Bestimmung der maximalen Bestandesgrundfläche}

Wie im vorherigen Kapitel dargelegt, ist das Ziel der Quantilsregression die Bildung einer Umhüllenden der beobachteten Bestandesgrundflächen, um die maximalen Werte schätzen zu können. Wie in Abbildung $3.3 \mathrm{zu}$ sehen ist, werden die Daten mit diesem Konzept sehr gut beschrieben. Darüber hinaus werden auch die Extremwerte durch die Approximation des 0,95-Quantils, mit Hilfe der kumulativen Weibullverteilungsfunktion, gut erklärt.

Eichenbestände können demnach eine maximale Bestandesgrundfläche von $52 \mathrm{~m}^{2} \mathrm{ha}^{-1}$ erreichen. Dies sind $12 \mathrm{~m}^{2} \mathrm{ha}^{-1}$ mehr als sie sich nach Wenk et al. (1990) in über 130jährigen Eichenbeständen durch die Multiplikation eines Faktors von 1,2 bis 1,25 mit der optimalen Grundfläche ergeben würden. Buchenbestände erreichen eine maximale Bestandesgrundfläche von etwa $55 \mathrm{~m}^{2} \mathrm{ha}^{-1}$. Annähernd diesen Wert hat Assmann (1961) auch für über 100-jährige Buchenbestände auf guten Standorten angegeben (45 bis $\left.50 \mathrm{~m}^{2} \mathrm{ha}^{-1}\right)$. Fichtenbestände können eine maximale Bestandesgrundfläche von ca. $71 \mathrm{~m}^{2} \mathrm{ha}^{-1}$ erreichen. Ähnlich hoch ist sie allerdings auch im Alter von rund 120 Jahren bei einer Oberhöhenbonität von $40 \mathrm{~m}$ nach der Ertragstafel von Assmann und Franz (1963) (Wenk et al., 1990). Die maximale Bestandesgrundfläche der Kiefernbestände beträgt hier etwa $47 \mathrm{~m}^{2} \mathrm{ha}^{-1}$. Dieses Ergebnis wird durch Wenk et al. (1990) mit einer maximal möglichen Grundfläche für Kiefernbestände von rund $48 \mathrm{~m}^{2} \mathrm{ha}^{-1}$ bestätigt. In Douglasienbeständen sind nach der hier entwickelten Methode rund $89 \mathrm{~m}^{2} \mathrm{ha}^{-1}$ als maximale Bestandesgrundfläche möglich.

Bei der Anwendung der Methode nach Sterba $(1975,1981,1987)$ mit den Koeffizienten von Döbbeler (2004) für Nordwestdeutschland, ist die maximale Bestandesgrundfläche bei einer Spitzenhöhe von $35 \mathrm{~m}$ für Eichen- und Buchenbestände geringer als mit der Methode der Quantilsregression. Für die Nadelbaumarten stimmen die Angaben bei einer Spitzenhöhe von ca. 30 bis $40 \mathrm{~m}$ zwischen den beiden Methoden nahezu überein (vgl. Abb. 3.1 u. 3.3). Ausschlaggebend ist der Unterschied der beiden Schätzverfahren in den ersten, waldbaulich sehr entscheidenden Pflegephasen. Überwiegend in diesem Bereich konnte durch die Integration jüngerer Versuchsflächendaten und die Anwendung der Quantilsregression eine deutlich bessere Schätzung der maximalen Bestandesgrundflächen erreicht werden.

Das Zusammenspiel der maximalen Bestandesgrundfläche mit dem natürlichen Bestockungsgrad führt, durch das erstellte Nutzungskonzept (s. Tab. 3.8), zu gestaffelten Grundflächenhaltungen. Beispielsweise sind bei der Buche vier Pflegephasen vorgesehen. Die erste Pflegephase reicht von 16 bis $22 \mathrm{~m}$ Bestandesspitzenhöhe, was etwa der natürlichen Altersstufe geringes Baumholz entspricht. Dabei wird die vorhandene Bestandesgrundfläche auf $55 \%$ der maximal möglichen abgesenkt, das sind je nach Bestandeshöhe etwa 18 bis $25 \mathrm{~m}^{2} \mathrm{ha}^{-1}$. In der Buchenertragstafel, mäßige Durchforstung, liegen die Grundflächen für einen ähnlichen Spitzenhöhenbereich, je nach Ertragsklasse, zwischen rund 18 bis $29 \mathrm{~m}^{2} \mathrm{ha}^{-1}$ (Schober, 1995). In der zweiten Pflegephase, ab 22 
bis $26 \mathrm{~m}$, wird die Bestandesgrundfläche auf $60 \%$ der maximalen Grundfläche reduziert, d. h. je nach Höhe auf ca. 26 bis $30 \mathrm{~m}^{2} \mathrm{ha}^{-1}$. Ab einer Spitzenhöhe des Bestandes von 26 bis $32 \mathrm{~m}$, geringes bis mittleres Baumholz, wird die Bestandesgrundfläche bei rund 31 bis $36 \mathrm{~m}^{2} \mathrm{ha}^{-1}$ gehalten. In der letzten Pflegephase (Spitzenhöhe $>32 \mathrm{~m}$, mittleres bis starkes Baumholz) erhöht sich der natürliche Bestockungsgrad auf 0,7 und es sollte mit der Nutzung zielstarker Bäume begonnen werden. Die Grundfläche wird dabei zwischen rund 36 und maximal $38 \mathrm{~m}^{2} \mathrm{ha}^{-1}$ gehalten.

Exemplarisch für die Nadelbaumarten werden hier die Ergebnisse für die Kiefer vorgestellt. Bei dieser Art sind drei Pflegephasen vorgesehen, wobei das rasche Jugendwachstum der Kiefer im Vergleich zur Buche, früher einsetzende Durchforstungseingriffe notwendig macht. Die erste Pflegephase beginnt bei einer Spitzenhöhe von $12 \mathrm{~m}$ und senkt die Bestandesgrundfläche auf $60 \%$ der maximal möglichen ab (ca. 22 bis $26 \mathrm{~m}^{2} \mathrm{ha}^{-1}$ ). Ab einer Höhe von 18 bis $22 \mathrm{~m}$ wird die Grundfläche bei rund 27 bis $31 \mathrm{~m}^{2} \mathrm{ha}^{-1}$ gehalten. In der letzten Pflegephase (Spitzenhöhe $>22 \mathrm{~m}$ ) beträgt der natürliche Bestockungsgrad 0,7 (ca. $32 \mathrm{~m}^{2} \mathrm{ha}^{-1}$ ) und es kann eine räumliche verteilte Zielstärkennutzung, bei entsprechenden Durchmessern und Qualitäten, beginnen.

Für die anderen Baumarten sind vier bzw. für Douglasie fünf Pflegephasen vorgesehen und die Bestandesgrundflächen lassen sich, wie eben für Buche und Kiefer beschrieben, ableiten. Durch die Variation der Pflegephasen und der natürlichen Bestockungsgrade können unterschiedliche Konzepte der grundflächengesteuerten Durchforstung dargestellt werden.

Die Ausgleichsfunktion (s. Gl. 3.10) mit den entsprechenden Koeffizienten zur Schätzung der maximalen Bestandesgrundfläche und das Nutzungskonzept wurden in den WaldPlaner (Hansen, 2012; Hansen und Nagel, 2014) integriert. Somit ist eine objektive, bonitätsunabhängige Grundlage vorhanden, um grundflächengesteuerte und praxisbezogene Eingriffe abzubilden. 


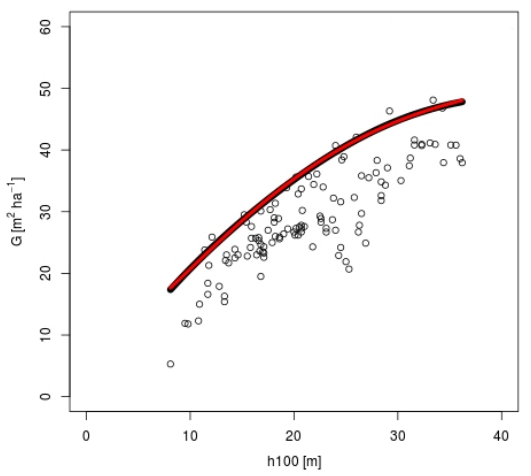

(a)

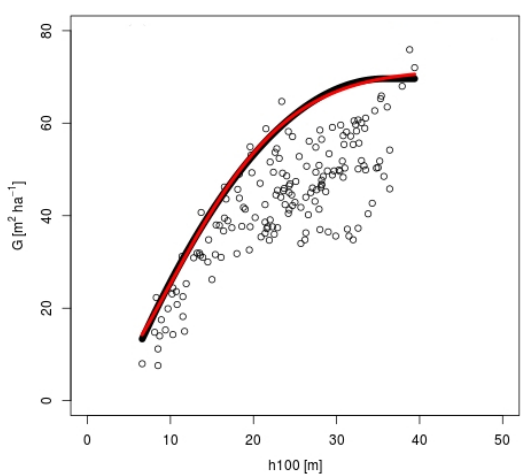

(c)

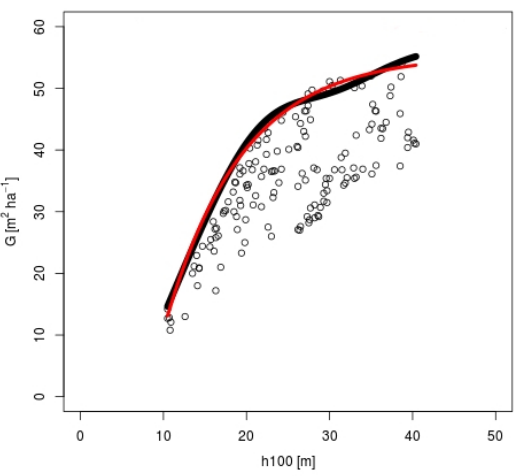

(b)

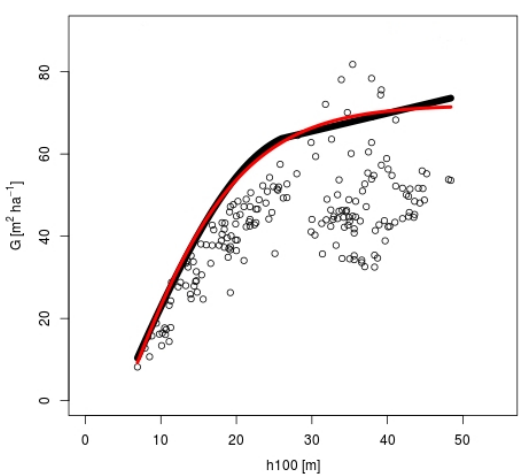

(d)

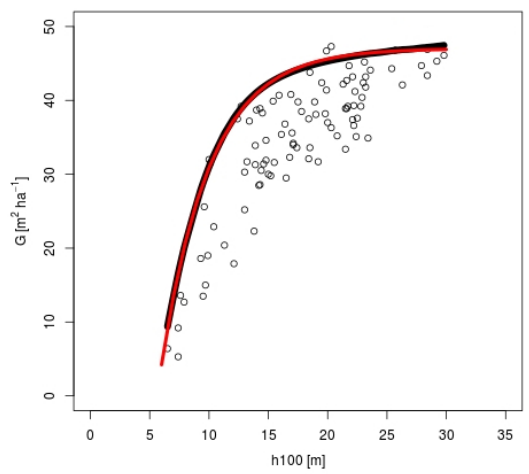

(e)

Abbildung 3.3: Vergleich der Versuchsflächendaten mit dem Funktionsverlauf des 0,95-Quantils der Box-Cox-Cole-Green-Verteilung (BCCG-Verteilung, schwarze Kurve) zur Schätzung der maximalen Bestandesgrundfläche $\left(\left[\mathrm{m}^{2} \mathrm{ha}^{-1}\right]\right)$ sowie der ausgleichenden Funktion (rote Kurve) über der Spitzenhöhe (h100 [m]) für Reinbestände der Hauptwirtschaftsbaumarten in Nordwestdeutschland $(\mathrm{a}=$ Eiche, $\mathrm{b}$ $=$ Buche, $\mathrm{c}=$ Fichte, $\mathrm{d}=$ Douglasie, $\mathrm{e}=$ Kiefer ) 


\subsubsection{Sortierungsvorgaben}

Die Sortierungsvorgaben sind für beide Behandlungsvarianten gleich. Dabei wird beim Stammholz zwischen Laub- und Nadelholz unterschieden. Der Mindestzopfdurchmesser beträgt für Laubstammholz $28 \mathrm{~cm}$ und für Nadelstammholz $9 \mathrm{~cm}$. Die Länge dieser Sortimente liegt jeweils zwischen 3 und $18 \mathrm{~m}$ mit Zugabe. Für das Industrieholz werden, unabhängig von der Holzart, ein Mindestdurchmesser von $7 \mathrm{~cm}$ und eine Länge von wenigstens einem Meter angenommen. Für jeden genutzten Baum wird das im Wald verbleibende Volumen der Stubben mit einer Höhe von $30 \mathrm{~cm}$ quantifiziert (s. Tab. 3.9). Eine Sortierung nach Qualitäten findet nicht statt, da entsprechende Informationen nicht vorliegen und die Entwicklung der Holzqualität bzw. deren Modellierung von vielen verschiedenen Faktoren abhängt.

Tabelle 3.9: Verwendete Sortierungsvorgaben.

\begin{tabular}{lcc}
\hline Sortiment & Mindestdurchmesser $[\mathrm{cm}]$ & Länge $[\mathrm{m}]$ \\
\hline Laubstammholz & 28 & $3-18+$ mind. 0,1 Zugabe \\
Nadelstammholz & 9 & $3-18+$ mind. 0,1 Zugabe \\
Industrieholz & 7 & mind. 1,0 \\
Stubben & - & 0,3 \\
\hline
\end{tabular}

\subsection{Ermittlung der Kohlenstoffvorräte der betrachteten Speicher}

\subsubsection{Lebende Baumbiomasse}

Holz besteht aus organischen Verbindungen, welche aus den chemischen Elementen Kohlenstoff (C), Wasserstoff (H), Sauerstoff (O) und Stickstoff (N) aufgebaut sind. Darrtrockenes Holz (0\% Holzfeuchte) besteht zu etwa $50 \%$ aus Kohlenstoff, zu $6 \%$ aus Wasserstoff, zu $42 \%$ aus Sauerstoff und zu rund 1\% aus Stickstoff (Knigge und Schulz, 1966). Da im Allgemeinen die Kohlenstoffkonzentration zwischen den Baumarten nur wenig variiert, wird eine mittlere Konzentration von $50 \%$ unterstellt (Burschel et al., 1993; Bauer et al., 2000; Wirth et al., 2004a; Pretzsch, 2009; West, 2009). Die Umrechnung von Kohlenstoff in Kohlenstoffdioxid kann mit Hilfe des Atommassenverhältnisses (1:3,67) erfolgen, denn $12 \mathrm{~kg}$ Kohlenstoff $(\mathrm{C})$ verbinden sich mit $32 \mathrm{~kg}$ Sauerstoff $\left(\mathrm{O}_{2}\right) \mathrm{zu}$ $44 \mathrm{~kg}$ Kohlenstoffdioxid $\left(\mathrm{CO}_{2}\right)$.

Zur Herleitung des Kohlenstoffvorrates der lebenden Baumbiomasse können Expansionsfaktoren verwendet werden. Dabei wird das Derbholzvolumen um das Volumen der Grobwurzeln, Äste und Blätter mittels der genannten Faktoren erweitert, und mit 
der baumartenspezifischen Raumdichte (s. Tab. 1.2) sowie dem Kohlenstoffgehalt multipliziert. Die Expansionsfaktoren, Raumdichten und Kohlenstoffgehalte können je nach Untersuchung variieren. Eine andere Möglichkeit zur Schätzung der lebenden ober- und unterirdischen Baumbiomasse besteht in der Anwendung von Biomassefunktionen, welche als Eingangsparameter meist den Durchmesser und die Höhe des Baumes haben. Anschließend wird die Biomasse mit dem mittleren Kohlenstoffgehalt der Holzmasse multipliziert, um den Kohlenstoffvorrat zu erhalten.

Bei den durchgeführten Simulationen werden Einzelbaumdaten in Fünfjahresschritten ausgegeben. Diese dienen zur Berechnung der oberirdischen lebenden Baumbiomasse mit den Funktionen von Rumpf et al. (2012) ( $\left.\hat{Y}_{o}[\mathrm{~kg}]\right)$ für Eiche (3.11), Buche (3.12), Fichte (3.13), Douglasie (3.14) und Kiefer (3.15), in Abhängigkeit vom Durchmesser in 1,3 m Höhe $(d[\mathrm{~cm}])$ und der Baumhöhe $(h[\mathrm{~m}])$. Allerdings wird bei diesen Funktionen die Blattbiomasse der Laubbäume nicht mitberechnet. Für die Ermittlung der unterirdischen lebenden Baumbiomasse wird die Funktion von Dieter und Elsasser (2002) $\left(\hat{Y}_{u}\right.$ $\left.\left[t h a^{-1}\right]\right)$, abhängig von der oberirdisch lebenden Bestandesbiomasse $\left(\hat{Y}_{o}\left[t h a^{-1}\right]\right)$, mit den in Tabelle 3.10 aufgeführten Koeffizienten verwendet.

$$
\begin{aligned}
& \hat{Y}_{\text {o Eiche }}=\exp (-3.786878+1.960767 \times \ln (d)+1.105109 \times \ln (h)) \\
& \hat{Y}_{\text {o Buche }}=\exp (-3.538373+2.031439 \times \ln (d)+0.954456 \times \ln (h)) \\
& \hat{Y}_{\text {o Fichte }}=0.1558394 \times\left(d^{1.9157968}\right) \times\left(h^{0.4193738}\right) \\
& \hat{Y}_{\text {Douglasie }}=\exp (-1.533214+2.229548 \times \ln (d)) \\
& \hat{Y}_{\text {Kiefer }}=0.0566125 \times\left(d^{1.9543788}\right) \times\left(h^{0.679117}\right) \\
& \sqrt{\hat{Y}_{u}}=\beta \times \sqrt{\hat{Y}_{o}} \times \delta_{\text {Baumart }}
\end{aligned}
$$


Tabelle 3.10: Koeffizienten der verwendeten Funktion zur Schätzung der unterirdisch lebenden Baumbiomasse (s. Gl. 3.16) (Quelle: Dieter und Elsasser (2002))

\begin{tabular}{ccc}
\hline Baumart & $\beta$ & $\delta$ \\
\hline Buche & & 0 \\
Douglasie & & 0,4738 \\
Eiche & 0,4259 & 0 \\
Fichte & & 1,169 \\
Kiefer & & 0,2864 \\
\hline
\end{tabular}

\subsubsection{Tote Baumbiomasse}

Der Kohlenstoffvorrat des Totholzes wird meist unter der Annahme einer mittleren Raumdichte, teilweise differenziert nach Zersetzungsgraden, und unter Berücksichtigung des mittleren Kohlenstoffgehaltes berechnet. Während der Simulation wird ein Totholzvorrat von bis zu $20 \mathrm{~m}^{3}$ ha $^{-1}$ angestrebt (s. Kap. 3.2.2). Zu diesem, aktiv vom Prognosesystem erzeugten Totholz, werden die Stubben der geernteten Bäume sowie deren weitere Ernteverluste hinzu addiert.

Der Totholzabbau wird mit Hilfe der von Rock et al. (2008) ermittelten Zersetzungskonstanten, einer eigens hergeleiteten Zersetzungskonstanten für Douglasie auf Grundlage der Arbeit von Laiho und Prescott (2004) sowie eines einfachen exponentiellen Modells quantifiziert (s. Gl. 3.17). Dabei beziehen sich die Totholzzersetzungskonstanten auf die Holzdichte. Somit bewegen sich die Lebensdauern $\left(t_{95}\right)$, d. h. $95 \%$ der Dichte im Vergleich zum Anfangszustand sind zersetzt, zwischen rund 45 Jahren bei Buche und bis zu 125 Jahren bei Douglasie (s. Tab. 3.11).

Das anfallende Totholzvolumen wird mit dem funktionalisierten Zusammenhang, zwischen dem relativen Anteil der Dichte des im Bestand verbliebenen Totholzes und der vergangenen Zeit (s. Abb. 3.4), sowie der baumartenspezifischen Raumdichte (s. Tab. 1.2) multipliziert, um die Masse des Totholzes zu einem bestimmten Zeitpunkt zu ermitteln. Dieser Totholzmasse wird eine mittlere Kohlenstoffkonzentration von $50 \%$ unterstellt.

$$
\rho_{i}=\frac{-\left(\ln \left(1-\frac{i}{100}\right)\right)}{k}
$$

mit:

$\rho_{i}=$ Holzdichte des verbleibenden Totholzes $\left[\mathrm{t}^{-3}\right]$

$i=$ abgebauter Anteil 


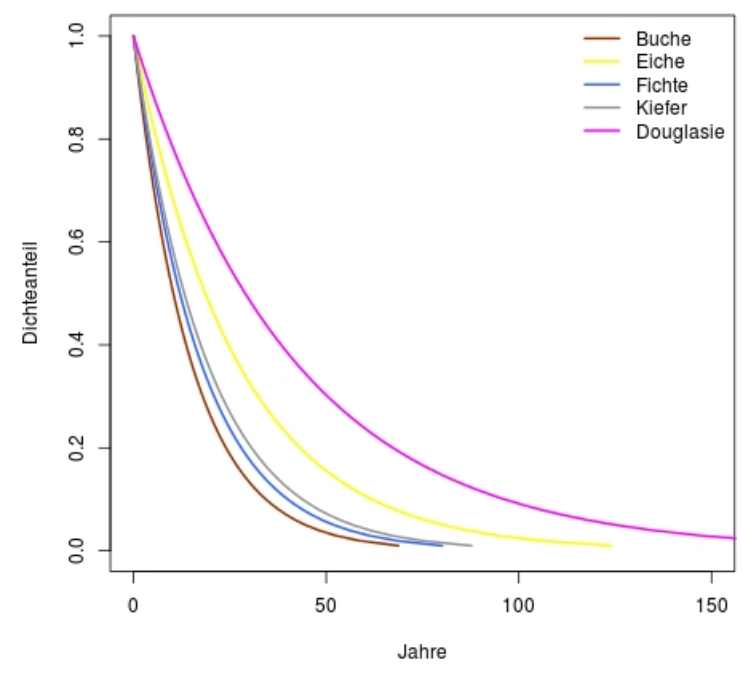

Abbildung 3.4: Zeitliche Entwicklung des Holzdichteanteils des im Bestand verbleibenden Totholzes getrennt nach Baumarten.

Tabelle 3.11: Totholzzersetzungskonstanten $(\mathrm{k})$ und entsprechende Lebensdauern $\left(\mathrm{t}_{95}\right)$ des Totholzes, bezogen auf die Holzdichte (Quellen: Rock et al. (2008), Douglasie eigene Herleitung).

\begin{tabular}{lcc}
\hline Baumart & $\mathrm{k}\left[\mathrm{a}^{-1}\right]$ & $\mathrm{t}_{95}[\mathrm{a}]$ \\
\hline Eiche & 0,0372 & 80,5 \\
Buche & 0,0670 & 44,7 \\
Fichte & 0,0575 & 52,1 \\
Kiefer & 0,0525 & 57,1 \\
Douglasie & 0,0239 & 125,3 \\
\hline
\end{tabular}

\subsubsection{Holzprodukte}

Um die schier unendliche Vielfalt der Holzprodukte abbilden zu können, wurden in einem ersten Schritt Holzproduktklassen erstellt, die sich aus verschiedenen Hauptprodukten zusammensetzen (s. Tab. 3.12). Bei den vier Holzproduktklassen handelt es sich um Produkte mit langer Lebensdauer (PlL), dazu zählt insbesondere Bauholz, Produkte mit mittlerer Lebensdauer (PmL), vornehmlich Span- und Tischlerplatten, Furniere sowie Vollholzmöbel. Des Weiteren sind es Produkte mit kurzer Lebensdauer (PkL), vor allem Papier, Pappe und Kartonagen, sowie Energieholz (E), insbesondere Brennholz und Pellets. Diesen Produktklassen wurden Zersetzungskonstanten (k) auf Grundlage der Angaben von Wirth et al. (2004a) und Pistorius (2007a) zugeordnet. Damit war es 
Tabelle 3.12: Holzproduktklassen (PlL, PmL, PkL = Produkte mit langer, mittlerer und kurzer Lebensdauer, $\mathrm{E}=$ Energieholz) mit den entsprechenden Zersetzungskonstanten $(\mathrm{k})$, Halbwertszeiten $\left(\mathrm{t}_{50}\right)$ Lebensdauern $\left(\mathrm{t}_{63}, \mathrm{t}_{95}\right)$ und Hauptprodukten (Quellen: Wirth et al. (2004a); Pistorius (2007a)).

\begin{tabular}{lccccl}
\hline Produktklasse & $\mathrm{k}\left[\mathrm{a}^{-1}\right]$ & $\mathrm{t}_{50}[\mathrm{a}]$ & $\mathrm{t}_{63}[\mathrm{a}]$ & $\mathrm{t}_{95}[\mathrm{a}]$ & Hauptprodukte \\
\hline PlL & 0,020 & 35 & 50 & 150 & Bauholz \\
PmL & 0,039 & 18 & 25 & 77 & $\begin{array}{l}\text { Span- und Tischlerplatten, } \\
\text { Furniere, Vollholzmöbel }\end{array}$ \\
PkL & 0,320 & 2 & 3 & 9 & Papier, Pappe, Kartonagen \\
E & 0,700 & 1 & 1 & 4 & Brennholz, Pellets \\
\hline
\end{tabular}

möglich, für die einzelnen Klassen Halbwertszeiten $\left(t_{50}\right)$, mittlere Lebensdauern $\left(t_{63}\right)$ und Lebensdauern $\left(t_{95}\right)$ zu berechnen (s. Gl. 3.18). Die Lebensdauern (t) entsprechen einem exponentiellen Abbau über der Zeit, in der 50\%, $63 \%$ und $95 \%$ (i) des organischen Kohlenstoffs abgebaut sind (Mund et al., 2006). Hier wird die mittlere Lebensdauer $\left(\mathrm{t}_{63}\right)$ als Nutzungsdauer der Holzprodukte in den verschiedenen Kategorien unterstellt.

$$
t_{i}=\frac{-\left(\ln \left(1-\frac{i}{100}\right)\right)}{k}
$$

Die Summe der eingeschlagenen Holzsortimente wird den verschiedenen Produkten und Produktklassen mit Hilfe eines Holzverwendungsschlüssels zugeordnet. Dieser stellt hohe Ansprüche an die Datengrundlage und ist in seiner Herleitung sehr aufwendig, da sich die Wege der verschiedenen ausgehaltenen Sortimente häufig nicht vom Wald zum Verwerter nachvollziehen lassen (Verkäufer $\rightarrow$ Vertreter oder Holzhandel $\rightarrow$ Verarbeitung oder Weiterverkauf). Dieses Problem ließe sich gegebenenfalls mit einer umfassenden anonymen Befragung der Unternehmen der holzbe- und verarbeitenden Industrie eines Landes lösen (vgl. Profft et al., 2009). Allerdings war dies im Rahmen dieser Arbeit nicht möglich. Daher wurde folgender Ansatz gewählt: Den Produktklassen wurde ausgehend von den Hauptprodukten das eingeschlagene Stamm-, Industrie- und Energieholz sowie das nicht verwertbare Holz zugeordnet. Stammholz wird zumeist an Säger verkauft, welche daraus Produkte für den Hausbau o. ä. anfertigen. Folglich wird dieses Sortiment den Produkten mit langer Lebensdauer (PlL) zugeordnet. Der Ausbeutungsgrad der Schnittholzproduktion beträgt bundesweit bei Nadelholz ca. $61 \%$ und bei Laubholz etwa $64 \%$ (Mantau, 2008). Die als Koppelprodukt entstehenden Sägenebenprodukte werden, unabhängig ob es sich um Laub- oder Nadelholz handelt, zu $44 \%$ den Produkten mit mittlerer Lebensdauer, zu $18 \%$ den Produkten mit kurzer Lebensdauer und zu $38 \%$ dem Energieholz zugeordnet. Das eingeschlagene Industrieholz wird zu unterschiedlichen Anteilen den Produkten mit mittlerer (PmL) und kurzer Lebensdauer 
(PkL) zugewiesen. Um den Anteil von Laub- und Nadelindustrieholz an der Klasse Produkte mit kurzer Lebensdauer, hauptsächlich Papier und Pappe, zu bestimmen, wurde auf bundesweite Zahlen des Verbandes Deutscher Papierfabriken e. V. zurückgegriffen (VDP, 2007, 2010, 2011, 2012), da Daten auf Landesebene nicht vorlagen. Das Nadelindustrieholz wurde demnach zu $65 \%$ und das Laubindustrieholz zu $26 \%$ der kurzlebigen Produktklasse $(\mathrm{PkL})$ zugeordnet, die restlichen Anteile den Produkten mit mittlerer Lebensdauer (PmL). Ausgehaltenes Energieholz und nicht verwertbares Holz wurde der Produktgruppe Energieholz zugeordnet. Die Datengrundlage für die Sortimente stammt aus den Holzmarktberichten für die Jahre 2006, 2009, 2010 und 2011 (BMELV, 2007, 2010, 2011, 2012b). Diese Jahre wurden gewählt, da sie von Stürmen und Wirtschaftsschwankungen weitgehend unbeeinflusst waren. Der mit diesen Annahmen hergeleitete und sehr vereinfachte Holzverwendungsschlüssel ist in Tabelle 3.13 dargestellt.

Der Abbildung einer Kaskadennutzung (s. Abb. 3.5) liegen folgende Überlegungen zugrunde: Produkte der Klasse Energieholz werden nur einmal energetisch genutzt. Dieser Pool wird allerdings auch durch die Be- und Verarbeitung anderer Produktklassen aufgefüllt. Die Produkte mit kurzer Lebensdauer, überwiegend Papier, werden zu $71 \%$ als Altpapier von Neuem zur Papierherstellung (VDP, 2012) in der Folgeperiode, d. h. stofflich, genutzt. Der restliche Anteil dieser Produktgruppe wird weiterhin benutzt und zersetzt sich nach Gleichung 3.18 bis er seine mittlere Lebensdauer erreicht hat. Der Gesetzgeber sieht vor, dass diese Produktklasse hauptsächlich stofflich genutzt werden soll ( $\$ 14 \mathrm{KrWG}$ ), wobei eine energetische Nutzung nach Erreichen der mittleren Lebensdauer nicht ausgeschlossen ist. Produkte mit langer und mittlerer Lebensdauer können mehrfach, insbesondere über den Zwischenspeicher Altholz, stofflich genutzt werden. Dabei wird angenommen, dass im ersten Schritt $23 \%$ der Produkte mit langer Lebensdauer stofflich zu Produkten mit mittlerer Lebensdauer weiter verwertet werden, $33 \%$ energetisch genutzt werden und $44 \%$ dem Altholz zuzurechnen sind. Das Altholz wird zu $34 \%$ energetisch und zu 38\% stofflich zur Herstellung von Produkten mit mittlerer Lebensdauer genutzt. Die restlichen $28 \%$ werden anderweitig verwertet, d. h. kompostiert, gemulcht oder auch deponiert (Trähn, 2009). Somit steht dieser Anteil für keine weitere Nutzung zur Verfügung. Die Produkte der Klasse mit mittlerer Lebensdauer werden zu $44 \%$ als Altholz stofflich und zu $56 \%$ energetisch verwendet. Durch die Einbeziehung einer Kaskade werden die produktklassenspezifischen Verweildauern anteilig um den Nutzungszeitraum eines möglichen Folgeproduktes verlängert und die energetischen Nutzungsmöglichkeiten von Nebenprodukten beachtet. Hinzu kommt, dass mit einer Kaskadennutzung von Holzprodukten der Konflikt zwischen stofflicher und energetischer Holznutzung entschärft werden kann, ohne den Holzeinschlag zu erhöhen. Die aufgeführten Anteile basieren auf den Arbeiten von Frühwald et al. (2010) und Trähn (2009) sowie eigenen Überlegungen. Sie werden im Rahmen der Simulationsläufe als statisch angesehen. Darüber hinaus wird weder Import noch Export von Holz bzw. Althölzern mit in die Betrachtungen einbezogen.

Die stoffliche und energetische Nutzung von Holz bzw. Produkten aus Holz hat positive ökologische Effekte. Durch ihre Verwendung wird die Freisetzung von Kohlenstoff 
Tabelle 3.13: Holzverwendungsschlüssel zur Verteilung von Laub- und Nadelholz auf die Produktklassen (PlL, PmL, PkL = Produkte mit langer, mittlerer und kurzer Lebensdauer, $\mathrm{E}=$ Energieholz) in Niedersachsen.

\begin{tabular}{lcccc}
\hline Art & PlL & PmL & PkL & E \\
\hline Laubholz & $22 \%$ & $24 \%$ & $9 \%$ & $45 \%$ \\
Nadelholz & $34 \%$ & $23 \%$ & $30 \%$ & $13 \%$ \\
\hline
\end{tabular}

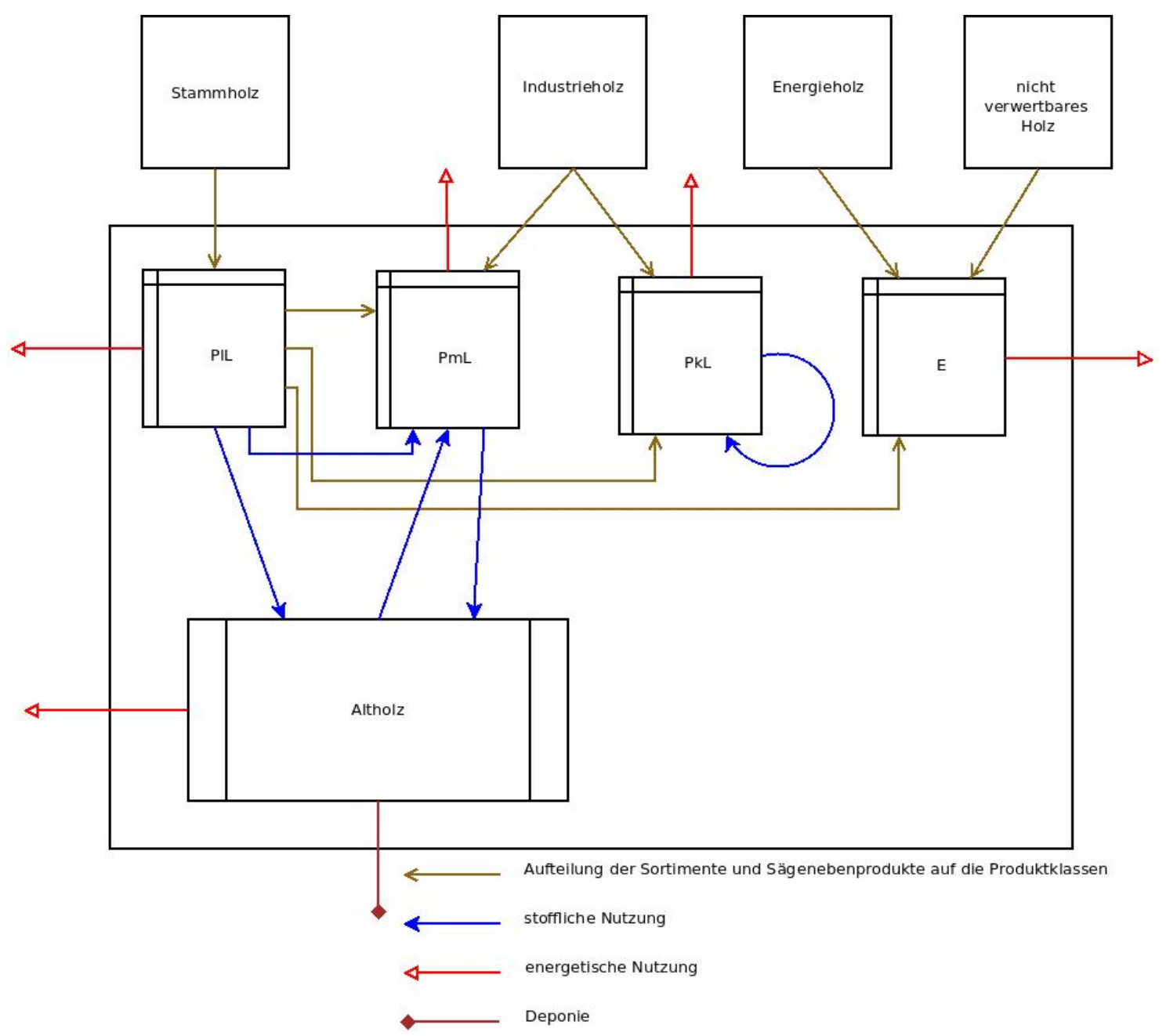

Abbildung 3.5: Aufteilung der Sortimente auf Produktklassen und das entwickelte Modell zur Kaskadennutzung von Holzprodukten. 
verzögert, energieaufwendig herzustellende Produkte werden ersetzt und Treibhausgasemissionen durch die Nutzung fossiler Energieträger vermieden (Rüter, 2011). In verschiedenen Arbeiten zu Ökobilanzen von Holzprodukten wurden Faktoren zur Berechnung der Substitutionswirkung abgeleitet (Fürstenau, 2008; Sathre und O'Connor, 2010; Rüter, 2011; Knauf et al., 2013). Um hier diese Wirkung der Holzprodukte abbilden zu können, werden in Anlehnung an die Studie von Knauf et al. (2013), folgende Faktoren benutzt. Für die Materialsubstitution dient der Faktor $1,5 \mathrm{t} \mathrm{C} \mathrm{t}^{-1} \mathrm{C}^{-1}$ und für die energetische Substitution der Faktor $0,67 \mathrm{t} \mathrm{C} \mathrm{t}^{-1} \mathrm{C}^{-1}$. Wobei beispielsweise der Faktor für die Materialsubstitution besagt, dass jede Tonne Kohlenstoff eines Holzproduktes, welches ein Nicht-Holzprodukt ersetzt, 1,5 Tonnen Kohlenstoff an Emissionen einspart (Mund et al., 2015). Zur Berechnung der Kohlenstoffmengen, die in den Holzproduktpool eingehen, werden den ermittelten Erntemengen (s. Kap. 3.2.4) die baumartenspezifischen Raumdichten (s. Tab. 1.2) und eine mittlere Kohlenstoffkonzentration von $50 \%$ unterstellt. Die Materialsubstitution wird durch die Verrechnung der Kohlenstoffmengen der Produkte mit langer und mittlerer Lebensdauer mit dem entsprechenden Faktor abgebildet. In die Berechnung der energetischen Substitution gehen die Kohlenstoffmengen der Klassen Energieholz, die Produkte mit kurzer Lebensdauer und die energetischen Nutzungen aus der Kaskade ein. Da die Gruppe der Produkte mit kurzer Lebensdauer kaum substituierbare Erzeugnisse wie Papier oder Pappe enthält, können auch keine Alternativprodukte aus anderen Materialien ersetzt werden (Rock und Bolte, 2011). Daher wird hier nach Erreichen der mittleren Lebensdauer von einer energetischen Nutzung ausgegangen.

\subsubsection{Ableitung der Speicherraten}

Aus den Kohlenstoffvorräten zu verschiedenen Zeitpunkten kann eine Speicherrate berechnet werden. Die Berechnung der Kohlenstoffbindungsrate der lebenden Baumbiomasse erfolgt in Bezug auf den Zeitpunkt der maximalen Produktionszeit bzw. der Räumung des Bestandes. Dabei wird der Kohlenstoffvorrat nach Beginn der Zielstärkennutzung um den Beitrag einer ideellen Verjüngung erhöht, die auf Grundlage der jungen Weiserbestände bzw. junger simulierter Bestände hergeleitet wurde. Damit wird die Überlappung von Alt- und Folgebestand für den Zeitraum zwischen dem Beginn der Zielstärkennutzung und dem Ende der spezifischen Produktionszeit berücksichtigt. Nur so lassen sich die Unterschiede der Baumarten auf gleichem Standort darstellen, auch wenn die jungen Weiserbestände nur eingeschränkt einen realen Unterbau oder eine Naturverjüngung abbilden. Der Totholzspeicher baut sich erst im Laufe der Simulationen auf und daher wird für diesen Pool die Simulationsdauer zur Berechnung einer Rate herangezogen.

Der Holzproduktspeicher wird durch die generierten Erntemengen bis zum Ende der Simulationen befüllt. Dabei addieren sich die Nutzungsmengen bei den Produkten mit langer und mittlerer Lebensdauer bis zur jeweiligen mittleren Lebensdauer auf und gehen anschließend in eine Kaskadennutzung über. Da die Baumarten verschieden lange Simu- 
lationsdauern haben (s. Tab. 3.3), durch eine unterschiedliche Anzahl von Beständen repräsentiert werden (s. Tab. 3.4) und der Holzverwendungsschlüssel die Nutzungsmengen je nach Holzart unterschiedlich auf die Produktklassen verteilt (s. Tab. 3.13), wird zur Berechnung einer Rate des additiven Holzproduktspeichers und der damit einhergehenden Substitutionswirkung eine einheitliche Bezugsbasis benötigt. Hier dient die Eiche (maximaler Produktionszeitraum 200 Jahre) als Referenz und dementsprechend durchlaufen die anderen Baumarten aufgrund ihrer geringeren Produktionszeiträume (s. Tab. 3.3) diesen Zeitraum mehr als einmal. 


\section{Kohlenstoffvorräte und Speicherraten der betrachteten Speicher}

\subsection{Lebende Baumbiomasse}

Ausgehend von den Weiserbeständen (s. Kap. 2.3), werden die waldbaulichen Behandlungen wie im Kapitel 3.2.2 beschrieben, im Prognosesystem WaldPlaner (Hansen, 2012; Hansen und Nagel, 2014) umgesetzt. Diese haben je nach Baumart, Alter, Leistung und Standort unterschiedliche initiale Kohlenstoffvorräte (s. Tab. A.22). Die Tabelle 4.1 zeigt die mittleren ober- und unterirdisch lebenden Baumbiomassen sowie die sich daraus ergebenden Kohlenstoffvorräte der nach Kapitel 2.3 erstellten Weiserbestände getrennt nach Region, Baumart und Alter. Daraus ist ersichtlich wie die ober- und unterirdisch lebende Baumbiomasse der Bestände mit dem Alter ansteigt und sich somit höhere Kohlenstoffvorräte in den älteren Beständen ergeben als in den jüngeren. Darüber hinaus sind die Werte der Baumarten Eiche, Buche und Fichte im Bergland im Mittel etwas höher als im Tiefland. Werden die mittleren Kohlenstoffvorräte der lebenden Baumbiomasse der jungen Weiserbestände im Startalter (s. Tab. 3.3) in Beziehung zueinander gesetzt, ergibt sich im Bergland folgende Baumartenreihung: Fichte $>$ Buche $>$ Eiche. Im Tiefland ist die Reihung: Douglasie, Fichte $>$ Kiefer, Buche $>$ Eiche.

Die Auswirkungen der waldbaulichen Behandlungen werden beispielhaft an der Baumart Buche dargestellt und sind in der Abbildung 4.1 sehen. Dabei sind deutlich die Zeitpunkte der Initialisierung der Weiserbestände durch Verschiebungen der Kohlenstoffvorräte erkennbar. Ausgehend von den Weiserbeständen werden in den ersten 60 Jahren (Alter 40 bis 100) die Buchenbestände bei beiden waldbaulichen Szenarien ähnlich behandelt und es finden Durchforstungen statt. Die Kohlenstoffvorräte betragen hier anfangs im Mittel ca. $45 \mathrm{t} \mathrm{Cha}^{-1}$ und am Ende der zweiten Periode je nach Leistungsfähigkeit zwischen rund 107 und etwa $237 \mathrm{t} \mathrm{C} \mathrm{ha}^{-1}$. In den folgenden drei Jahrzehnten (ab Alter 100 bis 130) sind erste Unterschiede in der Behandlung ersichtlich. Einige Bestände in der Ost-Heide, der Hohen Heide und dem Unteren Solling haben eine schlechte Bonität (vgl. Tab. A.4) und daher ist der Zieldurchmesser bei der kohlenstofforientierten Behandlung geringer als bei der naturnahen Variante. Darum setzt in diesen Beständen die Zielstärkennutzung vergleichsweise früh ein und der Kohlenstoffvorrat sinkt. Bei der naturnahen Variante setzt die Zielstärkennutzung in Beständen mit guter Bonität in den 
Tabelle 4.1: Mittlere ober- (OB) und unterirdisch (UB) lebende Baumbiomassen sowie daraus resultierende Kohlenstoffvorräte $(\mathrm{CV})$ der erstellten Weiserbestände getrennt nach Region $(\mathrm{BL}=$ Bergland, $\mathrm{TL}=$ Tiefland $)$, Art $(110=$ Eiche, $211=$ Buche, $511=$ Fichte, $611=$ Douglasie, $711=$ Kiefer $)$ und Alter.

\begin{tabular}{|c|c|c|c|c|c|}
\hline Region & Art & Alter & $\mathrm{OB}\left[\mathrm{tha}^{-1}\right.$ & $\mathrm{UB}\left[\mathrm{t} \mathrm{ha}^{-1}\right.$ & $\mathrm{CV}\left[\mathrm{t} \mathrm{Cha}^{-1}\right]$ \\
\hline \multirow{13}{*}{$\mathrm{BL}$} & \multirow{6}{*}{110} & 35 & 65,1 & 11,8 & 38,5 \\
\hline & & 65 & 114,6 & 20,8 & 67,7 \\
\hline & & 95 & 154,8 & 28,1 & 91,5 \\
\hline & & 125 & 192,4 & 34,9 & 113,6 \\
\hline & & 155 & 237,7 & 43,1 & 140,4 \\
\hline & & 185 & 283,2 & 51,4 & 167,3 \\
\hline & \multirow{4}{*}{211} & 40 & 79,1 & 14,3 & 46,7 \\
\hline & & 70 & 151,8 & 27,5 & 89,6 \\
\hline & & 100 & 220,7 & 40,0 & 130,4 \\
\hline & & 130 & 269,1 & 48,8 & 159,0 \\
\hline & \multirow{3}{*}{511} & 30 & 121,9 & 30,2 & 76,1 \\
\hline & & 60 & 180,9 & 44,8 & 112,9 \\
\hline & & 90 & 220,2 & 54,6 & 137,4 \\
\hline \multirow{22}{*}{ TL } & \multirow{6}{*}{110} & 35 & 58,1 & 10,5 & 34,3 \\
\hline & & 65 & 104,3 & 18,9 & 61,6 \\
\hline & & 95 & 142,5 & 25,8 & 84,2 \\
\hline & & 125 & 179,8 & 32,6 & 106,2 \\
\hline & & 155 & 218,4 & 39,6 & 129,0 \\
\hline & & 185 & 263,0 & 47,7 & 155,4 \\
\hline & \multirow{4}{*}{211} & 40 & 73,1 & 13,3 & 43,2 \\
\hline & & 70 & 143,7 & 26,1 & 84,9 \\
\hline & & 100 & 210,9 & 38,2 & 124,6 \\
\hline & & 130 & 261,6 & 47,5 & 154,5 \\
\hline & \multirow{3}{*}{511} & 30 & 121,2 & 30,0 & 75,6 \\
\hline & & 60 & 180,4 & 44,7 & 112,6 \\
\hline & & 90 & 218,8 & 54,2 & 136,5 \\
\hline & \multirow{4}{*}{611} & 25 & 117,6 & 4,8 & 61,2 \\
\hline & & 55 & 170,9 & 7,0 & 88,9 \\
\hline & & 85 & 260,0 & 10,6 & 135,3 \\
\hline & & 115 & 301,7 & 12,3 & 157,0 \\
\hline & \multirow{5}{*}{711} & 30 & 83,0 & 1,2 & 42,1 \\
\hline & & 60 & 101,1 & 1,5 & 51,3 \\
\hline & & 90 & 120,7 & 1,8 & 61,3 \\
\hline & & 120 & 129,7 & 1,9 & 65,8 \\
\hline & & 150 & 126,3 & 1,9 & 64,1 \\
\hline
\end{tabular}




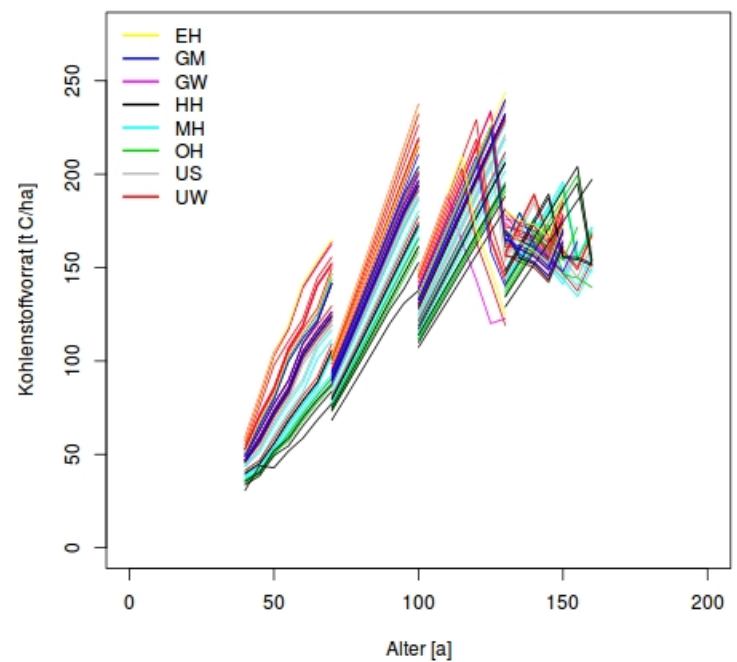

(a)

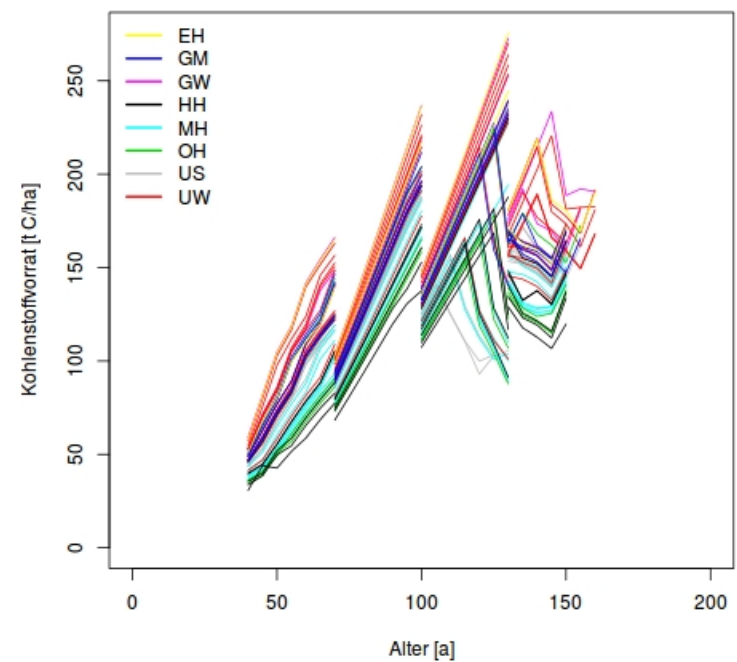

(b)

Abbildung 4.1: Entwicklung des Kohlenstoffvorrates [t C ha-1] der lebenden Baumbiomasse von Buchenreinbeständen bei naturnaher (a) und kohlenstofforientierter (b) Behandlung getrennt nach Wuchsbezirken (EH = Ems-Hase-Hunte-Geest, GM $=$ Geest-Mitte, $\mathrm{GW}=$ Göttinger Wald, $\mathrm{HH}=$ Hohe Heide, $\mathrm{MH}=$ Montaner Mittel-und Oberharz, $\mathrm{OH}=$ Ost-Heide, US $=$ Unterer Solling, $\mathrm{UW}=$ Unteres Weser-Leine-Bergland).

Wuchsbezirken Göttinger Wald, Unteres Weser-Leine-Bergland und Ems-Hase-HunteGeest ebenfalls früh ein, da sie den angestrebten Zieldurchmesser schon erreicht haben. Am Ende dieser Phase schwanken die Kohlenstoffvorräte bei der naturnahen Behandlung zwischen rund 120 und $234 \mathrm{t} \mathrm{Cha}^{-1}$ sowie bei der kohlenstofforientierten Variante zwischen etwa 100 und $254 \mathrm{t} \mathrm{C} \mathrm{ha}^{-1}$. Bei erneuter Initialisierung (Alter 130) werden durch den Waldwachstumssimulator alle Bestände als Endnutzungsbestände angesehen, wobei hier folglich auch der Kohlenstoffvorrat junger Weiserbestände zum Tragen kommt. Die Kohlenstoffvorräte betragen in der letzten Altersphase (130 bis 160 Jahre) durchschnittlich ca. $164 \mathrm{t} \mathrm{Cha}^{-1}$ bei naturnaher und etwa $163 \mathrm{t} \mathrm{C} \mathrm{ha}^{-1}$ bei kohlenstofforientierter Behandlung. Durch den WaldPlaner werden in dieser Periode auch Bestände mit schlechter bis mittlerer Bonität vor dem Ende der Produktionszeit geräumt, da sie den letzten Abschnitt im Verjüngungsgang (s. Tab. 3.1) erreicht haben. Wenn Bestände am Ende der Produktionszeit nicht geräumt waren wurden sie nachträglich dem Holzproduktspeicher zugeführt. Insgesamt gesehen sind die Unterschiede der mittleren Kohlenstoffvorräte der lebenden Baumbiomasse zwischen den beiden waldbaulichen Szenarien gering. Die Abbildungen zur Entwicklung des Kohlenstoffvorrates der lebenden Baumbiomasse der anderen Baumarten sind im Anhang A.5 zu finden. 
Die Verschiebungen zwischen den modellgestützt fortgeschriebenen Kohlenstoffvorräten der Weiserbestände am Ende einer 30-jährigen Simulationsperiode und den gewählten Startwerten der folgenden Simulationsperiode wurden in Kauf genommen, um die Simulationsläufe periodisch an die realen Gegebenheiten rückzukoppeln. Hierdurch wird die von den Modellen abweichende waldbauliche Behandlung der Weiserbestände (reale Behandlung versus Waldbauregeln des Waldwachstumssimulators) ebenso berücksichtigt, wie die besseren Bonitäten der jüngeren Bestände im Vergleich zu den älteren Beständen auf gleichem Standort. Die Bonitätsunterschiede sind durch die unterschiedlich lange Einwirkungsdauer der Stickstoffeinträge seit den 1960er Jahren des 20. Jahrhunderts bedingt, welche zu verbesserten Wuchsbedingungen geführt haben. Zugleich werden durch dieses Vorgehen die Simulationsfehler über einen Zeitraum von bis zu 200 Jahren minimiert. Insofern ist die vorliegende Analyse als konservative Einschätzung der Kohlenstoffspeicherleistung zu verstehen.

\subsection{Tote Baumbiomasse}

Der Totholzspeicher wird durch die Zersetzungs- und Nachlieferungsdynamik der toten Baumbiomasse bestimmt. Allgemein kann gesagt werden, dass sich das Totholz von Buche, Fichte sowie Kiefer schnell zersetzt, das von Eiche und Douglasie langsamer (s. Abb. 3.4). In der Abbildung 4.2 ist die baumartenspezifische Entwicklung des Kohlenstoffvorrates der toten Baumbiomasse in Beständen aus Buche und Fichte zu sehen und es wird der Einfluss der Nutzungen deutlich sichtbar. Die entsprechenden Abbildungen für die anderen Baumarten sind im Anhang A.6 zu finden.

$\mathrm{Zu}$ Beginn des Simulationszeitraumes muss sich zunächst ein gewisser Totholzvorrat und damit Kohlenstoffvorrat aufbauen. Dies geschieht aktiv durch die natürliche Mortalität und die Ernteverluste, welche bei der Feinerschließung und den Durchforstungen anfallen. Insbesondere bei der Fichte ist die simulierte natürliche Mortalität sehr hoch. Der Totholzvorrat beträgt im Mittel bei beiden Varianten rund $28 \mathrm{~m}^{3} \mathrm{ha}^{-1}$ und liegt über dem angestrebten Totholzvolumen von $20 \mathrm{~m}^{3} \mathrm{ha}^{-1}$ (s. Kap. 3.2.2). Die kohlenstofforientierte Behandlung führt im Mittel zu einem Kohlenstoffvorrat im Totholz von 6,8 t $\mathrm{C}^{-1}$ in Eichenbeständen, 4,6 t C ha ${ }^{-1}$ in Buchenbeständen, 5,3 t $\mathrm{Cha}^{-1}$ in Fichtenbeständen, $14,3 \mathrm{t} \mathrm{Cha}^{-1}$ in Douglasienbeständen und $6,0 \mathrm{t} \mathrm{C} \mathrm{ha}^{-1}$ in Kiefernbeständen. Die entsprechenden Werte für die naturnahe Variante sind 6,7, 4,4, 3,8, 13,9 und 5,9 t Cha ${ }^{-1}$. Sie sind demnach etwas geringer als bei der kohlenstofforientierten Variante. Auf einzelnen Standorten werden jedoch zum Ende der Produktionszeit stellenweise höhere Kohlenstoffvorräte in diesem Speicher durch die naturnahe waldbauliche Behandlung erzielt als durch die kohlenstofforientierte (s. Abb. A.18). Auffällig sind die hohen Werte beim Douglasientotholz, welche sich durch die vergleichsweise geringe Zersetzungsrate und damit einer relativ langsamen, lang andauernden Dekomposition erklären lassen. 


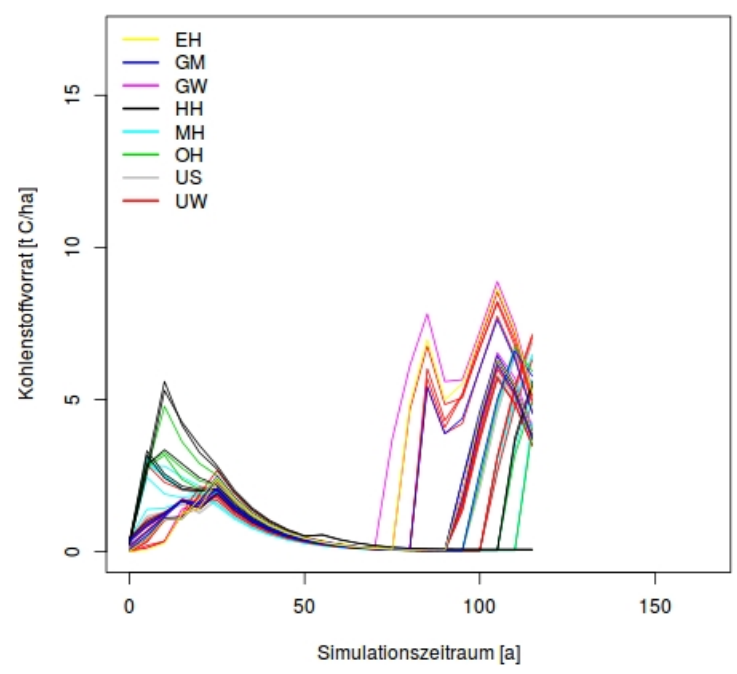

(a)

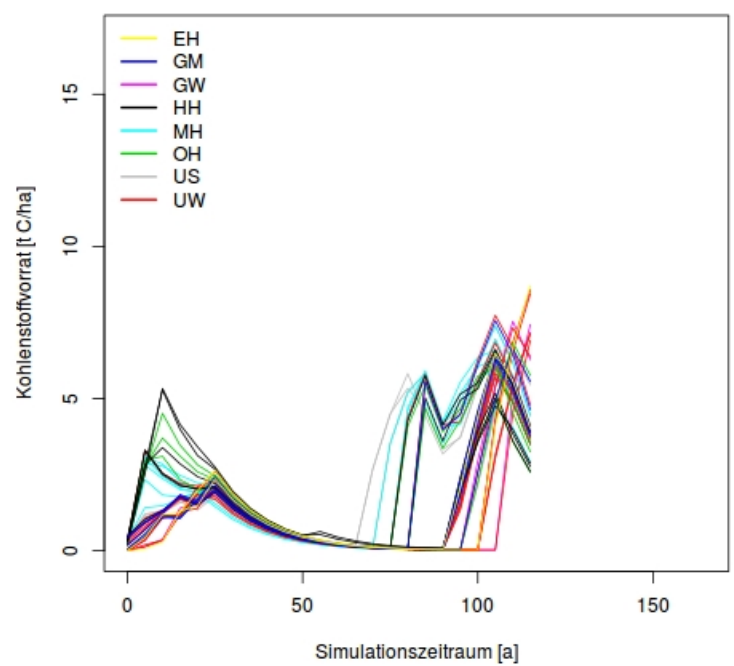

(c)

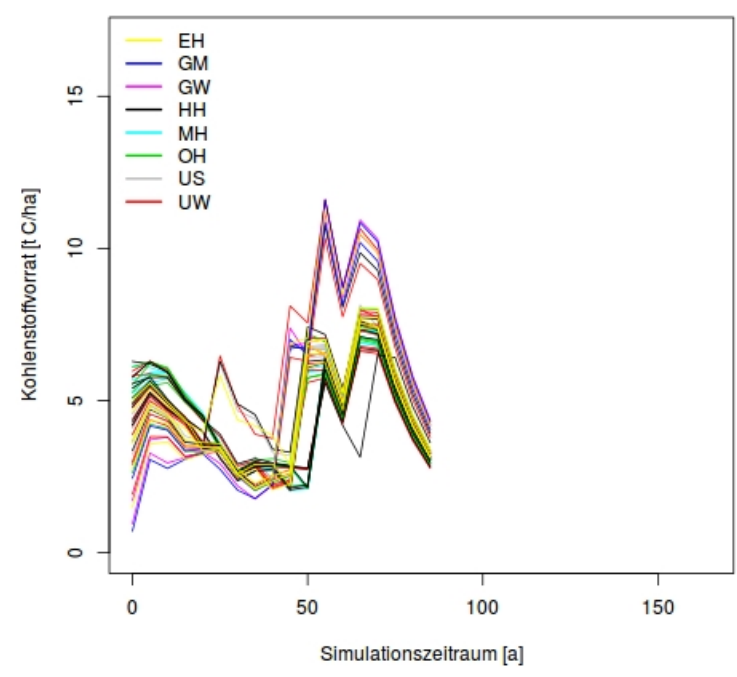

(b)

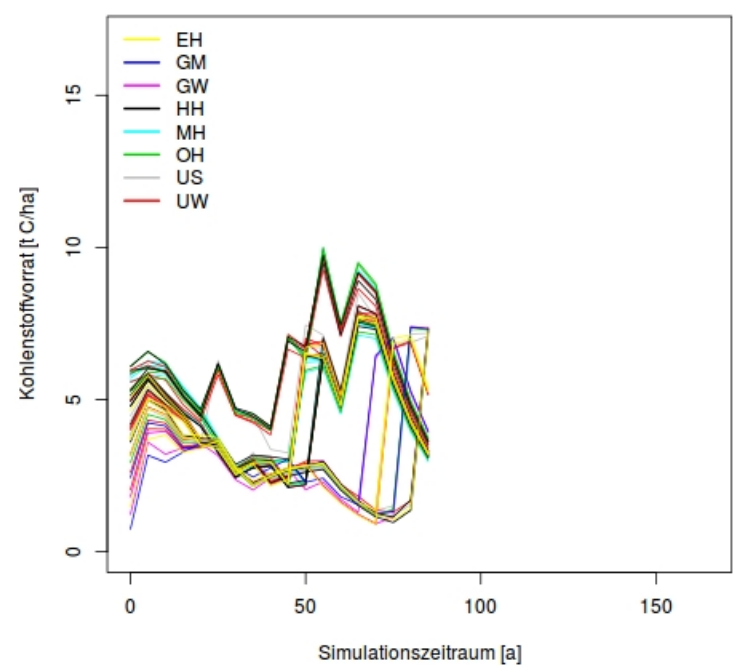

(d)

Abbildung 4.2: Entwicklung des Kohlenstoffvorrates [t $\left.\mathrm{C} \mathrm{ha}^{-1}\right]$ der toten Baumbiomasse in Reinbeständen aus Buche $(\mathrm{a}, \mathrm{c})$ und Fichte $(\mathrm{b}, \mathrm{d})$ getrennt nach Wuchsbezirken $(\mathrm{EH}$ $=$ Ems-Hase-Hunte-Geest, GM = Geest-Mitte, GW = Göttinger Wald, HH = Hohe Heide, $\mathrm{MH}=$ Montaner Mittel-und Oberharz, $\mathrm{OH}=$ Ost-Heide, US = Unterer Solling, UW = Unteres Weser-Leine-Bergland) bei naturnaher $(\mathrm{a}, \mathrm{b})$ und kohlenstofforientierter (c, d) Behandlung. 


\subsection{Holzprodukte}

In den Holzprodukten sind die Kohlenstoffmengen der geernteten Bäume gespeichert. Gesamt betrachtet werden durch die kohlenstofforientierte Bewirtschaftung der Weiserbestände rund $75.448 \mathrm{~m}^{3}$ Laub- bzw. $129.052 \mathrm{~m}^{3}$ Nadelholz dem Produktspeicher zugeführt. Bei naturnaher Behandlung sind es $72.674 \mathrm{~m}^{3}$ und $138.395 \mathrm{~m}^{3}$. In Abbildung 4.3 ist das im Verlauf der Simulation periodisch anfallende Laub- und Nadelholz getrennt nach waldbaulicher Behandlung zu sehen. Dabei wird deutlich, dass der Nutzungsverlauf der Behandlungsszenarien meist gleich ist. Im Laufe der Simulation werden die ausgehaltenen Sortimente den Produktklassen zugeordnet und es finden Nachlieferungen, auch in Form einer Kaskadennutzung, statt (s. Kap. 3.3.3).

In Abbildung 4.4 ist die summarische Entwicklung des Kohlenstoffvorrates im Produktspeicher getrennt nach Produktklassen, Holzart und waldbaulicher Behandlung dargestellt. Dabei bezieht sich der angegebene Kohlenstoffvorrat auf die Gesamtfläche der jeweils ein Hektar großen Weiserbestände (Laubwaldfläche: 418 ha, Nadelwaldfläche: 437 ha, Gesamtfläche: 855 ha). Es fällt auf, dass sich dieser Speicher bei beiden Behandlungsszenarien ähnlich entwickelt und es einen gravierenden Unterschied zwischen Laub- und Nadelholz gibt. Maximal werden bei kohlenstofforientierter bzw. naturnaher Behandlung rund 8.838 bzw. 9.222 t C durch Nadelholz und etwa 4.965 bzw. 4.689 t C mittels Laubholz dem Produktpool zugeführt. Dabei ist zu beachten, dass der betrachtete Zeitraum beim Laubholz 40 Jahre länger ist als beim Nadelholz und die starke Absenkung der Kohlenstoffvorräte gegen Ende des Betrachtungszeitraumes durch die individuelle Simulationszeit der verschiedenen Baumarten in den Kategorien Laub- und Nadelholz sowie die produktklassenspezifischen mittleren Lebensdauern, die in der Kaskade Beachtung finden, bedingt ist.

Holzprodukte substituieren andere Materialien und Energieträger. Um diesen Effekt darzustellen, wurden die in Kapitel 3.3.3 genannten Faktoren genutzt. Da die Substitution nicht umkehrbar ist, addieren sich die Effekte über den gesamten Simulationszeitraum auf. In Abbildung 4.5 ist die Entwicklung der Substitutionswirkung der Holzprodukte über den gesamten Zeitraum, getrennt nach Laub- und Nadelholz sowie waldbaulicher Behandlung zu sehen. Dabei basiert die Substitutionsleistung auf den generierten Erntemengen und den geräumten Beständen. Der auf dieser Grundlage ermittelte Substitutionseffekt der energetischen Nutzung ist bei Nadelholz höher als bei Laubholz. Dies lässt sich durch die höhere Rohholzmenge und der somit auch größeren Menge an Sägenebenprodukten, welche zu großen Teilen der energetischen Nutzung zugeführt werden (s. Kap. 3.3.3), erklären. Bis zum Ende des Produktionszeitraumes werden durch die kohlenstofforientierte Behandlung der Nadelholzbestände und deren energetische Nutzung rund 18.042 t C substituiert. Bei gleicher Behandlung der Laubholzbestände werden durch die energetische Nutzung nur etwa 9.197 t C ersetzt. Ungleich höher ist der Beitrag der Holzprodukte zur Materialsubstitution. Bis zum Ende des Betrachtungszeitraumes werden bei kohlenstofforientierter Behandlung der Bestände und der Herstellung von Holzprodukten aus Laub- bzw. Nadelholz mit langer und mittlerer Lebensdauer etwa 


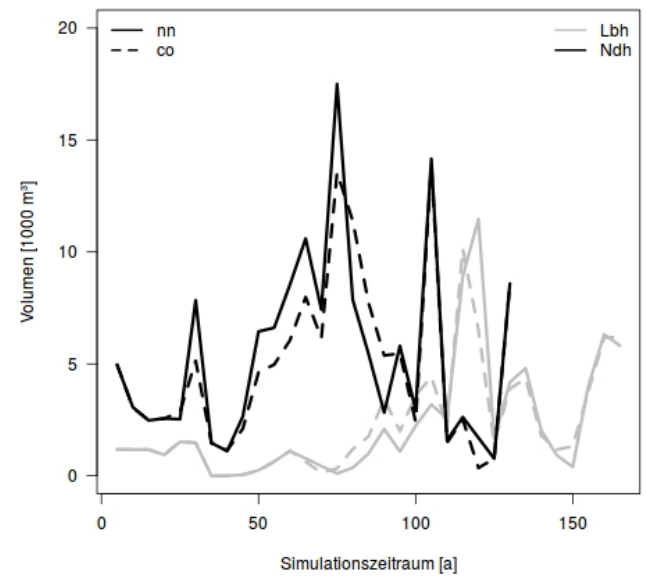

Abbildung 4.3: Im Verlauf der Simulationen anfallende Nutzungsmengen aus den Weiserbeständen, getrennt nach Laub- (Lbh) und Nadelholz (Ndh) sowie waldbaulicher Behandlung $(\mathrm{nn}=$ naturnah, $\mathrm{co}=$ kohlenstofforientiert $)$.

51.314 bzw. 98.347t C substituiert. Die naturnahe waldbauliche Behandlung der Bestände führt zu einem ähnlich hohen Beitrag zur energetischen (Laubholz: 8.364tC; Nadelholz: 19.632 t C) und materiellen Substitution (Laubholz: 46.556 t C; Nadelholz: 107.262 t C). Zu beachten ist auch hier, dass unterschiedlich lange Zeiträume betrachtet werden und verschiedene Bestandesanzahlen (s. Tab. 3.4) zugrunde liegen. Im Mittel beträgt hier das Verhältnis von energetischer zu materieller Substitution am Ende der Betrachtungszeit etwa $1 \mathrm{zu}$ 5. Daraus wird der immense Beitrag der stofflichen Holznutzung sowie dem Substitutionseffekt zur Vermeidung von Kohlenstoffdioxidemissionen in die Atmosphäre ersichtlich. 


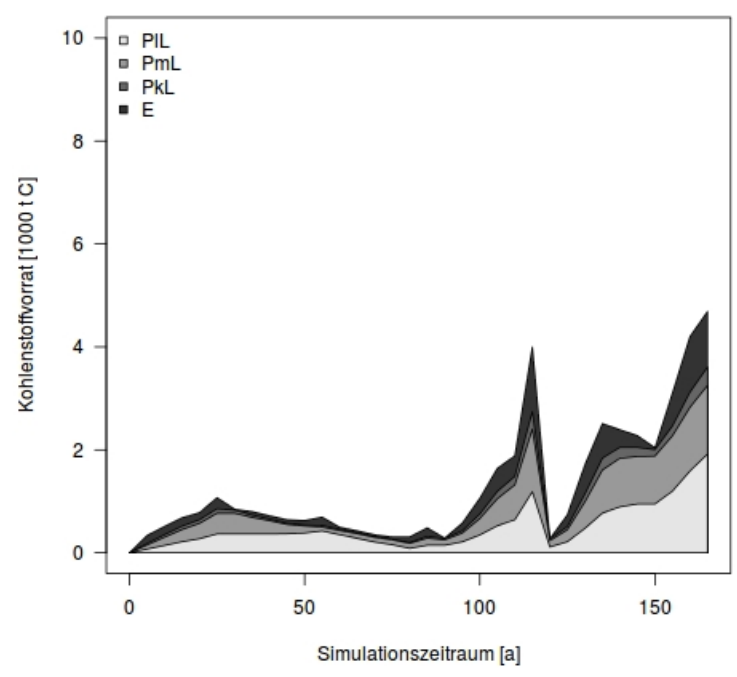

(a)

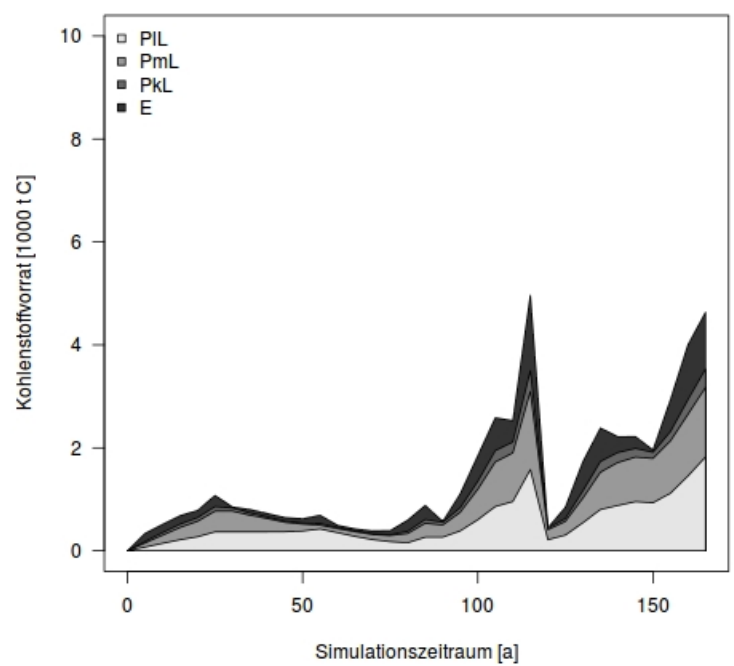

(c)

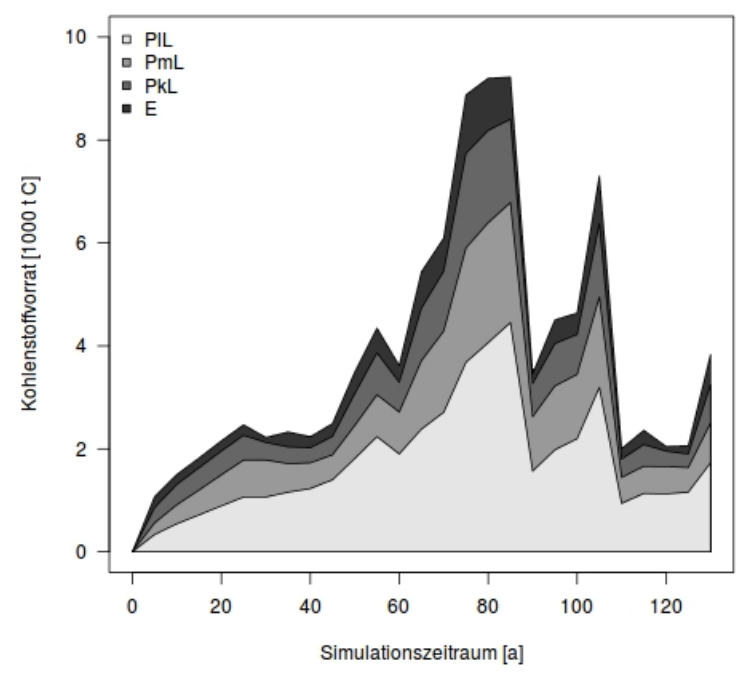

(b)

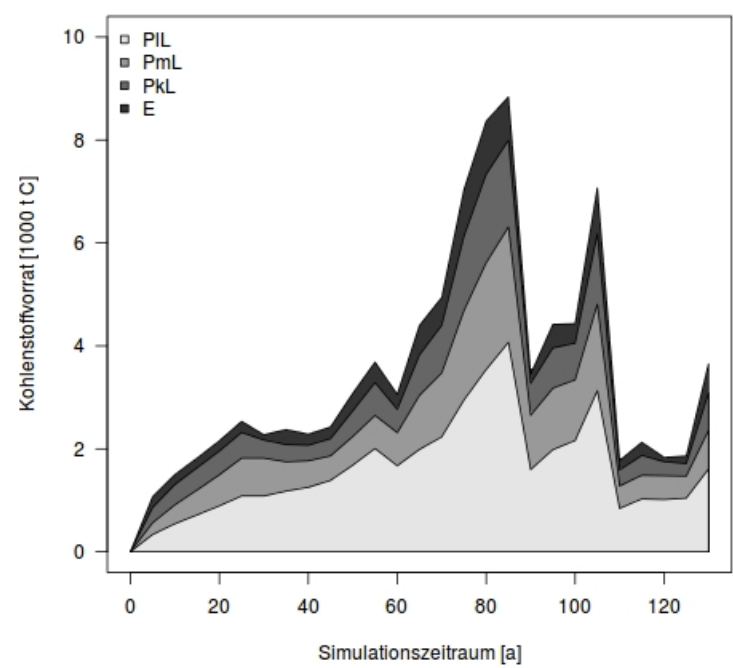

(d)

Abbildung 4.4: Entwicklung des gesamten Kohlenstoffvorrates im Produktspeicher [1000 t C], basierend auf den anfallenden Nutzungsmengen der jeweils ein Hektar großen Weiserbestände, getrennt nach Produktklassen (PlL, PmL, PkL = Produkte mit langer, mittlerer und kurzer Lebensdauer, $\mathrm{E}=$ Energieholz) sowie Laub- (a, c) und Nadelholz (b, d) bei naturnaher (a, b) und kohlenstofforientierter (c, d) waldbaulicher Behandlung (Laubwaldfläche: 418 ha, Nadelwaldfläche: 437 ha). 


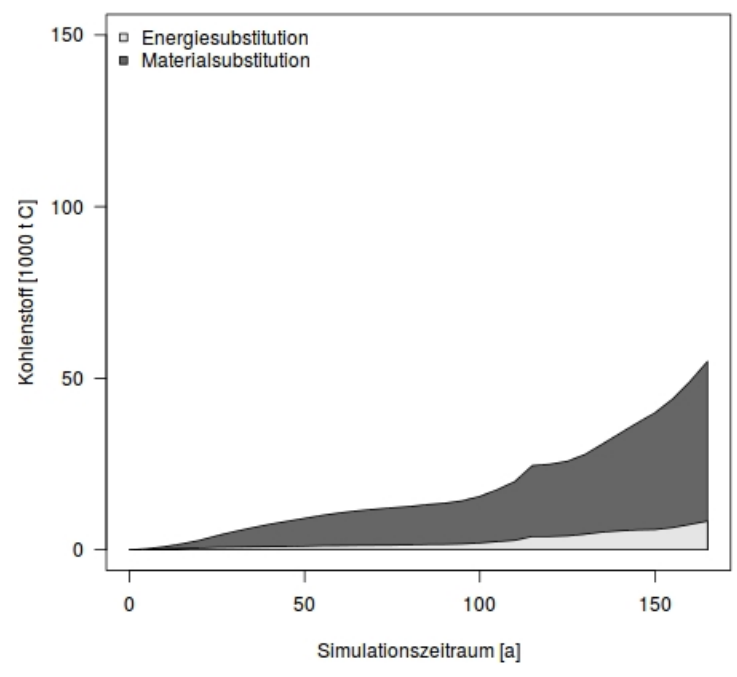

(a)

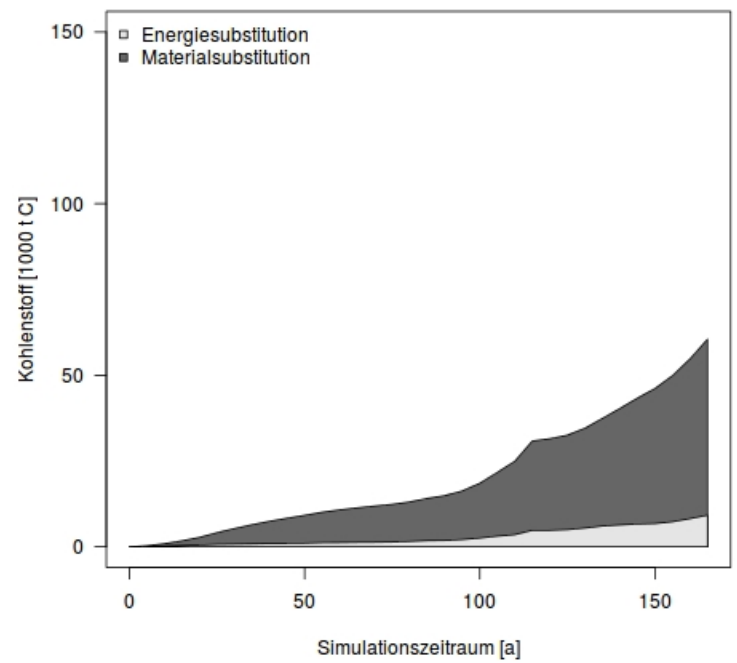

(c)

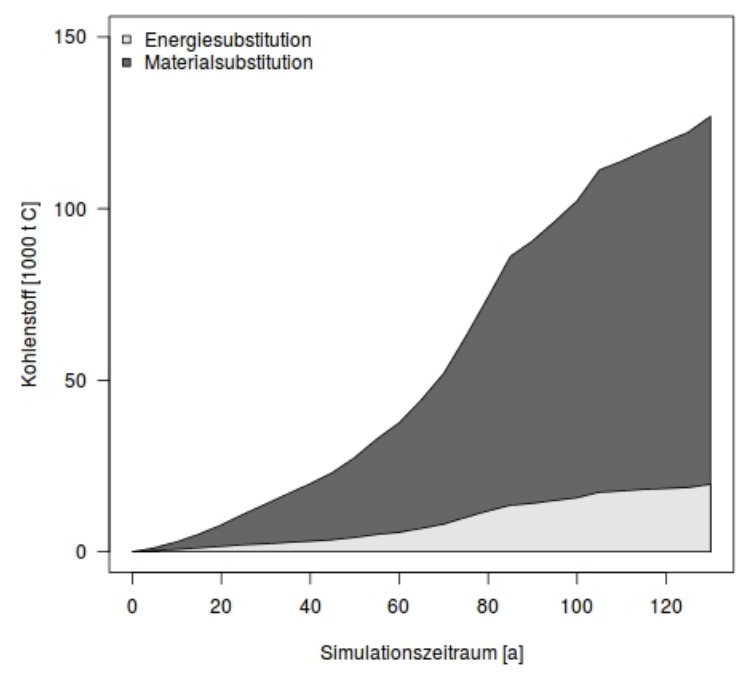

(b)

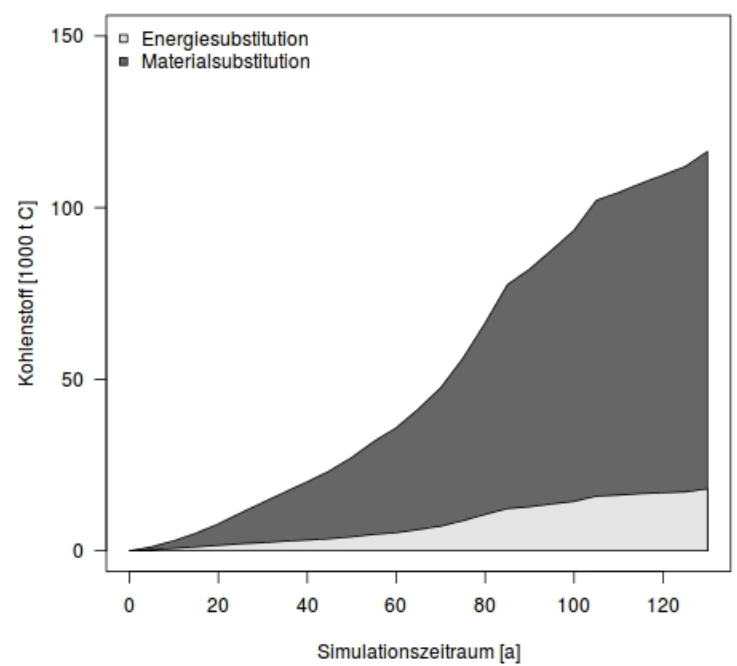

(d)

Abbildung 4.5: Summarische Entwicklung der Substitutionswirkung des Produktspeichers [1000 t C], basierend auf den angefallenen, den Produktklassen zugeordneten Nutzungsmengen der jeweils ein Hektar großen Weiserbestände, getrennt nach Laub- $(\mathrm{a}, \mathrm{c})$ und Nadelholz $(\mathrm{b}, \mathrm{d})$ bei naturnaher $(\mathrm{a}, \mathrm{b})$ und kohlenstofforientierter (c, d) waldbaulicher Behandlung (Laubwaldfläche: 418 ha, Nadelwaldfläche: 437 ha). 


\subsection{Speicherraten der betrachteten Speicher}

Ein besserer Vergleich der Baumarten auf den verschiedene Standorten und der Möglichkeiten Kohlenstoff zu speichern, ist mit dem Bilden von Raten möglich. Dabei wird die Kohlenstoffbindungsrate der lebenden Baumbiomasse auf den Produktionszeitraum bezogen, wobei es an dessen Ende einen Überlappungszeitraum gibt (s. Kap. 3.1). Allerdings muss das Ende der Produktionszeit nicht gleichzeitig der Zeitpunkt der höchsten Kohlenstoffvorräte sein.

Die baumartenspezifischen Kohlenstoffspeicherraten der lebenden Baumbiomasse innerhalb eines Wuchsbezirkes, abhängig von Wasser- und Nährstoffversorgung, lassen direkte Rückschlüsse über den Einfluss des Standortes auf die Sequestrierung von Kohlenstoff zu. Im Göttinger Wald sind Buche und Fichte auf frischen, ziemlich gut nährstoffversorgten basenarmen Silikatgesteinen und auf mäßig frischen, sehr gut mit Nährstoffen versorgten Kalkgesteinen vertreten (s. Abb. 4.6(a)). Dabei ist die Speicherrate der Fichte höher (1,5 bis 1,7 t $\mathrm{C} \mathrm{ha}^{-1} \mathrm{a}^{-1}$ ) als die der Buche (etwa 1,2 $\mathrm{t} \mathrm{Cha}^{-1} \mathrm{a}^{-1}$ ). Auf den mäßig frischen Kalkgesteinen mit sehr guter Nährstoffversorgung ist die Speicherrate der Buchenbestände etwas geringer (rund $1,17 \mathrm{t} \mathrm{Cha}^{-1} \mathrm{a}^{-1}$ ) als auf den besser wasser- jedoch schlechter nährstoffversorgten basenarmen Silikatgesteinen. Umgekehrt ist es bei der Fichte, die auf den Kalkgesteinen Speicherraten von bis zu etwa 1,8 t $\mathrm{C} \mathrm{ha}^{-1} \mathrm{a}^{-1}$ erreicht. Hinsichtlich der Behandlungsvarianten führt die naturnahe Behandlung der Bestände auf den genannten Standorten im Wuchsbezirk Göttinger Wald im Mittel zu einer leicht höheren Speicherrate (ca. 1,5 $\mathrm{t} \mathrm{Cha}^{-1} \mathrm{a}^{-1}$ ) als bei der kohlenstofforientierten Behandlung (ca. 1,4 t $\left.\mathrm{C} \mathrm{ha}^{-1} \mathrm{a}^{-1}\right)$.

Im Montanen Mittel- und Oberharz, klimatisch gekennzeichnet durch hohe Niederschlagsmengen und niedrige Temperaturen im Vergleich zu den anderen Wuchsbezirken in der Berglandregion, wurden Buchen- und Fichtenbestände auf basenarmen sowie -reichen Silikatgesteinen analysiert (s. Abb. 4.6(b)). Im Allgemeinen ist die Kohlenstoffsequestrierung in der lebenden Baumbiomasse bei Fichte höher ( $\left.\varnothing 1,4 \mathrm{t} \mathrm{C} \mathrm{ha}^{-1} \mathrm{a}^{-1}\right)$ als bei Buche (Ø 1,0 t $\mathrm{C} \mathrm{ha}^{-1} \mathrm{a}^{-1}$ ). Unabhängig von der Bodenart reagieren Fichte und $\mathrm{Bu}-$ che auf frischen Standorten mit einem Anstieg der Speicherrate bei besser werdender Nährstoffversorgung. Bei gleicher Nährstoffversorgung und abnehmender Wasserversorgung ist eine Abnahme der Speicherraten zu erkennen. Die Unterschiede zwischen den Behandlungsszenarien sind hier ebenfalls gering $\left(0,001\right.$ bis $\left.0,021 \mathrm{t} \mathrm{Cha}^{-1} \mathrm{a}^{-1}\right)$.

Im Unteren Solling stocken Eichen, Buchen und Fichten auf basenarmen Silikatgesteinen und Lössen (s. Abb. 4.6(c)). Wobei die Bindungsraten der lebenden Baumbiomasse der Fichten ( $\varnothing$ 1,6 $\left.\mathrm{tCha}^{-1} \mathrm{a}^{-1}\right)$ höher sind als die von Buchen $\left(\varnothing 1,0 \mathrm{t} \mathrm{Cha}^{-1} \mathrm{a}^{-1}\right)$ oder Eichen ( $\varnothing 0,8 \mathrm{tCha}^{-1} \mathrm{a}^{-1}$ ). Bei Eiche ist die Tendenz einer geringer werdenden Rate bei gleicher Wasserversorgung (frisch bzw. mäßig frisch) und abnehmender Nährstoffversorgung zu erkennen (ziemlich gut zu mäßig). Bei konstanter Nährstoffversorgung (mäßig) und abnehmender Wasserverfügbarkeit (frisch zu mäßig frisch) kann ein leichter Anstieg der Bindungsrate erkannt werden. Die unterschiedlichen Behandlungen haben bei Eiche keinen nennenswerten Einfluss. Die höchsten Speicherraten in diesem Wuchsgebiet 
sind in Fichtenbeständen auf frischen und nachhaltig frischen Standorten mit ziemlich guter Nährstoffversorgung zu finden $\left(\varnothing 1,7 \mathrm{t} \mathrm{C} \mathrm{ha}^{-1} \mathrm{a}^{-1}\right)$. Hinsichtlich der Variante ist die naturnahe Behandlung der Fichtenbestände etwas vorteilhafter in Bezug auf die Kohlenstoffspeicherung $\left(\varnothing+0,08\right.$ t ha $\left.^{-1} \mathrm{a}^{-1}\right)$ als das kohlenstofforientierte Szenario. Da die Fichtenbestände bei der kohlenstofforientierten Behandlung einer guten Bonität zugeordnet werden, haben sie einen größeren Zieldurchmesser als bei der naturnahen (s. Tab. 3.5). Somit beginnt die Zielstärkennutzung später und der Überlappungszeitraum ist entsprechend geringer. Bei den anderen Baumarten sind die Unterschiede zwischen den Behandlungsvarianten noch geringer.

Im Unteren Weser-Leine-Bergland ist die Variation der Standorte, im Vergleich zu den anderen ausgesuchten Wuchsbezirken im Bergland, am größten. Auch hier ist die Kohlenstoffspeicherrate der lebenden Baumbiomasse bei Fichte $\left(\varnothing 1,5 \mathrm{t} \mathrm{C} \mathrm{ha}^{-1} \mathrm{a}^{-1}\right)$ höher als bei Buche $\left(\varnothing 1,1 \mathrm{t} \mathrm{Cha}^{-1} \mathrm{a}^{-1}\right.$ ) oder Eiche $\left(\varnothing 0,8 \mathrm{t} \mathrm{Cha}^{-1} \mathrm{a}^{-1}\right)$ (s. Abb. 4.6(d)). In diesem Wuchsgebiet sind die wichtigsten Standortstypenobergruppen basenarme Silikatgesteine, Kalkgesteine und Lösse. Auf den unterschiedlich gut nährstoffversorgten Standorten ist bei den drei vorkommenden Baumarten eine Abnahme der Speicherrate mit abnehmender Wasserversorgung zu erkennen. Auf den guten Standorten (nachhaltig frisch oder frisch, ziemlich bis sehr gut nährstoffversorgt) in diesem Wuchsbezirk, erweist sich die naturnahe Behandlung der Fichtenbestände vorteilhafter $\left(\varnothing+0,12 \mathrm{t} \mathrm{Cha}^{-1} \mathrm{a}^{-1}\right)$ als die kohlenstofforientierte. Bei den anderen Baumarten bzw. bei den anderen Standortskombinationen ist kaum ein Unterschied zwischen den verschiedenen Behandlungen festzustellen.

Die ausgesuchten Wuchsbezirke im niedersächsischen Tiefland sind geprägt durch Geschiebelehme und Sande (s. Kap. 2.2.1) und in jedem der ausgesuchten Wuchsbezirke kommen alle fünf Hauptwirtschaftsbaumarten vor. Wobei im Tiefland die Kohlenstoffspeicherraten der Bestände aus Douglasie am größten sind, gefolgt von den Beständen aus Fichte, Buche, Eiche und Kiefer. Im Wuchsbezirk Ems-Hase-Hunte-Geest kommen auf den schwach grundwasserbeeinflussten, schwach bis mäßig mit Nährstoffen versorgten Standorten auf Flugsanden Eiche, Douglasie und Kiefer vor (s. Abb.4.7(a)). Hier ist die Speicherrate der Douglasie $\left(\varnothing 1,7 \mathrm{t} \mathrm{Cha}^{-1} \mathrm{a}^{-1}\right)$ mehr als doppelt so hoch wie die der Eiche $\left(\varnothing 0,7 \mathrm{tCha}^{-1} \mathrm{a}^{-1}\right)$ und mehr als dreimal so hoch wie die der Kiefer $(\varnothing$ 0,5 t Cha $\left.^{-1} \mathrm{a}^{-1}\right)$. Auf den frischen Geschiebelehmen mit ziemlich guter Nährstoffversorgung ist die höchste Speicherrate der Buche zu finden (Ø 1,2 $\mathrm{t} \mathrm{C} \mathrm{ha}^{-1} \mathrm{a}^{-1}$ ). Allerdings sind die Speicherraten der Fichten- und Douglasienbestände auf gleichem Standort um rund $34 \%$ höher als die der Buchenbestände. Die Speicherrate der Kiefernbestände steigt bei gleichbleibend mäßiger Nährstoffversorgung von den schwach grundwasserbeeinflussten Standorten zu den frischen über die mäßig frischen bis frischen Standorte an, um bei sich verschlechternder Wasserversorgung wiederum abzufallen. Hinsichtlich der unterschiedlichen waldbaulichen Behandlung kann kein eindeutiger Trend festgestellt werden.

Im Wuchsbezirk Geest Mitte kommen, im Vergleich zum Wuchsbezirk Ems-HaseHunte-Geest, auch stark und mäßig grundwasserbeeinflusste Standorte, mit der Standortstypenobergruppe Sande im Grundwasserbereich, vor (s. Abb. 4.7(b)). Auf diesen 


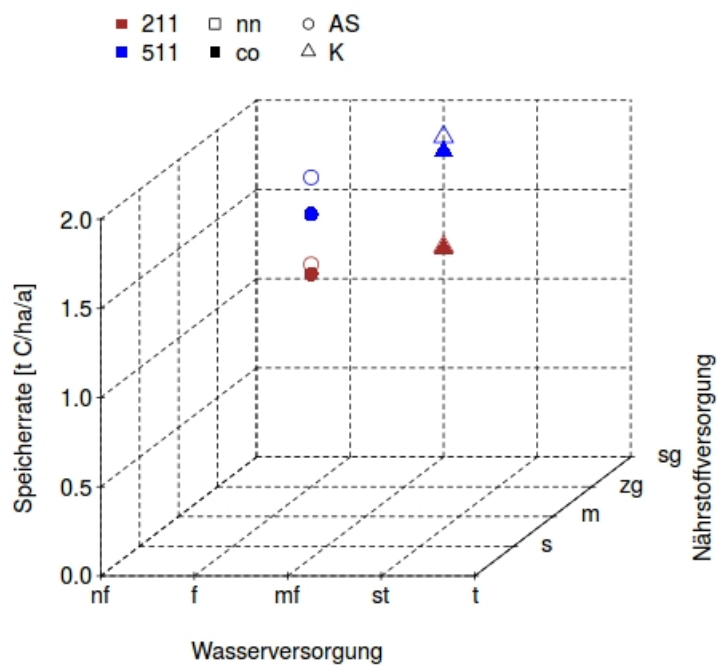

(a)

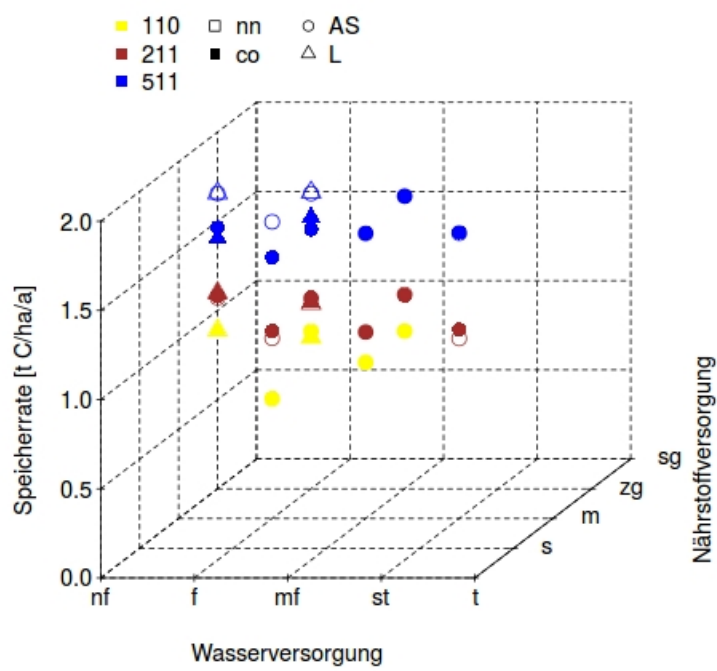

(c)

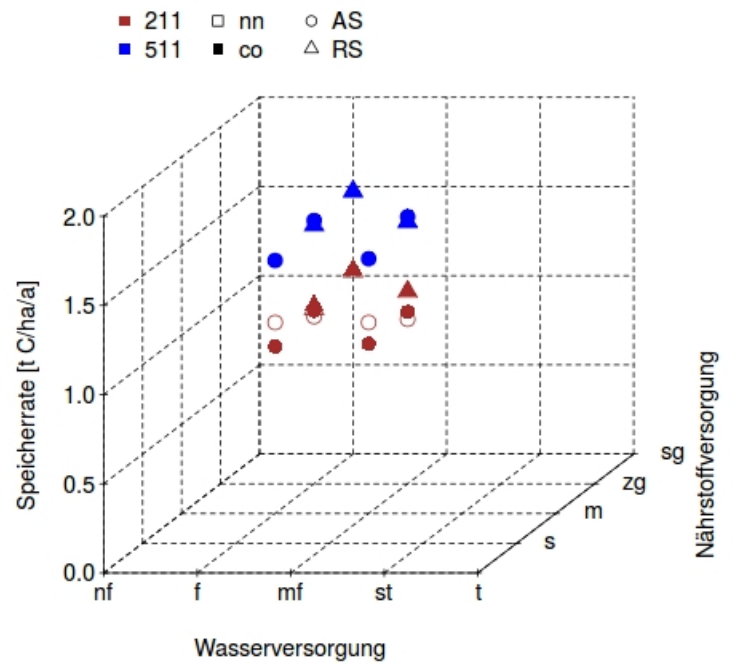

(b)

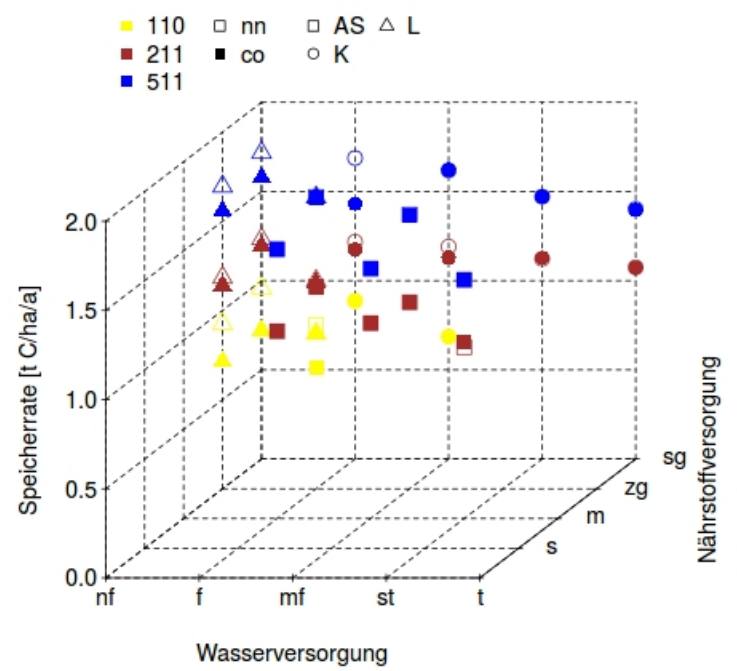

(d)

Abbildung 4.6: Kohlenstoffspeicherrate der lebenden Baumbiomasse $\left[\mathrm{t} \mathrm{C} \mathrm{ha}^{-1} \mathrm{a}^{-1}\right]$ in Abhängigkeit von Wasser- $(\mathrm{nf}=$ nachhaltig frisch, $\mathrm{f}=$ frisch, $\mathrm{mf}=$ mäßig frisch, $\mathrm{st}=$ sommertrocken, $\mathrm{t}=$ trocken) und Nährstoffversorgung ( $\mathrm{s}=$ schwach, $\mathrm{m}=$ mäBig, $\mathrm{zg}=$ ziemlich gut, $\mathrm{sg}=\mathrm{sehr}$ gut $)$, getrennt nach Baumart $(110=$ Eiche, $211=$ Buche, $511=$ Fichte $)$, Behandlung $(\mathrm{co}=$ kohlenstofforientiert, $\mathrm{nn}=$ naturnah) und Standortstypenobergruppe (AS = basenarme Silikatgesteine, RS = basenreiche Silikatgesteine, $\mathrm{K}=$ Kalkgesteine, $\mathrm{L}=$ Lösse) in den Wuchsbezirken Göttinger Wald (a), Montaner Mittel- und Oberharz (b), Unterer Solling (c) sowie Unteres Weser-Leine-Bergland (d). 
Standorten stocken Kiefern- und Eichenbestände, wobei bei gleicher Nährstoffversorgung und abnehmenden Grundwassereinfluss die Bindungsrate der lebenden Baumbiomasse der Kiefern, unabhängig von der Behandlung, leicht ansteigt $\left(\varnothing+0,04\right.$ t ha $\left.^{-1} \mathrm{a}^{-1}\right)$ und die der Eichenbestände bei naturnaher Behandlung abfällt (von 0,9 auf $0,8 \mathrm{t} \mathrm{C} \mathrm{ha}^{-1} \mathrm{a}^{-1}$ ) bzw. bei kohlenstofforientierter Behandlung ansteigt (von 0,7 auf $0,8 \mathrm{t} \mathrm{Cha}^{-1} \mathrm{a}^{-1}$ ). Die Fichte stockt auf frischen und ziemlich gut versorgten Geschiebelehmen sowie Sandlössen, mit der höchsten Speicherrate auf Sandlöss $\left(\varnothing 1,7 \mathrm{t} \mathrm{Cha}^{-1} \mathrm{a}^{-1}\right)$. Die Douglasienbestände erreichen im Mittel über alle möglichen Standortskombinationen ebenfalls sehr hohe Bindungsraten ( $\left.\varnothing 1,7 \mathrm{t} \mathrm{C} \mathrm{ha}^{-1} \mathrm{a}^{-1}\right)$. Die Buche stockt hier auf Geschiebelehmen, Sanden sowie Sandlössen und erreicht bei ziemlich guter Versorgung mit Nährstoffen und einem als frisch einzustufenden Wasserhaushalt ihre höchsten Speicherraten auf den Sandlössen und Geschiebelehmen (Ø 1,14 $\mathrm{tCha}^{-1} \mathrm{a}^{-1}$ ). Hinsichtlich der Behandlung ist festzuhalten, dass es auch hier nur geringe Unterschiede zwischen den Behandlungsvarianten gibt. Die Differenzen zwischen den Baumarten sind dafür umso größer und es ergibt sich folgende Reihung: Douglasie, Fichte $\left(\varnothing 1,6 \mathrm{t} \mathrm{Cha}^{-1} \mathrm{a}^{-1}\right)>\operatorname{Buche}\left(\varnothing 1,1 \mathrm{t} \mathrm{Cha-1} \mathrm{a}^{-1}\right)$ $>$ Eiche $\left(\varnothing 0,8 \mathrm{tC} \mathrm{ha}^{-1} \mathrm{a}^{-1}\right)>\operatorname{Kiefer}\left(\varnothing 0,5 \mathrm{t} \mathrm{Cha}^{-1} \mathrm{a}^{-1}\right)$.

Die Eichenbestände im Wuchsbezirk Hohe Heide stocken auf Geschiebelehmen und Sanden, wobei sich im Mittel, mit abnehmender Nährstoff- und Wasserversorgung, auch die Kohlenstoffspeicherrate der lebenden Baumbiomasse verringert (s. Abb. 4.7(c)). Allerdings wird durch die kohlenstofforientierte Behandlung der Eichenbestände auf Sanden eine deutlich höhere Speicherrate erbracht ( $\left.\varnothing 0,8 \mathrm{tC} \mathrm{ha}^{-1} \mathrm{a}^{-1}\right)$ als durch die naturnahe Bewirtschaftung $\left(\varnothing 0,5 \mathrm{t} \mathrm{Cha} \mathrm{C}^{-1} \mathrm{a}^{-1}\right)$. Eine vergleichsweise hohe Speicherrate ergibt sich bei der naturnahen Behandlung der Buche auf schlecht versorgten, trockenen Sanden. Da die Wuchsleistung auf diesem Standort gering ist und der Zieldurchmesser vom Waldwachstumssimulator strikt eingehalten wird, baut sich mehr Biomasse in dem Bestand auf als bei kohlenstofforientierter Behandlung mit einem sehr viel geringeren Zieldurchmesser. In Fichtenbeständen wird am wenigsten Kohlenstoff in der lebenden Baumbiomasse auf trockenen und schwach versorgten Sanden gespeichert. Mit ansteigender Wasser- und Nährstoffversorgung zeigt sich der Trend zu steigenden Speicherraten (1,3 bis $\left.1,7 \mathrm{t} \mathrm{Cha}^{-1} \mathrm{a}^{-1}\right)$. Ein anscheinend ungerichtetes Verhalten ist bei den Douglasienbeständen zu beobachten. Ihre Speicherraten schwanken in einem Bereich von 1,0 bis 1,6 t C ha ${ }^{-1} \mathrm{a}^{-1}$. Eine hohe Speicherrate ergibt sich auf den schlecht versorgten, trockenen Sanden bei kohlenstofforientierter Behandlung durch einen großen Überlappungszeitraum. Aufgrund der geringen Wuchsleistung auf diesem Standort ist angestrebte Zieldurchmesser geringer als bei der naturnahen Behandlung. Die Zielstärkennutzung setzt früher ein und junge Bestände tragen einen Großteil zur Erhöhung der Speicherrate bei. Auch in diesem Wuchsbezirk sind bei den Kiefernbeständen vergleichsweise geringe Speicherraten auf den verschiedenen Standorten festzustellen $\left(\varnothing 0,5 \mathrm{t} \mathrm{C} \mathrm{ha}^{-1} \mathrm{a}^{-1}\right)$. Im Mittel sind auch hier nur geringe Unterschiede zwischen den Behandlungsvarianten bei den verschiedenen Baumarten festzustellen. Allerdings sind die Kontraste zwischen den untersuchten Baumarten sehr viel größer als in den anderen Wuchsbezirken.

Der Wuchsbezirk Ost-Heide ist durch geringe Niederschläge geprägt (s. Tab. 2.2). 
Eichenbestände sind hier auf schlechter nährstoffversorgten Sanden, auch mit starkem Grundwassereinfluss, oder auf mäßig bis ziemlich gut versorgten Geschiebelehmen zu finden (s. Abb. 4.7(d)). Ihre Kohlenstoffspeicherrate nimmt, bei gleicher Nährstoffversorgung und abnehmender Wasserversorgung, ab. Die Buchenbestände sind ebenfalls auf den besser versorgten Geschiebelehmen und auf den mäßig nährstoffversorgten Sanden zu finden. Hier zeigt sich, dass bei kohlenstofforientierter Behandlung der Bestände sowie abnehmender Wasser- und Nährstoffversorgung auch die Speicherrate der lebenden Baumbiomasse abnimmt. Allerdings ist bei der naturnahen Behandlung der Buchenbestände ein deutlicher Anstieg der Speicherrate, bei gleicher Nährstoff- jedoch abnehmender Wasserversorgung, auf den Sandstandorten festzustellen. Da der Waldwachstumssimulator erst vergleichsweise spät nutzt, ist hier die Biomassenallokation sehr hoch und somit auch die entsprechende Rate. Die Speicherraten der Fichten- und Douglasienbestände sind sehr viel höher als die der anderen Baumarten und erreichen ihr Maximum auf den frischen, ziemlich gut versorgten Geschiebelehmen ( $\left.\varnothing 1,7 \mathrm{tC} \mathrm{ha}^{-1} \mathrm{a}^{-1}\right)$. Die Kohlenstoffspeicherrate der lebenden Baumbiomasse der Kiefer schwankt in diesem Wuchsbezirk zwischen 0,5 und $0,7 \mathrm{tCha}^{-1} \mathrm{a}^{-1}$, mit einem Maximum auf den stark grundwasserbeeinflussten Sanden mit mäßiger Nährstoffversorgung. Insgesamt bleibt für diesen Wuchsbezirk festzuhalten, dass die Behandlungsvarianten im Mittel kaum voneinander abweichen (naturnah $\approx 0,9 \mathrm{t} \mathrm{Cha}^{-1} \mathrm{a}^{-1}$, kohlenstofforientiert $\approx 1,0 \mathrm{t} \mathrm{Cha}^{-1} \mathrm{a}^{-1}$ ), die Unterschiede zwischen den Baumarten sich hier auch sehr viel deutlicher (Douglasie, Fichte $\left(\varnothing 1,4 \mathrm{tCha}^{-1} \mathrm{a}^{-1}\right)>$ Buche $\left(\varnothing 1,0 \mathrm{t} \mathrm{Cha}^{-1} \mathrm{a}^{-1}\right)>$ Eiche $\left(\varnothing 0,7 \mathrm{t} \mathrm{Cha}^{-1} \mathrm{a}^{-1}\right)>$ Kiefer $(\varnothing$ 0,5 t $\left.\left.\mathrm{C} \mathrm{ha}^{-1} \mathrm{a}^{-1}\right)\right)$.

Die Auswirkungen der Behandlung auf die Kohlenstoffspeicherraten der toten Baumbiomasse und des Holzproduktpools sowie dessen Substitutionspotenzial (s. Anh. A.7) soll an zwei Buchenbeständen auf verschiedenen Standorten näher erläutert werden. Dabei sie hier nochmal darauf verwiesen, dass die Speicherraten des Produktspeichers und der Substitutionswirkung ins Verhältnis zur Eiche gesetzt wurden (s. Kap. 3.3.4). In der Abbildung 4.8(a) sind die Bindungsraten der betrachteten Speicher eines Buchenbestandes auf mäßig nährstoffversorgten, mäßig frischen Sanden im Wuchsbezirk Ost-Heide zu sehen. Bei kohlenstofforientierter Behandlung beträgt hier die Zielstärke $50 \mathrm{~cm}$, mithin $10 \mathrm{~cm}$ weniger als bei naturnaher Behandlung (s. Tab. 3.5). Die lebende Baumbiomasse speichert bei kohlenstofforientierter Behandlungen etwa $0,95 \mathrm{t} \mathrm{Cha}^{-1} \mathrm{a}^{-1}$ und bei naturnaher Behandlung etwa 1,05 t $\mathrm{Cha}^{-1} \mathrm{a}^{-1}$. Die Speicherrate der toten Baumbiomasse ist ungleich geringer und beträgt durchschnittlich nur $0,03 \mathrm{t} \mathrm{Cha}^{-1} \mathrm{a}^{-1}$. Bedingt durch den geringeren Zieldurchmesser bei der kohlenstofforientierten als bei der naturnahen Behandlung, werden größere Mengen Rohholz zur Herstellung von Holzprodukten bereitgestellt. Daher ist die Speicherrate der Holzprodukte bei kohlenstofforientierter Behandlung um rund $1 \mathrm{t} \mathrm{Cha-1} \mathrm{a}^{-1}$ höher als bei der naturnahen Variante. Daraus ergibt sich bei kohlenstofforientierter Bestandesbehandlung eine fast fünffach höhere Substitutionswirkung der Holzprodukte als bei der naturnahen Behandlung. Umgekehrt sind die Verhältnisse im nächsten Beispiel, wobei dieser Buchenbestand auf frischen, ziemlich gut versorgten Geschiebelehmen im Wuchsbezirk Ems-Hase-Hunte-Geest stockt (s. Abb. 


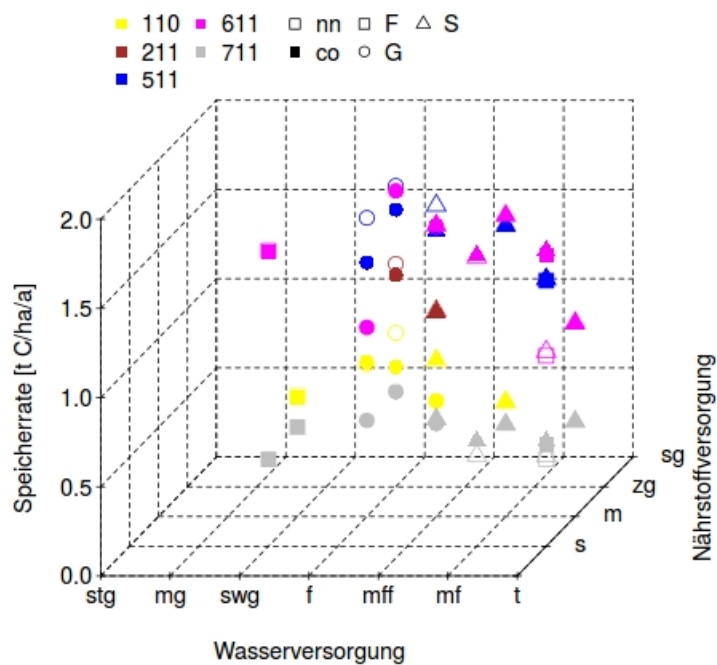

(a)

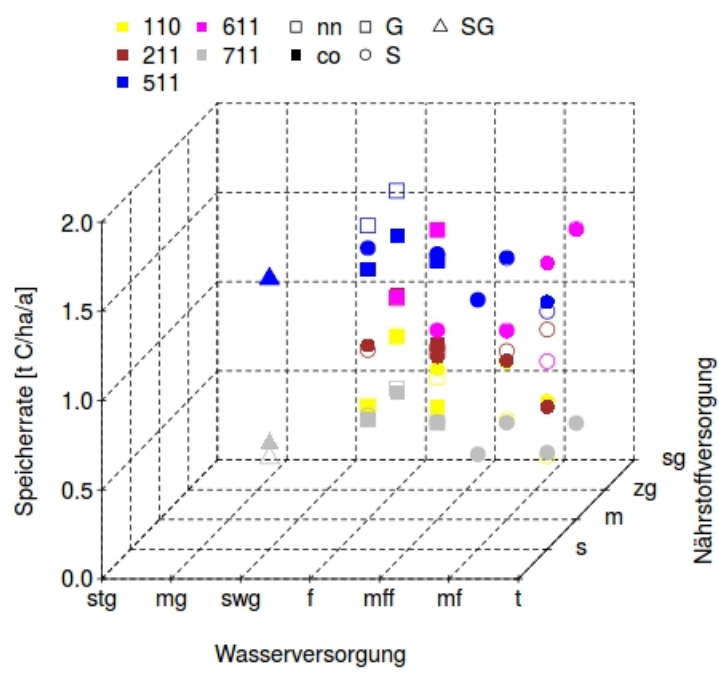

(c)

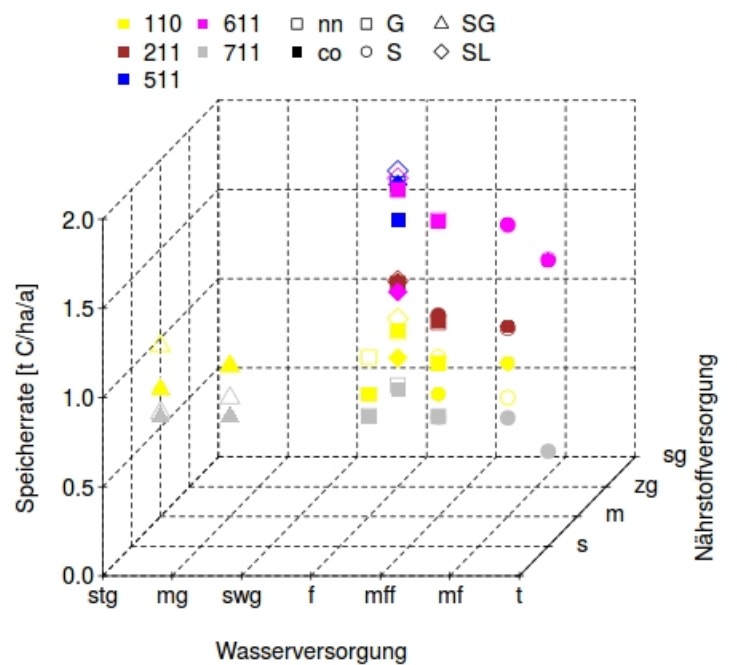

(b)

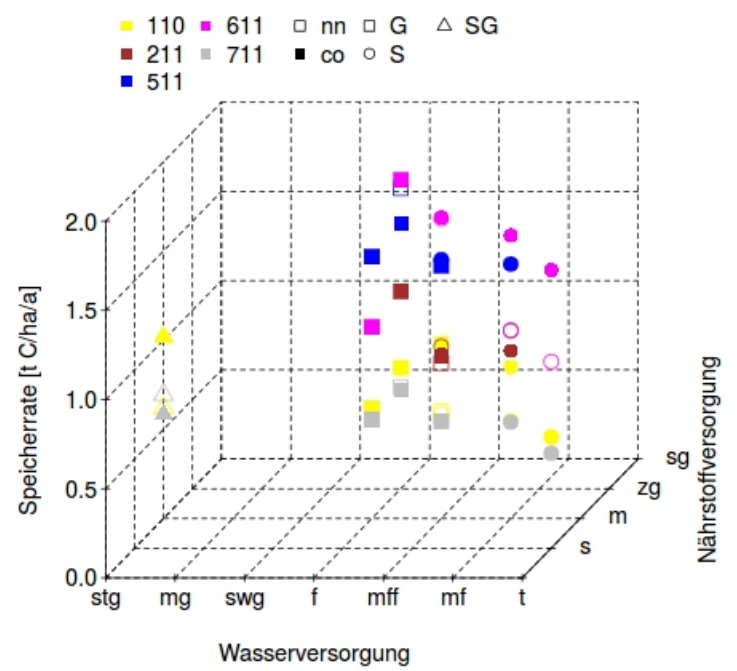

(d)

Abbildung 4.7: Kohlenstoffspeicherrate der lebenden Baumbiomasse $\left[\mathrm{t} \mathrm{C} \mathrm{ha}^{-1} \mathrm{a}^{-1}\right]$ in Abhängigkeit von Wasser- (stg, mg, swg = stark, mäßig, schwach grundwasserbeeinflusst, $\mathrm{f}=$ frisch, $\mathrm{mff}=$ mäßig frisch bis frisch, $\mathrm{mf}=$ mäßig frisch, $\mathrm{t}=$ trocken) und Nährstoffversorgung ( $\mathrm{s}=$ schwach, $\mathrm{m}=$ mäßig, $\mathrm{zg}=$ ziemlich gut, $\mathrm{sg}=\mathrm{sehr}$ gut $)$, getrennt nach Baumart $(110=$ Eiche, $211=$ Buche, $511=$ Fichte, $611=$ Douglasie, $711=$ Kiefer $)$, Behandlung $(\mathrm{co}=$ kohlenstofforientiert, $\mathrm{nn}=$ naturnah) und Standortstypenobergruppe $(\mathrm{F}=$ Flugsande, $\mathrm{G}=$ Geschiebelehm, $\mathrm{S}$ $=$ Sande, $\mathrm{SG}=$ Sande im Grundwasserbereich, $\mathrm{SL}=$ Sandlösse) in den Wuchsbezirken Ems-Hase-Hunte-Geest (a), Geest Mitte (b), Hohe Heide (c) sowie Ost-Heide (d). 


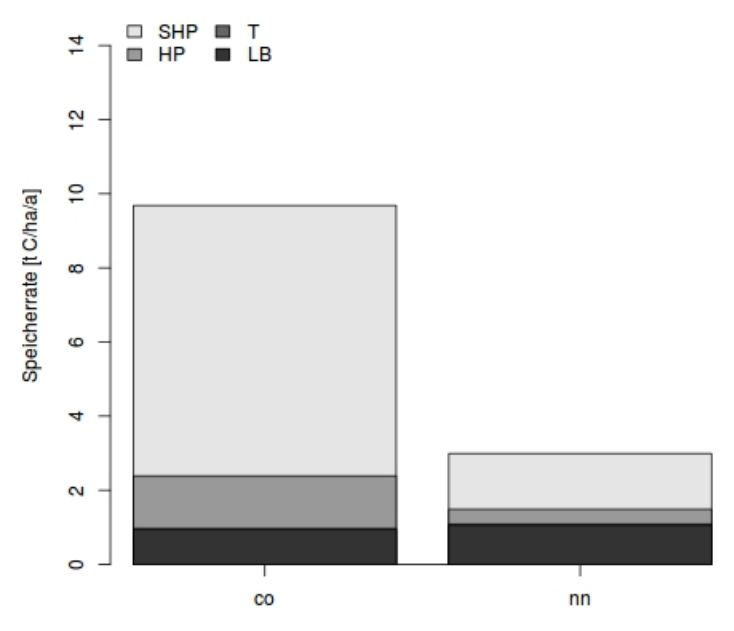

(a)

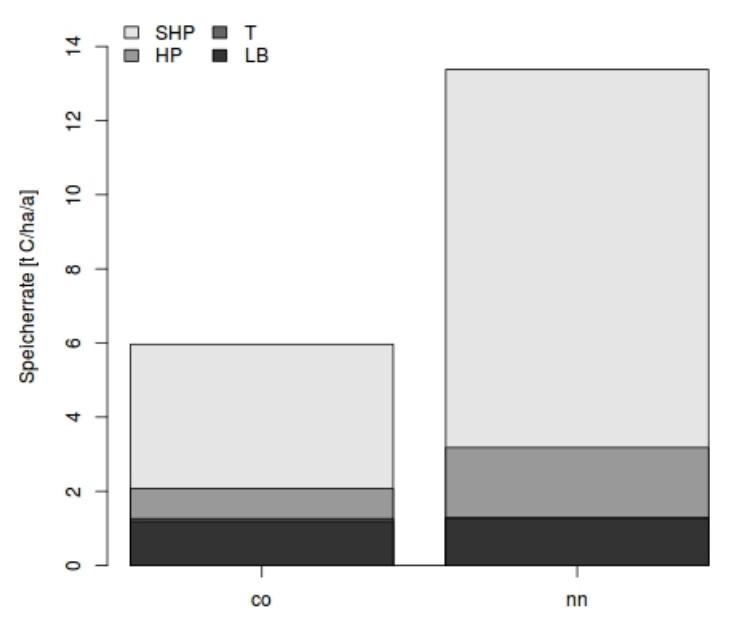

(b)

Abbildung 4.8: Kohlenstoffspeicherraten $\left[\mathrm{t} \mathrm{Cha}^{-1} \mathrm{a}^{-1}\right]$ der analysierten Speicher $(\mathrm{SHP}=\mathrm{Sub}-$ stitution der Holzprodukte, HP $=$ Holzprodukte, $\mathrm{T}=$ tote Baumbiomasse, $\mathrm{LB}=$ lebende Baumbiomasse) von zwei Buchenbeständen auf ausgewählten Standorten (s. Text) bei unterschiedlicher waldbaulicher Behandlung (co = kohlenstofforientiert, $\mathrm{nn}=$ naturnah).

4.8(b)). Auch hier sind die Kohlenstoffbindungsraten der lebenden Baumbiomasse bei beiden Varianten ähnlich hoch (ca. 1,2 $\mathrm{t} \mathrm{C} \mathrm{ha}^{-1} \mathrm{a}^{-1}$ ), allerdings beträgt der Zieldurchmesser bei der kohlenstofforientierten Behandlung hier $70 \mathrm{~cm}$, demnach $10 \mathrm{~cm}$ mehr als beim naturnahen Szenario (s. Tab. 3.5). Die Speicherrate der toten Baumbiomasse unterscheidet sich zwischen den Varianten ebenfalls nur wenig. Durch den geringeren Zieldurchmesser der naturnahen Behandlung beginnt die Endnutzung früher und größere Mengen Rohholz werden dem Holzproduktspeicher zur Verfügung gestellt als bei kohlenstofforientierter Behandlung. Daraus resultieren höhere Speicherraten des Holzproduktspeichers (kohlenstofforientiert $\approx 0,8 \mathrm{t} \mathrm{Cha}^{-1} \mathrm{a}^{-1}$, naturnah $\approx 1,8 \mathrm{t} \mathrm{Cha}^{-1} \mathrm{a}^{-1}$ ) und eine größere Substitutionswirkung (kohlenstofforientiert $\approx 3,9 \mathrm{t} \mathrm{C} \mathrm{ha}^{-1} \mathrm{a}^{-1}$, naturnah $\approx 10,2 \mathrm{t} \mathrm{Cha}^{-1} \mathrm{a}^{-1}$ ).

Werden nun verschiedene Quantile ${ }^{1}$ der Kohlenstoffsequestrierungsraten der lebenden und toten Baumbiomasse der Weiserbestände auf den exemplarisch ausgewählten Standortstypenobergruppen getrennt nach Szenarien und Baumarten betrachtet (s. Tab. 4.2), ergibt sich folgendes Bild. Wie schon oben festgestellt, sind die Unterschiede zwischen den beiden waldbaulichen Behandlungen und deren Auswirkungen auf die Spei-

\footnotetext{
${ }^{1}$ Ein Quantil ist ein Lagemaß der Statistik. Beispielsweise gilt für das 10\%-Quantil $\left(\mathrm{Q}_{10} \%\right)$, dass $10 \%$ der Werte kleiner sind als der angegebene Wert.
} 
cherrate der lebenden Baumbiomasse gering. So schwanken $80 \%$ der Kohlenstoffspeicherraten dieses Speichers bei Eiche zwischen rund 0,6 und etwa 0,9 t $\mathrm{C} \mathrm{ha}^{-1} \mathrm{a}^{-1}$ sowie bei Buche zwischen 0,9 und etwa 1,2 t C ha ${ }^{-1} \mathrm{a}^{-1}$. Das $10 \%$ - und $90 \%$-Quantil der Speicherraten der lebenden Baumbiomasse der Fichten- und Douglasienbestände ist höher als bei den betrachteten Laubbaumarten. Für Fichte betragen die Speicherraten ca. 1,4 bzw. 1,65 t Cha ha $\mathrm{a}^{-1}$ sowie für Douglasie rund 1,1 bzw. 1,7 t Cha $\mathrm{Ca}^{-1}$. Am geringsten sind die betrachteten Quantile für die Kiefer, wobei sie sich auf etwa 0,5 bzw. 0,6 t C ha ${ }^{-1} \mathrm{a}^{-1}$ belaufen.

Die Speicherrate der toten Baumbiomasse ist ungleich geringer als die der lebenden Baumbiomasse (s. Tab. 4.2). So liegen $80 \%$ der ermittelten Raten für diesen Pool bei den meisten analysierten Baumarten zwischen 0,03 und 0,06 t $\mathrm{C} \mathrm{ha}^{-1} \mathrm{a}^{-1}$. Die betrachteten Quantile der Douglasie sind allerdings höher als die der anderen Arten und betragen 0,14 und $0,17 \mathrm{t} \mathrm{Cha}^{-1} \mathrm{a}^{-1}$. Dies kann durch die relativ geringe Zersetzungsrate (s. Tab. 3.11) erklärt werden.

Da die Unterschiede zwischen den Baumarten sehr viel größer sind als zwischen den beiden waldbaulichen Szenarien, ergibt sich für die Kohlenstoffspeicherung in der lebenden und toten Baumbiomasse folgende Reihung der baumartenspezifischen Gesamtspeicherrate: Douglasie ${ }^{2}$, Fichte $>$ Buche $>$ Eiche $>$ Kiefer.

Auch bei der Kohlenstoffspeicherung in Holzprodukten und deren Substitutionswirkung ist kein eindeutiger Trend hinsichtlich der Behandlung festzustellen. Zu beachten ist hier, dass sich die baumartenspezifischen Speicherraten des Holzproduktpools und dessen Substitutionsleistung auf den Produktionszeitraum der Eiche beziehen (200 Jahre, s. Kap. 3.3.4). Somit vollzieht sich bei den anderen Baumarten mehr als nur ein Produktionszyklus. Bei Fichte führt die kohlenstofforientierte Variante zu einer deutlich geringeren Gesamtspeicherrate als die naturnahe Behandlung (s. Tab. 4.3). Da der Zieldurchmesser der naturnahen Behandlung in allen Fichtenbeständen gleich ist, kommt das bessere Wachstum der Bestände mit guter Bonität nicht zum Tragen. Bei einem Großteil dieser Fichtenbestände setzt allerdings bei kohlenstofforientierter Behandlung die Endnutzung im Waldwachstumssimulator vergleichsweise spät ein. Somit wird weniger Rohholz zur Holzproduktherstellung bereitgestellt und dementsprechend geringer sind die darauf basierenden Speicherraten. Die Differenzen zwischen den untersuchten Baumarten sind sehr deutlich. So liegt die jährliche Speicherrate (10\%- und 90\%-Quantil) des Produktpools befüllt durch die Nutzungen in den Eichenbeständen im Bereich von 0,63 bis $0,83 \mathrm{t} \mathrm{Cha} \mathrm{C}^{-1} \mathrm{a}^{-1}$. Die entsprechenden Raten in den Buchenbeständen betragen 0,40 bis $1,76 \mathrm{t} \mathrm{Cha}^{-1} \mathrm{a}^{-1}$. Die Verwendung von Fichten- und Douglasienholz in Holzprodukten führt zu einer wesentlich höheren Speicherrate in diesem Pool mit jährlich etwa 1,73 bis 3,07 t C ha ${ }^{-1} \mathrm{a}^{-1}$ bzw. 3,03 bis 3,41 t $\mathrm{C} \mathrm{ha}^{-1} \mathrm{a}^{-1}$. Der Beitrag der Kiefernbestände zum Produktpool ist mit 0,83 bis $1,17 \mathrm{t} \mathrm{Cha}^{-1} \mathrm{a}^{-1}$ mit dem der Eichen- und Buchenbestände vergleichbar. Die hergestellten Holzprodukte substituieren fossile Energieträger bzw.

\footnotetext{
${ }^{2}$ Das allgemein sehr hohe Leistungspotenzial der Douglasie wird aufgrund der Vorauswahl von Standorten nicht zutreffend dargestellt (s. Kap. 2.2.2).
} 
Tabelle 4.2: 10\%- $\left(\mathrm{Q}_{10 \%}\right)$ und 90\%-Quantil $\left(\mathrm{Q}_{90} \%\right)$ der Kohlenstoffbindungsrate $\left[\mathrm{t} \mathrm{Cha}^{-1} \mathrm{a}^{-1}\right]$ der lebenden und toten Baumbiomasse ( $\mathrm{LB}=$ lebende Baumbiomasse, $\mathrm{TB}=$ tote Baumbiomasse), getrennt nach Art und waldbaulicher Behandlung (Var, co = kohlenstofforientiert, $\mathrm{nn}=$ naturnah) für die Weiserbestände. Die Speicherrate der Douglasie wird aufgrund der Vorauswahl der Standorte unterschätzt (s. Kap. 2.2.2).

\begin{tabular}{cccccccc}
\hline \multirow{2}{*}{ Art } & \multirow{2}{*}{ Var } & \multicolumn{2}{c}{ LB } & \multicolumn{2}{c}{ TB } & \multicolumn{2}{c}{ Gesamt } \\
& & $\mathrm{Q}_{10 \%}$ & $\mathrm{Q}_{90 \%}$ & $\mathrm{Q}_{10 \%}$ & $\mathrm{Q}_{90 \%}$ & $\mathrm{Q}_{10 \%}$ & $\mathrm{Q}_{90 \%}$ \\
\hline \multirow{2}{*}{ Eiche } & $\mathrm{co}$ & 0,64 & 0,89 & 0,04 & 0,05 & 0,68 & 0,94 \\
& $\mathrm{nn}$ & 0,58 & 0,92 & 0,04 & 0,05 & 0,62 & 0,97 \\
\multirow{5}{*}{ Buche } & $\mathrm{co}$ & 0,93 & 1,16 & 0,03 & 0,06 & 0,96 & 1,22 \\
& $\mathrm{nn}$ & 0,95 & 1,20 & 0,03 & 0,06 & 0,98 & 1,26 \\
\multirow{2}{*}{ Fichte } & $\mathrm{co}$ & 1,40 & 1,63 & 0,04 & 0,09 & 1,44 & 1,72 \\
& $\mathrm{nn}$ & 1,42 & 1,70 & 0,03 & 0,05 & 1,45 & 1,75 \\
Douglasie & $\mathrm{co}$ & 1,07 & 1,68 & 0,14 & 0,17 & 1,21 & 1,85 \\
& $\mathrm{nn}$ & 1,06 & 1,68 & 0,14 & 0,17 & 1,20 & 1,85 \\
\multirow{2}{*}{ Kiefer } & $\mathrm{co}$ & 0,51 & 0,59 & 0,03 & 0,04 & 0,54 & 0,63 \\
& $\mathrm{nn}$ & 0,51 & 0,58 & 0,03 & 0,04 & 0,54 & 0,62 \\
\hline
\end{tabular}

Tabelle 4.3: $10 \%-\left(\mathrm{Q}_{10 \%}\right)$ und 90\%-Quantil $\left(\mathrm{Q}_{90 \%}\right)$ der Kohlenstoffbindungsrate $\left[\mathrm{t} \mathrm{Cha}{ }^{-1} \mathrm{a}^{-1}\right.$ ] des Holzproduktspeichers und dessen Substitutionswirkung (HP = Holzprodukte, E. Subs., M. Subs. = energetische und materielle Substitution durch Holzprodukte), getrennt nach Art und waldbaulicher Behandlung (Var, co = kohlenstofforientiert, $\mathrm{nn}=$ naturnah) für die Weiserbestände. Die Speicherrate der Douglasie wird aufgrund der Vorauswahl der Standorte unterschätzt (s. Kap. 2.2.2).

\begin{tabular}{|c|c|c|c|c|c|c|c|c|c|}
\hline \multirow{2}{*}{ Art } & \multirow{2}{*}{ Var } & \multicolumn{2}{|c|}{$\mathrm{HP}$} & \multicolumn{2}{|c|}{ E. Subs. } & \multicolumn{2}{|c|}{ M. Subs. } & \multicolumn{2}{|c|}{ Gesamt } \\
\hline & & $\mathrm{Q}_{10 \%}$ & $\mathrm{Q}_{90 \%}$ & $\mathrm{Q}_{10 \%}$ & $\mathrm{Q}_{90 \%}$ & $\mathrm{Q}_{10 \%}$ & $\mathrm{Q}_{90 \%}$ & $\mathrm{Q}_{10 \%}$ & $\mathrm{Q}_{90 \%}$ \\
\hline \multirow{2}{*}{ Eiche } & co & 0,63 & 0,83 & 0,65 & 1,21 & 3,31 & 7,89 & 4,59 & 9,93 \\
\hline & $\mathrm{nn}$ & 0,70 & 0,83 & 0,75 & 1,09 & 4,30 & 6,87 & 5,75 & 8,79 \\
\hline \multirow{2}{*}{ Buche } & co & 0,60 & 1,54 & 0,59 & 1,38 & 2,95 & 7,00 & 4,14 & 9,92 \\
\hline & nn & 0,40 & 1,76 & 0,27 & 1,38 & 1,26 & 6,94 & 1,93 & 10,08 \\
\hline \multirow{2}{*}{ Fichte } & $\mathrm{co}$ & 1,73 & 2,53 & 2,18 & 4,28 & 11,56 & 24,24 & 15,47 & 31,05 \\
\hline & $\mathrm{nn}$ & 2,06 & 3,07 & 3,15 & 5,27 & 17,04 & 29,48 & 22,25 & 37,82 \\
\hline \multirow{2}{*}{ Douglasie } & $\mathrm{co}$ & 3,04 & 3,40 & 3,08 & 3,53 & 15,83 & 19,23 & 21,95 & 26,16 \\
\hline & nn & 3,03 & 3,41 & 3,04 & 3,39 & 15,36 & 17,69 & 21,45 & 24,49 \\
\hline \multirow{2}{*}{ Kiefer } & co & 0,83 & 1,13 & 0,84 & 1,61 & 4,25 & 9,45 & 5,92 & 12,19 \\
\hline & $\mathrm{nn}$ & 0,90 & 1,17 & 1,01 & 1,72 & 5,38 & 9,92 & 7,29 & 12,81 \\
\hline
\end{tabular}


Nicht-Holzprodukte und vermeiden somit deren Treibhausgasemissionen. Das energetische Substitutionspotenzial ist bei der Buche etwas höher als bei Eiche (Q90\%: 1,38 zu etwa 1,15 $\mathrm{t} \mathrm{Cha}^{-1} \mathrm{a}^{-1}$ ) und bei den Nadelhölzern allgemein höher als bei den Laubhölzern. Denn durch einen hohen Nadelholzeinschlag und -verbrauch steigt der Nadelholzanteil an der Produktklasse Energieholz, da innerhalb der Kaskadennutzung bei vielen Herstellungsstufen Holzreste zur energetischen Verwendung anfallen (s. Abb. 3.5). Hinzu kommt, dass die Produktionszeit der Nadelbaumarten geringer ist als von Eiche und sie somit im gleichen Zeitraum einen vielfachen Beitrag zu diesem Speicher leisten können. Bei der Materialsubstitution verhalten sich die baumartenspezifischen Kohlenstoffspeicherraten ähnlich zueinander wie beim energetischen Substitutionspotenzial, wobei hier die Relation von Buche zu Eiche umgekehrt ist. Auch wird hier die überwiegende Verwendung des Nadelholzes als Bauholz mit einem hohen Substitutionspotenzial deutlich. Da nur vergleichsweise wenige Douglasienbestände mit überwiegend mittlerer Leistungsfähigkeit in die Analyse einbezogen wurden, wird das allgemein höhere Substitutionspotenzial dieser Baumart im Vergleich zu den anderen Baumarten eher unterschätzt. Nichtsdestotrotz ergibt sich bei einer Gesamtbetrachtung der Speicherraten des Holzproduktspeichers und dem Substitutionspotenzial folgende Baumartenreihung: Fichte, Douglasie $^{2}>$ Kiefer $>$ Eiche, Buche.

Bei den angeführten Reihungen ist zu beachten, dass die genannten Raten auf einer unterschiedlichen Anzahl von Vergleichsbeständen basieren und die analysierten Standorte ungleich mit Baumarten besetzt sind. Somit gibt auch die folgende Reihung nur näherungsweise die Gesamtspeicherleistung der Baumarten wieder. Für Eiche und Buche beträgt die durchschnittliche Gesamtspeicherrate über alle Kohlenstoffpools basierend auf den oben genannten Werten (s. Tab. 4.2 u. 4.3) rund $8 \mathrm{t} \mathrm{Cha}^{-1} \mathrm{a}^{-1}$, für Kiefer etwa $10 \mathrm{tCha}^{-1} \mathrm{a}^{-1}$ sowie für Fichte $28 \mathrm{t} \mathrm{Cha}^{-1} \mathrm{a}^{-1}$ und für Douglasie $25 \mathrm{t} \mathrm{Cha}^{-1} \mathrm{a}^{-1}$. Die Materialsubstitution hat an diesen Gesamtspeicherraten Anteile von 60 bis $73 \%$. Dadurch erklären sich auch die vergleichsweise hohen Gesamtspeicherraten von Douglasie und Fichte, denn sie durchlaufen im Gegensatz zu den anderen Baumarten den Bezugszeitraum mehr als einmal und werden überwiegend stofflich verwendet. Darüber hinaus erweitert die Kaskadennutzung das Kohlenstoffspeicherpotenzial des Holzproduktpools, indem gewisse Anteile der verschiedenen Produktklassen zur Herstellung weiterer Produkte verwendet werden (Erhöhung des Materialsubstitutionspotenzials) und auch die energetische Holznutzung, als Koppelprodukt der Holzproduktherstellung, hat eine gewisse Bedeutung (Erhöhung des Energiesubstitutionspotenzials). 


\section{Diskussion}

\subsection{Material und Methoden}

Die Baumartengruppen Eiche, Buche, Fichte, Kiefer und Douglasie bedecken zusammen ca. $75 \%$ des Holzbodens in Niedersachsen, bezogen auf den Landeswald sind es rund $84 \%$ (ML, 2014). Allein aufgrund ihrer Flächenanteile sind die gewählten Baumarten von großer ökologischer, ökonomischer und sozioökonomischer Bedeutung. Die verschiedenen analysierten Wuchsbezirke bilden einerseits einen Temperatur- und Niederschlagsgradienten ab, andererseits sind die geographischen und geologischen Merkmale zwischen den Wuchsbezirken verschieden (s. Tab. 2.3 u. 2.2). Sie sind geprägt durch unterschiedliche Standortstypenobergruppen, deren wichtigste Vertreter mit Hilfe der Auswertungen von Otto $(1989,1991)$ identifiziert wurden. Diese Analysen bilden eine detaillierte und zuverlässige Datengrundlage für große Teile des niedersächsischen Landeswaldes.

Die genutzten Standortsdaten und die Informationen über die aufstockenden Bestände wurden von den Niedersächsischen Landesforsten bereitgestellt. Daher beziehen sich die Ergebnisse in erster Linie auf den Landeswald. Sie lassen sich jedoch bis zu einem gewissen Maße auch auf Flächen anderer Waldbesitzarten mit vergleichbaren Standorten übertragen, soweit deren waldbauliche Ausgangssituationen und Zielvorstellungen nicht zu stark von denjenigen im Landeswald abweichen.

Der Waldwachstumssimulator WaldPlaner (Hansen, 2012; Hansen und Nagel, 2014) ist für Nordwestdeutschland parametrisiert und ein geeignetes Instrument, um die Auswirkungen waldbaulicher Behandlungen zu beurteilen. Dies haben unter anderem die Arbeiten von Rüther et al. (2007, 2008a,b), Hansen et al. (2008), Hansen (2012) oder der NW-FVA (2013b) gezeigt. Die Simulationsergebnisse bilden eine gute Grundlage zur Identifikation der strategischen Potenziale, mit denen sich die Klimaschutzleistungen des Landeswaldes durch Kohlenstoffspeicherung in der ober- und unterirdisch lebenden Baumbiomasse, in der toten Baumbiomasse und in den aus dem bereitgestellten Rohholz produzierbaren Holzprodukten sichern bzw. erhöhen lassen. Die Eingangswerte und Steuerungswerte der Simulationen bilden ein weites Spektrum praxisnaher waldbaulicher Strategien ab. Die waldbaulichen Regeln für die einzelnen Baumarten basieren auf den Pflege- und Entwicklungszielen der Merkblätter der Niedersächsischen Landesforsten (NLF, 2014b,c,d,e, 2015) sowie auf Erfahrungswerten aus dem Versuchswesen.

Die maximale Bestandesgrundfläche ist eine geeignete Bezugsgröße zur zahlenmäßigen Steuerung der Durchforstung. In Form des natürlichen Bestockungsgrades ist eine eindeutige Kennzeichnung der Durchforstungsstärke möglich (Assmann, 1956). Im Ver- 
gleich zu dem herkömmlichen Bestockungsgrad auf Basis der Ertragstafeln ist der natürliche Bestockungsgrad ein standörtlich-ökologisches Maß, welches eindeutig biometrisch definiert ist. Damit kann die Dichte der Bestockung auf standörtlicher Grundlage beurteilt werden und es sind verhältnismäßig sichere Voraussagen über die zu erwartenden Leistungen der Bestände möglich (Assmann, 1956; Franz, 1965). Um den natürlichen Bestockungsgrad zu bestimmen, ist es notwendig die standörtlich maximal mögliche Grundfläche der lebenden Bäume zu ermitteln. Deren Bestimmung erfolgte hier mit Hilfe der Methode der Quantilsregression. Die Datengrundlage bildeten Versuchsflächen der Nordwestdeutschen Forstlichen Versuchsanstalt. Zur Verbreiterung der Datenbasis wurden neben Nullflächen (ohne Durchforstung) und schwach durchforsteten Flächen (A-Grad) auch einige mäßig durchforstete Flächen (B-Grad) in die Auswertungen einbezogen (s. Tab. A.16). Im Vergleich zu früheren Untersuchungen von Spellmann et al. (1999) und Döbbeler (2004), die einem Ansatz von Sterba (1975, 1981, 1987) folgten, konnten im Rahmen dieser Arbeit mehr Informationen aus jungen Beständen genutzt und mit Hilfe der Methode der Quantilsregression bessere Anpassungen erzielt werden. Die maximalen Bestandesgrundflächen bilden in Verbindung mit den definierten Pflegephasen und den angestrebten natürlichen Bestockungsgraden (s. Tab. 3.8) ein verbessertes System zur Steuerung der Durchforstung, vor allem in der waldbaulich sehr wichtigen Jungdurchforstungsphase. Durch die Variation der Pflegephasen und ihrer angestrebten natürlichen Bestockungsgrade lassen sich auch andere Pflegekonzepte umsetzen, als die in dieser Untersuchung implementierte gestaffelte Durchforstung.

Zur Generierung der Ausgangsbestände wurden die umfangreichen Daten der Betriebsinventuren und Forsteinrichtungen der Niedersächsischen Landesforsten genutzt. Mit den entwickelten Methoden zur Bestandesgenerierung lassen sich mit wenigen Eingangsgrößen (Baumart, Alter, absolute Mittelhöhe im Alter 100) Einzelbaumdaten generieren. Problematisch ist jedoch die Generierung sehr junger Bestände, da keine Daten unter $7 \mathrm{~cm}$ Durchmesser, d.h. unterhalb der Derbholzgrenze, aus den Aufnahmen vorliegen. Dies kann dazu führen, dass die Durchmesserverteilungen und die dazugehörige Bestandesgrundflächen der sehr jungen Bestände unter Umständen unvollständig sind. Diesem Mangel wurde durch die Anwendung einer linksseitig trunkierten Form, der den Durchmessern zugrundeliegenden Box-Cox-Cole-Green-Verteilung, begegnet. Die hier unterstellten Produktionszeiträume für die jeweiligen Baumarten (s. Tab. 3.3) sind gleichzeitig als Gültigkeitsbereich für die verwendeten Methoden zur Bestandesgenerierung anzusehen. Die erstellten Weiserbestände weichen hinsichtlich ihrer Stammzahlen und der Durchmesser des Grundflächenmittelstammes von den Angaben der verschiedenen Ertragstafeln ab (s. Kap. 2.3). Diese Unterschiede sind durch veränderte Wuchsbedingungen sowie andere Durchforstungsarten und -stärken zu erklären.

Die Wachstumsdynamik und die Berechnung weiterer Einzelbaumdaten wurde mit den im WaldPlaner (Hansen, 2012; Hansen und Nagel, 2014) implementierten Methoden umgesetzt. Sie sind für längere Simulationen geeignet, allerdings nehmen mit der Simulationsdauer die Unsicherheiten zu. Damit diese relativ gering bleiben, wurden Weiserbestände im Abstand von 30 Jahren generiert und für die Simulationen genutzt (s. 
Kap. 3.1). Die so entstandenen Ergebnisse sind vergleichbar mit einer Wuchsreihe und wesentlich realistischer als längere Simulationen mit Zeiträumen von über 30 Jahren.

Die Größen der analysierten Kohlenstoffspeicher sind stark von den Produktionszeiten der Baumarten überprägt. Daher war es für einen Vergleich notwendig, eine Stromgröße zu berechnen. Dies wurde mit der Bildung von Speicherraten umgesetzt.

Die eingangs gestellten Fragen zur Überprüfung der Arbeitshypothesen (s. Kap. 1.4) konnten mit dem gewählten Forschungsansatz dieser Untersuchung beantwortet werden. Die mittlere Leistungsfähigkeit der Hauptwirtschaftsbaumarten auf verschiedenen Standorten in ausgesuchten Wuchsbezirken wurde ermittelt, der Holzverwendungsschlüssel verbessert und eine Kaskadennutzung abgebildet. Darüber hinaus konnten auch neue Methoden zur Schätzung der maximalen Bestandesgrundfläche sowie zur Generierung von Weiserbeständen unterschiedlicher Bonität erarbeitet und angewendet werden. Die Ergebnisse zeigen auch, wie verschieden die Kohlenstoffsenkenleistungen der Baumarten bei gleicher Wasser- und Nährstoffversorgung sind und das sie bei Beachtung der spezifischen Wuchsleistung erhöht werden können.

\subsection{Entwicklung der Kohlenstoffspeicher}

Die Ermittlung der Kohlenstoffvorräte der lebenden ober- und unterirdischen Baumbiomasse basiert auf zuverlässigen Verfahren. Zur Berechnung der lebenden oberirdischen Biomasse wurden die für Nordwestdeutschland von Rumpf et al. (2012) entwickelten Funktionen benutzt. Zur Berechnung der unterirdischen Baumbiomasse fand die Funktion von Dieter und Elsasser (2002) Anwendung. Aus der Biomasse konnte mit Hilfe des Kohlenstoffgehalts des Holzes der Kohlenstoffvorrat hergeleitet werden. Da mit dem Beginn der Zielstärkennutzung auch gleichzeitig die Bestandesverjüngung einsetzt und der Kohlenstoffvorrat des Bestandes meist abnimmt, wurde ab diesem Zeitpunkt eine baumartenspezifische Bindungsrate junger Weiserbestände genutzt, um den Kohlenstoffvorrat in diesem Überlappungszeitraum abzubilden.

Die Kohlenstoffvorräte der lebenden Baumbiomasse verschiedener Untersuchungen (s. Kap. 1.2.2) unterliegen vielfältigen Einflussgrößen. Wie beispielsweise unterschiedlichen Berechnungsweisen, Ertragsniveaus, Altersklassenverteilungen oder Formen der Waldbewirtschaftung und sollten nur als Orientierung dienen. Die unter anderem von Wirth et al. (2004a), Pistorius (2007b) und Wördehoff et al. (2011) festgestellte Reihung der Hauptwirtschaftsbaumarten nach ihren mittleren Kohlenstoffvorräten (Buche $>$ Eiche $>$ Fichte $>$ Kiefer) kann ebenfalls nur eine Orientierungshilfe sein. Normalerweise werden in Deutschland Bestände in Abhängigkeit von der Baumart mit einer Umtriebszeit zwischen 80 und 200 Jahren bewirtschaftet. Ist die Endnutzungsphase erreicht und die Verjüngung etabliert, wird der noch aufstockende Altbestand allmählich geräumt und somit ist die Häufigkeit alter Bestände mit einem sehr hohen Kohlenstoffvorrat gering (Klein und Schulz, 2012). Hinzu kommen die im Vergleich zu den Nadelholzbeständen verhältnismäßig langen Produktionszeiten der Laubholzbestände. Der mittlere Kohlen- 
stoffvorrat der jüngeren Nadelholzbestände ist anfangs teilweise doppelt so hoch (Fichte etwa $76 \mathrm{t} \mathrm{Cha}^{-1}$ im Alter 30 Jahre, Douglasie rund $61 \mathrm{t} \mathrm{Cha}^{-1}$ im Alter 25 Jahre, Kiefer ca. $42 \mathrm{t} \mathrm{Cha}^{-1}$ im Alter 30 Jahre) als derjenige, der etwas älteren Laubholzbestände (Eiche rund $35 \mathrm{t} \mathrm{C} \mathrm{ha}^{-1}$ im Alter 35 Jahre, Buche ca. $45 \mathrm{t} \mathrm{C} \mathrm{ha}^{-1}$ im Alter 40 Jahre). Daraus lässt sich ein großer Beitrag junger Nadelholzbestände zur Kohlenstoffspeicherung in lebender Baumbiomasse ableiten.

Die derzeitige Altersklassenverteilung der untersuchten Baumarten ist in Niedersachsen bei den Laubbaumarten relativ ausgeglichen. Dabei führt die Stark- und Wertholzproduktion bei Eiche zu großen Flächenanteilen in den hohen Altersklassen $(44 \%>100$ Jahre, $21 \%>140$ Jahre) und bei Buche ist der hohe Anteil der verjüngten Flächen unter Schirm zu beachten. Die Nadelbaumarten sind ungleichmäßig über dem Alter verteilt. Die Kiefer ist zu $51 \%$ jünger als 61 Jahre, bei der Fichte sind es $61 \%$. Die Douglasien sind zu ca. $72 \%$ jünger als 41 Jahre (vTI, 2015). Die großen Flächenanteile der jungen Nadelholzbestände werden sich zukünftig in höhere Altersklassen verschieben und ihr Kohlenstoffvorrat wird sich dabei erhöhen. Allerdings wird er bei weiter fortschreitendem Alter und dem Beginn der Endnutzung wiederum sinken. Auch die hohen Flächenanteile der momentan alten Laubbaumbestände werden nur noch begrenzt in höhere Altersklassen wechseln und somit stärker dem Produktpool zugeführt. Damit ergibt sich die Möglichkeit einer großen Substitutionswirkung. Vorausgesetzt es treten keine großflächigen Störungen wie Sturmwürfe, Waldbrände o. ä. auf, wodurch Kohlenstoff freigesetzt wird.

Bisher sind Risiken nicht berücksichtigt, obwohl sie ein grundlegender Bestandteil der Bestandesentwicklung und daher auch eine wichtige Komponente bei der Beurteilung des waldbaulichen Handelns sind (Schall und Ammer, 2013). Beispielsweise kann die Schädigung von Eichen durch die Eichenfraßgesellschaft, den Hallimasch oder den Eichenprozessionsspinner in Verbindung mit Witterungsextremen bis zum Absterben der betroffenen Bäume führen. Bei der Buche kann die Buchenkomplexkrankheit Schäden sowie eine erhöhte Mortalität im Bestand und damit finanzielle Verluste der Forstbetriebe verursachen. Die Risiken der Fichte sind hauptsächlich durch Windwurf und Insektenbefall bedingt. In Douglasienbeständen der Inlandsform kann die rostige Douglasienschütte (Rhabdocline pseudotsugae) bei starker Infektion und gravierendem Krankheitsverlauf zum Verlust ganzer Nadeljahrgänge führen. Von den Kieferngroßschädlingen (Kiefernspanner, Forleule, Kiefernspinner, Kiefern-Buschhornblattwespe) und der Nonne gehen bei Massenvermehrungen erhebliche Risiken für Kiefernbestände aus. Aber auch das Wild hat oftmals einen negativen Einfluss auf die lebende Baumbiomasse des Nachwuchses und somit den Kohlenstoffvorrat, wie Ammer et al. (2010) darstellen. Diese nur wenigen Beispiele zeigen die vielfältigen biotischen und abiotischen Risiken, denen ein Wald ausgesetzt sein kann. Hinzu kommen die Unsicherheiten aufgrund zukünftiger klimatischer Veränderungen, sich ändernder gesellschaftlicher Ansprüche an den Wald oder schwankenden Marktbedingungen. Diese sollten allerdings möglichst mit beachtet werden, wie unter anderem Höllerl und Bork (2013), Klein und Schulz (2012) oder Seidl et al. (2008) zeigen. Denn starke Störungen verschieben zunächst große Mengen 
Kohlenstoff von der lebenden zur toten Baumbiomasse. Darüber hinaus entscheidet die Verwendung des Schadholzes, welche Produktklassen aufgefüllt werden und wie sich die eingesparte Menge an Kohlenstoffemissionen entwickelt (Mund et al., 2015).

Der Vergleich der Kohlenstoffbindungsraten der lebenden Baumbiomasse auf den Standorten der ausgesuchten Wuchsbezirke (s. Kap. 4.4) zeigt, dass sie direkt von der Baumart sowie der Nährstoff- und Wasserversorgung abhängen. Auch Shanin et al. (2014) kommen zu dem Ergebnis, dass die Kohlenstoffvorräte in der lebenden Baumbiomasse von schlecht zu gut versorgten Standorten ansteigen. Für einen weiteren Vergleich der Bindungsraten der lebenden Baumbiomasse wurden diese, unabhängig vom Wuchsbezirk, der Standortstypenobergruppe und Variante, über die gesamte individuelle Produktionszeit gemittelt. Anschließend konnten die vorkommenden Baumarten ins Verhältnis zur Fichte im Bergland bzw. zur Kiefer im Tiefland auf den verschiedenen Standorten gesetzt werden.

Im Bergland ist bei dieser Betrachtungsweise zu erkennen, dass die Fichte im Mittel rund 30 bis $50 \%$ mehr Kohlenstoff in der lebenden Baumbiomasse speichert als Eiche und Buche (s. Abb. 5.1). Steigt die Nährstoffversorgung an, verringern sich die Unterschiede zwischen Buche, Eiche und der Referenzbaumart Fichte nur wenig. Wird allerdings die Wasserversorgung auf den sommertrockenen und trockenen, sehr gut nährstoffversorgten Standorten zum limitierenden Faktor für das Wachstum, steigt die Speicherleistung der lebenden Baumbiomasse der Buchenbestände an bzw. das Leistungspotenzial der Fichte nimmt ab. Somit erreicht die Buche ca. $76 \%$ der Speicherleistung der Fichtenbestände. Die Speicherrate der Eiche sinkt, im Vergleich zu derjenigen der Fichte, bei mäßiger bis ziemlich guter Nährstoffversorgung mit zunehmender Wasserverfügbarkeit (s. Abb. $5.1(\mathrm{a})$ u. 5.1(b)).

Im Tiefland, mit der Bezugsbaumart Kiefer (s. Abb. 5.2), wird deutlich, dass die anderen Baumarten höhere Speicherraten haben als die Kiefer. Insbesondere Douglasie und Fichte speichern etwa zweieinhalbmal bis dreimal mehr Kohlenstoff in ihrer lebenden Baumbiomasse als die Kiefer. Die mittlere Speicherfähigkeit der Laubbäume ist im Vergleich zu Douglasie und Fichte geringer, wobei Eiche und Buche etwa 35 bzw. $90 \%$ mehr Kohlenstoff binden als Kiefer. Bei steigender Nährstoffversorgung erhöht sich die Wachstumsleistung der Baumarten und somit steigt auch ihr Vermögen Kohlenstoff zu binden. Mit zunehmender Trockenheit nimmt die Bindungsrate der lebenden Baumbiomasse insgesamt ab.

In Niedersachsen stocken die Nadelbaumarten auf den eher schlechten und die Laubbaumarten auf den eher besseren Standorten (Wördehoff et al., 2011). In Anbetracht des eben dargestellten, hohen Kohlenstoffbindungsvermögen der lebenden Baumbiomasse der Nadelbaumarten im Vergleich zu den Laubbaumarten, sollten die Nadelbaumarten bei der Baumartenwahl im Rahmen der Produktionsplanung stärker, auch auf den ziemlich gut nährstoffversorgten Standorten, berücksichtigt werden. Insbesondere wenn im Rahmen einer nachhaltigen multifunktionalen Forstwirtschaft dem Teilziel der Kohlenstoffspeicherung Vorrang vor anderen Teilzielen eingeräumt wird. Darüber hinaus sind die Substitutionseffekte der Produkte aus Nadelholz höher als bei Produkten aus 


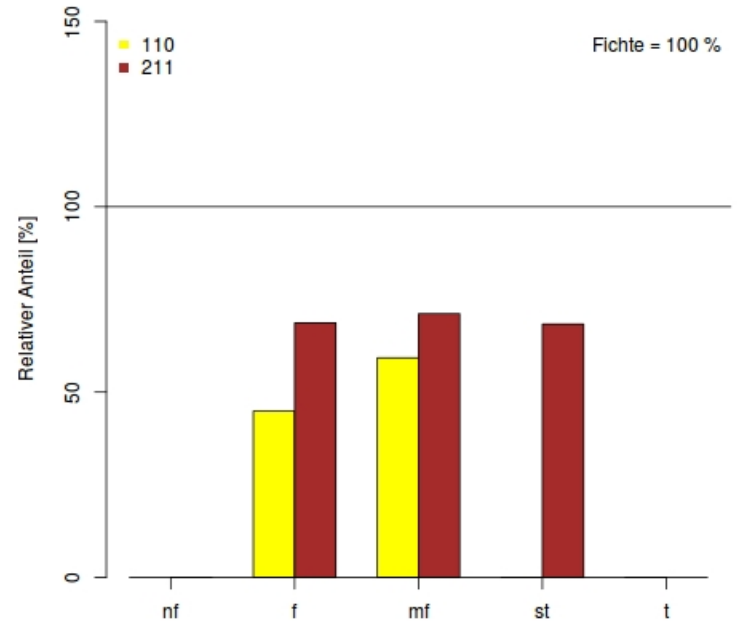

(a)

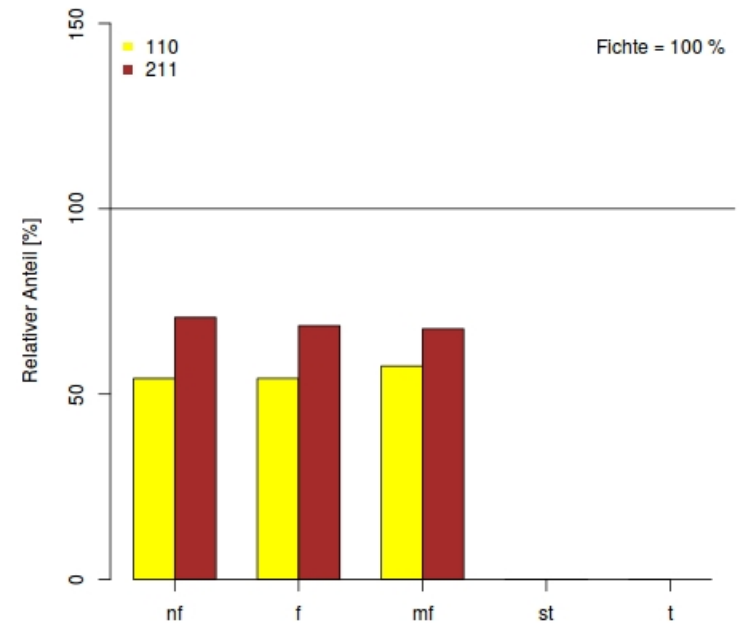

(b)

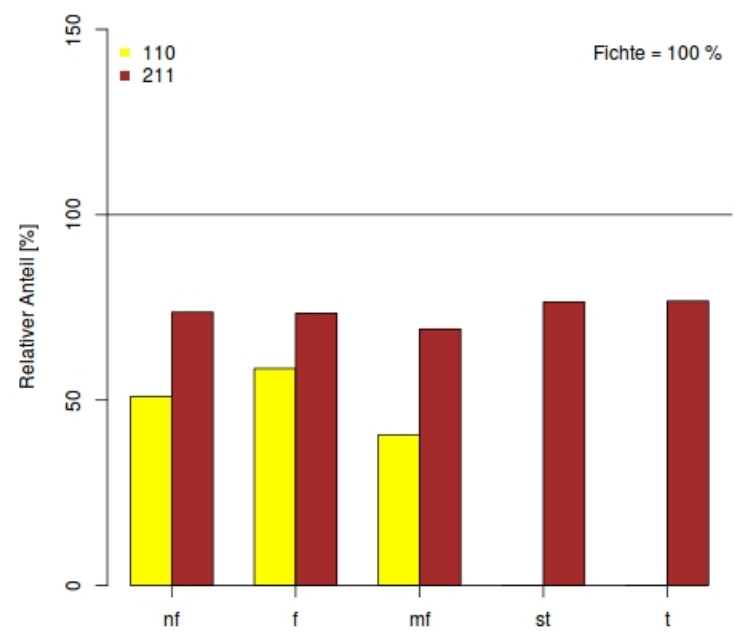

(c)

Abbildung 5.1: Relativer Anteil [\%] der baumartenspezifischen mittleren Kohlenstoffbindungsraten der lebenden Baumbiomasse $(110=$ Eiche, $211=$ Buche $)$ in Bezug zur Fichte $(100 \%)$ bei unterschiedlicher Wasserversorgung (nf = nachhaltig frisch, $\mathrm{f}=$ frisch, $\mathrm{mf}=$ mäßig frisch, $\mathrm{st}=$ sommertrocken, $\mathrm{t}=$ trocken) sowie mäßiger (a), ziemlich guter (b) und sehr guter Nährstoffversorgung (c) im niedersächsischen Bergland. 


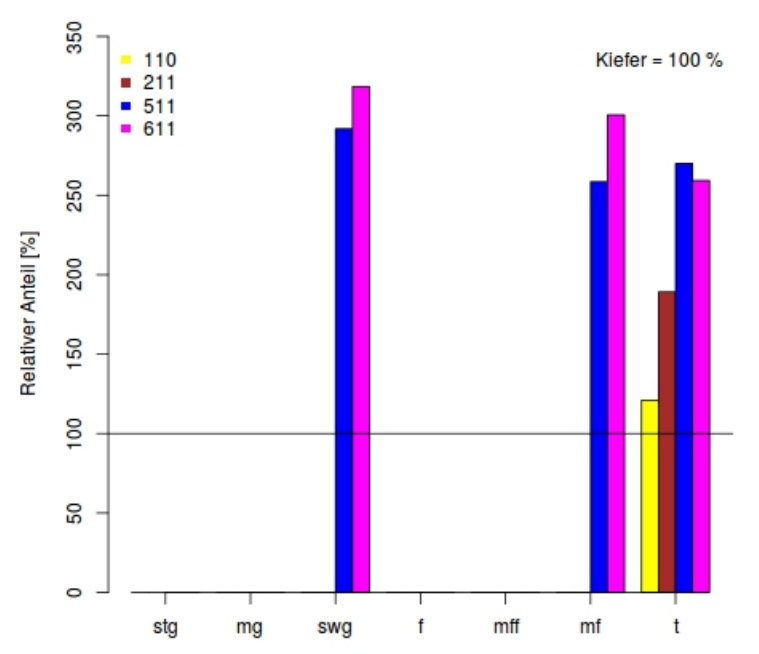

(a)

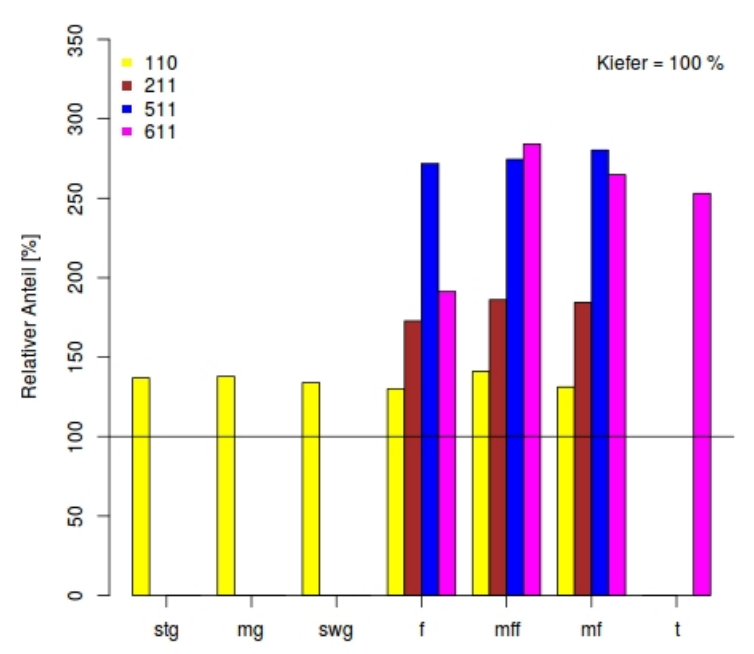

(b)

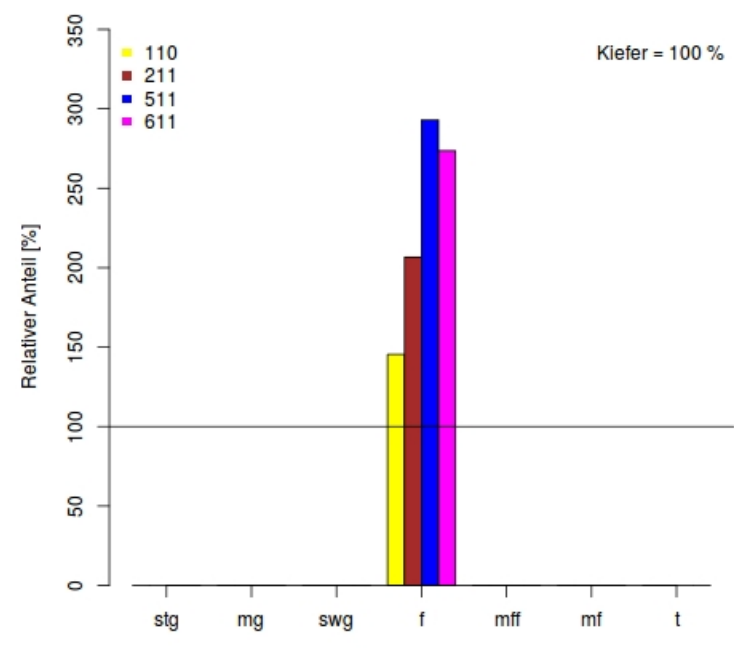

(c)

Abbildung 5.2: Relativer Anteil [\%] der baumartenspezifischen mittleren Kohlenstoffbindungsraten der lebenden Baumbiomasse $(110=$ Eiche, $211=$ Buche, $511=$ Fichte, $611=$ Douglasie) in Bezug zur Kiefer $(100 \%)$ bei unterschiedlicher Wasserversorgung (stg, mg, swg = stark, mäßig, schwach grundwasserbeeinflusst, $\mathrm{f}$ $=$ frisch, $\mathrm{mff}=$ mäßig frisch bis frisch, $\mathrm{mf}=$ mäßig frisch, $\mathrm{t}=$ trocken $)$ sowie schwacher (a), mäßiger (b) und ziemlich guter Nährstoffversorgung (c) im niedersächsischen Tiefland. 
Laubholz (s. Kap. 4.4). Vor diesem Hintergrund und den bisherigen Ergebnissen ist die momentan geringe Verjüngungsfläche der Nadelbaumarten in Niedersachsen (ML, 2014), mit Blick auf den Klimaschutz, als ungünstig zu bezeichnen.

Die Bestimmung der Totholzzersetzung und -nachlieferung ist problematisch, da sie von vielen biotischen und abiotischen Faktoren abhängt (Mackensen et al., 2003; Zell et al., 2009; Meyer et al., 2009), welche allerdings nicht bei den Simulationen berücksichtigt werden konnten. Die Zersetzungskonstanten für Totholz von Rock et al. (2008) erscheinen allerdings für mitteleuropäische Verhältnisse geeignet. Es fehlt jedoch die Vergleichsmöglichkeit für die eigens für Douglasie erstellte Konstante. Wobei Wirth et al. (2004a) und Kahl (2003) ähnlich hohe Zersetzungsraten für Nadel- bzw. Fichtentotholz ermittelt haben. Die Entwicklung des Totholzspeichers wird hauptsächlich durch die Nachlieferung von totem Holz in Form von Ernteresten und natürlicher Mortalität beeinflusst. Dabei liegt der Beitrag der mit dem WaldPlaner simulierten natürlichen Mortalität am Aufbau des Kohlenstoffvorrates dieses Speichers je nach Baumart zwischen durchschnittlich 30 bis $50 \%$. Die Kohlenstoffvorräte des Totholzes sind am Ende der Produktionszeit zwischen den verschiedenen Baumarten unterschiedlich hoch (s. Abb. 4.2 u. A.18). Unabhängig von der Behandlungsvariante ergibt sich ein Kohlenstoffvorrat in den Eichenbeständen von rund $6,8 \mathrm{t} \mathrm{Cha}^{-1}$, in den Buchenbeständen von ca. 4,5 $\mathrm{t} \mathrm{Cha}^{-1}$, in den Fichtenbeständen von etwa $3,8 \mathrm{t} \mathrm{Cha}^{-1}$, in den Douglasienbeständen annähernd von 14,3 $\mathrm{t} \mathrm{Cha}^{-1}$ und in den Kiefernbeständen von ca. 5,8 t $\mathrm{C} \mathrm{ha}^{-1}$. Die ermittelten Kohlenstoffvorräte liegen oberhalb des für Wirtschaftswälder typischen Bereich von 2,1 $\pm 1,4$ t C ha $^{-1}$ (Wirth et al., 2004a). Auch für den Totholzspeicher im Wald von Niedersachsen wurde ein geringerer mittlerer Kohlenstoffvorrat von 2,2 $\mathrm{t} \mathrm{C} \mathrm{ha}^{-1}$ berechnet (Wördehoff et al., 2011). Demgegenüber ermittelten Mund et al. (2015) bei der Simulation des Totholzspeichers für ihre Nutzungsvariante höhere Kohlenstoffvorräte von 8,4 bis $12,1 \mathrm{t} \mathrm{Cha}^{-1}$. Gründe hierfür sind andere verwendete Abbauraten und die Ermittlung eines Startvorrates für Totholz mittels Inventuren. Dieser Startvorrat fehlt in der vorliegenden Arbeit. Basierend auf einem Simulationszeitraum von 50 Jahren können aus den Kohlenstoffvorräten im Totholz von Mund et al. (2015) Speicherraten von etwa 0,126 bis $0,188 \mathrm{t} \mathrm{Cha}^{-1} \mathrm{a}^{-1}$ hergeleitet werden. Die hier identifizierten Quantile der Kohlenstoffbindungsraten (s. Tab. 4.2) sind allerdings mit rund 0,03 bis 0,09 t $\mathrm{C} \mathrm{ha}^{-1} \mathrm{a}^{-1}$ bei Eiche, Buche, Fichte und Kiefer geringer. Auch Köhl et al. (2008) solch eine geringe Speicherrate, bei einer exponentiellen Abbaurate und einer Lebensspanne von 50 Jahren, ermitteln. Dunger et al. (2014) haben auf Grundlage der Bundeswaldinventuren und der Zwischeninventur von 2008 eine mittlere Speicherrate der toten Baumbiomasse von 0,0368 t $\mathrm{C} \mathrm{ha}^{-1} \mathrm{a}^{-1}$ berechnet. Dieses Ergebnis deckt sich, abgesehen von Douglasie, gut mit den hier ermittelten Bindungsraten für diesen Pool.

Der Produktpool wird bestimmt durch die zur Verfügung stehenden Rohholzmengen und Sortimente, die jeweilige Holzverwendung sowie die mittlere Lebensdauer der einzelnen Holzprodukte. Mittels eines vereinfachten Holzverwendungsschlüssels wurden die verschiedenen Sortimente unterschiedlichen Produkten und assoziierten Klassen zugeordnet. Anschließend wurden sie in einer Kaskade genutzt. Hierdurch wird berücksich- 
tigt, dass Teile des Sägeholzes in Form von Sägeresten stofflich und energetisch genutzt werden. Auch fungiert ein Altholzspeicher als Rückkopplungselement innerhalb der Kaskade für die Produkte mit mittlerer Lebensdauer sowie die energetische Nutzung. Eine ähnliche Funktion erfüllt die Altholzkomponente für die Produkte mit kurzer Lebensdauer. Somit wird mit der entwickelten Kaskade die Rohstoffverfügbarkeit sowie das Substitutionspotenzial erhöht ohne den Einschlag zu steigern. Die statische Berechnung der Holzverwendung ist in Hinblick auf die steigende energetische Holznutzung (Mantau, 2012), die auf allen Ebenen der Kaskade stattfindet, kritisch zu sehen. Ebenso die invarianten Anteile der Kaskadennutzung der einzelnen Holzproduktkategorien. Die Ergebnisse sind daher mit Unsicherheiten behaftet, jedoch gibt es bisher keine dynamischen Modelle zur Lösung dieses Problems. Hier besteht weiterhin großer Forschungsbedarf. Festzuhalten ist allerdings, dass eine weitestgehend stoffliche Mehrfachnutzung des geernteten Holzes und eine abschließende energetische Verwendung, d.h. die Nutzung aller Substitutionseffekte von Holzprodukten, eine Voraussetzung des „kohlenstoffökologischen Königsweges“ der Speicherung von Kohlenstoff im Forst-Holz-Sektor darstellt (Klein und Schulz, 2012). Mund et al. (2015) gehen allerdings davon aus, dass nahezu das gesamte stofflich genutzte Holz schon am Ende der ersten Verarbeitungsstufe energetisch genutzt wird und damit keine Kaskadennutzung im Sinne von Frühwald et al. (2010) stattfindet. Hinzu kommt, dass die Holzverwendung in Deutschland durch Nadelholz geprägt ist und dieser Rohstoff zukünftig weniger verfügbar sein wird (Mantau, 2015b; Spellmann et al., 2015a). Da viele Holzprodukte im Baugewerbe aus Nadelholz gefertigt sind, wird das künftige Potential Kohlenstoff durch Holznutzung langfristig in Gebäuden zu speichern ebenfalls sinken. Laubholz hingegen wird kaum stofflich verwendet und somit findet meist nur eine kurzfristige Kohlenstoffbindung statt (Mantau, 2015b). Hier sind innovative Holzprodukte aus oder mit Laubholz notwendig, um dieses Problem zu lösen.

Die Substitutionswirkung wurde in dieser Untersuchung mit Faktoren aus der Literatur hergeleitet. Diese sind im Vergleich zu anderen Faktoren, insbesondere für die stoffliche Nutzung, als konservativ einzuschätzen (Knauf et al., 2013). Die Substitutionswirkung der Produkte aus Nadelholz ist insgesamt größer im Vergleich zu Produkten aus Laubholz, da ein größerer Anteil des eingeschlagenen Nadelrohholzes der Materialsubstitution zugeordnet wird (Laubholz: $46 \%$; Nadelholz: $57 \%$ ) und das Potenzial für die holzverarbeitende Industrie aufgrund der derzeitigen Waldverhältnisse momentan noch größer ist. Aus der zukünftig geringeren Nadelrohholzmenge ergibt sich nach Mantau (2015b) und den hier ermittelten Ergebnissen eine verminderte Kohlenstoffspeicherung im Forst-Holz-Sektor. Weiterhin kommt es, bei einem Ausbau der Laubholzbestände über das waldbaulich notwendige Maß hinaus, zu Versorgungsengpässen bei ökologischen Holzprodukten aus Nadelholz, einem insgesamt geringeren Substitutionspotenzial von Holzprodukten, einer Abhängigkeit von Importen aus anderen Regionen mit möglicherweise geringeren ökologischen Standards und einem Verlust von Arbeitsplätzen in der deutschen Holzindustrie (Mantau, 2015b). 


\section{Schlussfolgerungen}

\subsection{Kohlenstoffspeicherung}

Die Forstwirtschaft muss ökologische, soziale und wirtschaftliche Funktionen der Wälder sichern (BMELV, 2013). Aus dieser Forderung ergeben sich verschiedene forstbetriebliche und -politische Konsequenzen. Bei der Kohlenstoffsequestrierung geht es sowohl darum, im Wald angemessene Speicher zu erhalten, als auch darum, durch eine intelligente Nutzung die Speicherung von Kohlenstoff in Holzprodukten sowie die energetische und stoffliche Substitution zu optimieren.

Die von Umweltverbänden geforderte Einschränkung der Nutzung der Wälder als Beitrag zum Klimaschutz beruht auf der Betrachtung des Waldes als geschlossenes System (Köhl, 2013). Dabei werden der Aufbau und der Erhalt des Waldspeichers favorisiert. Im Gegensatz dazu ergänzt der Sektoransatz den Waldspeicher um den Holzproduktspeicher und die Substitutionseffekte durch die stoffliche sowie energetische Holzverwendung. Aufgrund der unterschiedlichen Sichtweisen ergeben sich daraus verschiedene Konsequenzen für die Waldbewirtschaftung und die Nutzung des Rohstoffes Holz. So wird von Vertretern des Ökosystemansatzes der Verzicht auf Holznutzungen und eine Verlängerung der Umtriebszeiten gefordert, von Fürsprechern des Sektoransatzes hingegen die Erhöhung der Produktivität und der Nutzung der Wälder. Im Spannungsfeld dieser Gegensätze ist die optimale Strategie zur Erhöhung der positiven Beiträge des Waldes zum Klimaschutz zu suchen. Im Naturschutzbereich wird natürlichen Waldökosystemen und damit dem Aufbau des Waldspeichers durch Nutzungsverzicht der Vorzug gegeben. Der Holzsektor ist hingegen an einer hohen Wertschöpfung interessiert und betont die positiven Klimaschutzwirkungen durch die Verwendung von Holzprodukten und deren Substitutionseffekte. Zwischen diesen Akteuren ist der Forstsektor zu finden. Einerseits ist er der nachhaltigen Sicherung der Multifunktionalität der Wälder unter Beachtung unterschiedlicher biotischer und abiotischer Risiken verpflichtet, andererseits auf die Nutzung des Waldes und den Verkauf von Holz als wichtigste Einnahmequelle angewiesen. Dazu ist in einer Kulturlandschaft wie in Deutschland ein integratives Konzept der Waldbewirtschaftung notwendig. Hinzu kommt, dass nachhaltig genutzte Wälder zuwachsstark und somit langfristig eine Senke für Kohlenstoff bleiben, wo hingegen Urwälder in der Zerfallsphase zur Kohlenstoffquelle werden, wie Hasenauer (2011) zeigen konnte. Auch entstehen durch die Herstellung und die Nutzung von Holzprodukten immense Substitutionspotenziale, welche bei einer Nutzungseinschränkung nur teilweise realisiert werden können. Zusätzlich müsste für die Herstellung von Holzprodukten demzufolge 
Holz, gegebenenfalls auch aus nicht nachhaltiger Produktion, importiert und Teile der in Deutschland ansässigen Holzindustrie aufgegeben werden (Mantau, 2015b). Somit ergibt sich die Herausforderung, eine Balance zwischen der Kohlenstoffspeicherung im Wald sowie in Holzprodukten, und anderen ökologischen, ökonomischen und sozioökonomischen Funktionen des Waldes zu finden (Köhl, 2013). Das bedeutet, dass das Teilziel der Kohlenstoffspeicherung in Wäldern gegebenenfalls neu bewertet und die Pools regelmäßig evaluiert werden müssen. Vor allem in Hinblick auf zunehmende Risiken ist dies wichtig, denn mit zunehmender Erderwärmung kommt dem Erhalt und der Sicherung der Wälder gegen abiotische und biotische Gefahren eine steigende Bedeutung zu (Bösch et al., 2015). Auch ist die Kohlenstoffspeicherung in Wäldern nur ein Kriterium einer nachhaltigen Forstwirtschaft (vgl. MCPFE, 2003) und die Mehrzweckforstwirtschaft zur Erfüllung der verschiedenen Waldfunktionen gesetzlich vorgegeben (§ 1 BWaldG).

Die Höhe der Kohlenstoffbindungsraten der lebenden Baumbiomasse hängt von der Baumart ebenso ab wie von den zur Verfügung stehenden Wasser- und Nährstoffressourcen. Auch die waldbauliche Behandlung, die einen gewissen Vorratsaufbau ermöglicht und Rohholz zur Herstellung von Holzprodukten zur stofflichen und abschließenden energetischen Nutzung bereitstellt sowie gleichzeitig Substitutionswirkungen ermöglicht, beeinflusst die Speicherung von Kohlenstoff im Forst-Holz-Sektor positiv (Klein und Schulz, 2012). Entscheidend ist dabei die Verwendung des Holzes, denn damit wird der mögliche Zeitraum der produktspezifischen Kohlenstoffbindung, die Verwendung innerhalb einer Nutzungskaskade und das Substitutionspotenzial bestimmt. In dieser Arbeit konnten nur geringe Unterschiede zwischen den Gesamtspeicherraten der beiden untersuchten Behandlungsvarianten festgestellt werden. Die Differenzen zwischen den Baumarten sind demgegenüber deutlich größer. So binden in der Gesamtbetrachtung aller analysierten Speicher Douglasie und Fichte mehr Kohlenstoff als Kiefer und Buche oder Eiche. Sehr wichtig sind hierbei die stoffliche Verwendung und die Materialsubstitution, welche an der Gesamtspeicherrate einen Anteil von rund 60 bis $73 \%$ hat. Ein weiterer wichtiger Speicher im Wald ist der Boden, welcher in Niedersachsen zwischen den beiden Bodenzustandserhebungen rund 1,35 $\mathrm{t} \mathrm{ha}^{-1} \mathrm{a}^{-1}$ (Bezugstiefe 0 bis $90 \mathrm{~cm}$ ) gespeichert hat (Evers, 2015). Bundesweit wird allerdings von einer geringeren Speicherrate $\left(0,41 \mathrm{tC} \mathrm{ha}^{-1} \mathrm{a}^{-1}\right.$, Bezugstiefe 0 bis $30 \mathrm{~cm}$ ) mit starken regionalen Unterschieden ausgegangen (Grüneberg et al., 2014, 2015). Die Bodenvegetation hat eine vergleichsweise geringe Speicherleistung von durchschnittlich $0,15 \mathrm{tC} \mathrm{ha}^{-1} \mathrm{a}^{-1}$, wobei diese stark von der auf den Boden treffende Strahlungsmenge abhängt. Die Emissionen aus der Waldbewirtschaftung und dem Holztransport sind in den Substitutionsfaktoren von Knauf et al. (2013) enthalten und haben nach Berechnungen von Mund et al. (2015) einen Anteil von etwa 0,6 bis 1\% an der Kohlenstoffbilanz der Holzverwendung. Auch bei der Drainage von organischen Böden, der Kalkung und durch Brände wird im Wald Kohlenstoff freigesetzt, wobei dieser Anteil an der Treibhausgasbilanz des Waldes bundesweit rund $7 \%$ beträgt (Dunger et al., 2014). 


\subsection{Forstbetriebliche Ebene}

Die Baumartenwahl ist die wichtigste ökologische und ökonomische Entscheidung im Forstbetrieb mit vielfältigen Auswirkungen auf Aufwand und Ertrag, die Lieferung von Infrastrukturleistungen, die Gestaltung des Landschaftsbildes und die Übernahme bestimmter Produktionsrisiken (Röhrig et al., 2006; Speidel, 1972). Auch der Kohlenstoffvorrat im Forst-Holz-Sektor kann langfristig damit am stärksten beeinflusst werden. Die Waldbewirtschaftung trägt dazu bei, den Holzvorrat aufzubauen und gleichzeitig den Rohstoff zur Herstellung von Holzprodukten bereitzustellen. Die Niedersächsischen Landesforsten realisieren dies mit der naturnahen Bewirtschaftung der ihr anvertrauten Wälder. Aus dem standörtlichen Vergleich der Kohlenstoffbindungsraten der einzelnen Baumarten ergibt sich folgende Konsequenz: Zur Förderung des Klimaschutzes sollten bei der Produktionsplanung standortgemäße Nadelbaumarten aufgrund ihrer höheren Gesamtspeicherraten gegenüber den Laubbaumarten wieder stärker berücksichtigt werden. Dieser Effekt könnte durch den Anbau von Nadelbaumarten auf Standorten mit etwas besserer Wasserversorgung und ziemlich guter Versorgung mit Nährstoffen noch erhöht werden. Von einem Nadelholzanbau auf Standorten mit guter bis sehr guter Nährstoffversorgung ist hingegen abzusehen, da die Qualität des dort produzierten Nadelholzes meist gering ist und es zu Stabilitätsproblemen kommen kann. Ein weiterer betrieblicher Aspekt ist die Sicherung standortgemäßer Naturverjüngung von Nadelbaumarten in Laubholzbeständen, wobei der Anteil der Nadelhölzer im Jungwuchs in Niedersachsens Wäldern momentan weniger als $20 \%$ beträgt (ML, 2014). Dieses wichtige Potential der Nadelbaumarten muss in Anbetracht ihrer großen Klimaschutzwirkung, aus ökonomischer Sicht und mit Blick auf die Risikoverteilung (Spellmann, 2015) erhalten und angehoben werden. Sind standortgemäße Nadelbaumanteile gesichert, muss infolgedessen in der Pflegephase zielstrebig auf einen Waldentwicklungstyp mit führendem Nadelholz hingearbeitet werden. Ferner ist der Altersklassenaufbau der Nadelholzbestände für die Rohholzbereitstellung und damit für die Holzproduktherstellung ein entscheidender Faktor. In den Niedersächsischen Landesforsten sind die Nadelholzbestände überwiegend 41 bis 80 Jahre alt. Daher besteht in den nächsten Jahren ein gutes Nutzungspotenzial im Nadelstammholzbereich (Spellmann, 2015), welches zur Herstellung von langlebigen Holzprodukten mit einem hohen materiellen Substitutionseffekt dienen kann. Dazu sollte es aus Gründen der Risikominderung, der Liquiditätssicherung und einer zuverlässigen Rohholzversorgung zeitlich gestreckt genutzt werden. Hierfür müssen sich die angestrebten Zielstärken für die Nadelaltholzbestände stärker an der Stabilität, Wuchsleistung sowie Qualität orientieren, wobei bei den Endnutzungen streng auf die räumliche Ordnung zu achten ist (Spellmann et al., 2015a). Allerdings wird dieses Potenzial in Niedersachsen langfristig zurückgehen und beim Laubholz wird das Rohholzangebot die Nachfrage voraussichtlich übersteigen (Spellmann, 2015; Spellmann et al., 2015a). Somit gehen auch die Möglichkeiten der Kohlenstoffspeicherung in Produkten aus Nadelholz sowie deren Substitutionseffekte zurück, welche momentan nicht durch die Herstellung von Produkten aus Laubholz kompensiert werden können. 
Das Teilziel der Kohlenstoffspeicherung im Wald und in Holzprodukten muss insgesamt mit anderen Regelungs- (ökosystemare Kreisläufe), Lebensraum- (Biodiversität), Nutz- (Holz, Wasser Jagd) sowie Kultur- und Sozialfunktionen (Arbeit, Einkommen, Kulturerbe, Erholung) im Einklang gehalten werden (Beese, 1996). Für einen Forstbetrieb sind im Hinblick auf den Erhalt und die Erhöhung der Klimaschutzleistungen die Handlungsfelder Baumartenwahl (Leistung und Verwendungsmöglichkeiten), Vorratssteuerung (ein durchschnittlicher Vorrat mit möglichst hohen Zuwächsen), Risikominimierung (kein Vorratsabbau durch Kalamitäten), Bodenschutz (Erhalt des Bodens und der Humusauflage), Nutzholzproduktion (möglichst stofflich verwertbare Holzsortimente) und der Walderhalt von großer Bedeutung (Bösch et al., 2015). Allerdings ist der Gestaltungsspielraum der Forstbetriebe durch unterschiedliche Naturschutzauflagen eingeschränkt.

Durch die Mischung verschiedener Baumarten können standortabhängig höhere Bestandesbiomassen und somit größere Kohlenstoffvorräte erreicht werden. Dieser Effekt tritt jedoch nicht zwangsläufig ein bzw. es können bei der Mischung von Baumarten mit der gleichen oder ähnlichen ökologischen Nische Produktivitätsverluste von bis zu $20 \%$ auftreten. Werden jedoch Pionier- und Klimaxbaumarten, in ihrer Entwicklung früh und spät kulminierende Arten oder Licht- und Schattbaumarten gemischt, kann die Produktivität dieser Mischbestände auf bestimmten Standorten um bis zu $30 \%$ größer sein, als in Reinbeständen (Pretzsch, 2009; Pretzsch und Schütz, 2016). Die Mischungsform auf gegebenem Standort ist demnach für die Produktivität von herausragender Bedeutung (Assmann, 1961; von Lüpke und Spellmann, 1997; Spellmann, 2010; Pretzsch et al., 2010, 2013) und ermöglicht einen höheren Kohlenstoffvorrat im Bestand.

Weiterhin wird in Mischbeständen durch die unterschiedliche Wurzelbildung der beteiligten Baumarten das Wasser- und Nährstoffangebot der jeweiligen Standorte oft besser ausgenutzt (Kramer, 1988), die Bestandesstabilität sowie das Lebensraumangebot erhöht und Risiken werden verteilt bzw. verringert (Röhrig et al., 2006). Dieser Aspekt wird mit fortschreitendem Klimawandel und der damit steigenden Eintrittswahrscheinlichkeit von Kalamitäten immer wichtiger. Die Mischwaldvermehrung und -erhaltung bietet dem Forstbetrieb die Chance, einen Kompromiss zwischen ökologischen sowie ökonomischen Anforderungen zu finden (Spellmann, 2013) und sie ermöglicht die erforderliche Beachtung der oben genannten Handlungsfelder. Um den Totholzvorrat im Bestand und damit den Kohlenstoffvorrat in diesem Speicher sowie die biologische Vielfalt im Wald zu erhöhen, können bei der Waldbewirtschaftung Erntereste bewusst im Wald belassen werden.

Die standortgemäße Baumartenwahl sowie die Mischung der Arten sind wichtige Maßnahmen der strategischen Waldbauplanung, mit denen der Forstbetrieb den Kohlenstoffvorrat im Bestand halten respektive erhöhen kann. Wobei in Hinblick auf die Ökosystemleistung des Waldes Kohlenstoff zu speichern, ein angemessener Anteil standortgerechter Nadelhölzer bei möglichst geringen Risiken angestrebt werden sollte. Allerdings müssen die festgelegten Strategien, als Leitplanken des täglichen Handelns, beispielsweise im Rahmen der Forsteinrichtung und unter Berücksichtigung neuer wissenschaftlicher Er- 
kenntnisse regelmäßig kritisch hinterfragt und gegebenenfalls angepasst werden, um unerwünschten Entwicklungen entgegenwirken zu können. Gleichzeitig müssen die Umsetzungsmaßnahmen im Sinne eines adaptiven Managements adjustiert werden. Zukünftig wird jede Landfläche der Erde von Klimaänderungen und deren Auswirkungen beeinflusst sein (Birdsey und Pan, 2015). So wird beispielsweise im norddeutschen Tiefland ein beschleunigter Humusabbau und ein Anstieg der Waldbrandfläche infolge der zukünftigen Klimaveränderungen mit negativen Auswirkungen auf den Kohlenstoffvorrat im Wald erwartet (Fleck und Meesenburg, 2015). Demzufolge müssen einmal gewählte Strategien angepasst werden. Darüber hinaus wird durch eine leistungsgerechte und ordnungsgemäße Waldbewirtschaftung der Rohstoff Holz der holzverarbeitenden Industrie sowie der Energiewirtschaft zur Verfügung gestellt und es können damit andere, in der Herstellung energieintensivere Materialien bzw. fossile Brennstoffe, substituiert werden. Damit wird ein umfassender Beitrag zum gesellschaftlich gewünschten und geforderten Klimaschutz geleistet, welcher bei einer Nichtnutzung von Wäldern nicht realisiert werden kann.

\subsection{Forstpolitische Ebene}

Der Forderung nach der Einbindung der stofflichen Nutzung in die Treibhausgasbilanzierung wurde auf der 17. Vertragsstaatenkonferenz der Klimarahmenkonvention in Durban entsprochen (BMELV, 2012a). Somit kommt eine stoffliche Holznutzung nicht mehr einer Kohlenstofffreisetzung gleich und das betrachtete System bezieht sich auf die Senkenwirkung der Wälder und die Speicherung von Kohlenstoff in Holzprodukten. Daher kann eine Stilllegung von Wäldern nicht überzeugend alleinig mit Klimaschutzaspekten begründet werden, da keinerlei Einbindung von Kohlenstoff in Holzprodukte sowie keine materielle und energetische Substitution durch deren Nutzung stattfindet (Köhl, 2013; Mund et al., 2015).

Global betrachtet, relativiert sich der Beitrag der deutschen Wälder zur Kohlenstoffbindung, da der Großteil der weltweiten Treibhausgasemissionen bisher keiner Begrenzung unterlag. Bezogen auf die Bundesrepublik speichert der deutsche Wald jährlich rund $6 \%$ der emittierten Treibhausgase (s. Kap. 1.2). Auf der 21. Vertragsstaatenkonferenz der Klimarahmenkonvention in Paris Ende 2015 wurde erstmals eine Begrenzung des Anstiegs der Erderwärmung auf zwei Grad Celsius in einem völkerrechtlichen Abkommen verankert, welches von den einzelnen Staaten allerdings noch in nationales Recht überführt werden muss. Dieses Ziel soll durch Treibhausgasneutralität in der zweiten Hälfte des Jahrhunderts, vor allem durch den Verzicht auf fossile Energien (Dekarbonisierung), erreicht werden (BMUB, 2015). Allerdings beträgt die durchschnittliche Verweildauer von Kohlenstoffdioxid in der Atmosphäre 120 Jahre und daher ist eine alleinige Begrenzung der Treibhausgasemission nicht ausreichend, um die Folgen des Klimawandels zu bewältigen.

Zugleich enthält die nationale Klimapolitik keine konkreten oder mit anderen Pro- 
zessen (z. B. Nationale Biodiversitätsstrategie, Waldstrategie 2020) abgestimmte Maßnahmen bzw. nur wenige Politikinstrumente, um bestehende Zielkonflikte, beispielsweise zwischen Erhöhung der Kohlenstoffvorräte im Bestand und gleichzeitiger Steigerung der Substitutionsmöglichkeiten durch Holzprodukte oder die Ausweitung der Nutzung von Biomasse und den ökologischen Auswirkungen, zu lösen. Vielmehr beeinflusste die Förderung der erneuerbaren Energien durch Preissteigerungen für Heizöl die Nachfrage nach Holz als Energieträger (Bösch et al., 2015). Momentan ist dies allerdings aufgrund des geringen Ölpreises nicht gegeben. Faktisch spielen Wälder und Holzverwendung in der deutschen Klimaschutzpolitik kaum eine Rolle, was Bösch et al. (2015) auf folgende Umstände zurückführen: Bundesregierung sowie Europäische Union legen ihren Fokus auf die Reduktion der fossilen Emissionen; der Kohlenstoffkreislauf und die Wechselwirkungen der einzelnen Speicher untereinander sind sehr komplex; die Speicherung von Kohlenstoff ist aufgrund der sich ändernden klimatischen Bedingungen und der geringen Flächenverfügbarkeit nicht steuerbar.

Da das Kyoto-Protokoll die Emissionsrechte der teilnehmenden Staaten begrenzt, sind diese mittels Zertifikaten entsprechend handelbar. Dadurch ist es möglich, Erträge zu erwirtschaften und die Senkenleistung der Wälder zu honorieren. Aber das Kyoto-Protokoll regelt einzig das Rechtsverhältnis zwischen Staaten und daher können nur sie Erträge aus dem Emissionshandel erzielen. Senkenzertifikate die den Handel auf Betriebsebene ermöglichen, wurden bisher nicht in das Europäische Emissionshandelssystem integriert. Allerdings sollen mindestens 50 \% der Einnahmen aus dem Verkauf von Emissionszertifikaten für Zwecke des Klimaschutzes und der Klimaanpassung, unter anderem die Kohlenstoffspeicherung durch die Forstwirtschaft in der Europäischen Gemeinschaft, verwendet werden (Bösch et al., 2015).

Ein bestehendes, relativ neues, konkretes Politikinstrument ist der Waldklimafonds, welcher durch Erlöse des Europäischen Zertifikatehandels finanziert wird. Durch die Anpassung der Wälder an den Klimawandel sowie der Förderung von Maßnahmen zur Erschließung des Minderungs-, Energie- und Substitutionspotenzials von Wald und Holz, soll er zum Erreichen der Klimaziele der Bundesregierung beitragen (WKF, 2015). Welche Ergebnisse die einzelnen Projekte liefern und wie diese letztendlich umgesetzt werden bleibt abzuwarten, da bisher kein gefördertes Vorhaben abgeschlossen wurde.

Eine sehr effektive Maßnahme zur Erhöhung des Kohlenstoffvorrates im Waldspeicher ist die Vergrößerung der Waldfläche, wobei eine starke Konkurrenz mit landwirtschaftlichen Flächen besteht. Während die globale Waldfläche in den letzten Jahrzehnten deutlich abgenommen hat (s. Kap. 1.2), ist sie in Deutschland in den letzten Jahren leicht angestiegen (BMEL, 2014). Dies ist vor allem der gesetzlichen Sicherung des Waldes zu verdanken ( $\$ 9$ BWaldG). Aber auch der Schutz der Moore, mit ihren hohen Bodenkohlenstoffvorräten, ist eine wichtige Maßnahme zur Begrenzung der Treibhausgasemissionen. Weiterhin könnten durch vertraglich geregelte Methoden, ähnlich dem Vertragsnaturschutz, Anreize für Waldbesitzer geschaffen werden, ihren Wald so zu bewirtschaften bzw. bestimmte Maßnahmen wie die Zielstärkennutzung so durchzuführen, dass ein hoher Effekt für den Klimaschutz erzielt wird. Auf Grundlage der Gemeinschafts- 
aufgabe Agrarstruktur und Küstenschutz (GAK) erfolgt eine Förderung der forstwirtschaftlichen Zusammenschlüsse sowie der naturnahen Waldbewirtschaftung, welche vornehmlich den Klein- und Kleinstprivatwald einbezieht. Dabei werden die Vorgaben des GAK-Rahmenplanes von den einzelnen Bundesländern konkretisiert und Zuwendungen gewährt (BMEL, 2015). Die Minderung des Kohlenstoffdioxids in der Atmosphäre könnte auch durch den vermehrten Anbau von Douglasie und Küstentanne gefördert werden. Dabei gelten beide Baumarten als nicht invasiv, standortgemäß und als Resistent gegen Sommertrockenheit (Vor et al., 2015), was in Hinblick auf die Risikominderung und Klimaanpassung der Wälder von hoher Relevanz ist. Eine Änderung der zurzeit einseitig auf Naturnähe ausgerichteten Förderpolitik (Laubwaldvermehrung) zur Nachzucht von Nadelbaumanteilen könnte das Minderungspotenzial erhöhen und würde auch ökonomische Effekte haben. Ein anderes Handlungsfeld ist die Begrenzung der nach wie vor hohen Stickstoffeinträge in Waldökosysteme, wobei die Hauptemittenten die Bereiche Landwirtschaft, Energie und Verkehr sind. Der überschüssige Stickstoff führt dazu, dass er im Waldboden in sehr klimaschädliches Lachgas umgewandelt und freigesetzt wird (Bösch et al., 2015).

Um die Wechselwirkungen zwischen Klimapolitik und Klimawandel sowie unseren natürlichen Lebensgrundlagen zu betrachten, bietet sich das Konzept der Ökosystemleistungen an. Dabei werden Ökosysteme, ihre Strukturen sowie Prozesse, und das menschliche Wohlergehen in Verbindung zueinander gesetzt. Ökosysteme stiften individuellen bzw. gesellschaftlichen Nutzen, erfahren allerdings von den Menschen unterschiedliche Wertschätzung. Mit einer ökonomischen Bewertung der Ökosystemleistungen kann die Bedeutung der Waldökosysteme für den Klimaschutz gestärkt werden. Momentan werden vor allem die Kosten des Klimawandels auf Grundlage der Quantifizierung von Schäden betrachtet. Dabei sind diejenigen Kosten eingeschlossen, die unmittelbar auf Schäden infolge des Klimawandels zurückzuführen sind. Auch wenn die Berechnung der Schadenskosten mit erheblichen Unsicherheiten und methodischen Problemen behaftet ist, schlagen Bonn et al. (2015) zur Bewertung der Ökosystemleistung Klimaschutz Schadenskosten in Höhe von 80 bis $120 € \mathrm{t}^{-1} \mathrm{CO}_{2}^{-1}$ vor. In Verbindung mit den oben genannten mittleren Speicherraten würde die baumartenspezifische Senkenleistung der Wälder sowie der Holzprodukte und der Substitutionseffekte im Durchschnitt mit rund 3.000 bis $10.000 €$ ha $^{-1} \mathrm{a}^{-1}$ bewertet werden. Für die Gesellschaft in Deutschland erbringen die Wälder verschiedene Ökosystemleistungen mit erheblichem Nutzen. Wobei die Klimaschutzleistung des Waldes in Deutschland, d.h. die zusätzliche jährliche Einbindung von Kohlenstoff in die lebende Baumbiomasse der Wälder und in Holzprodukte, im Vergleich zu anderen Ökosystemleistungen, wie der Erholungsleistung oder der Holzproduktion, eine geringere gesellschaftliche Wertschätzung erfährt (Bösch et al., 2015). Weltweit gesehen steht dies allerdings im Gegensatz zu den Ergebnissen der Vertragsstaatenkonferenzen der Klimarahmenkonvention in Durban und Paris, welche zeigen, dass die globale Gesellschaft dem Klimaschutz eine hohe Bedeutung beimisst.

Hier ist die Forstpolitik gefragt die Rahmenbedingungen der nationalen wie internationalen Klimapolitik zu verbessern. Insbesondere müssen bestehende Zielkonflikte 
zwischen Waldnaturschutz- und Energiewendezielen gelöst werden (Bösch et al., 2015). Ebenso muss die Bedeutung der Wälder und deren ordnungsgemäße Bewirtschaftung für den Klimaschutz, auf nationaler wie auch auf internationaler Ebene, der Gesellschaft stärker aufgezeigt werden. Außerdem ist der Beitrag der Kaskadennutzung zur Entschärfung bestehender Konkurrenzen bezüglich der Verwendung des Rohstoffes Holz und zum Erhalt des Waldspeichers weiter zu analysieren und zu intensivieren. 


\section{Literaturverzeichnis}

3N, 2014. Feuerstättenzählung Niedersachsen 2013. Kompetenzzentrum Niedersachsen Netzwerk Nachwachsende Rohstoffe e.V., 26 S.

Albert, M., Schmidt, M., 2009. Beurteilung der Anbauwürdigkeit von Baumarten unter Klimawandel mittels dreidimensionaler Ökogramme. In: Nagel, J. (Hrsg.): Deutscher Verband Forstlicher Forschungsanstalten, Sektion Ertragskunde. Jahrestagung 25. bis 27. Mai 2009, Ascona, 83-94

Albrecht, S., Rüter, S., Welling, J., Knauf, M., Mantau, U., Braune, A., Baitz, M., Weimar, H., Sörgel, S., Kreissig, J., Deimling, J., Hellwig, S., 2008. ÖkoPot - Ökologische Potenziale durch Holznutzung gezielt fördern. ÖkoPot-Abschlussbericht zum BMBF-Projekt FKZ 0330545. Stuttgart, 298 S.

Ammer, C., Vor, T., Knoke, T., Wagner, S., 2010. Der Wald-Wild-Konflikt. Analyse und Lösungsansätze vor dem Hintergrund rechtlicher, ökologischer und ökonomischer Zusammenhänge. Göttinger Forstwissenschaften, Universitätsverlag Göttingen, 5, 199 S.

Ammer, S., Huber, C., 2007. Die Regenwurmlebensgemeinschaft im Höglwaldexperiment 21 Jahre nach der Kalkung. Allgemeine Forst- und Jagdzeitung, 178, 213-220

Ando, T., 1968. Ecological studies on the stand density control in even-aged pure stand. Tokyo Government Forest Experiment Station Bulletin, 210, 1-153

Anonymus, 1902. Beratungen der vom Verein Forstlicher Versuchsanstalten eingesetzten Kommission zur Feststellung des neuen Arbeitsplanes für Durchforstungs- und Lichtungsversuche. Allgemeine Forst- und Jagdzeitung, 78, 180-184

Ashton, M.S., Tyrell, M.L., Spalding, D., Gentry, B. (Hrsg.), 2012. Managing Forest Carbon in a Changing Climate. Springer, Dordrecht, 414 S.

Assmann, E., 1956. Natürlicher Bestockungsgrad und Zuwachs. Forstwissenschaftliches Centralblatt, 75, 257-265

Assmann, E., 1961. Waldertragskunde. BLV Verlagsgesellschaft, München, 490 S.

Assmann, E., Franz, F., 1963. Vorläufige Fichten-Ertragstafel für Bayern. Institut für Ertragskunde der Forstlichen Forschungsanstalt, München, 104 S. 
Bauer, G.A., Persson, H., Perrson, T., Mund, M., Hein, M., Kummetz, E., Matteucci, G., Oene, H.v., Scarascia-Mugnozza, G., Schulze, E.D., 2000. Linking Plant Nutrition and Ecosystem Processes. In: Schulze, E.D. (Hrsg.): Carbon and Nitrogen Cycling in European Forest Ecosystems. Springer, Berlin, 63-98

Beese, F., 1996. Indikatoren für eine multifunktionelle Waldnutzung. Forstwissenschaftliches Centralblatt, 115, 65-79

Bergel, D., 1985. Douglasien-Ertragstafel für Nordwestdeutschland. Niedersächsische Forstliche Versuchsanstalt, Göttingen, 72 S.

Birdsey, R., Pan, Y., 2015. Trends in management of the world's forest and impacts on carbon stocks. Forest Ecology and Management, 355, 83-90

Bitter, A., 2004. Strategische Planung als Instrument der forstlichen Betriebsgestaltung. In: Löwenstein, W. (Hrsg.): Perspektiven forstökonomischer Forschung. Sauerländer, Frankfurt am Main, 1-13

BMEL, 2014. Der Wald in Deutschland - Ausgewählte Ergebnisse der dritten Bundeswaldinventur. Bundesministerium für Ernährung und Landwirtschaft, 56 S.

BMEL, 2015. GAK-Rahmenplan ab 2015 - Förderbereich: Forsten. Bundesministerium für Ernährung und Landwirtschaft. URL: http://www.bmel.de/DE/Landwirtschaft/ Foerderung-Agrarsozialpolitik/GAK/_Texte/Foerdergrundsaetze2015.html, aufgerufen am 03.12.2015

BMELV, 2005. Die zweite Bundeswaldinventur - Der Inventurbericht. Bundesministerium für Ernährung, Landwirtschaft und Verbraucherschutz, 231 S.

BMELV, 2007. Holzmarktbericht 2006. Abschlussergebnisse für die Forst- und Holzwirtschaft des Wirtschaftsjahres 2006. Bundesministerium für Ernährung, Landwirtschaft und Verbraucherschutz. URL: http://www.bmelv-statistik.de/de/fachstatistiken/ forst-und-holzwirtschaft/, aufgerufen am 09.12.2012

BMELV, 2010. Holzmarktbericht 2009. Abschlussergebnisse für die Forst- und Holzwirtschaft des Wirtschaftsjahres 2009. Bundesministerium für Ernährung, Landwirtschaft und Verbraucherschutz. URL: http://www.bmelv-statistik.de/de/fachstatistiken/ forst-und-holzwirtschaft/, aufgerufen am 09.12.2012

BMELV, 2011. Holzmarktbericht 2010. Abschlussergebnisse für die Forst- und Holzwirtschaft des Wirtschaftsjahres 2010. Bundesministerium für Ernährung, Landwirtschaft und Verbraucherschutz. URL: http://www.bmelv-statistik.de/de/fachstatistiken/ forst-und-holzwirtschaft/, aufgerufen am 09.12.2012 
BMELV, 2012a. Ergebnisse der 17. Vertragsstaatenkonferenz der Klimarahmenkonvention. Bericht des Bundesministeriums für Ernährung, Landwirtschaft und Verbraucherschutz. Amtschefkonferenz vom 18. bis 19. Januar 2012 in Berlin, 8 S. URL: http: //www.klimawandel-und-klimaschutz.de/amtschefkonferenz-im-januar-2012/, aufgerufen am 10.4.2012

BMELV, 2012b. Holzmarktbericht 2011. Abschlussergebnisse für die Forst- und Holzwirtschaft des Wirtschaftsjahres 2011. Bundesministerium für Ernährung, Landwirtschaft und Verbraucherschutz. URL: http://www.bmelv-statistik.de/de/ fachstatistiken/forst-und-holzwirtschaft/, aufgerufen am 09.12.2012

BMELV, 2013. Elemente der internationalen Waldpolitik. Bundesministerium für Ernährung, Landwirtschaft und Verbraucherschutz. URL: http: //www.bmelv.de/SharedDocs/Dossier/Landwirtschaft/InternationaleWaldpolitik. html?notFirst $=$ true\&docId=1641306, aufgerufen am 07.02.2013

BMUB, 2015. Klimaschützer schreiben Geschichte. Bundesministerium für Umwelt, Naturschutz, Bau und Reaktorsicherheit, Presseinfo Nr. 344/15, Berlin, 12.12.2015. URL: http://www.bmub.bund.de/N52703/, aufgerufen am 14.12.2015

Böckmann, T., Saborowski, J., Dahm, S., Nagel, J., Spellmann, H., 1998. Die Weiterentwicklung der Betriebsinventur in Niedersachsen. Forst und Holz, 8, 219-226

Böckmann, T., Spellmann, H., Hüsing, F., 1998. Neukonzeption und Weiterentwicklung der Forsteinrichtung in Niedersachsen. Forst und Holz, 10, 298-302

Bolte, A., 1999. Abschätzung von Trockensubstanz-, Kohlenstoff- und Nährelementvorräten der Waldbodenflora - Verfahren, Anwendung und Schätztafeln. Dissertation, Techn. Univ. Dresden, Fachrichtung Forstwiss. Tharandt, 285 S.

Bolte, A., Wellbrock, N., Dunger, K., 2011. Wälder, Klimaschutz und Klimaanpassung: welche Maßnahmen sind umsetzbar? AFZ-Der Wald, 66, 27-29

Bonn, A., Grunewald, K., Hampicke, U., Hartje, V., Jax, K., Marzelli, S., Meyerhoff, J., Schweppe-Kraft, B., 2015. Methodische Grundlagen zu Ökosystemleistungen und Ökonomischer Bewertung. In: Hartje, V., Wüstemann, H., Bonn, A. (Hrsg.): TEEB DE Naturkapital und Klimapolitik - Synergien und Konflikte. Technische Universität Berlin, Helmholtz-Zentrum für Umweltforschung. Berlin, Leipzig, 21-64

Borchers, K., 1952. Grundsätzliches zu einer waldbaulichen Planung in Niedersachsen. Forst und Holz, 11, 147-151, Fortsetzung: 12, 162-165

Borys, A., Lasch, P., Suckow, F., Reyer, C., 2013. Kohlenstoffspeicherung in Buchenbeständen in Abhängigkeit von Waldpflege und Klimawandel. Allgemeine Forst- und Jagdzeitung, 184, 26-35 
Bösch, M., Leefken, G., Möhring, B., Pistorius, T., Rock, J., Rüter, S., Schröppel, B., 2015. Klimaschutz als Ökosystemdienstleistung des Waldes in Deutschland: Wie tragen deutsche Wälder zum Schutz der Atmosphäre bei? In: Hartje, V., Wüstemann, H., Bonn, A. (Hrsg.): TEEB DE Naturkapital und Klimapolitik - Synergien und Konflikte. Technische Universität Berlin, Helmholtz-Zentrum für Umweltforschung. Berlin, Leipzig, 148-171

Böswald, K., 1996. Zur Bedeutung des Waldes und der Forstwirtschaft im Kohlenstoffhaushalt, eine Analyse am Beispiel des Bundeslandes Bayern. Forstliche Forschungsberichte, München, 159, 147 S.

Böswald, K., Wierling, R., 1997. Wald und Forstwirtschaft Niedersachsens im Kohlenstoffhaushalt. Aus dem Walde - Mitteilungen aus der Niedersächsischen Landesforstverwaltung, Niedersächsisches Ministerium für Ernährung, Landwirtschaft und Forsten (Hrsg.), 50, $333 \mathrm{~S}$.

Burschel, P., Kürsten, E., Larson, B., 1993. Die Rolle von Wald und Forstwirtschaft im Kohlenstoffhaushalt - eine Betrachtung für die Bundesrepublik Deutschland. Forstliche Forschungsberichte, München, 126, 135 S.

Burschel, P., Huss, J., 2003. Grundriss des Waldbaus. Ulmer, Stuttgart, 487 S.

BWaldG, 2015. Bundeswaldgesetz vom 2. Mai 1975 (BGBl. I S. 1037), das zuletzt durch Artikel 413 der Verordnung vom 31. August 2015 (BGBl. I S. 1474) geändert worden ist. URL: http://www.gesetze-im-internet.de/bwaldg/, aufgerufen am 01.12.2015

Christophel, D., Spengler, S., Schmidt, B., Ewald, J., Prietzel, J., 2013. Customary selective harvesting has considerably decreased organic carbon and nitrogen stocks in forest soils of the Bavarian Limestone Alps. Forest Ecology and Management, 305, $167-176$

Dieter, M., Elsasser, P., 2002. Carbon stocks and carbon stock changes in the tree biomass of Germany's forests. Forstwissenschaftliches Centralblatt, 121, 195-210

Dittmar, O., Knapp, E., Lembcke, G., 1983. DDR-Buchenertragstafel. Institut für Forstwissenschaften Eberswalde, Abt. Information (IFE), $57 \mathrm{~S}$.

Döbbeler, H., 2004. Simulation und Bewertung von Nutzungsstrategien unter heutigen und veränderten Klimabedingungen mit dem Wuchsmodell SILVA 2.2. Dissertation, Fakultät für Forstwissenschaften und Waldökologie der Georg-August-Universität Göttingen, $494 \mathrm{~S}$.

Dunger, K., Wolfgang, S., Oehmichen, K., Riedel, T., Ziche, D., Grüneberg, E., Wellbrock, N., 2014. Kap. 7.2: Wälder. In: Umweltbundesamt (Hrsg.): Inventarbericht zum Deutschen Treibhausgasinventar 1990 - 2012, 524-571 
Erteld, W., 1962. Wachstumsgang und Vorratsbehandlung der Eiche im norddeutschen Diluvium. Archiv für Forstwesen, 11, 1155-1176

Evers, J., Dammann, I., Noltensmeier, A., Nagel, R.-V., 2008. Auswirkungen von Bodenschutzkalkungen auf Buchenwälder (Fagus sylvatica L.). In: Nordwestdeutsche Forstliche Versuchsanstalt (Hrsg.): Ergebnisse angewandter Forschung zur Buche. Beiträge aus der Nordwestdeutschen Forstlichen Versuchsanstalt, Universitätsverlag Göttingen, $3,21-50$

Evers, J., Paar, U., Schulze, A., 2011. Kohlenstoffspeicherung in hessischen Waldböden Ergebnisse der Bodenzustandserhebung II. In: Nordwestdeutsche Forstliche Versuchsanstalt (Hrsg.): Waldzustandsbericht 2011 für Hessen, 23-25

Evers, J., Paar, U., 2012. Bodenzustandserhebungen (BZE I und BZE II) - Wie hat sich der Bodenzustand in Niedersachsens Waldböden verändert? In: Nordwestdeutsche Forstliche Versuchsanstalt (Hrsg.): Waldzustandsbericht 2012 für Niedersachsen, 22-28

Evers, J., 2015. Kohlenstoff und Bodenversauerung. Wie verändern sich die Waldböden in Sachsen-Anhalt? Ergebnisse der Bodenzustandserhebung. Beitrag zu: Internationales Jahr des Bodens 2015: Schwarzerde und Co. Die Böden Sachsen-Anhalts unter Beobachtung. 25. bis 26. November 2015, Halle. URL: http://www.lagb.sachsen-anhalt.de/fileadmin/Bibliothek/LaGB/aktuelles/ tagung_schwarzerde/2015_1125_Kohlenstoff_Bodenversauerung.pdf, aufgerufen am 22.01 .2016

Evers, J., 2015a. Vorräte organischer Substanz in Waldböden. Umweltbericht Niedersachsen 2015 URL: http://www.umwelt.niedersachsen. de/umweltbericht/boden/vorraete_organischer_substanz_waldboeden/

vorraete-organischer-substanz-in-waldboeden-139271.html, aufgerufen am 17.02.2016

Fahrmeir, L., Kneib, T., Lang, S., 2009. Regression: Modelle, Methoden und Anwendungen. 2. Aufl., Springer, Berlin, 502 S.

FAO, 2010. Global Forest Ressource Assessment 2010. Food and Agriculture Organization of the United Nations, Rome, FAO Forestry Paper 163, 378 S.

FAO, 2012. Global forest land-use change from 1990 to 2005. Food and Agriculture Organization of the United Nations, Rome, FAO Forestry Paper 169, 53 S.

Fleck, S., Meesenburg, H., 2015. Klimawandel und Nachhaltiges Landmanagement im norddeutschen Tiefland. In: Nordwestdeutsche Forstliche Versuchanstalt (Hrsg.): Waldzustandsbericht 2015 für Niedersachsen, 36-38

Franz, F., 1965. Ermittlung von Schätzwerten der natürlichen Grundfläche mit Hilfe ertragskundlicher Bestimmungsgrößen des verbleibenden Bestandes. Forstwissenschaftliches Centralblatt, 84, 357-386 
Frühwald, A., Diederichs, S., Morgan, R.M., 2010. Verwendungspotentiale heben durch Kaskadennutzung am Beispiel Holz. In: Thomé-Kozmiensky, K.J., Beckmann, M. (Hrsg.): Erneuerbare Energien, Band 4 - Biomasse und Biogas, Ersatzbrennstoffe, Solar- und Windenergie. TK Verlag, Neuruppin, 37-50

Fürstenau, C., Badeck, F., Lasch, P., Lexer, M., Lindner, M., Mohr, P., Suckow, F., 2007. Multiple-use forest management in consideration of climate change and the interests of stakeholder groups. European Journal of Forest Research, 126, 225-239

Fürstenau, C., 2008. The impact of silvicultural strategies and climate change on carbon sequestration and other forest ecosystem functions. Dissertation, MathematischNaturwissenschaftliche Fakultät der Universität Potsdam, 153 S.

Gadow, K. v., 2005. Forsteinrichtung: Analyse und Entwurf der Waldentwicklung. Universitätsverlag Göttingen, $342 \mathrm{~S}$.

Grothendieck, G., 2010. nls2: Non-linear regression with brute force. R package version $0.1-3$

Grüneberg, E., Ziche, D., Wellbrock, N., 2014. Organic carbon stocks and sequestration rates of forest soils in Germany. Global Change Biology, 20, 2644-2662

Grüneberg, E., Höhle, J., Ziche, D., Wellbrock, N., 2015. Kohlenstoffspeicherung in Deutschlands Waldböden. Johann Heinrich von Thünen-Institut, Braunschweig, 6 S.

Hansen, J., Ludwig, A., Spellmann, H., Nagel, J., Möhring, B., von Lüpke, N., SchmidtWalter, P., 2008. Rohholzpotentiale und ihre Verfügbarkeit in Hessen. Nordwestdeutsche Forstliche Versuchsanstalt, $47 \mathrm{~S}$.

Hansen, J., 2012. Modellbasierte Entscheidungsunterstützung für den Forstbetrieb: Optimierung kurzfristiger Nutzungsoptionen und mittelfristiger Strategien unter Verwendung metaheuristischer Verfahren und parallelen Rechnens. Dissertation, Fakultät für Forstwissenschaften und Waldökologie der Georg-August-Universität Göttingen, 216 S.

Hansen, J., Nagel, J., 2014. Waldwachstumskundliche Softwaresysteme auf Basis von TreeGrOSS - Anwendung und theoretische Grundlagen. Beiträge aus der Nordwestdeutschen Forstlichen Versuchsanstalt, Universitätsverlag Göttingen, 11, 224 S.

Hartig, G.L., 1804. Anweisung zur Taxation und Beschreibung der Forste. Gießen und Darmstadt, bey Georg Friedrich Heyer, 208 S.

Hartmann, H., 2009. Grundlagen der thermo-chemischen Umwandlung biogener Festbrennstoffe. In: Kaltschmitt, M., Hartmann, H., Hofbauer, H. (Hrsg.): Energie aus Biomasse. 2., neu bearb. und erw. Aufl., korrigierter Nachdr., Springer, Berlin, 333461 
Hasenauer, H., 2011. Überlegungen zur $\mathrm{CO}_{2}$-Bilanz von Waldökosystemen. Austrian Journal of Forest Science, 128, 33-52

Heinsdorf, D., Krauß, H.H., 1990. Schätztafeln für Trockenmasse und Nährstoffspeicherung von Kiefernbeständen. Institut für Forstwissenschaften Eberswalde, Abt. Information (IFE), $77 \mathrm{~S}$.

Heyer, C., 1841. Die Waldertrags-Regelung. Ferber, Gießen, 264 S.

Höllerl, S., Neuner, M., 2011. Kohlenstoffbilanz des Wald- und Holzsektors bewirtschafteter und unbewirtschafteter Bergmischwälder der Bayerischen Alpen. Forstarchiv, 82, $142-154$

Höllerl, S., Bork, J., 2013. Die Kohlenstoffspeicherung von bewirtschafteten und unbewirtschafteten Fichtenbeständen unter Berücksichtigung von Ausfallrisiken - Aussagen nicht nur über Bestände der montanen Zone. Forstarchiv, 84, 52-64

Ilg, S., 2002. Erhebung der oberirdischen Biomasse- und Elementvorräte eines Fichtenbestandes (Picea abies (L.) Karst) im Bereich der Waldklimastation Zusmarshausen. Masterarbeit, Fachhochschule Weihenstephan, Fachbereich Forstwirtschaft, 98 S.

IPCC, 2000. Land Use, Land-Use Change, and Forestry: A Special Report of the Intergovernmental Panel on Climate Change. Cambridge University Press, 377 S.

IPCC, 2007. Climate Change 2007: Synthesis Report. Contribution of Working Groups I, II and III to the Fourth Assessment Report of the Intergovernmental Panel on Climate Change. Intergovernmental Panel on Climate Change, Geneva, Switzerland, 104 S.

IPCC, 2013. Klimaänderung 2013: Wissenschaftliche Grundlagen. Zusammenfassung für politische Entscheidungsträger. Cambridge University Press, 36 S.

Jacobsen, C., Rademacher, P., Meesenburg, H., Meiwes, K.J., 2003. Gehalte chemischer Elemente in den Baumkompartimenten - Literaturstudie und Datensammlung. Forschungszentrum Waldökosysteme der Universität Göttingen, 96, 81 S.

Jandl, R., Lindner, M., Vesterdal, L., Bauwens, B., Baritz, R., Hagedorn, F., Johnson, D., Minkkinen, K., Byrne, K., 2007. How strongly can forest management influence soil carbon sequestration? Geoderma, 137, 253-268

Jarvis, P.G., Ibrom, A., Linder, S., 2005. Carbon forestry: managing forests to conserve carbon. In: Griffiths, H., Jarvis, P.G. (Hrsg.): The Carbon Balance of Forest Biomes. Taylor \& Francis Group, Oxford, 331-349

Joosten, R., Schumacher, J., Wirth, C., Schulte, A., 2004. Evaluating tree carbon predictions for beech (Fagus sylvatica L.) in western Germany. Forest Ecology and Management, 189, 87-96 
Kahl, T., 2003. Abbauraten von Fichtentotholz (Picea abies (L.) Karst.) - Bohrwiderstandsmessungen als neuer Ansatz zur Bestimmung des Totholzabbaus, einer wichtigen Größe im Kohlenstoffhaushalt mitteleuropäischer Wälder. Magisterarbeit, FriedrichSchiller-Universität Jena, 98 S.

Kira, T., Ogawa, H., Sakazaki, N., 1953. Intraspecific competition among higher plants. I. Competition-yield-density interrelationship in regularly dispersed populations. Journal of the Institute of Polytechnics, Osaka City University, D4, 1-16

Klein, D., Schulz, C., 2012. Die Kohlenstoffbilanz der bayerischen Forst- und Holzwirtschaft. Bayerische Landesanstalt für Wald und Forstwirtschaft, Freising, 184 S.

Knauf, M., Frühwald, A., Köhl, M., 2013. Beitrag des NRW Clusters ForstHolz zum Klimaschutz. Landesbetrieb Wald und Holz Nordrhein-Westfalen, Münster, 200 S.

Knigge, W., Schulz, H., 1966. Grundriss der Forstbenutzung. Parey, Hamburg, 584 S.

Knohl, A., Schulze, E., Kolle, O., Buchmann, N., 2003. Large carbon uptake by an unmanaged 250-year-old deciduous forest in Central Germany. Agricultural and Forest Meteorology, 118, 151-167

Knoke, T., Schneider, T., Hahn, A., Griess, V.C., Rößiger, J., 2012. Forstbetriebsplanung als Entscheidungshilfe. Ulmer, Stuttgart, 408 S.

Koenker, R., 2012. quantreg: Quantile Regression. R package version 4.79

Köhl, M., Stümer, W., Kenter, B., Riedel, T., 2008. Effect of the estimation of forest management and decay of dead woody material on the reliability of carbon stock and carbon stock changes - A simulation study. Forest Ecology and Management, 256, $229-236$

Köhl, M., Frühwald, A., Kenter, B., Olschofsky, K., Köhler, R., Köthke, M., Rüter, S., Pretzsch, H., Rötzer, T., Makeschin, F., Abiy, M., Dieter, M., 2009. Potenzial und Dynamik der Kohlenstoffspeicherung in Wald und Holz: Beitrag des deutschen Forstund Holzsektors zum Klimaschutz. In: Seintsch, B., Dieter, M. (Hrsg.): Waldstrategie 2020. Tagungsband zum Symposium des BMELV, 10.-11. Dez. 2008, Berlin. Landbauforschung - vTI Agriculture and Forestry Research. Sonderheft 327, Johann Heinrich von Thünen Institut, Braunschweig, 103-109

Köhl, M., 2013. Klimaschutz - Aus der Sicht der Forstwissenschaft - Umweltgerechte Waldnutzung im Diskurs. AFZ-Der Wald, 17, 34-36

Kownatzki, D., Kriebitzsch, W.U., Bolte, A., Liesebach, H., Schmitt, U., Elsasser, P., 2011. Zum Douglasienanbau in Deutschland. Landbauforschung - vTI Agriculture and Forestry Research. Sonderheft 344, Johann Heinrich von Thünen Institut, Braunschweig, $78 \mathrm{~S}$. 
Kramer, H., 1988. Waldwachstumslehre. Parey, Hamburg, 374 S.

Kramer, H., Akca, A., 2008. Leitfaden zur Waldmesslehre. 5. überarb. Aufl., Sauerländer, Frankfurt am Main, 226 S.

Krug, J., Köhl, M., Kownatzki, D., 2012. Revaluing unmanaged forests for climate change mitigation. Carbon Balance and Management, 7:11, $8 \mathrm{~S}$.

KrWG, 2012. Gesetz zur Förderung der Kreislaufwirtschaft und Sicherung der umweltverträglichen Bewirtschaftung von Abfällen (Kreislaufwirtschaftsgesetz - KrWG) vom 24. Februar 2012 (BGBl. I S. 212), das zuletzt durch § 44 Absatz 4 des Gesetzes vom 22. Mai 2013 (BGBl. I S. 1324) geändert worden ist. URL: http: //www.gesetze-im-internet.de/krwg/, aufgerufen am 30.11.2015

Laiho, R., Prescott, C., 2004. Decay and nutrient dynamics of coarse woody debris in northern coniferous forests: a synthesis. Canadian Journal of Forest Research, 34, 763-777

Lembcke, G., Knapp, E., Dittmar, O., 2000. Ertragstafel für die Kiefer (Pinus sylvestris L.) im nordostdeutschen Tiefland. Landesforstanstalt Eberswalde, 107 S.

Liski, J., Palosuo, T., Peltoniemi, M., Sievänen, R., 2005. Carbon and decomposition model Yasso for forest soils. Ecological Modelling, 189, 168-182

Lorenz, K., Lal, R., 2010. Carbon Sequestration in Forest Ecosystems. Springer, Dordrecht, $279 \mathrm{~S}$.

Luyssaert, S., Inglima, I., Jung, M., Richardson, A.D., Reichstein, M., Papale, D., Piao, S.L., Schulze, E.D., Wingate, L., Matteucci, G., Aragao, L., Aubinet, M., Beer, C., Bernhofer, C., Black, K.G., Bonal, D., Bonnefond, J.M., Chambers, J., Ciais, P., Cook, B., Davis, K.J., Dolman, A.J., Gielen, B., Goulden, M., Grace, J., Granier, A., Grelle, A., Griffis, T., Grünewald, T., Guidolotti, G., Hanson, P.J., Harding, R., Hollinger, D.Y., Hutyra, L.R., Kolari, P., Kruijt, B., Kutsch, W., Lagergren, F., Laurila, T., Law, B.E., Le Maire, G., Lindroth, A., Loustau, D., Malhi, Y., Mateus, J., Migliavacca, M., Misson, L., Montagnani, L., Moncrieff, J., Moors, E., Munger, J.W., Nikinmaa, E., Ollinger, S.V., Pita, G., Rebmann, C., Roupsard, O., Saigusa, N., Sanz, M.J., Seufert, G., Sierra, C., Smith, M.L., Tang, J., Valentini, R., Vesala, T., Janssens, I.A., 2007. $\mathrm{CO}_{2}$ balance of boreal, temperate, and tropical forests derived from a global database. Global Change Biology, 13, 2509-2537

Luyssaert, S., Schulze, E.D., Börner, A., Knohl, A., Hessenmöller, D., Law, B., Ciais, P., Grace, J., 2008. Old-growth forests as global carbon sinks. Nature, 455, 213-215

Luyssaert, S., Ciais, P., Piao, S.L., Schulze, E.D., Jung, M., Zaehle, S., Schelhaas, M.J., Reichstein, M., Churkina, G., Papale, D., Abril, G. Beer, C., Grace, J., Loustau, D., 
Matteucci, G., Magnani, F., Nabuurs, G.J. Verbeeck, H., Sulkava, M., Werf, G.R. v. d., Janssens, I.A., 2010. The European carbon balance. Part 3: forests. Global Change Biology, 16, 1429-1450

Mackensen, J., Bauhus, J., Webber, E., 2003. Decomposition rates of coarse woody debris - A review with particular emphasis on Australian tree species. Australian Journal of Botany, 51, 27-37

Mantau, U., 2004. Holzrohstoffbilanz für Deutschland - Holzrohstoffaufkommen und dessen Verwendung im Jahr 2002. Holz-Zentralblatt, 76, 1026-1028

Mantau, U., 2008. Holzrohstoffbilanz Deutschland, Szenarien des Holzaufkommens und der Holzverwendung bis 2012. Hamburg, $79 \mathrm{~S}$.

Mantau, U., 2012. Holzrohstoffbilanz Deutschland, Entwicklungen und Szenarien des Holzaufkommens und der Holzverwendung von 1987 bis 2015. Hamburg, 65 S.

Mantau, U., 2015a. Verbundvorhaben Sicherung der Nadelrohholzversorgung in Norddeutschland; Teilvorhaben 1b: Nadelholz-Bedarfsanalyse der Holzindustrie in Norddeutschland - Regionale Holzrohstoffbilanz. Schlussbericht Nordwestdeutsche Forstliche Versuchsanstalt und Informationssysteme für Rohstoffe, Hamburg, 42 S.

Mantau, U., 2015b. Die überragende Bedeutung des Nadelholzes - der Laubholz-Irrweg. AFZ-Der Wald, 17, 24-26

Mayer, H., 1992. Waldbau auf soziologisch-ökologischer Grundlage. 4., tlw. neu bearb. Aufl., G. Fischer, Stuttgart, 522 S.

MCPFE, 1993. Helsinki Resolution H1: General Guidelines for the Sustainable Management of Forests in Europe. URL: http://www.foresteurope.org/docs/MC/MC helsinki_resolutionH1.pdf, aufgerufen am 07.03.2013

MCPFE, 2003. State of Europe's Forests 2003: The MCPFE Report on Sustainable Forest Management in Europe. Ministerial Conference on the Protection of Forests in Europe, Wien, $126 \mathrm{~S}$.

MCPFE, 2011. State of Europe's Forests 2011: Status and Trends in Sustainable Forest Management in Europe. Forest Europe, UNECE and FAO, Oslo, 344 S.

Merker, K., 2015. Wald in guten Händen. AFZ-Der Wald, 18, 13-15

Meyer, P., Menke, N., Nagel, J., Hansen, J., Kawaletz, H., Paar, U., Evers, J., 2009. Abschlussbericht des von der Deutschen Bundesstiftung Umwelt geförderten Projekts: Entwicklung eines Managementmoduls für Totholz im Forstbetrieb. Nordwestdeutsche Forstliche Versuchsanstalt (Hrsg.), 110 S. 
ML, 2013. Langfristige, ökologische Waldentwicklung in den Niedersächsischen Landesforsten (LÖWE-Erlass). Niedersächsisches Ministerialblatt, RdErl. 27. 2. 2013, $214-220$

ML, 2014. Der Wald in Niedersachsen - Ergebnisse der Bundeswaldinventur 3. Niedersächsisches Ministerium für Ernährung, Landwirtschaft und Verbraucherschutz (Hrsg.), 55 S.

Monni, S., Peltoniemi, M., Palosuo, T., Lehtonen, A., Mäkipää, R., Savolainen, I., 2007. Uncertainty of forest carbon stock changes - implications to the total uncertainty of GHG inventory of Finland. Climatic Change, 81, 391-413

Mund, M., Kummetz, E., Hein, M., Bauer, G., Schulze, E.D., 2002. Growth and carbon stocks of a spruce forest chronosequence in central Europe. Forest Ecology and Management, 171, 275-296

Mund, M., Profft, I., Wutzler, T., Schulze, E.D., Weber, G., Weller, E., 2006. Vorbereitungen auf eine laufende Fortschreibung der Kohlenstoffvorräte in den Wäldern Thüringens. Thüringer Landesanstalt für Wald, Jagd und Fischerei, 26, 128 S.

Mund, M., Schulze, E.D., 2006. Impacts of forest management on the carbon budget of European beech (Fagus sylvatica) forests. Allgemeine Forst- und Jagdzeitung, 177, 47-63

Mund, M., Frischbier, N., Profft, I., Raacke, J., Richter, F., Ammer, C., 2015. Klimaschutzwirkung des Wald- und Holzsektors: Schutz- und Nutzungsszenarien für drei Modellregionen in Thüringen. BfN-Skripten 396, Bundesamt für Naturschutz, Bonn, $168 \mathrm{~S}$.

Nabuurs, G., Thürig, E., Heidema, N., Armolaitis, K., Biber, P., Cienciala, E., Kaufmann, E., Mäkipää, R., Nilsen, P., Petritsch, R., Pristova, T., Rock, J., Schelhaas, M.J., Sievanen, R., Somogyi, Z., Vallet, P., 2008. Hotspots of the European forests carbon cycle. Forest Ecology and Management, 256, 194-200

Nagel, J., 1997. Bwin Programm zur Bestandesanalyse und Prognose. Handbuch zur Version 3.0. Niedersächsische Forstliche Versuchsanstalt Göttingen

Nagel, J., 1999. Konzeptionelle Überlegungen zum schrittweisen Aufbau eines waldwachstumskundlichen Simulationssystems für Nordwestdeutschland. Schriften aus der Forstlichen Fakultät der Universität Göttingen und der Niedersächsischen Forstlichen Versuchsanstalt, 128, Sauerländer, Frankfurt am Main, 122 S.

NFP, 2009a. Technische Anweisung zur Bestandesinventur. Niedersächsisches Forstplanungsamt (Hrsg.), $78 \mathrm{~S}$. 
NFP, 2009b. Technische Anweisung zur Betriebsinventur. Niedersächsisches Forstplanungsamt (Hrsg.), $68 \mathrm{~S}$.

NFP, 2012. Forsteinrichtung in den Niedersächsischen Landesforsten. Niedersächsisches Forstplanungsamt (Hrsg.), $48 \mathrm{~S}$.

NFP, 2013. Informationen zur Betriebsinventur. Niedersächsisches Forstplanungsamt (Hrsg.), 11 S.

NLF, 2005. Wertästung. Niedersächsische Landesforsten (Hrsg.), 26 S.

NLF, 2011. 20 Jahre langfristige ökologische Waldentwicklung. Das LÖWE-Programm. Niedersächsische Landesforsten (Hrsg.), 32 S.

NLF, 2014a. Die Wälder der Landesforsten in Zahlen - Ergebnisse der Bundeswaldinventur 3. Niedersächsische Landesforsten (Hrsg.), 5 S.

NLF, 2014b. Entscheidungshilfen zur Begründung und Behandlung von Stiel- und Traubeneichen. Niedersächsische Landesforsten (Hrsg.), 11 S.

NLF, 2014c. Entscheidungshilfen zur Behandlung und Entwicklung von Buchenbeständen. Niedersächsische Landesforsten (Hrsg.), 12 S.

NLF, 2014d. Entscheidungshilfen zur Behandlung und Entwicklung von Fichtenbeständen. Niedersächsische Landesforsten (Hrsg.), 19 S.

NLF, 2014e. Entscheidungshilfen zur Behandlung und Entwicklung von Kiefernbeständen. Niedersächsische Landesforsten (Hrsg.), 14 S.

NLF, 2015. Entscheidungshilfen zur Bewirtschaftung von Douglasienbeständen. Niedersächsische Landesforsten (Hrsg.), 10 S.

NOAA, 2016. Trends in Atmospheric Carbon Dioxide. URL: http://www.esrl.noaa.gov/ gmd/ccgg/trends/\#global, aufgerufen am 19.01.2016

Nultsch, W., 2001. Allgemeine Botanik. 11., völlig neubearb. und erw. Aufl., Thieme, Stuttgart, 663 S.

NW-FVA, 2013a. Der Waldplaner 2.0. URL: http://www.nw-fva.de/?id=216, aufgerufen am 11.02.2013

NW-FVA (Hrsg.), 2013b. Waldentwicklungsszenarien für das Hessische Ried Entscheidungsunterstützung vor dem Hintergrund sich beschleunigt ändernder Wasserhaushalts- und Klimabedingungen und den Anforderungen aus dem europäischen Schutzgebietssystem Natura 2000. Beiträge aus der Nordwestdeutsche Forstliche Versuchsanstalt, Universitätsverlag Göttingen, 10, 398 S. 
NWaldLG, 2011. Niedersächsisches Gesetz über den Wald und die Landschaftsordnung (NWaldLG). Vom 21. März 2002. Nds. GVBl. 2002, 112, letzte berücksichtigte Änderung: $\S 43$ geändert durch Artikel 16 des Gesetzes vom 13.10.2011 (Nds. GVBl. S. 353)

Oehmichen, K., Demant, B., Dunger, K., Grüneberg, E., Hennig, P., Kroiher, F., Neubauer, M., Polley, H., Riedel, T., Rock, J., Schwitzgebel, F., Stümer, W., Wellbrock, N., Ziche, D., Bolte, A., 2011. Inventurstudie 2008 und Treibhausgasinventar Wald. Landbauforschung - vTI Agriculture and Forestry Research. Sonderheft 343, Johann Heinrich von Thünen-Institut, Braunschweig, 164 S.

Oesten, G., Roeder, A., 2012. Management von Forstbetrieben. Band 1. Grundlagen, Betriebspolitik. 3. Aufl., Institut für Forstökonomie der Universität Freiburg, 388 S.

Ostwald, R., 1931. Grundlagen einer Waldrententheorie, d.h. einer im Anhalt an das relative Waldrenten-Maximum entwickelten forstlichen Reinertragstheorie. Verlag W. F. Häcker, Riga

Otto, H.J., 1972. Die Ergebnisse der Standortskartierung im pleistozänen Flachland Niedersachsens - Grundlage waldbaulicher Vorstellungen. Aus dem Walde - Mitteilungen aus der Niedersächsischen Landesforstverwaltung, Niedersächsisches Ministerium für Ernährung, Landwirtschaft und Forsten (Hrsg.), 19, 191 S.

Otto, H.J., 1989. Langfristige, ökologische Waldbauplanung für die Niedersächsischen Landesforsten - Band 1. Aus dem Walde - Mitteilungen aus der Niedersächsischen Landesforstverwaltung, Niedersächsisches Ministerium für Ernährung, Landwirtschaft und Forsten (Hrsg.), 42, $442 \mathrm{~S}$.

Otto, H.J., 1991. Langfristige, ökologische Waldbauplanung für die Niedersächsischen Landesforsten - Band 2. Aus dem Walde - Mitteilungen aus der Niedersächsischen Landesforstverwaltung, Niedersächsisches Ministerium für Ernährung, Landwirtschaft und Forsten (Hrsg.), 43, 527 S.

Otto, H.J., 1994. Waldökologie. Ulmer, Stuttgart, 391 S.

Otto, H.J., Wagner, S., Schüler, G., 2007. Standortansprüche der wichtigsten Waldbaumarten. 9., überarb. Aufl., AID-Infodienst, Bonn, 47 S.

Overbeck, M., Schmidt, M., Fischer, C., Evers, J., Schulze, A., Hövelmann, T., Spellmann, H., 2011. Ein statistisches Modell zur Regionalisierung der nutzbaren Feldkapazität von Waldstandorten in Niedersachsen. Forstarchiv, 82, 92-100

Pan, Y., Birdsey, R., Fang, J., Houghton, R., Kauppi, P., Kurz, W., Phillips, O., Shvidenko, A., Lewis, S., Canadell, J., et al., 2011. A Large and Persistent Carbon Sink in the World's Forests. Science, 19, 988-993 
Peltoniemi, M., Palosuo, T., Monni, S., Mäkipää, R., 2006. Factors affecting the uncertainty of sinks and stocks of carbon in Finnish forests soils and vegetation. Forest Ecology and Management, 232, 75-85

Pinheiro, J., Bates, D., DebRoy, S., Sarkar, D., 2011. nlme: Linear and Nonlinear Mixed Effects Models. R package version 3.1-102

Pistorius, T., Zell, J., 2005. Die Dynamik der Kohlenstoffvorräte in Baden-Württemberg zwischen 1987 und 2002. Veränderungen der Waldbiomasse und Modellierung der Holzproduktspeicher. Allgemeine Forst- und Jagdzeitung, 176, 111-119

Pistorius, T., Zell, J., Hartebrodt, C., 2006. Untersuchungen zur Rolle des Waldes und der Forstwirtschaft im Kohlenstoffhaushalt des Landes Baden-Württemberg. Forschungsbericht FZKA-BWPLUS. Forstliche Versuchs- und Forschungsanstalt BadenWürttemberg, Abt. Forstökonomie, Freiburg, 182 S.

Pistorius, T., 2007a. Die Bedeutung von Kohlenstoffbilanzen im Diskurs über die Einbindung der Forstwirtschaft in die nationale Klimapolitik. Dissertation, Fakultät für Forst- und Umweltwissenschaften der Albert-Ludwigs-Universität zu Freiburg im Breisgau, $255 \mathrm{~S}$.

Pistorius, T., 2007b. Kohlenstoffspeicherung in Wald und Holzprodukten. Forstliche Versuchsanstalt Baden-Württemberg, Abt. Forstökonomie, Freiburg, 10 S.

Polley, H., Henning, P., Kroiher, F., 2009. Baumarten, Altersstruktur und Totholz in Deutschland. AFZ-Der Wald, 20, 1074-1075

Pregitzer, K., Euskirchen, E., 2004. Carbon cycling and storage in world forests: biome patterns related to forest age. Global Change Biology, 10, 2052-2077

Pretzsch, H., 2003. Strategische Planung der Nachhaltigkeit auf Forstbetriebsebene. Beitrag der Waldwachstumsforschung. Forstwissenschaftliches Centralblatt, 122, 231249

Pretzsch, H., Pott, M., 2004. Strategische Planung der Nachhaltigkeit auf Forstbetriebsebene - Beitrag der Waldwachstumsforschung. In: Deutsche Bundesstiftung Umwelt (Hrsg.): Nachhaltige und multifunktionale Forstwirtschaft: ein Verfahrens- und Zustandsvergleich für verschiedene Testregionen. E. Schmidt, Berlin, 233-254

Pretzsch, H., 2009. Forest Dynamics, Growth and Yield: From Measurement to Model. Springer, Berlin, $664 \mathrm{~S}$.

Pretzsch, H., Block, J., Dieler, J., Dong, P., Kohnle, U., Nagel, J., Spellmann, H., Zingg, A., 2010. Comparison between the productivity of pure and mixed stands of Norway spruce and European beech along an ecological gradient. Annals of Forest Science, 67, 712 
Pretzsch, H., Bielak, K., Block, J., Bruchwald, A., Dieler, J., Ehrhart, H.P., Kohnle, U., Nagel, J., Spellmann, H., Zasada, M., Zingg, A., 2013. Productivity of mixed versus pure stands of oak (Quercus petraea (Matt.) Liebl. and Quercus robur L.) and European beech (Fagus sylvatica L.) along an ecological gradient. European Journal of Forest Research, 132, 263-280

Pretzsch, H., Schütz, G., 2016. Effect of tree species mixing on the size structure, density, and yield of forest stands. European Journal of Forest Research, 135, 1-22

Profft, I., Mund, M., Weber, G., Weller, E., Schulze, E.D., 2009. Forest management and carbon sequestration in wood products. European Journal of Forest Research, $128,399-413$

R Core Team, 2015. R: A Language and Environment for Statistical Computing. R Foundation for Statistical Computing, Vienna, Austria. URL: https://www.R-project. org/

R Development Core Team, 2011. R: A Language and Environment for Statistical Computing. R Foundation for Statistical Computing, Vienna, Austria, URL: http: //www.R-project.org/

R Development Core Team, 2012. R: A Language and Environment for Statistical Computing. R Foundation for Statistical Computing, Vienna, Austria. URL: http: //www.R-project.org/

Rademacher, P., Khanna, P., Eichhorn, J., Guericke, M., 2009. Tree Growth, Biomass, and Elements in Tree Components of Three Beech Sites. In: Brumme, R., Khanna, P.K. (Hrsg.): Functioning and Management of European Beech Ecosystems. Ecological Studies 208, Springer, Berlin, 105-136

Reineke, L., 1933. Perfecting a stand-density index for even-aged forest. Journal of Agricultural Research, 46, 627-638

Riechsteiner, D., Lemm, R., 2009. Grundlagen zur Konzeption einer zeitgemässen forstlichen Betriebsplanung. In: Thees, O., Lemm, R. (Hrsg.): Management zukunftsfähige Waldnutzung. Grundlagen, Methoden und Instrumente. 1. Aufl., Eidg. Forschungsanstalt für Wald, Schnee und Landschaft. vdf Hochschulverlag, Zürich, 321-359

Rigby, R.A., Stasinopoulos, D.M., 2005. Generalized additive models for location, scale and shape (with discussion). Applied Statistics, 54, 507-554

Robinson, D., 2007. Implications of a large global root biomass for carbon sink estimates and for soil carbon dynamics. Proceedings of the Royal Society B: Biological Sciences, 274, 2753-2759 
Rock, J., 2008. Klimaschutz und Kohlenstoff in Holz : Vergleich verschiedener Strategien. Dissertation, Mathematisch-Naturwissenschaftliche Fakultät der Universität Potsdam, $162 \mathrm{~S}$.

Rock, J., Badeck, F., Harmon, M., 2008. Estimating decomposition rate constants for European tree species from literature sources. European Journal of Forest Research, $127,301-313$

Rock, J., Bolte, A., 2011. Auswirkungen der Waldbewirtschaftung 2002 bis 2008 auf die $\mathrm{CO}_{2}$-Bilanz. AFZ-Der Wald, 66, 22-24

Röhrig, E., Bartsch, N., 1992. Waldbau auf ökologischer Grundlage - Der Wald als Vegetationsform und seine Bedeutung für den Menschen. 6., völlig neu bearb. Aufl., Parey, Hamburg, 350 S.

Röhrig, E., Bartsch, N., von Lüpke, B., 2006. Waldbau auf ökologischer Grundlage. 7., vollst. aktual. Aufl., UTB, Stuttgart, 479 S.

Rumpf, S., Nagel, J., Schmidt, M., 2012. Biomasseschätzfunktionen von Fichte (Picea abies L.), Kiefer (Pinus sylvestris L.), Buche (Fagus sylvatica L.), Eiche (textitQuercus robur und petraea L.) und Douglasie (Pseudotsuga menziesii L.) für Nordwestdeutschland. In: Nordwestdeutsche Forstliche Versuchsanstalt (Hrsg.): Abschlussbericht des Projektes Möglichkeiten und Grenzen der Vollbaumnutzung. Fachagentur Nachwachsende Rohstoffe e.V., FKZ 22015407, 25-124

Russ, A., Rieck, W., Martin, J., 2011. Zustand und Wandel der Böden MecklenburgVorpommerns. Mitteilungen aus dem Forstlichen Versuchswesen MecklenburgVorpommern, 9, $180 \mathrm{~S}$.

Rüter, S., 2010. Einbeziehung von Holzprodukten in die Klimapolitik. Holz-Zentralblatt, $25,623-624$

Rüter, S., 2011. Welchen Beitrag leisten Holzprodukte zur $\mathrm{CO}_{2}$-Bilanz? AFZ-Der Wald, $15,15-18$

Rüther, B., Hansen, J., Ludwig, A., Spellmann, H., Nagel, J., Möhring, B., Dieter, M., 2007. Clusterstudie Forst und Holz Niedersachsen. Beiträge aus der Nordwestdeutschen Forstlichen Versuchsanstalt, Universitätsverlag Göttingen, 1, 92 S.

Rüther, B., Hansen, J., Ludwig, A., Spellmann, H., Nagel, J., Möhring, B., von Lüpke, N., Schmidt-Walter, P., Dieter, M., 2008a. Clusterstudie Forst und Holz SchleswigHolstein. Nordwestdeutsche Forstliche Versuchsanstalt (Hrsg.), 78 S.

Rüther, B., Hansen, J., Spellmann, H., Nagel, J., Möhring, B., Schmidt-Walter, P., Dieter, M., 2008b. Clusterstudie Forst und Holz Sachsen-Anhalt. Nordwestdeutsche Forstliche Versuchsanstalt (Hrsg.), 64 S. 
Sachs, L., Hedderich, J., 2006. Angewandte Statistik. 12., vollst. neu bearb. Aufl., Springer, Berlin, $702 \mathrm{~S}$.

Sathre, R., O'Connor, J., 2010. Meta-analysis of greenhouse gas displacement factors of wood product substitution. Environmental Science and Policy, 13, 104-114

Saugier, B., Roy, J., Mooney, H.A., 2001. Estimations of global terrestrial productivity: converging to a single number? In: Saugier, B., Roy, J., Mooney, H.A. (Hrsg.): Terrestrial global productivity. 1. Aufl., Academic Press, San Diego, 543-557

Schall, P., Ammer, C., 2013. How to quantify forest management intensity in Central European forests. European Journal of Forest Research, 132, 379-396

Schmithüsen, F., Kaiser, B., Schmidhauser, A., Mellinghoff, S., Kammerhofer, A.W. (Hrsg.), 2009. Unternehmerisches Handeln in der Wald- und Holzwirtschaft. 2., aktualisierte und erw. Aufl., Deutscher Betriebswirte-Verlag, Gernsbach, 610 S.

Schmitz, F., Polley, H., Hennig, P., Dunger, K., Schwitzgebel, F., 2008. Die zweite Bundeswaldinventur. Inventur- und Auswertungsmethoden. Zu den Bundeswaldinventuren 2001 bis 2002 und 1986 bis 1988. Johann Heinrich von Thünen-Institut, Arbeitsbericht, $85 \mathrm{~S}$.

Schober, R., 1995. Ertragstafeln wichtiger Baumarten bei verschiedener Durchforstung. 4. Aufl., Sauerländer, Frankfurt am Main, 166 S.

Schulze, E.D. (Hrsg.), 2000. Carbon and Nitrogen Cycling in European Forest Ecosystems. Springer, Berlin, $500 \mathrm{~S}$.

Schulze, E.D., Wirth, C., Heimann, M., 2002. Carbon Fluxes of the Eurosiberian Region. Environment Control in Biology, 40, 249-258

Schwarzmeier, M., 2000. Erhebung der oberirdischen Biomassevorräte von Fichtenbeständen (Picea abies (L.) Karst) im Bereich der Waldklimastationen Ebersberg und Flossenbürg. Masterarbeit, Fachhochschule Weihenstephan, Fachbereich Forstwirtschaft, $155 \mathrm{~S}$.

Seidl, R., Rammer, W., Jäger, D., Lexer, M., 2008. Impact of bark beetle (Ips typographus L.) disturbance on timber production and carbon sequestration in different management strategies under climate change. Forest Ecology and Management, 256, $209-220$

Shanin, V., Komarov, A., Mäkipää, R., 2014. Tree species composition affects productivity and carbon dynamics of different site types in boreal forests. European Journal of Forest Research, 133, 273-286 
Sloboda, B., Gaffrey, D., Matsu, 1993. Regionale und lokale Systeme von Höhenkurven für gleichaltrige Waldbestände. Allgemeine Forst- und Jagdzeitung, 164, 225-228

Speidel, G., 1972. Planung im Forstbetrieb: Grundlagen und Methoden der Forsteinrichtung. Parey, Hamburg, 267 S.

Spellmann, H., 1997. Ertragsentwicklung im LÖWE-Wald der Niedersächsischen Landesforstverwaltung. Forst und Holz, 24, 711-718

Spellmann, H., Nagel, J., Böckmann, T., 1999. Summarische Nutzungsplanung auf der Basis von Betriebsinventurdaten. Allgemeine Forst- und Jagdzeitung, 170, 122-128.

Spellmann, H., 2003. Sicherung einer nachhaltigen Waldentwicklung auf überbetrieblichen Ebenen. Forstwissenschaftliches Centralblatt, 122, 250-257

Spellmann, H., Kehr, I., Hillebrand, K., Schütze, C., 2004. Niedersächsische Beiträge. In: Deutsche Bundesstiftung Umwelt (Hrsg.): Nachhaltige und multifunktionale Forstwirtschaft: ein Verfahrens- und Zustandsvergleich für verschiedene Testregionen. E. Schmidt, Berlin, 283 S.

Spellmann, H., 2010. Waldbauliche Möglichkeiten zur Sicherung der Nadelrohholzversorgung. Vortrag im Rahmen der Tagung: Sicherung der Nadelrohholzversorgung, Kompetenznetz für Nachhaltige Holznutzung e. V., 12.11.2010, Göttingen URL: http: //www.kompetenznetz-holz.de/aktuelles/nadelholz/8_Spellmann.pdf, aufgerufen am 12.8.2012

Spellmann, H., 2013. Waldbauliche Konsequenzen aus einer veränderten Rohholznachfrage. AFZ-Der Wald, 9, 10-15

Spellmann, H., 2015. Es grünt so grün,...wenn Nachhaltigkeit uns leitet. AFZ-Der Wald, $18,20-23$

Spellmann, H., Döbbeler, H, Rudolph, J. 2015a. Entwicklung des NadelrohholzAngebotes in Norddeutschland. AFZ-Der Wald, 17, 16-19

Spellmann, H., Rudolph, J., Döbbeler, H., Nagel, J., 2015b. Verbundvorhaben: Sicherung der Nadelrohholzversorgung in Norddeutschland; Teilvorhaben 1a: Holzaufkommen und verwendungsorientierte Waldbauplanung für Nadelbaumarten. Schlussbericht der Nordwestdeutschen Forstlichen Versuchsanstalt, Fachagentur Nachwachsende Rohstoffe e. V., Förderkennzeichen: 22009510, 106 S.

Stasinopoulos, M., Rigby, B., Akantziliotou, C., 2012. Instructions on how to use the gamlss package in R - Second Edition, February 25, 2012. URL: http://www.gamlss. org/ 
Sterba, H., 1975. Assmanns Theorie der Grundflächenhaltung und die Competition Density - Rule der Japaner Kira, Ando und Tadaki. Centralblatt für das gesamte Forstwesen, 92, 46-62

Sterba, H., 1981. Natürlicher Bestockungsgrad und Reinekes SDI. Centralblatt für das gesamte Forstwesen, 98, 101-116

Sterba, H., 1987. Estimating potential density from thinning experiments and inventory data. Forest Science, 33, 1022-1034

Tadaki, Y., 1963. The pre-estimating of stem yield, based on the competition-density effect. Tokyo Government Forest Experiment Station Bulletin, 154, 1-19

Taverna, R., Hofer, P., Werner, F., Kaufmann, E., Thürig, E., 2007. $\mathrm{CO}_{2}$-Effekte der Schweizer Wald- und Holzwirtschaft. Szenarien zukünftiger Beiträge zum Klimaschutz. Umwelt-Wissen Nr. 0739. Bundesamt für Umwelt, Bern, 102 S.

Trähn, D., 2009. Altholz. In: Kaltschmitt, M., Hartmann, H., Hofbauer, H. (Hrsg.): Energie aus Biomasse. 2., neu bearb. und erw. Aufl., korrigierter Nachdr., Springer, Berlin, 143-184

UBA, 2014. Nationaler Inventarbericht zum Deutschen Treibhausgasinventar 1990 2012. Umweltbundesamt (Hrsg.), $965 \mathrm{~S}$.

UBA, 2015. UBA-Emissionsdaten 2014 zeigen Trendwende beim Klimaschutz. Umweltbundesamt, Presseinfo Nr. 14 vom 31.03.2015

VDP, 2007. Papier: die Zellstoff-, Holzstoff-, Papier- u. Pappenindustrie im Jahre 2007; ein Leistungsbericht. Verband Deutscher Papierfabriken e.V., Bonn

VDP, 2010. Papier: die Zellstoff-, Holzstoff-, Papier- u. Pappenindustrie im Jahre 2010; ein Leistungsbericht. Verband Deutscher Papierfabriken e.V., Bonn

VDP, 2011. Papier: ein Leistungsbericht. Verband Deutscher Papierfabriken e.V., Bonn VDP, 2012. Papier: ein Leistungsbericht. Verband Deutscher Papierfabriken e.V., Bonn

von Lüpke, B., Spellmann, H., 1997. Aspekte der Stabilität und des Wachstums von Mischbeständen aus Fichte und Buche als Grundlage für waldbauliche Entscheidungen. Forstarchiv, 68, 167-179

vTI, 2015. Ergebnisdatenbank der dritten Bundeswaldinventur. Johann Heinrich von Thünen-Institut. URL: https://bwi.info/start.aspx, aufgerufen am 23.11.2015

Vor, T., Spellmann, H., Bolte, A., Ammer, C. (Hrsg.), 2015. Potenziale und Risiken eingeführter Baumarten. Göttinger Forstwissenschaften, Universitätsverlag Göttingen, 7, 269 S. 
Wäldchen, J., Schulze, E.D., Schöning, I., Schrumpf, M., Sierra, C., 2013. The influence of changes in forest management over the past 200 years on present soil organic carbon stocks. Forest Ecology and Management, 289, 243-254

WBGU, 1998. Die Anrechnung biologischer Quellen und Senken im Kyoto-Protokoll: Fortschritt oder Rückschlag für den globalen Umweltschutz. Sondergutachten 1998, Wissenschaftlicher Beirat der Bundesregierung Globale Umweltveränderungen, Bremerhaven, $76 \mathrm{~S}$.

Weimar, H., 2014. Holzbilanzen 2012 und 2013 für die Bundesrepublik Deutschland. Thünen Working Paper 31. Johann Heinrich von Thünen-Institut, Hamburg, 37 S.

Weis, W., Göttlein, A., 2002. Vergleich von Biomasse, Elementgehalten und Elementvorräten von Fichte (Picea abies L. Karst.) und Buche (Fagus sylvatica L.) am Standort Höglwald zu Zeiten der Vegetationsruhe. Forstliche Forschungsberichte, München, 186, $163-167$

Wellbrock, N., Grüneberg, E., Ziche, D., Holzhausen, M., 2010. Die Bodenzustandserhebung im Wald (BioSoil/BZE) als Grundlage für den Bodenschutz insbesondere der C-Speicherfunktion. Beitrag zu: 6. Marktredwitzer Bodenschutztage am 06.10.2010

Wenk, G., Römisch, K., Gerold, D., 1984. DDR-Fichtenertragstafel. Agrarwissenschaftliche Gesellschaft der Deutschen Demokratischen Republik. TU Dresden, Sektion Forstwirtschaft Tharandt, Wissenschaftsbereich Forsteinrichtung und Forstliche Ertragskunde, Tharandt, $64 \mathrm{~S}$.

Wenk, G., Antanaitis, V., Smelko, S., 1990. Waldertragslehre. Deutscher Landwirtschaftsverlag, Berlin, 448 S.

West, P., 2009. Tree and forest measurement. 2. Aufl., Springer, Dordrecht, 192 S.

Wiedemann, E., 1935. Zur Klärung der Durchforstungsbegriffe. Zeitschrift für Forstund Jagdwesen, 67, 56-64

Wirth, C., Schulze, E.D., Schwalbe, G., Tomczyk, S., Weber, G.E., Weller, E., 2004a. Dynamik der Kohlenstoffvorräte in den Wäldern Thüringens. Thüringer Landesanstalt für Wald, Jagd und Fischerei, $308 \mathrm{~S}$.

Wirth, C., Schumacher, J., Schulze, E.D., 2004b. Generic biomass functions for Norway spruce in Central Europe - A meta-analysis approach toward prediction and uncertainty estimation. Tree Physiology, 24, 121-139

WKF, 2015. Waldklimafonds. URL: http://www.waldklimafonds.de/, aufgerufen am 03.12 .2015 
Wood, S.N., 2011. Fast stable restricted maximum likelihood and marginal likelihood estimation of semiparametric generalized linear models. Journal of the Royal Statistical Society (B), 73, 3-36

Wördehoff, R., Spellmann, H., Evers, J., Nagel, J., 2011. Kohlenstoffstudie Forst und Holz Niedersachsen. Beiträge aus der Nordwestdeutschen Forstlichen Versuchsanstalt, Universitätsverlag Göttingen, 6, $92 \mathrm{~S}$.

Wördehoff, R., Spellmann, H., Evers, J., Cihan, T.A., Nagel, J., 2012a. Kohlenstoffstudie Forst und Holz Schleswig-Holstein. Nordwestdeutsche Forstliche Versuchsanstalt (Hrsg.), 104 S.

Wördehoff, R., Spellmann, H., Evers, J., Nagel, J., Gaertner, U., 2012b. Kohlenstoffstudie Forst und Holz Sachsen-Anhalt. Nordwestdeutsche Forstliche Versuchsanstalt (Hrsg.), 105 S.

WRI, 2011. A World of Opportunity. World Resource Institute, Forest landscapes initiative. URL: http://www.wri.org/sites/default/files/world_of_opportunity_ brochure_2011-09.pdf, aufgerufen am 11.12.2012

Zell, J., Kändler, G., Hanewinkel, M., 2009. Predicting constant decay rates of coarse woody debris - A meta-analysis approach with a mixed model. Ecological Modelling, 220, 904-912 


\section{A Anhang}

\section{A.1 Leistungsklassen der Hauptwirtschaftsbaumarten auf ausgesuchten Standorten im niedersächsischen Landeswald}

Tabelle A.1: Mittlere Leistungsklassen (LKL) der Hauptwirtschaftsbaumarten auf ausgesuchten Standorten getrennt nach Wasserhaushalt und Nährstoffversorgung im Wuchsbezirk Montaner Mittel- und Oberharz.

\begin{tabular}{|c|c|c|c|c|}
\hline $\begin{array}{l}\text { Standortstypen- } \\
\text { obergruppe }\end{array}$ & Wasserhaushalt & $\begin{array}{l}\text { Nährstoffver- } \\
\text { sorgung }\end{array}$ & Baumart & $\begin{array}{c}\text { LKL } \\
{\left[\mathrm{m}^{3} \mathrm{ha}^{-1} \mathrm{a}^{-1}\right]}\end{array}$ \\
\hline \multirow{4}{*}{$\begin{array}{l}\text { basenarme } \\
\text { Silikatgesteine }\end{array}$} & \multirow{2}{*}{ frisch } & mäßig & $\begin{array}{c}\mathrm{Bu} \\
\mathrm{Fi}\end{array}$ & $\begin{array}{l}6,5 \\
9,7\end{array}$ \\
\hline & & ziemlich gut & $\begin{array}{l}\mathrm{Bu} \\
\mathrm{Fi}\end{array}$ & $\begin{array}{c}6,7 \\
10,2\end{array}$ \\
\hline & \multirow{2}{*}{ mäßig frisch } & mäßig & $\begin{array}{c}\mathrm{Bu} \\
\mathrm{Fi}\end{array}$ & $\begin{array}{l}6,6 \\
9,8\end{array}$ \\
\hline & & ziemlich gut & $\begin{array}{l}\mathrm{Bu} \\
\mathrm{Fi}\end{array}$ & $\begin{array}{c}6,7 \\
10,4\end{array}$ \\
\hline \multirow{3}{*}{$\begin{array}{l}\text { basenreiche } \\
\text { Silikatgesteine }\end{array}$} & \multirow{2}{*}{ frisch } & sehr gut & $\begin{array}{c}\mathrm{Bu} \\
\mathrm{Fi}\end{array}$ & $\begin{array}{c}7,2 \\
10,2\end{array}$ \\
\hline & & ziemlich gut & $\begin{array}{l}\mathrm{Bu} \\
\mathrm{Fi}\end{array}$ & $\begin{array}{c}7,0 \\
10,0\end{array}$ \\
\hline & mäßig frisch & ziemlich gut & $\begin{array}{c}\mathrm{Bu} \\
\mathrm{Fi}\end{array}$ & $\begin{array}{c}7,4 \\
10,2\end{array}$ \\
\hline
\end{tabular}


Tabelle A.2: Mittlere Leistungsklassen (LKL) der Hauptwirtschaftsbaumarten auf ausgesuchten Standorten getrennt nach Wasserhaushalt und Nährstoffversorgung im Wuchsbezirk Unterer Solling.

\begin{tabular}{|c|c|c|c|c|}
\hline $\begin{array}{l}\text { Standortstypen- } \\
\text { obergruppe }\end{array}$ & Wasserhaushalt & $\begin{array}{l}\text { Nährstoffver- } \\
\text { sorgung }\end{array}$ & Baumart & $\begin{array}{c}\text { LKL } \\
{\left[\mathrm{m}^{3} \mathrm{ha}^{-1} \mathrm{a}^{-1}\right]}\end{array}$ \\
\hline \multirow{16}{*}{$\begin{array}{l}\text { basenarme } \\
\text { Silikatgesteine }\end{array}$} & \multirow{6}{*}{ frisch } & \multirow{3}{*}{ mäßig } & $\mathrm{Bu}$ & 7,3 \\
\hline & & & $\mathrm{Ei}$ & 6,0 \\
\hline & & & $\mathrm{Fi}$ & 11,4 \\
\hline & & \multirow{3}{*}{ ziemlich gut } & $\mathrm{Bu}$ & 7,5 \\
\hline & & & $\mathrm{Ei}$ & 6,2 \\
\hline & & & $\mathrm{Fi}$ & 11,4 \\
\hline & \multirow{6}{*}{ mäßig frisch } & \multirow{3}{*}{ mäßig } & $\mathrm{Bu}$ & 7,4 \\
\hline & & & $\mathrm{Ei}$ & 6,1 \\
\hline & & & $\mathrm{Fi}$ & 10,9 \\
\hline & & \multirow{3}{*}{ ziemlich gut } & $\mathrm{Bu}$ & 7,5 \\
\hline & & & $\mathrm{Ei}$ & 6,2 \\
\hline & & & $\mathrm{Fi}$ & 11,2 \\
\hline & \multirow{2}{*}{ nachhaltig frisch } & \multirow{2}{*}{ ziemlich gut } & $\mathrm{Bu}$ & 7,4 \\
\hline & & & $\mathrm{Fi}$ & 11,5 \\
\hline & \multirow{2}{*}{ sommertrocken } & \multirow{2}{*}{ mäßig } & $\mathrm{Bu}$ & 7,3 \\
\hline & & & $\mathrm{Fi}$ & 10,8 \\
\hline \multirow{6}{*}{ Lösse } & \multirow{3}{*}{ frisch } & \multirow{3}{*}{ ziemlich gut } & $\mathrm{Bu}$ & 7,3 \\
\hline & & & $\mathrm{Ei}$ & 5,9 \\
\hline & & & $\mathrm{Fi}$ & 11,5 \\
\hline & \multirow{3}{*}{ nachhaltig frisch } & \multirow{3}{*}{ ziemlich gut } & $\mathrm{Bu}$ & 7,6 \\
\hline & & & $\mathrm{Ei}$ & 6,2 \\
\hline & & & $\mathrm{Fi}$ & 11,4 \\
\hline
\end{tabular}


Tabelle A.3: Mittlere Leistungsklassen (LKL) der Hauptwirtschaftsbaumarten auf ausgesuchten Standorten getrennt nach Wasserhaushalt und Nährstoffversorgung im Wuchsbezirk Unteres Weser-Leine-Bergland.

\begin{tabular}{|c|c|c|c|c|}
\hline $\begin{array}{l}\text { Standortstypen- } \\
\text { obergruppe }\end{array}$ & Wasserhaushalt & $\begin{array}{l}\text { Nährstoffver- } \\
\text { sorgung }\end{array}$ & Baumart & $\begin{array}{c}\text { LKL } \\
{\left[\mathrm{m}^{3} \mathrm{ha}^{-1} \mathrm{a}^{-1}\right]}\end{array}$ \\
\hline \multirow{10}{*}{$\begin{array}{l}\text { basenarme } \\
\text { Silikatgesteine }\end{array}$} & \multirow{4}{*}{ frisch } & mäßig & $\begin{array}{c}\mathrm{Bu} \\
\mathrm{Fi}\end{array}$ & $\begin{array}{c}7,6 \\
10,6\end{array}$ \\
\hline & & \multirow{3}{*}{ ziemlich gut } & $\mathrm{Bu}$ & 7,8 \\
\hline & & & $\mathrm{Ei}$ & 6,4 \\
\hline & & & $\mathrm{Fi}$ & 11,2 \\
\hline & \multirow{4}{*}{ mäßig frisch } & \multirow{2}{*}{ mäßig } & $\mathrm{Bu}$ & 7,5 \\
\hline & & & $\mathrm{Fi}$ & 9,5 \\
\hline & & \multirow{2}{*}{ ziemlich gut } & $\mathrm{Bu}$ & 7,6 \\
\hline & & & $\mathrm{Fi}$ & 10,8 \\
\hline & \multirow{2}{*}{ sommertrocken } & \multirow{2}{*}{ mäßig } & $\mathrm{Bu}$ & 6,9 \\
\hline & & & $\mathrm{Fi}$ & 9,0 \\
\hline \multirow{10}{*}{ Kalkgesteine } & \multirow{3}{*}{ frisch } & \multirow{3}{*}{ sehr gut } & $\mathrm{Bu}$ & 8,4 \\
\hline & & & $\mathrm{Ei}$ & 6,2 \\
\hline & & & $\mathrm{Fi}$ & 11,8 \\
\hline & \multirow{3}{*}{ mäßig frisch } & \multirow{3}{*}{ sehr gut } & $\mathrm{Bu}$ & 8,2 \\
\hline & & & $\mathrm{Ei}$ & 6,2 \\
\hline & & & $\mathrm{Fi}$ & 11,1 \\
\hline & \multirow{2}{*}{ sommertrocken } & \multirow{2}{*}{ sehr gut } & $\mathrm{Bu}$ & 7,8 \\
\hline & & & $\mathrm{Fi}$ & 10,2 \\
\hline & \multirow{2}{*}{ trocken } & \multirow{2}{*}{ sehr gut } & $\mathrm{Bu}$ & 7,4 \\
\hline & & & $\mathrm{Fi}$ & 9,5 \\
\hline \multirow{9}{*}{ Lösse } & \multirow{3}{*}{ frisch } & \multirow{3}{*}{ ziemlich gut } & $\mathrm{Bu}$ & 8,1 \\
\hline & & & $\mathrm{Ei}$ & 6,1 \\
\hline & & & $\mathrm{Fi}$ & 11,2 \\
\hline & \multirow{6}{*}{ nachhaltig frisch } & \multirow{3}{*}{ sehr gut } & $\mathrm{Bu}$ & 8,6 \\
\hline & & & $\mathrm{Ei}$ & 6,7 \\
\hline & & & $\mathrm{Fi}$ & 12,2 \\
\hline & & \multirow{3}{*}{ ziemlich gut } & $\mathrm{Bu}$ & 8,2 \\
\hline & & & $\mathrm{Ei}$ & 6,5 \\
\hline & & & $\mathrm{Fi}$ & 11,8 \\
\hline
\end{tabular}


Tabelle A.4: Mittlere Leistungsklassen (LKL) der Hauptwirtschaftsbaumarten auf ausgesuchten Standorten getrennt nach Wasserhaushalt und Nährstoffversorgung im Wuchsbezirk Ost-Heide.

\begin{tabular}{|c|c|c|c|c|}
\hline $\begin{array}{l}\text { Standortstypen- } \\
\text { obergruppe }\end{array}$ & Wasserhaushalt & $\begin{array}{l}\text { Nährstoffver- } \\
\text { sorgung }\end{array}$ & Baumart & $\begin{array}{c}\text { LKL } \\
{\left[\mathrm{m}^{3} \mathrm{ha}^{-1} \mathrm{a}^{-1}\right]}\end{array}$ \\
\hline \multirow{3}{*}{ Geschiebelehme } & \multirow[b]{2}{*}{ frisch } & mäßig & $\begin{array}{c}\mathrm{Dgl} \\
\mathrm{Ei} \\
\mathrm{Fi} \\
\mathrm{Ki}\end{array}$ & $\begin{array}{c}14,1 \\
5,4 \\
10,2 \\
7,1\end{array}$ \\
\hline & & ziemlich gut & $\begin{array}{c}\mathrm{Bu} \\
\mathrm{Dgl} \\
\mathrm{Ei} \\
\mathrm{Fi} \\
\mathrm{Ki}\end{array}$ & $\begin{array}{c}7,9 \\
15,3 \\
6,1 \\
11,8 \\
7,5\end{array}$ \\
\hline & mäßig frisch bis frisch & mäßig & $\begin{array}{c}\mathrm{Bu} \\
\mathrm{Ei} \\
\mathrm{Fi} \\
\mathrm{Ki}\end{array}$ & $\begin{array}{l}6,3 \\
5,1 \\
9,7 \\
6,9\end{array}$ \\
\hline $\begin{array}{l}\text { Sande im } \\
\text { Grundwasser- } \\
\text { bereich }\end{array}$ & $\begin{array}{l}\text { stark grundwasserbe- } \\
\text { einflusst }\end{array}$ & mäßig & $\begin{array}{l}\mathrm{Ei} \\
\mathrm{Ki}\end{array}$ & $\begin{array}{l}5,3 \\
8,3\end{array}$ \\
\hline \multirow{3}{*}{ Sande } & mäßig frisch bis frisch & mäßig & $\begin{array}{c}\mathrm{Bu} \\
\mathrm{Dgl} \\
\mathrm{Ei} \\
\mathrm{Fi} \\
\mathrm{Ki}\end{array}$ & $\begin{array}{c}6,4 \\
14,6 \\
5,0 \\
10,0 \\
6,9\end{array}$ \\
\hline & mäßig frisch & mäßig & $\begin{array}{c}\mathrm{Bu} \\
\mathrm{Dgl} \\
\mathrm{Ei} \\
\mathrm{Fi} \\
\mathrm{Ki}\end{array}$ & $\begin{array}{c}6,5 \\
13,0 \\
4,5 \\
9,7 \\
6,8\end{array}$ \\
\hline & trocken & schwach & $\begin{array}{c}\mathrm{Dgl} \\
\mathrm{Ei} \\
\mathrm{Ki}\end{array}$ & $\begin{array}{l}12,3 \\
5,4 \\
6,7\end{array}$ \\
\hline
\end{tabular}


Tabelle A.5: Mittlere Leistungsklassen (LKL) der Hauptwirtschaftsbaumarten auf ausgesuchten Standorten getrennt nach Wasserhaushalt und Nährstoffversorgung im Wuchsbezirk Geest-Mitte.

\begin{tabular}{|c|c|c|c|c|}
\hline $\begin{array}{l}\text { Standortstypen- } \\
\text { obergruppe }\end{array}$ & Wasserhaushalt & $\begin{array}{l}\text { Nährstoffver- } \\
\text { sorgung }\end{array}$ & Baumart & $\begin{array}{c}\text { LKL } \\
{\left[\mathrm{m}^{3} \mathrm{ha}^{-1} \mathrm{a}^{-1}\right]}\end{array}$ \\
\hline \multirow{9}{*}{ Geschiebelehme } & \multirow{5}{*}{ frisch } & mäßig & $\begin{array}{l}\mathrm{Ei} \\
\mathrm{Ki}\end{array}$ & $\begin{array}{l}6,3 \\
7,3\end{array}$ \\
\hline & & \multirow{4}{*}{ ziemlich gut } & $\begin{array}{c}\mathrm{Bu} \\
\mathrm{Dol}\end{array}$ & $\begin{array}{c}7,9 \\
145\end{array}$ \\
\hline & & & $\mathrm{Ei}$ & 6,1 \\
\hline & & & $\mathrm{Fi}$ & 11,9 \\
\hline & & & $\mathrm{Ki}$ & 7,4 \\
\hline & \multirow{4}{*}{ mäßig frisch bis frisch } & \multirow{4}{*}{ mäßig } & $\mathrm{Bu}$ & 7,6 \\
\hline & & & Dgl & 14,3 \\
\hline & & & $\mathrm{Ei}$ & 6,0 \\
\hline & & & $\mathrm{Ki}$ & 7,2 \\
\hline \multirow{4}{*}{$\begin{array}{l}\text { Sand im Grund- } \\
\text { wasserbereich }\end{array}$} & \multirow{2}{*}{$\begin{array}{l}\text { mäßig grundwasserbe- } \\
\text { einflusst }\end{array}$} & \multirow{2}{*}{ mäßig } & $\mathrm{Ei}$ & 5,9 \\
\hline & & & $\mathrm{Ki}$ & 7,8 \\
\hline & \multirow{2}{*}{$\begin{array}{l}\text { stark grundwasserbe- } \\
\text { einflusst }\end{array}$} & \multirow{2}{*}{ mäßig } & $\mathrm{Ei}$ & 6,7 \\
\hline & & & $\mathrm{Ki}$ & 7,7 \\
\hline \multirow{9}{*}{ Sande } & \multirow{3}{*}{ mäßig frisch bis frisch } & \multirow{3}{*}{ mäßig } & $\mathrm{Bu}$ & 7,8 \\
\hline & & & $\mathrm{Ei}$ & 6,3 \\
\hline & & & Ki & 7,2 \\
\hline & \multirow{4}{*}{ mäßig frisch } & \multirow{4}{*}{ mäßig } & $\mathrm{Bu}$ & 7,5 \\
\hline & & & Dgl & 13,9 \\
\hline & & & $\mathrm{Ei}$ & 6,0 \\
\hline & & & Ki & 7,1 \\
\hline & \multirow{2}{*}{ trocken } & \multirow{2}{*}{ schwach } & Dgl & 13,3 \\
\hline & & & $\mathrm{Ki}$ & 6,7 \\
\hline \multirow{4}{*}{ Sandlösse } & \multirow{4}{*}{ frisch } & \multirow{4}{*}{ ziemlich gut } & $\mathrm{Bu}$ & 8,0 \\
\hline & & & Dgl & 15,8 \\
\hline & & & $\mathrm{Ei}$ & 6,7 \\
\hline & & & $\mathrm{Fi}$ & 12,9 \\
\hline
\end{tabular}


Tabelle A.6: Mittlere Leistungsklassen (LKL) der Hauptwirtschaftsbaumarten auf ausgesuchten Standorten getrennt nach Wasserhaushalt und Nährstoffversorgung im Wuchsbezirk Hohe Heide.

\begin{tabular}{|c|c|c|c|c|}
\hline $\begin{array}{l}\text { Standortstypen- } \\
\text { obergruppe }\end{array}$ & Wasserhaushalt & $\begin{array}{l}\text { Nährstoffver- } \\
\text { sorgung }\end{array}$ & Baumart & $\begin{array}{c}\text { LKL } \\
{\left[\mathrm{m}^{3} \mathrm{ha}^{-1} \mathrm{a}^{-1}\right]}\end{array}$ \\
\hline \multirow{3}{*}{ Geschiebelehme } & \multirow[b]{2}{*}{ frisch } & mäßig & $\begin{array}{l}\mathrm{Ei} \\
\mathrm{Fi} \\
\mathrm{Ki}\end{array}$ & $\begin{array}{c}5,6 \\
11,3 \\
7,2 \\
\end{array}$ \\
\hline & & ziemlich gut & $\begin{array}{c}\mathrm{Bu} \\
\mathrm{Dgl} \\
\mathrm{Ei} \\
\mathrm{Fi} \\
\mathrm{Ki}\end{array}$ & $\begin{array}{c}7,5 \\
14,6 \\
6,0 \\
11,6 \\
7,4\end{array}$ \\
\hline & mäßig frisch bis frisch & mäßig & $\begin{array}{c}\mathrm{Bu} \\
\mathrm{Dgl} \\
\mathrm{Ei} \\
\mathrm{Fi} \\
\mathrm{Ki}\end{array}$ & $\begin{array}{c}6,8 \\
13,6 \\
5,5 \\
10,0 \\
6,8 \\
\end{array}$ \\
\hline $\begin{array}{l}\text { Sande im } \\
\text { Grundwasser- } \\
\text { bereich }\end{array}$ & $\begin{array}{l}\text { schwach grundwasser- } \\
\text { beeinflusst }\end{array}$ & schwach & $\begin{array}{l}\mathrm{Fi} \\
\mathrm{Ki}\end{array}$ & $\begin{array}{c}10,6 \\
6,3\end{array}$ \\
\hline \multirow{4}{*}{ Sande } & frisch & mäßig & $\begin{array}{c}\mathrm{Bu} \\
\mathrm{Fi} \\
\mathrm{Ki}\end{array}$ & $\begin{array}{c}6,8 \\
10,7 \\
7,7 \\
\end{array}$ \\
\hline & mäßig frisch bis frisch & mäßig & $\begin{array}{c}\mathrm{Bu} \\
\mathrm{Dgl} \\
\mathrm{Ei} \\
\mathrm{Fi} \\
\mathrm{Ki}\end{array}$ & $\begin{array}{c}6,4 \\
13,2 \\
4,5 \\
10,4 \\
7,0\end{array}$ \\
\hline & \multirow[t]{2}{*}{ mäßig frisch } & mäßig & $\begin{array}{c}\mathrm{Bu} \\
\mathrm{Dgl} \\
\mathrm{Ei} \\
\mathrm{Fi} \\
\mathrm{Ki}\end{array}$ & $\begin{array}{c}6,2 \\
13,2 \\
4,7 \\
10,2 \\
6,9\end{array}$ \\
\hline & & schwach & $\begin{array}{l}\mathrm{Fi} \\
\mathrm{Ki}\end{array}$ & $\begin{array}{l}9,5 \\
6,7\end{array}$ \\
\hline
\end{tabular}

Fortsetzung auf nächster Seite. 
Tabelle A.6

\begin{tabular}{lllcc}
\hline $\begin{array}{l}\text { Standortstypen- } \\
\text { obergruppe }\end{array}$ & Wasserhaushalt & $\begin{array}{l}\text { Nährstoffver- } \\
\text { sorgung }\end{array}$ & Baumart & $\begin{array}{c}\text { LKL } \\
{\left[\mathrm{m}^{3} \mathrm{ha}^{-1} \mathrm{a}^{-1}\right]}\end{array}$ \\
\hline & \multirow{2}{*}{ mäßig } & $\mathrm{Dgl}$ & 13,7 \\
& & & $\mathrm{Ki}$ & 6,8 \\
\cline { 3 - 5 } Sande & trocken & $\mathrm{Bu}$ & 5,9 \\
& & $\mathrm{Dgl}$ & 13,0 \\
& & $\mathrm{Ei}$ & 4,4 \\
& & $\mathrm{Fi}$ & 9,3 \\
& & $\mathrm{Ki}$ & 6,8 \\
\hline
\end{tabular}




\section{A.2 Generierung von Weiserbeständen}

\section{A.2.1 Angaben zu den Durchmesserverteilungen}

Tabelle A.7: Koeffizienten und statistische Eigenschaften der Parameter der Box-Cox-ColeGreen-Verteilung zur Schätzung der Durchmesserverteilung von Eichenreinbeständen.

\begin{tabular}{lrrrr}
\hline Parameterkoeffizienten & Schätzwert & Std. Fehler & t-Wert & $\operatorname{Pr}(>|\mathrm{t}|)$ \\
\hline Koeffizienten von $\mu$ & & & & \\
Interzept & 4.24574 & $8.477 \mathrm{e}-04$ & 5008.3 & 0 \\
Alter & 0.01562 & $9.036 \mathrm{e}-06$ & 1728.3 & 0 \\
hg100 (<32) & -0.02785 & $1.376 \mathrm{e}-04$ & -202.5 & 0 \\
Koeffizienten von $\sigma$ & & & & \\
Interzept & -1.000567 & $1.629 \mathrm{e}-03$ & -614.1 & 0 \\
Alter & -0.004043 & $2.461 \mathrm{e}-05$ & -164.3 & 0 \\
Koeffizienten von $\nu$ & & & & \\
Interzept & 0.137463 & 0.0058136 & 23.64 & $1.588 \mathrm{e}-123$ \\
Alter & 0.001579 & 0.0001169 & 13.50 & $1.505 \mathrm{e}-41$ \\
\hline
\end{tabular}

Tabelle A.8: Koeffizienten und statistische Eigenschaften der Parameter der Box-Cox-ColeGreen-Verteilung zur Schätzung der Durchmesserverteilung von Buchenreinbeständen.

\begin{tabular}{lrrrr}
\hline Parameterkoeffizienten & Schätzwert & Std. Fehler & t-Wert & $\operatorname{Pr}(>|\mathrm{t}|)$ \\
\hline Koeffizienten von $\mu$ & & & & \\
Interzept & 3.51154 & $3.099 \mathrm{e}-03$ & 1133.0 & 0 \\
Alter & 0.01824 & $1.853 \mathrm{e}-05$ & 984.5 & 0 \\
hg100 (> 17) & 0.03290 & $1.106 \mathrm{e}-04$ & 297.5 & 0 \\
Koeffizienten von $\sigma$ & & & & \\
Interzept & -1.099430 & $1.662 \mathrm{e}-03$ & -661.6 & 0 \\
Alter $(>60)$ & -0.005133 & $4.042 \mathrm{e}-05$ & -127.0 & 0 \\
Koeffizienten von $\nu$ & & & & \\
Interzept & 0.50401 & 0.0048533 & 103.85 & 0 \\
Alter $(<40)$ & -0.05165 & 0.0008902 & -58.02 & 0 \\
\hline
\end{tabular}


Tabelle A.9: Koeffizienten und statistische Eigenschaften der Parameter der Box-Cox-ColeGreen-Verteilung zur Schätzung der Durchmesserverteilung von Fichtenreinbeständen.

\begin{tabular}{lrrrr}
\hline Parameterkoeffizienten & Schätzwert & Std. Fehler & t-Wert & $\operatorname{Pr}(>|\mathrm{t}|)$ \\
\hline Koeffizienten von $\mu$ & & & & \\
Interzept & 4.60431 & $3.399 \mathrm{e}-03$ & 1354.8 & 0 \\
Alter & 0.01272 & $4.548 \mathrm{e}-05$ & 279.7 & 0 \\
hg100 (> 22) & 0.02255 & $6.308 \mathrm{e}-05$ & 357.4 & 0 \\
Koeffizienten von $\sigma$ & & & & \\
Interzept & -1.60933 & 0.0023247 & -692.28 & 0 \\
Alter (<55) & 0.01219 & 0.0001475 & 82.64 & 0 \\
Koeffizienten von $\nu$ & & & & \\
Interzept & 0.49338 & 0.0025269 & 195.25 & 0 \\
Alter $(>140)$ & -0.04238 & 0.0005672 & -74.72 & 0 \\
\hline
\end{tabular}

Tabelle A.10: Koeffizienten und statistische Eigenschaften der Parameter der Box-Cox-ColeGreen-Verteilung zur Schätzung der Durchmesserverteilung von Douglasienreinbeständen.

\begin{tabular}{lrrrr}
\hline Parameterkoeffizienten & Schätzwert & Std. Fehler & t-Wert & $\operatorname{Pr}(>|\mathrm{t}|)$ \\
\hline Koeffizienten von $\mu$ & & & & \\
Interzept & 4.45313 & 0.0044289 & 1005.5 & 0 \\
Alter & 0.02515 & 0.0001076 & 233.7 & 0 \\
hg100 (> 36) & 0.02203 & 0.0001860 & 118.5 & 0 \\
Koeffizienten von $\sigma$ & & & & \\
Interzept & -1.043797 & 0.0063329 & -164.82 & 0 \\
Alter & -0.006423 & 0.0001745 & -36.81 & $2.609 \mathrm{e}-295$ \\
Koeffizienten von $\nu$ & & & & \\
Interzept & 0.644468 & 0.0193770 & 33.259 & $1.178 \mathrm{e}-241$ \\
Alter & 0.002612 & 0.0003851 & 6.783 & $1.178 \mathrm{e}-11$ \\
\hline
\end{tabular}


Tabelle A.11: Koeffizienten und statistische Eigenschaften der Parameter der Box-Cox-ColeGreen-Verteilung zur Schätzung der Durchmesserverteilung von Kiefernreinbeständen.

\begin{tabular}{lrrrr}
\hline Parameterkoeffizienten & Schätzwert & Std. Fehler & t-Wert & $\operatorname{Pr}(>|\mathrm{t}|)$ \\
\hline Koeffizienten von $\mu$ & & & & \\
Interzept & 4.20277 & $7.959 \mathrm{e}-04$ & 5280.50 & 0 \\
Alter & 0.01571 & $6.669 \mathrm{e}-06$ & 2355.05 & 0 \\
hy100 $(<16)$ & -0.02597 & $1.178 \mathrm{e}-03$ & -22.05 & $1.003 \mathrm{e}-107$ \\
hy100 $>$ > 16) & 0.02419 & $3.861 \mathrm{e}-05$ & 626.41 & 0 \\
Koeffizienten von $\sigma$ & & & & \\
Interzept & -1.722184 & $1.166 \mathrm{e}-03$ & -1477.3 & 0 \\
Alter $(<80)$ & 0.009264 & $3.058 \mathrm{e}-05$ & 303.0 & 0 \\
Alter $(>80)$ & 0.002050 & $4.746 \mathrm{e}-05$ & 43.2 & 0 \\
Koeffizienten von $\nu$ & & & & \\
Interzept & -0.002589 & $4.558 \mathrm{e}-03$ & -0.5681 & 0.57 \\
Alter & 0.008289 & $9.068 \mathrm{e}-05$ & 91.4126 & 0.00 \\
\hline
\end{tabular}




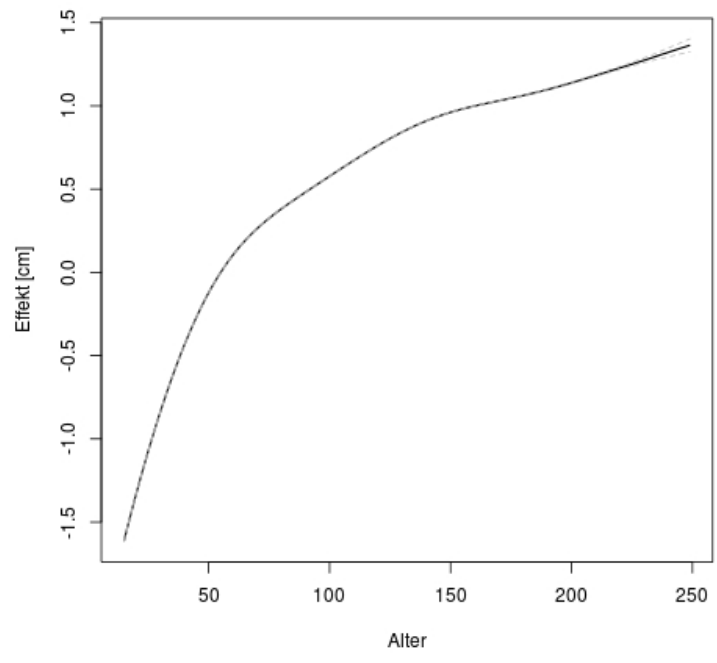

(a)

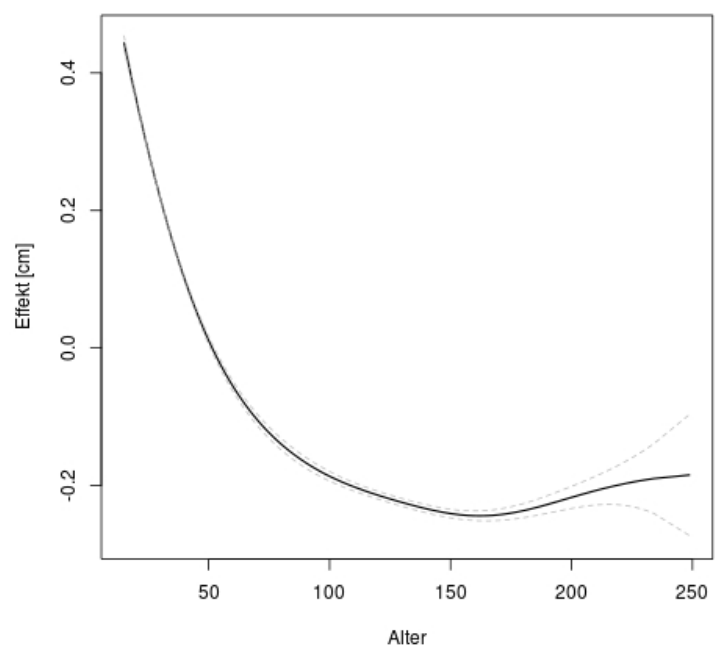

(c)

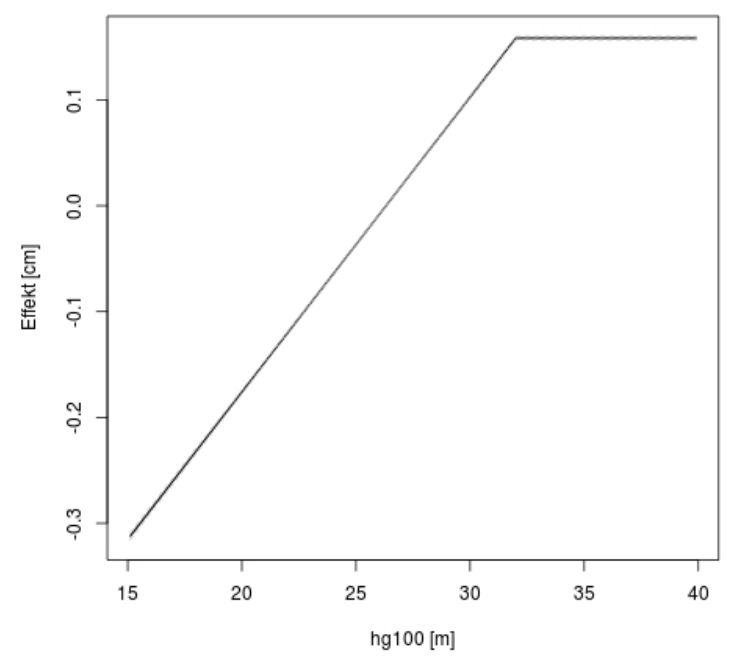

(b)

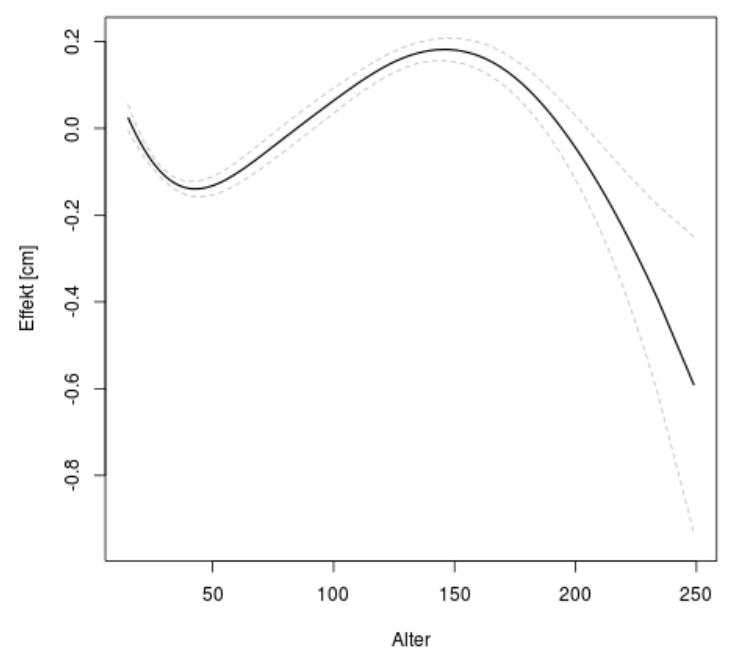

(d)

Abbildung A.1: Effekt des Alters und der absoluten Mittelhöhenbonität (hg100 [m]) auf den Mittelwert $\mu(\mathrm{a}, \mathrm{b})$ sowie des Alters auf den Variationskoeffizienten $\sigma(\mathrm{c})$ und die Schiefe $\nu$ (d) der Box-Cox-Cole-Green-Verteilung zur Schätzung der Durchmesserverteilung von Eichenreinbeständen 


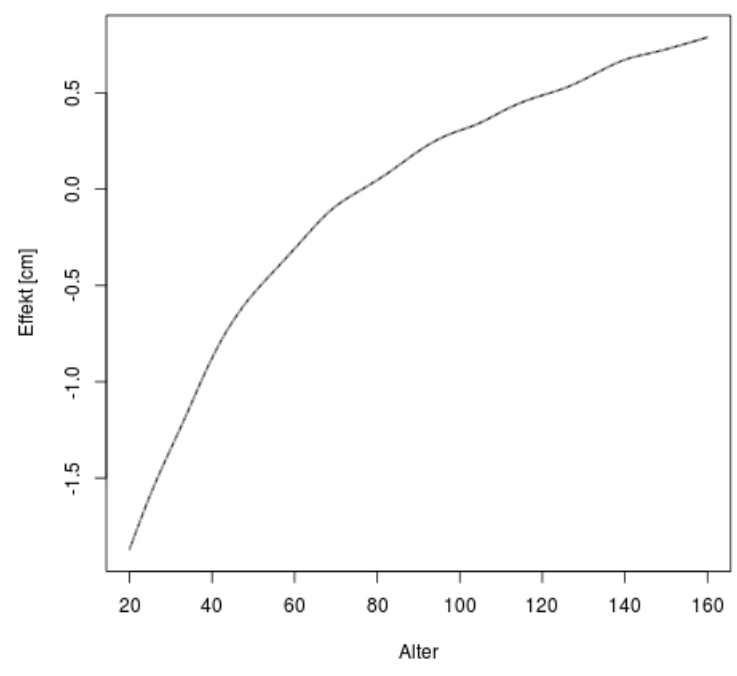

(a)

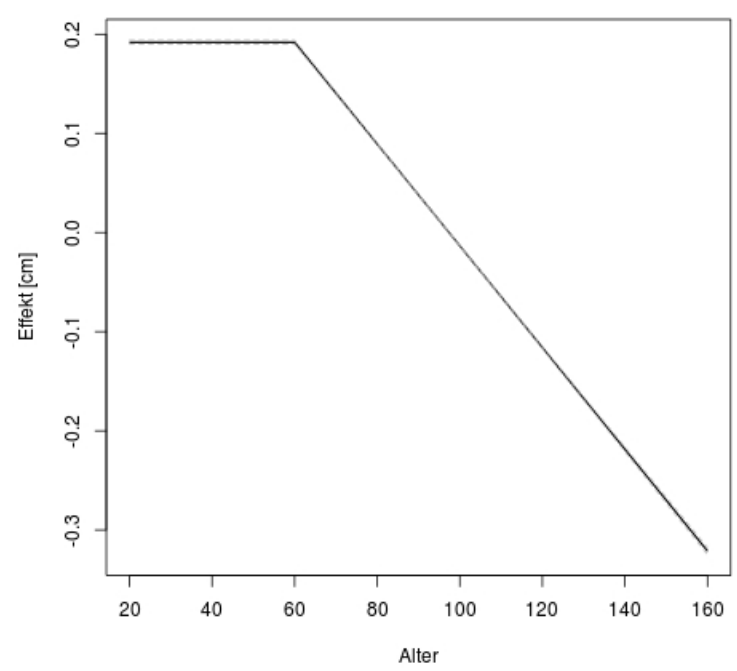

(c)

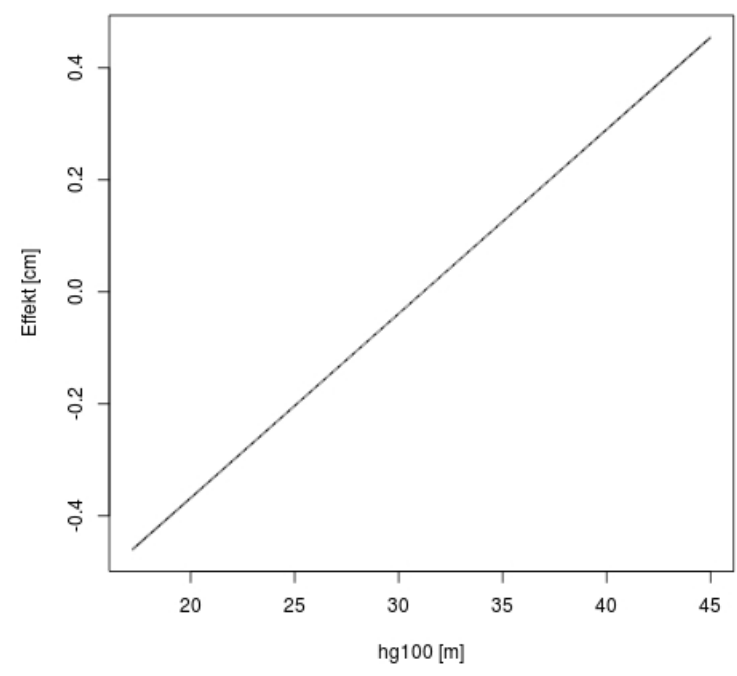

(b)

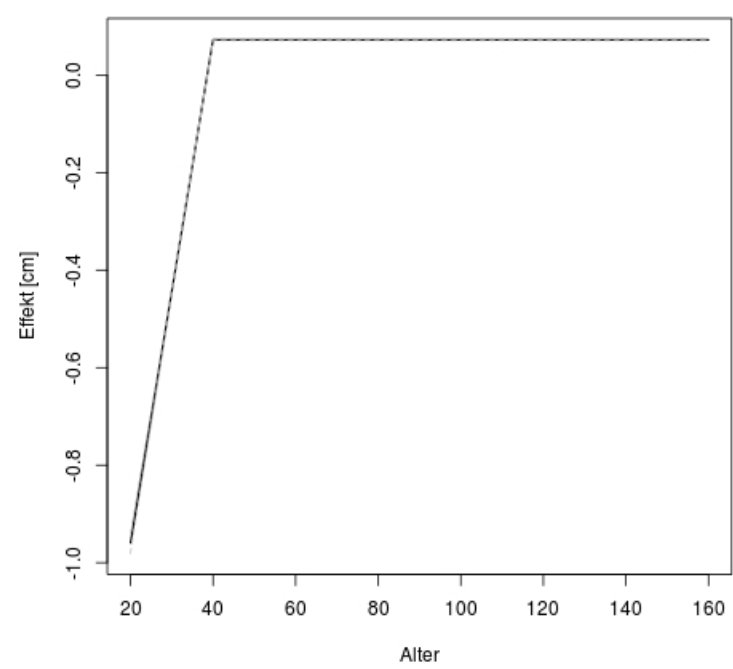

(d)

Abbildung A.2: Effekt des Alters und der absoluten Mittelhöhenbonität (hg100 [m]) auf den Mittelwert $\mu(\mathrm{a}, \mathrm{b})$ sowie des Alters auf den Variationskoeffizienten $\sigma(\mathrm{c})$ und die Schiefe $\nu$ (d) der Box-Cox-Cole-Green-Verteilung zur Schätzung der Durchmesserverteilung von Buchenreinbeständen. 


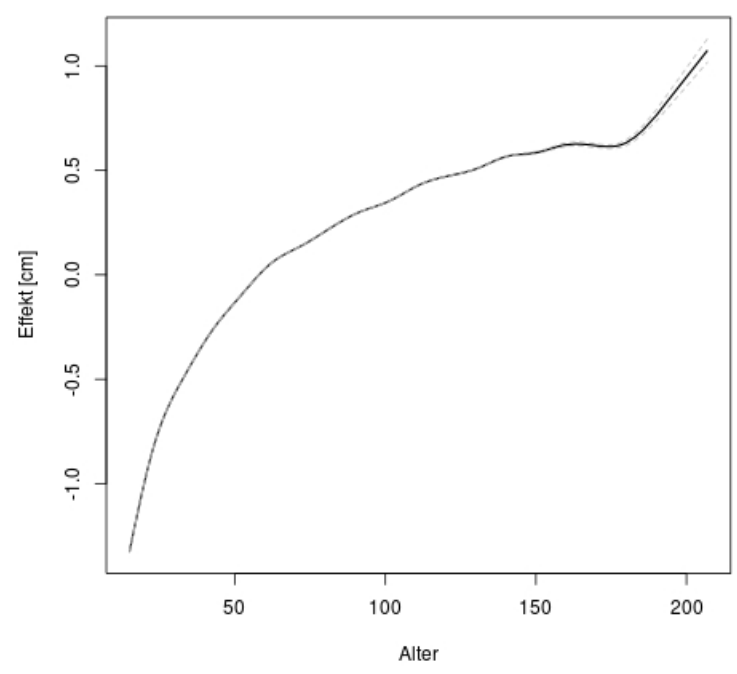

(a)

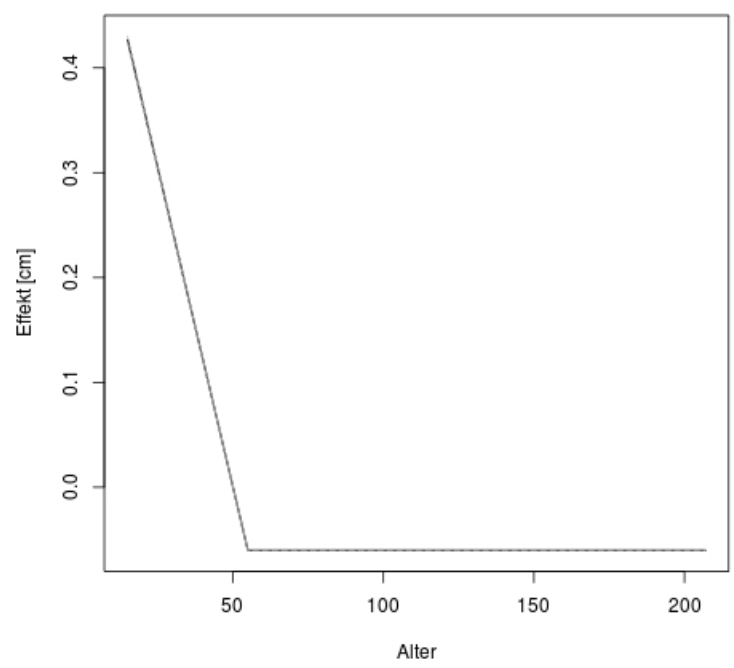

(c)

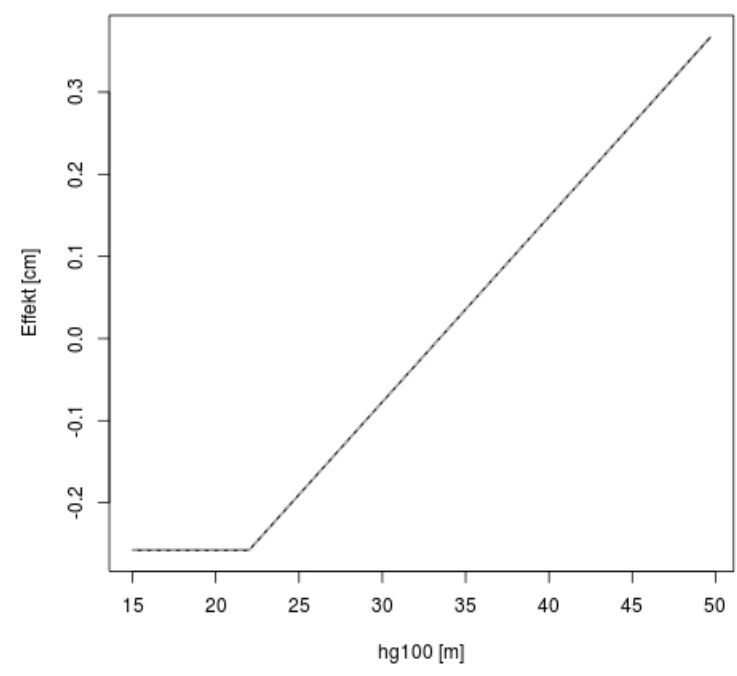

(b)

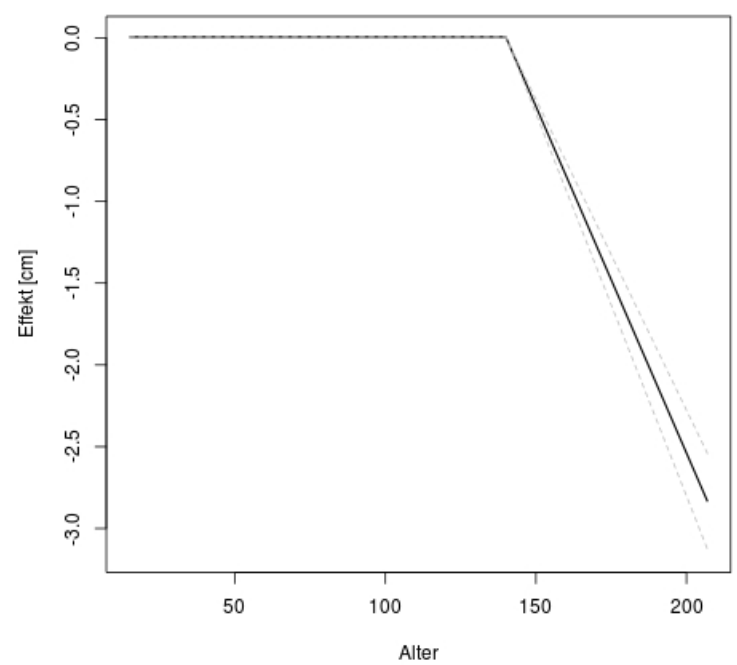

(d)

Abbildung A.3: Effekt des Alters und der absoluten Mittelhöhenbonität (hg100 [m]) auf den Mittelwert $\mu(\mathrm{a}, \mathrm{b})$ sowie des Alters auf den Variationskoeffizienten $\sigma$ (c) und die Schiefe $\nu$ (d) der Box-Cox-Cole-Green-Verteilung zur Schätzung der Durchmesserverteilung von Fichtenreinbeständen. 


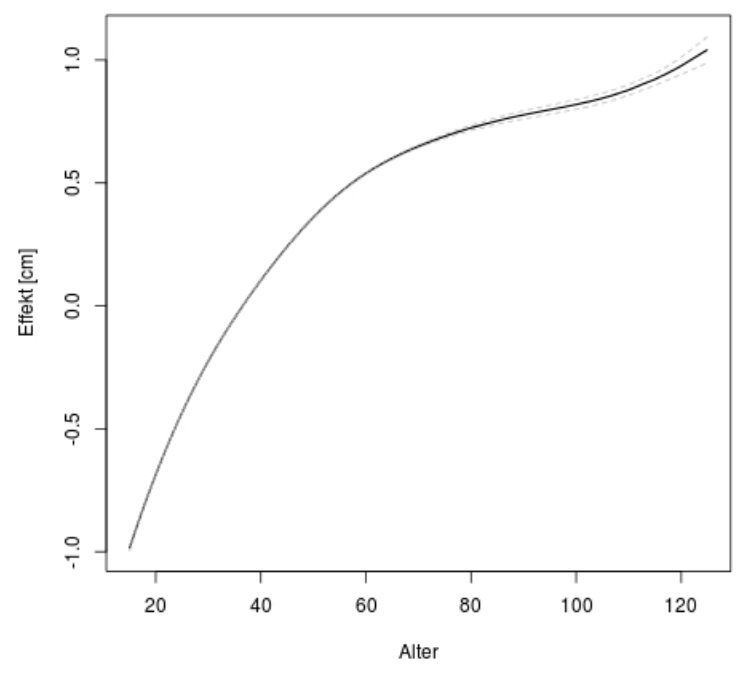

(a)

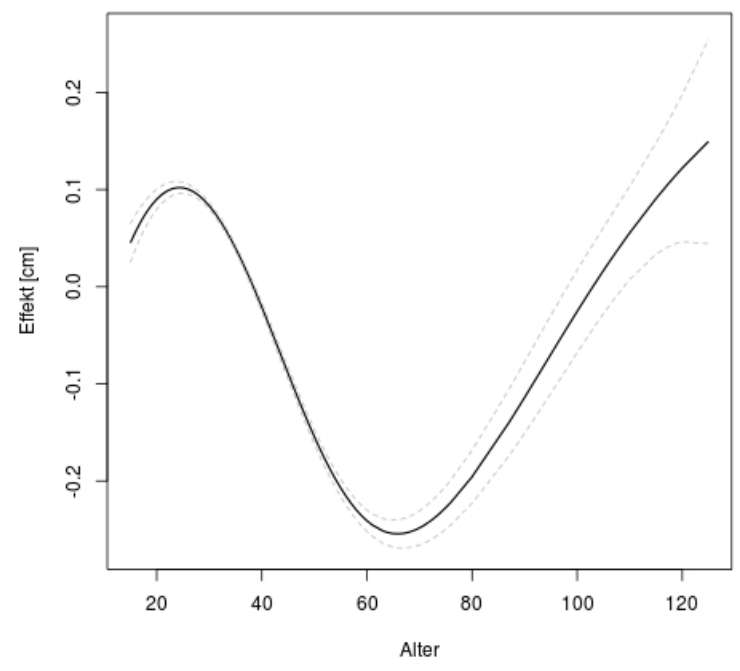

(c)

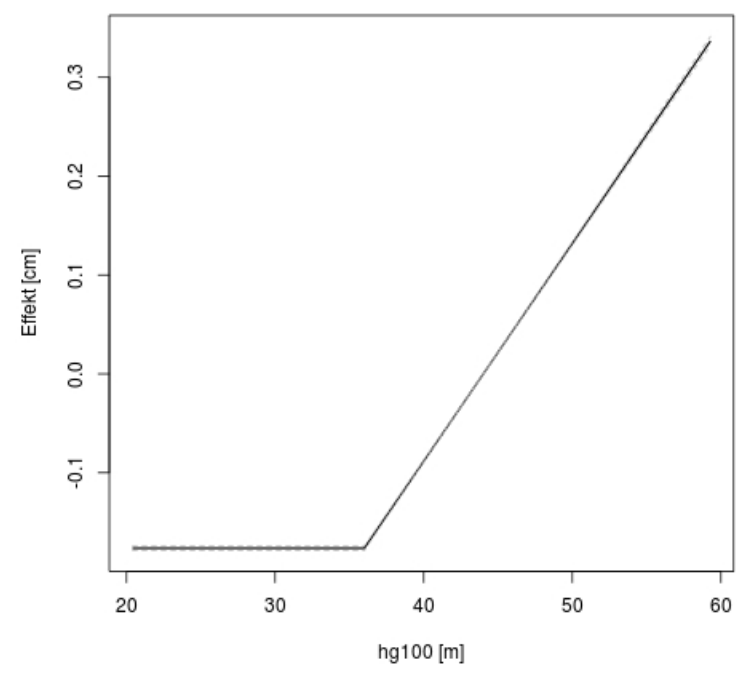

(b)

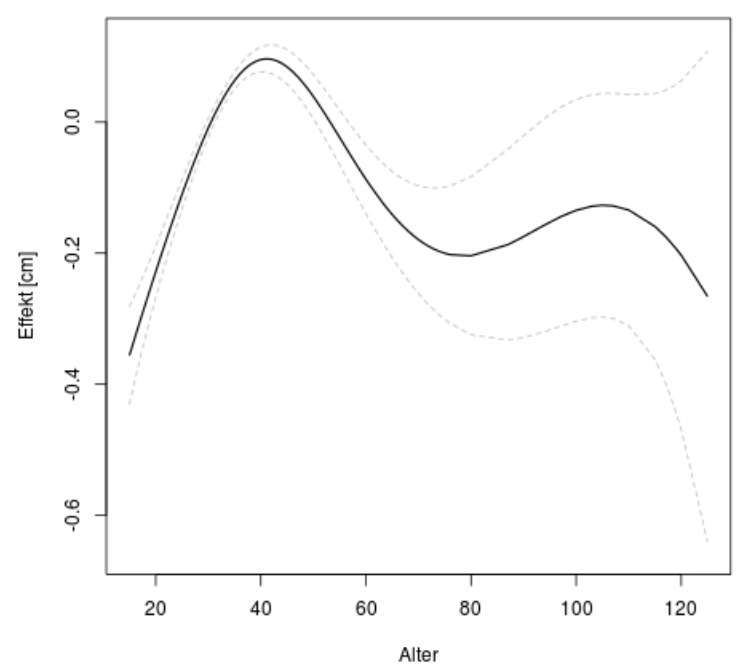

(d)

Abbildung A.4: Effekt des Alters und der absoluten Mittelhöhenbonität (hg100 [m]) auf den Mittelwert $\mu(\mathrm{a}, \mathrm{b})$ sowie des Alters auf den Variationskoeffizienten $\sigma(\mathrm{c})$ und die Schiefe $\nu$ (d) der Box-Cox-Cole-Green-Verteilung zur Schätzung der Durchmesserverteilung von Douglasienreinbeständen. 


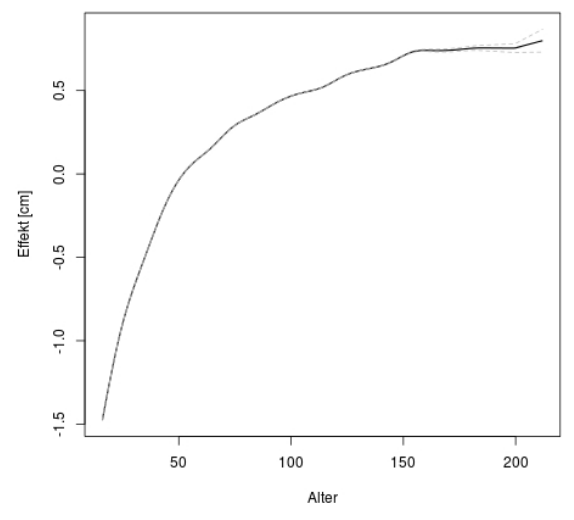

(a)

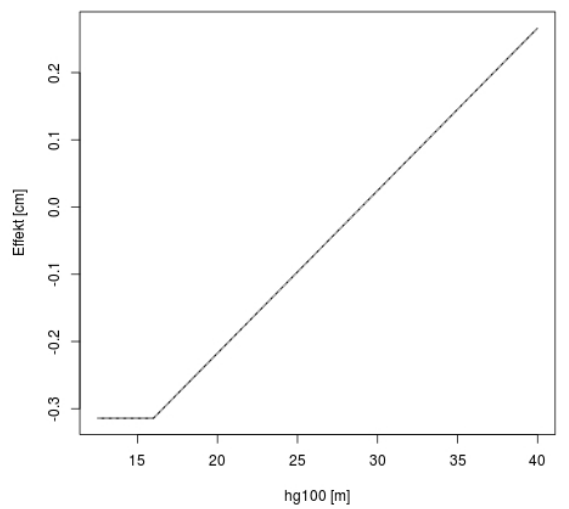

(c)

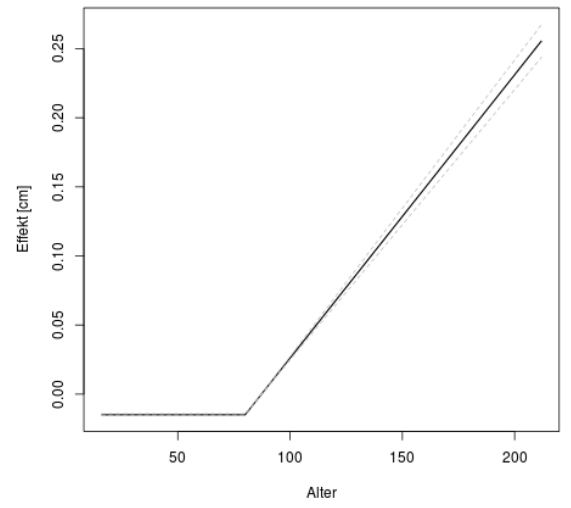

(e)

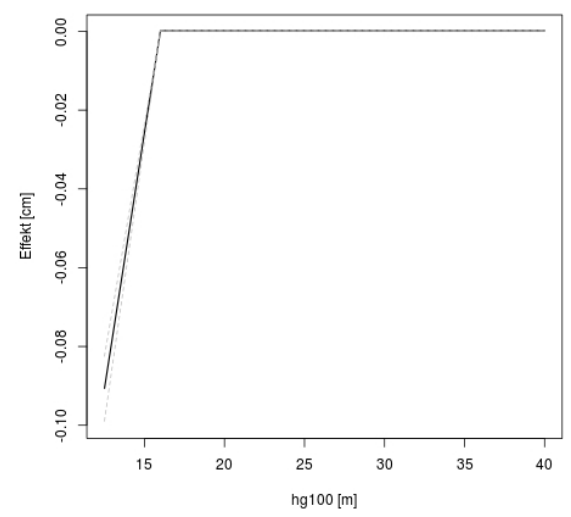

(b)

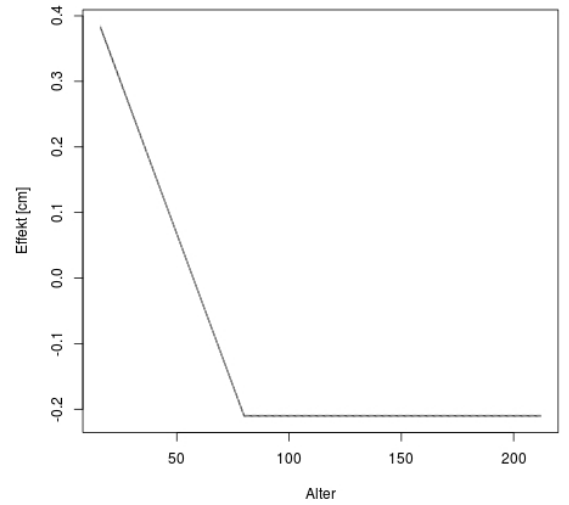

(d)

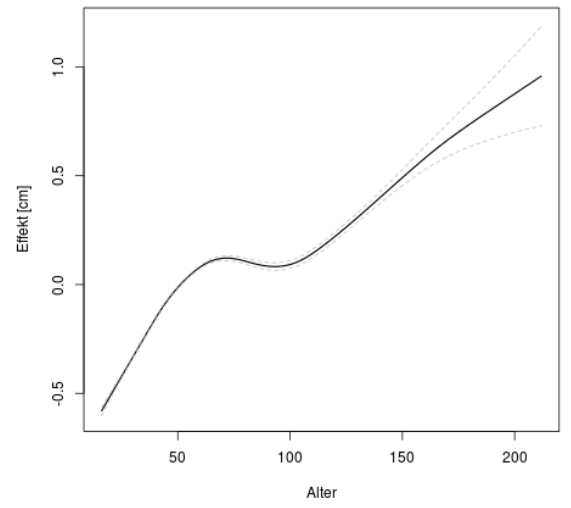

(f)

Abbildung A.5: Effekt des Alters und der absoluten Mittelhöhenbonität (hg100 [m]) auf den Mittelwert $\mu$ (a, b, c) sowie des Alters auf den Variationskoeffizienten $\sigma$ (d, e) und die Schiefe $\nu$ (f) der Box-Cox-Cole-Green-Verteilung zur Schätzung der Durchmesserverteilung von Kiefernreinbeständen. 


\section{A.2.2 Angaben zu den Grundflächenmodellen}

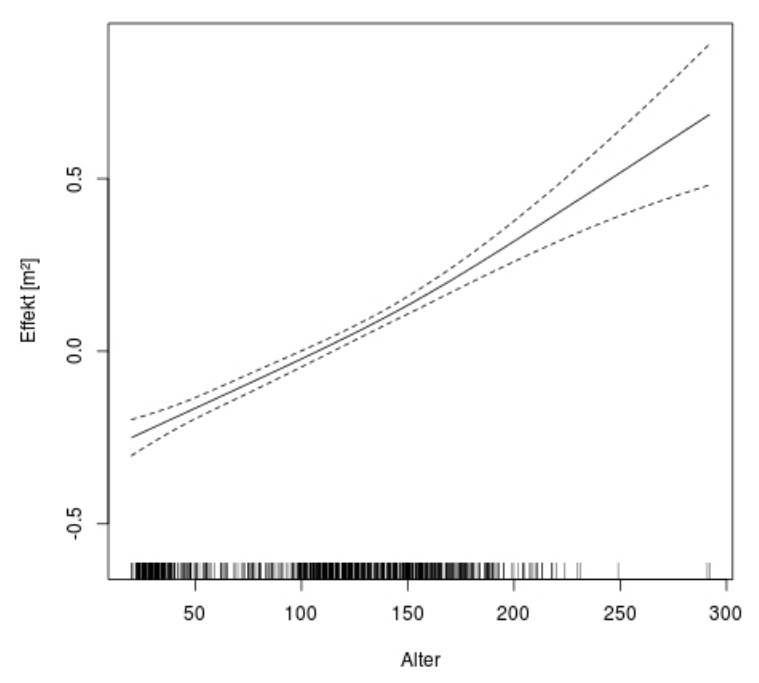

(a)

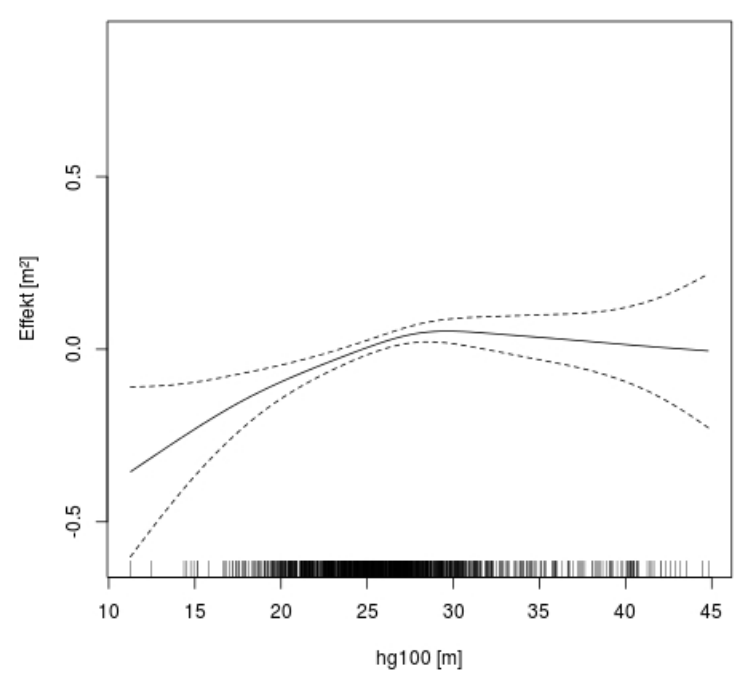

(b)

Abbildung A.6: Effekt des Alters (a) und der absoluten Mittelhöhenbonität (hg100 [m]) (b) auf die Schätzung der Bestandesgrundfläche von Eichenreinbeständen.

Tabelle A.12: Parameter und statistische Eigenschaften des Grundflächenmodells für Eichenreinbestände.

\begin{tabular}{lllll}
\hline \multicolumn{2}{l}{ Parameterkoeffizienten } \\
& Schätzwert & Std. Fehler & t-Wert & $\operatorname{Pr}(>|\mathrm{t}|)$ \\
\hline Interzept & 2.91201 & 0.01206 & 241.4 & $<2 \mathrm{e}-16$ \\
\hline \multicolumn{4}{l}{ Approximierte Signifikanz der glättenden Terme } \\
& edf & Ref.df & F & p-Wert \\
\hline Alter & 1.923 & 2.384 & 68.084 & $<2 \mathrm{e}-16$ \\
hg100 & 3.118 & 3.953 & 4.875 & 0.00073 \\
\hline
\end{tabular}



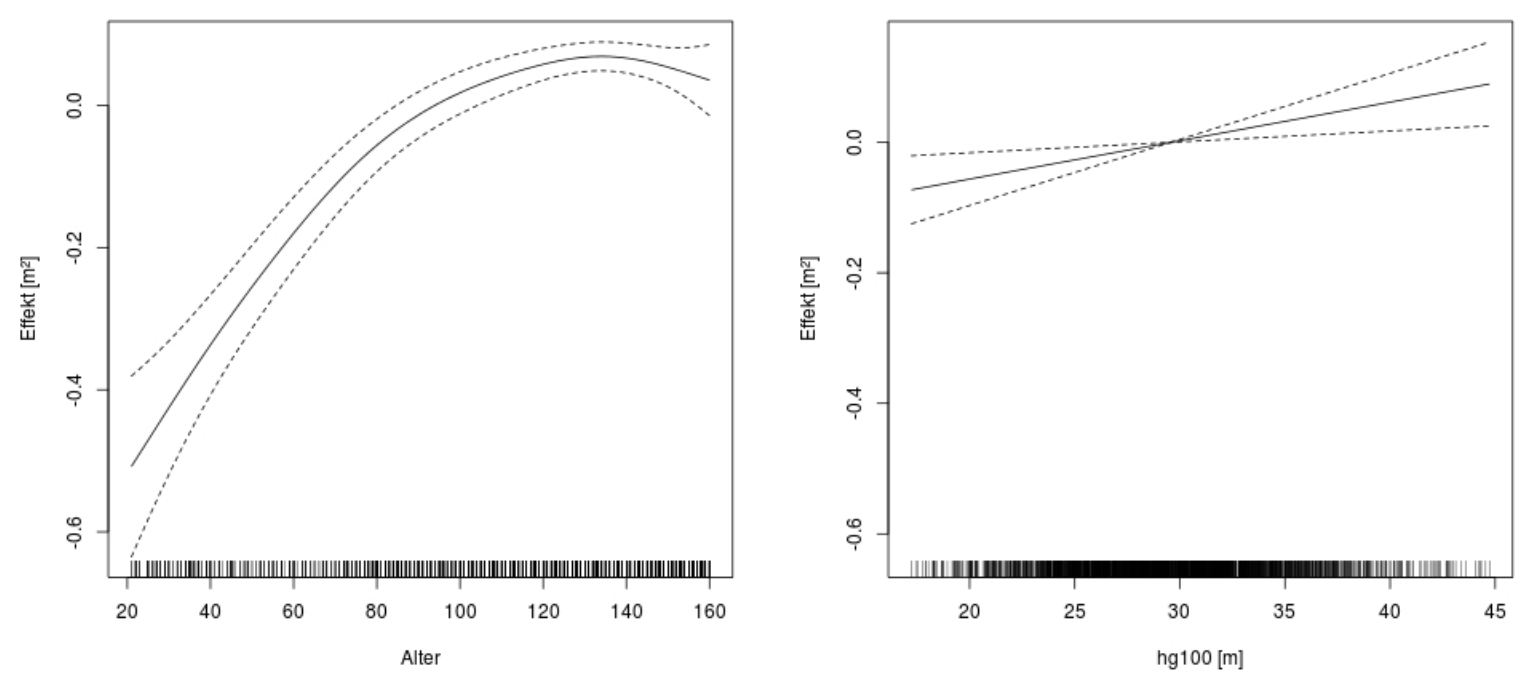

Abbildung A.7: Effekt des Alters (a) und der absoluten Mittelhöhenbonität (hg100 [m]) (b) auf die Schätzung der Bestandesgrundfläche von Buchenreinbeständen.

Tabelle A.13: Parameter und statistische Eigenschaften des Grundflächenmodells für Buchenreinbestände.

\begin{tabular}{|c|c|c|c|c|}
\hline Paramete & $\begin{array}{l}\text { xoeffizienten } \\
\text { Schätzwert }\end{array}$ & Std. Fehler & t-Wert & $\operatorname{Pr}(>|t|)$ \\
\hline Interzept & 2.870530 & 0.063456 & 45.24 & $<2 \mathrm{e}-16$ \\
\hline hg100 & 0.005896 & 0.002113 & 2.79 & 0.00533 \\
\hline \multicolumn{5}{|c|}{ Approximierte Signifikanz der glättenden Terme } \\
\hline & edf & Ref.df & $\mathrm{F}$ & p-Wert \\
\hline Alter & 3.299 & 4.089 & 25.79 & $<2 \mathrm{e}-16$ \\
\hline
\end{tabular}




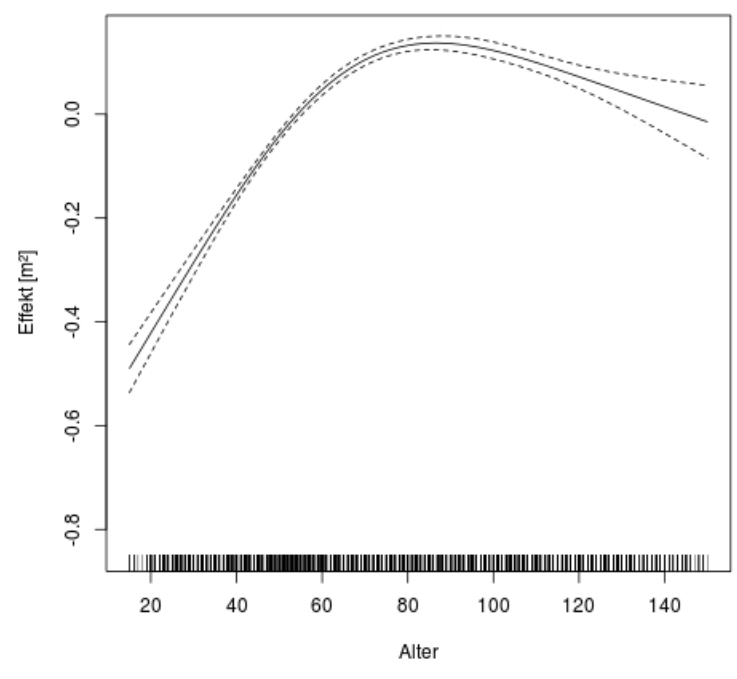

(a)

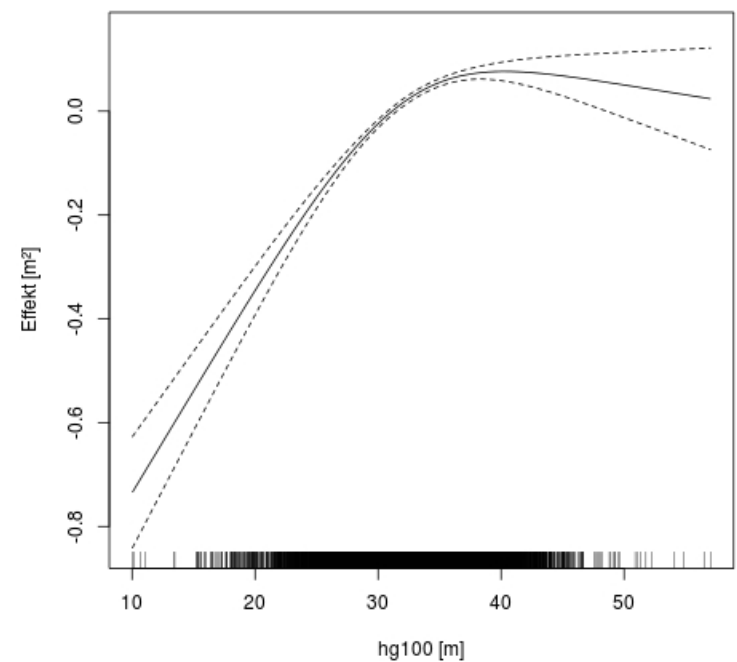

(b)

Abbildung A.8: Effekt des Alters (a) und der absoluten Mittelhöhenbonität (hg100 [m]) (b) auf die Schätzung der Bestandesgrundfläche von Fichtenreinbeständen.

Tabelle A.14: Parameter und statistische Eigenschaften des Grundflächenmodells für Fichtenreinbestände.

\begin{tabular}{lllll}
\hline \multicolumn{5}{c}{ Parameterkoeffizienten } \\
& Schätzwert & Std. Fehler & t-Wert & $\operatorname{Pr}(>|\mathrm{t}|)$ \\
\hline Interzept & 3.456723 & 0.004332 & 798 & $<2 \mathrm{e}-16$ \\
\hline \multicolumn{5}{c}{ Approximierte Signifikanz der glättenden Terme } \\
& edf & Ref.df & F & p-Wert \\
\hline Alter & 2.933 & 2.997 & 216.4 & $<2 \mathrm{e}-16$ \\
hg100 & 1.981 & 2.000 & 122.5 & $<2 \mathrm{e}-16$ \\
\hline
\end{tabular}




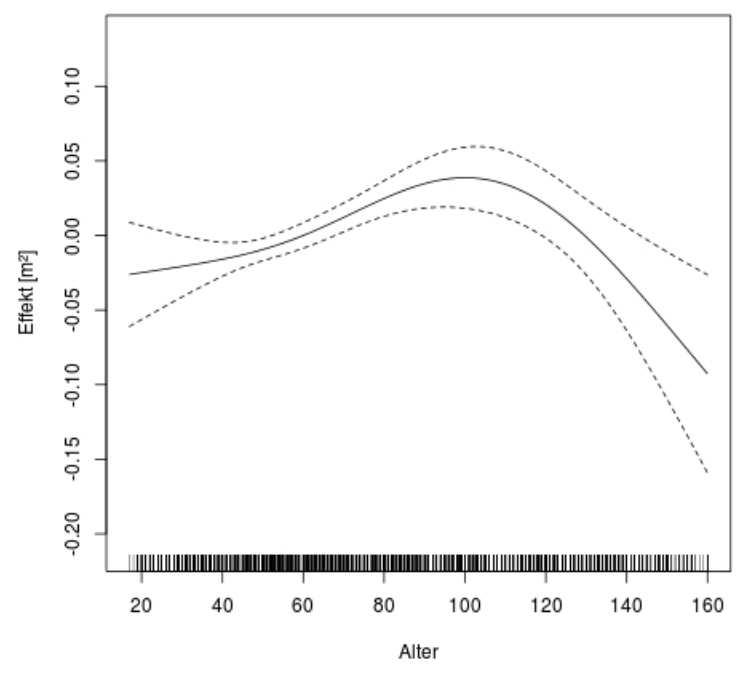

(a)

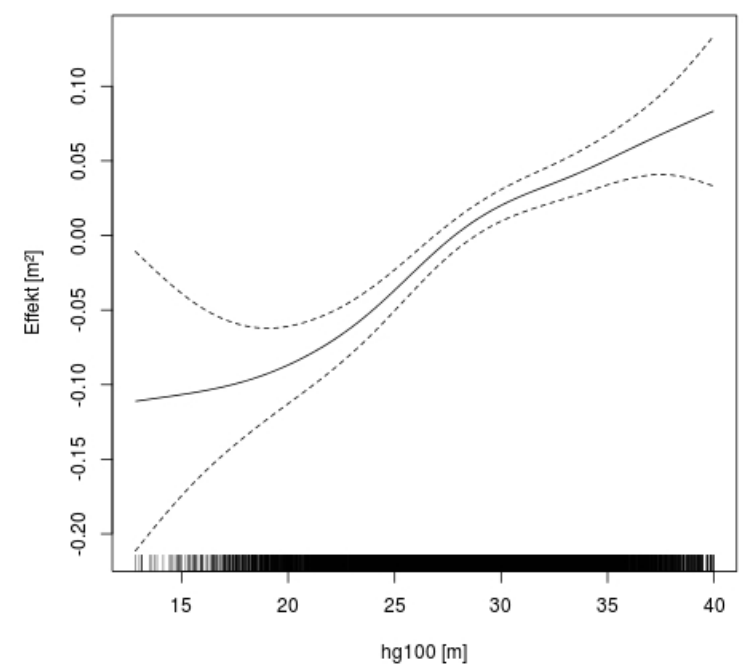

(b)

Abbildung A.9: Effekt des Alters (a) und der absoluten Mittelhöhenbonität (hg100 [m]) (b) auf die Schätzung der Bestandesgrundfläche von Kiefernreinbeständen.

Tabelle A.15: Parameter und statistische Eigenschaften des Grundflächenmodells für Kiefernreinbestände.

\begin{tabular}{lllll}
\hline \multicolumn{2}{l}{ Parameterkoeffizienten } \\
& Schätzwert & Std. Fehler & t-Wert & $\operatorname{Pr}(>|\mathrm{t}|)$ \\
\hline Interzept & 3.099527 & 0.004237 & 731.6 & $<2 \mathrm{e}-16$ \\
\hline \multicolumn{5}{c}{ Approximierte Signifikanz der glättenden Terme } \\
& edf & Ref.df & F & p-Wert \\
\hline Alter & 2.861 & 2.984 & 6.539 & 0.000217 \\
hg100 & 3.496 & 4.409 & 16.732 & $2.01 \mathrm{e}-14$ \\
\hline
\end{tabular}




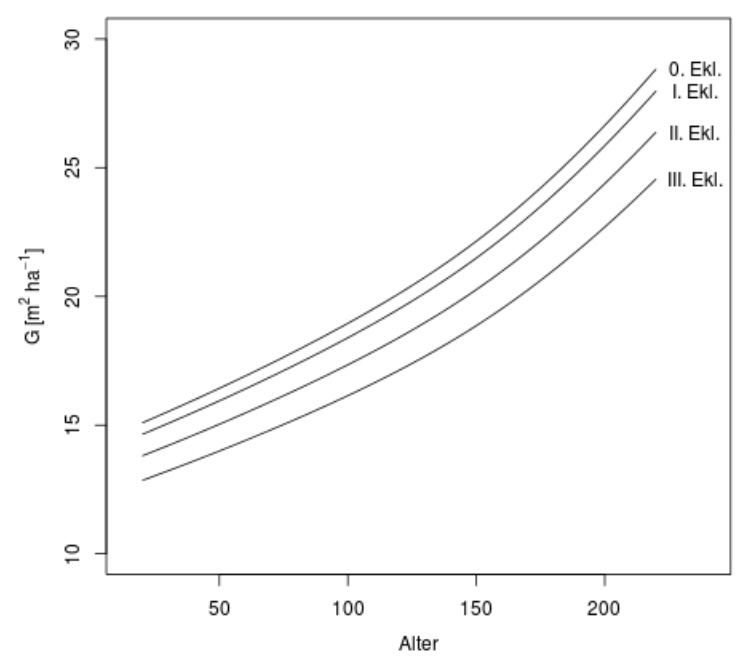

(a)

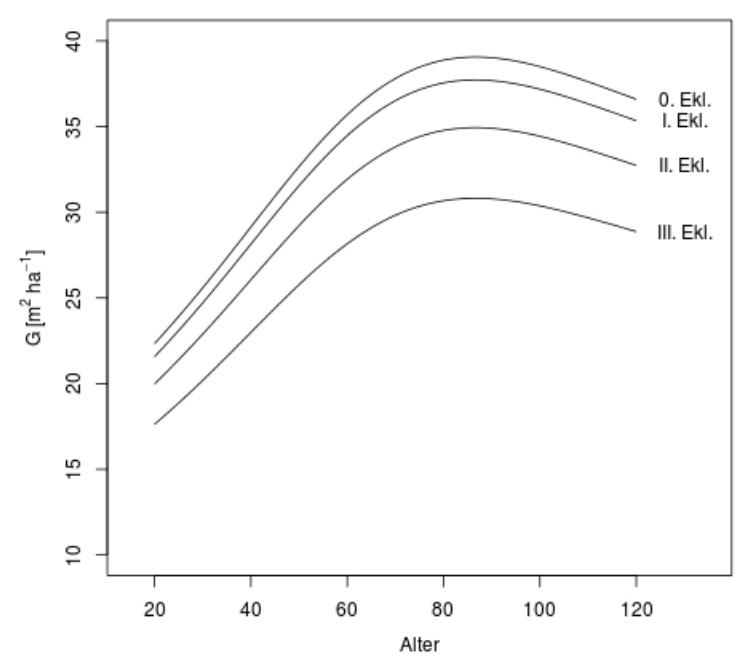

(c)

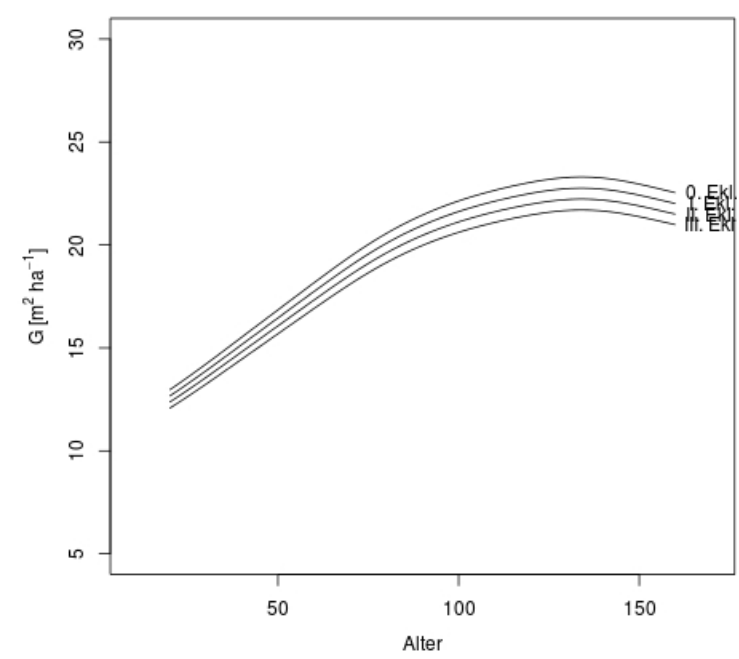

(b)

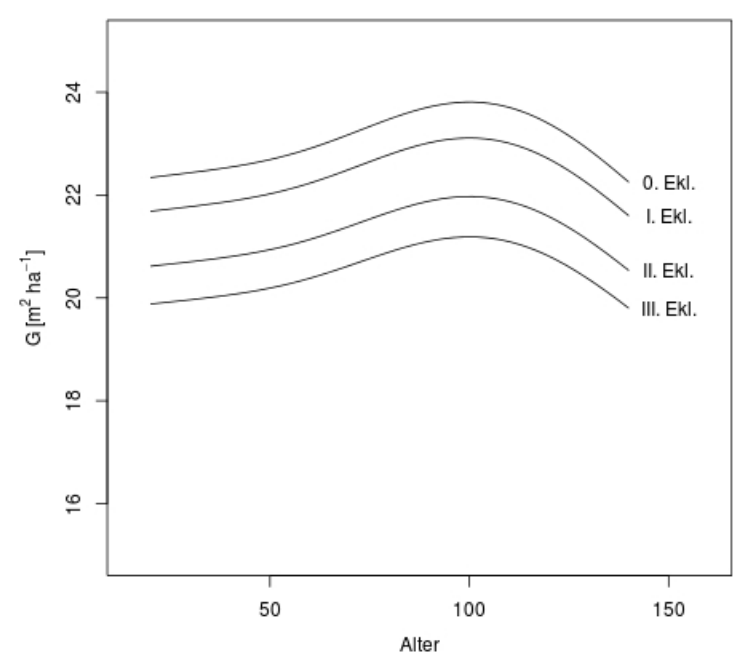

(d)

Abbildung A.10: Geschätzte Bestandesgrundflächen von Eichen- (a), Buchen- (b), Fichten- (c) und Kiefernreinbeständen (d) über dem Bestandesalter bei unterschiedlichen Ertragsklassen. 


\section{A.3 Herleitung der maximalen Bestandesgrundfläche}

Tabelle A.16: Verwendete Versuchsflächen der Nordwestdeutschen Forstlichen Versuchsanstalt zur Ermittlung der maximalen Bestandesgrundfläche von Reinbeständen der Hauptwirtschaftsbaumarten in Nordwestdeutschland.

\begin{tabular}{|c|c|c|}
\hline Art & Flächenbezeichnung & Durchforstungsart / sonstiges \\
\hline Eiche & $\begin{array}{l}\text { Ankum } 565 \\
\text { Saarforst } 3246 \\
\text { Herborn } 1169 \\
\text { Fuhrberg } 1231 \\
\text { Neuhaus } 1278 \\
\text { Wolfenbüttel } 2201 \\
\text { Rotenburg } 1079 \\
\text { Neuenburg } 317 \\
\text { Ahlhorn } 21 \\
\text { Göhrde } 1346 \\
\text { Rotenburg } 49 \\
\text { SHLF3779 } \\
\text { Münden } 2009 \\
\text { Jossgrund } 1787 \\
\text { Groß-Gerau } 332 \\
\text { LSA, BT Süd } 67 \mathrm{~b} 2 / \mathrm{b} 4 \\
\end{array}$ & $\begin{array}{l}\text { mäßige Niederdurchforstung } \\
\text { mäßige Niederdurchforstung } \\
\text { mäßige Niederdurchforstung } \\
\text { Nullfläche } \\
\text { Nullfläche } \\
\text { Nullfläche } \\
\text { Nullfläche } \\
\text { Nullfläche } \\
\text { Nullfläche } \\
\text { mäßige Niederdurchforstung } \\
\text { Nullfläche } \\
\text { Nullfläche } \\
\text { Nullfläche } \\
\text { Nullfläche } \\
\text { Nullfläche } \\
\text { erste Aufnahme }\end{array}$ \\
\hline Buche & $\begin{array}{l}\text { Saarf. (fr. Fischb.) } 1606 \\
\text { Herborn } 1333 \\
\text { Hochstift } 616 / 618 \\
\text { Ahlhorn } 106 \\
\text { Unterlüss } 138 \\
\text { Münden } 2027 \\
\text { Münden } 2028 \\
\text { Lauterberg } 368 \\
\text { Reinhausen } 3018 \\
\text { Saarforst } 1668 \\
\text { Lahnstein 9 } \\
\text { Bleicherode-Südharz } 701 \\
\text { Laubach (Stadtwald) } 130 \\
\text { Schotten } 393 / 395 \\
\text { Wiesbaden-Chausseeh. } 116 \\
\text { Dassel 4251 } \\
\text { Reinhausen } 166\end{array}$ & $\begin{array}{l}\text { schwache Niederdurchforstung } \\
\text { schwache Niederdurchforstung } \\
\text { schwache Niederdurchforstung } \\
\text { Nullfläche } \\
\text { Nullfläche } \\
\text { Nullfläche } \\
\text { Nullfläche } \\
\text { Nullfläche } \\
\text { Kernfläche Naturwald } \\
\text { Nullfläche } \\
\text { Nullfläche } \\
\text { Nullfläche } \\
\text { schwache Niederdurchforstung } \\
\text { Nullfläche } \\
\text { schwache Niederdurchforstung } \\
\text { Level II, ohne Durchforstung } \\
\text { Level II, ohne Durchforstung }\end{array}$ \\
\hline
\end{tabular}

Fortsetzung auf nächster Seite. 
Tabelle A.16

\begin{tabular}{|c|c|c|}
\hline Art & Flächenbezeichnung & Durchforstungsart / sonstiges \\
\hline \multirow{17}{*}{ Fichte } & Hochstift 990 & schwache Niederdurchforstung \\
\hline & Hochstift 697 & schwache Niederdurchforstung \\
\hline & Westerhof 131 & schwache Niederdurchforstung \\
\hline & NP Harz 683 & Imm.-DBO \\
\hline & Grünenplan 66 & Klon-Fichte, unbehandelt \\
\hline & Neuhaus 2271 & Nullfläche \\
\hline & Clausthal 1408/1411 & Nullfläche \\
\hline & Dassel 28 & Level II, ohne Durchforstung \\
\hline & NP Harz 358 & Imm.-DBO \\
\hline & Neuhaus 2146/2149 & Nullfäche \\
\hline & Oberharz 332/334 & schwache Niederdurchforstung \\
\hline & NP Harz 137 & schwache Niederdurchforstung \\
\hline & Ostharz 91 & schwache Niederdurchforstung \\
\hline & Bad Hersfeld 190 & Klon-Fi., unbehandelt \\
\hline & Wehretal 2588 & Klon-Fi., unbehandelt \\
\hline & Romrod 1301 & Nullfläche \\
\hline & SHLF 3532 & Klon-Fi., unbehandelt \\
\hline \multirow{21}{*}{ Douglasie } & Riefensbek 135 & schwache und mäßige Niederdurchforstung \\
\hline & Lauterberg 1317 & mäßige Niederdurchforstung \\
\hline & NP Harz 707 & ab 1980 unbehandelt \\
\hline & Lauterberg 225 & mäßige Niederdurchforstung \\
\hline & Lauterberg 211 & mäßige Niederdurchforstung \\
\hline & Ahlhorn 107 & Nullfläche \\
\hline & Neuenburg 1466 & Nullfäche \\
\hline & Harsefeld 164 & Nullfläche \\
\hline & Nienburg 1130 & Nullfläche \\
\hline & Arenberg-Nordkirchen 957 & Nullfläche \\
\hline & Fuhrberg 128 & Nullfläche \\
\hline & Göhrde 68 & Nullfläche \\
\hline & Dassel 3042 & Nullfläche \\
\hline & Rotenburg 1027 & Nullfläche \\
\hline & Ahlhorn 2138/2139 & Nullfläche \\
\hline & Idarwald 187 & Nullfläche \\
\hline & Sobernheim 219 & Nullfläche \\
\hline & Idarwald 282 & Nullfläche \\
\hline & Kaiserslautern XI 7a2 & Nullfläche \\
\hline & Klosterforsten 411 & Nullfläche und mäßige Niederdurchforstung \\
\hline & Sellhorn 9 & mäßige Niederdurchforstung \\
\hline
\end{tabular}

Fortsetzung auf nächster Seite. 


\begin{tabular}{|c|c|c|}
\hline Art & Flächenbezeichnung & Durchforstungsart / sonstiges \\
\hline Douglasie & $\begin{array}{l}\text { Niederrhein } 122 \mathrm{~F} \\
\text { Ankum } 1124 \\
\text { Dassel } 1597 \\
\text { Riefensbek } 135 \\
\text { Melsungen } 1083 \\
\text { Biedenkopf } 114\end{array}$ & $\begin{array}{l}\text { Nullfläche } \\
\text { Nullfläche } \\
\text { Nullfläche } \\
\text { mäßige Niederdurchforstung } \\
\text { Nullfläche } \\
\text { Nullfläche }\end{array}$ \\
\hline Kiefer & $\begin{array}{l}\text { Fuhrberg } 239 \\
\text { Göhrde } 1 \\
\text { Unterlüss } 262 \\
\text { Göhrde } 1262 \\
\text { Gifhorn (Steinhorst) } \\
\text { Auermühle (Schrader) } \\
\text { Romrod } 314 \\
\text { Romrod } 1007 \\
\text { Romrod } 1055 \\
\text { Anhalt } 1818 \\
\text { Südostheide (LWK) } 13 \\
\text { Anhalt } 1260\end{array}$ & $\begin{array}{l}\text { Nullfläche } \\
\text { Nullfläche } \\
\text { Nullfläche } \\
\text { Nullfläche } \\
\text { Nullfläche } \\
\text { Nullfläche } \\
\text { schwache und mäßige Niederdurchforstung } \\
\text { schwache Niederdurchforstung } \\
\text { schwache und mäßige Niederdurchforstung } \\
\text { Nullfläche } \\
\text { Nullfläche } \\
\text { Level II, mäßige Niederdurchforstung }\end{array}$ \\
\hline
\end{tabular}

Tabelle A.17: Koeffizienten und statistische Eigenschaften der Parameter der Box-Cox-ColeGreen-Verteilung zur Schätzung der maximalen Bestandesgrundfläche von Eichenreinbeständen.

\begin{tabular}{lrrrr}
\hline Parameterkoeffizienten & Schätzwert & Std. Fehler & $\mathrm{t}$-Wert & $\operatorname{Pr}(>|\mathrm{t}|)$ \\
\hline Koeffizienten von $\mu$ & & & & \\
Interzept & 6.148 & 1.267 & 4.852 & $4.06 \mathrm{e}-06$ \\
h100 & 1.076 & 0.057 & 18.637 & $<2 \mathrm{e}-16$ \\
Koeffizienten von $\sigma$ & & & & \\
Interzept & -1.421 & 0.234 & -6.063 & $1.92 \mathrm{e}-08$ \\
h100 & -0.023 & 0.010 & -2.257 & 0.026 \\
Koeffizienten von $\nu$ & & & & \\
Interzept & 1.382 & 0.461 & 2.999 & 0.003 \\
\hline
\end{tabular}


Tabelle A.18: Koeffizienten und statistische Eigenschaften der Parameter der Box-Cox-ColeGreen-Verteilung zur Schätzung der maximalen Bestandesgrundfläche von Buchenreinbeständen.

\begin{tabular}{lrrrr}
\hline Parameterkoeffizienten & Schätzwert & Std. Fehler & t-Wert & $\operatorname{Pr}(>|\mathrm{t}|)$ \\
\hline $\begin{array}{l}\text { Koeffizienten von } \mu \\
\text { Interzept }\end{array}$ & & & & \\
h100 & 1.039 & 0.973 & 8.856 & $2.81 \mathrm{e}-15$ \\
Koeffizienten von $\sigma$ & & & & \\
Interzept & -1.889 & 0.061 & -30.88 & $<2 \mathrm{e}-16$ \\
Koeffizienten von $\nu$ & & & & \\
Interzept & 1.220 & 0.521 & 2.341 & 0.020 \\
\hline
\end{tabular}

Tabelle A.19: Koeffizienten und statistische Eigenschaften der Parameter der Box-Cox-ColeGreen-Verteilung zur Schätzung der maximalen Bestandesgrundfläche von Fichtenreinbeständen.

\begin{tabular}{lrrrr}
\hline Parameterkoeffizienten & Schätzwert & Std. Fehler & t-Wert & $\operatorname{Pr}(>|\mathrm{t}|)$ \\
\hline Koeffizienten von $\mu$ & & & & \\
Interzept & 7.088 & 1.183 & 5.991 & $1.35 \mathrm{e}-08$ \\
h100 & 1.508 & 0.057 & 26.402 & $<2 \mathrm{e}-16$ \\
Koeffizienten von $\sigma$ & & & & \\
Interzept & -1.732 & 0.057 & -30.21 & $<2 \mathrm{e}-16$ \\
Koeffizienten von $\nu$ & & & & \\
Interzept & 0.835 & 0.370 & 2.257 & 0.025 \\
\hline
\end{tabular}


Tabelle A.20: Koeffizienten und statistische Eigenschaften der Parameter der Box-Cox-ColeGreen-Verteilung zur Schätzung der maximalen Bestandesgrundfläche von Douglasienreinbeständen.

\begin{tabular}{lrrrr}
\hline Parameterkoeffizienten & Schätzwert & Std. Fehler & t-Wert & $\operatorname{Pr}(>|\mathrm{t}|)$ \\
\hline Koeffizienten von $\mu$ & & & & \\
Interzept & 15.477 & 0.733 & 21.10 & $<2 \mathrm{e}-16$ \\
h100 & 0.941 & 0.038 & 24.29 & $<2 \mathrm{e}-16$ \\
Koeffizienten von $\sigma$ & & & & \\
Interzept & -2.058 & 0.144 & -14.213 & $<2 \mathrm{e}-16$ \\
h100 & 0.010 & 0.004 & 2.204 & 0.028 \\
Koeffizienten von $\nu$ & & & & \\
Interzept & -0.137 & 0.313 & -0.411 & 0.681 \\
\hline
\end{tabular}

Tabelle A.21: Koeffizienten und statistische Eigenschaften der Parameter der Box-Cox-ColeGreen-Verteilung zur Schätzung der maximalen Bestandesgrundfläche von Kiefernreinbeständen.

\begin{tabular}{lrrrr}
\hline Parameterkoeffizienten & Schätzwert & Std. Fehler & t-Wert & $\operatorname{Pr}(>|\mathrm{t}|)$ \\
\hline $\begin{array}{l}\text { Koeffizienten von } \mu \\
\text { Interzept }\end{array}$ & & & & \\
h100 & 1.861 & 1.161 & 2.464 & 0.015 \\
Koeffizienten von $\sigma$ & & 0.048 & 36.501 & $<2 \mathrm{e}-16$ \\
Interzept & -0.293 & 0.249 & -1.175 & 0.243 \\
h100 & -0.104 & 0.013 & -7.749 & $1.92 \mathrm{e}-11$ \\
Koeffizienten von $\nu$ & & & & \\
Interzept & 0.436 & 0.597 & 0.729 & 0.468 \\
\hline
\end{tabular}




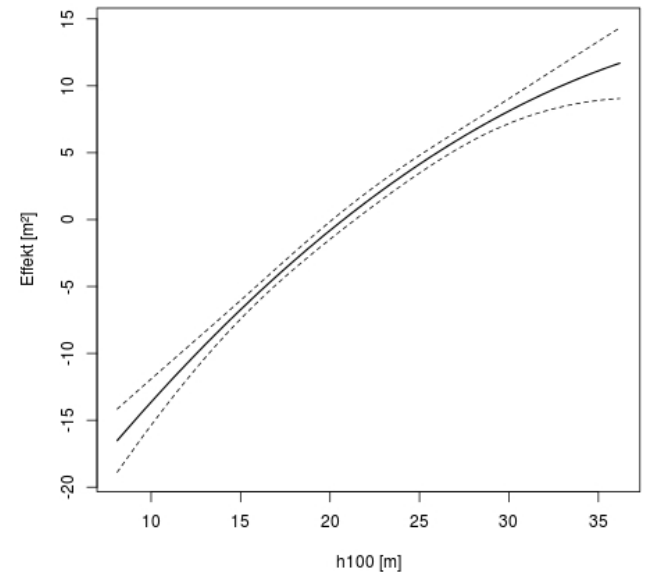

Abbildung A.11: Effekt der Spitzenhöhe (h100 [m]) auf den Mittelwert $\mu$ der Box-Cox-ColeGreen-Verteilung zur Schätzung der maximalen Bestandesgrundfläche von Eichenreinbeständen.

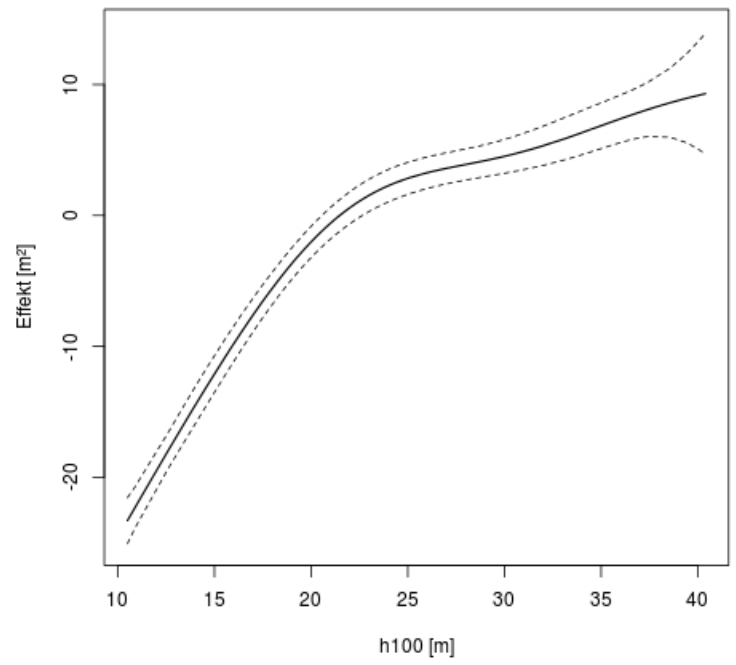

Abbildung A.12: Effekt der Spitzenhöhe (h100 [m]) auf den Mittelwert $\mu$ der Box-Cox-ColeGreen-Verteilung zur Schätzung der maximalen Bestandesgrundfläche von Buchenreinbeständen. 


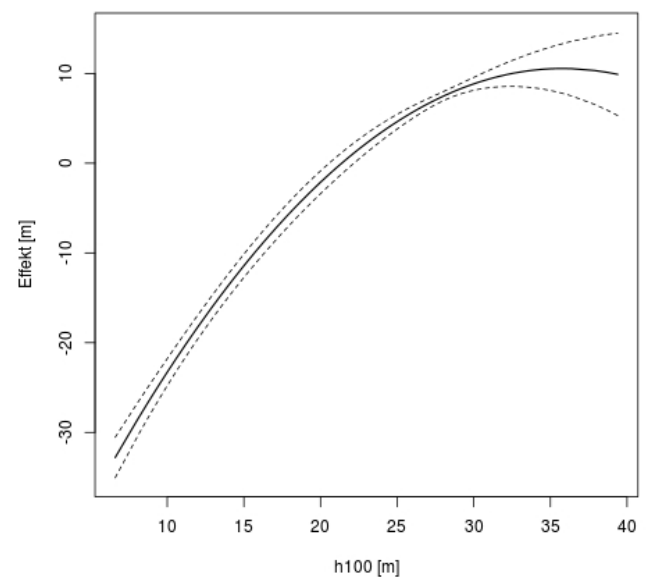

Abbildung A.13: Effekt der Spitzenhöhe (h100 [m]) auf den Mittelwert $\mu$ der Box-Cox-ColeGreen-Verteilung zur Schätzung der maximalen Bestandesgrundfläche von Fichtenreinbeständen.

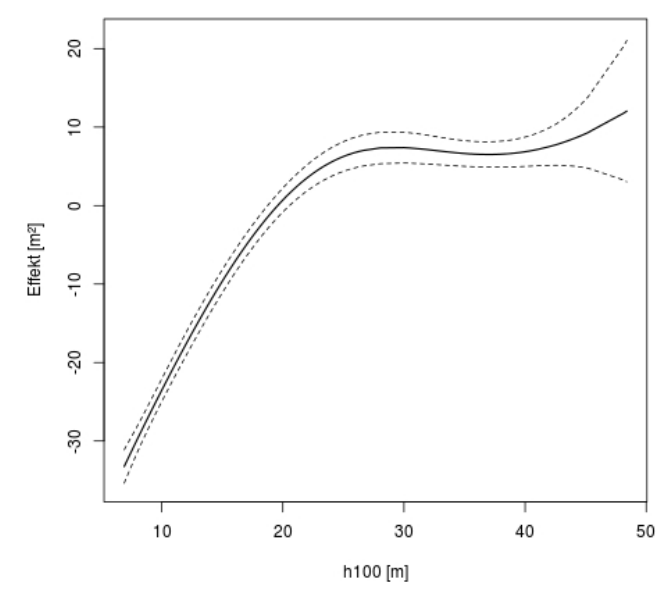

(a)

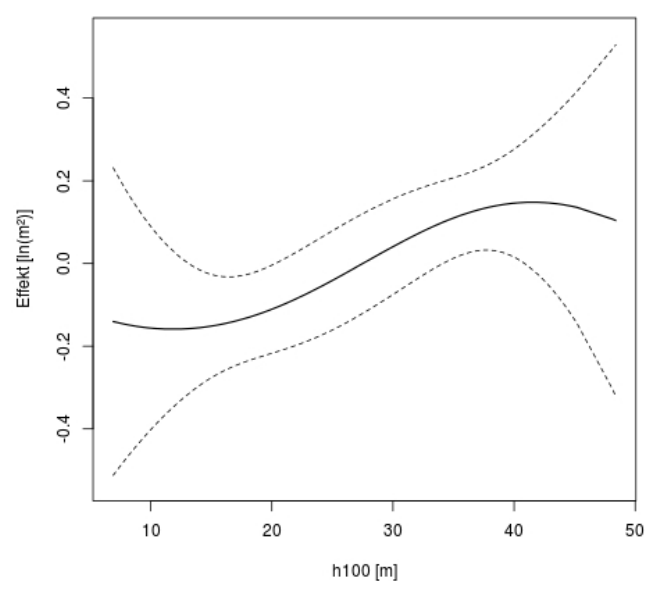

(b)

Abbildung A.14: Effekt der Spitzenhöhe (h100 [m]) auf den Mittelwert $\mu$ (a) sowie auf den Variationskoeffizienten $\sigma$ (b) der Box-Cox-Cole-Green-Verteilung zur Schätzung der maximalen Bestandesgrundfläche von Douglasienreinbeständen. 


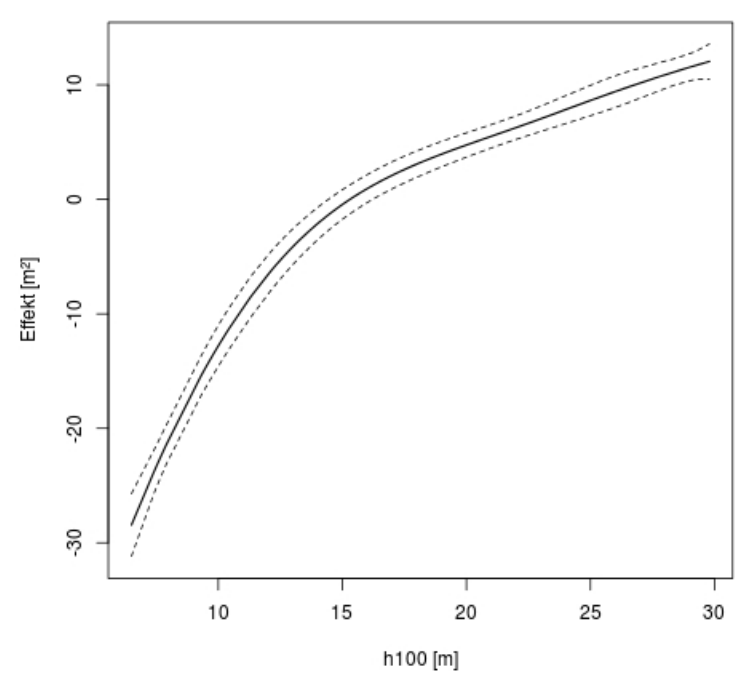

(a)

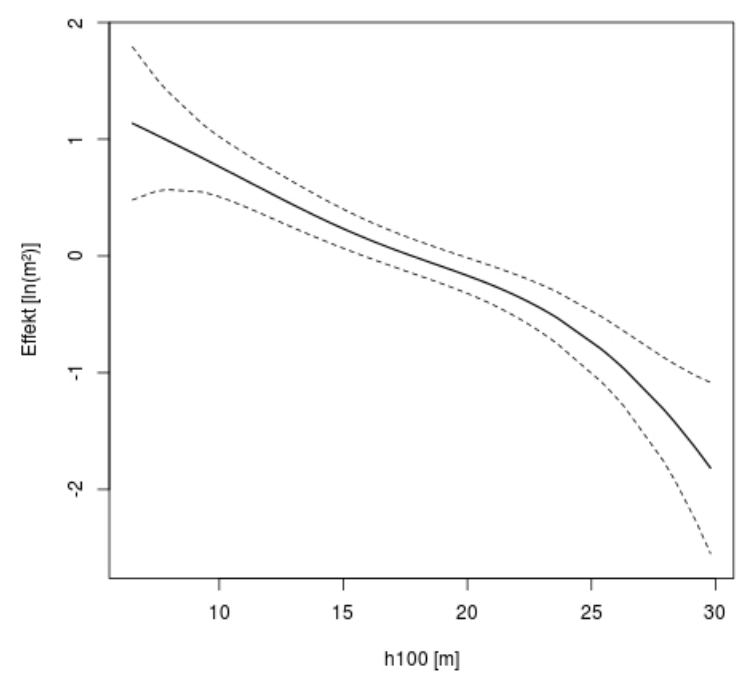

(b)

Abbildung A.15: Effekt der Spitzenhöhe (h100 [m]) auf den Mittelwert $\mu$ (a) sowie auf den Variationskoeffizienten $\sigma$ (b) der Box-Cox-Cole-Green-Verteilung zur Schätzung der maximalen Bestandesgrundfläche von Kiefernreinbeständen. 


\section{A.4 Kennwerte der Weiserbestände}

Tabelle A.22: Kennwerte (LKL $=$ Leistungsklasse $\left[\mathrm{m}^{3} \mathrm{ha}^{-1} \mathrm{a}^{-1}\right], \mathrm{N}=$ Stammzahl $\left[\mathrm{nha}^{-1}\right]$, dg $=$ Durchmesser des Grundflächenmittelstamms $[\mathrm{cm}], \mathrm{hg}=$ Höhe des Grundflächenmittelstamms $[\mathrm{m}], \mathrm{G}=$ Bestandesgrundfläche $\left[\mathrm{m}^{2} \mathrm{ha}^{-1}\right], \mathrm{V}=$ Derbholzvolumen $\left[\mathrm{m}^{3} \mathrm{ha}^{-1}\right], \mathrm{CV}=$ Kohlenstoffvorrat der lebenden Baumbiomasse $\left.\left[\mathrm{t} \mathrm{C} \mathrm{ha}^{-1}\right]\right)$ der erstellten Weiserbestände getrennt nach Wuchsbezirk (WBZ, EH = Ems-HaseHunte-Geest, GM = Geest-Mitte, GW = Göttinger Wald, HH = Hohe Heide, $\mathrm{MH}=$ Montaner Mittel-und Oberharz, $\mathrm{OH}=$ Ost-Heide, US = Unterer Solling, $\mathrm{UW}=$ Unteres Weser-Leine-Bergland), Standortstypenobergruppe (SOGR, $1=$ basenarme Silikatgesteine, 2 = basenreiche Silikatgesteine, $3=$ Flugsande, $4=$ Geschiebelehme, $5=$ Kalkgesteine, $6=$ Lösse, $7=$ Sande, $8=$ Sande im Grundwasserbereich, 9 = Sandlösse), Wasserhaushalts- (WHZ, 1 bis $3=$ stark, mäßig, schwach grundwasserbeeinflusst, $4=$ nachhaltig frisch, $5=$ frisch, $6=$ mäßig frisch bis frisch, $7=$ mäßig frisch, $8=$ sommertrocken, $9=$ trocken) und Nährstoffgruppe (NZ, $1=$ sehr gut, $2=$ ziemlich gut, $3=$ mäßig, $4=$ schwach) sowie Art $(110=$ Eiche, $211=$ Buche, $511=$ Fichte, $611=$ Douglasie, $711=$ Kiefer $)$, Alter (Alt) und Bonitätsgruppe (BG, $\mathrm{g}=$ gut, $\mathrm{m}=$ mittel, $\mathrm{s}=$ schlecht).

\begin{tabular}{cccccccccccccc}
\hline WBZ & SOGR & WHZ & NZ & Art & Alt & BG & LKL & N & dg & hg & V & G & CV \\
\hline EH & 3 & 3 & 3 & 110 & 35 & $\mathrm{~m}$ & 6,0 & 1168 & 12,8 & 12,0 & 87,2 & 15,1 & 37,2 \\
EH & 3 & 3 & 3 & 110 & 65 & $\mathrm{~m}$ & 6,0 & 284 & 27,0 & 19,3 & 168,0 & 16,2 & 64,6 \\
EH & 3 & 3 & 3 & 110 & 95 & $\mathrm{~m}$ & 6,0 & 162 & 37,7 & 23,8 & 230,8 & 18,1 & 89,5 \\
$\mathrm{EH}$ & 3 & 3 & 3 & 110 & 125 & $\mathrm{~m}$ & 6,0 & 100 & 50,0 & 27,1 & 284,1 & 19,7 & 110,6 \\
$\mathrm{EH}$ & 3 & 3 & 3 & 110 & 155 & $\mathrm{~m}$ & 6,0 & 82 & 57,5 & 29,6 & 334,4 & 21,3 & 131,2 \\
$\mathrm{EH}$ & 3 & 3 & 3 & 110 & 185 & $\mathrm{~m}$ & 6,0 & 72 & 64,9 & 31,7 & 400,9 & 23,9 & 158,1 \\
$\mathrm{EH}$ & 3 & 3 & 3 & 711 & 30 & $\mathrm{~s}$ & 6,3 & 2030 & 12,6 & 9,9 & 116,2 & 25,1 & 39,0 \\
$\mathrm{EH}$ & 3 & 3 & 3 & 711 & 60 & $\mathrm{~s}$ & 6,3 & 409 & 25,6 & 17,9 & 172,5 & 21,1 & 47,4 \\
$\mathrm{EH}$ & 3 & 3 & 3 & 711 & 90 & $\mathrm{~s}$ & 6,3 & 234 & 34,5 & 22,5 & 217,8 & 21,9 & 56,6 \\
$\mathrm{EH}$ & 3 & 3 & 3 & 711 & 120 & $\mathrm{~s}$ & 6,3 & 173 & 40,4 & 25,7 & 248,3 & 22,2 & 62,3 \\
$\mathrm{EH}$ & 3 & 3 & 3 & 711 & 150 & $\mathrm{~s}$ & 6,3 & 117 & 46,5 & 28,2 & 241,1 & 19,8 & 58,9 \\
$\mathrm{EH}$ & 3 & 3 & 4 & 611 & 25 & $\mathrm{~m}$ & 14,1 & 875 & 17,8 & 12,2 & 119,2 & 21,7 & 61,3 \\
$\mathrm{EH}$ & 3 & 3 & 4 & 611 & 55 & $\mathrm{~m}$ & 14,1 & 193 & 41,5 & 27,8 & 298,8 & 26,0 & 88,5 \\
$\mathrm{EH}$ & 3 & 3 & 4 & 611 & 85 & $\mathrm{~m}$ & 14,1 & 143 & 57,5 & 36,7 & 535,7 & 37,1 & 136,3 \\
$\mathrm{EH}$ & 3 & 3 & 4 & 611 & 115 & $\mathrm{~m}$ & 14,1 & 103 & 71,4 & 43,0 & 673,7 & 41,3 & 160,5 \\
$\mathrm{EH}$ & 3 & 3 & 4 & 711 & 30 & $\mathrm{~s}$ & 5,9 & 2081 & 12,3 & 9,6 & 110,4 & 24,7 & 37,6 \\
$\mathrm{EH}$ & 3 & 3 & 4 & 711 & 60 & $\mathrm{~s}$ & 5,9 & 413 & 25,6 & 17,4 & 168,8 & 21,2 & 46,6 \\
$\mathrm{EH}$ & 3 & 3 & 4 & 711 & 90 & $\mathrm{~s}$ & 5,9 & 245 & 33,6 & 21,8 & 209,9 & 21,7 & 55,0 \\
$\mathrm{EH}$ & 3 & 3 & 4 & 711 & 120 & $\mathrm{~s}$ & 5,9 & 177 & 39,2 & 25,0 & 233,4 & 21,4 & 59,0 \\
$\mathrm{EH}$ & 3 & 3 & 4 & 711 & 150 & $\mathrm{~s}$ & 5,9 & 116 & 46,5 & 27,4 & 233,3 & 19,7 & 57,5 \\
$\mathrm{EH}$ & 3 & 9 & 4 & 511 & 30 & $\mathrm{~m}$ & 10,4 & 1202 & 17,3 & 10,2 & 132,3 & 28,2 & 72,9 \\
\hline For & 3 & & 3 & & & & & & & & & &
\end{tabular}

Fortsetzung auf nächster Seite. 
Tabelle A.22

\begin{tabular}{|c|c|c|c|c|c|c|c|c|c|c|c|c|c|}
\hline WBZ & SOGR & WHZ & $\mathrm{NZ}$ & Art & Alt & $\mathrm{BG}$ & LKL & $\mathrm{N}$ & $\mathrm{dg}$ & hg & $\mathrm{V}$ & G & $\mathrm{CV}$ \\
\hline $\mathrm{EH}$ & 3 & 9 & 4 & 511 & 60 & $\mathrm{~m}$ & 10,4 & 439 & 30,9 & 21,0 & 307,6 & 32,8 & 109,2 \\
\hline $\mathrm{EH}$ & 3 & 9 & 4 & 511 & 90 & $\mathrm{~m}$ & 10,4 & 294 & 39,6 & 28,4 & 445,7 & 36,3 & 133,9 \\
\hline $\mathrm{EH}$ & 3 & 9 & 4 & 611 & 25 & $\mathrm{~m}$ & 13,7 & 893 & 17,5 & 11,9 & 115,2 & 21,5 & 60,6 \\
\hline $\mathrm{EH}$ & 3 & 9 & 4 & 611 & 55 & $\mathrm{~m}$ & 13,7 & 188 & 41,9 & 27,5 & 294,6 & 26,0 & 88,6 \\
\hline $\mathrm{EH}$ & 3 & 9 & 4 & 611 & 85 & $\mathrm{~m}$ & 13,7 & 147 & 56,6 & 36,3 & 529,1 & 37,0 & 135,4 \\
\hline $\mathrm{EH}$ & 3 & 9 & 4 & 611 & 115 & $\mathrm{~m}$ & 13,7 & 107 & 69,2 & 42,6 & 653,8 & 40,2 & 154,7 \\
\hline $\mathrm{EH}$ & 3 & 9 & 4 & 711 & 30 & $\mathrm{~S}$ & 5,9 & 2077 & 12,3 & 9,6 & 110,3 & 24,6 & 37,5 \\
\hline $\mathrm{EH}$ & 3 & 9 & 4 & 711 & 60 & $\mathrm{~s}$ & 5,9 & 408 & 25,5 & 17,4 & 166,5 & 20,9 & 46,0 \\
\hline $\mathrm{EH}$ & 3 & 9 & 4 & 711 & 90 & $\mathrm{~s}$ & 5,9 & 242 & 34,4 & 21,8 & 217,1 & 22,5 & 56,9 \\
\hline $\mathrm{EH}$ & 3 & 9 & 4 & 711 & 120 & $\mathrm{~S}$ & 5,9 & 173 & 39,8 & 25,0 & 234,6 & 21,5 & 59,3 \\
\hline $\mathrm{EH}$ & 3 & 9 & 4 & 711 & 150 & $\mathrm{~S}$ & 5,9 & 115 & 46,7 & 27,4 & 233,1 & 19,7 & 57,4 \\
\hline $\mathrm{EH}$ & 4 & 5 & 2 & 110 & 35 & $\mathrm{~m}$ & 6,0 & 1179 & 12,8 & 12,0 & 86,1 & 15,1 & 36,9 \\
\hline $\mathrm{EH}$ & 4 & 5 & 2 & 110 & 65 & $\mathrm{~m}$ & 6,0 & 282 & 27,1 & 19,3 & 168,7 & 16,3 & 64,9 \\
\hline $\mathrm{EH}$ & 4 & 5 & 2 & 110 & 95 & $\mathrm{~m}$ & 6,0 & 154 & 38,4 & 23,8 & 227,2 & 17,8 & 88,0 \\
\hline $\mathrm{EH}$ & 4 & 5 & 2 & 110 & 125 & $\mathrm{~m}$ & 6,0 & 99 & 50,5 & 27,1 & 287,0 & 19,8 & 111,6 \\
\hline $\mathrm{EH}$ & 4 & 5 & 2 & 110 & 155 & $\mathrm{~m}$ & 6,0 & 76 & 60,2 & 29,6 & 341,1 & 21,7 & 133,4 \\
\hline $\mathrm{EH}$ & 4 & 5 & 2 & 110 & 185 & $\mathrm{~m}$ & 6,0 & 72 & 64,8 & 31,7 & 398,8 & 23,8 & 157,5 \\
\hline $\mathrm{EH}$ & 4 & 5 & 2 & 211 & 40 & $\mathrm{~g}$ & 8,8 & 1262 & 13,6 & 14,6 & 126,0 & 18,2 & 57,9 \\
\hline $\mathrm{EH}$ & 4 & 5 & 2 & 211 & 70 & $\mathrm{~g}$ & 8,8 & 290 & 29,0 & 25,0 & 242,2 & 19,2 & 103,3 \\
\hline $\mathrm{EH}$ & 4 & 5 & 2 & 211 & 100 & $\mathrm{~g}$ & 8,8 & 156 & 42,1 & 31,6 & 352,5 & 21,7 & 147,1 \\
\hline $\mathrm{EH}$ & 4 & 5 & 2 & 211 & 130 & $\mathrm{~g}$ & 8,8 & 103 & 53,7 & 36,4 & 438,9 & 23,3 & 180,5 \\
\hline $\mathrm{EH}$ & 4 & 5 & 2 & 511 & 30 & $\mathrm{~g}$ & 11,6 & 1140 & 18,2 & 12,1 & 167,5 & 29,8 & 82,3 \\
\hline $\mathrm{EH}$ & 4 & 5 & 2 & 511 & 60 & $\mathrm{~g}$ & 11,6 & 418 & 32,4 & 23,4 & 359,2 & 34,4 & 119,3 \\
\hline $\mathrm{EH}$ & 4 & 5 & 2 & 511 & 90 & $\mathrm{~g}$ & 11,6 & 275 & 42,3 & 31,0 & 513,8 & 38,6 & 147,1 \\
\hline $\mathrm{EH}$ & 4 & 5 & 2 & 611 & 25 & $\mathrm{~m}$ & 14,4 & 876 & 17,8 & 12,4 & 122,1 & 21,8 & 61,7 \\
\hline $\mathrm{EH}$ & 4 & 5 & 2 & 611 & 55 & $\mathrm{~m}$ & 14,4 & 190 & 41,9 & 28,1 & 304,0 & 26,2 & 89,4 \\
\hline $\mathrm{EH}$ & 4 & 5 & 2 & 611 & 85 & $\mathrm{~m}$ & 14,4 & 138 & 59,0 & 37,0 & 546,7 & 37,7 & 139,4 \\
\hline $\mathrm{EH}$ & 4 & 5 & 2 & 611 & 115 & $\mathrm{~m}$ & 14,4 & 107 & 69,8 & 43,3 & 676,8 & 41,0 & 158,3 \\
\hline $\mathrm{EH}$ & 4 & 5 & 2 & 711 & 30 & $\mathrm{~m}$ & 6,6 & 1960 & 12,8 & 10,6 & 124,1 & 25,2 & 40,9 \\
\hline $\mathrm{EH}$ & 4 & 5 & 2 & 711 & 60 & $\mathrm{~m}$ & 6,6 & 381 & 26,7 & 18,9 & 183,2 & 21,4 & 49,7 \\
\hline $\mathrm{EH}$ & 4 & 5 & 2 & 711 & 90 & $\mathrm{~m}$ & 6,6 & 224 & 35,6 & 23,7 & 231,5 & 22,2 & 59,4 \\
\hline $\mathrm{EH}$ & 4 & 5 & 2 & 711 & 120 & $\mathrm{~m}$ & 6,6 & 159 & 41,8 & 27,0 & 255,8 & 21,8 & 63,3 \\
\hline $\mathrm{EH}$ & 4 & 5 & 2 & 711 & 150 & $\mathrm{~m}$ & 6,6 & 113 & 47,6 & 29,6 & 256,6 & 20,1 & 61,8 \\
\hline $\mathrm{EH}$ & 4 & 5 & 3 & 110 & 35 & $\mathrm{~m}$ & 6,0 & 1169 & 12,8 & 12,0 & 85,5 & 15,0 & 36,6 \\
\hline $\mathrm{EH}$ & 4 & 5 & 3 & 110 & 65 & $\mathrm{~m}$ & 6,0 & 281 & 27,1 & 19,3 & 167,7 & 16,2 & 64,5 \\
\hline $\mathrm{EH}$ & 4 & 5 & 3 & 110 & 95 & $\mathrm{~m}$ & 6,0 & 160 & 37,6 & 23,8 & 226,0 & 17,8 & 87,7 \\
\hline $\mathrm{EH}$ & 4 & 5 & 3 & 110 & 125 & $\mathrm{~m}$ & 6,0 & 102 & 50,3 & 27,1 & 293,7 & 20,3 & 114,2 \\
\hline $\mathrm{EH}$ & 4 & 5 & 3 & 110 & 155 & $\mathrm{~m}$ & 6,0 & 81 & 58,7 & 29,6 & 344,7 & 21,9 & 135,1 \\
\hline
\end{tabular}

Fortsetzung auf nächster Seite. 
Tabelle A.22

\begin{tabular}{|c|c|c|c|c|c|c|c|c|c|c|c|c|c|}
\hline WBZ & SOGR & WHZ & $\mathrm{NZ}$ & Art & Alt & $\mathrm{BG}$ & LKL & $\mathrm{N}$ & $\mathrm{dg}$ & hg & $\mathrm{V}$ & G & $\mathrm{CV}$ \\
\hline $\mathrm{EH}$ & 4 & 5 & 3 & 110 & 185 & $\mathrm{~m}$ & 6,0 & 73 & 65,7 & 31,7 & 414,7 & 24,7 & 163,6 \\
\hline $\mathrm{EH}$ & 4 & 5 & 3 & 511 & 30 & $\mathrm{~g}$ & 11,6 & 1158 & 18,1 & 11,8 & 162,9 & 29,8 & 81,4 \\
\hline $\mathrm{EH}$ & 4 & 5 & 3 & 511 & 60 & g & 11,6 & 427 & 32,1 & 23,0 & 353,7 & 34,5 & 118,7 \\
\hline $\mathrm{EH}$ & 4 & 5 & 3 & 511 & 90 & $\mathrm{~g}$ & 11,6 & 281 & 41,2 & 30,6 & 495,0 & 37,5 & 142,3 \\
\hline $\mathrm{EH}$ & 4 & 5 & 3 & 611 & 25 & $\mathrm{~m}$ & 13,7 & 868 & 17,8 & 11,8 & 113,8 & 21,5 & 60,5 \\
\hline $\mathrm{EH}$ & 4 & 5 & 3 & 611 & 55 & $\mathrm{~m}$ & 13,7 & 183 & 42,4 & 27,3 & 290,4 & 25,9 & 88,4 \\
\hline $\mathrm{EH}$ & 4 & 5 & 3 & 611 & 85 & $\mathrm{~m}$ & 13,7 & 147 & 56,3 & 36,2 & 523,0 & 36,6 & 134,0 \\
\hline $\mathrm{EH}$ & 4 & 5 & 3 & 611 & 115 & $\mathrm{~m}$ & 13,7 & 105 & 69,5 & 42,4 & 644,0 & 39,8 & 153,2 \\
\hline $\mathrm{EH}$ & 4 & 5 & 3 & 711 & 30 & $\mathrm{~m}$ & 6,8 & 1945 & 12,9 & 10,8 & 126,7 & 25,2 & 41,5 \\
\hline $\mathrm{EH}$ & 4 & 5 & 3 & 711 & 60 & $\mathrm{~m}$ & 6,8 & 382 & 27,0 & 19,1 & 188,4 & 21,8 & 51,0 \\
\hline $\mathrm{EH}$ & 4 & 5 & 3 & 711 & 90 & $\mathrm{~m}$ & 6,8 & 225 & 35,5 & 24,0 & 235,4 & 22,3 & 60,2 \\
\hline $\mathrm{EH}$ & 4 & 5 & 3 & 711 & 120 & $\mathrm{~m}$ & 6,8 & 160 & 42,5 & 27,3 & 268,5 & 22,7 & 66,3 \\
\hline $\mathrm{EH}$ & 4 & 5 & 3 & 711 & 150 & $\mathrm{~m}$ & 6,8 & 108 & 49,8 & 29,9 & 270,1 & 21,1 & 64,9 \\
\hline $\mathrm{EH}$ & 4 & 6 & 3 & 110 & 35 & $\mathrm{~m}$ & 5,7 & 1207 & 12,5 & 11,5 & 79,3 & 14,7 & 34,4 \\
\hline $\mathrm{EH}$ & 4 & 6 & 3 & 110 & 65 & $\mathrm{~m}$ & 5,7 & 287 & 26,7 & 18,7 & 162,0 & 16,1 & 61,9 \\
\hline $\mathrm{EH}$ & 4 & 6 & 3 & 110 & 95 & $\mathrm{~m}$ & 5,7 & 163 & 37,0 & 23,1 & 217,7 & 17,6 & 84,0 \\
\hline $\mathrm{EH}$ & 4 & 6 & 3 & 110 & 125 & $\mathrm{~m}$ & 5,7 & 105 & 49,0 & 26,3 & 278,2 & 19,8 & 107,7 \\
\hline $\mathrm{EH}$ & 4 & 6 & 3 & 110 & 155 & $\mathrm{~m}$ & 5,7 & 86 & 58,3 & 28,9 & 353,9 & 23,0 & 138,0 \\
\hline $\mathrm{EH}$ & 4 & 6 & 3 & 110 & 185 & $\mathrm{~m}$ & 5,7 & 77 & 62,5 & 30,9 & 386,0 & 23,6 & 152,0 \\
\hline $\mathrm{EH}$ & 4 & 6 & 3 & 611 & 25 & $\mathrm{~m}$ & 13,7 & 896 & 17,5 & 11,8 & 113,6 & 21,4 & 60,2 \\
\hline $\mathrm{EH}$ & 4 & 6 & 3 & 611 & 55 & $\mathrm{~m}$ & 13,7 & 189 & 41,8 & 27,3 & 291,8 & 25,9 & 88,2 \\
\hline $\mathrm{EH}$ & 4 & 6 & 3 & 611 & 85 & $\mathrm{~m}$ & 13,7 & 145 & 56,3 & 36,2 & 515,2 & 36,1 & 132,0 \\
\hline $\mathrm{EH}$ & 4 & 6 & 3 & 611 & 115 & $\mathrm{~m}$ & 13,7 & 108 & 69,0 & 42,4 & 653,9 & 40,4 & 155,5 \\
\hline $\mathrm{EH}$ & 4 & 6 & 3 & 711 & 30 & $\mathrm{~m}$ & 6,6 & 1991 & 12,7 & 10,3 & 120,9 & 25,2 & 40,2 \\
\hline $\mathrm{EH}$ & 4 & 6 & 3 & 711 & 60 & $\mathrm{~m}$ & 6,6 & 389 & 26,4 & 18,5 & 178,7 & 21,3 & 48,7 \\
\hline $\mathrm{EH}$ & 4 & 6 & 3 & 711 & 90 & $\mathrm{~m}$ & 6,6 & 232 & 35,4 & 23,2 & 233,5 & 22,8 & 60,2 \\
\hline $\mathrm{EH}$ & 4 & 6 & 3 & 711 & 120 & $\mathrm{~m}$ & 6,6 & 167 & 41,3 & 26,5 & 257,4 & 22,4 & 64,1 \\
\hline $\mathrm{EH}$ & 4 & 6 & 3 & 711 & 150 & $\mathrm{~m}$ & 6,6 & 111 & 48,7 & 29,0 & 257,4 & 20,7 & 62,4 \\
\hline $\mathrm{EH}$ & 7 & 6 & 3 & 110 & 35 & $\mathrm{~m}$ & 6,0 & 1165 & 12,8 & 12,2 & 87,2 & 15,0 & 37,3 \\
\hline $\mathrm{EH}$ & 7 & 6 & 3 & 110 & 65 & $\mathrm{~m}$ & 6,0 & 274 & 27,6 & 19,5 & 171,5 & 16,4 & 65,9 \\
\hline $\mathrm{EH}$ & 7 & 6 & 3 & 110 & 95 & $\mathrm{~m}$ & 6,0 & 159 & 37,7 & 24,0 & 226,9 & 17,7 & 88,2 \\
\hline $\mathrm{EH}$ & 7 & 6 & 3 & 110 & 125 & $\mathrm{~m}$ & 6,0 & 101 & 49,6 & 27,3 & 283,9 & 19,5 & 110,7 \\
\hline $\mathrm{EH}$ & 7 & 6 & 3 & 110 & 155 & $\mathrm{~m}$ & 6,0 & 82 & 59,4 & 29,8 & 359,4 & 22,7 & 140,9 \\
\hline $\mathrm{EH}$ & 7 & 6 & 3 & 110 & 185 & $\mathrm{~m}$ & 6,0 & 73 & 65,0 & 32,0 & 409,1 & 24,2 & 161,8 \\
\hline $\mathrm{EH}$ & 7 & 6 & 3 & 211 & 40 & $\mathrm{~m}$ & 8,0 & 1397 & 12,8 & 12,9 & 110,0 & 18,1 & 51,0 \\
\hline $\mathrm{EH}$ & 7 & 6 & 3 & 211 & 70 & $\mathrm{~m}$ & 8,0 & 334 & 27,5 & 23,1 & 230,4 & 19,8 & 98,8 \\
\hline $\mathrm{EH}$ & 7 & 6 & 3 & 211 & 100 & $\mathrm{~m}$ & 8,0 & 179 & 39,0 & 29,5 & 320,8 & 21,4 & 135,1 \\
\hline $\mathrm{EH}$ & 7 & 6 & 3 & 211 & 130 & $\mathrm{~m}$ & 8,0 & 107 & 52,9 & 34,2 & 413,2 & 23,5 & 171,0 \\
\hline
\end{tabular}

Fortsetzung auf nächster Seite. 
Tabelle A.22

\begin{tabular}{|c|c|c|c|c|c|c|c|c|c|c|c|c|c|}
\hline WBZ & SOGR & WHZ & $\mathrm{NZ}$ & Art & Alt & $\mathrm{BG}$ & LKL & $\mathrm{N}$ & $\mathrm{dg}$ & hg & $\mathrm{V}$ & G & CV \\
\hline EH & 7 & 6 & 3 & 511 & 30 & $\mathrm{~g}$ & 12,4 & 1090 & 18,8 & 13,0 & 182,8 & 30,2 & 85,7 \\
\hline EH & 7 & 6 & 3 & 511 & 60 & 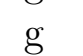 & 12,4 & 402 & 33,3 & 24,5 & 381,0 & 35,0 & 123,2 \\
\hline EH & 7 & 6 & 3 & 511 & 90 & $\mathrm{~g}$ & 12,4 & 256 & 43,7 & 32,3 & 530,0 & 38,4 & 148,4 \\
\hline $\mathrm{EH}$ & 7 & 6 & 3 & 611 & 25 & $\mathrm{~m}$ & 13,7 & 874 & 17,7 & 11,8 & 114,0 & 21,5 & 60,6 \\
\hline EH & 7 & 6 & 3 & 611 & 55 & $\mathrm{~m}$ & 13,7 & 192 & 41,4 & 27,3 & 290,8 & 25,8 & 87,8 \\
\hline EH & 7 & 6 & 3 & 611 & 85 & $\mathrm{~m}$ & 13,7 & 145 & 56,3 & 36,2 & 514,6 & 36,1 & 131,9 \\
\hline EH & 7 & 6 & 3 & 611 & 115 & $\mathrm{~m}$ & 13,7 & 108 & 68,4 & 42,4 & 644,0 & 39,7 & 153,1 \\
\hline $\mathrm{EH}$ & 7 & 6 & 3 & 711 & 30 & $\mathrm{~m}$ & 7,0 & 1917 & 13,0 & 10,9 & 129,5 & 25,5 & 42,2 \\
\hline $\mathrm{EH}$ & 7 & 6 & 3 & 711 & 60 & $\mathrm{~m}$ & 7,0 & 369 & 27,6 & 19,3 & 191,9 & 22,0 & 51,8 \\
\hline EH & 7 & 6 & 3 & 711 & 90 & $\mathrm{~m}$ & 7,0 & 221 & 35,9 & 24,2 & 238,3 & 22,4 & 60,8 \\
\hline $\mathrm{EH}$ & 7 & 6 & 3 & 711 & 120 & $\mathrm{~m}$ & 7,0 & 162 & 42,2 & 27,6 & 270,7 & 22,7 & 66,6 \\
\hline $\mathrm{EH}$ & 7 & 6 & 3 & 711 & 150 & $\mathrm{~m}$ & 7,0 & 111 & 48,9 & 30,2 & 270,4 & 20,8 & 64,8 \\
\hline $\mathrm{EH}$ & 7 & 7 & 3 & 110 & 35 & $\mathrm{~m}$ & 5,7 & 1218 & 12,4 & 11,5 & 79,3 & 14,7 & 34,5 \\
\hline EH & 7 & 7 & 3 & 110 & 65 & $\mathrm{~m}$ & 5,7 & 296 & 26,3 & 18,7 & 161,6 & 16,1 & 61,9 \\
\hline EH & 7 & 7 & 3 & 110 & 95 & $\mathrm{~m}$ & 5,7 & 162 & 37,3 & 23,1 & 219,5 & 17,7 & 84,6 \\
\hline EH & 7 & 7 & 3 & 110 & 125 & $\mathrm{~m}$ & 5,7 & 105 & 49,3 & 26,3 & 281,9 & 20,1 & 109,1 \\
\hline $\mathrm{EH}$ & 7 & 7 & 3 & 110 & 155 & $\mathrm{~m}$ & 5,7 & 83 & 57,6 & 28,9 & 332,2 & 21,6 & 129,7 \\
\hline $\mathrm{EH}$ & 7 & 7 & 3 & 110 & 185 & $\mathrm{~m}$ & 5,7 & 74 & 64,4 & 30,9 & 395,0 & 24,1 & 155,2 \\
\hline EH & 7 & 7 & 3 & 511 & 30 & $\mathrm{~m}$ & 11,1 & 1180 & 17,7 & 11,3 & 152,0 & 29,0 & 78,2 \\
\hline EH & 7 & 7 & 3 & 511 & 60 & $\mathrm{~m}$ & 11,1 & 441 & 31,3 & 22,3 & 337,5 & 33,9 & 115,2 \\
\hline EH & 7 & 7 & 2 & 511 & 90 & $\mathrm{~m}$ & 11,1 & 275 & 41,5 & 29,8 & 477,0 & 37,3 & 139,9 \\
\hline EH & 7 & 7 & 3 & 611 & 25 & $\mathrm{~m}$ & 14,4 & 852 & 18,1 & 12,6 & 125,2 & 22,0 & 62,4 \\
\hline $\mathrm{EH}$ & 7 & 7 & 3 & 611 & 55 & $\mathrm{~m}$ & 14,4 & 186 & 42,3 & 28,4 & 306,3 & 26,2 & 89,5 \\
\hline EH & 7 & - & 3 & 611 & 85 & $\mathrm{~m}$ & 14,4 & 147 & 56,7 & 37,3 & 544,6 & 37,1 & 135,7 \\
\hline $\mathrm{EH}$ & $=$ & 7 & 0 & 611 & 115 & $\mathrm{~m}$ & 14,4 & 103 & 72,7 & 43,6 & 706,2 & 42,8 & 167,1 \\
\hline EH & 7 & 7 & 3 & 711 & 30 & $\mathrm{~m}$ & 6,4 & 1979 & 12,7 & 10,2 & 118,5 & 24,9 & 39,5 \\
\hline $\mathrm{EH}$ & 7 & 7 & 3 & 711 & 60 & $\mathrm{~m}$ & 6,4 & 403 & 26,0 & 18,3 & 177,4 & 21,3 & 48,5 \\
\hline EH & - & 7 & 3 & 711 & 90 & $\mathrm{~m}$ & 6,4 & 233 & 35,3 & 23,0 & 231,1 & 22,8 & 59,7 \\
\hline $\mathrm{EH}$ & 7 & 1 & 3 & 711 & 120 & $\mathrm{~m}$ & 6,4 & 167 & 41,7 & 26,3 & 260,0 & 22,8 & 64,8 \\
\hline $\mathrm{EH}$ & 7 & 7 & 3 & 711 & 150 & $\mathrm{~m}$ & 6,4 & 116 & 47,5 & 28,8 & 255,5 & 20,6 & 62,0 \\
\hline $\mathrm{EH}$ & 7 & 7 & 4 & 611 & 25 & $\mathrm{~m}$ & 13,7 & 879 & 17,6 & 11,7 & 112,0 & 21,3 & 59,9 \\
\hline $\mathrm{EH}$ & 7 & 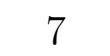 & 4 & 611 & 55 & $\mathrm{~m}$ & 13,7 & 186 & 42,2 & 27,1 & 291,0 & 26,1 & 89,0 \\
\hline $\mathrm{EH}$ & 7 & 7 & 4 & 611 & 85 & $\mathrm{~m}$ & 13,7 & 145 & 56,2 & 36,0 & 510,4 & 35,9 & 131,2 \\
\hline EH & 7 & 7 & 4 & 611 & 115 & $\mathrm{~m}$ & 13,7 & 107 & 69,8 & 42,2 & 659,5 & 41,0 & 158,5 \\
\hline $\mathrm{EH}$ & 7 & 7 & 4 & 711 & 30 & $\mathrm{~s}$ & 6,3 & 2008 & 12,6 & 10,1 & 117,0 & 24,9 & 39,1 \\
\hline EH & 7 & 7 & 4 & 711 & 60 & $\mathrm{~s}$ & 6,3 & 397 & 26,0 & 18,1 & 174,3 & 21,1 & 47,7 \\
\hline $\mathrm{EH}$ & 7 & 7 & 4 & 711 & 90 & $\mathrm{~s}$ & 6,3 & 235 & 34,6 & 22,7 & 222,1 & 22,1 & 57,6 \\
\hline EH & 7 & 7 & 4 & 711 & 120 & $\mathrm{~s}$ & 6,3 & 166 & 41,0 & 25,9 & 247,2 & 21,9 & 61,9 \\
\hline
\end{tabular}

Fortsetzung auf nächster Seite. 
Tabelle A.22

\begin{tabular}{|c|c|c|c|c|c|c|c|c|c|c|c|c|c|}
\hline WBZ & SOGR & WHZ & $\mathrm{NZ}$ & Art & Alt & $\mathrm{BG}$ & LKL & $\mathrm{N}$ & $\mathrm{dg}$ & hg & $\mathrm{V}$ & G & $\mathrm{CV}$ \\
\hline $\mathrm{EH}$ & 7 & 7 & 4 & 711 & 150 & $\mathrm{~s}$ & 6,3 & 116 & 47,2 & 28,4 & 248,0 & 20,3 & 60,5 \\
\hline $\mathrm{EH}$ & 7 & 9 & 3 & 611 & 25 & $\mathrm{~m}$ & 14,4 & 856 & 18,0 & 12,6 & 124,5 & 21,9 & 61,9 \\
\hline $\mathrm{EH}$ & 7 & 9 & 3 & 611 & 55 & $\mathrm{~m}$ & 14,4 & 182 & 43,3 & 28,4 & 311,7 & 26,8 & 92,0 \\
\hline $\mathrm{EH}$ & 7 & 9 & 3 & 611 & 85 & $\mathrm{~m}$ & 14,4 & 144 & 57,3 & 37,3 & 544,3 & 37,1 & 136,1 \\
\hline $\mathrm{EH}$ & 7 & 9 & 3 & 611 & 115 & $\mathrm{~m}$ & 14,4 & 106 & 71,4 & 43,6 & 702,8 & 42,4 & 165,1 \\
\hline $\mathrm{EH}$ & 7 & 9 & 3 & 711 & 30 & $\mathrm{~m}$ & 6,6 & 1932 & 12,9 & 10,6 & 124,3 & 25,1 & 40,8 \\
\hline $\mathrm{EH}$ & 7 & 9 & 3 & 711 & 60 & $\mathrm{~m}$ & 6,6 & 382 & 26,9 & 18,9 & 185,4 & 21,7 & 50,3 \\
\hline $\mathrm{EH}$ & 7 & 9 & 3 & 711 & 90 & $\mathrm{~m}$ & 6,6 & 224 & 35,5 & 23,7 & 231,0 & 22,2 & 59,3 \\
\hline $\mathrm{EH}$ & 7 & 9 & 3 & 711 & 120 & $\mathrm{~m}$ & 6,6 & 163 & 41,6 & 27,0 & 258,9 & 22,1 & 64,1 \\
\hline $\mathrm{EH}$ & 7 & 9 & 3 & 711 & 150 & $\mathrm{~m}$ & 6,6 & 108 & 49,3 & 29,6 & 261,9 & 20,6 & 63,1 \\
\hline $\mathrm{EH}$ & 7 & 9 & 4 & 511 & 30 & $\mathrm{~m}$ & 10,7 & 1207 & 17,3 & 10,3 & 134,6 & 28,4 & 73,7 \\
\hline $\mathrm{EH}$ & 7 & 9 & 4 & 511 & 60 & $\mathrm{~m}$ & 10,7 & 452 & 30,6 & 21,2 & 315,6 & 33,3 & 111,1 \\
\hline $\mathrm{EH}$ & 7 & 9 & 4 & 511 & 90 & $\mathrm{~m}$ & 10,7 & 287 & 40,2 & 28,6 & 450,7 & 36,5 & 135,1 \\
\hline $\mathrm{EH}$ & 7 & 9 & 4 & 611 & 25 & $\mathrm{~m}$ & 13,7 & 893 & 17,5 & 11,9 & 115,3 & 21,5 & 60,5 \\
\hline $\mathrm{EH}$ & 7 & 9 & 4 & 611 & 55 & $\mathrm{~m}$ & 13,7 & 187 & 42,0 & 27,5 & 294,3 & 26,0 & 88,6 \\
\hline $\mathrm{EH}$ & 7 & 9 & 4 & 611 & 85 & $\mathrm{~m}$ & 13,7 & 148 & 56,2 & 36,3 & 525,7 & 36,7 & 134,7 \\
\hline $\mathrm{EH}$ & 7 & 9 & 4 & 611 & 115 & $\mathrm{~m}$ & 13,7 & 92 & 75,4 & 42,6 & 659,5 & 41,1 & 161,7 \\
\hline $\mathrm{EH}$ & 7 & 9 & 4 & 711 & 30 & $\mathrm{~s}$ & 6,3 & 2026 & 12,5 & 9,9 & 115,6 & 25,0 & 38,8 \\
\hline $\mathrm{EH}$ & 7 & 9 & 4 & 711 & 60 & $\mathrm{~S}$ & 6,3 & 408 & 25,7 & 17,9 & 172,6 & 21,1 & 47,4 \\
\hline $\mathrm{EH}$ & 7 & 9 & 4 & 711 & 90 & $\mathrm{~s}$ & 6,3 & 236 & 34,4 & 22,5 & 218,5 & 22,0 & 56,8 \\
\hline $\mathrm{EH}$ & 7 & 9 & 4 & 711 & 120 & $\mathrm{~s}$ & 6,3 & 171 & 40,2 & 25,7 & 243,5 & 21,7 & 61,1 \\
\hline $\mathrm{EH}$ & 7 & 9 & 4 & 711 & 150 & $\mathrm{~s}$ & 6,3 & 116 & 46,6 & 28,2 & 240,9 & 19,8 & 58,9 \\
\hline GM & 4 & 5 & 2 & 110 & 35 & $\mathrm{~m}$ & 6,0 & 1168 & 12,8 & 12,2 & 87,7 & 15,0 & 37,5 \\
\hline GM & 4 & 5 & 2 & 110 & 65 & $\mathrm{~m}$ & 6,0 & 287 & 27,1 & 19,5 & 173,3 & 16,6 & 66,8 \\
\hline GM & 4 & 5 & 2 & 110 & 95 & $\mathrm{~m}$ & 6,0 & 161 & 37,6 & 24,0 & 228,9 & 17,9 & 89,0 \\
\hline GM & 4 & 5 & 2 & 110 & 125 & $\mathrm{~m}$ & 6,0 & 102 & 49,7 & 27,3 & 288,3 & 19,8 & 112,5 \\
\hline GM & 4 & 5 & 2 & 110 & 155 & $\mathrm{~m}$ & 6,0 & 80 & 59,5 & 29,8 & 353,0 & 22,3 & 138,3 \\
\hline GM & 4 & 5 & 2 & 110 & 185 & $\mathrm{~m}$ & 6,0 & 73 & 66,0 & 32,0 & 422,6 & 25,0 & 166,9 \\
\hline GM & 4 & 5 & 2 & 211 & 40 & $\mathrm{~m}$ & 8,0 & 1432 & 12,7 & 12,7 & 107,5 & 18,1 & 50,0 \\
\hline GM & 4 & 5 & 2 & 211 & 70 & $\mathrm{~m}$ & 8,0 & 336 & 26,9 & 22,8 & 218,4 & 19,0 & 93,7 \\
\hline GM & 4 & 5 & 2 & 211 & 100 & $\mathrm{~m}$ & 8,0 & 181 & 39,5 & 29,2 & 328,3 & 22,2 & 138,3 \\
\hline GM & 4 & 5 & 2 & 211 & 130 & $\mathrm{~m}$ & 8,0 & 117 & 49,9 & 33,9 & 397,8 & 22,8 & 165,0 \\
\hline GM & 4 & 5 & 2 & 511 & 30 & g & 11,9 & 1122 & 18,4 & 12,3 & 169,9 & 29,7 & 82,6 \\
\hline GM & 4 & 5 & 2 & 511 & 60 & g & 11,9 & 418 & 32,6 & 23,5 & 364,6 & 34,9 & 120,9 \\
\hline GM & 4 & 5 & 2 & 511 & 90 & g & 11,9 & 272 & 42,4 & 31,2 & 513,3 & 38,3 & 146,4 \\
\hline GM & 4 & 5 & 2 & 611 & 25 & $\mathrm{~m}$ & 14,4 & 867 & 18,0 & 12,6 & 125,0 & 22,0 & 62,3 \\
\hline GM & 4 & 5 & 2 & 611 & 55 & $\mathrm{~m}$ & 14,4 & 179 & 43,5 & 28,4 & 310,0 & 26,6 & 91,6 \\
\hline GM & 4 & 5 & 2 & 611 & 85 & $\mathrm{~m}$ & 14,4 & 137 & 58,9 & 37,3 & 544,3 & 37,3 & 137,4 \\
\hline
\end{tabular}

Fortsetzung auf nächster Seite. 
Tabelle A.22

\begin{tabular}{|c|c|c|c|c|c|c|c|c|c|c|c|c|c|}
\hline WBZ & SOGR & WHZ & $\mathrm{NZ}$ & Art & Alt & $\mathrm{BG}$ & LKL & $\mathrm{N}$ & $\mathrm{dg}$ & hg & $\mathrm{V}$ & $\mathrm{G}$ & $\mathrm{CV}$ \\
\hline GM & 4 & 5 & 2 & 611 & 115 & $\mathrm{~m}$ & 14,4 & 102 & 70,8 & 43,6 & 667,0 & 40,2 & 155,9 \\
\hline GM & 4 & 5 & 2 & 711 & 30 & g & 7,5 & 1848 & 13,3 & 11,5 & 137,5 & 25,7 & 44,1 \\
\hline GM & 4 & 5 & 2 & 711 & 60 & $\mathrm{~g}$ & 7,5 & 365 & 27,7 & 20,3 & 200,1 & 21,9 & 53,4 \\
\hline GM & 4 & 5 & 2 & 711 & 90 & $\mathrm{~g}$ & 7,5 & 212 & 36,9 & 25,3 & 250,3 & 22,7 & 63,1 \\
\hline GM & 4 & 5 & 2 & 711 & 120 & $\mathrm{~g}$ & 7,5 & 155 & 42,9 & 28,8 & 278,8 & 22,4 & 67,8 \\
\hline GM & 4 & 5 & 2 & 711 & 150 & g & 7,5 & 103 & 51,0 & 31,5 & 284,1 & 21,1 & 67,2 \\
\hline GM & 4 & 5 & 3 & 110 & 35 & g & 6,3 & 1165 & 12,8 & 12,5 & 90,0 & 15,0 & 38,5 \\
\hline GM & 4 & 5 & 3 & 110 & 65 & g & 6,3 & 272 & 27,7 & 19,8 & 174,1 & 16,4 & 67,1 \\
\hline GM & 4 & 5 & 3 & 110 & 95 & g & 6,3 & 160 & 38,2 & 24,4 & 239,4 & 18,4 & 93,2 \\
\hline GM & 4 & 5 & 3 & 110 & 125 & $\mathrm{~g}$ & 6,3 & 98 & 51,2 & 27,7 & 298,5 & 20,2 & 116,5 \\
\hline GM & 4 & 5 & 3 & 110 & 155 & $\mathrm{~g}$ & 6,3 & 78 & 60,0 & 30,3 & 354,0 & 22,1 & 139,2 \\
\hline GM & 4 & 5 & 3 & 110 & 185 & $\mathrm{~g}$ & 6,3 & 70 & 66,6 & 32,4 & 416,9 & 24,4 & 165,1 \\
\hline GM & 4 & 5 & 3 & 711 & 30 & $\mathrm{~m}$ & 7,3 & 1867 & 13,2 & 11,4 & 136,6 & 25,7 & 43,9 \\
\hline GM & 4 & 5 & 3 & 711 & 60 & $\mathrm{~m}$ & 7,3 & 355 & 28,1 & 20,1 & 198,2 & 21,9 & 53,0 \\
\hline GM & 4 & 5 & 3 & 711 & 90 & $\mathrm{~m}$ & 7,3 & 214 & 36,9 & 25,1 & 251,5 & 22,9 & 63,6 \\
\hline GM & 4 & 5 & 3 & 711 & 120 & $\mathrm{~m}$ & 7,3 & 155 & 43,2 & 28,6 & 281,1 & 22,8 & 68,5 \\
\hline GM & 4 & 5 & 3 & 711 & 150 & $\mathrm{~m}$ & 7,3 & 97 & 51,9 & 31,3 & 274,7 & 20,5 & 65,2 \\
\hline GM & 4 & 6 & 3 & 110 & 35 & $\mathrm{~m}$ & 6,0 & 1184 & 12,7 & 12,0 & 85,6 & 15,0 & 36,7 \\
\hline GM & 4 & 6 & 3 & 110 & 65 & $\mathrm{~m}$ & 6,0 & 278 & 27,2 & 19,3 & 168,1 & 16,2 & 64,5 \\
\hline GM & 4 & 6 & 3 & 110 & 95 & $\mathrm{~m}$ & 6,0 & 163 & 37,2 & 23,8 & 225,0 & 17,7 & 87,4 \\
\hline GM & 4 & 6 & 3 & 110 & 125 & $\mathrm{~m}$ & 6,0 & 100 & 49,8 & 27,1 & 280,9 & 19,4 & 109,4 \\
\hline GM & 4 & 6 & 3 & 110 & 155 & $\mathrm{~m}$ & 6,0 & 83 & 58,3 & 29,6 & 348,8 & 22,2 & 136,8 \\
\hline GM & 4 & 6 & 3 & 110 & 185 & $\mathrm{~m}$ & 6,0 & 75 & 66,0 & 31,7 & 430,0 & 25,6 & 169,6 \\
\hline GM & 4 & 6 & 3 & 211 & 40 & $\mathrm{~m}$ & 7,6 & 1480 & 12,5 & 12,0 & 101,6 & 18,0 & 47,3 \\
\hline GM & 4 & 6 & 3 & 211 & 70 & $\mathrm{~m}$ & 7,6 & 331 & 26,9 & 22,0 & 208,7 & 18,8 & 89,6 \\
\hline GM & 4 & 6 & 3 & 211 & 100 & $\mathrm{~m}$ & 7,6 & 196 & 37,4 & 28,3 & 309,3 & 21,6 & 130,7 \\
\hline GM & 4 & 6 & 3 & 211 & 130 & $\mathrm{~m}$ & 7,6 & 119 & 51,0 & 32,9 & 410,8 & 24,3 & 170,7 \\
\hline GM & 4 & 6 & 3 & 611 & 25 & $\mathrm{~m}$ & 14,4 & 866 & 18,1 & 12,4 & 124,8 & 22,3 & 63,3 \\
\hline GM & 4 & 6 & 3 & 611 & 55 & $\mathrm{~m}$ & 14,4 & 182 & 43,0 & 28,1 & 304,6 & 26,4 & 90,4 \\
\hline GM & 4 & 6 & 3 & 611 & 85 & $\mathrm{~m}$ & 14,4 & 139 & 58,5 & 37,0 & 542,5 & 37,4 & 137,9 \\
\hline GM & 4 & 6 & 3 & 611 & 115 & $\mathrm{~m}$ & 14,4 & 103 & 70,3 & 43,3 & 658,8 & 40,0 & 155,2 \\
\hline GM & 4 & 6 & 3 & 711 & 30 & $\mathrm{~m}$ & 7,1 & 1887 & 13,2 & 11,3 & 136,1 & 25,9 & 43,9 \\
\hline GM & 4 & 6 & 3 & 711 & 60 & $\mathrm{~m}$ & 7,1 & 369 & 27,4 & 19,9 & 194,7 & 21,7 & 52,2 \\
\hline GM & 4 & 6 & 3 & 711 & 90 & $\mathrm{~m}$ & 7,1 & 215 & 36,5 & 24,8 & 244,3 & 22,5 & 62,0 \\
\hline GM & 4 & 6 & 3 & 711 & 120 & $\mathrm{~m}$ & 7,1 & 157 & 42,5 & 28,3 & 271,9 & 22,2 & 66,5 \\
\hline GM & 4 & 6 & 3 & 711 & 150 & $\mathrm{~m}$ & 7,1 & 107 & 49,4 & 31,0 & 273,0 & 20,5 & 64,9 \\
\hline GM & 7 & 6 & 3 & 110 & 35 & $\mathrm{~g}$ & 6,3 & 1168 & 12,8 & 12,5 & 90,6 & 15,1 & 38,8 \\
\hline GM & 7 & 6 & 3 & 110 & 65 & g & 6,3 & 275 & 27,6 & 19,8 & 174,6 & 16,5 & 67,3 \\
\hline
\end{tabular}

Fortsetzung auf nächster Seite. 
Tabelle A.22

\begin{tabular}{|c|c|c|c|c|c|c|c|c|c|c|c|c|c|}
\hline WBZ & SOGR & WHZ & $\mathrm{NZ}$ & Art & Alt & $\mathrm{BG}$ & LKL & $\mathrm{N}$ & $\mathrm{dg}$ & hg & $\mathrm{V}$ & G & $\mathrm{CV}$ \\
\hline GM & 7 & 6 & 3 & 110 & 95 & $\mathrm{~g}$ & 6,3 & 150 & 39,2 & 24,4 & 236,4 & 18,1 & 91,9 \\
\hline GM & 7 & 6 & 3 & 110 & 125 & g & 6,3 & 101 & 50,5 & 27,7 & 298,6 & 20,2 & 116,7 \\
\hline GM & 7 & 6 & 3 & 110 & 155 & g & 6,3 & 82 & 60,7 & 30,3 & 380,5 & 23,7 & 149,5 \\
\hline GM & 7 & 6 & 3 & 110 & 185 & $\mathrm{~g}$ & 6,3 & 69 & 67,1 & 32,4 & 418,1 & 24,4 & 165,4 \\
\hline GM & 7 & 6 & 3 & 211 & 40 & $\mathrm{~m}$ & 7,8 & 1450 & 12,6 & 12,4 & 105,0 & 18,0 & 48,8 \\
\hline GM & 7 & 6 & 3 & 211 & 70 & $\mathrm{~m}$ & 7,8 & 341 & 26,6 & 22,5 & 213,3 & 18,9 & 91,6 \\
\hline GM & 7 & 6 & 3 & 211 & 100 & $\mathrm{~m}$ & 7,8 & 182 & 38,8 & 28,9 & 315,1 & 21,5 & 132,8 \\
\hline GM & 7 & 6 & 3 & 211 & 130 & $\mathrm{~m}$ & 7,8 & 117 & 50,2 & 33,6 & 398,6 & 23,1 & 165,5 \\
\hline GM & 7 & 6 & 3 & 711 & 30 & $\mathrm{~m}$ & 7,1 & 1893 & 13,1 & 11,3 & 134,6 & 25,6 & 43,5 \\
\hline GM & 7 & 6 & 3 & 711 & 60 & $\mathrm{~m}$ & 7,1 & 372 & 27,3 & 19,9 & 195,4 & 21,8 & 52,4 \\
\hline GM & 7 & 6 & 3 & 711 & 90 & $\mathrm{~m}$ & 7,1 & 212 & 37,2 & 24,8 & 249,8 & 23,0 & 63,3 \\
\hline GM & 7 & 6 & 3 & 711 & 120 & $\mathrm{~m}$ & 7,1 & 151 & 43,6 & 28,3 & 275,5 & 22,5 & 67,3 \\
\hline GM & 7 & 6 & 3 & 711 & 150 & $\mathrm{~m}$ & 7,1 & 106 & 49,9 & 31,0 & 274,7 & 20,7 & 65,4 \\
\hline GM & 7 & 7 & 3 & 110 & 35 & $\mathrm{~m}$ & 6,0 & 1174 & 12,7 & 12,0 & 85,2 & 14,9 & 36,5 \\
\hline GM & 7 & 7 & 3 & 110 & 65 & $\mathrm{~m}$ & 6,0 & 291 & 26,7 & 19,3 & 168,9 & 16,3 & 65,1 \\
\hline GM & 7 & 7 & 3 & 110 & 95 & $\mathrm{~m}$ & 6,0 & 162 & 37,5 & 23,8 & 228,2 & 17,9 & 88,6 \\
\hline GM & 7 & 7 & 3 & 110 & 125 & $\mathrm{~m}$ & 6,0 & 104 & 48,8 & 27,1 & 280,7 & 19,4 & 109,5 \\
\hline GM & 7 & 7 & 3 & 110 & 155 & $\mathrm{~m}$ & 6,0 & 83 & 57,9 & 29,6 & 343,6 & 21,9 & 134,8 \\
\hline GM & 7 & 7 & 3 & 110 & 185 & $\mathrm{~m}$ & 6,0 & 74 & 65,0 & 31,7 & 412,0 & 24,6 & 162,6 \\
\hline GM & 7 & 7 & 3 & 211 & 40 & $\mathrm{~m}$ & 7,6 & 1507 & 12,3 & 11,8 & 99,3 & 17,9 & 46,4 \\
\hline GM & 7 & 7 & 3 & 211 & 70 & $\mathrm{~m}$ & 7,6 & 364 & 25,7 & 21,8 & 205,8 & 18,8 & 88,5 \\
\hline GM & 7 & 7 & 3 & 211 & 100 & $\mathrm{~m}$ & 7,6 & 185 & 39,0 & 28,1 & 313,6 & 22,0 & 132,6 \\
\hline GM & 7 & 7 & 3 & 211 & 130 & $\mathrm{~m}$ & 7,6 & 124 & 49,0 & 32,7 & 392,3 & 23,4 & 163,3 \\
\hline GM & 7 & 7 & 3 & 611 & 25 & $\mathrm{~m}$ & 14,1 & 894 & 17,5 & 12,0 & 116,3 & 21,5 & 60,5 \\
\hline GM & 7 & 7 & 3 & 611 & 55 & $\mathrm{~m}$ & 14,1 & 191 & 41,5 & 27,6 & 294,8 & 25,9 & 88,1 \\
\hline GM & 7 & 7 & 3 & 611 & 85 & $\mathrm{~m}$ & 14,1 & 134 & 58,8 & 36,4 & 518,0 & 36,3 & 133,9 \\
\hline GM & 7 & 7 & 3 & 611 & 115 & $\mathrm{~m}$ & 14,1 & 108 & 69,9 & 42,7 & 675,0 & 41,5 & 160,2 \\
\hline GM & 7 & 7 & 3 & 711 & 30 & $\mathrm{~m}$ & 7,0 & 1901 & 13,1 & 11,2 & 134,3 & 25,8 & 43,5 \\
\hline GM & 7 & 7 & 3 & 711 & 60 & $\mathrm{~m}$ & 7,0 & 374 & 27,2 & 19,7 & 193,7 & 21,8 & 52,1 \\
\hline GM & 7 & 7 & 3 & 711 & 90 & $\mathrm{~m}$ & 7,0 & 218 & 36,6 & 24,6 & 247,2 & 22,9 & 62,8 \\
\hline GM & 7 & 7 & 3 & 711 & 120 & $\mathrm{~m}$ & 7,0 & 153 & 43,1 & 28,1 & 271,0 & 22,3 & 66,4 \\
\hline GM & 7 & 7 & 3 & 711 & 150 & $\mathrm{~m}$ & 7,0 & 106 & 49,6 & 30,7 & 269,7 & 20,5 & 64,3 \\
\hline GM & 7 & 9 & 4 & 611 & 25 & $\mathrm{~m}$ & 13,3 & 901 & 17,4 & 11,4 & 109,4 & 21,4 & 60,0 \\
\hline GM & 7 & 9 & 4 & 611 & 55 & $\mathrm{~m}$ & 13,3 & 190 & 41,4 & 26,9 & 284,5 & 25,6 & 87,2 \\
\hline GM & 7 & 9 & 4 & 611 & 85 & $\mathrm{~m}$ & 13,3 & 141 & 58,1 & 35,7 & 524,0 & 37,4 & 137,9 \\
\hline GM & 7 & 9 & 4 & 611 & 115 & $\mathrm{~m}$ & 13,3 & 113 & 66,2 & 41,9 & 627,3 & 38,9 & 148,7 \\
\hline GM & 7 & 9 & 4 & 711 & 30 & $\mathrm{~m}$ & 6,6 & 1963 & 12,8 & 10,6 & 125,5 & 25,4 & 41,3 \\
\hline GM & 7 & 9 & 4 & 711 & 60 & $\mathrm{~m}$ & 6,6 & 388 & 26,8 & 18,9 & 187,7 & 21,9 & 50,9 \\
\hline
\end{tabular}

Fortsetzung auf nächster Seite. 
Tabelle A.22

\begin{tabular}{|c|c|c|c|c|c|c|c|c|c|c|c|c|c|}
\hline WBZ & SOGR & WHZ & $\mathrm{NZ}$ & Art & Alt & $\mathrm{BG}$ & LKL & $\mathrm{N}$ & $\mathrm{dg}$ & hg & $\mathrm{V}$ & G & $\mathrm{CV}$ \\
\hline GM & 7 & 9 & 4 & 711 & 90 & $\mathrm{~m}$ & 6,6 & 225 & 35,7 & 23,7 & 234,3 & 22,5 & 60,1 \\
\hline GM & 7 & 9 & 4 & 711 & 120 & $\mathrm{~m}$ & 6,6 & 159 & 41,9 & 27,0 & 256,3 & 21,9 & 63,5 \\
\hline GM & 7 & 9 & 4 & 711 & 150 & $\mathrm{~m}$ & 6,6 & 110 & 48,8 & 29,6 & 262,4 & 20,6 & 63,2 \\
\hline GM & 8 & 1 & 3 & 110 & 35 & g & 6,8 & 1121 & 13,3 & 13,3 & 100,3 & 15,5 & 42,4 \\
\hline GM & 8 & 1 & 3 & 110 & 65 & g & 6,8 & 268 & 28,2 & 20,8 & 185,5 & 16,8 & 72,2 \\
\hline GM & 8 & 1 & 3 & 110 & 95 & g & 6,8 & 151 & 39,5 & 25,4 & 249,6 & 18,5 & 97,8 \\
\hline GM & 8 & 1 & 3 & 110 & 125 & g & 6,8 & 98 & 51,3 & 28,7 & 308,7 & 20,3 & 121,4 \\
\hline GM & 8 & 1 & 3 & 110 & 155 & g & 6,8 & 75 & 61,7 & 31,4 & 372,1 & 22,4 & 147,1 \\
\hline GM & 8 & 1 & 3 & 110 & 185 & g & 6,8 & 69 & 67,4 & 33,6 & 435,4 & 24,6 & 173,6 \\
\hline GM & 8 & 1 & 3 & 711 & 30 & g & 7,7 & 1813 & 13,5 & 11,9 & 143,5 & 26,0 & 45,6 \\
\hline GM & 8 & 1 & 3 & 711 & 60 & $\mathrm{~g}$ & 7,7 & 354 & 28,6 & 20,8 & 212,7 & 22,8 & 56,4 \\
\hline GM & 8 & 1 & 3 & 711 & 90 & g & 7,7 & 212 & 37,0 & 26,0 & 259,2 & 22,8 & 64,9 \\
\hline GM & 8 & 1 & 3 & 711 & 120 & g & 7,7 & 150 & 44,1 & 29,6 & 292,4 & 22,9 & 70,6 \\
\hline GM & 8 & 1 & 3 & 711 & 150 & g & 7,7 & 99 & 52,7 & 32,4 & 299,0 & 21,6 & 70,2 \\
\hline GM & 8 & 2 & 3 & 110 & 35 & $\mathrm{~m}$ & 5,8 & 1202 & 12,5 & 11,8 & 82,6 & 14,8 & 35,7 \\
\hline GM & 8 & 2 & 3 & 110 & 65 & $\mathrm{~m}$ & 5,8 & 289 & 26,9 & 19,0 & 168,0 & 16,5 & 64,4 \\
\hline GM & 8 & 2 & 3 & 110 & 95 & $\mathrm{~m}$ & 5,8 & 163 & 37,4 & 23,5 & 225,0 & 17,9 & 87,1 \\
\hline GM & 8 & 2 & 3 & 110 & 125 & $\mathrm{~m}$ & 5,8 & 102 & 50,1 & 26,7 & 287,0 & 20,1 & 111,3 \\
\hline GM & 8 & 2 & 3 & 110 & 155 & $\mathrm{~m}$ & 5,8 & 69 & 63,0 & 29,3 & 336,0 & 21,5 & 130,6 \\
\hline GM & 8 & 2 & 3 & 110 & 185 & $\mathrm{~m}$ & 5,8 & 75 & 63,9 & 31,4 & 399,7 & 24,1 & 157,7 \\
\hline GM & 8 & 2 & 3 & 711 & 30 & g & 7,7 & 1799 & 13,6 & 12,1 & 146,9 & 26,1 & 46,4 \\
\hline GM & 8 & 2 & 3 & 711 & 60 & g & 7,7 & 356 & 28,2 & 21,1 & 209,5 & 22,2 & 55,4 \\
\hline GM & 8 & 2 & 3 & 711 & 90 & $\mathrm{~g}$ & 7,7 & 203 & 38,1 & 26,3 & 264,6 & 23,1 & 66,1 \\
\hline GM & 8 & 2 & 3 & 711 & 120 & g & 7,7 & 146 & 44,7 & 29,9 & 294,6 & 22,9 & 70,9 \\
\hline GM & 8 & 2 & 3 & 711 & 150 & g & 7,7 & 100 & 52,1 & 32,7 & 297,4 & 21,3 & 69,7 \\
\hline GM & 9 & 5 & 2 & 110 & 35 & g & 6,8 & 1092 & 13,4 & 13,3 & 99,9 & 15,4 & 42,1 \\
\hline GM & 9 & 5 & 2 & 110 & 65 & g & 6,8 & 264 & 28,7 & 20,8 & 189,6 & 17,1 & 73,6 \\
\hline GM & 9 & 5 & 2 & 110 & 95 & g & 6,8 & 152 & 39,0 & 25,4 & 245,6 & 18,2 & 96,4 \\
\hline GM & 9 & 5 & 2 & 110 & 125 & g & 6,8 & 98 & 51,3 & 28,7 & 308,4 & 20,2 & 121,3 \\
\hline GM & 9 & 5 & 2 & 110 & 155 & g & 6,8 & 72 & 62,5 & 31,4 & 366,4 & 22,1 & 144,8 \\
\hline GM & 9 & 5 & 2 & 110 & 185 & g & 6,8 & 66 & 69,3 & 33,6 & 440,7 & 24,9 & 175,4 \\
\hline GM & 9 & 5 & 2 & 211 & 40 & $\mathrm{~m}$ & 8,0 & 1379 & 12,9 & 12,9 & 109,8 & 18,0 & 50,8 \\
\hline GM & 9 & 5 & 2 & 211 & 70 & $\mathrm{~m}$ & 8,0 & 332 & 26,9 & 23,1 & 218,9 & 18,8 & 93,9 \\
\hline GM & 9 & 5 & 2 & 211 & 100 & $\mathrm{~m}$ & 8,0 & 174 & 39,7 & 29,5 & 321,4 & 21,5 & 135,4 \\
\hline GM & 9 & 5 & 2 & 211 & 130 & $\mathrm{~m}$ & 8,0 & 116 & 50,6 & 34,2 & 410,6 & 23,3 & 169,9 \\
\hline GM & 9 & 5 & 2 & 511 & 30 & g & 12,6 & 1068 & 19,0 & 13,6 & 193,0 & 30,4 & 87,7 \\
\hline GM & 9 & 5 & 2 & 511 & 60 & g & 12,6 & 397 & 33,9 & 25,2 & 402,0 & 35,9 & 127,9 \\
\hline GM & 9 & 5 & 2 & 511 & 90 & $\mathrm{~g}$ & 12,6 & 248 & 44,8 & 33,0 & 548,1 & 39,0 & 151,9 \\
\hline
\end{tabular}

Fortsetzung auf nächster Seite. 
Tabelle A.22

\begin{tabular}{|c|c|c|c|c|c|c|c|c|c|c|c|c|c|}
\hline WBZ & SOGR & WHZ & $\mathrm{NZ}$ & Art & Alt & BG & LKL & $\mathrm{N}$ & $\mathrm{dg}$ & hg & $\mathrm{V}$ & $\mathrm{G}$ & $\mathrm{CV}$ \\
\hline GM & 9 & 5 & 2 & 611 & 25 & $\mathrm{~g}$ & 16,1 & 828 & 18,7 & 13,9 & 143,4 & 22,8 & 65,1 \\
\hline GM & 9 & 5 & 2 & 611 & 55 & g & 16,1 & 178 & 44,3 & 29,9 & 335,5 & 27,5 & 94,8 \\
\hline GM & 9 & 5 & 2 & 611 & 85 & $\mathrm{~g}$ & 16,1 & 139 & 59,5 & 39,0 & 589,6 & 38,7 & 142,9 \\
\hline GM & 9 & 5 & 2 & 611 & 115 & $\mathrm{~g}$ & 16,1 & 98 & 74,7 & 45,5 & 736,1 & 42,9 & 168,0 \\
\hline GW & 1 & 5 & 2 & 211 & 40 & g & 8,8 & 1254 & 13,7 & 14,6 & 128,9 & 18,5 & 59,1 \\
\hline GW & 1 & 5 & 2 & 211 & 70 & g & 8,8 & 291 & 29,1 & 25,0 & 242,7 & 19,3 & 103,6 \\
\hline GW & 1 & 5 & 2 & 211 & 100 & $\mathrm{~g}$ & 8,8 & 155 & 42,4 & 31,6 & 353,6 & 21,9 & 147,9 \\
\hline GW & 1 & 5 & 2 & 211 & 130 & $\mathrm{~g}$ & 8,8 & 100 & 53,9 & 36,4 & 427,9 & 22,8 & 176,0 \\
\hline GW & 1 & 5 & 2 & 511 & 30 & g & 12,4 & 1090 & 18,8 & 12,9 & 180,8 & 30,1 & 85,2 \\
\hline GW & 1 & 5 & 2 & 511 & 60 & $\mathrm{~g}$ & 12,4 & 405 & 33,2 & 24,3 & 379,7 & 35,1 & 123,4 \\
\hline GW & 1 & 5 & 2 & 511 & 90 & $\mathrm{~g}$ & 12,4 & 268 & 42,9 & 32,1 & 533,0 & 38,7 & 149,4 \\
\hline GW & 5 & 7 & 1 & 211 & 40 & g & 8,2 & 1351 & 13,1 & 13,4 & 114,6 & 18,1 & 53,0 \\
\hline GW & 5 & 7 & 1 & 211 & 70 & $\mathrm{~g}$ & 8,2 & 313 & 28,1 & 23,6 & 229,3 & 19,4 & 98,2 \\
\hline GW & 5 & 7 & 1 & 211 & 100 & $\mathrm{~g}$ & 8,2 & 171 & 40,2 & 30,1 & 332,2 & 21,7 & 139,7 \\
\hline GW & 5 & 7 & 1 & 211 & 130 & $\mathrm{~g}$ & 8,2 & 104 & 53,6 & 34,8 & 422,0 & 23,5 & 174,1 \\
\hline GW & 5 & 7 & 1 & 511 & 30 & g & 12,9 & 1059 & 19,3 & 13,8 & 199,4 & 30,9 & 89,8 \\
\hline GW & 5 & 7 & 1 & 511 & 60 & $\mathrm{~g}$ & 12,9 & 396 & 33,8 & 25,4 & 401,6 & 35,6 & 127,0 \\
\hline GW & 5 & 7 & 1 & 511 & 90 & $\mathrm{~g}$ & 12,9 & 256 & 44,2 & 33,2 & 557,7 & 39,3 & 153,6 \\
\hline $\mathrm{HH}$ & 4 & 5 & 2 & 110 & 35 & $\mathrm{~m}$ & 6,0 & 1163 & 12,8 & 12,0 & 85,1 & 14,9 & 36,4 \\
\hline $\mathrm{HH}$ & 4 & 5 & 2 & 110 & 65 & $\mathrm{~m}$ & 6,0 & 277 & 27,3 & 19,3 & 168,8 & 16,3 & 64,8 \\
\hline $\mathrm{HH}$ & 4 & 5 & 2 & 110 & 95 & $\mathrm{~m}$ & 6,0 & 159 & 38,3 & 23,8 & 232,8 & 18,3 & 90,2 \\
\hline $\mathrm{HH}$ & 4 & 5 & 2 & 110 & 125 & $\mathrm{~m}$ & 6,0 & 104 & 48,6 & 27,1 & 278,4 & 19,3 & 108,6 \\
\hline $\mathrm{HH}$ & 4 & 5 & 2 & 110 & 155 & $\mathrm{~m}$ & 6,0 & 77 & 59,8 & 29,6 & 341,0 & 21,6 & 133,4 \\
\hline $\mathrm{HH}$ & 4 & 5 & 2 & 110 & 185 & $\mathrm{~m}$ & 6,0 & 75 & 65,7 & 31,7 & 425,9 & 25,4 & 168,0 \\
\hline $\mathrm{HH}$ & 4 & 5 & 2 & 211 & 40 & $\mathrm{~m}$ & 7,6 & 1515 & 12,3 & 11,8 & 98,5 & 17,9 & 46,0 \\
\hline $\mathrm{HH}$ & 4 & 5 & 2 & 211 & 70 & $\mathrm{~m}$ & 7,6 & 361 & 26,0 & 21,8 & 209,9 & 19,2 & 90,3 \\
\hline HH & 4 & 5 & 2 & 211 & 100 & $\mathrm{~m}$ & 7,6 & 189 & 37,8 & 28,1 & 302,9 & 21,2 & 128,0 \\
\hline $\mathrm{HH}$ & 4 & 5 & 2 & 211 & 130 & $\mathrm{~m}$ & 7,6 & 122 & 50,3 & 32,7 & 406,0 & 24,2 & 168,9 \\
\hline $\mathrm{HH}$ & 4 & 5 & 2 & 511 & 30 & $\mathrm{~g}$ & 11,6 & 1153 & 18,1 & 11,8 & 162,8 & 29,7 & 81,3 \\
\hline $\mathrm{HH}$ & 4 & 5 & 2 & 511 & 60 & $\mathrm{~g}$ & 11,6 & 425 & 32,1 & 23,0 & 352,1 & 34,3 & 118,2 \\
\hline $\mathrm{HH}$ & 4 & 5 & 2 & 511 & 90 & g & 11,6 & 273 & 41,8 & 30,6 & 492,5 & 37,4 & 142,0 \\
\hline $\mathrm{HH}$ & 4 & 5 & 2 & 611 & 25 & $\mathrm{~m}$ & 14,4 & 845 & 18,2 & 12,7 & 125,8 & 22,0 & 62,4 \\
\hline $\mathrm{HH}$ & 4 & 5 & 2 & 611 & 55 & $\mathrm{~m}$ & 14,4 & 179 & 43,2 & 28,4 & 306,1 & 26,3 & 90,2 \\
\hline HH & 4 & 5 & 2 & 611 & 85 & $\mathrm{~m}$ & 14,4 & 144 & 57,4 & 37,4 & 548,2 & 37,3 & 136,5 \\
\hline $\mathrm{HH}$ & 4 & 5 & 2 & 611 & 115 & $\mathrm{~m}$ & 14,4 & 108 & 69,5 & 43,7 & 683,4 & 41,0 & 158,7 \\
\hline $\mathrm{HH}$ & 4 & 5 & 2 & 711 & 30 & $\mathrm{~g}$ & 7,5 & 1869 & 13,2 & 11,5 & 137,5 & 25,7 & 44,2 \\
\hline $\mathrm{HH}$ & 4 & 5 & 2 & 711 & 60 & g & 7,5 & 355 & 28,3 & 20,3 & 203,1 & 22,3 & 54,2 \\
\hline $\mathrm{HH}$ & 4 & 5 & 2 & 711 & 90 & $\mathrm{~g}$ & 7,5 & 211 & 37,6 & 25,3 & 258,4 & 23,4 & 65,2 \\
\hline
\end{tabular}

Fortsetzung auf nächster Seite. 
Tabelle A.22

\begin{tabular}{|c|c|c|c|c|c|c|c|c|c|c|c|c|c|}
\hline WBZ & SOGR & WHZ & $\mathrm{NZ}$ & Art & Alt & $\mathrm{BG}$ & LKL & $\mathrm{N}$ & $\mathrm{dg}$ & hg & $\mathrm{V}$ & $\mathrm{G}$ & $\mathrm{CV}$ \\
\hline $\mathrm{HH}$ & 4 & 5 & 2 & 711 & 120 & $\mathrm{~g}$ & 7,5 & 151 & 43,5 & 28,8 & 278,5 & 22,4 & 67,8 \\
\hline $\mathrm{HH}$ & 4 & 5 & 2 & 711 & 150 & g & 7,5 & 103 & 50,9 & 31,5 & 282,2 & 20,9 & 66,8 \\
\hline $\mathrm{HH}$ & 4 & 5 & 3 & 110 & 35 & $\mathrm{~m}$ & 5,5 & 1206 & 12,5 & 11,3 & 78,0 & 14,7 & 33,8 \\
\hline $\mathrm{HH}$ & 4 & 5 & 3 & 110 & 65 & $\mathrm{~m}$ & 5,5 & 287 & 26,6 & 18,5 & 158,8 & 15,9 & 60,6 \\
\hline $\mathrm{HH}$ & 4 & 5 & 3 & 110 & 95 & $\mathrm{~m}$ & 5,5 & 170 & 36,3 & 22,9 & 216,5 & 17,6 & 83,5 \\
\hline $\mathrm{HH}$ & 4 & 5 & 3 & 110 & 125 & $\mathrm{~m}$ & 5,5 & 109 & 47,6 & 26,1 & 271,0 & 19,4 & 105,0 \\
\hline $\mathrm{HH}$ & 4 & 5 & 3 & 110 & 155 & $\mathrm{~m}$ & 5,5 & 79 & 58,2 & 28,6 & 320,1 & 21,0 & 124,6 \\
\hline $\mathrm{HH}$ & 4 & 5 & 3 & 110 & 185 & $\mathrm{~m}$ & 5,5 & 74 & 64,8 & 30,7 & 398,1 & 24,4 & 156,0 \\
\hline $\mathrm{HH}$ & 4 & 5 & 3 & 511 & 30 & g & 11,4 & 1171 & 17,9 & 11,4 & 155,1 & 29,4 & 79,3 \\
\hline $\mathrm{HH}$ & 4 & 5 & 3 & 511 & 60 & $\mathrm{~g}$ & 11,4 & 425 & 31,9 & 22,5 & 339,9 & 33,9 & 115,7 \\
\hline $\mathrm{HH}$ & 4 & 5 & 3 & 511 & 90 & $\mathrm{~g}$ & 11,4 & 282 & 40,9 & 30,0 & 480,1 & 37,1 & 139,8 \\
\hline $\mathrm{HH}$ & 4 & 5 & 3 & 711 & 30 & $\mathrm{~m}$ & 7,1 & 1887 & 13,2 & 11,3 & 134,6 & 25,6 & 43,4 \\
\hline $\mathrm{HH}$ & 4 & 5 & 3 & 711 & 60 & $\mathrm{~m}$ & 7,1 & 374 & 27,2 & 19,9 & 195,2 & 21,8 & 52,3 \\
\hline $\mathrm{HH}$ & 4 & 5 & 3 & 711 & 90 & $\mathrm{~m}$ & 7,1 & 222 & 36,7 & 24,8 & 254,3 & 23,4 & 64,5 \\
\hline $\mathrm{HH}$ & 4 & 5 & 3 & 711 & 120 & $\mathrm{~m}$ & 7,1 & 155 & 43,6 & 28,3 & 283,5 & 23,2 & 69,3 \\
\hline $\mathrm{HH}$ & 4 & 5 & 3 & 711 & 150 & $\mathrm{~m}$ & 7,1 & 104 & 50,4 & 31,0 & 275,8 & 20,8 & 65,6 \\
\hline $\mathrm{HH}$ & 4 & 6 & 3 & 110 & 35 & $\mathrm{~m}$ & 5,4 & 1223 & 12,4 & 11,1 & 75,8 & 14,7 & 33,0 \\
\hline $\mathrm{HH}$ & 4 & 6 & 3 & 110 & 65 & $\mathrm{~m}$ & 5,4 & 296 & 26,4 & 18,2 & 158,5 & 16,2 & 60,4 \\
\hline $\mathrm{HH}$ & 4 & 6 & 3 & 110 & 95 & $\mathrm{~m}$ & 5,4 & 171 & 36,1 & 22,6 & 212,4 & 17,5 & 81,8 \\
\hline $\mathrm{HH}$ & 4 & 6 & 3 & 110 & 125 & $\mathrm{~m}$ & 5,4 & 107 & 48,2 & 25,8 & 270,0 & 19,5 & 104,2 \\
\hline $\mathrm{HH}$ & 4 & 6 & 3 & 110 & 155 & $\mathrm{~m}$ & 5,4 & 81 & 57,5 & 28,3 & 318,5 & 21,0 & 123,7 \\
\hline $\mathrm{HH}$ & 4 & 6 & 3 & 110 & 185 & $\mathrm{~m}$ & 5,4 & 79 & 61,5 & 30,4 & 377,9 & 23,4 & 148,4 \\
\hline $\mathrm{HH}$ & 4 & 6 & 3 & 211 & 40 & $\mathrm{~S}$ & 6,7 & 1657 & 11,7 & 10,1 & 84,7 & 17,8 & 39,6 \\
\hline $\mathrm{HH}$ & 4 & 6 & 3 & 211 & 70 & $\mathrm{~S}$ & 6,7 & 401 & 24,3 & 19,8 & 184,3 & 18,5 & 79,5 \\
\hline $\mathrm{HH}$ & 4 & 6 & 3 & 211 & 100 & $\mathrm{~S}$ & 6,7 & 212 & 35,8 & 26,0 & 279,4 & 21,3 & 118,8 \\
\hline $\mathrm{HH}$ & 4 & 6 & 3 & 211 & 130 & $\mathrm{~S}$ & 6,7 & 145 & 44,5 & 30,5 & 349,3 & 22,5 & 146,5 \\
\hline $\mathrm{HH}$ & 4 & 6 & 3 & 511 & 30 & $\mathrm{~m}$ & 10,2 & 1240 & 16,9 & 9,6 & 122,8 & 27,9 & 70,4 \\
\hline $\mathrm{HH}$ & 4 & 6 & 3 & 511 & 60 & $\mathrm{~m}$ & 10,2 & 467 & 29,9 & 20,3 & 298,6 & 32,8 & 107,9 \\
\hline $\mathrm{HH}$ & 4 & 6 & 3 & 511 & 90 & $\mathrm{~m}$ & 10,2 & 302 & 38,6 & 27,6 & 423,6 & 35,4 & 129,3 \\
\hline $\mathrm{HH}$ & 4 & 6 & 3 & 611 & 25 & $\mathrm{~m}$ & 13,7 & 899 & 17,6 & 11,7 & 114,5 & 21,8 & 61,3 \\
\hline $\mathrm{HH}$ & 4 & 6 & 3 & 611 & 55 & $\mathrm{~m}$ & 13,7 & 185 & 42,1 & 27,2 & 288,1 & 25,7 & 87,8 \\
\hline $\mathrm{HH}$ & 4 & 6 & 3 & 611 & 85 & $\mathrm{~m}$ & 13,7 & 149 & 57,3 & 36,1 & 545,0 & 38,4 & 141,2 \\
\hline $\mathrm{HH}$ & 4 & 6 & 3 & 611 & 115 & $\mathrm{~m}$ & 13,7 & 102 & 70,1 & 42,3 & 634,7 & 39,4 & 152,5 \\
\hline $\mathrm{HH}$ & 4 & 6 & 3 & 711 & 30 & $\mathrm{~m}$ & 6,8 & 1934 & 12,9 & 10,8 & 127,9 & 25,4 & 41,9 \\
\hline $\mathrm{HH}$ & 4 & 6 & 3 & 711 & 60 & $\mathrm{~m}$ & 6,8 & 378 & 27,2 & 19,1 & 189,1 & 21,9 & 51,2 \\
\hline $\mathrm{HH}$ & 4 & 6 & 3 & 711 & 90 & $\mathrm{~m}$ & 6,8 & 225 & 35,9 & 24,0 & 240,2 & 22,8 & 61,4 \\
\hline $\mathrm{HH}$ & 4 & 6 & 3 & 711 & 120 & $\mathrm{~m}$ & 6,8 & 162 & 42,0 & 27,3 & 265,7 & 22,5 & 65,6 \\
\hline $\mathrm{HH}$ & 4 & 6 & 3 & 711 & 150 & $\mathrm{~m}$ & 6,8 & 108 & 48,9 & 29,9 & 260,1 & 20,2 & 62,5 \\
\hline
\end{tabular}

Fortsetzung auf nächster Seite. 
Tabelle A.22

\begin{tabular}{|c|c|c|c|c|c|c|c|c|c|c|c|c|c|}
\hline WBZ & SOGR & WHZ & $\mathrm{NZ}$ & Art & Alt & $\mathrm{BG}$ & LKL & $\mathrm{N}$ & $\mathrm{dg}$ & hg & $\mathrm{V}$ & G & CV \\
\hline $\mathrm{HH}$ & 7 & 5 & 3 & 211 & 40 & $\mathrm{~s}$ & 6,7 & 1661 & 11,8 & 10,1 & 85,9 & 18,1 & 40,1 \\
\hline $\mathrm{HH}$ & 7 & 5 & 3 & 211 & 70 & $\mathrm{~s}$ & 6,7 & 394 & 24,4 & 19,8 & 184,4 & 18,5 & 79,5 \\
\hline $\mathrm{HH}$ & 7 & 5 & 3 & 211 & 100 & $\mathrm{~s}$ & 6,7 & 222 & 34,7 & 26,0 & 275,3 & 21,0 & 117,1 \\
\hline $\mathrm{HH}$ & 7 & 5 & 3 & 211 & 130 & $\mathrm{~s}$ & 6,7 & 140 & 45,5 & 30,5 & 353,3 & 22,8 & 148,2 \\
\hline $\mathrm{HH}$ & 7 & 5 & 3 & 511 & 30 & $\mathrm{~m}$ & 10,7 & 1198 & 17,5 & 10,6 & 139,9 & 28,7 & 75,2 \\
\hline $\mathrm{HH}$ & 7 & 5 & 3 & 511 & 60 & $\mathrm{~m}$ & 10,7 & 443 & 31,0 & 21,5 & 320,7 & 33,4 & 112,1 \\
\hline $\mathrm{HH}$ & 7 & 5 & 3 & 511 & 90 & $\mathrm{~m}$ & 10,7 & 290 & 39,9 & 28,9 & 453,3 & 36,3 & 134,8 \\
\hline $\mathrm{HH}$ & 7 & 5 & 3 & 711 & 30 & g & 7,7 & 1810 & 13,5 & 11,9 & 143,4 & 25,9 & 45,5 \\
\hline $\mathrm{HH}$ & 7 & 5 & 3 & 711 & 60 & $\mathrm{~g}$ & 7,7 & 358 & 28,1 & 20,8 & 206,4 & 22,1 & 54,8 \\
\hline $\mathrm{HH}$ & 7 & 5 & 3 & 711 & 90 & $\mathrm{~g}$ & 7,7 & 209 & 37,3 & 26,0 & 259,0 & 22,9 & 64,9 \\
\hline $\mathrm{HH}$ & 7 & 5 & 3 & 711 & 120 & $\mathrm{~g}$ & 7,7 & 149 & 43,9 & 29,6 & 287,7 & 22,6 & 69,5 \\
\hline $\mathrm{HH}$ & 7 & 5 & 3 & 711 & 150 & $\mathrm{~g}$ & 7,7 & 105 & 50,4 & 32,4 & 290,0 & 20,9 & 68,1 \\
\hline $\mathrm{HH}$ & 7 & 6 & 3 & 110 & 35 & $\mathrm{~s}$ & 4,5 & 1287 & 11,7 & 9,3 & 55,8 & 13,9 & 25,9 \\
\hline $\mathrm{HH}$ & 7 & 6 & 3 & 110 & 65 & $\mathrm{~s}$ & 4,5 & 320 & 24,5 & 16,1 & 131,6 & 15,1 & 49,3 \\
\hline $\mathrm{HH}$ & 7 & 6 & 3 & 110 & 95 & $\mathrm{~s}$ & 4,5 & 181 & 34,4 & 20,3 & 185,8 & 16,8 & 70,1 \\
\hline $\mathrm{HH}$ & 7 & 6 & 3 & 110 & 125 & $\mathrm{~s}$ & 4,5 & 113 & 46,3 & 23,4 & 241,3 & 19,0 & 91,3 \\
\hline $\mathrm{HH}$ & - & 6 & 3 & 110 & 155 & $\mathrm{~s}$ & 4,5 & 91 & 53,2 & 25,8 & 280,9 & 20,2 & 107,5 \\
\hline $\mathrm{HH}$ & 7 & 6 & 3 & 110 & 185 & $\mathrm{~s}$ & 4,5 & 81 & 60,6 & 27,8 & 348,5 & 23,4 & 134,2 \\
\hline $\mathrm{HH}$ & 7 & 6 & 3 & 211 & 40 & $\mathrm{~s}$ & 6,5 & 1735 & 11,4 & 9,2 & 75,9 & 17,6 & 35,5 \\
\hline $\mathrm{HH}$ & 7 & 6 & 3 & 211 & 70 & $\mathrm{~s}$ & 6,5 & 438 & 23,2 & 18,7 & 173,5 & 18,5 & 75,0 \\
\hline $\mathrm{HH}$ & 7 & 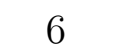 & 2 & 211 & 100 & $\mathrm{~s}$ & 6,5 & 231 & 34,4 & 24,8 & 267,2 & 21,4 & 113,9 \\
\hline $\mathrm{HH}$ & 7 & 6 & 3 & 211 & 130 & $\mathrm{~s}$ & 6,5 & 153 & 43,4 & 29,2 & 333,9 & 22,6 & 140,7 \\
\hline $\mathrm{HH}$ & 7 & 6 & 3 & 511 & 30 & $\mathrm{~m}$ & 10,4 & 1196 & 17,4 & 10,2 & 132,4 & 28,3 & 73,0 \\
\hline $\mathrm{HH}$ & 7 & 6 & 3 & 511 & 60 & $\mathrm{~m}$ & 10,4 & 452 & 30,4 & 21,0 & 308,5 & 32,8 & 109,2 \\
\hline $\mathrm{HH}$ & $=$ & 6 & 0 & 511 & 90 & $\mathrm{~m}$ & 10,4 & 290 & 39,9 & 28,4 & 444,3 & 36,2 & 133,7 \\
\hline $\mathrm{HH}$ & 7 & 6 & 3 & 611 & 25 & $\mathrm{~m}$ & 13,3 & 902 & 17,5 & 11,3 & 109,5 & 21,6 & 60,8 \\
\hline $\mathrm{HH}$ & 7 & 6 & 3 & 611 & 55 & $\mathrm{~m}$ & 13,3 & 192 & 41,2 & 26,7 & 281,9 & 25,6 & 86,8 \\
\hline $\mathrm{HH}$ & - & 6 & 3 & 611 & 85 & $\mathrm{~m}$ & 13,3 & 154 & 54,8 & 35,5 & 510,8 & 36,3 & 132,0 \\
\hline $\mathrm{HH}$ & 7 & 0 & 3 & 611 & 115 & $\mathrm{~m}$ & 13,3 & 108 & 67,8 & 41,7 & 621,8 & 38,9 & 150,2 \\
\hline $\mathrm{HH}$ & 7 & 6 & 3 & 711 & 30 & $\mathrm{~m}$ & 7,0 & 1907 & 13,0 & 11,0 & 129,9 & 25,4 & 42,3 \\
\hline $\mathrm{HH}$ & 7 & 6 & 3 & 711 & 60 & $\mathrm{~m}$ & 7,0 & 381 & 27,1 & 19,5 & 194,0 & 22,0 & 52,2 \\
\hline $\mathrm{HH}$ & 7 & , & 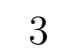 & 711 & 90 & $\mathrm{~m}$ & 7,0 & 218 & 36,4 & 24,3 & 241,4 & 22,7 & 61,5 \\
\hline $\mathrm{HH}$ & 7 & 6 & 3 & 711 & 120 & $\mathrm{~m}$ & 7,0 & 159 & 42,1 & 27,8 & 266,1 & 22,1 & 65,4 \\
\hline $\mathrm{HH}$ & 7 & 6 & 3 & 711 & 150 & $\mathrm{~m}$ & 7,0 & 114 & 48,3 & 30,4 & 272,8 & 20,9 & 65,3 \\
\hline $\mathrm{HH}$ & 7 & 7 & 3 & 110 & 35 & $\mathrm{~s}$ & 4,8 & 1263 & 11,9 & 9,7 & 59,8 & 14,0 & 27,2 \\
\hline $\mathrm{HH}$ & 7 & 7 & 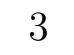 & 110 & 65 & $\mathrm{~s}$ & 4,8 & 311 & 25,2 & 16,6 & 139,0 & 15,5 & 52,2 \\
\hline $\mathrm{HH}$ & 7 & 7 & 3 & 110 & 95 & $\mathrm{~s}$ & 4,8 & 177 & 35,0 & 20,8 & 192,0 & 17,0 & 72,7 \\
\hline $\mathrm{HH}$ & 7 & 7 & 3 & 110 & 125 & $\mathrm{~s}$ & 4,8 & 118 & 44,4 & 23,9 & 235,1 & 18,2 & 89,8 \\
\hline
\end{tabular}

Fortsetzung auf nächster Seite. 
Tabelle A.22

\begin{tabular}{|c|c|c|c|c|c|c|c|c|c|c|c|c|c|}
\hline WBZ & SOGR & WHZ & $\mathrm{NZ}$ & Art & Alt & $\mathrm{BG}$ & LKL & $\mathrm{N}$ & $\mathrm{dg}$ & hg & $\mathrm{V}$ & G & CV \\
\hline $\mathrm{HH}$ & 7 & 7 & 3 & 110 & 155 & $\mathrm{~s}$ & 4,8 & 86 & 55,5 & 26,3 & 295,0 & 20,8 & 113,0 \\
\hline $\mathrm{HH}$ & 7 & 7 & 3 & 110 & 185 & $\mathrm{~s}$ & 4,8 & 78 & 60,7 & 28,3 & 342,3 & 22,6 & 132,4 \\
\hline $\mathrm{HH}$ & 7 & 7 & 3 & 211 & 40 & $\mathrm{~s}$ & 6,2 & 1804 & 11,1 & 8,7 & 72,2 & 17,6 & 33,8 \\
\hline $\mathrm{HH}$ & 7 & 7 & 3 & 211 & 70 & $\mathrm{~s}$ & 6,2 & 445 & 23,1 & 18,2 & 169,6 & 18,6 & 73,3 \\
\hline $\mathrm{HH}$ & 7 & 7 & 3 & 211 & 100 & $\mathrm{~s}$ & 6,2 & 253 & 32,6 & 24,2 & 257,5 & 21,1 & 109,9 \\
\hline $\mathrm{HH}$ & 7 & 7 & 3 & 211 & 130 & $\mathrm{~s}$ & 6,2 & 154 & 42,6 & 28,6 & 318,4 & 22,0 & 134,3 \\
\hline $\mathrm{HH}$ & 7 & 7 & 3 & 511 & 30 & $\mathrm{~m}$ & 10,2 & 1229 & 17,0 & 9,9 & 127,2 & 28,0 & 71,4 \\
\hline $\mathrm{HH}$ & 7 & 7 & 3 & 511 & 60 & $\mathrm{~m}$ & 10,2 & 451 & 30,3 & 20,6 & 300,7 & 32,6 & 107,7 \\
\hline $\mathrm{HH}$ & 7 & 7 & 3 & 511 & 90 & $\mathrm{~m}$ & 10,2 & 291 & 39,5 & 28,0 & 431,9 & 35,7 & 131,0 \\
\hline $\mathrm{HH}$ & 7 & 7 & 3 & 611 & 25 & $\mathrm{~m}$ & 13,3 & 902 & 17,3 & 11,3 & 107,0 & 21,1 & 59,2 \\
\hline $\mathrm{HH}$ & 7 & 7 & 3 & 611 & 55 & $\mathrm{~m}$ & 13,3 & 199 & 40,4 & 26,7 & 282,5 & 25,5 & 86,6 \\
\hline $\mathrm{HH}$ & 7 & 7 & 3 & 611 & 85 & $\mathrm{~m}$ & 13,3 & 150 & 55,2 & 35,5 & 503,2 & 35,8 & 130,5 \\
\hline $\mathrm{HH}$ & 7 & 7 & 3 & 611 & 115 & $\mathrm{~m}$ & 13,3 & 105 & 68,4 & 41,7 & 615,9 & 38,6 & 148,9 \\
\hline $\mathrm{HH}$ & 7 & 7 & 3 & 711 & 30 & $\mathrm{~m}$ & 7,0 & 1928 & 12,9 & 10,9 & 128,3 & 25,3 & 41,9 \\
\hline $\mathrm{HH}$ & 7 & 7 & 3 & 711 & 60 & $\mathrm{~m}$ & 7,0 & 380 & 26,9 & 19,3 & 188,8 & 21,6 & 51,0 \\
\hline $\mathrm{HH}$ & 7 & 7 & 3 & 711 & 90 & $\mathrm{~m}$ & 7,0 & 228 & 35,3 & 24,2 & 237,1 & 22,3 & 60,5 \\
\hline $\mathrm{HH}$ & 7 & 7 & 3 & 711 & 120 & $\mathrm{~m}$ & 7,0 & 163 & 42,0 & 27,6 & 270,0 & 22,6 & 66,5 \\
\hline $\mathrm{HH}$ & 7 & 7 & 3 & 711 & 150 & $\mathrm{~m}$ & 7,0 & 112 & 48,7 & 30,2 & 270,7 & 20,9 & 64,9 \\
\hline $\mathrm{HH}$ & 7 & 7 & 4 & 511 & 30 & $\mathrm{~s}$ & 9,7 & 1267 & 16,5 & 9,0 & 111,3 & 27,1 & 66,7 \\
\hline $\mathrm{HH}$ & 7 & 7 & 4 & 511 & 60 & $\mathrm{~s}$ & 9,7 & 470 & 29,5 & 19,5 & 279,8 & 32,1 & 103,7 \\
\hline $\mathrm{HH}$ & 7 & r & 4 & 511 & 90 & $\mathrm{~s}$ & 9,7 & 303 & 38,1 & 26,7 & 400,5 & 34,5 & 124,7 \\
\hline $\mathrm{HH}$ & 7 & 7 & 4 & 711 & 30 & $\mathrm{~m}$ & 6,6 & 1946 & 12,9 & 10,6 & 125,8 & 25,4 & 41,3 \\
\hline $\mathrm{HH}$ & 7 & 7 & 4 & 711 & 60 & $\mathrm{~m}$ & 6,6 & 384 & 26,6 & 18,9 & 183,5 & 21,4 & 49,8 \\
\hline $\mathrm{HH}$ & 7 & 7 & 4 & 711 & 90 & $\mathrm{~m}$ & 6,6 & 226 & 35,4 & 23,7 & 231,2 & 22,2 & 59,3 \\
\hline $\mathrm{HH}$ & $=$ & 7 & 4 & 711 & 120 & $\mathrm{~m}$ & 6,6 & 157 & 42,8 & 27,0 & 263,7 & 22,6 & 65,3 \\
\hline $\mathrm{HH}$ & 7 & 7 & 4 & 711 & 150 & $\mathrm{~m}$ & 6,6 & 107 & 49,6 & 29,6 & 263,0 & 20,7 & 63,4 \\
\hline $\mathrm{HH}$ & 7 & 9 & 3 & 611 & 25 & $\mathrm{~m}$ & 13,7 & 882 & 17,7 & 11,8 & 115,1 & 21,7 & 61,2 \\
\hline $\mathrm{HH}$ & - & 9 & 3 & 611 & 55 & $\mathrm{~m}$ & 13,7 & 191 & 42,0 & 27,3 & 297,5 & 26,5 & 90,3 \\
\hline $\mathrm{HH}$ & 7 & 9 & 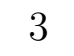 & 611 & 85 & $\mathrm{~m}$ & 13,7 & 150 & 56,2 & 36,2 & 530,8 & 37,2 & 136,0 \\
\hline $\mathrm{HH}$ & 7 & 9 & 3 & 611 & 115 & $\mathrm{~m}$ & 13,7 & 108 & 68,0 & 42,4 & 635,9 & 39,2 & 150,7 \\
\hline $\mathrm{HH}$ & 7 & 9 & 3 & 711 & 30 & $\mathrm{~m}$ & 6,8 & 1919 & 13,0 & 10,8 & 127,4 & 25,3 & 41,6 \\
\hline $\mathrm{HH}$ & 7 & 9 & 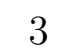 & 711 & 60 & $\mathrm{~m}$ & 6,8 & 390 & 26,6 & 19,1 & 187,7 & 21,7 & 50,8 \\
\hline $\mathrm{HH}$ & 7 & $y$ & 3 & 711 & 90 & $\mathrm{~m}$ & 6,8 & 221 & 36,0 & 24,0 & 237,7 & 22,6 & 60,8 \\
\hline $\mathrm{HH}$ & 7 & 9 & 3 & 711 & 120 & $\mathrm{~m}$ & 6,8 & 163 & 42,6 & 27,3 & 273,8 & 23,2 & 67,6 \\
\hline $\mathrm{HH}$ & 7 & 9 & 3 & 711 & 150 & $\mathrm{~m}$ & 6,8 & 111 & 49,4 & 29,9 & 273,3 & 21,3 & 65,6 \\
\hline $\mathrm{HH}$ & 7 & 9 & 4 & 110 & 35 & $\mathrm{~s}$ & 4,5 & 1312 & 11,6 & 9,2 & 54,6 & 13,9 & 25,6 \\
\hline $\mathrm{HH}$ & 7 & 9 & 4 & 110 & 65 & $\mathrm{~s}$ & 4,5 & 326 & 24,5 & 15,9 & 132,2 & 15,3 & 49,4 \\
\hline $\mathrm{HH}$ & 7 & 9 & 4 & 110 & 95 & $\mathrm{~s}$ & 4,5 & 183 & 33,9 & 20,1 & 179,8 & 16,5 & 67,8 \\
\hline
\end{tabular}

Fortsetzung auf nächster Seite. 
Tabelle A.22

\begin{tabular}{|c|c|c|c|c|c|c|c|c|c|c|c|c|c|}
\hline WBZ & SOGR & WHZ & $\mathrm{NZ}$ & Art & Alt & $\mathrm{BG}$ & LKL & $\mathrm{N}$ & $\mathrm{dg}$ & hg & $\mathrm{V}$ & $\mathrm{G}$ & $\mathrm{CV}$ \\
\hline $\mathrm{HH}$ & 7 & 9 & 4 & 110 & 125 & $\mathrm{~s}$ & 4,5 & 121 & 43,5 & 23,2 & 225,2 & 18,0 & 85,6 \\
\hline $\mathrm{HH}$ & 7 & 9 & 4 & 110 & 155 & $\mathrm{~s}$ & 4,5 & 95 & 51,6 & 25,6 & 273,6 & 19,9 & 104,8 \\
\hline $\mathrm{HH}$ & 7 & 9 & 4 & 110 & 185 & $\mathrm{~s}$ & 4,5 & 83 & 59,0 & 27,5 & 334,6 & 22,7 & 128,9 \\
\hline $\mathrm{HH}$ & 7 & 9 & 4 & 211 & 40 & $\mathrm{~s}$ & 5,9 & 1847 & 10,9 & 8,0 & 66,0 & 17,4 & 30,7 \\
\hline $\mathrm{HH}$ & 7 & 9 & 4 & 211 & 70 & $\mathrm{~S}$ & 5,9 & 479 & 22,0 & 17,4 & 157,9 & 18,1 & 68,4 \\
\hline $\mathrm{HH}$ & 7 & 9 & 4 & 211 & 100 & $\mathrm{~s}$ & 5,9 & 249 & 33,1 & 23,3 & 251,3 & 21,4 & 107,4 \\
\hline $\mathrm{HH}$ & 7 & 9 & 4 & 211 & 130 & $\mathrm{~S}$ & 5,9 & 165 & 41,0 & 27,6 & 304,9 & 21,8 & 129,0 \\
\hline $\mathrm{HH}$ & 7 & 9 & 4 & 511 & 30 & $\mathrm{~s}$ & 9,5 & 1283 & 16,4 & 8,7 & 108,1 & 27,3 & 66,2 \\
\hline $\mathrm{HH}$ & 7 & 9 & 4 & 511 & 60 & $\mathrm{~s}$ & 9,5 & 473 & 29,0 & 19,1 & 268,4 & 31,3 & 100,6 \\
\hline $\mathrm{HH}$ & 7 & 9 & 4 & 511 & 90 & $\mathrm{~S}$ & 9,5 & 311 & 37,5 & 26,3 & 393,1 & 34,3 & 123,3 \\
\hline $\mathrm{HH}$ & 7 & 9 & 4 & 611 & 25 & $\mathrm{~s}$ & 13,0 & 910 & 17,3 & 11,2 & 107,0 & 21,3 & 59,7 \\
\hline $\mathrm{HH}$ & 7 & 9 & 4 & 611 & 55 & $\mathrm{~S}$ & 13,0 & 193 & 40,8 & 26,5 & 276,8 & 25,2 & 85,5 \\
\hline $\mathrm{HH}$ & 7 & 9 & 4 & 611 & 85 & $\mathrm{~S}$ & 13,0 & 147 & 56,3 & 35,3 & 510,2 & 36,7 & 134,1 \\
\hline $\mathrm{HH}$ & 7 & 9 & 4 & 611 & 115 & $\mathrm{~s}$ & 13,0 & 107 & 67,9 & 41,5 & 614,3 & 38,7 & 149,3 \\
\hline $\mathrm{HH}$ & 7 & 9 & 4 & 711 & 30 & $\mathrm{~m}$ & 6,8 & 1941 & 12,9 & 10,8 & 126,9 & 25,3 & 41,6 \\
\hline $\mathrm{HH}$ & 7 & 9 & 4 & 711 & 60 & $\mathrm{~m}$ & 6,8 & 383 & 27,1 & 19,1 & 190,3 & 22,0 & 51,5 \\
\hline $\mathrm{HH}$ & 7 & 9 & 4 & 711 & 90 & $\mathrm{~m}$ & 6,8 & 221 & 35,9 & 24,0 & 235,4 & 22,3 & 60,2 \\
\hline $\mathrm{HH}$ & 7 & 9 & 4 & 711 & 120 & $\mathrm{~m}$ & 6,8 & 163 & 42,3 & 27,3 & 270,7 & 22,9 & 66,8 \\
\hline $\mathrm{HH}$ & 7 & 9 & 4 & 711 & 150 & $\mathrm{~m}$ & 6,8 & 112 & 47,9 & 29,9 & 259,8 & 20,2 & 62,4 \\
\hline $\mathrm{HH}$ & 8 & 3 & 4 & 511 & 30 & $\mathrm{~m}$ & 10,7 & 1226 & 17,3 & 10,5 & 139,0 & 28,7 & 75,1 \\
\hline $\mathrm{HH}$ & 8 & 3 & 4 & 511 & 60 & $\mathrm{~m}$ & 10,7 & 441 & 30,9 & 21,3 & 315,7 & 33,2 & 110,9 \\
\hline $\mathrm{HH}$ & 8 & 3 & 4 & 511 & 90 & $\mathrm{~m}$ & 10,7 & 287 & 40,2 & 28,7 & 450,3 & 36,4 & 134,7 \\
\hline $\mathrm{HH}$ & 8 & 3 & 4 & 711 & 30 & $\mathrm{~S}$ & 6,3 & 2010 & 12,6 & 10,1 & 117,2 & 24,9 & 39,2 \\
\hline $\mathrm{HH}$ & 8 & 3 & 4 & 711 & 60 & $\mathrm{~S}$ & 6,3 & 403 & 26,0 & 18,1 & 175,9 & 21,3 & 48,2 \\
\hline $\mathrm{HH}$ & 8 & 3 & 4 & 711 & 90 & $\mathrm{~s}$ & 6,3 & 232 & 34,8 & 22,7 & 220,4 & 22,0 & 57,2 \\
\hline $\mathrm{HH}$ & 8 & 3 & 4 & 711 & 120 & $\mathrm{~S}$ & 6,3 & 167 & 40,8 & 25,9 & 246,2 & 21,8 & 61,6 \\
\hline $\mathrm{HH}$ & 8 & 3 & 4 & 711 & 150 & $\mathrm{~s}$ & 6,3 & 116 & 47,0 & 28,4 & 245,9 & 20,1 & 60,0 \\
\hline $\mathrm{MH}$ & 1 & 5 & 2 & 211 & 40 & $\mathrm{~s}$ & 6,7 & 1678 & 11,6 & 9,9 & 81,5 & 17,6 & 38,2 \\
\hline $\mathrm{MH}$ & 1 & 5 & 2 & 211 & 70 & $\mathrm{~S}$ & 6,7 & 422 & 23,6 & 19,6 & 181,7 & 18,5 & 78,5 \\
\hline $\mathrm{MH}$ & 1 & 5 & 2 & 211 & 100 & $\mathrm{~S}$ & 6,7 & 226 & 35,3 & 25,7 & 287,2 & 22,2 & 122,2 \\
\hline $\mathrm{MH}$ & 1 & 5 & 2 & 211 & 130 & $\mathrm{~S}$ & 6,7 & 141 & 45,6 & 30,2 & 354,7 & 23,1 & 148,8 \\
\hline $\mathrm{MH}$ & 1 & 5 & 2 & 511 & 30 & $\mathrm{~m}$ & 10,2 & 1241 & 17,0 & 9,9 & 128,5 & 28,3 & 72,2 \\
\hline $\mathrm{MH}$ & 1 & 5 & 2 & 511 & 60 & $\mathrm{~m}$ & 10,2 & 449 & 30,4 & 20,6 & 299,4 & 32,5 & 107,4 \\
\hline $\mathrm{MH}$ & 1 & 5 & 2 & 511 & 90 & $\mathrm{~m}$ & 10,2 & 298 & 39,2 & 28,0 & 436,3 & 36,0 & 132,2 \\
\hline $\mathrm{MH}$ & 1 & 5 & 3 & 211 & 40 & $\mathrm{~s}$ & 6,5 & 1716 & 11,4 & 9,4 & 77,6 & 17,6 & 36,4 \\
\hline $\mathrm{MH}$ & 1 & 5 & 3 & 211 & 70 & $\mathrm{~S}$ & 6,5 & 429 & 23,5 & 19,0 & 178,5 & 18,7 & 77,1 \\
\hline $\mathrm{MH}$ & 1 & 5 & 3 & 211 & 100 & $\mathrm{~S}$ & 6,5 & 234 & 33,7 & 25,1 & 263,9 & 20,9 & 112,4 \\
\hline $\mathrm{MH}$ & 1 & 5 & 3 & 211 & 130 & $\mathrm{~S}$ & 6,5 & 146 & 44,0 & 29,6 & 335,7 & 22,2 & 141,1 \\
\hline
\end{tabular}

Fortsetzung auf nächster Seite. 
Tabelle A.22

\begin{tabular}{|c|c|c|c|c|c|c|c|c|c|c|c|c|c|}
\hline WBZ & SOGR & WHZ & $\mathrm{NZ}$ & Art & Alt & $\mathrm{BG}$ & LKL & $\mathrm{N}$ & $\mathrm{dg}$ & hg & $\mathrm{V}$ & G & $\mathrm{CV}$ \\
\hline $\mathrm{MH}$ & 1 & 5 & 3 & 511 & 30 & $\mathrm{~s}$ & 9,7 & 1256 & 16,7 & 9,2 & 115,7 & 27,5 & 68,3 \\
\hline $\mathrm{MH}$ & 1 & 5 & 3 & 511 & 60 & $\mathrm{~S}$ & 9,7 & 466 & 29,4 & 19,8 & 281,7 & 31,7 & 103,3 \\
\hline $\mathrm{MH}$ & 1 & 5 & 3 & 511 & 90 & $\mathrm{~S}$ & 9,7 & 301 & 38,3 & 27,0 & 406,6 & 34,7 & 125,7 \\
\hline $\mathrm{MH}$ & 1 & 7 & 2 & 211 & 40 & $\mathrm{~s}$ & 6,7 & 1671 & 11,6 & 9,9 & 82,9 & 17,8 & 38,8 \\
\hline $\mathrm{MH}$ & 1 & 7 & 2 & 211 & 70 & $\mathrm{~S}$ & 6,7 & 395 & 24,4 & 19,6 & 182,4 & 18,5 & 78,7 \\
\hline $\mathrm{MH}$ & 1 & 7 & 2 & 211 & 100 & $\mathrm{~S}$ & 6,7 & 228 & 34,2 & 25,7 & 271,4 & 20,9 & 115,5 \\
\hline $\mathrm{MH}$ & 1 & 7 & 2 & 211 & 130 & $\mathrm{~s}$ & 6,7 & 138 & 45,1 & 30,2 & 337,4 & 22,0 & 141,7 \\
\hline $\mathrm{MH}$ & 1 & 7 & 2 & 511 & 30 & $\mathrm{~m}$ & 10,4 & 1226 & 17,2 & 10,2 & 133,6 & 28,5 & 73,6 \\
\hline $\mathrm{MH}$ & 1 & 7 & 2 & 511 & 60 & $\mathrm{~m}$ & 10,4 & 452 & 30,4 & 21,0 & 308,2 & 32,8 & 109,1 \\
\hline $\mathrm{MH}$ & 1 & 7 & 2 & 511 & 90 & $\mathrm{~m}$ & 10,4 & 294 & 39,6 & 28,4 & 444,4 & 36,2 & 133,6 \\
\hline $\mathrm{MH}$ & 1 & 7 & 3 & 211 & 40 & $\mathrm{~s}$ & 6,6 & 1714 & 11,4 & 9,6 & 79,3 & 17,6 & 37,3 \\
\hline MH & 1 & 7 & 3 & 211 & 70 & $\mathrm{~S}$ & 6,6 & 407 & 24,0 & 19,3 & 177,7 & 18,4 & 76,7 \\
\hline $\mathrm{MH}$ & 1 & 7 & 3 & 211 & 100 & $\mathrm{~s}$ & 6,6 & 225 & 35,5 & 25,4 & 284,9 & 22,2 & 121,2 \\
\hline $\mathrm{MH}$ & 1 & 7 & 3 & 211 & 130 & $\mathrm{~s}$ & 6,6 & 144 & 44,6 & 29,9 & 341,3 & 22,5 & 143,4 \\
\hline $\mathrm{MH}$ & 1 & 7 & 3 & 511 & 30 & $\mathrm{~m}$ & 10,0 & 1258 & 16,7 & 9,3 & 117,6 & 27,7 & 68,9 \\
\hline $\mathrm{MH}$ & 1 & 7 & 3 & 511 & 60 & $\mathrm{~m}$ & 10,0 & 467 & 29,6 & 19,9 & 286,8 & 32,2 & 104,9 \\
\hline $\mathrm{MH}$ & 1 & 7 & 3 & 511 & 90 & $\mathrm{~m}$ & 10,0 & 295 & 38,8 & 27,2 & 411,0 & 34,9 & 126,7 \\
\hline $\mathrm{MH}$ & 2 & 5 & 1 & 211 & 40 & $\mathrm{~s}$ & 7,1 & 1572 & 12,1 & 11,1 & 93,1 & 17,9 & 43,5 \\
\hline $\mathrm{MH}$ & 2 & 5 & 1 & 211 & 70 & $\mathrm{~S}$ & 7,1 & 370 & 25,5 & 21,0 & 199,7 & 18,9 & 86,0 \\
\hline $\mathrm{MH}$ & 2 & 5 & 1 & 211 & 100 & $\mathrm{~s}$ & 7,1 & 208 & 35,9 & 27,2 & 289,9 & 21,1 & 122,9 \\
\hline $\mathrm{MH}$ & 2 & 5 & 1 & 211 & 130 & $\mathrm{~S}$ & 7,1 & 134 & 47,3 & 31,8 & 381,9 & 23,5 & 159,5 \\
\hline $\mathrm{MH}$ & 2 & 5 & 1 & 511 & 30 & $\mathrm{~m}$ & 10,2 & 1242 & 16,9 & 9,9 & 127,5 & 28,0 & 71,6 \\
\hline $\mathrm{MH}$ & 2 & 5 & 1 & 511 & 60 & $\mathrm{~m}$ & 10,2 & 458 & 30,1 & 20,6 & 300,6 & 32,6 & 107,6 \\
\hline $\mathrm{MH}$ & 2 & 5 & 1 & 511 & 90 & $\mathrm{~m}$ & 10,2 & 296 & 39,5 & 28,0 & 439,1 & 36,3 & 133,2 \\
\hline $\mathrm{MH}$ & 2 & 5 & 2 & 211 & 40 & $\mathrm{~S}$ & 7,0 & 1623 & 11,8 & 10,6 & 88,9 & 17,9 & 41,7 \\
\hline $\mathrm{MH}$ & 2 & 5 & 2 & 211 & 70 & $\mathrm{~S}$ & 7,0 & 368 & 25,4 & 20,4 & 191,5 & 18,6 & 82,5 \\
\hline $\mathrm{MH}$ & 2 & 5 & 2 & 211 & 100 & $\mathrm{~S}$ & 7,0 & 212 & 35,6 & 26,6 & 283,5 & 21,1 & 120,4 \\
\hline $\mathrm{MH}$ & 2 & 5 & 2 & 211 & 130 & $\mathrm{~S}$ & 7,0 & 133 & 46,1 & 31,1 & 352,6 & 22,2 & 147,6 \\
\hline $\mathrm{MH}$ & 2 & 5 & 2 & 511 & 30 & $\mathrm{~m}$ & 10,2 & 1252 & 16,8 & 9,6 & 121,8 & 27,7 & 69,9 \\
\hline $\mathrm{MH}$ & 2 & 5 & 2 & 511 & 60 & $\mathrm{~m}$ & 10,2 & 463 & 30,0 & 20,3 & 296,7 & 32,7 & 107,3 \\
\hline $\mathrm{MH}$ & 2 & 5 & 2 & 511 & 90 & $\mathrm{~m}$ & 10,2 & 302 & 38,5 & 27,6 & 422,0 & 35,2 & 128,7 \\
\hline $\mathrm{MH}$ & 2 & 7 & 2 & 211 & 40 & $\mathrm{~m}$ & 7,4 & 1525 & 12,2 & 11,6 & 97,4 & 17,9 & 45,5 \\
\hline $\mathrm{MH}$ & 2 & 7 & 2 & 211 & 70 & $\mathrm{~m}$ & 7,4 & 345 & 26,5 & 21,5 & 206,6 & 19,1 & 88,8 \\
\hline $\mathrm{MH}$ & 2 & 7 & 2 & 211 & 100 & $\mathrm{~m}$ & 7,4 & 193 & 37,5 & 27,8 & 301,1 & 21,3 & 127,3 \\
\hline $\mathrm{MH}$ & 2 & 7 & 2 & 211 & 130 & $\mathrm{~m}$ & 7,4 & 120 & 48,9 & 32,4 & 374,9 & 22,6 & 156,1 \\
\hline $\mathrm{MH}$ & 2 & 7 & 2 & 511 & 30 & $\mathrm{~m}$ & 10,2 & 1238 & 17,0 & 9,9 & 127,7 & 28,1 & 71,8 \\
\hline $\mathrm{MH}$ & 2 & 7 & 2 & 511 & 60 & $\mathrm{~m}$ & 10,2 & 460 & 30,1 & 20,6 & 301,2 & 32,7 & 107,9 \\
\hline $\mathrm{MH}$ & 2 & 7 & 2 & 511 & 90 & $\mathrm{~m}$ & 10,2 & 292 & 39,9 & 28,0 & 440,1 & 36,5 & 133,7 \\
\hline
\end{tabular}

Fortsetzung auf nächster Seite. 
Tabelle A.22

\begin{tabular}{|c|c|c|c|c|c|c|c|c|c|c|c|c|c|}
\hline WBZ & SOGR & WHZ & $\mathrm{NZ}$ & Art & Alt & $B G$ & LKL & $\mathrm{N}$ & $\mathrm{dg}$ & hg & $\mathrm{V}$ & G & $\mathrm{CV}$ \\
\hline $\mathrm{OH}$ & 4 & 5 & 2 & 110 & 35 & $\mathrm{~m}$ & 6,0 & 1165 & 12,8 & 12,2 & 86,9 & 14,9 & 37,2 \\
\hline $\mathrm{OH}$ & 4 & 5 & 2 & 110 & 65 & $\mathrm{~m}$ & 6,0 & 283 & 27,1 & 19,5 & 170,2 & 16,3 & 65,6 \\
\hline $\mathrm{OH}$ & 4 & 5 & 2 & 110 & 95 & $\mathrm{~m}$ & 6,0 & 156 & 39,2 & 24,0 & 241,5 & 18,8 & 93,4 \\
\hline $\mathrm{OH}$ & 4 & 5 & 2 & 110 & 125 & $\mathrm{~m}$ & 6,0 & 98 & 50,7 & 27,3 & 289,0 & 19,8 & 112,4 \\
\hline $\mathrm{OH}$ & 4 & 5 & 2 & 110 & 155 & $\mathrm{~m}$ & 6,0 & 80 & 58,6 & 29,8 & 341,4 & 21,6 & 134,0 \\
\hline $\mathrm{OH}$ & 4 & 5 & 2 & 110 & 185 & $\mathrm{~m}$ & 6,0 & 67 & 67,8 & 32,0 & 410,0 & 24,2 & 161,7 \\
\hline $\mathrm{OH}$ & 4 & 5 & 2 & 211 & 40 & $\mathrm{~m}$ & 8,0 & 1431 & 12,7 & 12,7 & 107,0 & 18,0 & 49,8 \\
\hline $\mathrm{OH}$ & 4 & 5 & 2 & 211 & 70 & $\mathrm{~m}$ & 8,0 & 331 & 27,0 & 22,8 & 217,1 & 18,9 & 93,2 \\
\hline $\mathrm{OH}$ & 4 & 5 & 2 & 211 & 100 & $\mathrm{~m}$ & 8,0 & 176 & 39,4 & 29,2 & 317,6 & 21,5 & 133,9 \\
\hline $\mathrm{OH}$ & 4 & 5 & 2 & 211 & 130 & $\mathrm{~m}$ & 8,0 & 118 & 49,4 & 33,9 & 391,9 & 22,6 & 162,6 \\
\hline $\mathrm{OH}$ & 4 & 5 & 2 & 511 & 30 & g & 11,6 & 1136 & 18,2 & 12,1 & 166,5 & 29,6 & 81,8 \\
\hline $\mathrm{OH}$ & 4 & 5 & 2 & 511 & 60 & g & 11,6 & 424 & 32,2 & 23,4 & 360,1 & 34,5 & 119,5 \\
\hline $\mathrm{OH}$ & 4 & 5 & 2 & 511 & 90 & $\mathrm{~g}$ & 11,6 & 271 & 42,6 & 31,0 & 513,8 & 38,7 & 147,3 \\
\hline $\mathrm{OH}$ & 4 & 5 & 2 & 611 & 25 & $\mathrm{~m}$ & 15,2 & 849 & 18,5 & 13,4 & 137,6 & 22,7 & 64,8 \\
\hline $\mathrm{OH}$ & 4 & 5 & 2 & 611 & 55 & $\mathrm{~m}$ & 15,2 & 176 & 44,1 & 29,3 & 321,7 & 26,8 & 92,6 \\
\hline $\mathrm{OH}$ & 4 & 5 & 2 & 611 & 85 & $\mathrm{~m}$ & 15,2 & 135 & 60,1 & 38,4 & 573,6 & 38,3 & 142,2 \\
\hline $\mathrm{OH}$ & 4 & 5 & 2 & 611 & 115 & $\mathrm{~m}$ & 15,2 & 99 & 75,3 & 44,8 & 744,5 & 44,1 & 173,7 \\
\hline $\mathrm{OH}$ & 4 & 5 & 2 & 711 & 30 & g & 7,5 & 1852 & 13,4 & 11,6 & 142,1 & 26,3 & 45,4 \\
\hline $\mathrm{OH}$ & 4 & 5 & 2 & 711 & 60 & $\mathrm{~g}$ & 7,5 & 364 & 27,9 & 20,4 & 204,4 & 22,3 & 54,5 \\
\hline $\mathrm{OH}$ & 4 & 5 & 2 & 711 & 90 & g & 7,5 & 209 & 37,4 & 25,5 & 255,5 & 22,9 & 64,3 \\
\hline $\mathrm{OH}$ & 4 & 5 & 2 & 711 & 120 & $\mathrm{~g}$ & 7,5 & 150 & 44,1 & 29,0 & 287,4 & 23,0 & 69,7 \\
\hline $\mathrm{OH}$ & 4 & 5 & 2 & 711 & 150 & g & 7,5 & 103 & 50,7 & 31,8 & 283,2 & 20,8 & 66,9 \\
\hline $\mathrm{OH}$ & 4 & 5 & 3 & 110 & 35 & $\mathrm{~m}$ & 5,4 & 1232 & 12,3 & 11,0 & 73,9 & 14,5 & 32,3 \\
\hline $\mathrm{OH}$ & 4 & 5 & 3 & 110 & 65 & $\mathrm{~m}$ & 5,4 & 299 & 26,1 & 18,0 & 155,8 & 16,0 & 59,2 \\
\hline $\mathrm{OH}$ & 4 & 5 & 3 & 110 & 95 & $\mathrm{~m}$ & 5,4 & 170 & 36,0 & 22,4 & 208,7 & 17,3 & 80,2 \\
\hline $\mathrm{OH}$ & 4 & 5 & 3 & 110 & 125 & $\mathrm{~m}$ & 5,4 & 107 & 47,7 & 25,6 & 263,2 & 19,2 & 101,5 \\
\hline $\mathrm{OH}$ & 4 & 5 & 3 & 110 & 155 & $\mathrm{~m}$ & 5,4 & 87 & 55,6 & 28,1 & 317,4 & 21,2 & 123,4 \\
\hline $\mathrm{OH}$ & 4 & 5 & 3 & 110 & 185 & $\mathrm{~m}$ & 5,4 & 74 & 63,5 & 30,2 & 375,4 & 23,4 & 146,9 \\
\hline $\mathrm{OH}$ & 4 & 5 & 3 & 511 & 30 & $\mathrm{~m}$ & 10,2 & 1238 & 17,0 & 9,9 & 127,2 & 28,0 & 71,6 \\
\hline $\mathrm{OH}$ & 4 & 5 & 3 & 511 & 60 & $\mathrm{~m}$ & 10,2 & 464 & 30,0 & 20,6 & 302,7 & 32,8 & 108,4 \\
\hline $\mathrm{OH}$ & 4 & 5 & 3 & 511 & 90 & $\mathrm{~m}$ & 10,2 & 293 & 39,4 & 28,0 & 433,6 & 35,8 & 131,4 \\
\hline $\mathrm{OH}$ & 4 & 5 & 3 & 611 & 25 & $\mathrm{~m}$ & 14,1 & 871 & 17,9 & 12,2 & 119,7 & 21,8 & 61,7 \\
\hline $\mathrm{OH}$ & 4 & 5 & 3 & 611 & 55 & $\mathrm{~m}$ & 14,1 & 189 & 42,4 & 27,8 & 304,5 & 26,6 & 91,0 \\
\hline $\mathrm{OH}$ & 4 & 5 & 3 & 611 & 85 & $\mathrm{~m}$ & 14,1 & 147 & 56,5 & 36,7 & 533,2 & 36,9 & 134,6 \\
\hline $\mathrm{OH}$ & 4 & 5 & 3 & 611 & 115 & $\mathrm{~m}$ & 14,1 & 103 & 71,5 & 43,0 & 675,0 & 41,4 & 161,0 \\
\hline $\mathrm{OH}$ & 4 & 5 & 3 & 711 & 30 & $\mathrm{~m}$ & 7,0 & 1902 & 13,1 & 11,2 & 133,3 & 25,6 & 43,2 \\
\hline $\mathrm{OH}$ & 4 & 5 & 3 & 711 & 60 & $\mathrm{~m}$ & 7,0 & 371 & 27,4 & 19,7 & 194,6 & 21,9 & 52,3 \\
\hline $\mathrm{OH}$ & 4 & 5 & 3 & 711 & 90 & $\mathrm{~m}$ & 7,0 & 214 & 37,0 & 24,6 & 248,0 & 23,0 & 63,0 \\
\hline
\end{tabular}

Fortsetzung auf nächster Seite. 
Tabelle A.22

\begin{tabular}{|c|c|c|c|c|c|c|c|c|c|c|c|c|c|}
\hline WBZ & SOGR & WHZ & $\mathrm{NZ}$ & Art & Alt & $B G$ & LKL & $\mathrm{N}$ & $\mathrm{dg}$ & hg & $\mathrm{V}$ & G & $\mathrm{CV}$ \\
\hline $\mathrm{OH}$ & 4 & 5 & 3 & 711 & 120 & $\mathrm{~m}$ & 7,0 & 152 & 43,1 & 28,1 & 269,5 & 22,2 & 66,0 \\
\hline $\mathrm{OH}$ & 4 & 5 & 3 & 711 & 150 & $\mathrm{~m}$ & 7,0 & 105 & 51,1 & 30,7 & 283,1 & 21,5 & 67,5 \\
\hline $\mathrm{OH}$ & 4 & 6 & 3 & 110 & 35 & $\mathrm{~s}$ & 5,1 & 1258 & 12,1 & 10,4 & 67,6 & 14,4 & 30,1 \\
\hline $\mathrm{OH}$ & 4 & 6 & 3 & 110 & 65 & $\mathrm{~s}$ & 5,1 & 300 & 25,7 & 17,4 & 146,5 & 15,6 & 55,4 \\
\hline $\mathrm{OH}$ & 4 & 6 & 3 & 110 & 95 & $\mathrm{~s}$ & 5,1 & 173 & 35,5 & 21,7 & 200,7 & 17,2 & 76,6 \\
\hline $\mathrm{OH}$ & 4 & 6 & 3 & 110 & 125 & $\mathrm{~s}$ & 5,1 & 111 & 46,9 & 24,9 & 256,9 & 19,2 & 98,6 \\
\hline $\mathrm{OH}$ & 4 & 6 & 3 & 110 & 155 & $\mathrm{~s}$ & 5,1 & 89 & 55,0 & 27,3 & 309,4 & 21,1 & 119,6 \\
\hline $\mathrm{OH}$ & 4 & 6 & 3 & 110 & 185 & $\mathrm{~S}$ & 5,1 & 77 & 63,0 & 29,4 & 375,9 & 24,0 & 146,2 \\
\hline $\mathrm{OH}$ & 4 & 6 & 3 & 211 & 40 & $\mathrm{~s}$ & 6,3 & 1775 & 11,2 & 8,9 & 73,3 & 17,5 & 34,4 \\
\hline $\mathrm{OH}$ & 4 & 6 & 3 & 211 & 70 & $\mathrm{~s}$ & 6,3 & 433 & 23,2 & 18,5 & 170,0 & 18,3 & 73,5 \\
\hline $\mathrm{OH}$ & 4 & 6 & 3 & 211 & 100 & $\mathrm{~S}$ & 6,3 & 240 & 33,5 & 24,5 & 261,3 & 21,2 & 111,5 \\
\hline $\mathrm{OH}$ & 4 & 6 & 3 & 211 & 130 & $\mathrm{~S}$ & 6,3 & 146 & 43,7 & 28,9 & 320,6 & 21,8 & 135,1 \\
\hline $\mathrm{OH}$ & 4 & 6 & 3 & 511 & 30 & $\mathrm{~S}$ & 9,7 & 1250 & 16,7 & 9,2 & 115,4 & 27,5 & 68,1 \\
\hline $\mathrm{OH}$ & 4 & 6 & 3 & 511 & 60 & $\mathrm{~S}$ & 9,7 & 468 & 29,8 & 19,8 & 289,4 & 32,7 & 106,3 \\
\hline $\mathrm{OH}$ & 4 & 6 & 3 & 511 & 90 & $\mathrm{~S}$ & 9,7 & 300 & 38,9 & 27,0 & 415,5 & 35,6 & 128,8 \\
\hline $\mathrm{OH}$ & 4 & 6 & 3 & 711 & 30 & $\mathrm{~m}$ & 7,0 & 1925 & 12,9 & 10,9 & 128,6 & 25,3 & 42,0 \\
\hline $\mathrm{OH}$ & 4 & 6 & 3 & 711 & 60 & $\mathrm{~m}$ & 7,0 & 384 & 27,0 & 19,3 & 191,4 & 21,9 & 51,7 \\
\hline $\mathrm{OH}$ & 4 & 6 & 3 & 711 & 90 & $\mathrm{~m}$ & 7,0 & 218 & 36,3 & 24,2 & 239,1 & 22,5 & 61,0 \\
\hline $\mathrm{OH}$ & 4 & 6 & 3 & 711 & 120 & $\mathrm{~m}$ & 7,0 & 156 & 42,4 & 27,6 & 262,7 & 22,0 & 64,7 \\
\hline $\mathrm{OH}$ & 4 & 6 & 3 & 711 & 150 & $\mathrm{~m}$ & 7,0 & 103 & 50,1 & 30,2 & 263,0 & 20,3 & 63,0 \\
\hline $\mathrm{OH}$ & 7 & 6 & 3 & 110 & 35 & $\mathrm{~s}$ & 4,9 & 1251 & 12,0 & 10,3 & 66,5 & 14,3 & 29,6 \\
\hline $\mathrm{OH}$ & 7 & 6 & 3 & 110 & 65 & $\mathrm{~S}$ & 4,9 & 303 & 25,6 & 17,2 & 145,2 & 15,6 & 54,8 \\
\hline $\mathrm{OH}$ & 7 & 6 & 3 & 110 & 95 & $\mathrm{~s}$ & 4,9 & 167 & 35,9 & 21,5 & 196,3 & 16,9 & 74,7 \\
\hline $\mathrm{OH}$ & 7 & 6 & 3 & 110 & 125 & $\mathrm{~S}$ & 4,9 & 113 & 46,3 & 24,6 & 251,8 & 19,0 & 96,4 \\
\hline $\mathrm{OH}$ & 7 & 6 & 3 & 110 & 155 & $\mathrm{~S}$ & 4,9 & 90 & 53,9 & 27,1 & 298,8 & 20,6 & 115,5 \\
\hline $\mathrm{OH}$ & 7 & 6 & 3 & 110 & 185 & $\mathrm{~S}$ & 4,9 & 79 & 62,8 & 29,1 & 379,7 & 24,4 & 147,4 \\
\hline $\mathrm{OH}$ & 7 & 6 & 3 & 211 & 40 & $\mathrm{~S}$ & 6,5 & 1750 & 11,3 & 9,2 & 76,6 & 17,7 & 35,9 \\
\hline $\mathrm{OH}$ & 7 & 6 & 3 & 211 & 70 & $\mathrm{~S}$ & 6,5 & 416 & 23,7 & 18,7 & 172,5 & 18,3 & 74,5 \\
\hline $\mathrm{OH}$ & 7 & 6 & 3 & 211 & 100 & $\mathrm{~s}$ & 6,5 & 229 & 34,0 & 24,8 & 261,2 & 20,8 & 111,3 \\
\hline $\mathrm{OH}$ & 7 & 6 & 3 & 211 & 130 & $\mathrm{~S}$ & 6,5 & 153 & 42,7 & 29,2 & 325,0 & 21,9 & 136,8 \\
\hline $\mathrm{OH}$ & 7 & 6 & 3 & 511 & 30 & $\mathrm{~m}$ & 10,2 & 1223 & 17,0 & 9,6 & 122,2 & 27,8 & 70,1 \\
\hline $\mathrm{OH}$ & 7 & 6 & 3 & 511 & 60 & $\mathrm{~m}$ & 10,2 & 459 & 30,1 & 20,3 & 297,0 & 32,7 & 107,4 \\
\hline $\mathrm{OH}$ & 7 & 6 & 3 & 511 & 90 & $\mathrm{~m}$ & 10,2 & 300 & 38,7 & 27,6 & 421,5 & 35,2 & 128,7 \\
\hline $\mathrm{OH}$ & 7 & 6 & 3 & 611 & 25 & $\mathrm{~m}$ & 14,4 & 860 & 18,0 & 12,7 & 125,5 & 21,9 & 62,0 \\
\hline $\mathrm{OH}$ & 7 & 6 & 3 & 611 & 55 & $\mathrm{~m}$ & 14,4 & 183 & 42,7 & 28,4 & 306,4 & 26,2 & 89,8 \\
\hline $\mathrm{OH}$ & 7 & 6 & 3 & 611 & 85 & $\mathrm{~m}$ & 14,4 & 136 & 58,9 & 37,4 & 541,9 & 37,0 & 136,4 \\
\hline $\mathrm{OH}$ & 7 & 6 & 3 & 611 & 115 & $\mathrm{~m}$ & 14,4 & 105 & 71,0 & 43,7 & 689,9 & 41,5 & 161,1 \\
\hline $\mathrm{OH}$ & 7 & 6 & 3 & 711 & 30 & $\mathrm{~m}$ & 7,0 & 1929 & 12,9 & 10,9 & 128,7 & 25,4 & 42,0 \\
\hline
\end{tabular}

Fortsetzung auf nächster Seite. 
Tabelle A.22

\begin{tabular}{|c|c|c|c|c|c|c|c|c|c|c|c|c|c|}
\hline WBZ & SOGR & WHZ & $\mathrm{NZ}$ & Art & Alt & $B G$ & LKL & $\mathrm{N}$ & $\mathrm{dg}$ & hg & $\mathrm{V}$ & G & $\mathrm{CV}$ \\
\hline $\mathrm{OH}$ & 7 & 6 & 3 & 711 & 60 & $\mathrm{~m}$ & 7,0 & 385 & 27,0 & 19,3 & 192,8 & 22,1 & 52,0 \\
\hline $\mathrm{OH}$ & 7 & 6 & 3 & 711 & 90 & $\mathrm{~m}$ & 7,0 & 221 & 36,0 & 24,2 & 239,3 & 22,5 & 61,0 \\
\hline $\mathrm{OH}$ & 7 & 6 & 3 & 711 & 120 & $\mathrm{~m}$ & 7,0 & 153 & 43,3 & 27,6 & 268,7 & 22,5 & 66,1 \\
\hline $\mathrm{OH}$ & 7 & 6 & 3 & 711 & 150 & $\mathrm{~m}$ & 7,0 & 113 & 48,2 & 30,2 & 267,1 & 20,6 & 64,0 \\
\hline $\mathrm{OH}$ & 7 & 7 & 3 & 110 & 35 & $\mathrm{~S}$ & 4,5 & 1269 & 11,8 & 9,3 & 56,1 & 13,9 & 25,9 \\
\hline $\mathrm{OH}$ & 7 & 7 & 3 & 110 & 65 & $\mathrm{~S}$ & 4,5 & 323 & 24,7 & 16,1 & 134,7 & 15,4 & 50,4 \\
\hline $\mathrm{OH}$ & 7 & 7 & 3 & 110 & 95 & $\mathrm{~S}$ & 4,5 & 180 & 34,2 & 20,3 & 181,6 & 16,5 & 68,6 \\
\hline $\mathrm{OH}$ & 7 & 7 & 3 & 110 & 125 & $\mathrm{~S}$ & 4,5 & 115 & 45,0 & 23,4 & 231,9 & 18,3 & 88,0 \\
\hline $\mathrm{OH}$ & 7 & 7 & 3 & 110 & 155 & $\mathrm{~S}$ & 4,5 & 92 & 52,6 & 25,8 & 277,7 & 20,0 & 106,4 \\
\hline $\mathrm{OH}$ & 7 & 7 & 3 & 110 & 185 & $\mathrm{~S}$ & 4,5 & 86 & 60,6 & 27,8 & 370,3 & 24,8 & 142,7 \\
\hline $\mathrm{OH}$ & 7 & 7 & 3 & 211 & 40 & $\mathrm{~S}$ & 6,5 & 1720 & 11,4 & 9,4 & 77,3 & 17,5 & 36,3 \\
\hline $\mathrm{OH}$ & 7 & 7 & 3 & 211 & 70 & $\mathrm{~S}$ & 6,5 & 434 & 23,3 & 19,0 & 176,5 & 18,5 & 76,3 \\
\hline $\mathrm{OH}$ & 7 & 7 & 3 & 211 & 100 & $\mathrm{~S}$ & 6,5 & 214 & 35,4 & 25,1 & 267,3 & 21,1 & 113,8 \\
\hline $\mathrm{OH}$ & 7 & 7 & 3 & 211 & 130 & $\mathrm{~S}$ & 6,5 & 146 & 43,8 & 29,6 & 330,4 & 22,0 & 138,9 \\
\hline $\mathrm{OH}$ & 7 & 7 & 3 & 511 & 30 & $\mathrm{~S}$ & 9,7 & 1273 & 16,7 & 9,2 & 117,0 & 27,9 & 69,1 \\
\hline $\mathrm{OH}$ & 7 & 7 & 3 & 511 & 60 & $\mathrm{~S}$ & 9,7 & 471 & 29,4 & 19,8 & 283,5 & 31,9 & 104,0 \\
\hline $\mathrm{OH}$ & 7 & 7 & 3 & 511 & 90 & $\mathrm{~S}$ & 9,7 & 302 & 38,7 & 27,0 & 414,6 & 35,5 & 128,5 \\
\hline $\mathrm{OH}$ & 7 & 7 & 3 & 611 & 25 & $\mathrm{~S}$ & 13,0 & 898 & 17,2 & 11,2 & 105,5 & 21,0 & 58,8 \\
\hline $\mathrm{OH}$ & 7 & 7 & 3 & 611 & 55 & $\mathrm{~S}$ & 13,0 & 196 & 40,4 & 26,5 & 275,5 & 25,1 & 84,8 \\
\hline $\mathrm{OH}$ & 7 & 7 & 3 & 611 & 85 & $\mathrm{~S}$ & 13,0 & 152 & 54,7 & 35,3 & 500,0 & 35,8 & 130,3 \\
\hline $\mathrm{OH}$ & 7 & 7 & 3 & 611 & 115 & $\mathrm{~S}$ & 13,0 & 111 & 67,4 & 41,5 & 630,1 & 39,6 & 151,5 \\
\hline $\mathrm{OH}$ & 7 & 7 & 3 & 711 & 30 & $\mathrm{~m}$ & 6,8 & 1914 & 13,0 & 10,8 & 127,1 & 25,3 & 41,6 \\
\hline $\mathrm{OH}$ & 7 & 7 & 3 & 711 & 60 & $\mathrm{~m}$ & 6,8 & 389 & 26,7 & 19,1 & 187,7 & 21,7 & 50,8 \\
\hline $\mathrm{OH}$ & 7 & 7 & 3 & 711 & 90 & $\mathrm{~m}$ & 6,8 & 226 & 35,8 & 24,0 & 240,1 & 22,8 & 61,4 \\
\hline $\mathrm{OH}$ & 7 & 7 & 3 & 711 & 120 & $\mathrm{~m}$ & 6,8 & 159 & 42,0 & 27,3 & 259,9 & 22,0 & 64,2 \\
\hline $\mathrm{OH}$ & 7 & 7 & 3 & 711 & 150 & $\mathrm{~m}$ & 6,8 & 111 & 48,2 & 29,9 & 259,6 & 20,2 & 62,4 \\
\hline $\mathrm{OH}$ & 7 & 9 & 4 & 110 & 35 & $\mathrm{~m}$ & 5,4 & 1197 & 12,5 & 11,0 & 75,0 & 14,6 & 32,6 \\
\hline $\mathrm{OH}$ & 7 & 9 & 4 & 110 & 65 & $\mathrm{~m}$ & 5,4 & 295 & 26,1 & 18,0 & 153,6 & 15,8 & 58,4 \\
\hline $\mathrm{OH}$ & 7 & 9 & 4 & 110 & 95 & $\mathrm{~m}$ & 5,4 & 170 & 36,3 & 22,4 & 211,4 & 17,6 & 81,2 \\
\hline $\mathrm{OH}$ & 7 & 9 & 4 & 110 & 125 & $\mathrm{~m}$ & 5,4 & 104 & 48,7 & 25,6 & 266,6 & 19,4 & 102,5 \\
\hline $\mathrm{OH}$ & 7 & 9 & 4 & 110 & 155 & $\mathrm{~m}$ & 5,4 & 84 & 56,4 & 28,1 & 314,4 & 21,0 & 122,2 \\
\hline $\mathrm{OH}$ & 7 & 9 & 4 & 110 & 185 & $\mathrm{~m}$ & 5,4 & 79 & 62,6 & 30,2 & 389,8 & 24,3 & 152,7 \\
\hline $\mathrm{OH}$ & 7 & 9 & 4 & 611 & 25 & $\mathrm{~S}$ & 12,3 & 923 & 16,9 & 10,5 & 97,3 & 20,8 & 57,9 \\
\hline $\mathrm{OH}$ & 7 & 9 & 4 & 611 & 55 & $\mathrm{~S}$ & 12,3 & 196 & 40,3 & 25,7 & 265,8 & 24,9 & 84,3 \\
\hline $\mathrm{OH}$ & 7 & 9 & 4 & 611 & 85 & $\mathrm{~S}$ & 12,3 & 156 & 53,5 & 34,3 & 478,4 & 35,1 & 126,8 \\
\hline $\mathrm{OH}$ & 7 & 9 & 4 & 611 & 115 & $\mathrm{~S}$ & 12,3 & 108 & 67,5 & 40,4 & 598,0 & 38,6 & 148,7 \\
\hline $\mathrm{OH}$ & 7 & 9 & 4 & 711 & 30 & $\mathrm{~m}$ & 6,6 & 1955 & 12,9 & 10,6 & 125,5 & 25,4 & 41,3 \\
\hline $\mathrm{OH}$ & 7 & 9 & 4 & 711 & 60 & $\mathrm{~m}$ & 6,6 & 382 & 27,1 & 18,9 & 188,0 & 22,0 & 51,0 \\
\hline
\end{tabular}

Fortsetzung auf nächster Seite. 
Tabelle A.22

\begin{tabular}{|c|c|c|c|c|c|c|c|c|c|c|c|c|c|}
\hline WBZ & SOGR & WHZ & $\mathrm{NZ}$ & Art & Alt & $\mathrm{BG}$ & LKL & $\mathrm{N}$ & $\mathrm{dg}$ & hg & $\mathrm{V}$ & G & $\mathrm{CV}$ \\
\hline $\mathrm{OH}$ & 7 & 9 & 4 & 711 & 90 & $\mathrm{~m}$ & 6,6 & 226 & 35,6 & 23,7 & 235,0 & 22,6 & 60,3 \\
\hline $\mathrm{OH}$ & 7 & 9 & 4 & 711 & 120 & $\mathrm{~m}$ & 6,6 & 161 & 42,2 & 27,0 & 263,7 & 22,5 & 65,3 \\
\hline $\mathrm{OH}$ & 7 & 9 & 4 & 711 & 150 & $\mathrm{~m}$ & 6,6 & 109 & 48,6 & 29,6 & 256,4 & 20,2 & 61,8 \\
\hline $\mathrm{OH}$ & 8 & 1 & 3 & 110 & 35 & $\mathrm{~s}$ & 5,2 & 1216 & 12,3 & 10,8 & 71,9 & 14,4 & 31,5 \\
\hline $\mathrm{OH}$ & 8 & 1 & 3 & 110 & 65 & $\mathrm{~S}$ & 5,2 & 301 & 26,0 & 17,8 & 153,1 & 15,9 & 58,1 \\
\hline $\mathrm{OH}$ & 8 & 1 & 3 & 110 & 95 & $\mathrm{~S}$ & 5,2 & 174 & 35,4 & 22,2 & 204,3 & 17,1 & 78,5 \\
\hline $\mathrm{OH}$ & 8 & 1 & 3 & 110 & 125 & $\mathrm{~S}$ & 5,2 & 108 & 47,8 & 25,4 & 263,7 & 19,3 & 101,5 \\
\hline $\mathrm{OH}$ & 8 & 1 & 3 & 110 & 155 & $\mathrm{~S}$ & 5,2 & 85 & 57,3 & 27,9 & 326,9 & 22,0 & 126,7 \\
\hline $\mathrm{OH}$ & 8 & 1 & 3 & 110 & 185 & $\mathrm{~S}$ & 5,2 & 76 & 62,4 & 29,9 & 371,0 & 23,3 & 144,9 \\
\hline $\mathrm{OH}$ & 8 & 1 & 3 & 711 & 30 & g & 8,4 & 1719 & 14,0 & 12,7 & 155,2 & 26,4 & 48,3 \\
\hline $\mathrm{OH}$ & 8 & 1 & 3 & 711 & 60 & g & 8,4 & 335 & 29,3 & 22,1 & 222,3 & 22,6 & 58,1 \\
\hline $\mathrm{OH}$ & 8 & 1 & 3 & 711 & 90 & g & 8,4 & 198 & 39,3 & 27,4 & 286,1 & 24,1 & 70,6 \\
\hline $\mathrm{OH}$ & 8 & 1 & 3 & 711 & 120 & g & 8,4 & 142 & 45,5 & 31,2 & 308,8 & 23,1 & 73,5 \\
\hline $\mathrm{OH}$ & 8 & 1 & 3 & 711 & 150 & g & 8,4 & 98 & 53,6 & 34,1 & 321,3 & 22,1 & 74,3 \\
\hline US & 1 & 4 & 2 & 211 & 40 & $\mathrm{~m}$ & 7,4 & 1525 & 12,3 & 11,6 & 98,5 & 18,1 & 46,0 \\
\hline US & 1 & 4 & 2 & 211 & 70 & $\mathrm{~m}$ & 7,4 & 367 & 25,4 & 21,5 & 201,0 & 18,6 & 86,5 \\
\hline US & 1 & 4 & 2 & 211 & 100 & $\mathrm{~m}$ & 7,4 & 193 & 37,9 & 27,8 & 306,1 & 21,7 & 129,5 \\
\hline US & 1 & 4 & 2 & 211 & 130 & $\mathrm{~m}$ & 7,4 & 128 & 47,5 & 32,4 & 376,9 & 22,7 & 157,0 \\
\hline US & 1 & 4 & 2 & 511 & 30 & g & 11,4 & 1164 & 17,9 & 11,7 & 159,5 & 29,4 & 80,1 \\
\hline US & 1 & 4 & 2 & 511 & 60 & g & 11,4 & 428 & 31,9 & 22,8 & 347,4 & 34,2 & 117,2 \\
\hline US & 1 & 4 & 2 & 511 & 90 & g & 11,4 & 274 & 41,8 & 30,4 & 490,3 & 37,6 & 142,1 \\
\hline US & 1 & 5 & 2 & 110 & 35 & $\mathrm{~m}$ & 6,2 & 1155 & 12,9 & 12,3 & 89,5 & 15,1 & 38,1 \\
\hline US & 1 & 5 & 2 & 110 & 65 & $\mathrm{~m}$ & 6,2 & 275 & 27,5 & 19,7 & 172,7 & 16,4 & 66,6 \\
\hline US & 1 & 5 & 2 & 110 & 95 & $\mathrm{~m}$ & 6,2 & 156 & 38,6 & 24,2 & 236,0 & 18,2 & 91,6 \\
\hline US & 1 & 5 & 2 & 110 & 125 & $\mathrm{~m}$ & 6,2 & 102 & 51,0 & 27,5 & 305,8 & 20,9 & 119,2 \\
\hline US & 1 & 5 & 2 & 110 & 155 & $\mathrm{~m}$ & 6,2 & 83 & 59,1 & 30,1 & 363,3 & 22,8 & 142,8 \\
\hline US & 1 & 5 & 2 & 110 & 185 & $\mathrm{~m}$ & 6,2 & 73 & 65,3 & 32,2 & 415,0 & 24,4 & 164,3 \\
\hline US & 1 & 5 & 2 & 211 & 40 & $\mathrm{~m}$ & 7,6 & 1504 & 12,4 & 11,8 & 100,8 & 18,2 & 46,9 \\
\hline US & 1 & 5 & 2 & 211 & 70 & $\mathrm{~m}$ & 7,6 & 353 & 26,1 & 21,8 & 207,0 & 19,0 & 89,0 \\
\hline US & 1 & 5 & 2 & 211 & 100 & $\mathrm{~m}$ & 7,6 & 195 & 38,0 & 28,1 & 316,4 & 22,2 & 133,7 \\
\hline US & 1 & 5 & 2 & 211 & 130 & $\mathrm{~m}$ & 7,6 & 123 & 48,2 & 32,7 & 374,9 & 22,4 & 156,2 \\
\hline US & 1 & 5 & 2 & 511 & 30 & $\mathrm{~g}$ & 11,4 & 1169 & 17,9 & 11,5 & 157,1 & 29,5 & 79,8 \\
\hline US & 1 & 5 & 2 & 511 & 60 & $\mathrm{~g}$ & 11,4 & 435 & 31,6 & 22,7 & 345,6 & 34,1 & 116,7 \\
\hline US & 1 & 5 & 2 & 511 & 90 & $\mathrm{~g}$ & 11,4 & 275 & 42,2 & 30,2 & 498,1 & 38,5 & 145,1 \\
\hline US & 1 & 5 & 3 & 110 & 35 & $\mathrm{~m}$ & 6,0 & 1180 & 12,7 & 12,0 & 85,3 & 14,9 & 36,6 \\
\hline US & 1 & 5 & 3 & 110 & 65 & $\mathrm{~m}$ & 6,0 & 291 & 26,6 & 19,3 & 167,4 & 16,2 & 64,5 \\
\hline US & 1 & 5 & 3 & 110 & 95 & $\mathrm{~m}$ & 6,0 & 157 & 37,9 & 23,8 & 224,8 & 17,7 & 87,2 \\
\hline US & 1 & 5 & 3 & 110 & 125 & $\mathrm{~m}$ & 6,0 & 103 & 49,3 & 27,1 & 283,6 & 19,6 & 110,5 \\
\hline
\end{tabular}

Fortsetzung auf nächster Seite. 
Tabelle A.22

\begin{tabular}{|c|c|c|c|c|c|c|c|c|c|c|c|c|c|}
\hline WBZ & SOGR & WHZ & $\mathrm{NZ}$ & Art & Alt & $\mathrm{BG}$ & LKL & $\mathrm{N}$ & $\mathrm{dg}$ & hg & $\mathrm{V}$ & G & $\mathrm{CV}$ \\
\hline US & 1 & 5 & 3 & 110 & 155 & $\mathrm{~m}$ & 6,0 & 78 & 60,2 & 29,6 & 348,9 & 22,2 & 136,5 \\
\hline US & 1 & 5 & 3 & 110 & 185 & $\mathrm{~m}$ & 6,0 & 72 & 65,8 & 31,7 & 411,1 & 24,5 & 162,0 \\
\hline US & 1 & 5 & 3 & 211 & 40 & $\mathrm{~s}$ & 7,3 & 1515 & 12,2 & 11,3 & 94,5 & 17,8 & 44,1 \\
\hline US & 1 & 5 & 3 & 211 & 70 & S & 7,3 & 379 & 25,2 & 21,2 & 201,3 & 18,9 & 86,7 \\
\hline US & 1 & 5 & 3 & 211 & 100 & $\mathrm{~S}$ & 7,3 & 201 & 36,6 & 27,5 & 294,6 & 21,2 & 124,7 \\
\hline US & 1 & 5 & 3 & 211 & 130 & $\mathrm{~s}$ & 7,3 & 131 & 46,9 & 32,1 & 371,5 & 22,7 & 155,1 \\
\hline US & 1 & 5 & 3 & 511 & 30 & g & 11,4 & 1181 & 17,9 & 11,5 & 158,4 & 29,7 & 80,5 \\
\hline US & 1 & 5 & 3 & 511 & 60 & g & 11,4 & 435 & 31,5 & 22,7 & 345,2 & 34,0 & 116,6 \\
\hline US & 1 & 5 & 3 & 511 & 90 & g & 11,4 & 277 & 41,8 & 30,2 & 493,3 & 38,0 & 143,5 \\
\hline US & 1 & 7 & 2 & 110 & 35 & $\mathrm{~m}$ & 6,2 & 1162 & 12,8 & 12,3 & 88,6 & 15,0 & 37,9 \\
\hline US & 1 & 7 & 2 & 110 & 65 & $\mathrm{~m}$ & 6,2 & 276 & 27,7 & 19,7 & 175,9 & 16,6 & 67,7 \\
\hline US & 1 & 7 & 2 & 110 & 95 & $\mathrm{~m}$ & 6,2 & 162 & 37,5 & 24,2 & 230,8 & 17,9 & 89,9 \\
\hline US & 1 & 7 & 2 & 110 & 125 & $\mathrm{~m}$ & 6,2 & 102 & 49,8 & 27,5 & 291,1 & 19,9 & 113,7 \\
\hline US & 1 & 7 & 2 & 110 & 155 & $\mathrm{~m}$ & 6,2 & 82 & 59,8 & 30,1 & 368,1 & 23,0 & 144,6 \\
\hline US & 1 & 7 & 2 & 110 & 185 & $\mathrm{~m}$ & 6,2 & 69 & 67,9 & 32,2 & 425,6 & 25,0 & 168,0 \\
\hline US & 1 & 7 & 2 & 211 & 40 & $\mathrm{~m}$ & 7,6 & 1500 & 12,4 & 11,8 & 99,7 & 18,0 & 46,4 \\
\hline US & 1 & 7 & 2 & 211 & 70 & $\mathrm{~m}$ & 7,6 & 355 & 26,1 & 21,8 & 207,8 & 18,9 & 89,3 \\
\hline US & 1 & 7 & 2 & 211 & 100 & $\mathrm{~m}$ & 7,6 & 191 & 38,1 & 28,1 & 309,1 & 21,8 & 130,7 \\
\hline US & 1 & 7 & 2 & 211 & 130 & $\mathrm{~m}$ & 7,6 & 123 & 49,0 & 32,7 & 390,2 & 23,2 & 162,3 \\
\hline US & 1 & 7 & 2 & 511 & 30 & $\mathrm{~m}$ & 11,1 & 1185 & 17,8 & 11,3 & 153,5 & 29,4 & 79,0 \\
\hline US & 1 & 7 & 2 & 511 & 60 & $\mathrm{~m}$ & 11,1 & 442 & 31,2 & 22,3 & 338,4 & 33,9 & 115,4 \\
\hline US & 1 & 7 & 2 & 511 & 90 & $\mathrm{~m}$ & 11,1 & 284 & 40,9 & 29,8 & 479,3 & 37,3 & 140,2 \\
\hline US & 1 & 7 & 3 & 110 & 35 & $\mathrm{~m}$ & 6,0 & 1185 & 12,7 & 12,2 & 87,0 & 15,0 & 37,4 \\
\hline US & 1 & 7 & 3 & 110 & 65 & $\mathrm{~m}$ & 6,0 & 281 & 27,3 & 19,5 & 172,9 & 16,5 & 66,5 \\
\hline US & 1 & 7 & 3 & 110 & 95 & $\mathrm{~m}$ & 6,0 & 161 & 37,7 & 24,0 & 230,6 & 18,0 & 89,6 \\
\hline US & 1 & 7 & 3 & 110 & 125 & $\mathrm{~m}$ & 6,0 & 107 & 48,4 & 27,3 & 286,3 & 19,7 & 111,9 \\
\hline US & 1 & 7 & 3 & 110 & 155 & $\mathrm{~m}$ & 6,0 & 80 & 59,1 & 29,8 & 347,3 & 21,9 & 136,2 \\
\hline US & 1 & 7 & 3 & 110 & 185 & $\mathrm{~m}$ & 6,0 & 71 & 65,6 & 32,0 & 406,1 & 24,0 & 160,5 \\
\hline US & 1 & 7 & 3 & 211 & 40 & $\mathrm{~m}$ & 7,4 & 1518 & 12,2 & 11,6 & 97,4 & 17,9 & 45,5 \\
\hline US & 1 & 7 & 3 & 211 & 70 & $\mathrm{~m}$ & 7,4 & 356 & 26,2 & 21,5 & 206,8 & 19,1 & 88,9 \\
\hline US & 1 & 7 & 3 & 211 & 100 & $\mathrm{~m}$ & 7,4 & 200 & 37,2 & 27,8 & 306,2 & 21,8 & 129,6 \\
\hline US & 1 & 7 & 3 & 211 & 130 & $\mathrm{~m}$ & 7,4 & 127 & 47,5 & 32,4 & 373,4 & 22,5 & 155,6 \\
\hline US & 1 & 7 & 3 & 511 & 30 & $\mathrm{~m}$ & 10,9 & 1205 & 17,4 & 10,8 & 143,7 & 28,8 & 76,1 \\
\hline US & 1 & 7 & 3 & 511 & 60 & $\mathrm{~m}$ & 10,9 & 447 & 30,9 & 21,8 & 326,1 & 33,4 & 112,8 \\
\hline US & 1 & 7 & 3 & 511 & 90 & $\mathrm{~m}$ & 10,9 & 286 & 40,4 & 29,2 & 461,1 & 36,6 & 136,5 \\
\hline US & 1 & 8 & 3 & 211 & 40 & $\mathrm{~s}$ & 7,3 & 1549 & 12,1 & 11,3 & 94,4 & 17,8 & 44,1 \\
\hline US & 1 & 8 & 3 & 211 & 70 & $\mathrm{~S}$ & 7,3 & 369 & 25,4 & 21,2 & 200,0 & 18,8 & 86,1 \\
\hline US & 1 & 8 & 3 & 211 & 100 & $\mathrm{~S}$ & 7,3 & 189 & 37,8 & 27,5 & 296,0 & 21,2 & 125,2 \\
\hline
\end{tabular}

Fortsetzung auf nächster Seite. 
Tabelle A.22

\begin{tabular}{|c|c|c|c|c|c|c|c|c|c|c|c|c|c|}
\hline WBZ & SOGR & WHZ & $\mathrm{NZ}$ & Art & Alt & $\mathrm{BG}$ & LKL & $\mathrm{N}$ & $\mathrm{dg}$ & hg & $\mathrm{V}$ & G & $\mathrm{CV}$ \\
\hline US & 1 & 8 & 3 & 211 & 130 & $\mathrm{~s}$ & 7,3 & 131 & 46,7 & 32,1 & 367,7 & 22,4 & 153,4 \\
\hline US & 1 & 8 & 3 & 511 & 30 & $\mathrm{~m}$ & 10,9 & 1206 & 17,5 & 10,8 & 144,7 & 29,0 & 76,7 \\
\hline US & 1 & 8 & 3 & 511 & 60 & $\mathrm{~m}$ & 10,9 & 436 & 31,2 & 21,7 & 322,9 & 33,3 & 112,2 \\
\hline US & 1 & 8 & 3 & 511 & 90 & $\mathrm{~m}$ & 10,9 & 285 & 40,7 & 29,1 & 465,7 & 37,2 & 138,3 \\
\hline US & 6 & 4 & 2 & 110 & 35 & $\mathrm{~m}$ & 6,2 & 1173 & 12,9 & 12,3 & 90,5 & 15,3 & 38,6 \\
\hline US & 6 & 4 & 2 & 110 & 65 & $\mathrm{~m}$ & 6,2 & 284 & 27,1 & 19,7 & 172,5 & 16,4 & 66,6 \\
\hline US & 6 & 4 & 2 & 110 & 95 & $\mathrm{~m}$ & 6,2 & 161 & 37,7 & 24,2 & 231,3 & 17,9 & 90,1 \\
\hline US & 6 & 4 & 2 & 110 & 125 & $\mathrm{~m}$ & 6,2 & 103 & 49,7 & 27,5 & 292,7 & 20,0 & 114,4 \\
\hline US & 6 & 4 & 2 & 110 & 155 & $\mathrm{~m}$ & 6,2 & 78 & 60,0 & 30,1 & 352,7 & 22,1 & 138,5 \\
\hline US & 6 & 4 & 2 & 110 & 185 & $\mathrm{~m}$ & 6,2 & 73 & 67,4 & 32,2 & 442,5 & 26,0 & 174,9 \\
\hline US & 6 & 4 & 2 & 211 & 40 & $\mathrm{~m}$ & 7,6 & 1482 & 12,4 & 12,0 & 100,7 & 17,9 & 46,9 \\
\hline US & 6 & 4 & 2 & 211 & 70 & $\mathrm{~m}$ & 7,6 & 357 & 26,1 & 22,0 & 211,8 & 19,1 & 91,0 \\
\hline US & 6 & 4 & 2 & 211 & 100 & $\mathrm{~m}$ & 7,6 & 192 & 38,3 & 28,3 & 317,6 & 22,2 & 134,2 \\
\hline US & 6 & 4 & 2 & 211 & 130 & $\mathrm{~m}$ & 7,6 & 118 & 49,5 & 32,9 & 384,3 & 22,7 & 159,6 \\
\hline US & 6 & 4 & 2 & 511 & 30 & g & 11,4 & 1168 & 18,0 & 11,5 & 158,0 & 29,7 & 80,3 \\
\hline US & 6 & 4 & 2 & 511 & 60 & g & 11,4 & 437 & 31,9 & 22,7 & 353,5 & 34,9 & 119,6 \\
\hline US & 6 & 4 & 2 & 511 & 90 & g & 11,4 & 285 & 41,1 & 30,2 & 492,9 & 37,9 & 143,0 \\
\hline US & 6 & 5 & 2 & 110 & 35 & $\mathrm{~m}$ & 5,8 & 1200 & 12,6 & 11,8 & 84,0 & 15,0 & 36,1 \\
\hline US & 6 & 5 & 2 & 110 & 65 & $\mathrm{~m}$ & 5,8 & 278 & 27,2 & 19,0 & 165,7 & 16,2 & 63,4 \\
\hline US & 6 & 5 & 2 & 110 & 95 & $\mathrm{~m}$ & 5,8 & 160 & 37,6 & 23,5 & 223,5 & 17,8 & 86,4 \\
\hline US & 6 & 5 & 2 & 110 & 125 & $\mathrm{~m}$ & 5,8 & 99 & 49,8 & 26,7 & 275,3 & 19,3 & 106,8 \\
\hline US & 6 & 5 & 2 & 110 & 155 & $\mathrm{~m}$ & 5,8 & 81 & 58,9 & 29,3 & 344,0 & 22,0 & 134,4 \\
\hline US & 6 & 5 & 2 & 110 & 185 & $\mathrm{~m}$ & 5,8 & 76 & 63,6 & 31,4 & 400,5 & 24,1 & 158,1 \\
\hline US & 6 & 5 & 2 & 211 & 40 & $\mathrm{~S}$ & 7,3 & 1541 & 12,2 & 11,3 & 94,5 & 17,9 & 44,1 \\
\hline US & 6 & 5 & 2 & 211 & 70 & $\mathrm{~S}$ & 7,3 & 369 & 25,6 & 21,2 & 201,5 & 19,0 & 86,8 \\
\hline US & 6 & 5 & 2 & 211 & 100 & $\mathrm{~S}$ & 7,3 & 194 & 37,3 & 27,5 & 295,0 & 21,2 & 124,9 \\
\hline US & 6 & 5 & 2 & 211 & 130 & $\mathrm{~S}$ & 7,3 & 133 & 47,1 & 32,1 & 379,7 & 23,1 & 158,4 \\
\hline US & 6 & 5 & 2 & 511 & 30 & g & 11,4 & 1141 & 18,2 & 11,7 & 159,9 & 29,5 & 80,4 \\
\hline US & 6 & 5 & 2 & 511 & 60 & g & 11,4 & 426 & 32,2 & 22,8 & 351,5 & 34,6 & 118,7 \\
\hline US & 6 & 5 & 2 & 511 & 90 & g & 11,4 & 270 & 42,5 & 30,4 & 497,9 & 38,3 & 144,6 \\
\hline UW & 1 & 5 & 2 & 110 & 35 & g & 6,5 & 1138 & 13,1 & 12,7 & 93,9 & 15,3 & 39,9 \\
\hline UW & 1 & 5 & 2 & 110 & 65 & g & 6,5 & 268 & 28,2 & 20,1 & 180,7 & 16,8 & 69,7 \\
\hline UW & 1 & 5 & 2 & 110 & 95 & g & 6,5 & 157 & 38,6 & 24,7 & 242,2 & 18,4 & 94,5 \\
\hline UW & 1 & 5 & 2 & 110 & 125 & g & 6,5 & 98 & 50,5 & 28,0 & 291,9 & 19,6 & 114,4 \\
\hline UW & 1 & 5 & 2 & 110 & 155 & $\mathrm{~g}$ & 6,5 & 79 & 60,1 & 30,6 & 364,0 & 22,4 & 143,4 \\
\hline UW & 1 & 5 & 2 & 110 & 185 & g & 6,5 & 74 & 65,2 & 32,8 & 426,6 & 24,7 & 169,6 \\
\hline UW & 1 & 5 & 2 & 211 & 40 & $\mathrm{~m}$ & 7,8 & 1447 & 12,6 & 12,4 & 105,5 & 18,1 & 49,0 \\
\hline UW & 1 & 5 & 2 & 211 & 70 & $\mathrm{~m}$ & 7,8 & 337 & 27,2 & 22,5 & 221,3 & 19,5 & 95,0 \\
\hline
\end{tabular}

Fortsetzung auf nächster Seite. 
Tabelle A.22

\begin{tabular}{|c|c|c|c|c|c|c|c|c|c|c|c|c|c|}
\hline WBZ & SOGR & WHZ & $\mathrm{NZ}$ & Art & Alt & $\mathrm{BG}$ & LKL & $\mathrm{N}$ & $\mathrm{dg}$ & hg & $\mathrm{V}$ & G & $\mathrm{CV}$ \\
\hline UW & 1 & 5 & 2 & 211 & 100 & $\mathrm{~m}$ & 7,8 & 179 & 39,4 & 28,9 & 320,2 & 21,9 & 135,0 \\
\hline UW & 1 & 5 & 2 & 211 & 130 & $\mathrm{~m}$ & 7,8 & 115 & 50,2 & 33,6 & 393,2 & 22,8 & 163,1 \\
\hline UW & 1 & 5 & 2 & 511 & 30 & $\mathrm{~m}$ & 11,1 & 1174 & 17,8 & 11,3 & 152,6 & 29,2 & 78,5 \\
\hline UW & 1 & 5 & 2 & 511 & 60 & $\mathrm{~m}$ & 11,1 & 420 & 32,1 & 22,3 & 335,9 & 33,9 & 115,1 \\
\hline UW & 1 & 5 & 2 & 511 & 90 & $\mathrm{~m}$ & 11,1 & 282 & 41,0 & 29,8 & 477,7 & 37,2 & 139,7 \\
\hline UW & 1 & 5 & 3 & 211 & 40 & $\mathrm{~m}$ & 7,6 & 1479 & 12,4 & 12,0 & 101,2 & 17,9 & 47,2 \\
\hline UW & 1 & 5 & 3 & 211 & 70 & $\mathrm{~m}$ & 7,6 & 348 & 26,8 & 22,0 & 218,0 & 19,7 & 93,7 \\
\hline UW & 1 & 5 & 3 & 211 & 100 & $\mathrm{~m}$ & 7,6 & 187 & 38,1 & 28,3 & 305,5 & 21,3 & 129,0 \\
\hline UW & 1 & 5 & 3 & 211 & 130 & $\mathrm{~m}$ & 7,6 & 125 & 47,8 & 32,9 & 377,1 & 22,4 & 157,0 \\
\hline UW & 1 & 5 & 3 & 511 & 30 & $\mathrm{~m}$ & 10,7 & 1207 & 17,3 & 10,5 & 137,6 & 28,4 & 74,3 \\
\hline UW & 1 & 5 & 3 & 511 & 60 & $\mathrm{~m}$ & 10,7 & 451 & 30,6 & 21,3 & 315,2 & 33,1 & 110,7 \\
\hline UW & 1 & 5 & 3 & 511 & 90 & $\mathrm{~m}$ & 10,7 & 285 & 40,6 & 28,7 & 454,8 & 36,8 & 136,3 \\
\hline UW & 1 & 7 & 2 & 211 & 40 & $\mathrm{~m}$ & 7,6 & 1491 & 12,4 & 12,0 & 100,9 & 17,9 & 47,1 \\
\hline UW & 1 & 7 & 2 & 211 & 70 & $\mathrm{~m}$ & 7,6 & 358 & 26,0 & 22,0 & 210,7 & 19,1 & 90,6 \\
\hline UW & 1 & 7 & 2 & 211 & 100 & $\mathrm{~m}$ & 7,6 & 190 & 37,9 & 28,3 & 307,4 & 21,4 & 129,9 \\
\hline UW & 1 & 7 & 2 & 211 & 130 & $\mathrm{~m}$ & 7,6 & 125 & 47,9 & 32,9 & 379,3 & 22,6 & 157,9 \\
\hline UW & 1 & 7 & 2 & 511 & 30 & $\mathrm{~m}$ & 10,9 & 1198 & 17,6 & 10,8 & 144,5 & 29,0 & 76,6 \\
\hline UW & 1 & 7 & 2 & 511 & 60 & $\mathrm{~m}$ & 10,9 & 441 & 31,2 & 21,7 & 326,4 & 33,7 & 113,4 \\
\hline UW & 1 & 7 & 2 & 511 & 90 & $\mathrm{~m}$ & 10,9 & 282 & 41,2 & 29,1 & 469,7 & 37,6 & 139,7 \\
\hline UW & 1 & 7 & 3 & 211 & 40 & $\mathrm{~m}$ & 7,6 & 1507 & 12,4 & 11,8 & 100,1 & 18,1 & 46,7 \\
\hline UW & 1 & 7 & 3 & 211 & 70 & $\mathrm{~m}$ & 7,6 & 363 & 25,6 & 21,8 & 204,3 & 18,7 & 87,9 \\
\hline UW & 1 & 7 & 3 & 211 & 100 & $\mathrm{~m}$ & 7,6 & 196 & 37,4 & 28,1 & 306,0 & 21,5 & 129,4 \\
\hline UW & 1 & 7 & 3 & 211 & 130 & $\mathrm{~m}$ & 7,6 & 119 & 49,0 & 32,7 & 375,8 & 22,4 & 156,3 \\
\hline UW & 1 & 7 & 3 & 511 & 30 & $\mathrm{~s}$ & 9,7 & 1275 & 16,5 & 9,0 & 112,1 & 27,3 & 67,2 \\
\hline UW & 1 & 7 & 3 & 511 & 60 & $\mathrm{~S}$ & 9,7 & 475 & 29,4 & 19,5 & 281,0 & 32,2 & 104,1 \\
\hline UW & 1 & 7 & 3 & 511 & 90 & $\mathrm{~S}$ & 9,7 & 296 & 38,6 & 26,7 & 399,8 & 34,6 & 124,8 \\
\hline UW & 1 & 8 & 3 & 211 & 40 & $\mathrm{~S}$ & 6,9 & 1640 & 11,9 & 10,4 & 88,7 & 18,1 & 41,4 \\
\hline UW & 1 & 8 & 3 & 211 & 70 & $\mathrm{~S}$ & 6,9 & 388 & 24,6 & 20,1 & 187,6 & 18,5 & 80,9 \\
\hline UW & 1 & 8 & 3 & 211 & 100 & $\mathrm{~s}$ & 6,9 & 212 & 35,9 & 26,3 & 286,3 & 21,5 & 121,5 \\
\hline UW & 1 & 8 & 3 & 211 & 130 & $\mathrm{~s}$ & 6,9 & 131 & 46,4 & 30,8 & 347,5 & 22,1 & 145,5 \\
\hline UW & 1 & 8 & 3 & 511 & 30 & $\mathrm{~S}$ & 9,1 & 1298 & 16,1 & 8,3 & 99,1 & 26,3 & 62,8 \\
\hline UW & 1 & 8 & 3 & 511 & 60 & $\mathrm{~s}$ & 9,1 & 481 & 28,5 & 18,6 & 256,4 & 30,7 & 97,6 \\
\hline UW & 1 & 8 & 3 & 511 & 90 & $\mathrm{~s}$ & 9,1 & 301 & 37,7 & 25,7 & 374,6 & 33,6 & 119,5 \\
\hline UW & 5 & 5 & 1 & 110 & 35 & $\mathrm{~m}$ & 6,2 & 1150 & 12,9 & 12,3 & 89,2 & 15,1 & 38,0 \\
\hline UW & 5 & 5 & 1 & 110 & 65 & $\mathrm{~m}$ & 6,2 & 279 & 27,5 & 19,7 & 175,3 & 16,6 & 67,6 \\
\hline UW & 5 & 5 & 1 & 110 & 95 & $\mathrm{~m}$ & 6,2 & 156 & 38,1 & 24,2 & 230,0 & 17,8 & 89,4 \\
\hline UW & 5 & 5 & 1 & 110 & 125 & $\mathrm{~m}$ & 6,2 & 99 & 50,4 & 27,5 & 290,1 & 19,8 & 113,1 \\
\hline UW & 5 & 5 & 1 & 110 & 155 & $\mathrm{~m}$ & 6,2 & 80 & 59,7 & 30,1 & 357,5 & 22,4 & 140,4 \\
\hline
\end{tabular}

Fortsetzung auf nächster Seite. 
Tabelle A.22

\begin{tabular}{|c|c|c|c|c|c|c|c|c|c|c|c|c|c|}
\hline WBZ & SOGR & WHZ & $\mathrm{NZ}$ & Art & Alt & $\mathrm{BG}$ & LKL & $\mathrm{N}$ & $\mathrm{dg}$ & hg & $\mathrm{V}$ & G & $\mathrm{CV}$ \\
\hline UW & 5 & 5 & 1 & 110 & 185 & $\mathrm{~m}$ & 6,2 & 71 & 65,5 & 32,2 & 406,5 & 23,9 & 160,9 \\
\hline UW & 5 & 5 & 1 & 211 & 40 & g & 8,4 & 1314 & 13,3 & 13,9 & 119,2 & 18,2 & 55,0 \\
\hline UW & 5 & 5 & 1 & 211 & 70 & g & 8,4 & 309 & 28,2 & 24,2 & 234,3 & 19,2 & 100,2 \\
\hline UW & 5 & 5 & 1 & 211 & 100 & $\mathrm{~g}$ & 8,4 & 166 & 41,1 & 30,7 & 344,5 & 22,1 & 144,6 \\
\hline UW & 5 & 5 & 1 & 211 & 130 & g & 8,4 & 102 & 53,3 & 35,5 & 417,2 & 22,8 & 171,9 \\
\hline UW & 5 & 5 & 1 & 511 & 30 & $\mathrm{~g}$ & 11,6 & 1120 & 18,3 & 12,1 & 166,5 & 29,6 & 81,7 \\
\hline UW & 5 & 5 & 1 & 511 & 60 & $\mathrm{~g}$ & 11,6 & 419 & 32,6 & 23,4 & 364,5 & 35,0 & 121,2 \\
\hline UW & 5 & 5 & 1 & 511 & 90 & g & 11,6 & 278 & 41,7 & 31,0 & 507,6 & 38,0 & 145,0 \\
\hline UW & 5 & 7 & 1 & 110 & 35 & $\mathrm{~m}$ & 6,2 & 1161 & 12,9 & 12,3 & 89,3 & 15,1 & 38,1 \\
\hline UW & 5 & 7 & 1 & 110 & 65 & $\mathrm{~m}$ & 6,2 & 272 & 27,7 & 19,7 & 172,5 & 16,3 & 66,4 \\
\hline UW & 5 & 7 & 1 & 110 & 95 & $\mathrm{~m}$ & 6,2 & 154 & 38,8 & 24,2 & 235,3 & 18,2 & 91,4 \\
\hline UW & 5 & 7 & 1 & 110 & 125 & $\mathrm{~m}$ & 6,2 & 97 & 50,6 & 27,5 & 286,0 & 19,5 & 111,5 \\
\hline UW & 5 & 7 & 1 & 110 & 155 & $\mathrm{~m}$ & 6,2 & 81 & 59,5 & 30,1 & 359,3 & 22,5 & 141,2 \\
\hline UW & 5 & 7 & 1 & 110 & 185 & $\mathrm{~m}$ & 6,2 & 72 & 65,7 & 32,2 & 415,1 & 24,4 & 164,2 \\
\hline UW & 5 & 7 & 1 & 211 & 40 & $\mathrm{~g}$ & 8,2 & 1351 & 13,0 & 13,4 & 114,6 & 18,1 & 53,0 \\
\hline UW & 5 & 7 & 1 & 211 & 70 & $\mathrm{~g}$ & 8,2 & 319 & 27,8 & 23,6 & 230,3 & 19,4 & 98,6 \\
\hline UW & 5 & 7 & 1 & 211 & 100 & $\mathrm{~g}$ & 8,2 & 174 & 40,1 & 30,1 & 338,0 & 22,0 & 142,0 \\
\hline UW & 5 & 7 & 1 & 211 & 130 & $\mathrm{~g}$ & 8,2 & 114 & 51,8 & 34,8 & 431,7 & 24,0 & 178,2 \\
\hline UW & 5 & 7 & 1 & 511 & 30 & $\mathrm{~m}$ & 11,1 & 1181 & 17,7 & 11,1 & 148,9 & 29,0 & 77,5 \\
\hline UW & 5 & 7 & 1 & 511 & 60 & $\mathrm{~m}$ & 11,1 & 435 & 31,4 & 22,1 & 332,0 & 33,6 & 114,1 \\
\hline UW & 5 & 7 & 1 & 511 & 90 & $\mathrm{~m}$ & 11,1 & 284 & 40,7 & 29,6 & 472,4 & 37,0 & 138,7 \\
\hline UW & 5 & 8 & 1 & 211 & 40 & $\mathrm{~m}$ & 7,8 & 1442 & 12,7 & 12,4 & 105,8 & 18,1 & 49,1 \\
\hline UW & 5 & 8 & 1 & 211 & 70 & $\mathrm{~m}$ & 7,8 & 339 & 26,8 & 22,5 & 215,7 & 19,1 & 92,6 \\
\hline UW & 5 & 8 & 1 & 211 & 100 & $\mathrm{~m}$ & 7,8 & 184 & 38,5 & 28,9 & 314,8 & 21,4 & 132,7 \\
\hline UW & 5 & 8 & 1 & 211 & 130 & $\mathrm{~m}$ & 7,8 & 112 & 50,6 & 33,6 & 389,4 & 22,5 & 161,5 \\
\hline UW & 5 & 8 & 1 & 511 & 30 & $\mathrm{~m}$ & 10,2 & 1238 & 17,0 & 9,9 & 127,9 & 28,1 & 71,9 \\
\hline UW & 5 & 8 & 1 & 511 & 60 & $\mathrm{~m}$ & 10,2 & 453 & 30,2 & 20,6 & 299,5 & 32,5 & 107,3 \\
\hline UW & 5 & 8 & 1 & 511 & 90 & $\mathrm{~m}$ & 10,2 & 298 & 39,3 & 28,0 & 437,1 & 36,1 & 132,5 \\
\hline UW & 5 & 9 & 1 & 211 & 40 & $\mathrm{~m}$ & 7,4 & 1522 & 12,3 & 11,6 & 98,5 & 18,1 & 45,9 \\
\hline UW & 5 & 9 & 1 & 211 & 70 & $\mathrm{~m}$ & 7,4 & 363 & 26,0 & 21,5 & 209,2 & 19,3 & 90,0 \\
\hline UW & 5 & 9 & 1 & 211 & 100 & $\mathrm{~m}$ & 7,4 & 195 & 37,8 & 27,8 & 307,8 & 21,9 & 130,2 \\
\hline UW & 5 & 9 & 1 & 211 & 130 & $\mathrm{~m}$ & 7,4 & 122 & 48,7 & 32,4 & 376,9 & 22,8 & 157,1 \\
\hline UW & 5 & 9 & 1 & 511 & 30 & s & 9,7 & 1253 & 16,6 & 9,0 & 111,4 & 27,1 & 66,8 \\
\hline UW & 5 & 9 & 1 & 511 & 60 & s & 9,7 & 470 & 29,3 & 19,5 & 277,6 & 31,8 & 102,8 \\
\hline UW & 5 & 9 & 1 & 511 & 90 & s & 9,7 & 304 & 38,2 & 26,7 & 403,9 & 34,9 & 125,8 \\
\hline UW & 6 & 4 & 1 & 110 & 35 & g & 6,8 & 1140 & 13,3 & 13,3 & 102,1 & 15,7 & 43,2 \\
\hline UW & 6 & 4 & 1 & 110 & 65 & g & 6,8 & 269 & 28,8 & 20,8 & 194,7 & 17,5 & 75,5 \\
\hline UW & 6 & 4 & 1 & 110 & 95 & g & 6,8 & 155 & 39,4 & 25,4 & 254,9 & 18,9 & 99,9 \\
\hline
\end{tabular}

Fortsetzung auf nächster Seite. 
Tabelle A.22

\begin{tabular}{|c|c|c|c|c|c|c|c|c|c|c|c|c|c|}
\hline WBZ & SOGR & WHZ & $\mathrm{NZ}$ & Art & Alt & $\mathrm{BG}$ & LKL & $\mathrm{N}$ & $\mathrm{dg}$ & hg & $\mathrm{V}$ & G & $\mathrm{CV}$ \\
\hline UW & 6 & 4 & 1 & 110 & 125 & $\mathrm{~g}$ & 6,8 & 95 & 51,9 & 28,7 & 306,6 & 20,1 & 120,5 \\
\hline UW & 6 & 4 & 1 & 110 & 155 & $\mathrm{~g}$ & 6,8 & 76 & 61,3 & 31,4 & 371,7 & 22,4 & 147,1 \\
\hline UW & 6 & 4 & 1 & 110 & 185 & $\mathrm{~g}$ & 6,8 & 71 & 69,0 & 33,6 & 468,6 & 26,5 & 186,6 \\
\hline UW & 6 & 4 & 1 & 211 & 40 & g & 8,6 & 1288 & 13,4 & 14,4 & 124,5 & 18,3 & 57,3 \\
\hline UW & 6 & 4 & 1 & 211 & 70 & $\mathrm{~g}$ & 8,6 & 298 & 28,6 & 24,7 & 237,5 & 19,1 & 101,5 \\
\hline UW & 6 & 4 & 1 & 211 & 100 & $\mathrm{~g}$ & 8,6 & 164 & 41,1 & 31,3 & 348,2 & 21,8 & 145,8 \\
\hline UW & 6 & 4 & 1 & 211 & 130 & $\mathrm{~g}$ & 8,6 & 106 & 53,2 & 36,1 & 438,8 & 23,6 & 180,7 \\
\hline UW & 6 & 4 & 1 & 511 & 30 & $\mathrm{~g}$ & 12,1 & 1120 & 18,4 & 12,6 & 175,8 & 29,9 & 84,0 \\
\hline UW & 6 & 4 & 1 & 511 & 60 & $\mathrm{~g}$ & 12,1 & 410 & 33,1 & 24,0 & 376,4 & 35,3 & 123,3 \\
\hline UW & 6 & 4 & 1 & 511 & 90 & $\mathrm{~g}$ & 12,1 & 263 & 43,1 & 31,7 & 521,3 & 38,4 & 147,5 \\
\hline UW & 6 & 4 & 2 & 110 & 35 & $\mathrm{~g}$ & 6,5 & 1141 & 13,0 & 12,9 & 95,3 & 15,2 & 40,5 \\
\hline UW & 6 & 4 & 2 & 110 & 65 & $\mathrm{~g}$ & 6,5 & 273 & 28,3 & 20,3 & 186,7 & 17,2 & 72,2 \\
\hline UW & 6 & 4 & 2 & 110 & 95 & g & 6,5 & 155 & 39,0 & 24,9 & 245,2 & 18,5 & 95,7 \\
\hline UW & 6 & 4 & 2 & 110 & 125 & $\mathrm{~g}$ & 6,5 & 93 & 52,0 & 28,2 & 295,0 & 19,7 & 115,6 \\
\hline UW & 6 & 4 & 2 & 110 & 155 & $\mathrm{~g}$ & 6,5 & 81 & 59,2 & 30,8 & 362,8 & 22,3 & 143,3 \\
\hline UW & 6 & 4 & 2 & 110 & 185 & g & 6,5 & 71 & 67,9 & 33,0 & 446,9 & 25,7 & 177,4 \\
\hline UW & 6 & 4 & 2 & 211 & 40 & $\mathrm{~g}$ & 8,2 & 1363 & 13,1 & 13,4 & 115,1 & 18,3 & 53,2 \\
\hline UW & 6 & 4 & 2 & 211 & 70 & $\mathrm{~g}$ & 8,2 & 317 & 27,9 & 23,6 & 230,0 & 19,4 & 98,5 \\
\hline UW & 6 & 4 & 2 & 211 & 100 & $\mathrm{~g}$ & 8,2 & 161 & 41,3 & 30,1 & 330,9 & 21,6 & 139,0 \\
\hline UW & 6 & 4 & 2 & 211 & 130 & $\mathrm{~g}$ & 8,2 & 107 & 51,9 & 34,8 & 405,2 & 22,6 & 167,4 \\
\hline UW & 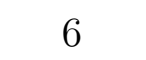 & 4 & 2 & 511 & 30 & $\mathrm{~g}$ & 11,6 & 1136 & 18,3 & 12,1 & 168,5 & 30,0 & 82,7 \\
\hline UW & $c$ & 4 & 2 & 511 & 60 & $\mathrm{~g}$ & 11,6 & 420 & 32,4 & 23,4 & 360,1 & 34,5 & 119,6 \\
\hline UW & 6 & 4 & 2 & 511 & 90 & $\mathrm{~g}$ & 11,6 & 261 & 42,8 & 31,0 & 498,8 & 37,6 & 143,2 \\
\hline UW & 6 & 5 & 2 & 110 & 35 & $\mathrm{~m}$ & 6,0 & 1163 & 12,8 & 12,2 & 87,0 & 14,9 & 37,2 \\
\hline UW & 6 & 5 & 2 & 110 & 65 & $\mathrm{~m}$ & 6,0 & 279 & 27,3 & 19,5 & 170,3 & 16,3 & 65,6 \\
\hline UW & 6 & 5 & 2 & 110 & 95 & $\mathrm{~m}$ & 6,0 & 159 & 38,4 & 24,0 & 236,6 & 18,4 & 91,8 \\
\hline UW & 6 & 5 & 2 & 110 & 125 & $\mathrm{~m}$ & 6,0 & 106 & 48,7 & 27,3 & 286,9 & 19,7 & 112,1 \\
\hline UW & $c$ & 5 & 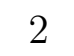 & 110 & 155 & $\mathrm{~m}$ & 6,0 & 79 & 59,5 & 29,8 & 348,4 & 22,0 & 136,5 \\
\hline UW & 6 & 5 & 2 & 110 & 185 & $\mathrm{~m}$ & 6,0 & 73 & 64,7 & 32,0 & 406,0 & 24,0 & 160,6 \\
\hline UW & 6 & 5 & 2 & 211 & 40 & $\mathrm{~m}$ & 8,1 & 1365 & 13,0 & 13,2 & 113,0 & 18,1 & 52,2 \\
\hline UW & 6 & 5 & 2 & 211 & 70 & $\mathrm{~m}$ & 8,1 & 313 & 27,8 & 23,4 & 222,9 & 18,9 & 95,5 \\
\hline UW & 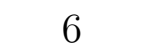 & 5 & 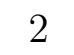 & 211 & 100 & $\mathrm{~m}$ & 8,1 & 175 & 39,8 & 29,8 & 330,8 & 21,8 & 139,1 \\
\hline UW & 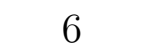 & 5 & 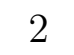 & 211 & 130 & $\mathrm{~m}$ & 8,1 & 113 & 50,8 & 34,5 & 405,1 & 22,9 & 167,7 \\
\hline UW & 6 & 5 & 2 & 511 & 30 & $\mathrm{~m}$ & 11,1 & 1182 & 17,7 & 11,3 & 151,8 & 29,0 & 78,1 \\
\hline UW & 6 & 5 & 2 & 511 & 60 & $\mathrm{~m}$ & 11,1 & 443 & 31,4 & 22,3 & 341,5 & 34,3 & 116,7 \\
\hline UW & 6 & 5 & 2 & 511 & 90 & $\mathrm{~m}$ & 11,1 & 284 & 41,1 & 29,8 & 483,3 & 37,7 & 141,5 \\
\hline
\end{tabular}




\section{A.5 Entwicklung des Kohlenstoffvorrates der lebenden Biomasse}

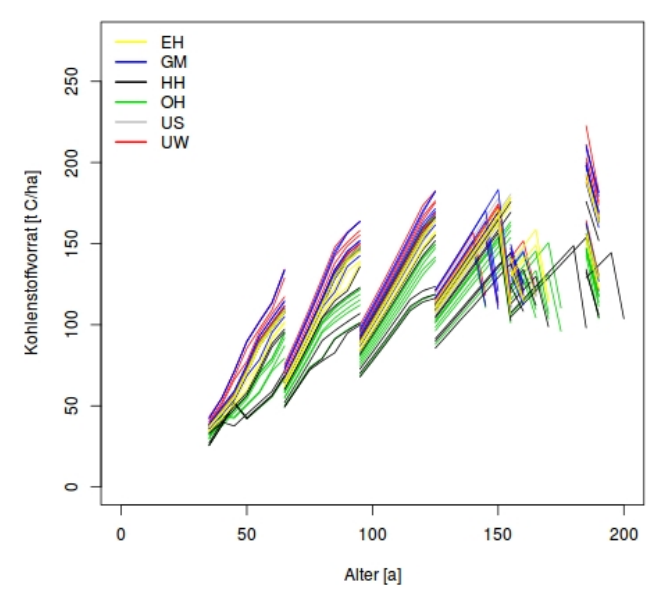

(a)

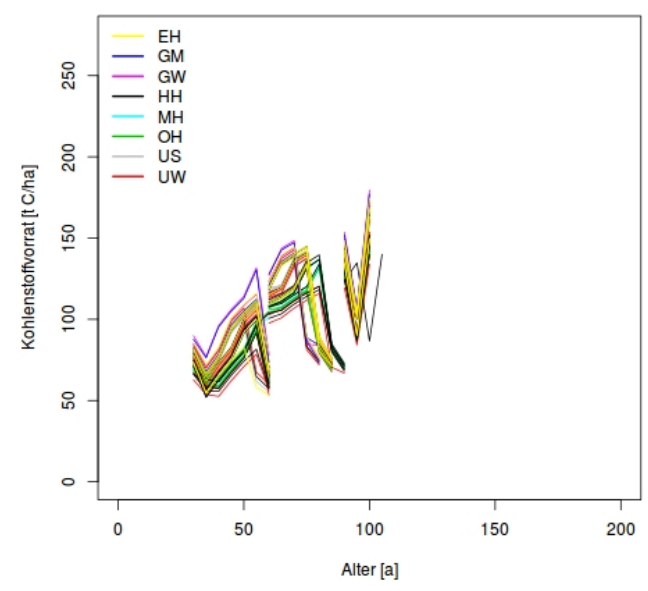

(c)

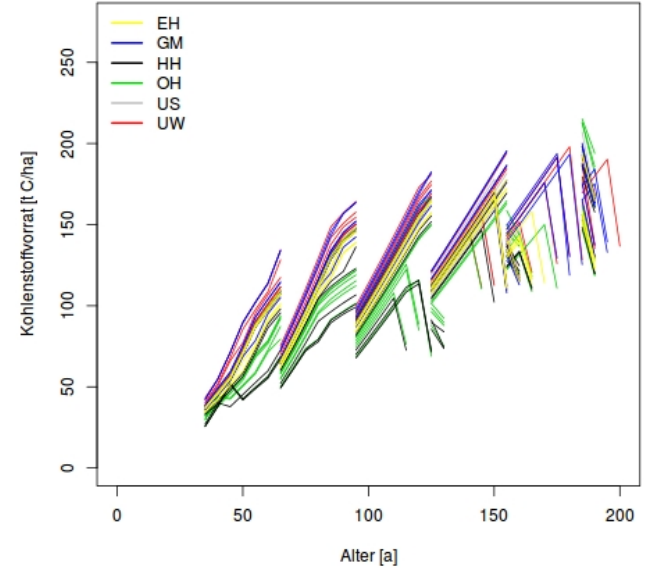

(b)

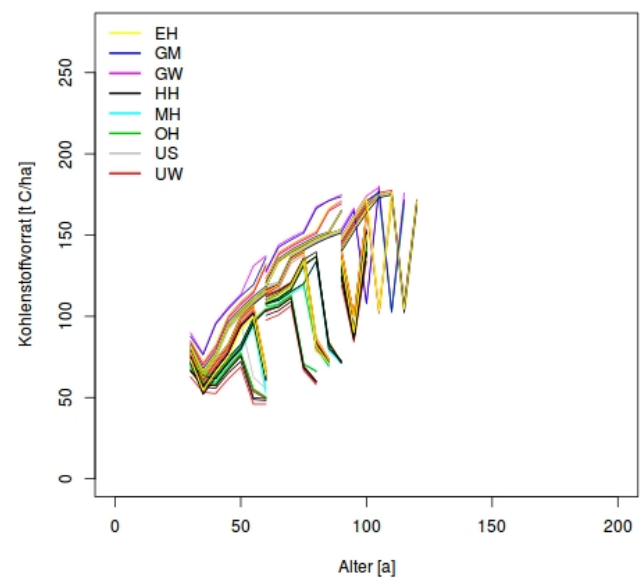

(d)

Abbildung A.16: Entwicklung des Kohlenstoffvorrates $\left[\mathrm{t} \mathrm{Cha}^{-1}\right]$ der lebenden Baumbiomasse von Eichen- $(\mathrm{a}, \mathrm{b})$ und Fichtenreinbeständen $(\mathrm{c}, \mathrm{d})$ bei naturnaher $(\mathrm{a}, \mathrm{c})$ und kohlenstofforientierter (b, d) Behandlung getrennt nach Wuchsbezirken (EH $=$ Ems-Hase-Hunte-Geest, GM = Geest-Mitte, GW = Göttinger Wald, $\mathrm{HH}$

$=$ Hohe Heide, $\mathrm{MH}=$ Montaner Mittel-und Oberharz, $\mathrm{OH}=$ Ost-Heide, US $=$ Unterer Solling, $\mathrm{UW}=$ Unteres Weser-Leine-Bergland). 


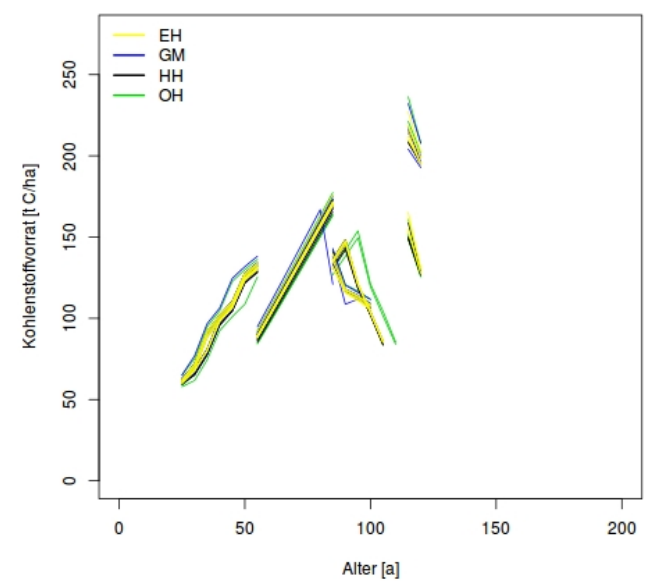

(a)

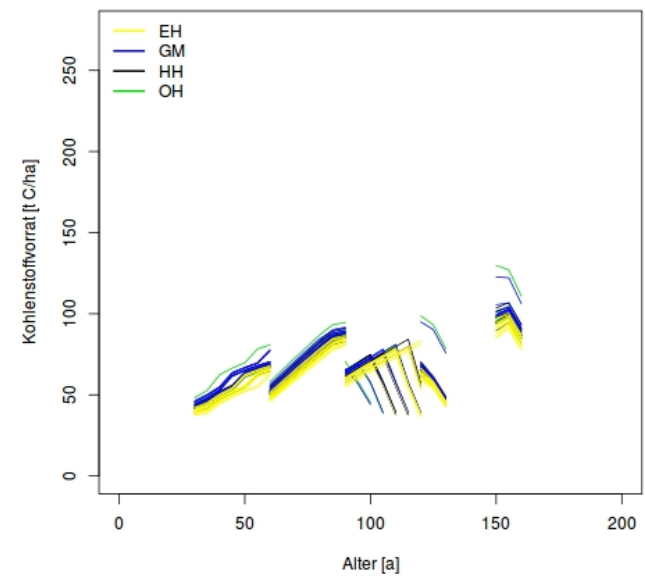

(c)

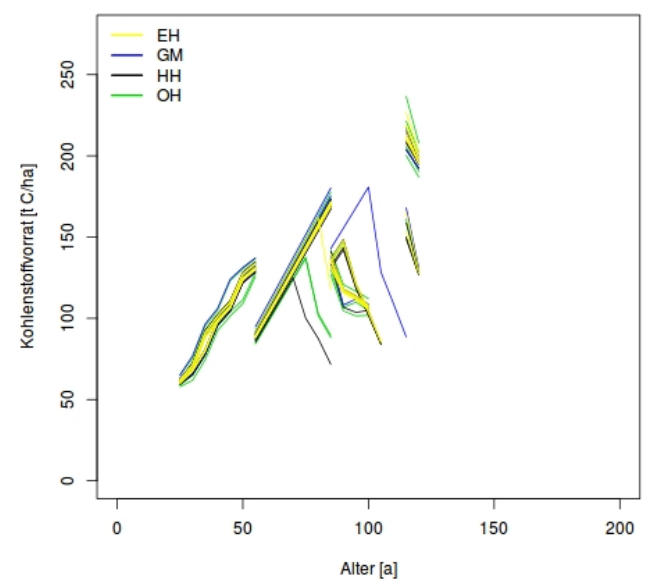

(b)

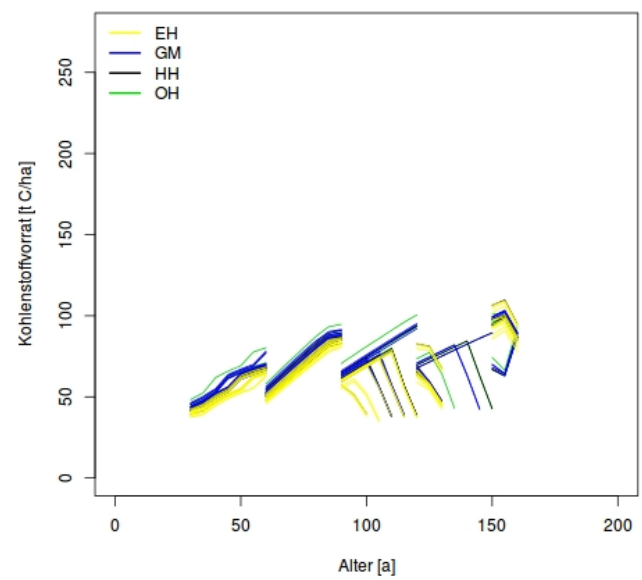

(d)

Abbildung A.17: Entwicklung des Kohlenstoffvorrates [ $\left.\mathrm{t} \mathrm{Cha}^{-1}\right]$ der lebenden Baumbiomasse von Douglasien- $(\mathrm{a}, \mathrm{b})$ und Kiefernreinbeständen $(\mathrm{c}, \mathrm{d})$ bei naturnaher $(\mathrm{a}, \mathrm{c})$ und kohlenstofforientierter (b, d) Behandlung getrennt nach Wuchsbezirken $(\mathrm{EH}=$ Ems-Hase-Hunte-Geest, GM = Geest-Mitte, $\mathrm{HH}=$ Hohe Heide, $\mathrm{OH}$ $=$ Ost-Heide). 


\section{A.6 Entwicklung des Kohlenstoffvorrates der toten Baumbiomasse}

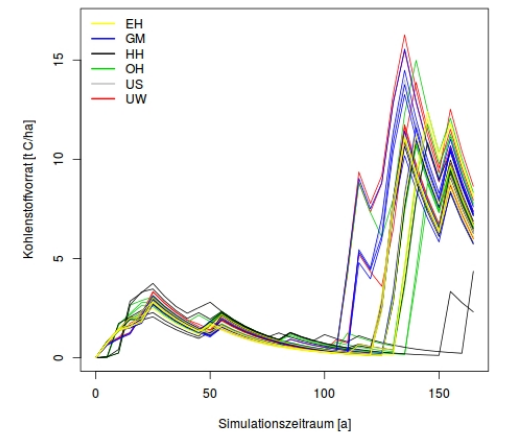

(a)

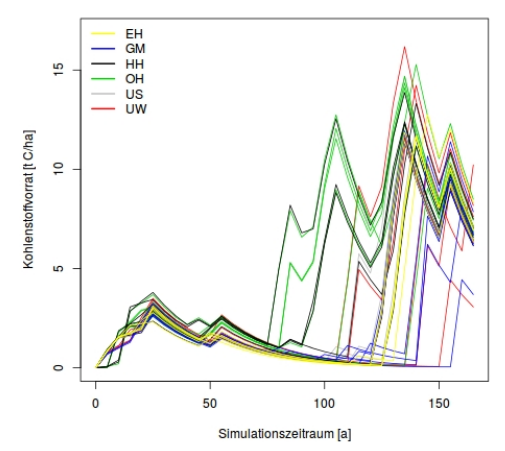

(d)

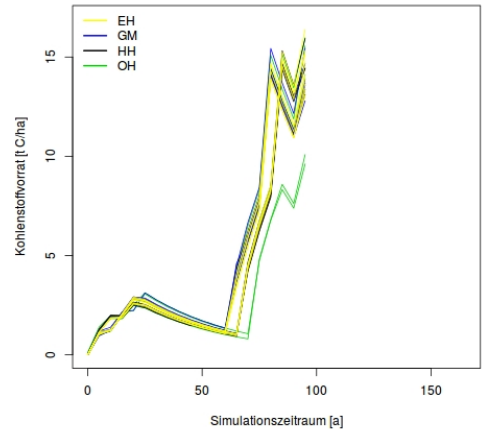

(b)

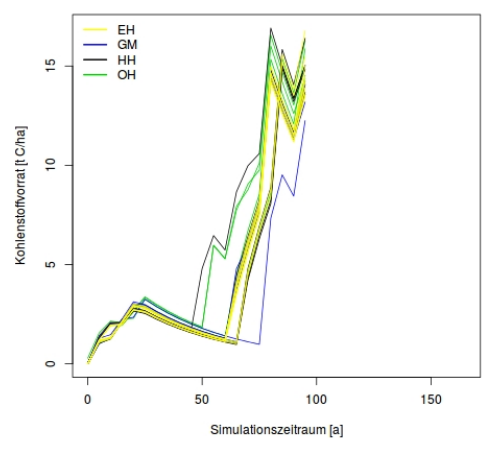

(e)

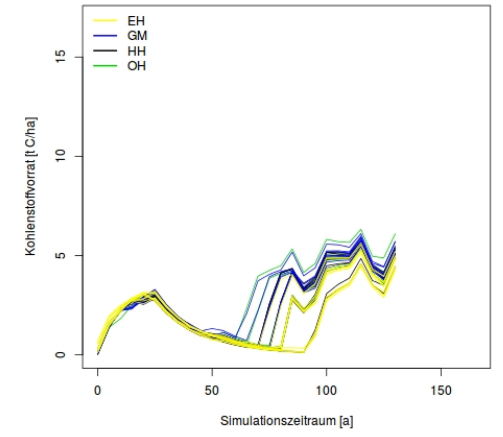

(c)

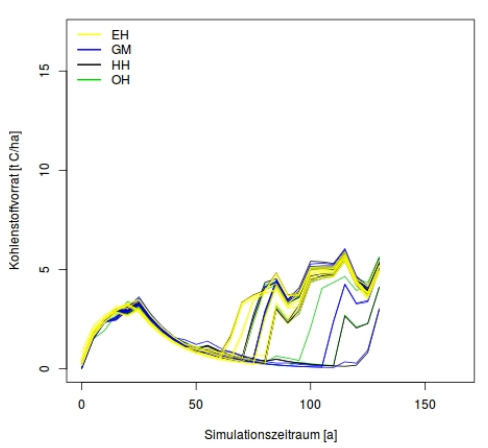

(f)

Abbildung A.18: Entwicklung des Kohlenstoffvorrates $\left[\mathrm{t} \mathrm{Cha}^{-1}\right]$ der toten Baumbiomasse in Reinbeständen aus Eiche (a, d), Douglasie (b, e) und Kiefer (c, f) getrennt nach Wuchsbezirken $(\mathrm{EH}=$ Ems-Hase-Hunte-Geest, GM = Geest-Mitte, GW $=$ Göttinger Wald, $\mathrm{HH}=$ Hohe Heide, $\mathrm{MH}=$ Montaner Mittel-und Oberharz, $\mathrm{OH}=$ Ost-Heide, US = Unterer Solling, UW = Unteres Weser-LeineBergland) bei naturnaher ( $a, b, c)$ und kohlenstofforientierter (d, e, f) Behandlung. 


\section{A.7 Kohlenstoffspeicherraten}

Tabelle A.23: Kohlenstoffbindungsraten $\left[\mathrm{t} \mathrm{Cha}^{-1} \mathrm{a}^{-1}\right]$ der einzelnen Speicher (LB = lebende Baumbiomasse, $\mathrm{T}=$ tote Baumbiomasse, $\mathrm{HP}=$ Holzprodukte, $\mathrm{SHP}=$ Substitution durch Holzprodukte) getrennt nach Wuchsbezirk (WBZ, EH = Ems-HaseHunte-Geest, GM = Geest-Mitte, GW = Göttinger Wald, HH = Hohe Heide, $\mathrm{MH}=$ Montaner Mittel-und Oberharz, $\mathrm{OH}=$ Ost-Heide, US $=$ Unterer Solling, $\mathrm{UW}=$ Unteres Weser-Leine-Bergland), Standortstypenobergruppe (SOGR, $1=$ basenarme Silikatgesteine, $2=$ basenreiche Silikatgesteine, $3=$ Flugsande, $4=$ Geschiebelehme, $5=$ Kalkgesteine, $6=$ Lösse, $7=$ Sande, $8=$ Sande im Grundwasserbereich, 9 = Sandlösse), Wasserhaushalts- (WHZ, 1 bis 3 = stark, mäßig, schwach grundwasserbeeinflusst, $4=$ nachhaltig frisch, $5=$ frisch, $6=$ mäßig frisch bis frisch, $7=$ mäßig frisch, $8=$ sommertrocken, $9=$ trocken) und Nährstoffgruppe (NZ, $1=$ sehr gut, $2=$ ziemlich gut, $3=$ mäßig, $4=$ schwach) sowie Art $(110=$ Eiche, $211=$ Buche, $511=$ Fichte, $611=$ Douglasie, $711=$ Kiefer $)$ und waldbauliche Behandlung (Var, co $=$ kohlenstofforientiert, $\mathrm{nn}=$ naturnah).

\begin{tabular}{ccccccccccc}
\hline WBZ & SOGR & WHZ & NZ & Art & Var & LB & T & HP & SHP & Gesamt \\
\hline EH & 3 & 3 & 3 & 110 & co & 0,671 & 0,042 & 0,799 & 5,508 & 7,019 \\
EH & 3 & 3 & 3 & 110 & nn & 0,676 & 0,040 & 0,783 & 5,470 & 6,969 \\
EH & 3 & 3 & 3 & 711 & co & 0,504 & 0,038 & 1,031 & 10,284 & 11,858 \\
EH & 3 & 3 & 3 & 711 & nn & 0,500 & 0,034 & 0,901 & 6,387 & 7,823 \\
EH & 3 & 3 & 4 & 611 & co & 1,654 & 0,152 & 3,168 & 20,408 & 25,381 \\
EH & 3 & 3 & 4 & 611 & nn & 1,657 & 0,147 & 3,123 & 20,324 & 25,252 \\
EH & 3 & 3 & 4 & 711 & co & 0,487 & 0,037 & 0,993 & 10,098 & 11,615 \\
EH & 3 & 3 & 4 & 711 & nn & 0,488 & 0,033 & 0,871 & 6,369 & 7,761 \\
EH & 3 & 9 & 4 & 511 & co & 1,489 & 0,037 & 2,179 & 23,475 & 27,180 \\
EH & 3 & 9 & 4 & 511 & nn & 1,489 & 0,037 & 2,222 & 26,970 & 30,718 \\
EH & 3 & 9 & 4 & 611 & co & 1,629 & 0,147 & 3,076 & 20,039 & 24,890 \\
EH & 3 & 9 & 4 & 611 & nn & 1,068 & 0,160 & 3,301 & 19,567 & 24,096 \\
EH & 3 & 9 & 4 & 711 & co & 0,572 & 0,038 & 0,998 & 11,099 & 12,707 \\
EH & 3 & 9 & 4 & 711 & nn & 0,488 & 0,033 & 0,866 & 6,395 & 7,783 \\
EH & 4 & 5 & 2 & 110 & co & 0,672 & 0,042 & 0,776 & 5,493 & 6,983 \\
EH & 4 & 5 & 2 & 110 & nn & 0,863 & 0,036 & 0,713 & 5,625 & 7,236 \\
EH & 4 & 5 & 2 & 211 & co & 1,189 & 0,076 & 0,812 & 3,890 & 5,966 \\
EH & 4 & 5 & 2 & 211 & nn & 1,250 & 0,045 & 1,886 & 10,196 & 13,377 \\
EH & 4 & 5 & 2 & 511 & co & 1,554 & 0,063 & 2,957 & 17,387 & 21,961 \\
EH & 4 & 5 & 2 & 511 & nn & 1,687 & 0,040 & 2,573 & 28,351 & 32,652 \\
EH & 4 & 5 & 2 & 611 & co & 1,657 & 0,153 & 3,200 & 20,767 & 25,776 \\
EH & 4 & 5 & 2 & 611 & nn & 1,661 & 0,148 & 3,198 & 20,876 & 25,883 \\
EH & 4 & 5 & 2 & 711 & co & 0,531 & 0,040 & 1,067 & 9,673 & 11,310 \\
\hline Fortstzin
\end{tabular}

Fortsetzung auf nächster Seite. 
Tabelle A.23

\begin{tabular}{|c|c|c|c|c|c|c|c|c|c|c|}
\hline WBZ & SOGR & WHZ & $\mathrm{NZ}$ & Art & Var & LB & $\mathrm{T}$ & HP & SHP & Gesamt \\
\hline EH & 4 & 5 & 2 & 711 & $\mathrm{nn}$ & 0,535 & 0,037 & 1,013 & 8,302 & 9,887 \\
\hline EH & 4 & 5 & 3 & 110 & co & 0,865 & 0,039 & 0,730 & 5,698 & 7,333 \\
\hline EH & 4 & 5 & 3 & 110 & $\mathrm{nn}$ & 0,854 & 0,038 & 0,721 & 5,668 & 7,282 \\
\hline $\mathrm{EH}$ & 4 & 5 & 3 & 511 & co & 1,424 & 0,086 & 1,729 & 12,694 & 15,933 \\
\hline $\mathrm{EH}$ & 4 & 5 & 3 & 511 & $\mathrm{nn}$ & 1,675 & 0,039 & 2,506 & 27,494 & 31,713 \\
\hline EH & 4 & 5 & 3 & 611 & co & 1,061 & 0,163 & 3,258 & 19,191 & 23,673 \\
\hline $\mathrm{EH}$ & 4 & 5 & 3 & 611 & $\mathrm{nn}$ & 1,060 & 0,159 & 3,275 & 19,207 & 23,701 \\
\hline $\mathrm{EH}$ & 4 & 5 & 3 & 711 & co & 0,539 & 0,042 & 1,094 & 9,994 & 11,669 \\
\hline $\mathrm{EH}$ & 4 & 5 & 3 & 711 & $\mathrm{nn}$ & 0,539 & 0,040 & 1,090 & 9,864 & 11,534 \\
\hline EH & 4 & 6 & 3 & 110 & co & 0,649 & 0,050 & 0,832 & 5,401 & 6,932 \\
\hline EH & 4 & 6 & 3 & 110 & $\mathrm{nn}$ & 0,647 & 0,049 & 0,833 & 5,671 & 7,201 \\
\hline EH & 4 & 6 & 3 & 611 & co & 1,634 & 0,143 & 3,060 & 19,332 & 24,169 \\
\hline EH & 4 & 6 & 3 & 611 & $\mathrm{nn}$ & 1,625 & 0,141 & 3,108 & 19,448 & 24,322 \\
\hline EH & 4 & 6 & 3 & 711 & co & 0,519 & 0,040 & 1,018 & 8,530 & 10,106 \\
\hline EH & 4 & 6 & 3 & 711 & $\mathrm{nn}$ & 0,522 & 0,038 & 1,016 & 8,375 & 9,951 \\
\hline $\mathrm{EH}$ & 7 & 6 & 3 & 110 & co & 0,879 & 0,038 & 0,730 & 5,861 & 7,508 \\
\hline $\mathrm{EH}$ & 7 & 6 & 3 & 110 & $\mathrm{nn}$ & 0,872 & 0,037 & 0,737 & 5,768 & 7,413 \\
\hline EH & 7 & 6 & 3 & 211 & co & 1,145 & 0,035 & 1,452 & 5,991 & 8,623 \\
\hline EH & 7 & 6 & 3 & 211 & $\mathrm{nn}$ & 1,151 & 0,034 & 1,469 & 5,634 & 8,289 \\
\hline EH & 7 & 6 & 3 & 511 & co & 1,598 & 0,062 & 2,969 & 17,697 & 22,326 \\
\hline $\mathrm{EH}$ & 7 & 6 & 3 & 511 & $\mathrm{nn}$ & 1,744 & 0,049 & 3,199 & 36,756 & 41,748 \\
\hline $\mathrm{EH}$ & 7 & 6 & 3 & 611 & co & 1,632 & 0,142 & 3,084 & 19,449 & 24,306 \\
\hline EH & 7 & 6 & 3 & 611 & $\mathrm{nn}$ & 1,632 & 0,138 & 3,073 & 19,332 & 24,175 \\
\hline $\mathrm{EH}$ & 7 & 6 & 3 & 711 & co & 0,544 & 0,042 & 1,125 & 10,515 & 12,226 \\
\hline $\mathrm{EH}$ & 7 & 6 & 3 & 711 & $\mathrm{nn}$ & 0,545 & 0,040 & 1,114 & 10,635 & 12,334 \\
\hline $\mathrm{EH}$ & 7 & 7 & 3 & 110 & co & 0,644 & 0,043 & 0,768 & 5,443 & 6,898 \\
\hline EH & 7 & 7 & 3 & 110 & $\mathrm{nn}$ & 0,641 & 0,050 & 0,803 & 5,239 & 6,734 \\
\hline $\mathrm{EH}$ & 7 & 7 & 3 & 511 & co & 1,629 & 0,038 & 2,396 & 25,998 & 30,061 \\
\hline $\mathrm{EH}$ & 7 & 7 & 3 & 511 & $\mathrm{nn}$ & 1,632 & 0,037 & 2,378 & 25,856 & 29,902 \\
\hline $\mathrm{EH}$ & 7 & 7 & 3 & 611 & co & 1,686 & 0,159 & 3,208 & 21,043 & 26,096 \\
\hline EH & 7 & 7 & 3 & 611 & $\mathrm{nn}$ & 1,687 & 0,156 & 3,277 & 21,196 & 26,316 \\
\hline $\mathrm{EH}$ & 7 & 7 & 3 & 711 & co & 0,516 & 0,040 & 1,014 & 8,398 & 9,969 \\
\hline $\mathrm{EH}$ & 7 & 7 & 3 & 711 & $\mathrm{nn}$ & 0,516 & 0,038 & 1,019 & 8,339 & 9,912 \\
\hline $\mathrm{EH}$ & 7 & 7 & 4 & 611 & co & 1,630 & 0,146 & 3,051 & 19,331 & 24,158 \\
\hline $\mathrm{EH}$ & 7 & 7 & 4 & 611 & $\mathrm{nn}$ & 1,621 & 0,144 & 3,098 & 19,478 & 24,342 \\
\hline $\mathrm{EH}$ & 7 & 7 & 4 & 711 & co & 0,591 & 0,040 & 1,044 & 11,270 & 12,944 \\
\hline $\mathrm{EH}$ & 7 & 7 & 4 & 711 & $\mathrm{nn}$ & 0,508 & 0,037 & 0,979 & 8,088 & 9,613 \\
\hline EH & 7 & 9 & 3 & 611 & co & 1,083 & 0,177 & 3,468 & 20,493 & 25,220 \\
\hline
\end{tabular}

Fortsetzung auf nächster Seite. 
Tabelle A.23

\begin{tabular}{|c|c|c|c|c|c|c|c|c|c|c|}
\hline WBZ & SOGR & WHZ & $\mathrm{NZ}$ & Art & Var & LB & $\mathrm{T}$ & HP & SHP & Gesamt \\
\hline EH & 7 & 9 & 3 & 611 & $\mathrm{nn}$ & 1,085 & 0,173 & 3,428 & 20,528 & 25,214 \\
\hline EH & 7 & 9 & 3 & 711 & $\mathrm{co}$ & 0,532 & 0,040 & 1,025 & 8,371 & 9,967 \\
\hline $\mathrm{EH}$ & 7 & 9 & 3 & 711 & nn & 0,532 & 0,038 & 1,024 & 8,341 & 9,934 \\
\hline $\mathrm{EH}$ & 7 & 9 & 4 & 511 & $\mathrm{co}$ & 1,500 & 0,038 & 2,226 & 24,206 & 27,970 \\
\hline $\mathrm{EH}$ & 7 & 9 & 4 & 511 & $\mathrm{nn}$ & 1,497 & 0,037 & 2,220 & 23,990 & 27,743 \\
\hline $\mathrm{EH}$ & 7 & 9 & 4 & 611 & $\mathrm{co}$ & 1,658 & 0,150 & 3,079 & 20,082 & 24,969 \\
\hline $\mathrm{EH}$ & 7 & 9 & 4 & 611 & nn & 1,091 & 0,161 & 3,236 & 19,453 & 23,941 \\
\hline $\mathrm{EH}$ & 7 & 9 & 4 & 711 & $\mathrm{co}$ & 0,588 & 0,038 & 1,024 & 11,051 & 12,701 \\
\hline EH & 7 & 9 & 4 & 711 & $\mathrm{nn}$ & 0,506 & 0,035 & 0,894 & 6,229 & 7,663 \\
\hline GM & 4 & 5 & 2 & 110 & $\mathrm{co}$ & 0,874 & 0,040 & 0,733 & 5,909 & 7,556 \\
\hline GM & 4 & 5 & 2 & 110 & $\mathrm{nn}$ & 0,869 & 0,039 & 0,732 & 5,755 & 7,394 \\
\hline GM & 4 & 5 & 2 & 211 & co & 1,137 & 0,040 & 1,710 & 8,255 & 11,143 \\
\hline GM & 4 & 5 & 2 & 211 & $\mathrm{nn}$ & 1,139 & 0,039 & 1,695 & 8,246 & 11,119 \\
\hline GM & 4 & 5 & 2 & 511 & $\mathrm{co}$ & 1,494 & 0,086 & 1,791 & 14,975 & 18,346 \\
\hline GM & 4 & 5 & 2 & 511 & $\mathrm{nn}$ & 1,697 & 0,048 & 3,075 & 34,749 & 39,568 \\
\hline GM & 4 & 5 & 2 & 611 & $\mathrm{co}$ & 1,665 & 0,148 & 3,131 & 20,565 & 25,509 \\
\hline GM & 4 & 5 & 2 & 611 & $\mathrm{nn}$ & 1,666 & 0,144 & 3,143 & 20,518 & 25,470 \\
\hline GM & 4 & 5 & 2 & 711 & $\mathrm{co}$ & 0,545 & 0,023 & 0,609 & 3,531 & 4,708 \\
\hline GM & 4 & 5 & 2 & 711 & $\mathrm{nn}$ & 0,570 & 0,041 & 1,140 & 10,393 & 12,145 \\
\hline GM & 4 & 5 & 3 & 110 & $\mathrm{co}$ & 0,682 & 0,022 & 0,666 & 2,039 & 3,409 \\
\hline GM & 4 & 5 & 3 & 110 & $\mathrm{nn}$ & 0,891 & 0,046 & 0,837 & 8,892 & 10,666 \\
\hline GM & 4 & 5 & 3 & 711 & co & 0,562 & 0,043 & 1,125 & 10,491 & 12,220 \\
\hline GM & 4 & 5 & 3 & 711 & $\mathrm{nn}$ & 0,565 & 0,041 & 1,119 & 10,372 & 12,097 \\
\hline GM & 4 & 6 & 3 & 110 & $\mathrm{co}$ & 0,859 & 0,043 & 0,752 & 5,851 & 7,505 \\
\hline GM & 4 & 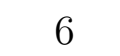 & 3 & 110 & nn & 0,859 & 0,045 & 0,781 & 7,221 & 8,906 \\
\hline GM & 4 & 6 & 3 & 211 & $\mathrm{co}$ & 1,095 & 0,034 & 1,422 & 5,527 & 8,079 \\
\hline GM & 4 & 6 & 3 & 211 & $\mathrm{nn}$ & 1,089 & 0,034 & 1,434 & 5,416 & 7,973 \\
\hline GM & 4 & 6 & 3 & 611 & co & 1,658 & 0,148 & 3,117 & 20,591 & 25,514 \\
\hline GM & 4 & 6 & 3 & 611 & $\mathrm{nn}$ & 1,660 & 0,143 & 3,123 & 20,530 & 25,456 \\
\hline GM & 4 & 6 & 3 & 711 & $\mathrm{co}$ & 0,558 & 0,042 & 1,107 & 10,054 & 11,762 \\
\hline GM & 4 & 6 & 3 & 711 & $\mathrm{nn}$ & 0,561 & 0,039 & 1,058 & 8,874 & 10,532 \\
\hline GM & 7 & 6 & 3 & 110 & $\mathrm{co}$ & 0,687 & 0,038 & 0,694 & 3,793 & 5,212 \\
\hline GM & 7 & 6 & 3 & 110 & $\mathrm{nn}$ & 0,894 & 0,044 & 0,812 & 7,746 & 9,496 \\
\hline GM & 7 & 6 & 3 & 211 & $\mathrm{co}$ & 1,126 & 0,033 & 1,403 & 5,292 & 7,854 \\
\hline GM & 7 & 6 & 3 & 211 & nn & 1,128 & 0,033 & 1,393 & 5,249 & 7,804 \\
\hline GM & 7 & 6 & 3 & 711 & $\mathrm{co}$ & 0,557 & 0,042 & 1,138 & 10,784 & 12,521 \\
\hline GM & 7 & 6 & 3 & 711 & nn & 0,558 & 0,041 & 1,140 & 11,004 & 12,742 \\
\hline GM & 7 & 7 & 3 & 110 & $\mathrm{co}$ & 0,858 & 0,039 & 0,728 & 5,719 & 7,344 \\
\hline
\end{tabular}

Fortsetzung auf nächster Seite. 
Tabelle A.23

\begin{tabular}{|c|c|c|c|c|c|c|c|c|c|c|}
\hline WBZ & SOGR & WHZ & $\mathrm{NZ}$ & Art & Var & LB & $\mathrm{T}$ & HP & SHP & Gesamt \\
\hline GM & 7 & 7 & 3 & 110 & $\mathrm{nn}$ & 0,667 & 0,044 & 0,797 & 5,630 & 7,137 \\
\hline GM & 7 & 7 & 3 & 211 & $\mathrm{co}$ & 1,066 & 0,048 & 0,528 & 3,777 & 5,419 \\
\hline GM & 7 & 7 & 3 & 211 & nn & 1,056 & 0,050 & 0,556 & 3,864 & 5,527 \\
\hline GM & 7 & 7 & 3 & 611 & $\mathrm{co}$ & 1,637 & 0,150 & 3,139 & 20,009 & 24,934 \\
\hline GM & 7 & 7 & 3 & 611 & $\mathrm{nn}$ & 1,635 & 0,146 & 3,152 & 20,034 & 24,968 \\
\hline GM & 7 & 7 & 3 & 711 & $\mathrm{co}$ & 0,553 & 0,042 & 1,119 & 10,842 & 12,556 \\
\hline GM & 7 & 7 & 3 & 711 & nn & 0,553 & 0,040 & 1,120 & 10,928 & 12,641 \\
\hline GM & 7 & 9 & 4 & 611 & $\mathrm{co}$ & 1,602 & 0,139 & 3,044 & 19,391 & 24,176 \\
\hline GM & 7 & 9 & 4 & 611 & $\mathrm{nn}$ & 1,607 & 0,135 & 3,047 & 19,389 & 24,178 \\
\hline GM & 7 & 9 & 4 & 711 & $\mathrm{co}$ & 0,533 & 0,039 & 1,022 & 8,596 & 10,190 \\
\hline GM & 7 & 9 & 4 & 711 & $\mathrm{nn}$ & 0,532 & 0,039 & 1,070 & 9,771 & 11,413 \\
\hline GM & 8 & 1 & 3 & 110 & co & 0,715 & 0,041 & 0,807 & 3,356 & 4,919 \\
\hline GM & 8 & 1 & 3 & 110 & $\mathrm{nn}$ & 0,955 & 0,038 & 0,750 & 6,107 & 7,849 \\
\hline GM & 8 & 1 & 3 & 711 & $\mathrm{co}$ & 0,559 & 0,039 & 0,893 & 5,677 & 7,167 \\
\hline GM & 8 & 1 & 3 & 711 & $\mathrm{nn}$ & 0,582 & 0,044 & 1,191 & 12,141 & 13,958 \\
\hline GM & 8 & 2 & 3 & 110 & $\mathrm{co}$ & 0,849 & 0,037 & 0,700 & 5,559 & 7,145 \\
\hline GM & 8 & 2 & 3 & 110 & $\mathrm{nn}$ & 0,842 & 0,035 & 0,703 & 5,396 & 6,976 \\
\hline GM & 8 & 2 & 3 & 711 & $\mathrm{co}$ & 0,562 & 0,038 & 0,894 & 5,558 & 7,052 \\
\hline GM & 8 & 2 & 3 & 711 & $\mathrm{nn}$ & 0,664 & 0,044 & 1,189 & 13,266 & 15,162 \\
\hline GM & 9 & 5 & 2 & 110 & $\mathrm{co}$ & 0,723 & 0,048 & 0,803 & 4,891 & 6,465 \\
\hline GM & 9 & 5 & 2 & 110 & $\mathrm{nn}$ & 0,944 & 0,044 & 0,829 & 7,815 & 9,632 \\
\hline GM & 9 & 5 & 2 & 211 & co & 1,146 & 0,035 & 1,471 & 5,587 & 8,239 \\
\hline GM & 9 & 5 & 2 & 211 & $\mathrm{nn}$ & 1,155 & 0,034 & 1,455 & 5,552 & 8,196 \\
\hline GM & 9 & 5 & 2 & 511 & $\mathrm{co}$ & 1,696 & 0,047 & 2,527 & 20,986 & 25,255 \\
\hline GM & 9 & 5 & 2 & 511 & nn & 1,771 & 0,051 & 3,333 & 37,549 & 42,704 \\
\hline GM & 9 & 5 & 2 & 611 & $\mathrm{co}$ & 1,093 & 0,129 & 3,019 & 15,253 & 19,495 \\
\hline GM & 9 & 5 & 2 & 611 & $\mathrm{nn}$ & 1,731 & 0,163 & 3,396 & 22,785 & 28,075 \\
\hline GW & 1 & 5 & 2 & 211 & co & 1,192 & 0,065 & 0,661 & 3,095 & 5,013 \\
\hline GW & 1 & 5 & 2 & 211 & $\mathrm{nn}$ & 1,247 & 0,046 & 1,904 & 11,653 & 14,850 \\
\hline GW & 1 & 5 & 2 & 511 & $\mathrm{co}$ & 1,530 & 0,086 & 1,756 & 14,939 & 18,311 \\
\hline GW & 1 & 5 & 2 & 511 & $\mathrm{nn}$ & 1,735 & 0,049 & 3,199 & 36,494 & 41,477 \\
\hline GW & 5 & 7 & 1 & 211 & $\mathrm{co}$ & 1,169 & 0,055 & 0,598 & 4,195 & 6,017 \\
\hline GW & 5 & 7 & 1 & 211 & $\mathrm{nn}$ & 1,179 & 0,036 & 1,477 & 5,420 & 8,112 \\
\hline GW & 5 & 7 & 1 & 511 & $\mathrm{co}$ & 1,717 & 0,046 & 2,527 & 19,869 & 24,159 \\
\hline GW & 5 & 7 & 1 & 511 & nn & 1,795 & 0,051 & 3,369 & 37,913 & 43,129 \\
\hline $\mathrm{HH}$ & 4 & 5 & 2 & 110 & $\mathrm{co}$ & 0,860 & 0,041 & 0,729 & 5,705 & 7,336 \\
\hline $\mathrm{HH}$ & 4 & 5 & 2 & 110 & nn & 0,859 & 0,040 & 0,728 & 5,691 & 7,318 \\
\hline $\mathrm{HH}$ & 4 & 5 & 2 & 211 & $\mathrm{co}$ & 1,090 & 0,034 & 1,427 & 5,291 & 7,842 \\
\hline
\end{tabular}

Fortsetzung auf nächster Seite. 
Tabelle A.23

\begin{tabular}{|c|c|c|c|c|c|c|c|c|c|c|}
\hline WBZ & SOGR & WHZ & $\mathrm{NZ}$ & Art & Var & LB & $\mathrm{T}$ & HP & SHP & Gesamt \\
\hline $\mathrm{HH}$ & 4 & 5 & 2 & 211 & nn & 1,075 & 0,034 & 1,380 & 5,229 & 7,717 \\
\hline HH & 4 & 5 & 2 & 511 & co & 1,423 & 0,086 & 1,696 & 12,597 & 15,801 \\
\hline $\mathrm{HH}$ & 4 & 5 & 2 & 511 & $\mathrm{nn}$ & 1,676 & 0,046 & 2,977 & 33,517 & 38,216 \\
\hline $\mathrm{HH}$ & 4 & 5 & 2 & 611 & $\mathrm{co}$ & 1,082 & 0,173 & 3,445 & 20,523 & 25,222 \\
\hline $\mathrm{HH}$ & 4 & 5 & 2 & 611 & nn & 1,077 & 0,168 & 3,428 & 20,432 & 25,106 \\
\hline $\mathrm{HH}$ & 4 & 5 & 2 & 711 & co & 0,542 & 0,023 & 0,607 & 3,693 & 4,865 \\
\hline $\mathrm{HH}$ & 4 & 5 & 2 & 711 & $\mathrm{nn}$ & 0,569 & 0,041 & 1,158 & 11,231 & 12,999 \\
\hline $\mathrm{HH}$ & 4 & 5 & 3 & 110 & $\mathrm{co}$ & 0,631 & 0,043 & 0,741 & 5,432 & 6,847 \\
\hline $\mathrm{HH}$ & 4 & 5 & 3 & 110 & $\mathrm{nn}$ & 0,637 & 0,042 & 0,774 & 5,524 & 6,976 \\
\hline HH & 4 & 5 & 3 & 511 & $\mathrm{co}$ & 1,400 & 0,085 & 1,698 & 11,927 & 15,110 \\
\hline HH & 4 & 5 & 3 & 511 & $\mathrm{nn}$ & 1,648 & 0,038 & 2,468 & 29,976 & 34,131 \\
\hline $\mathrm{HH}$ & 4 & 5 & 3 & 711 & $\mathrm{co}$ & 0,558 & 0,043 & 1,143 & 11,090 & 12,834 \\
\hline $\mathrm{HH}$ & 4 & 5 & 3 & 711 & nn & 0,560 & 0,041 & 1,145 & 11,088 & 12,834 \\
\hline HH & 4 & 6 & 3 & 110 & $\mathrm{co}$ & 0,631 & 0,045 & 0,805 & 6,918 & 8,400 \\
\hline HH & 4 & 6 & 3 & 110 & nn & 0,800 & 0,035 & 0,682 & 5,504 & 7,021 \\
\hline $\mathrm{HH}$ & 4 & 6 & 3 & 211 & co & 0,981 & 0,034 & 1,505 & 7,937 & 10,458 \\
\hline $\mathrm{HH}$ & 4 & 6 & 3 & 211 & $\mathrm{nn}$ & 0,945 & 0,048 & 0,479 & 2,230 & 3,702 \\
\hline HH & 4 & 6 & 3 & 511 & $\mathrm{co}$ & 1,452 & 0,036 & 2,158 & 21,214 & 24,860 \\
\hline HH & 4 & 6 & 3 & 511 & $\mathrm{nn}$ & 1,452 & 0,035 & 2,150 & 21,145 & 24,782 \\
\hline $\mathrm{HH}$ & 4 & 6 & 3 & 611 & $\mathrm{co}$ & 1,625 & 0,144 & 3,054 & 20,028 & 24,850 \\
\hline $\mathrm{HH}$ & 4 & 6 & 3 & 611 & $\mathrm{nn}$ & 1,623 & 0,141 & 3,043 & 20,054 & 24,861 \\
\hline HH & 4 & 6 & 3 & 711 & $\mathrm{co}$ & 0,542 & 0,041 & 1,071 & 9,923 & 11,576 \\
\hline HH & 4 & 6 & 3 & 711 & nn & 0,542 & 0,039 & 1,082 & 9,915 & 11,578 \\
\hline $\mathrm{HH}$ & 7 & 5 & 3 & 211 & co & 0,976 & 0,034 & 1,497 & 6,900 & 9,407 \\
\hline $\mathrm{HH}$ & 7 & 5 & 3 & 211 & nn & 0,951 & 0,049 & 0,491 & 2,252 & 3,743 \\
\hline HH & 7 & 5 & 3 & 511 & co & 1,523 & 0,038 & 2,247 & 24,418 & 28,226 \\
\hline HH & 7 & 5 & 3 & 511 & nn & 1,521 & 0,037 & 2,255 & 24,743 & 28,556 \\
\hline $\mathrm{HH}$ & 7 & 5 & 3 & 711 & co & 0,558 & 0,032 & 0,770 & 4,609 & 5,970 \\
\hline HH & 7 & 5 & 3 & 711 & nn & 0,584 & 0,042 & 1,184 & 11,281 & 13,091 \\
\hline HH & 7 & 6 & 3 & 110 & co & 0,844 & 0,039 & 0,607 & 8,273 & 9,763 \\
\hline HH & 7 & 6 & 3 & 110 & nn & 0,550 & 0,014 & 0,428 & 2,574 & 3,566 \\
\hline $\mathrm{HH}$ & 7 & 6 & 3 & 211 & co & 0,913 & 0,024 & 1,163 & 4,093 & 6,193 \\
\hline $\mathrm{HH}$ & 7 & S & 3 & 211 & $\mathrm{nn}$ & 0,961 & 0,000 & 0,000 & 1,088 & 2,049 \\
\hline HH & 7 & 6 & 3 & 511 & co & 1,490 & 0,037 & 2,167 & 23,575 & 27,270 \\
\hline HH & 7 & 6 & 3 & 511 & $\mathrm{nn}$ & 1,488 & 0,036 & 2,195 & 23,585 & 27,304 \\
\hline $\mathrm{HH}$ & 7 & 6 & 3 & 611 & $\mathrm{co}$ & 1,059 & 0,158 & 3,225 & 18,733 & 23,175 \\
\hline $\mathrm{HH}$ & 7 & 6 & 3 & 611 & $\mathrm{nn}$ & 1,061 & 0,154 & 3,215 & 18,778 & 23,209 \\
\hline HH & 7 & 6 & 3 & 711 & $\mathrm{co}$ & 0,548 & 0,042 & 1,120 & 10,549 & 12,259 \\
\hline
\end{tabular}

Fortsetzung auf nächster Seite. 
Tabelle A.23

\begin{tabular}{|c|c|c|c|c|c|c|c|c|c|c|}
\hline WBZ & SOGR & WHZ & $\mathrm{NZ}$ & Art & Var & LB & $\mathrm{T}$ & HP & SHP & Gesamt \\
\hline $\mathrm{HH}$ & 7 & 6 & 3 & 711 & $\mathrm{nn}$ & 0,548 & 0,040 & 1,105 & 9,980 & 11,673 \\
\hline $\mathrm{HH}$ & 7 & 7 & 3 & 110 & co & 0,882 & 0,040 & 0,624 & 10,626 & 12,171 \\
\hline $\mathrm{HH}$ & 7 & 7 & 3 & 110 & $\mathrm{nn}$ & 0,560 & 0,043 & 0,713 & 5,327 & 6,643 \\
\hline $\mathrm{HH}$ & 7 & 7 & 3 & 211 & co & 0,891 & 0,023 & 1,122 & 3,843 & 5,878 \\
\hline $\mathrm{HH}$ & 7 & 7 & 3 & 211 & $\mathrm{nn}$ & 0,945 & 0,001 & 0,002 & 0,951 & 1,899 \\
\hline $\mathrm{HH}$ & 7 & 7 & 3 & 511 & co & 1,470 & 0,037 & 2,192 & 21,576 & 25,275 \\
\hline $\mathrm{HH}$ & 7 & 7 & 3 & 511 & $\mathrm{nn}$ & 1,466 & 0,036 & 2,192 & 21,536 & 25,230 \\
\hline $\mathrm{HH}$ & 7 & 7 & 3 & 611 & co & 1,056 & 0,156 & 3,163 & 18,419 & 22,793 \\
\hline $\mathrm{HH}$ & 7 & 7 & 3 & 611 & $\mathrm{nn}$ & 1,060 & 0,152 & 3,161 & 18,340 & 22,713 \\
\hline $\mathrm{HH}$ & 7 & 7 & 3 & 711 & co & 0,543 & 0,041 & 1,048 & 8,741 & 10,373 \\
\hline $\mathrm{HH}$ & 7 & 7 & 3 & 711 & $\mathrm{nn}$ & 0,544 & 0,038 & 0,975 & 6,946 & 8,502 \\
\hline $\mathrm{HH}$ & 7 & 7 & 4 & 511 & co & 1,398 & 0,043 & 2,385 & 28,760 & 32,585 \\
\hline $\mathrm{HH}$ & 7 & 7 & 4 & 511 & $\mathrm{nn}$ & 1,399 & 0,033 & 1,987 & 19,578 & 22,998 \\
\hline $\mathrm{HH}$ & 7 & 7 & 4 & 711 & co & 0,531 & 0,040 & 1,028 & 8,459 & 10,058 \\
\hline $\mathrm{HH}$ & 7 & 7 & 4 & 711 & $\mathrm{nn}$ & 0,533 & 0,038 & 1,028 & 8,486 & 10,085 \\
\hline $\mathrm{HH}$ & 7 & 9 & 3 & 611 & co & 1,625 & 0,143 & 2,976 & 19,677 & 24,421 \\
\hline $\mathrm{HH}$ & 7 & 0 & 3 & 611 & $\mathrm{nn}$ & 1,629 & 0,138 & 3,038 & 19,617 & 24,422 \\
\hline $\mathrm{HH}$ & 7 & 9 & 3 & 711 & co & 0,539 & 0,042 & 1,046 & 8,741 & 10,368 \\
\hline $\mathrm{HH}$ & 7 & 9 & 3 & 711 & $\mathrm{nn}$ & 0,541 & 0,040 & 1,053 & 8,764 & 10,398 \\
\hline $\mathrm{HH}$ & 7 & 9 & 4 & 110 & co & 0,830 & 0,037 & 0,602 & 8,078 & 9,548 \\
\hline $\mathrm{HH}$ & 7 & 0 & 4 & 110 & $\mathrm{nn}$ & 0,519 & 0,026 & 0,605 & 2,147 & 3,297 \\
\hline $\mathrm{HH}$ & 7 & 9 & 4 & 211 & co & 0,798 & 0,025 & 1,052 & 3,658 & 5,533 \\
\hline $\mathrm{HH}$ & 7 & 9 & 4 & 211 & $\mathrm{nn}$ & 1,232 & 0,001 & 0,001 & 0,928 & 2,162 \\
\hline $\mathrm{HH}$ & 7 & 9 & 4 & 511 & co & 1,386 & 0,041 & 2,329 & 27,764 & 31,521 \\
\hline $\mathrm{HH}$ & 7 & 9 & 4 & 511 & $\mathrm{nn}$ & 1,333 & 0,043 & 2,172 & 18,312 & 21,861 \\
\hline $\mathrm{HH}$ & 7 & 9 & 4 & 611 & co & 1,604 & 0,158 & 3,091 & 25,019 & 29,872 \\
\hline $\mathrm{HH}$ & 7 & 9 & 4 & 611 & $\mathrm{nn}$ & 1,054 & 0,152 & 3,151 & 18,471 & 22,827 \\
\hline $\mathrm{HH}$ & 7 & 0 & 4 & 711 & co & 0,540 & 0,041 & 1,084 & 9,903 & 11,568 \\
\hline $\mathrm{HH}$ & 7 & 9 & 4 & 711 & $\mathrm{nn}$ & 0,541 & 0,039 & 1,075 & 9,908 & 11,564 \\
\hline $\mathrm{HH}$ & 8 & 3 & 4 & 511 & co & 1,519 & 0,039 & 2,351 & 23,407 & 27,315 \\
\hline $\mathrm{HH}$ & 8 & 3 & 4 & 511 & $\mathrm{nn}$ & 1,516 & 0,037 & 2,299 & 22,900 & 26,752 \\
\hline $\mathrm{HH}$ & 8 & 3 & 4 & 711 & co & 0,593 & 0,039 & 1,029 & 11,151 & 12,812 \\
\hline $\mathrm{HH}$ & 8 & 2 & 4 & 711 & $\mathrm{nn}$ & 0,512 & 0,034 & 0,907 & 6,370 & 7,824 \\
\hline MH & 1 & 5 & 2 & 211 & co & 0,970 & 0,033 & 1,487 & 8,160 & 10,650 \\
\hline MH & 1 & 5 & 2 & 211 & $\mathrm{nn}$ & 0,937 & 0,056 & 0,586 & 2,787 & 4,367 \\
\hline MH & 1 & 5 & 2 & 511 & co & 1,478 & 0,037 & 2,120 & 22,538 & 26,173 \\
\hline MH & 1 & - & 2 & 511 & $\mathrm{nn}$ & 1,476 & 0,036 & 2,226 & 21,732 & 25,470 \\
\hline MH & 1 & 5 & 3 & 211 & co & 0,936 & 0,025 & 1,206 & 4,170 & 6,338 \\
\hline
\end{tabular}

Fortsetzung auf nächster Seite. 
Tabelle A.23

\begin{tabular}{|c|c|c|c|c|c|c|c|c|c|c|}
\hline WBZ & SOGR & WHZ & $\mathrm{NZ}$ & Art & Var & $\mathrm{LB}$ & $\mathrm{T}$ & HP & SHP & Gesan \\
\hline $\mathrm{MH}$ & 1 & 5 & 3 & 211 & $\mathrm{nn}$ & 1,071 & 0,036 & 0,410 & 1,565 & 3,081 \\
\hline $\mathrm{MH}$ & 1 & 5 & 3 & 511 & co & 1,418 & 0,043 & 2,433 & 29,199 & 33,093 \\
\hline MH & 1 & 5 & 3 & 511 & $\mathrm{nn}$ & 1,419 & 0,034 & 2,064 & 19,944 & 23,460 \\
\hline MH & 1 & 7 & 2 & 211 & co & 0,963 & 0,032 & 1,464 & 6,656 & 9,116 \\
\hline $\mathrm{MH}$ & 1 & 7 & 2 & 211 & $\mathrm{nn}$ & 0,923 & 0,046 & 0,465 & 2,140 & 3,574 \\
\hline $\mathrm{MH}$ & 1 & 7 & 2 & 511 & co & 1,501 & 0,037 & 2,170 & 23,571 & 27,279 \\
\hline MH & 1 & 7 & 2 & 511 & $\mathrm{nn}$ & 1,497 & 0,036 & 2,188 & 23,632 & 27,354 \\
\hline MH & 1 & 7 & 3 & 211 & co & 0,951 & 0,032 & 1,450 & 7,815 & 10,249 \\
\hline MH & 1 & 7 & 3 & 211 & $\mathrm{nn}$ & 1,071 & 0,037 & 0,423 & 1,682 & 3,213 \\
\hline $\mathrm{MH}$ & 1 & 7 & 3 & 511 & co & 1,430 & 0,035 & 2,010 & 21,423 & 24,898 \\
\hline MH & 1 & 7 & 3 & 511 & $\mathrm{nn}$ & 1,428 & 0,034 & 2,072 & 20,252 & 23,786 \\
\hline MH & 2 & 5 & 1 & 211 & co & 1,036 & 0,039 & 1,597 & 9,193 & 11,865 \\
\hline $\mathrm{MH}$ & 2 & 5 & 1 & 211 & $\mathrm{nn}$ & 1,023 & 0,048 & 0,523 & 3,565 & 5,159 \\
\hline $\mathrm{MH}$ & 2 & 5 & 1 & 511 & co & 1,474 & 0,037 & 2,135 & 23,083 & 26,729 \\
\hline $\mathrm{MH}$ & 2 & 5 & 1 & 511 & $\mathrm{nn}$ & 1,472 & 0,036 & 2,137 & 23,067 & 26,713 \\
\hline MH & 2 & 5 & 2 & 211 & co & 1,003 & 0,036 & 1,524 & 7,751 & 10,314 \\
\hline MH & 2 & 5 & 2 & 211 & $\mathrm{nn}$ & 0,980 & 0,056 & 0,595 & 2,609 & 4,239 \\
\hline MH & 2 & 5 & 2 & 511 & co & 1,448 & 0,037 & 2,174 & 21,251 & 24,909 \\
\hline $\mathrm{MH}$ & 2 & 5 & 2 & 511 & $\mathrm{nn}$ & 1,450 & 0,035 & 2,152 & 21,004 & 24,640 \\
\hline MH & 2 & 7 & 2 & 211 & co & 1,080 & 0,030 & 1,357 & 4,952 & 7,419 \\
\hline MH & 2 & - & 2 & 211 & $\mathrm{nn}$ & 1,076 & 0,030 & 1,385 & 4,886 & 7,377 \\
\hline MH & 2 & 7 & 2 & 511 & $\mathrm{co}$ & 1,472 & 0,038 & 2,250 & 22,100 & 25,860 \\
\hline $\mathrm{MH}$ & 2 & 7 & 2 & 511 & $\mathrm{nn}$ & 1,468 & 0,036 & 2,205 & 21,780 & 25,489 \\
\hline $\mathrm{OH}$ & 4 & 5 & 2 & 110 & co & 0,681 & 0,051 & 0,875 & 8,524 & 10,131 \\
\hline $\mathrm{OH}$ & 4 & 5 & 2 & 110 & $\mathrm{nn}$ & 0,678 & 0,051 & 0,889 & 8,529 & 10,147 \\
\hline $\mathrm{OH}$ & 4 & 5 & 2 & 211 & co & 1,114 & 0,050 & 0,542 & 4,044 & 5,749 \\
\hline $\mathrm{OH}$ & 4 & 5 & 2 & 211 & $\mathrm{nn}$ & 1,107 & 0,052 & 0,568 & 4,166 & 5,892 \\
\hline $\mathrm{OH}$ & 4 & 5 & 2 & 511 & со & 1,485 & 0,086 & 1,767 & 14,652 & 17,989 \\
\hline $\mathrm{OH}$ & 4 & 5 & 2 & 511 & $\mathrm{nn}$ & 1,685 & 0,040 & 2,552 & 28,270 & 32,547 \\
\hline $\mathrm{OH}$ & 4 & 5 & 2 & 611 & со & 1,733 & 0,167 & 3,418 & 22,261 & 27,579 \\
\hline $\mathrm{OH}$ & 4 & 5 & 2 & 611 & $\mathrm{nn}$ & 1,732 & 0,164 & 3,426 & 22,418 & 27,741 \\
\hline $\mathrm{OH}$ & 4 & - & 2 & 711 & co & 0,552 & 0,031 & 0,749 & 4,479 & 5,812 \\
\hline $\mathrm{OH}$ & 4 & r & 2 & 711 & $\mathrm{nn}$ & 0,570 & 0,042 & 1,159 & 11,995 & 13,767 \\
\hline $\mathrm{OH}$ & 4 & 5 & 3 & 110 & со & 0,624 & 0,040 & 0,735 & 5,581 & 6,980 \\
\hline $\mathrm{OH}$ & 4 & 5 & 3 & 110 & $\mathrm{nn}$ & 0,618 & 0,039 & 0,723 & 5,503 & 6,883 \\
\hline $\mathrm{OH}$ & 4 & 5 & 3 & 511 & со & 1,468 & 0,037 & 2,195 & 21,482 & 25,182 \\
\hline $\mathrm{OH}$ & 4 & - & 3 & 511 & $\mathrm{nn}$ & 1,471 & 0,036 & 2,204 & 21,629 & 25,339 \\
\hline $\mathrm{OH}$ & 4 & 5 & 3 & 611 & со & 1,072 & 0,171 & 3,360 & 19,932 & 24,535 \\
\hline
\end{tabular}

Fortsetzung auf nächster Seite. 
Tabelle A.23

\begin{tabular}{|c|c|c|c|c|c|c|c|c|c|c|}
\hline WBZ & SOGR & WHZ & $\mathrm{NZ}$ & Art & Var & LB & $\mathrm{T}$ & HP & SHP & Gesamt \\
\hline $\mathrm{OH}$ & 4 & 5 & 3 & 611 & $\mathrm{nn}$ & 1,075 & 0,168 & 3,326 & 19,997 & 24,566 \\
\hline $\mathrm{OH}$ & 4 & 5 & 3 & 711 & $\mathrm{co}$ & 0,555 & 0,043 & 1,132 & 10,840 & 12,570 \\
\hline $\mathrm{OH}$ & 4 & 5 & 3 & 711 & nn & 0,556 & 0,042 & 1,136 & 10,827 & 12,561 \\
\hline $\mathrm{OH}$ & 4 & 6 & 3 & 110 & co & 0,977 & 0,043 & 0,668 & 9,986 & 11,674 \\
\hline $\mathrm{OH}$ & 4 & 6 & 3 & 110 & $\mathrm{nn}$ & 0,599 & 0,040 & 0,731 & 5,429 & 6,800 \\
\hline $\mathrm{OH}$ & 4 & 6 & 3 & 211 & co & 0,910 & 0,022 & 1,144 & 3,770 & 5,847 \\
\hline $\mathrm{OH}$ & 4 & 6 & 3 & 211 & $\mathrm{nn}$ & 0,871 & 0,043 & 0,424 & 1,698 & 3,037 \\
\hline $\mathrm{OH}$ & 4 & 6 & 3 & 511 & co & 1,417 & 0,044 & 2,477 & 29,473 & 33,411 \\
\hline $\mathrm{OH}$ & 4 & 6 & 3 & 511 & $\mathrm{nn}$ & 1,416 & 0,035 & 2,014 & 21,574 & 25,038 \\
\hline $\mathrm{OH}$ & 4 & 6 & 3 & 711 & $\mathrm{co}$ & 0,542 & 0,041 & 1,083 & 9,939 & 11,606 \\
\hline $\mathrm{OH}$ & 4 & 6 & 3 & 711 & $\mathrm{nn}$ & 0,545 & 0,039 & 1,081 & 9,861 & 11,526 \\
\hline $\mathrm{OH}$ & 7 & 6 & 3 & 110 & $\mathrm{co}$ & 0,957 & 0,045 & 0,678 & 10,922 & 12,603 \\
\hline $\mathrm{OH}$ & 7 & 6 & 3 & 110 & $\mathrm{nn}$ & 0,587 & 0,047 & 0,749 & 5,197 & 6,579 \\
\hline $\mathrm{OH}$ & 7 & 6 & 3 & 211 & $\mathrm{co}$ & 0,919 & 0,028 & 1,376 & 6,216 & 8,539 \\
\hline $\mathrm{OH}$ & 7 & 6 & 3 & 211 & $\mathrm{nn}$ & 0,964 & 0,000 & 0,000 & 1,128 & 2,093 \\
\hline $\mathrm{OH}$ & 7 & 6 & 3 & 511 & co & 1,449 & 0,036 & 2,071 & 22,185 & 25,741 \\
\hline $\mathrm{OH}$ & 7 & 6 & 3 & 511 & $\mathrm{nn}$ & 1,452 & 0,034 & 2,069 & 22,218 & 25,774 \\
\hline $\mathrm{OH}$ & 7 & 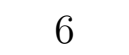 & 3 & 611 & $\mathrm{co}$ & 1,686 & 0,153 & 3,268 & 20,952 & 26,059 \\
\hline $\mathrm{OH}$ & 7 & 6 & 3 & 611 & $\mathrm{nn}$ & 1,682 & 0,149 & 3,225 & 20,775 & 25,830 \\
\hline $\mathrm{OH}$ & 7 & 6 & 3 & 711 & co & 0,545 & 0,041 & 1,113 & 10,649 & 12,348 \\
\hline $\mathrm{OH}$ & 7 & 6 & 3 & 711 & $\mathrm{nn}$ & 0,543 & 0,040 & 1,091 & 10,079 & 11,753 \\
\hline $\mathrm{OH}$ & 7 & 7 & 3 & 110 & $\mathrm{co}$ & 0,849 & 0,042 & 0,627 & 8,284 & 9,801 \\
\hline $\mathrm{OH}$ & 7 & 7 & 3 & 110 & $\mathrm{nn}$ & 0,547 & 0,040 & 0,632 & 4,448 & 5,667 \\
\hline $\mathrm{OH}$ & 7 & 7 & 3 & 211 & co & 0,940 & 0,031 & 1,418 & 7,294 & 9,684 \\
\hline $\mathrm{OH}$ & 7 & 7 & 3 & 211 & nn & 1,056 & 0,035 & 0,398 & 1,503 & 2,993 \\
\hline $\mathrm{OH}$ & 7 & -7 & 3 & 511 & co & 1,427 & 0,044 & 2,464 & 29,242 & 33,176 \\
\hline $\mathrm{OH}$ & 7 & 7 & 3 & 511 & nn & 1,427 & 0,035 & 2,081 & 20,187 & 23,729 \\
\hline $\mathrm{OH}$ & 7 & 7 & 3 & 611 & $\mathrm{co}$ & 1,587 & 0,158 & 3,170 & 24,458 & 29,373 \\
\hline $\mathrm{OH}$ & 7 & 7 & 3 & 611 & $\mathrm{nn}$ & 1,051 & 0,106 & 2,713 & 13,452 & 17,322 \\
\hline $\mathrm{OH}$ & 7 & 7 & 3 & 711 & $\mathrm{co}$ & 0,539 & 0,039 & 1,029 & 8,664 & 10,272 \\
\hline $\mathrm{OH}$ & 7 & 7 & 3 & 711 & $\mathrm{nn}$ & 0,540 & 0,038 & 1,029 & 8,612 & 10,219 \\
\hline $\mathrm{OH}$ & 7 & 9 & 4 & 110 & $\mathrm{co}$ & 0,626 & 0,042 & 0,698 & 4,497 & 5,863 \\
\hline $\mathrm{OH}$ & 7 & 9 & 4 & 110 & $\mathrm{nn}$ & 0,622 & 0,041 & 0,706 & 4,444 & 5,812 \\
\hline $\mathrm{OH}$ & 7 & 0 & 4 & 611 & $\mathrm{co}$ & 1,558 & 0,151 & 3,042 & 23,272 & 28,024 \\
\hline $\mathrm{OH}$ & 7 & 9 & 4 & 611 & $\mathrm{nn}$ & 1,046 & 0,101 & 2,598 & 13,477 & 17,222 \\
\hline $\mathrm{OH}$ & 7 & 9 & 4 & 711 & $\mathrm{co}$ & 0,533 & 0,039 & 1,019 & 8,576 & 10,168 \\
\hline $\mathrm{OH}$ & 7 & 9 & 4 & 711 & $\mathrm{nn}$ & 0,532 & 0,038 & 1,022 & 8,660 & 10,251 \\
\hline $\mathrm{OH}$ & 8 & 1 & 3 & 110 & co & 1,021 & 0,042 & 0,683 & 10,333 & 12,079 \\
\hline
\end{tabular}

Fortsetzung auf nächster Seite. 
Tabelle A.23

\begin{tabular}{|c|c|c|c|c|c|c|c|c|c|c|}
\hline WBZ & SOGR & WHZ & $\mathrm{NZ}$ & Art & Var & LB & $\mathrm{T}$ & HP & SHP & Gesamt \\
\hline $\mathrm{OH}$ & 8 & 1 & 3 & 110 & nn & 0,619 & 0,047 & 0,780 & 5,459 & 6,905 \\
\hline $\mathrm{OH}$ & 8 & 1 & 3 & 711 & $\mathrm{co}$ & 0,584 & 0,043 & 1,021 & 6,789 & 8,437 \\
\hline $\mathrm{OH}$ & 8 & 1 & 3 & 711 & nn & 0,693 & 0,047 & 1,245 & 13,532 & 15,517 \\
\hline US & 1 & 4 & 2 & 211 & $\mathrm{co}$ & 1,083 & 0,030 & 1,364 & 4,902 & 7,379 \\
\hline US & 1 & 4 & 2 & 211 & $\mathrm{nn}$ & 1,071 & 0,031 & 1,380 & 4,977 & 7,459 \\
\hline US & 1 & 4 & 2 & 511 & co & 1,465 & 0,083 & 1,701 & 13,947 & 17,197 \\
\hline US & 1 & 4 & 2 & 511 & nn & 1,656 & 0,038 & 2,455 & 27,068 & 31,218 \\
\hline US & 1 & 5 & 2 & 110 & $\mathrm{co}$ & 0,881 & 0,040 & 0,746 & 5,899 & 7,566 \\
\hline US & 1 & 5 & 2 & 110 & $\mathrm{nn}$ & 0,884 & 0,038 & 0,742 & 5,871 & 7,535 \\
\hline US & 1 & 5 & 2 & 211 & $\mathrm{co}$ & 1,072 & 0,047 & 0,510 & 3,645 & 5,275 \\
\hline US & 1 & 5 & 2 & 211 & $\mathrm{nn}$ & 1,066 & 0,046 & 0,505 & 3,603 & 5,221 \\
\hline US & 1 & 5 & 2 & 511 & $\mathrm{co}$ & 1,454 & 0,086 & 1,727 & 13,877 & 17,145 \\
\hline US & 1 & 5 & 2 & 511 & $\mathrm{nn}$ & 1,655 & 0,039 & 2,464 & 26,883 & 31,041 \\
\hline US & 1 & 5 & 3 & 110 & $\mathrm{co}$ & 0,675 & 0,045 & 0,799 & 5,684 & 7,203 \\
\hline US & 1 & 5 & 3 & 110 & nn & 0,670 & 0,044 & 0,818 & 5,722 & 7,253 \\
\hline US & 1 & 5 & 3 & 211 & co & 1,051 & 0,036 & 1,530 & 9,229 & 11,847 \\
\hline US & 1 & 5 & 3 & 211 & $\mathrm{nn}$ & 1,011 & 0,060 & 0,636 & 3,078 & 4,785 \\
\hline US & 1 & 5 & 3 & 511 & $\mathrm{co}$ & 1,464 & 0,085 & 1,705 & 13,742 & 16,995 \\
\hline US & 1 & 5 & 3 & 511 & $\mathrm{nn}$ & 1,663 & 0,038 & 2,449 & 26,751 & 30,902 \\
\hline US & 1 & 7 & 2 & 110 & $\mathrm{co}$ & 0,884 & 0,046 & 0,806 & 7,545 & 9,281 \\
\hline US & 1 & 7 & 2 & 110 & $\mathrm{nn}$ & 0,884 & 0,044 & 0,806 & 7,636 & 9,371 \\
\hline US & 1 & 7 & 2 & 211 & $\mathrm{co}$ & 1,084 & 0,034 & 1,389 & 5,094 & 7,601 \\
\hline US & 1 & 7 & 2 & 211 & $\mathrm{nn}$ & 1,090 & 0,032 & 1,365 & 5,030 & 7,517 \\
\hline US & 1 & 7 & 2 & 511 & $\mathrm{co}$ & 1,641 & 0,038 & 2,395 & 25,978 & 30,053 \\
\hline US & 1 & 7 & 2 & 511 & nn & 1,641 & 0,037 & 2,402 & 26,008 & 30,088 \\
\hline US & 1 & 7 & 3 & 110 & $\mathrm{co}$ & 0,874 & 0,038 & 0,729 & 5,774 & 7,415 \\
\hline US & 1 & 7 & 3 & 110 & $\mathrm{nn}$ & 0,876 & 0,037 & 0,722 & 5,736 & 7,371 \\
\hline US & 1 & 7 & 3 & 211 & $\mathrm{co}$ & 1,044 & 0,048 & 0,516 & 3,597 & 5,206 \\
\hline US & 1 & 7 & 3 & 211 & nn & 1,046 & 0,048 & 0,514 & 3,693 & 5,300 \\
\hline US & 1 & 7 & 3 & 511 & co & 1,600 & 0,036 & 2,304 & 25,005 & 28,946 \\
\hline US & 1 & 7 & 3 & 511 & $\mathrm{nn}$ & 1,598 & 0,035 & 2,287 & 24,883 & 28,803 \\
\hline US & 1 & 8 & 3 & 211 & co & 1,059 & 0,035 & 1,542 & 9,171 & 11,808 \\
\hline US & 1 & 8 & 3 & 211 & $\mathrm{nn}$ & 1,009 & 0,060 & 0,640 & 2,984 & 4,692 \\
\hline US & 1 & 8 & 3 & 511 & $\mathrm{co}$ & 1,604 & 0,038 & 2,348 & 28,534 & 32,525 \\
\hline US & 1 & 8 & 3 & 511 & $\mathrm{nn}$ & 1,600 & 0,037 & 2,362 & 28,597 & 32,596 \\
\hline US & 6 & 4 & 2 & 110 & co & 0,882 & 0,043 & 0,774 & 6,022 & 7,721 \\
\hline US & 6 & 4 & 2 & 110 & $\mathrm{nn}$ & 0,890 & 0,041 & 0,750 & 5,868 & 7,549 \\
\hline US & 6 & 4 & 2 & 211 & $\mathrm{co}$ & 1,094 & 0,032 & 1,386 & 5,001 & 7,513 \\
\hline
\end{tabular}

Fortsetzung auf nächster Seite. 
Tabelle A.23

\begin{tabular}{|c|c|c|c|c|c|c|c|c|c|c|}
\hline WBZ & SOGR & WHZ & $\mathrm{NZ}$ & Art & Var & LB & $\mathrm{T}$ & HP & SHP & Gesamt \\
\hline US & 6 & 4 & 2 & 211 & $\mathrm{nn}$ & 1,098 & 0,032 & 1,359 & 5,018 & 7,507 \\
\hline US & 6 & 4 & 2 & 511 & $\mathrm{co}$ & 1,405 & 0,084 & 1,697 & 12,736 & 15,922 \\
\hline US & 6 & 4 & 2 & 511 & nn & 1,657 & 0,038 & 2,472 & 27,144 & 31,312 \\
\hline US & 6 & 5 & 2 & 110 & $\mathrm{co}$ & 0,847 & 0,038 & 0,717 & 5,598 & 7,200 \\
\hline US & 6 & 5 & 2 & 110 & $\mathrm{nn}$ & 0,849 & 0,037 & 0,704 & 5,615 & 7,206 \\
\hline US & 6 & 5 & 2 & 211 & $\mathrm{co}$ & 1,048 & 0,039 & 1,601 & 9,157 & 11,846 \\
\hline US & 6 & 5 & 2 & 211 & nn & 1,038 & 0,047 & 0,517 & 3,489 & 5,092 \\
\hline US & 6 & 5 & 2 & 511 & $\mathrm{co}$ & 1,524 & 0,061 & 2,868 & 16,912 & 21,365 \\
\hline US & 6 & 5 & 2 & 511 & $\mathrm{nn}$ & 1,662 & 0,039 & 2,485 & 27,152 & 31,338 \\
\hline UW & 1 & 5 & 2 & 110 & $\mathrm{co}$ & 0,684 & 0,062 & 1,056 & 4,277 & 6,079 \\
\hline UW & 1 & 5 & 2 & 110 & $\mathrm{nn}$ & 0,916 & 0,039 & 0,770 & 6,121 & 7,847 \\
\hline UW & 1 & 5 & 2 & 211 & co & 1,131 & 0,033 & 1,361 & 5,251 & 7,775 \\
\hline UW & 1 & 5 & 2 & 211 & $\mathrm{nn}$ & 1,133 & 0,033 & 1,451 & 5,275 & 7,892 \\
\hline UW & 1 & 5 & 2 & 511 & $\mathrm{co}$ & 1,634 & 0,038 & 2,384 & 25,901 & 29,957 \\
\hline UW & 1 & 5 & 2 & 511 & $\mathrm{nn}$ & 1,636 & 0,045 & 2,847 & 31,724 & 36,252 \\
\hline UW & 1 & 5 & 3 & 211 & $\mathrm{co}$ & 1,051 & 0,062 & 0,654 & 3,253 & 5,019 \\
\hline UW & 1 & 5 & 3 & 211 & $\mathrm{nn}$ & 1,050 & 0,061 & 0,655 & 3,278 & 5,044 \\
\hline UW & 1 & 5 & 3 & 511 & $\mathrm{co}$ & 1,509 & 0,038 & 2,236 & 24,190 & 27,974 \\
\hline UW & 1 & 5 & 3 & 511 & $\mathrm{nn}$ & 1,509 & 0,037 & 2,228 & 24,116 & 27,890 \\
\hline UW & 1 & 7 & 2 & 211 & $\mathrm{co}$ & 1,048 & 0,062 & 0,659 & 3,308 & 5,078 \\
\hline UW & 1 & 7 & 2 & 211 & $\mathrm{nn}$ & 1,045 & 0,062 & 0,662 & 3,317 & 5,086 \\
\hline UW & 1 & 7 & 2 & 511 & co & 1,535 & 0,039 & 2,262 & 24,959 & 28,795 \\
\hline UW & 1 & 7 & 2 & 511 & $\mathrm{nn}$ & 1,537 & 0,038 & 2,306 & 25,246 & 29,128 \\
\hline UW & 1 & 7 & 3 & 211 & $\mathrm{co}$ & 1,096 & 0,030 & 1,363 & 4,866 & 7,356 \\
\hline UW & 1 & 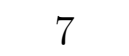 & 3 & 211 & nn & 1,095 & 0,030 & 1,360 & 4,841 & 7,327 \\
\hline UW & 1 & 7 & 3 & 511 & $\mathrm{co}$ & 1,402 & 0,042 & 2,400 & 28,619 & 32,463 \\
\hline UW & 1 & 7 & 3 & 511 & $\mathrm{nn}$ & 1,401 & 0,033 & 1,991 & 19,576 & 23,001 \\
\hline UW & 1 & 8 & 3 & 211 & co & 0,992 & 0,036 & 1,496 & 8,006 & 10,529 \\
\hline UW & 1 & 8 & 3 & 211 & $\mathrm{nn}$ & 0,960 & 0,055 & 0,576 & 2,872 & 4,463 \\
\hline UW & 1 & 8 & 3 & 511 & $\mathrm{co}$ & 1,341 & 0,040 & 2,228 & 26,541 & 30,150 \\
\hline UW & 1 & 8 & 3 & 511 & $\mathrm{nn}$ & 1,338 & 0,033 & 1,918 & 19,063 & 22,351 \\
\hline UW & 5 & 5 & 1 & 110 & $\mathrm{co}$ & 0,889 & 0,046 & 0,839 & 8,881 & 10,655 \\
\hline UW & 5 & 5 & 1 & 110 & $\mathrm{nn}$ & 0,886 & 0,045 & 0,821 & 8,794 & 10,545 \\
\hline UW & 5 & 5 & 1 & 211 & $\mathrm{co}$ & 1,175 & 0,055 & 0,608 & 4,073 & 5,911 \\
\hline UW & 5 & 5 & 1 & 211 & nn & 1,218 & 0,042 & 1,777 & 9,705 & 12,743 \\
\hline UW & 5 & 5 & 1 & 511 & $\mathrm{co}$ & 1,432 & 0,087 & 1,760 & 13,458 & 16,736 \\
\hline UW & 5 & 5 & 1 & 511 & nn & 1,688 & 0,039 & 2,570 & 28,389 & 32,686 \\
\hline UW & 5 & 7 & 1 & 110 & $\mathrm{co}$ & 0,686 & 0,050 & 0,869 & 7,278 & 8,882 \\
\hline
\end{tabular}

Fortsetzung auf nächster Seite. 
Tabelle A.23

\begin{tabular}{|c|c|c|c|c|c|c|c|c|c|c|}
\hline WBZ & SOGR & WHZ & $\mathrm{NZ}$ & Art & Var & LB & $\mathrm{T}$ & HP & SHP & Gesamt \\
\hline UW & 5 & 7 & 1 & 110 & $\mathrm{nn}$ & 0,687 & 0,048 & 0,859 & 7,222 & 8,816 \\
\hline UW & 5 & 7 & 1 & 211 & co & 1,130 & 0,074 & 0,786 & 3,584 & 5,574 \\
\hline UW & 5 & 7 & 1 & 211 & $\mathrm{nn}$ & 1,191 & 0,043 & 1,831 & 8,402 & 11,468 \\
\hline UW & 5 & 7 & 1 & 511 & co & 1,622 & 0,038 & 2,356 & 25,470 & 29,487 \\
\hline UW & 5 & 7 & 1 & 511 & $\mathrm{nn}$ & 1,619 & 0,037 & 2,364 & 25,469 & 29,488 \\
\hline UW & 5 & 8 & 1 & 211 & co & 1,128 & 0,032 & 1,382 & 5,177 & 7,719 \\
\hline UW & 5 & 8 & 1 & 211 & $\mathrm{nn}$ & 1,123 & 0,032 & 1,324 & 5,201 & 7,680 \\
\hline UW & 5 & 8 & 1 & 511 & co & 1,471 & 0,037 & 2,206 & 21,453 & 25,167 \\
\hline UW & 5 & 8 & 1 & 511 & $\mathrm{nn}$ & 1,471 & 0,036 & 2,129 & 22,969 & 26,606 \\
\hline UW & 5 & 9 & 1 & 211 & co & 1,073 & 0,031 & 1,358 & 4,876 & 7,338 \\
\hline UW & 5 & 9 & 1 & 211 & $\mathrm{nn}$ & 1,075 & 0,030 & 1,358 & 4,863 & 7,327 \\
\hline UW & 5 & , & 1 & 511 & co & 1,397 & 0,043 & 2,403 & 28,506 & 32,349 \\
\hline UW & 5 & 9 & 1 & 511 & $\mathrm{nn}$ & 1,402 & 0,034 & 1,964 & 20,655 & 24,054 \\
\hline UW & 6 & 4 & 1 & 110 & co & 0,724 & 0,039 & 0,709 & 4,002 & 5,474 \\
\hline UW & 6 & 4 & 1 & 110 & $\mathrm{nn}$ & 0,956 & 0,052 & 0,904 & 9,684 & 11,597 \\
\hline UW & 6 & 4 & 1 & 211 & со & 1,197 & 0,075 & 0,799 & 3,856 & 5,927 \\
\hline UW & 6 & 4 & 1 & 211 & $\mathrm{nn}$ & 1,234 & 0,045 & 1,876 & 8,851 & 12,005 \\
\hline UW & 6 & 4 & 1 & 511 & co & 1,579 & 0,061 & 2,918 & 17,333 & 21,892 \\
\hline UW & 6 & 4 & 1 & 511 & $\mathrm{nn}$ & 1,718 & 0,049 & 3,208 & 40,453 & 45,429 \\
\hline UW & 6 & 4 & 2 & 110 & co & 0,713 & 0,019 & 0,548 & 2,663 & 3,943 \\
\hline UW & 6 & 4 & 2 & 110 & $\mathrm{nn}$ & 0,926 & 0,041 & 0,781 & 6,266 & 8,014 \\
\hline UW & 6 & 4 & 2 & 211 & co & 1,142 & 0,060 & 0,615 & 2,730 & 4,547 \\
\hline UW & 6 & 4 & 2 & 211 & $\mathrm{nn}$ & 1,184 & 0,040 & 1,736 & 7,852 & 10,811 \\
\hline UW & 6 & 4 & 2 & 511 & co & 1,558 & 0,061 & 2,883 & 17,154 & 21,656 \\
\hline UW & 6 & 4 & 2 & 511 & $\mathrm{nn}$ & 1,695 & 0,039 & 2,515 & 28,000 & 32,249 \\
\hline UW & 6 & 5 & 2 & 110 & co & 0,867 & 0,038 & 0,723 & 5,736 & 7,365 \\
\hline UW & 6 & 5 & 2 & 110 & $\mathrm{nn}$ & 0,872 & 0,036 & 0,714 & 5,637 & 7,259 \\
\hline UW & 6 & 5 & 2 & 211 & co & 1,156 & 0,042 & 1,745 & 8,510 & 11,452 \\
\hline UW & 6 & 5 & 2 & 211 & $\mathrm{nn}$ & 1,166 & 0,034 & 1,407 & 5,622 & 8,229 \\
\hline UW & 6 & 5 & 2 & 511 & co & 1,628 & 0,039 & 2,421 & 26,229 & 30,317 \\
\hline UW & 6 & 5 & 2 & 511 & $\mathrm{nn}$ & 1,634 & 0,037 & 2,406 & 26,118 & 30,195 \\
\hline
\end{tabular}

